\title{
A Basic Three-Dimensional Turbulent Boundary Layer Experiment To Test Second-Moment Closure Models
}

\author{
Shereef A. Sadek \\ Dissertation submitted to the Faculty of the \\ Virginia Polytechnic Institute and State University \\ in partial fulfillment of the requirements for the degree of
}

\author{
Doctor of Philosophy \\ in \\ Aerospace Engineering
}
Roger L. Simpson, Chair
William J. Devenport
William H. Mason
James F. Marchman
Saad A. Ragab

September 10, 2008

Blacksburg, Virginia

Keywords: Turbulence Modeling, Three-Dimensional Boundary Layer, Turbulent Diffusion, Second-Moment Closure, Turbulent transport, Copyright 2008, Shereef A. Sadek 


\title{
A Basic Three-Dimensional Turbulent Boundary Layer Experiment To Test Second-Moment Closure Models
}

\author{
Shereef A. Sadek
}

\section{(ABSTRACT)}

In this work, a three-dimensional turbulent boundary layer experiment was set up with alternating stream-wise and span-wise pressure gradients. The pressure gradients are generated as a result of the test section wavy side wall shape. Each side had six sine waves with a trough to peak magnitude to wavelength ratio of 0.25 . Boundary layer control was used so that the flow over the side walls remains attached. The mean flow velocity components, static and total pressures were measured at six plane along the stream-wise direction. The alternating mean span-wise and stream-wise pressure gradients created alternating stream-wise and span-wise vorticity fluxes, respectively, along the test section. As the flow developed downstream the vorticity created at the tunnel floor and ceiling diffused away from the wall.

The vorticity components in the stream-wise and span-wise directions are strengthened due to stretching and tilting terms in the vorticity transport equaitons. The positive$z$ half of the test section contains large areas that generate positive vorticity flux in the trough region and smaller areas generating negative vorticity around the wave peak. The opposite is true for the negative-z half of the test-section. This results in a large positive stream-wise vorticity in the positive- $z$ half and negative stream-wise vorticity in the negative-z half of the test-section. The smaller regions of opposite sign vorticity in each half tend to mix the flow such that as they diffuse away from the wall, the turbulent stresses are more uniform.

Turbulent fluctuating velocity components were measured using Laser Doppler Velocimetery. Mean velocities as well as Reynolds stresses and triple velocity component correlations were measured at thirty stations along the last wave in the test section. Profiles at the center of the test section showed three dimensionality, but exhibited high turbulence intensities in the outer layer.

Profiles off the test section centerline are highly three dimensional with multiple peaks in the normal stress profiles. The flow also reaches a state where all the normal stresses have equal magnitudes while the shear stresses are non-zero. 
Flow angles, flow gradient angles and shear stress angles show very large differences between wall values and outer layer vlaues. The shear stress angle lagged the flow gradient angle indicating non-equilibrium.

A turbulent kinetic energy transport budget is performed for all profiles and the turbulence kinetic energy dissipation rate is estimated. Spectral measurements were also made and an independent estimate of the kinetic energy dissipation rate is made. These estimates agree very well with those estimates made by balancing the turbulence kinetic energy transport equation.

Multiple turbulent diffusion models are compared to measured quantities. The models varied in agreement with experimental data. However, fair agreement with turbulence kinetic energy turbulent diffusion is observed. A model for the dissipation rate tensor anisotropy is used to extract estimates of the pressure-strain tensor from the Reynolds stress transport equations. The pressure-strain estimates are compared with some of the models in the literature. The comparison showed poor agreement with estimated pressure-strain values extracted from experimental data.

A tentative model for the turbulent Reynolds shear stress angle is devloped that captures the shear stress angle near wall behvior to a very good extent. The model contains one constant that is related to mean flow variables. However, the developed expression needs modification so that the prediction is improved along the entire boundary layer thickness. 


\section{Dedication}

To my beloved parents, Fatemah and Aly

To my dear brother Mohammad

To my wonderful family, my wife Jennifer and my son Ibraheem

And to ALL who contributed to my education 


\section{Acknowledgments}

All praise is to GOD, Almighty, without His bounties and sustenance I could not have finished this work.

I would like to express my deep gratitude and appreciation to my advisor, Prof. Roger Simpson, for his continuous patience, guidance and support, both academically and financialy, throughout the duration of this project. I would like to thank him for giving me the opportunity to work on this project. I would also like to thank other members of the advisory committee, Prof. William Devenport, Prof. William Mason, Prof. James Marchman and Prof. Saad Ragab for sharing their thoughts about this project and for agreeing to be on the committee.

I am indebted to my parents, Aly and Fatemah, for their continuous support. No matter what I do or say I will never be able to repay them for all their love and care. I can only strive hard to bring happiness to their hearts.

I am also grateful to my brother, Mohammad, for his support and encouragement. I appreciate everything he has done for me to make my life easier and to focus on my studies.

I would like to thank my wonderful wife, Jennie, for supporting me during the entire period of this project. I highly appreciate all the sacrifices she has done so that I can work on this project without interruptions. I would also like to thank my son Ibraheem for forcing me to work very long hours at night.

Many thanks to everybody who helped me in this project, Dr. Gwibo Byun, Dr. Todd Lowe, Dr. Qing Tian, Mr. Edgar Orsi, Mr. Kevin Pisterman, Mr. Nathaniel Varano, Ms. Deirdre Hunter, Mr. Devin Stewart and Mr. Jeremy Bennegton. I would also like to thank the AOE staff, Mike Vaught, James Lambert and Bruce Stanger and our IT wizard Mr. Steve Edwards. Also, many thanks to the people in the AOE office for their support and care.

Finally, this work was partially supported by NASA Glenn Research Center Grant NAG 3 2733. We highly appreciate their support. 


\section{Contents}

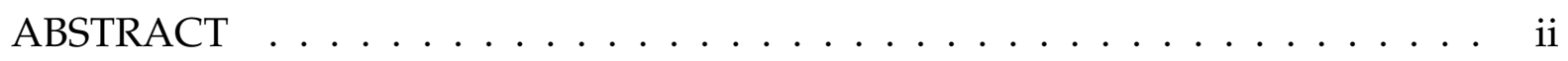

Acknowledgments ......................... iv

Table of Contents . . . . . . . . . . . . . . . . . . . . viii

List of Figures . . . . . . . . . . . . . . . . . . . . xxiii

List of Tables . . . . . . . . . . . . . . . . . . . . . xxiv

$\begin{array}{ll}\text { Nomenclature } & \mathbf{x x v}\end{array}$

$\begin{array}{llr}1 & \text { Introduction } & 1\end{array}$

1.1 Motivation . . . . . . . . . . . . . . . . . . . . . 1

1.2 Governing Equations $\ldots \ldots \ldots \ldots \ldots \ldots$

1.3 Discussion of Turbulent Transport Modeling $\ldots \ldots \ldots \ldots$. . . . . . . . 4

1.4 Dissertation Organization $\ldots \ldots \ldots \ldots \ldots$

$\begin{array}{|ll|}2 & \text { Experimental Setup and Instrumentation }\end{array}$

$2.1 \quad$ Experimental Facility $\ldots \ldots \ldots \ldots$

2.2 Wavy Wall Test Section Geometry . . . . . . . . . . . . . . . . . . . 10

2.3 Measuring Instruments and locations $\ldots \ldots \ldots \ldots$

$2.3 .1 \quad$ Total Pressure Rake $(16$ tubes $) . \ldots \ldots \ldots \ldots$

2.3 .2 Pitot-Static Tube . . . . . . . . . . . . . . . . . . . . . . 11

2.3 .3 Scannivalve system . . . . . . . . . . . . . . . . . . . 11

2.3 .4 Seven-Hole Pitot tube $\ldots \ldots \ldots \ldots$. . . . . . . . . . . . 12

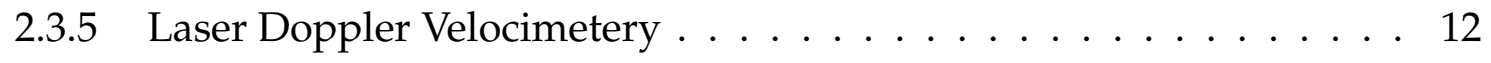


2.3 .6 Seeding System $\ldots \ldots \ldots \ldots$

2.3 .7 Data Acquisition and Processing . . . . . . . . . . . . . . . . 14

2.4 Boundary Layer Control . . . . . . . . . . . . . . . . . . . . . . . . 15

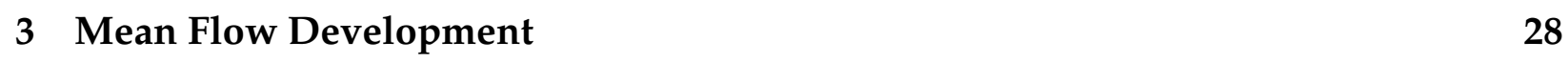

3.1 Measurement Locations . . . . . . . . . . . . . . . . . . . . . 28

3.2 Seven-Hole Probe Alignment $\ldots \ldots \ldots \ldots$

3.3 Uncertainty Estimates $\ldots . . \ldots 29$

3.4 Mean Flow Velocity . . . . . . . . . . . . . . . . . . . . . . . . . . . . 29

3.5 Centerline Flow Development . . . . . . . . . . . . . . . . . . . . 32

$\begin{array}{|ll|}4 & \text { LDV Measurement Results }\end{array}$

4.1 Measurement Locations . . . . . . . . . . . . . . . . . . . . . . 42

4.2 Velocity-Gradient Broadening . . . . . . . . . . . . . . . . . . . 43

4.3 Skin Friction velocity $u_{\tau} \ldots \ldots \ldots \ldots \ldots \ldots$

4.4 Entrance Profiles . . . . . . . . . . . . . . . . . . . . . . . . 44

4.5 Down-stream Profiles . . . . . . . . . . . . . . . . . . . . . . . 45

$4.5 .1 \quad \mathrm{P} 08$ Cluster $\ldots \ldots \ldots \ldots \ldots$

4.5 .2 P13 Cluster . . . . . . . . . . . . . . . . . 50

$4.5 .3 \quad \mathrm{P} 18$ Cluster $\ldots \ldots \ldots \ldots \ldots \ldots$

$4.5 .4 \quad$ P19 Cluster . . . . . . . . . . . . . . . . . . . . 58

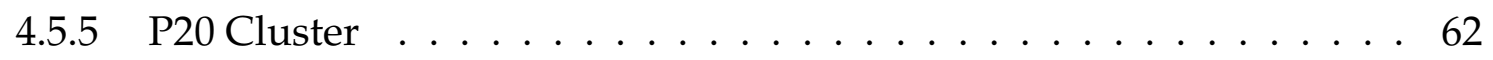

4.5 .6 P33 Cluster . . . . . . . . . . . . . . . . . . . . . . 65

4.6 Spectral Measurements . . . . . . . . . . . . . . . . . . . . . . . . . . . . . . 69

$\begin{array}{lll}5 \text { Discussion } & 140\end{array}$

5.1 Features of Interest $\ldots \ldots \ldots$

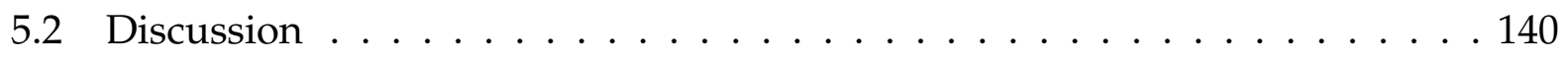

5.2 .1 Reynolds' Stress Transport Budget . . . . . . . . . . . . . . . . . . 141

5.2 .2 Stress Production . . . . . . . . . . . . . . . . . . . . . . . 144 


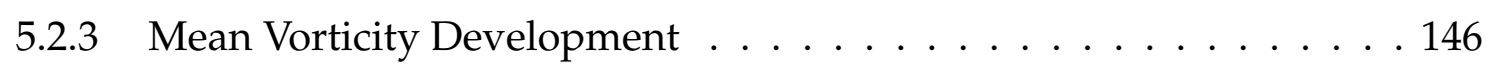

$\begin{array}{llr}6 & \text { Second-Moment Closure Models } & 170\end{array}$

6.1 Turbulence Closure . . . . . . . . . . . . . . . . . . . . . . . . 170

6.2 Turbulent Diffusion Models . . . . . . . . . . . . . . . . . . . . . . . . 171

6.2 .1 Results . . . . . . . . . . . . . . . . . . . . . . 173

6.3 Dissipation Rate Tensor Anisotropy Models . . . . . . . . . . . . . . . . . . . 175

6.4 Pressure-Strain Models . . . . . . . . . . . . . . . . . . . . . . . . . . . . 176

6.5 Reynolds Shear Stress Angle Lag Model . . . . . . . . . . . . . . . . . . . . 180

$6.5 .1 \quad$ Reynolds Shear Stress Angle Wall Limiting Value . . . . . . . . . 181

6.5 .2 Results . . . . . . . . . . . . . . . . . . . . . . . . . 185

\begin{tabular}{llr}
\hline 7 & Conclusions & 202
\end{tabular}

\begin{tabular}{lr}
\hline Bibliography & 204
\end{tabular}

\begin{tabular}{|lr}
\hline A Wavy Wall Geometry & 210
\end{tabular}

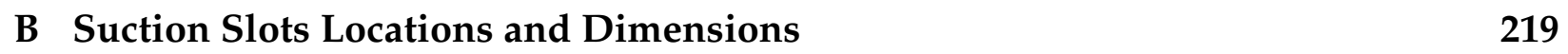

C Matching Short LDV System and CompLDV Profiles and Uncertainty Estimates 221

C.1 Matching Procedure . . . . . . . . . . . . . . . . . . . . . . . . . . 221

C.2 Uncertainty Estimates . . . . . . . . . . . . . . . . . . 222

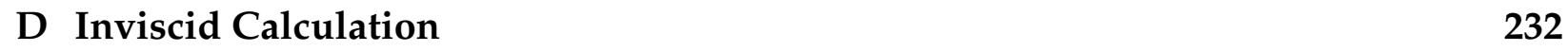




\section{List of Figures}

2.1 Wavy Wall Plan View . . . . . . . . . . . . . . . . . . . . . . 18

2.2 Wind Tunnel Side View Schematic . . . . . . . . . . . . . . . . . . . . . 19

2.3 Wavy Wall Right Side Wall . . . . . . . . . . . . . . . . . . . . . 20

2.416 hole Total Pressure Rake . . . . . . . . . . . . . . . . . . . 21

2.5 Rake-Pitot Tube assembly $\ldots \ldots \ldots$. . . . . . . . . . . . . . . . 21

2.6 Seven-Hole Probe . . . . . . . . . . . . . . . . . . . . . . . . . 22

2.7 CompLDV Probe Head Assembly $\ldots \ldots \ldots . \ldots . \ldots . \ldots 22$

2.8 Wavy Wall Test section Inlet Side Wall showing tufts attached to the side wall 23

2.9 Side Wall Boundary Layer Measurement Locations. . . . . . . . . . . . . . 24

2.10 Side Wall Suctoin Flow Rates ． . . . . . . . . . . . . . . . . . . . . . 25

2.11 Right side wall normalized velocity profiles vs normal distance . . . . . . 26

2.12 Left side wall normalized velocity profiles vs normal distance . . . . . . . 26

$2.13 C_{p}$ distribution along the side wall peaks $\ldots \ldots \ldots \ldots . \ldots 27$

$3.1 \quad$ U-velocity component contours at $x / L=0.00, Y_{\max }=10.5 \mathrm{in}$. . . . . . . 34

3.2 U-velocity component contours at $x / L=0.13, Y_{\max }=10.17 \mathrm{in}$. . . . . . 35

$3.3 \quad$ U-velocity component contours at $x / L=0.39, Y_{\max }=9.72 \mathrm{in}$. . . . . . 36

3.4 U-velocity component contours at $x / L=0.64, Y_{\max }=9.22 \mathrm{in}$. . . . . . . 37

3.5 U-velocity component contours at $x / L=0.79, Y_{\max }=8.92 \mathrm{in}$. . . . . . . 38

3.6 U-velocity component contours at $x / L=0.90, Y_{\max }=8.70 \mathrm{in}$. . . . . . . 39

3.7 U-velocity component contours at $5 \mathrm{x} / \mathrm{L}$ stations $\ldots \ldots \ldots$. . . . . . . . 40

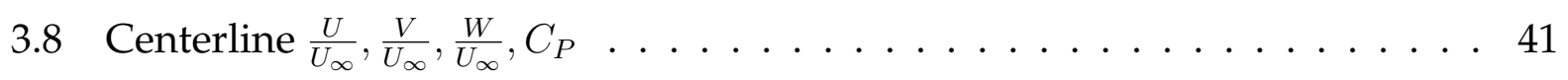


3.9 Boundary Layer Thickness along tunnel centerline . . . . . . . . . . . . . . 41

4.1 LDV Measurement Locations . . . . . . . . . . . . . . . . . . . . . . 72

4.2 Entrance Profiles Comparison with DNS data . . . . . . . . . . . . . . 73

(a) Entrance Profiles Comparison with DNS data . . . . . . . . . . 73

(b) Entrance Profiles Comparison with DNS data $\ldots . . . . . . . .773$

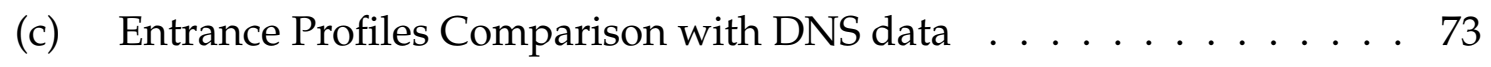

(d) Entrance Profiles Comparison with DNS data . . . . . . . . . . . 73

(e) Entrance Profiles Comparison with DNS data . . . . . . . . . . 73

(f) Entrance Profiles Comparison with DNS data $\ldots \ldots \ldots$. . . . . 73

$4.3 \quad P 08$ Cluster . . . . . . . . . . . . . . . . . . . . . . . . . . . . . . 74

(a) $\quad P 08$ Cluster, Mean Velocities $U^{+}, W^{+} \ldots \ldots \ldots \ldots \ldots . \ldots . . \ldots$

(b) $\quad P 08$ Cluster, Normal Stress ${\overline{u^{2}}}^{+} \ldots \ldots \ldots \ldots$. . . . . . . . 74

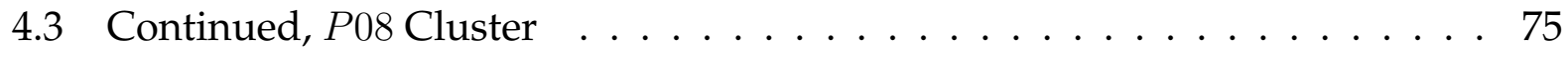

(c) $\quad P 08$ Cluster, Normal Stresses $\overline{v^{2}}$ and $\overline{w^{2}} \ldots \ldots \ldots \ldots$

(d) $\quad P 08$ Cluster,Reynolds' Shear Stress, $\overline{u v^{+}}$and $\overline{v w}+\ldots \ldots \ldots$

4.3 Continued $P 08$ Cluster $\ldots \ldots \ldots \ldots \ldots \ldots \ldots$

(e) $\quad P 08$ Cluster,Reynolds' Shear Stress, $\overline{u w^{+}} \ldots \ldots \ldots$. . . . . . 76

(f) $\quad P 08$ Cluster, Correlation Coefficient $\rho_{u v} \ldots \ldots \ldots \ldots$

4.3 Continued, $P 08$ Cluster $\ldots \ldots \ldots \ldots \ldots \ldots$

(g) $\quad P 08$ Cluster, 1 S Parameter $\ldots \ldots \ldots \ldots \ldots \ldots . \ldots . \ldots 77$

(h) $\quad P 08$ Cluster, Turbulent Kinetic Energy, TKE . . . . . . . . . . 77

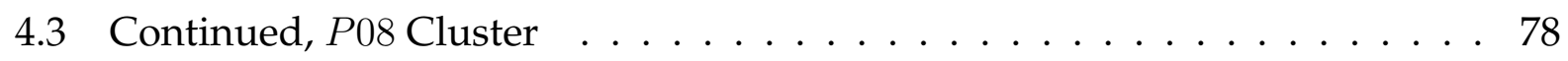

(i) $\quad P 08$ Cluster, Turbulent Diffusion Velocity component $V_{q x} \ldots \ldots 78$

(j) $\quad P 08$ Cluster, Turbulent Diffusion Velocity component $V_{q y} \ldots \ldots$

4.3 Continued, $P 08$ Cluster $\ldots \ldots \ldots \ldots$

(k) $\quad P 08$ Cluster, Turbulent Diffusion Velocity component $V_{q z} \ldots \ldots$. . . 79

(1) $\quad P 08$ Cluster, Townsend's Structural Parameter $A_{1} \ldots \ldots$. . . . . 79

4.3 Continued $P 08$ Cluster $\ldots \ldots \ldots \ldots$. . . . . . . . . . 80 
(m) $\quad P 08$ Cluster, Flow Angle $\gamma_{a} \ldots \ldots \ldots \ldots \ldots$

(n) $\quad P 08$ Cluster, Shear Stress Angle $\gamma_{s} \ldots \ldots \ldots \ldots$. . . . . . 80

4.3 Continued, $P 08$ Cluster $\ldots \ldots \ldots \ldots$. . . . . . . . . . . 81

(o) $\quad P 08$ Cluster, Flow Gradient Angle $\gamma_{g} . \ldots \ldots \ldots \ldots \ldots$

(p) P08 Cluster, Difference between Shear stress and Flow Gradient Angles 81

4.3 Continued, $P 08$ Cluster $\ldots \ldots \ldots$. . . . . . . . . . 82

(q) $\quad P 08$ Cluster, Mixing Length $L_{m}^{+} \ldots \ldots \ldots \ldots \ldots \ldots$

(r) $\quad P 08$ Cluster, Near Wall Mixing Length $L_{m}^{+} \ldots \ldots \ldots \ldots \ldots$

4.3 Concluded $P 08$ Cluster $\ldots \ldots \ldots$. . . . . . . . . . . 83

(s) P08 Cluster, Turbulent Eddy Viscosity $\nu_{T} x \ldots \ldots$. . . . . . . 83

(t) P08 Cluster, Near Wall Turbulent Eddy Viscosity $\nu_{T} x \ldots \ldots$. . . . . . 83

$4.4 \quad P 13$ Cluster . . . . . . . . . . . . . . . . . . . . . . . . . . . . . . 84

(a) P13 Cluster, MeanVelocities $U^{+}, W^{+} \ldots \ldots \ldots \ldots$. . . . . 84

(b) P13 Cluster, NormalStress $\overline{u^{2}} \ldots \ldots$. . . . . . . . . . . 84

4.4 Continued, $P 13$ Cluster $\ldots \ldots \ldots \ldots$

(c) P13 Cluster, NormalStresses ${\overline{v^{2}}}^{+}$and $\overline{w^{2}}+\ldots$. . . . . . . . 85

(d) $\quad P 13$ Cluster,Reynolds' Shear Stress, $\overline{u v}^{+}$and $\overline{v w} \bar{w}^{+} \ldots \ldots \ldots$

4.4 Continued,$P 13$ Cluster $\ldots \ldots \ldots$. . . . . . . . . . . 86

(e) $\quad P 13$ Cluster,Reynolds' Shear Stress, $\overline{u w^{+}} \ldots \ldots \ldots$. . . . . . . . 86

(f) $\quad P 13$ Cluster, Correlation Coefficient $\rho_{u v} \ldots \ldots \ldots \ldots$

4.4 Continued,$P 13$ Cluster $\ldots \ldots \ldots \ldots$. . . . . . . . . 87

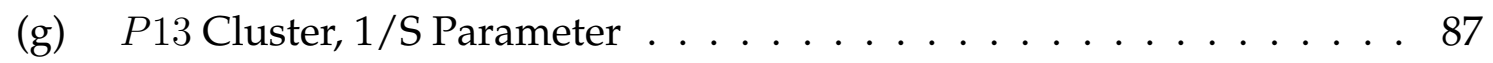

(h) $\quad P 13$ Cluster, Turbulent Kinetic Energy, $k \ldots \ldots \ldots$

4.4 Continued,$P 13$ Cluster $\ldots \ldots \ldots$. . . . . . . . . . . 88

(i) $\quad P 13$ Cluster, Turbulent Diffusion Velocity component $V_{q x} \ldots \ldots 88$

(j) $\quad P 13$ Cluster, Turbulent Diffusion Velocity component $V_{q y} \ldots$. . . . 88

4.4 Continued,$P 13$ Cluster $\ldots \ldots$. . . . . . . . . . . . . . . . . . . . . 89

(k) $\quad P 13$ Cluster, Turbulent Diffusion Velocity component $V_{q z} \ldots$. . . . 89 
(1) $\quad P 13$ Cluster, Townsend's Structural Parameter $A_{1} \ldots \ldots \ldots$

4.4 Continued, $P 13$ Cluster $\ldots \ldots \ldots$. . . . . . . . . . . 90

(m) $\quad P 13$ Cluster, Flow Angle $\gamma_{a} \ldots \ldots \ldots \ldots \ldots$

(n) $\quad P 13$ Cluster, Shear Stress Angle $\gamma_{s} \ldots \ldots \ldots \ldots$

4.4 Continued,$P 13$ Cluster $\ldots \ldots \ldots$. . . . . . . . . . . . 91

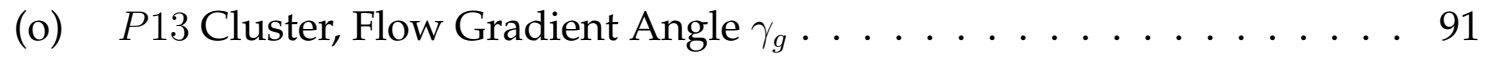

(p) P13 Cluster, Difference between Shear stress and Flow Gradient Angles 91

4.4 Continued, $P 13$ Cluster $\ldots \ldots \ldots$. . . . . . . . . . . . . . 92

(q) $\quad P 13$ Cluster, Mixing Length $L_{m}^{+} \ldots \ldots \ldots \ldots \ldots \ldots$. . . . . . 92

(r) $\quad P 13$ Cluster, Near Wall Mixing Length $L_{m}^{+} \quad \ldots \ldots \ldots \ldots \ldots$

4.4 Concluded,$P 13$ Cluster $\ldots \ldots \ldots$. . . . . . . . . . . . 93

(s) $\quad P 13$ Cluster, Turbulent Eddy Viscosity $\nu_{T x} \ldots \ldots \ldots$. . . . . 93

(t) $\quad P 13$ Cluster, Near Wall Turbulent Eddy Viscosity $\nu_{T x} \ldots \ldots \ldots$

$4.5 \quad P 18$ Cluster $\ldots \ldots \ldots \ldots \ldots$

(a) $\quad P 18$ Cluster, Mean Velocities $U^{+}, W^{+} \ldots \ldots \ldots \ldots$

(b) $\quad P 18$ Cluster, Normal Stress $\overline{u^{2}}$. . . . . . . . . . . . . . . . . . . 94

4.5 Continued, $P 18$ Cluster $\ldots \ldots \ldots$. . . . . . . . . . . . . . 95

(c) $\quad P 18$ Cluster, Normal Stresses ${\overline{v^{2}}}^{+}$and $\overline{w^{2}}$. . . . . . . . . . . . 95

(d) P18 Cluster,Reynolds' Shear Stress, $\overline{u v^{+}}$and $\overline{v w^{+}} \ldots \ldots$. . . . . . 95

4.5 Continued,$P 18$ Cluster $\ldots \ldots \ldots$. . . . . . . . . . . . 96

(e) $\quad P 18$ Cluster,Reynolds' Shear Stress, $\overline{u w}+\ldots \ldots$. . . . . . . 96

(f) $\quad P 18$ Cluster, Correlation Coefficient $\rho_{u v} \ldots \ldots \ldots \ldots$. . . . 96

4.5 Continued,$P 18$ Cluster $\ldots \ldots \ldots$. . . . . . . . . . 97

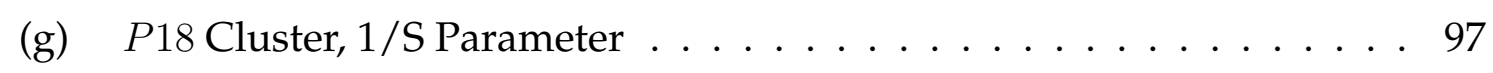

(h) $\quad P 18$ Cluster, Turbulent Kinetic Energy, TKE . . . . . . . . . . . . 97

4.5 Continued, $P 18$ Cluster $\ldots \ldots \ldots \ldots$

(i) $\quad P 18$ Cluster, Turbulent Diffusion Velocity component $V_{q x} \ldots \ldots$. . . 98

(j) $\quad P 18$ Cluster, Turbulent Diffusion Velocity component $V_{q y} \ldots$. . . . 98 
4.5 Continued,$P 18$ Cluster $\ldots \ldots \ldots 9$

(k) $\quad P 18$ Cluster, Turbulent Diffusion Velocity component $V_{q z} \ldots \ldots$. . . 99

(1) $\quad P 18$ Cluster, Townsend's Structural Parameter $A_{1} \ldots \ldots$. . . . . . . 99

4.5 Continued,$P 18$ Cluster $\ldots \ldots \ldots$. . . . . . . . . . . . 100

(m) P18 Cluster, Flow Angle $\gamma_{a} \ldots \ldots$. . . . . . . . . . . . . 100

(n) P18 Cluster, Shear Stress Angle $\gamma_{s} \ldots \ldots \ldots \ldots$

4.5 Continued, $P 18$ Cluster $\ldots \ldots \ldots 1$

(o) $\quad P 18$ Cluster, Flow Gradient Angle $\gamma_{g} \ldots \ldots \ldots \ldots$. . . . . . . . 101

(p) P18 Cluster, Difference between Shear stress and Flow Gradient Angles 101

4.5 Continued, $P 18$ Cluster $\ldots$. . . . . . . . . . . . . . . . . . . 102

(q) $\quad P 18$ Cluster, Mixing Length $L_{m}^{+} \ldots \ldots \ldots \ldots \ldots \ldots \ldots \ldots \ldots \ldots$

(r) $\quad P 18$ Cluster, Near Wall Mixing Length $L_{m}^{+} \quad \ldots \ldots \ldots \ldots \ldots \ldots$

4.5 Concluded,$P 18$ Cluster $\ldots \ldots$. . . . . . . . . . . . . . 103

(s) P18 Cluster, Turbulent Eddy Viscosity $\nu_{T} x \ldots \ldots \ldots$. . . . . 103

(t) $\quad$ P18 Cluster, Near Wall Turbulent Eddy Viscosity $\nu_{T} x \ldots \ldots$. . . . . . 103

$4.6 \quad P 19$ Cluster . . . . . . . . . . . . . . . . . . . . . . . . . . . . . . . . . . 104

(a) $\quad P 19$ Cluster, Mean Velocities $U^{+}, W^{+} \ldots \ldots \ldots \ldots$

(b) $\quad P 19$ Cluster, Normal Stress $\overline{u^{2}}$. . . . . . . . . . . . . . . . . 104

4.6 Continued P19 Cluster ． . . . . . . . . . . . . . . . . . . . . . . . 105

(c) P19 Cluster, Normal Stresses $\overline{v^{2}}$ and $\overline{w^{2}}$. . . . . . . . . . . . 105

(d) $\quad P 19$ Cluster,Reynolds' Shear Stress, $\overline{u v}^{+}$and $\overline{v w}+\ldots . . . . .105$

4.6 Continued, $P 19$ Cluster $\ldots \ldots \ldots 6$

(e) $\quad P 19$ Cluster,Reynolds' Shear Stress, $\overline{u w^{+}} \ldots \ldots$. . . . . . . . . . 106

(f) $\quad P 19$ Cluster, Correlation Coefficient $\rho_{u v} \ldots \ldots \ldots \ldots \ldots$

4.6 Continued, $P 19$ Cluster $\ldots \ldots \ldots 7$. . . . . . . . . . . 107

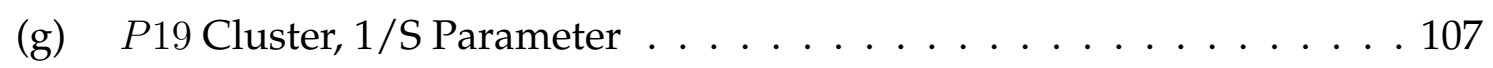

(h) P19 Cluster, Turbulent Kinetic Energy, TKE . . . . . . . . . . . . 107

4.6 Continued, $P 19$ Cluster $\ldots \ldots$. . . . . . . . . . . . . . . . 108 
(i) $\quad P 19$ Cluster, Turbulent Diffusion Velocity component $V_{q x} \ldots \ldots$. . . 108

(j) $\quad P 19$ Cluster, Turbulent Diffusion Velocity component $V_{q y} \ldots \ldots$. . . . 108

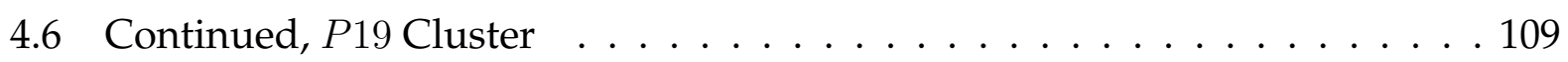

(k) $\quad P 19$ Cluster, Turbulent Diffusion Velocity component $V_{q z} \ldots \ldots$

(1) $\quad P 19$ Cluster, Townsend's Structural Parameter $A_{1}$. . . . . . . . . . . 109

4.6 Continued,$P 19$ Cluster $\ldots \ldots \ldots$

(m) $\quad P 19$ Cluster, Flow Angle $\gamma_{a} \ldots \ldots \ldots \ldots$

(n) $\quad P 19$ Cluster, Shear Stress Angle $\gamma_{s} \ldots \ldots \ldots \ldots \ldots$

4.6 Continued, $P 19$ Cluster $\ldots \ldots \ldots \ldots$

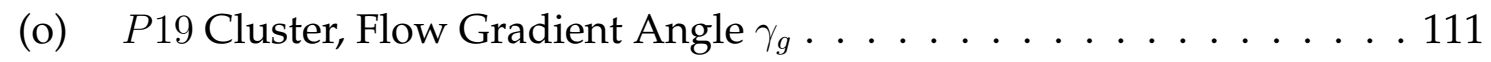

(p) P19 Cluster, Difference between Shear stress and Flow Gradient Angles 111

4.6 Continued, $P 19$ Cluster $\ldots \ldots$. . . . . . . . . . . . . . . . . . . . 112

(q) $\quad P 19$ Cluster, Mixing Length $L_{m}^{+} \ldots \ldots \ldots \ldots \ldots \ldots \ldots \ldots \ldots \ldots$

(r) $\quad P 19$ Cluster, Near Wall Mixing Length $L_{m}^{+} \quad \ldots \ldots \ldots \ldots \ldots \ldots \ldots$

4.6 Concluded, $P 19$ Cluster $\ldots \ldots \ldots$

(s) P19 Cluster, Turbulent Eddy Viscosity $\nu_{T} x \ldots \ldots$. . . . . . . 113

(t) $\quad$ P19 Cluster, Near Wall Turbulent Eddy Viscosity $\nu_{T} x \ldots \ldots$. . . . . 113

$4.7 \quad P 20$ Cluster . . . . . . . . . . . . . . . . . . . . . . . . . . . . . . . . . . 114

(a) $\quad P 20$ Cluster, Mean Velocities $U^{+}, W^{+} \ldots \ldots \ldots \ldots \ldots \ldots \ldots$

(b) $\quad P 20$ Cluster, Normal Stress $\overline{u^{2}}$. . . . . . . . . . . . . . . . . 114

4.7 Continued,$P 20$ Cluster $\ldots \ldots \ldots$

(c) P20 Cluster, Normal Stresses ${\overline{v^{2}}}^{+}$and ${\overline{w^{2}}}^{+} \ldots \ldots$. . . . . . . 115

(d) $\quad P 20$ Cluster,Reynolds' Shear Stress, $\overline{u v}^{+}$and $\overline{v w^{+}} \ldots \ldots . . . . .115$

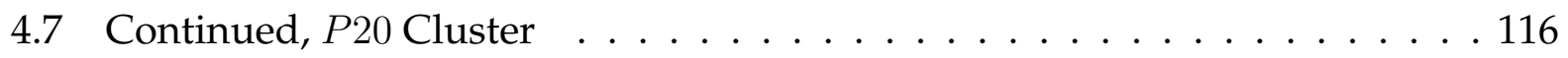

(e) $\quad P 20$ Cluster,Reynolds' Shear Stress, $\overline{u w^{+}} \ldots \ldots \ldots \ldots$

(f) $\quad P 20$ Cluster, Correlation Coefficient $\rho_{u v} \ldots \ldots \ldots \ldots$. . . . . 116

4.7 Continued, $P 20$ Cluster $\ldots \ldots \ldots \ldots$

(g) $\quad P 20$ Cluster $1 /$ S Parameter $\ldots \ldots \ldots \ldots \ldots$. . . . . . 117 
(h) P20 Cluster, Turbulent Kinetic Energy, TKE . . . . . . . . . . . 117

4.7 Continued, $P 20$ Cluster $\ldots \ldots \ldots \ldots \ldots$

(i) $\quad P 20$ Cluster, Turbulent Diffusion Velocity component $V_{q x} \ldots$. . . . 118

(j) $\quad P 20$ Cluster, Turbulent Diffusion Velocity component $V_{q y} \ldots \ldots$

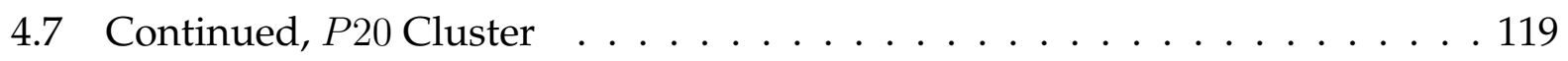

(k) $\quad P 20$ Cluster, Turbulent Diffusion Velocity component $V_{q z} \ldots \ldots$. . . 119

(l) $\quad P 20$ Cluster, Townsend's Structural Parameter $A_{1} \ldots \ldots$. . . . . . 119

4.7 Continued,$P 20$ Cluster $\ldots \ldots \ldots$

(m) $\quad P 20$ Cluster, Flow Angle $\gamma_{a} \ldots \ldots \ldots \ldots \ldots$

(n) $\quad P 20$ Cluster, Shear Stress Angle $\gamma_{s} \ldots \ldots \ldots \ldots \ldots \ldots$

4.7 Continued,$P 20$ Cluster $\ldots \ldots \ldots \ldots$

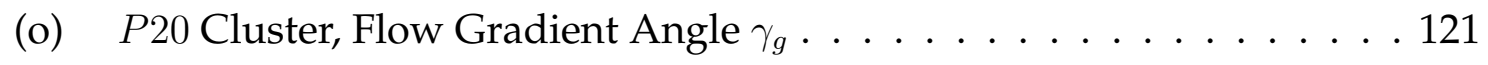

(p) P20 Cluster, Difference between Shear stress and Flow Gradient Angles 121

4.7 Continued,$P 20$ Cluster $\ldots \ldots \ldots$

(q) $\quad P 20$ Cluster, Mixing Length $L_{m}^{+} \ldots \ldots \ldots \ldots \ldots \ldots \ldots \ldots \ldots \ldots \ldots$

(r) $\quad P 20$ Cluster, Near Wall Mixing Length $L_{m}^{+} \ldots \ldots \ldots \ldots \ldots \ldots$

4.7 Concluded, $P 20$ Cluster $\ldots \ldots \ldots$. . . . . . . . . . . . . 123

(s) P20 Cluster, Near Wall Turbulent Eddy Viscosity $\nu_{T} x$. . . . . . . . 123

(t) P20 Cluster, Turbulent Eddy Viscosity $\nu_{T} x \ldots \ldots \ldots 123$

4.8 P33 Cluster . . . . . . . . . . . . . . . . . . . . . . . . . . . . . . . . . . . . 124

(a) $\quad P 33$ Cluster, Mean Velocities $U^{+}, W^{+} \ldots \ldots \ldots \ldots \ldots \ldots$

(b) $\quad P 33$ Cluster, Normal Stress ${\overline{u^{2}}}^{+} \ldots \ldots \ldots$. . . . . . . . . . . . 124

4.8 Continued, $P 33$ Cluster $\ldots \ldots \ldots \ldots$

(c) P33 Cluster, Normal Stresses $\overline{v^{2}}$ and $\overline{w^{2}}$. . . . . . . . . . . . . 125

(d) $\quad P 33$ Cluster,Reynolds' Shear Stress, $\overline{u v^{+}}$and $\overline{v w^{+}} \ldots \ldots . . . . .125$

4.8 Continued, $P 33$ Cluster $\ldots \ldots \ldots$

(e) $\quad P 33$ Cluster,Reynolds' Shear Stress, $\overline{u w^{+}} \ldots \ldots \ldots . . . . . . .126$

(f) $\quad P 33$ Cluster, Correlation Coefficient $\rho_{u v} \ldots \ldots \ldots \ldots \ldots$ 
4.8 Continued, $P 33$ Cluster $\ldots \ldots \ldots \ldots$

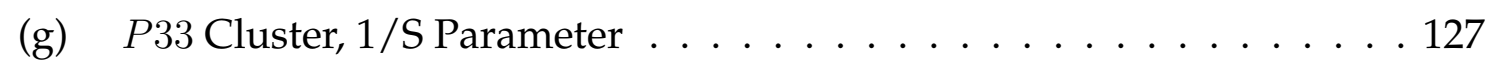

(h) $\quad P 33$ Cluster, Turbulent Kinetic Energy, TKE . . . . . . . . . . . . 127

4.8 Continued, $P 33$ Cluster $\ldots \ldots \ldots \ldots \ldots$

(i) $\quad P 33$ Cluster, Turbulent Diffusion Velocity component $V_{q x} \ldots \ldots$. . . 128

(j) $\quad P 33$ Cluster, Turbulent Diffusion Velocity component $V_{q y} \ldots \ldots . .128$

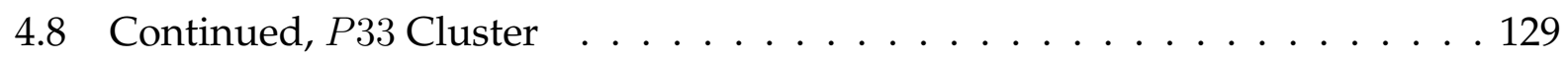

(k) $\quad P 33$ Cluster, Turbulent Diffusion Velocity component $V_{q z} \ldots \ldots$. . . . 129

(l) $\quad P 33$ Cluster, Townsend's Structural Parameter $A_{1} \ldots \ldots$. . . . . . 129

4.8 Continued, $P 33$ Cluster $\ldots \ldots$. . . . . . . . . . . . . . . . 130

(m) $\quad P 33$ Cluster, Flow Angle $\gamma_{a} \ldots \ldots \ldots \ldots$

(n) $\quad P 33$ Cluster, Shear Stress Angle $\gamma_{s} \ldots \ldots \ldots \ldots \ldots$

4.8 Continued, $P 33$ Cluster $\ldots \ldots \ldots \ldots$

(o) $\quad P 33$ Cluster, Flow Gradient Angle $\gamma_{g} \ldots \ldots \ldots \ldots$. . . . . . . 131

(p) P33 Cluster, Difference between Shear stress and Flow Gradient Angles 131

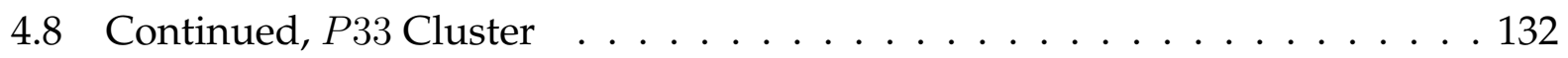

(q) $\quad P 33$ Cluster, Mixing Length $L_{m}^{+} \ldots \ldots \ldots \ldots \ldots \ldots \ldots \ldots \ldots \ldots$

(r) $\quad P 33$ Cluster, Near Wall Mixing Length $L_{m}^{+} \ldots \ldots \ldots \ldots$. . . . 132

4.8 Concluded, $P 33$ Cluster $\ldots \ldots \ldots$

(s) P33 Cluster, Turbulent Eddy Viscosity $\nu_{T} x \ldots \ldots$. . . . . . . 133

(t) P33 Cluster, Near Wall Turbulent Eddy Viscosity $\nu_{T} x . \ldots . . . . .133$

4.9 One-Dimensional Spectra of Reynolds Stresses at Profile P18. . . . . . . . . 134

(a) ${\overline{u^{2}}}^{+}$Normal Stress Power Spectra, $E_{11} \ldots$. . . . . . . . . . . . . 134

(b) ${\overline{v^{2}}}^{+}$Normal Stress Power Spectra, $E_{22}$. . . . . . . . . . . . . . . 134

(c) $\overline{w^{2}}$ Normal Stress Power Spectra, $E_{33} \ldots \ldots \ldots$. . . . . . . . 134

(d) $\overline{u v}{ }^{+}$Shear Stress Cross Spectra, $E_{12} \ldots \ldots \ldots \ldots$. . . . . . . . 134

(e) $\overline{u w^{+}}$Shear Stress Cross Spectra, $E_{13} \ldots \ldots \ldots \ldots$

(f) $\quad \overline{v w^{+}}$Shear Stress Cross Spectra, $E_{23} \ldots \ldots \ldots \ldots$. . . . . . . 134 
4.10 One-Dimensional Spectra of Reynolds Stresses at Profile $P 19 p$. . . . . . . 135

(a) ${\overline{u^{2}}}^{+}$Normal Stress Power Spectra, $E_{11} \ldots$. . . . . . . . . . . 135

(b) ${\overline{v^{2}}}^{+}$Normal Stress Power Spectra, $E_{22}$. . . . . . . . . . . . . . . 135

(c) ${\overline{w^{2}}}^{+}$Normal Stress Power Spectra, $E_{33}$. . . . . . . . . . . . . . . 135

(d) $\overline{u v}^{+}$Shear Stress Cross Spectra, $E_{12} \ldots \ldots \ldots$. . . . . . . . . 135

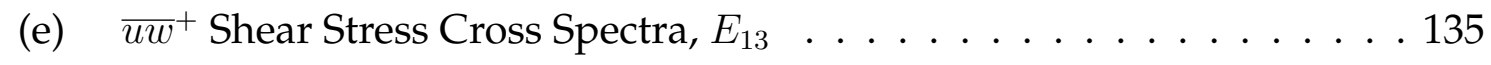

(f) $\quad \overline{v w^{+}}$Shear Stress Cross Spectra, $E_{23}$. . . . . . . . . . . . . . 135

4.11 One-Dimensional Spectra of Reynolds Stresses at Profile P19 . . . . . . . . 136

(a) ${\overline{u^{2}}}^{+}$Normal Stress Power Spectra, $E_{11} \ldots \ldots$. . . . . . . . 136

(b) ${\overline{v^{2}}}^{+}$Normal Stress Power Spectra, $E_{22} \ldots \ldots$. . . . . . . . 136

(c) $\overline{w^{2}}$ Normal Stress Power Spectra, $E_{33} \ldots \ldots \ldots$. . . . . . . 136

(d) $\overline{u v^{+}}$Shear Stress Cross Spectra, $E_{12} \ldots \ldots \ldots \ldots \ldots \ldots$

(e) $\overline{u w}{ }^{+}$Shear Stress Cross Spectra, $E_{13} \ldots \ldots \ldots \ldots$

(f) $\quad \overline{v w^{+}}$Shear Stress Cross Spectra, $E_{23} \ldots \ldots \ldots \ldots \ldots$

4.12 One-Dimensional Spectra of Reynolds Stresses at Profile $P 19_{1}$. . . . . . . 137

(a) ${\overline{u^{2}}}^{+}$Normal Stress Power Spectra, $E_{11} \ldots$. . . . . . . . . . . . 137

(b) ${\overline{v^{2}}}^{+}$Normal Stress Power Spectra, $E_{22}$. . . . . . . . . . . . . 137

(c) ${\overline{w^{2}}}^{+}$Normal Stress Power Spectra, $E_{33}$. . . . . . . . . . . . . 137

(d) $\overline{u v}{ }^{+}$Shear Stress Cross Spectra, $E_{12} \ldots \ldots \ldots \ldots$. . . . . . . . 137

(e) $\overline{u w^{+}}$Shear Stress Cross Spectra, $E_{13} \ldots \ldots \ldots \ldots$. . . . . . 137

(f) $\quad \overline{v w^{+}}$Shear Stress Cross Spectra, $E_{23} \ldots \ldots \ldots \ldots$

$4.13 \epsilon^{+}$Estimates . . . . . . . . . . . . . . . . . . . . . . . . 138

(a) $\quad P 18$, Turbulence Kinetic Energy Dissipation Rate $\epsilon^{+} \ldots \ldots$. . . . . . 138

(b) $P 19 p$, Turbulence Kinetic Energy Dissipation Rate $\epsilon^{+} \ldots \ldots$. . . . 138

$4.13 \epsilon^{+}$Estimates . . . . . . . . . . . . . . . . . . . . . . . . . . 139

(c) $\quad P 19$, Turbulence Kinetic Energy Dissipation Rate $\epsilon^{+} \ldots \ldots$. . . . . . 139

(d) $P 19_{1}$, Turbulence Kinetic Energy Dissipation Rate $\epsilon^{+} \ldots$. . . . . . . . 139 
5.1 Reynolds Stresses Transport Budgets at P07 . . . . . . . . . . . . . . . . . . 151

5.2 Reynolds Stresses Transport Budgets at P13 . . . . . . . . . . . . . . . . . 152

5.3 Reynolds Stresses Transport Budgets at P18 . . . . . . . . . . . . . . 153

5.4 Reynolds Stresses Transport Budgets at P19p . . . . . . . . . . . . . . . . 154

5.5 Reynolds Stresses Transport Budgets at P19 . . . . . . . . . . . . . . . . 155

5.6 Reynolds Stresses Transport Budgets at P20 . . . . . . . . . . . . . . . . . 156

5.7 Reynolds Stresses Transport Budgets at P33 . . . . . . . . . . . . . . 157

5.8 Reynolds Stresses Production at P08, DNS data by Iwamoto [20] . . . . . . . 158

5.9 Reynolds Stresses Production at P13, DNS data by Iwamoto [20] . . . . . . . 159

5.10 Reynolds Stresses Production at P18, DNS data by Iwamoto [20] . . . . . . . 160

5.11 Reynolds Stresses Production at P19, DNS data by Iwamoto [20] . . . . . . . 161

5.12 Reynolds Stresses Production at $P 19_{p}$, DNS data by Iwamoto [20] . . . . . 162

5.13 Reynolds Stresses Production at P20, DNS data by Iwamoto [20] . . . . . . . 163

5.14 Reynolds Stresses Production at P33, DNS data by Iwamoto [20] . . . . . . . 164

5.15 Contours of Span-wise Mean Vorticity, $\frac{\Omega_{z} H}{U_{\infty}}$, shown as colored contours and Stream-wise Mean Vorticity, $\frac{\Omega_{x} H}{U_{\infty}}$, shown as line contours along different stream-wise stations. Dashed lines denote negative values. Step between

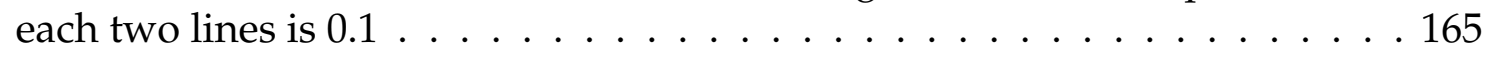

5.16 Stream-wise and Span-wise Coefficient of Pressure Gradients . . . . . . 166

5.17 Mean Rate of Strain, $S_{11}, S_{13}, S_{33} \ldots \ldots \ldots$. . . . . . . . . 167

5.18 Stream-wise Vorticity development . . . . . . . . . . . . . . . . . . 168

5.19 Stream-wise Vorticity interaction in $y$-z plane . . . . . . . . . . . . . . . 169

6.1 Comparison between measured and modeled triple-velocity correlations for Profile $P 07,(E x p)$ Experiment, $(H L)$ Hanjalić and Launder model, $(M k)$ Mackinnon model, $(D H)$ Daly-Harlow model and $(M R)$ Moore's model. $D_{i i}^{T} / 2$ is the Turbulence Kinetic Energy Diffusion. . . . . . . . . . . . . 187

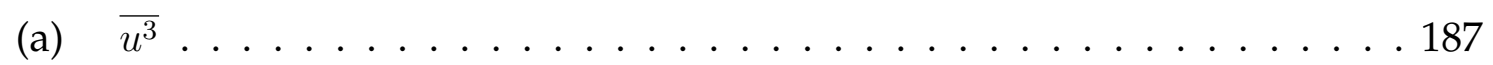

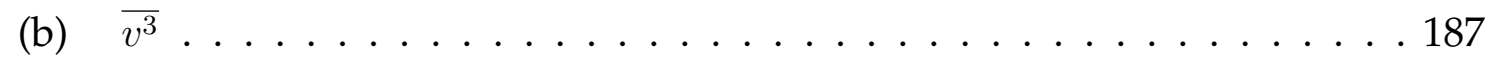

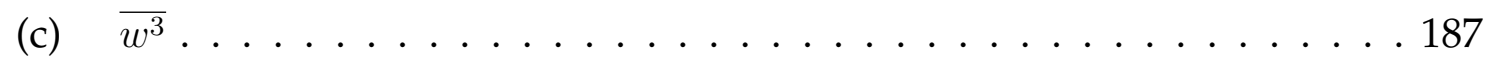

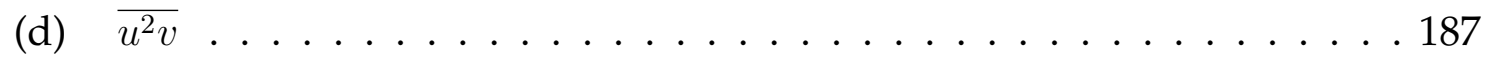




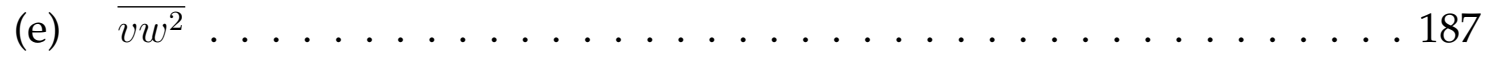

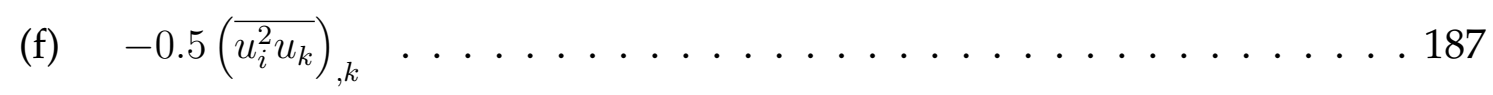

6.2 Comparison between measured and modeled triple-velocity correlations for Profile $P 13,($ Exp $)$ Experiment, $(H L)$ Hanjalić and Launder model, $(M k)$ Mackinnon model, $(D H)$ Daly-Harlow model and $(M R)$ Moore's model. $D_{i i}^{T} / 2$ is the Turbulence Kinetic Energy Diffusion. $\ldots \ldots \ldots$. . . . . 188

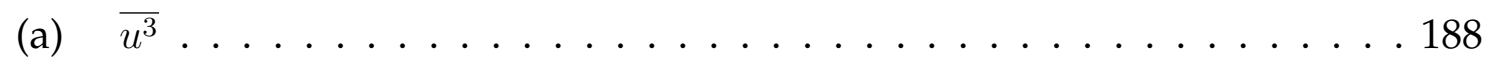

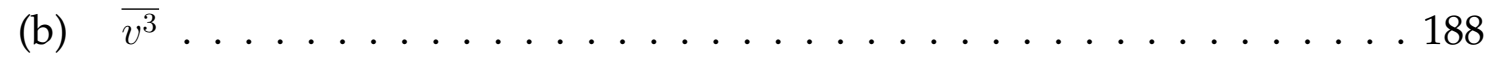

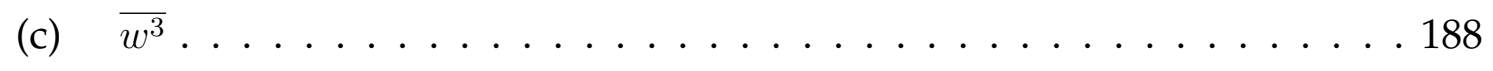

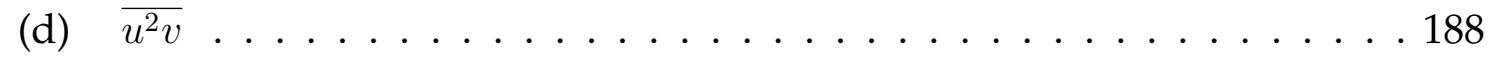

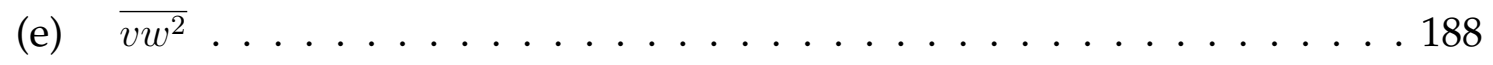

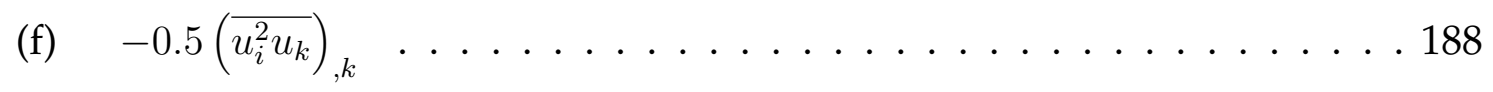

6.3 Comparison between measured and modeled triple-velocity correlations for Profile P19pp, (Exp) Experiment, $(H L)$ Hanjalić and Launder model, $(M k)$ Mackinnon model, $(D H)$ Daly-Harlow model and $(M R)$ Moore's model. $D_{i i}^{T} / 2$ is the Turbulence Kinetic Energy Diffusion. $\ldots$. . . . . . . . 189

(a) $\overline{u^{3}} \ldots \ldots \ldots$. . . . . . . . . . . . . . . . . . . . 189

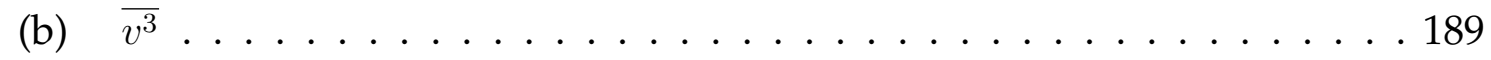

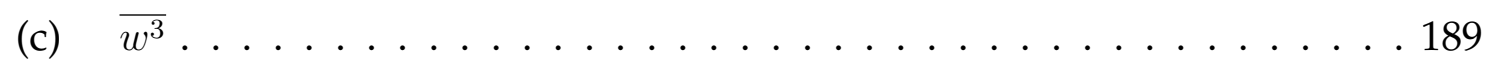

(d) $\overline{u^{2} v} \ldots \ldots \ldots \ldots \ldots \ldots$

(e) $\quad \overline{v w^{2}} \ldots \ldots \ldots \ldots \ldots \ldots \ldots$

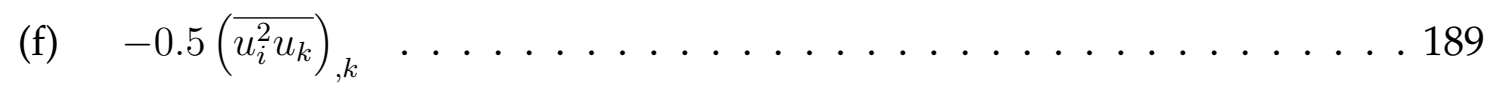

6.4 Comparison between measured and modeled triple-velocity correlations for Profile $P 32,(\operatorname{Exp})$ Experiment, $(H L)$ Hanjalić and Launder model, $(M k)$ Mackinnon model, $(D H)$ Daly-Harlow model and $(M R)$ Moore's model. $D_{i i}^{T} / 2$ is the Turbulence Kinetic Energy Diffusion. . . . . . . . . . . . . 190

(a) $\overline{u^{3}} \ldots \ldots \ldots \ldots$. . . . . . . . . . . . . . . . 190

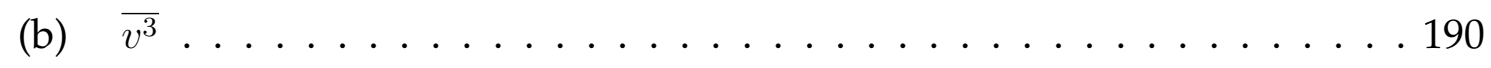

(c) $\overline{w^{3}} \ldots \ldots \ldots \ldots \ldots \ldots \ldots$

(d) $\overline{u^{2} v} \ldots \ldots \ldots \ldots \ldots \ldots \ldots$

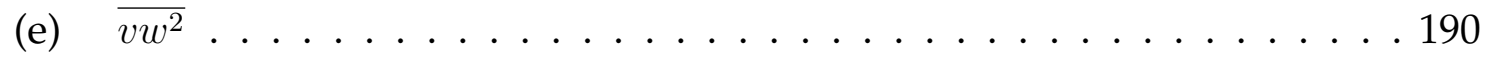


(f) $\quad-0.5\left(\overline{u_{i}^{2} u_{k}}\right)_{, k} \ldots \ldots \ldots \ldots \ldots \ldots$

6.5 Blending function, $f_{s}$, in Jakirlić and Hanjalić dissipation rate tensor anisotropy 191

6.6 Comparison between dissipation rate models at profile $P 10,(H J)$ Hallback and Johansson model, $(P N)$ Perot and Natu model, $(J H)$ Jakirlić and Hanjalić model $\ldots \ldots \ldots \ldots 19 \ldots \ldots \ldots$

(a) $\epsilon_{11} \ldots \ldots \ldots \ldots \ldots$

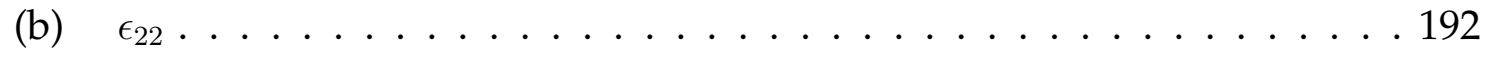

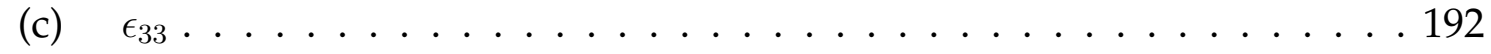

(d) $\epsilon_{12} \ldots \ldots \ldots \ldots$

(e) $\epsilon_{13} \ldots \ldots \ldots \ldots . \ldots \ldots \ldots$

(f) $\epsilon_{23} \ldots \ldots \ldots \ldots \ldots$

6.7 Comparison between dissipation rate models at profile $P 35,(H J)$ Hallback and Johansson model, $(P N)$ Perot and Natu model, $(J H)$ Jakirlić and Hanjalić model $\ldots \ldots \ldots \ldots 19 \ldots \ldots \ldots$

(a) $\epsilon_{11} \ldots \ldots \ldots \ldots$

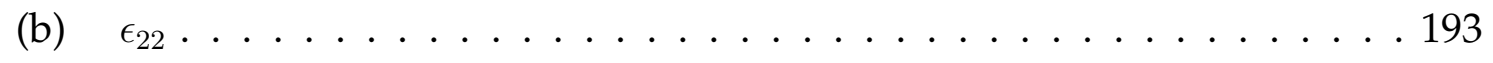

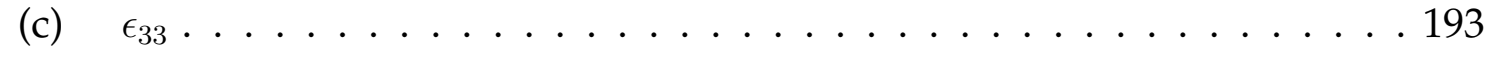

(d) $\epsilon_{12} \ldots \ldots \ldots \ldots \ldots \ldots$

(e) $\epsilon_{13} \ldots \ldots \ldots \ldots \ldots \ldots$

(f) $\epsilon_{23} \ldots \ldots \ldots \ldots \ldots \ldots$

6.8 Comparison between Pressure-Strain models at profile P07, (Exp) experiment, $(L R R)$ Launder, Reece and Rodi model, $(S S G)$ Sarkar, Speziale and Gatski model, $(S H L)$ Shih and Lumley model, $(F L T) \mathrm{Fu}$, Launder and Tselepidakis model $\ldots \ldots \ldots \ldots . \ldots \ldots$. . . . . . . . . . . . . . . .

(a) $\phi_{11} \ldots \ldots \ldots \ldots$

(b) $\phi_{22} \ldots \ldots \ldots \ldots \ldots \ldots \ldots$

(c) $\phi_{33} \ldots \ldots \ldots \ldots \ldots \ldots$

(d) $\phi_{12} \ldots \ldots \ldots \ldots \ldots \ldots$

(e) $\phi_{13} \ldots \ldots \ldots \ldots \ldots$

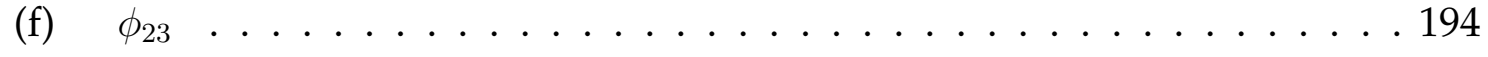


6.9 Comparison between Pressure-Strain models at profile $P 13,($ Exp $)$ experiment, $(L R R)$ Launder, Reece and Rodi model, $(S S G)$ Sarkar, Speziale and Gatski model, $(S H L)$ Shih and Lumley model, $(F L T) \mathrm{Fu}$, Launder and Tselepidakis model $\ldots \ldots \ldots \ldots \ldots$

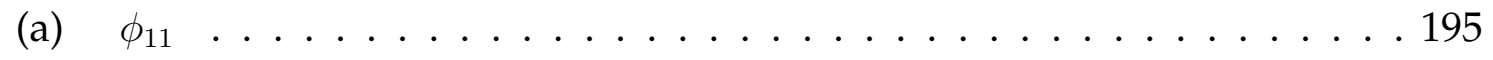

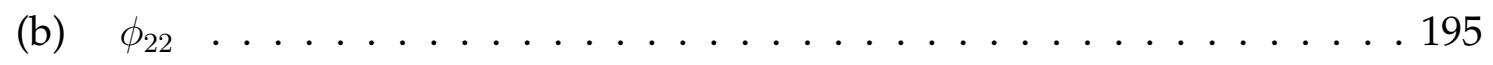

(c) $\phi_{33} \ldots \ldots \ldots \ldots \ldots$

(d) $\phi_{12} \ldots \ldots \ldots \ldots \ldots \ldots \ldots$

(e) $\phi_{13} \ldots \ldots \ldots \ldots \ldots$

(f) $\quad \phi_{23} \quad \ldots \ldots \ldots \ldots$

6.10 Comparison between Pressure-Strain models at profile $P 18,(E x p)$ experiment, $(L R R)$ Launder, Reece and Rodi model, $(S S G)$ Sarkar, Speziale and Gatski model, $(S H L)$ Shih and Lumley model, $(F L T) \mathrm{Fu}$, Launder and

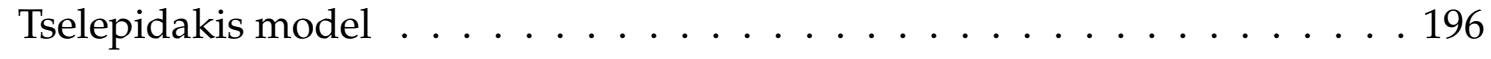

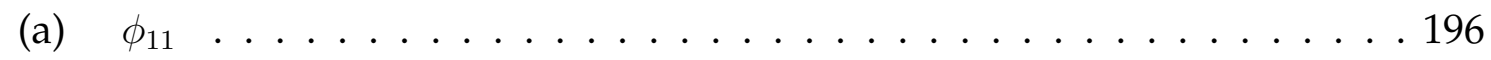

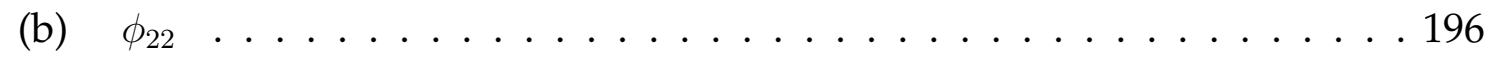

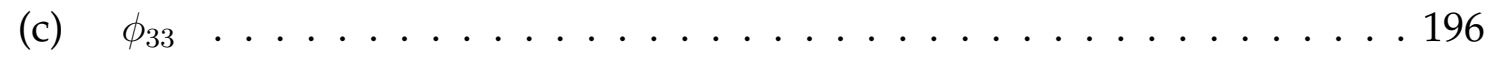

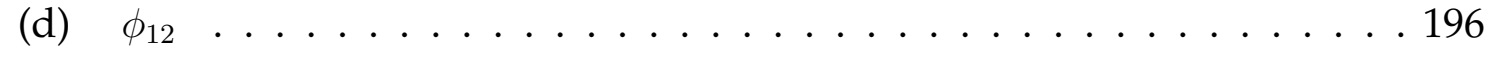

(e) $\phi_{13} \ldots \ldots \ldots \ldots \ldots$

(f) $\phi_{23} \ldots \ldots \ldots \ldots \ldots \ldots$

6.11 Comparison between Pressure-Strain models at profile P19, (Exp) experiment, $(L R R)$ Launder, Reece and Rodi model, $(S S G)$ Sarkar, Speziale and Gatski model, $(S H L)$ Shih and Lumley model, $(F L T)$ Fu, Launder and Tselepidakis model $\ldots \ldots \ldots \ldots 197$

(a) $\phi_{11} \ldots \ldots \ldots \ldots$. . . . . . . . . . . . . . . . . . . . . . 197

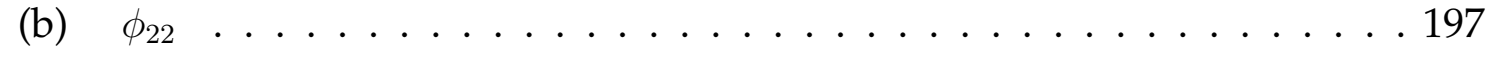

(c) $\phi_{33} \ldots \ldots \ldots \ldots$

(d) $\phi_{12} \ldots \ldots \ldots \ldots \ldots$

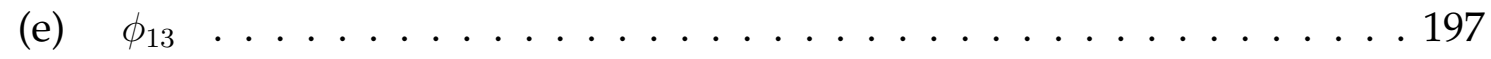

(f) $\phi_{23} \ldots \ldots \ldots \ldots \ldots$ 
6.12 Comparison between Pressure-Strain models at profile $P 20,(E x p)$ experiment, $(L R R)$ Launder, Reece and Rodi model, $(S S G)$ Sarkar, Speziale and Gatski model, $(S H L)$ Shih and Lumley model, $(F L T) \mathrm{Fu}$, Launder and Tselepidakis model $\ldots \ldots \ldots \ldots \ldots$. . . . . . . . . . . . . . . . . . . .

(a) $\phi_{11} \ldots \ldots \ldots \ldots \ldots \ldots$

(b) $\phi_{22} \ldots \ldots \ldots \ldots \ldots$

(c) $\phi_{33} \ldots \ldots \ldots \ldots \ldots$

(d) $\phi_{12} \ldots \ldots \ldots \ldots \ldots \ldots$

(e) $\phi_{13} \ldots \ldots \ldots \ldots \ldots$

(f) $\quad \phi_{23} \quad \ldots \ldots \ldots \ldots$

6.13 Comparison between Pressure-Strain models at profile P33, (Exp) experiment, $(L R R)$ Launder, Reece and Rodi model, $(S S G)$ Sarkar, Speziale and Gatski model, $(S H L)$ Shih and Lumley model, (FLT) Fu, Launder and Tselepidakis model $\ldots \ldots \ldots . \ldots \ldots 99$

(a) $\phi_{11} \ldots \ldots \ldots \ldots \ldots$

(b) $\phi_{22} \ldots \ldots \ldots \ldots \ldots$

(c) $\phi_{33} \ldots \ldots \ldots \ldots \ldots$

(d) $\phi_{12} \ldots \ldots \ldots \ldots \ldots$

(e) $\phi_{13} \ldots \ldots \ldots \ldots \ldots$

(f) $\phi_{23} \ldots \ldots \ldots \ldots \ldots$

6.14 Expression for Flow Gradient Angle Lag . . . . . . . . . . . . . . . . . . . 200

6.15 Comparison between Measured and modeled Reynolds Shear Stress Angle, $\nabla$ Experiment, - Current Lag Model and - - Rotta's Lag Model . . . . 201

(a) Profile $P 06 . \ldots \ldots \ldots$. . . . . . . . . . . . . . . . . 201

(b) Profile $P 13 \ldots \ldots \ldots$. . . . . . . . . . . . . . . . . . . . . 201

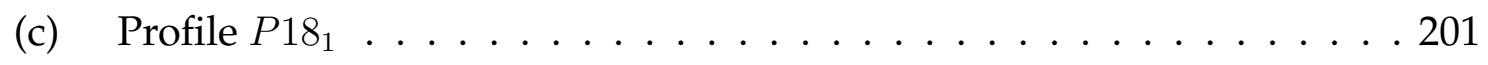

(d) Profile $P 19 p p \ldots \ldots \ldots \ldots$. . . . . . . . . . . . . . 201

(e) Profile $P 20_{1} \ldots \ldots \ldots \ldots$. . . . . . . . . . . . . . . . . . . 201

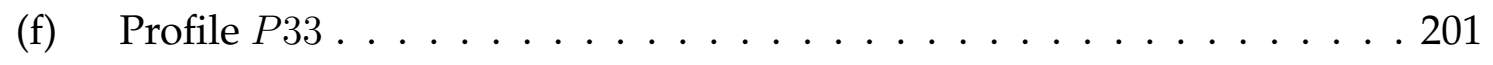

A.1 Wavy Wall Test Section Plan-view . . . . . . . . . . . . . . . . . . . . . . 218 
A.2 Side Wall Geometry Measurement Setup . . . . . . . . . . . . . . . . . . . 218

C.1 Matching Short and CompLDV Profiles at P07 . . . . . . . . . . . . . . . 227

C.2 Matching Short and CompLDV Profiles at $P 16$. . . . . . . . . . . . . 228

C.3 Matching Short and CompLDV Profiles at P19 . . . . . . . . . . . . . . . . 229

C.4 Matching Short and CompLDV Profiles at $P 19_{2}$. . . . . . . . . . . . . . 230

C.5 Matching Short and CompLDV Profiles at $P 31 \ldots$. . . . . . . . . . . 231 


\section{List of Tables}

2.1 Boundary Layer Suction Volume Flow Rate in $f t^{3} /$ min. . . . . . . . . . . 17

2.2 Location of Side Wall Velocity Profiles Maxima $\ldots \ldots \ldots \ldots$

3.1 Seven-Hole Probe Uncertainties . . . . . . . . . . . . . . . . . . . . . . . . . . . 29

3.2 Mean Flow Free Stream Conditions $\ldots . \ldots$. . . . . . . . . . 33

3.3 Maximum test section ceiling height at seven-hole probe measurement planes 33

3.4 Centerline Mean Edge Velocity and Boundary Layer Thickness . . . . . . . 33

$4.1 \quad$ Profiles' Coordinates, estimated Pressure gradients, y-Shift and Friction Ve-

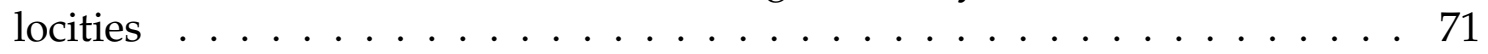

6.1 Comparison between measured and computed Reynolds Shear Stress an-

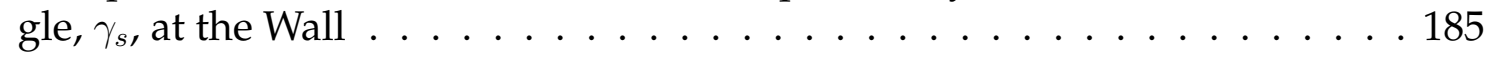

A.1 Wavy Wall Test Section Geometry . . . . . . . . . . . . . . . . . . . . . . 212

B.1 Suction Slots Location and Size . . . . . . . . . . . . . . . . . . . . . . . 219

C.1 Short system and CompLDV estimated friction velocity, $u_{\tau}$ and average value for each cluster . . . . . . . . . . . . . . . . . . . . . . 224

C.2 20:1 Uncertainties by matching Short system and CompLDV profiles using Peirce's Criterion . . . . . . . . . . . . . . . . . . . 225

C.3 20:1 Short system Uncertainties using Peirce's Criterion . . . . . . . . . . . . 225

C.4 20:1 CompLDV system Uncertainties using Peirce's Criterion. . . . . . . . . 225

C.5 Derived quantities uncertainties using formal sensitivity analysis and esti-
mated uncertainties in measured primary quantities. $\ldots \ldots \ldots \ldots . \ldots \ldots$ 


\section{Nomenclature}

$\alpha$

$\alpha$

$\delta y$

$\delta$

$\epsilon_{i j}$

$\gamma_{a}$

$\gamma_{g}$

$\gamma_{s}$

$\hat{i}, \hat{j}, \hat{k}$

$\hat{n}$

$\kappa, B$

$\nu$

$\nu_{T x}$

$\Omega_{k}$

$\overline{(\bullet)}$

$\bar{d}_{g}$

$\overline{u_{i} \frac{\partial p}{\partial x_{j}}}$

$\overline{u_{i} u_{j} u_{k}}$

$\overline{u_{i} u_{j}}$
A Constant in Tchen's high mean vorticity model, page 70

Constant in Hallback's dissipation rate anisotropy, page 175

Shift in the $y$-coordinates of LDV profiles, page 44

Boundary Layer Thickness, page 33

Turbulent Dissipation Rate, page 3

Flow Angle, page 49

Flow Gradient Angle, page 49

Shear Stress Angle, page 49

Unit vectors in stream-wise, vertical and span-wise direction respectively, page 146

Boundary Surface unit normal vector, page 233

Constants in the Law of the wall, page 45

Kinematic Viscosity, page 3

Turbulent Eddy Viscosity in the x-direction, page 50

Mean Vorticity Vector, page 143

Ensemble Average, page 3

Geometric Mean Particle Diameter, page 14

Velocity pressure-gradient correlation, page 3

Triple-velocity Correlation or Turbulent Transport, page 3

Reynolds' Turbulent Stresses, page 3 


\begin{tabular}{|c|c|}
\hline$\phi_{i j}$ & Pressure-Strain tensor, page 176 \\
\hline$\Pi_{i j}$ & Velocity-Pressure Gradient, page 4 \\
\hline$\rho_{u v}$ & Correlation Coefficient, page 47 \\
\hline$\sigma_{g}$ & Seeding Particle Diameter Geometric Standard Deviation, page 14 \\
\hline$\tau$ & Reynolds Shear Stress in a plane parallel to the wall, page 181 \\
\hline$\tau$ & Wall Total Shear Stress, page 43 \\
\hline$\theta$ & Momentum Thickness, page 44 \\
\hline$\varphi$ & Velocity Potential, page 232 \\
\hline$\vec{V}_{q}^{+}$ & Turbulent Diffusion Velocity non-dimensionalized by $u_{\tau}$, page 48 \\
\hline$\xi, \eta, \zeta$ & Generalized coordinates, page 232 \\
\hline$\xi_{i}$ & Generalized Coordinates in Tensor notation, page 233 \\
\hline$A$ & $\begin{array}{l}\text { Flatness parameter in Jakirlić and Hanjalić model dissipation rate anisotropy } \\
\text { model, page } 176\end{array}$ \\
\hline$A_{1}$ & Townsend's Structural Parameter, page 48 \\
\hline$a_{i j}$ & Reynolds stress anisotropy, page 175 \\
\hline$C$ & Constant in the $-5 / 3$ Power Spectrum law, page 70 \\
\hline$C^{*}$ & Constant in Perot and Natu dissipation rate anisotropy tensor, page 175 \\
\hline$C_{f}$ & Wall Skin-Friction Coefficient, $C_{f}=\left(u_{\tau} / U_{e}\right)^{2}$, page 140 \\
\hline$C_{P}$ & Pressure Coefficient, page 33 \\
\hline$C_{i j}$ & Reynolds' stresses Convective rate of Change, page 4 \\
\hline$D_{i j}^{\nu}$ & Reynolds' stresses Viscous Diffusion, page 4 \\
\hline$D_{i j}^{T}$ & Reynolds' stresses Turbulent Diffusion, page 4 \\
\hline$D_{i j}^{P}$ & Pressure-Diffusion tensor, page 176 \\
\hline$E_{i j}$ & One-dimensional $\overline{u_{i} u_{j}}$ Power Spectrum, page 70 \\
\hline$f$ & Frequency in the Power Spectrum law, page 70 \\
\hline
\end{tabular}


$f_{s} \quad$ Blending function in Jakirlić and Hanjalić model dissipation rate anisotropy model, page 176

$I I_{a} \quad$ Reynolds stress anisotropy invariant, page 175

L Wavy Wall Test Section Length, page 10

$L_{m} \quad$ Mixing Length, page 50

$L_{i j} \quad$ Reynolds' stresses local time rate of Change, page 4

Min Minimum Function, page 7

$P \quad$ Mean Static Pressure, page 3

$P_{i j} \quad$ Reynolds' stresses Production, page 4

$Q \quad$ Side Wall suction volume flow rate in $f t^{3} / \mathrm{min}$, page 28

$Q_{e} \quad$ Boundary Layer edge velocity parallel to Tunnel's floor, $\sqrt{U^{2}+W^{2}}$, page 33

$R \quad$ Correlation coefficient for fitting LDV data to Equation 4.2 , page 44

$R_{i j} \quad$ Rotation Rate Tensor, page 143

$R_{\theta} \quad$ Reynolds' Number based on Momentum Thickness, page 44

$S^{-1} \quad 1 / S$ parameter, page 47

$S_{i j} \quad$ Mean Rate of Strain, page 144

$s_{i j} \quad$ Fluctuating Rate of Strain, page 144

TKE, $k \quad$ Turbulent Kinetic Energy, page 47

$U, V, W \quad$ Mean Flow Velocity components in the stream, vertical and span-wise directions respectively, page 33

$u_{i} \quad$ Fluctuation velocity component in $x_{i}$ direction, page 3

$u_{\tau} \quad$ Friction Velocity, page 43

$U_{i} \quad$ Mean Flow Velocity Components, page 3

$U_{\max } \quad$ Maximum speed measured along normal direction to the side walls, page 26

$W_{i j} \quad$ Rotation tensor, page 177

$W_{x=0} \quad$ Wavy Wall Test Section Entrance Width, page 10 
$x, y, z \quad$ Spatial Coordinates in the stream, vertical and span-wise directions respectively, page 3

$x_{i}$ Spatial Coordinates in tensor notation, page 3

Function in Perot and Natu dissipation rate anisotropy tensor, page 175 


\section{Chapter 1}

\section{Introduction}

\subsection{Motivation}

Research has been going on for many decades for ways to predict turbulent flow fields. Turbulent flows appear in a wide range of engineering applications and more recently in bio-medical applications. Turbulent flow fields for Newtonian fluids are governed by the Navier-Stokes equations. These equations are a nonlinear set of time dependent partial differential equations. As a consequence of this nonlinearity, turbulent flow fields are chaotic and are very sensitive to initial and boundary conditions and external disturbances. Slight changes in initial and boundary conditions lead to completely different time histories of flow variables.

Different methods have been developed in the last four decades to solve these equations. The most commonly used methods are Reynolds-Averaged Navier-Stokes methods (RANS). Flow variables are assumed to be composed of a mean value and a fluctuating component with zero mean value. The Navier-Stokes equations are then time or ensemble averaged. Hence equations for mean flow variables are obtained. The effects of all turbulent flow scales, fluctuating components, appear as stress terms in these equations, called Reynolds stresses. These stresses are modeled either by simple algebraic formulas or by writing transport equations for each stress term. The resulting stress transport equations contain higher order expressions that need to be modeled in terms of known quantities. Modeling these terms have been the focus of research for over forty years. However, the prediction capabilities of any one model are not satisfactory for different flows and different configurations. This is in part due to the lack of experimental data for some of the terms that needs modeling. Despite the above fact these models are still used in industry extensively due to its ease of application and the need for fast solution methods that can be incorporated in design processes.

Another approach to predict turbulent flows is to simulate the turbulent flow fields 
directly using what is called Direct Numerical Simulations, DNS, Orszag and Patterson [45]. This category of methods uses a very fine grid in order to resolve all flow details. Unfortunately turbulent flows have a wide range of time and length scales that need to be resolved in order to predict the flow field adequately. This range is dependent on the flow Reynolds number, Re, to some power. Estimates of the required grid points to fully resolve turbulent flows are given by different authors, e.g. Moin and Mahesh [36], Dubois and Temam [12]. For a three dimensional flow, the number of points required to fully resolve the flow field is proportional to $R e^{2.25}$. For engineering applications the Reynolds number can be of the order of a few hundred thousand to a billion, which makes the applicability of this category of methods unfeasible in the foreseeable future for engineering and industrial applications where multiple simulations are often required. However these methods prove to be a valuable tool for fluid dynamics research where it can give a better understanding of turbulent flow phenomena. A review of the applications and potentials of DNS is given in Moin and Mahesh [36].

A different approach was also developed in the sixties, Large Eddy Simulations or LES, by Smagorinsky [62]. This approach is intermediate between RANS and DNS methods. It aims at resolving only large length scales of the flow field which would be Reynolds number and flow dependent and to model the effects of the smaller structures which would have a more universal nature. This methodology leads to a significant reduction in the number of points required to resolve the flow field. For example, for a channel flow the number of points needed to resolve the wall region is proportional to $R e^{1.8}$, which is a significantly smaller than the requirement for a DNS solution. Reviews of LES methods can be found in Lesieur and Metais [24], Piomelli [49], and Meneveau and Katz [34]. However, the applicability of LES is still limited to the lower end of engineering applications since the above requirement is still too demanding. Attempts have been made to reduce the grid size requirements by modeling the wall region by the so called Wall-Layer Models, in which the effects of the smaller eddies near the wall are modeled in an average sense. A review of these models can be found in Piomelli [48]. Although DNS and LES are more accurate techniques for solving turbulent flow problems, their applicability to engineering problems does not seem feasible in the near future, see Pope [51] and Moin [36].

Hybrid approaches are also developed where RANS models are used where the flow is attached and the boundary layer is thin and LES model is used where the flow is detached. Such approach is called Detached-Eddy Simulation, DES, and Delayed DetachedEddy Simulation, DDES. A description of the method is given by Spalart [64].

On the other hand RANS models are routinely carried out in industry, hence improving the models for different terms in the RANS equations might have a big impact on their predictive capability and hence on the design process in industry. This is the primary motivation for the present work, providing low uncertainty turbulent three dimensional flow data to help improve modeling of different terms appearing in the governing equations. In the next section the Reynolds Averaged Navier-Stokes equations are presented along 
with the transport equations for the Reynolds stresses.

\subsection{Governing Equations}

For incompressible isothermal flow the Reynolds Averaged Navier-Stokes (RANS) equations along with the continuity equation for incompressible flow are given by, Chou [6], Moore and Moore [38],

Continuity:

$$
\frac{\partial U_{i}}{\partial x_{i}}=0
$$

Momentum:

$$
\frac{\partial U_{i}}{\partial t}+U_{l} \frac{\partial U_{i}}{\partial x_{l}}=-\frac{\partial P}{\partial x_{i}}+\frac{\partial}{\partial x_{l}}\left(\nu \frac{\partial U_{i}}{\partial x_{l}}-\overline{u_{i} u_{l}}\right)
$$

Where, $U_{i}$ and $P$ are the mean velocity components and pressure respectively. The last term in the momentum equation is the Reynolds stress contribution to the mean flow equations. In order to be able to solve the governing equations, the Reynolds stresses have to be specified. We will consider the second-moment closure, where the transport equations for the Reynolds stresses, given in Equation 1.3, are solved along with the RANS equations.

$$
\frac{\partial \overline{u_{i} u_{j}}}{\partial t}+U_{l} \frac{\partial \overline{u_{i} u_{j}}}{\partial x_{l}}=-\overline{u_{i} u_{l}} \frac{\partial U_{j}}{\partial x_{l}}-\overline{u_{j} u_{l}} \frac{\partial U_{i}}{\partial x_{l}}-\overline{u_{i} \frac{\partial p}{\partial x_{j}}}-\overline{u_{j} \frac{\partial p}{\partial x_{i}}}+\nu \frac{\partial^{2} \overline{u_{i} u_{j}}}{\partial x_{l}^{2}}-\frac{\partial \overline{u_{i} u_{j} u_{k}}}{\partial x_{k}}-\epsilon_{i j}
$$

The above equation can be written symbolically as,

$$
L_{i j}+C_{i j}=P_{i j}+\Pi_{i j}+D_{i j}^{T}+D_{i j}^{\nu}-\epsilon_{i j}
$$

Where,

- $L_{i j}$ Local time rate of change, first term on left hand side of Equation 1.3

- $C_{i j}$ Convective Transport, second term on left hand side of Equation 1.3

- $P_{i j}$ Stress Production, first two terms on right hand side of Equation 1.3

- $\Pi_{i j}$ Velocity-Pressure Gradient, third and fourth terms on right hand side of Equation 1.3

- $D_{i j}^{\nu}$ Viscous Diffusion, fifth term on right hand side of Equation 1.3

- $D_{i j}^{T}$ Turbulent Diffusion, sixth term on right hand side of Equation 1.3

- $\epsilon_{i j}$ Dissipation, last term on right hand side of Equation 1.3 
An over bar denotes an ensemble or time average. All terms listed above can be computed except three terms, velocity-pressure gradient, turbulent dissipation rate and turbulent diffusion. The velocity-pressure gradient term can be broken into two terms, the pressure-strain correlation and the pressure-velocity correlation. Most frequently the pressure-velocity correlation is added in the turbulent diffusion term and both terms are modeled together. In the current work we will focus on modeling turbulent diffusion which involves the triple-velocity correlations. In general the magnitude of turbulent diffusion is smaller than production and dissipation; however in some situations it is a dominant term. For example, in separated flow the turbulent diffusion is important in the recirculation region as pointed out by Suga [67] by analyzing the flow over an obstacle in a channel. Turbulent diffusion term is also the easiest to measure among the three terms to be modeled and it seems that available models in general were based on assumptions that were valid only in the absence of anisotropy and viscous effects. Available models are discussed in the next section.

\subsection{Discussion of Turbulent Transport Modeling}

Transport equations for turbulent flow were first introduced by Chou [6], who used them to model the flow between two flat plates subject to a pressure gradient. These equations included the mean flow equations, Reynolds stresses transport equations and the triple-velocity correlations (turbulent transport) transport equation. In the equation for turbulent transport, Chou argued that the fourth order correlations together with velocity-dissipation rate correlations are small compared to the two velocity component pressure-gradient correlations in the context of parallel flow between two flat plates. Chou also neglected the velocity-instantaneous dissipation correlation compared to viscous diffusion term. These assumptions were made for the special case of flow between two parallel plates.

Davydov [9], further simplified the transport equations for the triple-velocity correlations by neglecting all viscous terms and modeled the two velocity component pressuregradient correlations as a function of third order moments. Davidov also used the Millionshchikov's, [35], approximation of fourth order correlation as the sum of second order correlations, assuming zero fourth order Cumulant. Again the above assumptions are valid only away from solid boundaries where viscous and anisotropy effects are negligible. These two pioneering models were differential in nature; simpler algebraic models were later obtained.

Daly and Harlow [8] proposed a simpler model using the analogy to the approximation for the flux of a scalar. The turbulent transport term was approximated using a gradient diffusion form. It is worth noting that in their paper they noted that the assumptions used to derive their model were partly correct. One drawback of this model is that 
it is not symmetric with respect to the indices in contrast with the term to be modeled. In other words it is not invariant with respect to coordinate transformation.

Mellor and Herring [33] developed another simple algebraic model of the gradient type in terms of gradients of the Reynolds stresses. The coefficients of the gradients were isotropic tensors and assumed symmetry in all directions. This model was based on purely mathematical considerations; no flow physics were included in the formulation. Hence it is not expected to portray the physics involved in turbulent transport process.

Hanjalic and Launder [18] developed a more rigorous model using the transport equations of the triple velocity correlations. Starting from the transport equation for the triple-velocity products, they arrived at their model by neglecting the convection term, viscous terms and using the Millionshchikov, [35], approximation of the fourth order correlations. In addition they expressed the two velocity component pressure gradient correlations as a function of the triple velocity correlations. Their model can be viewed as an isotropic version of Daly and Harlow model. Their model is intended for flow regions where the Reynolds number is high, away from solid boundaries, and assuming isotropy of small scales. They used this model successfully to compute turbulent flows in an annular channel and free shear flow. The agreement between this model and experimental data in general is good away from boundaries, in the core of channel flow, but departs from experimental data close to the wall as pointed out by Mansour and Kim [32]. This model is often used where the assumption made to derive it don't hold, this is due to its simple nature.

Another rigorous model was developed by Lumley and Khajeh-Nouri [28], where they developed a general approach to derive models of third order moments of any given order and for any order in Reynolds number. They assumed a tensor functional form of third order moments in terms of Reynolds stresses and dissipation rate, then Taylor series expansion of the arguments is formed and the coefficients are written in invariant form while expressing the stresses in terms of the anisotropy tensor. Finally the coefficients are determined by dimensional analysis.

Cormack et al. [7], extended Lumley and Khajeh-Nouri model to higher orders in anisotropy and in-homogeneity. The model contained four constants and can be considered as a generalization of all algebraic models stated above. Amano et al. [2] analyzed separating and reattaching flow behind a backward-facing step. In their calculations they solved the transport equation for triple velocity correlations directly. Several terms in the transport equations were modeled in order to close the system of equations. Their results showed some improvements over algebraic models; however it does not justify the added complexity of solving eleven partial differential equations.

Nagano and Tagawa [40], proposed a structural model based on the structure of turbulence, where they were able to compute third order moments of any order using skewness factors of the velocity components. They separated the contribution of different kinds of turbulent motion to turbulent transport (ejection, sweeps and interaction). 
They concluded that ejections and sweeps are the dominant contributors and hence they were able to relate different triple velocity correlations to the skewness of velocity components. However, in order to obtain the skewness of the velocity components the transport equations for the third order moments of these components need to be solved. Thus the number of equations to be solved is reduced.

Magnaudet [31], further extended Lumleys work to model turbulent transport for free surface flows. Magnaudet pointed out that all previous algebraic models didnt have the correct asymptotic behavior close to boundaries. The resulting model included gradients of the Reynolds stresses, turbulent kinetic energy and turbulent kinetic energy dissipation rate.

Shimomura [60], used a statistical approach to derive a model for turbulent diffusion. He used a two-scale direct interaction approximation where a scale parameter is introduced and the flow fluctuations are separated into rapid and slow variations using this scale parameter as a first approximation. The final expression obtained is similar to Hanjalic and Launder model except that it had a term containing the gradients of the dissipation rate. This model hasn $\mathrm{t}$ been popular in computations.

Mackinnon et al. [30], proposed two algebraic models for the triple velocity correlations. The first model was a modified gradient diffusion model and the second was an algebraic model derived from the triple velocity correlation transport equations. These models were evaluated and compared to the Daly and Harlow model and to experimental data for turbulent boundary layer with free-stream turbulence. In general both models were in good agreement with the data and responded correctly to changes in free-stream turbulence intensity. However, the gradients of predicted quantities differed significantly from the experimental data which would lead to large errors in computing the turbulent diffusion terms in the near wall region.

Straatman [66], modified Lumley's model by analyzing zero-mean shear turbulence and modifying the constants in the model. The model was then tested for jet flow, channel flow and a backward facing step. The model shows mild improvements over the original model.

Kurbatskii and Poroseva [22], modeled turbulent diffusion in a rotating cylindrical pipe flow. The model was differential where the triple velocity correlation transport equations were simplified and solved. The dissipation term was neglected and the two velocity pressure gradient correlations were expressed in terms of mean velocity gradients and triple velocity correlations and stress gradients. The results were compared with experimental data favorably; however other algebraic models gave a comparable result, which does not justify the added complexity of the differential model.

In a trial to find the most generalized model for third order moments and to include the effects of mean flow gradients, Younis et al. [72], formulated a generalized form of third order tensor functions in Reynolds stresses, their gradients and mean flow velocity 
gradients. Their work was based on the work of Smith [63]. They limited their formulation to linear and bilinear terms of Reynolds stresses, their gradients and mean flow velocity gradients. They also showed that all the above algebraic models were special cases from the general form that they have obtained except for Magnaudet 's model since it contained derivatives of the turbulent kinetic energy dissipation rate. Other algebraic models are also available; like Shir [61] and Wyngaard et al. [71] . These are basically modified versions of the above mentioned models. However they are less popular than the above mentioned ones. Despite the fact that efforts for modeling third order moments of turbulent fluctuations have been going on for almost six decades, there is no one model that performs well in all types of flow. Furthermore, all models perform poorly very close to solid boundaries.

There are some conditions that must be met by any model for it to be expected to perform well in different flow fields. These conditions were cited by Magnaudet, and repeated below,

- Invariant modeling: The closed form of third order moment correlations must be a fully symmetric third order tensor which vanishes in homogeneous situations. This requirement is purely mathematical which stems from the symmetry of the triple velocity correlations tensor. In reality in bounded flows for example there might be preferred directions like the free stream direction, however if the model equation contains the right physics it should detect these preferred directions.

- Realizability: If a velocity component fluctuations vanishes at a boundary, the third order moments normal to the boundary must vanish.

- Asymptotic consistency: If $u_{i}, u_{j}$ and $u_{k}$ decay as $y^{l}, y^{m}$ and $y^{n}$, respectively, then

$$
\overline{u_{i} u_{j} u_{k}} \propto y^{l+m+n}
$$

Other Realizability conditions were developed by Andre et al. [3], since most differential models neglect the viscous diffusion terms and as a consequence of quasi normality of fourth order correlations the third order moments tend to grow to high values which are not realistic. This condition is repeated below,

$$
\overline{u_{i} u_{j} u_{k}} \leq \operatorname{Min}\left\{\begin{array}{l}
\sqrt{\overline{\overline{u_{i}^{2}}}\left(\overline{u_{j}^{2}} \overline{u_{k}^{2}}+\overline{u_{j} u_{k}}\right)} \\
\sqrt{\overline{u_{j}^{2}}\left(\overline{u_{k}^{2}} \overline{u_{i}^{2}}+\overline{u_{k} u_{i}^{2}}\right)} \\
\sqrt{\overline{u_{k}^{2}}\left(\overline{u_{i}^{2}} \overline{u_{j}^{2}}+\overline{u_{i} u_{j}^{2}}\right)}
\end{array}\right\}
$$

The fact that available models do not satisfy the above conditions is clear when these models are used to predict turbulent transport for a given flow and compared to experimental data. For example, Mansour tested the Hanjalic and Launder model to predict 
turbulent transport in fully developed channel flow and compared it to their DNS simulations. The model seemed to work well far away from the wall, $y^{+}>100$ but close to the wall it seems to underpredict turbulent transport. More importantly the model doesn ' $t$ always have the right trend for some of the components of the turbulent transport tensor. This was revealed by the cross-streamwise turbulent transport of the shear stress.

Ölçmen and Simpson [44], also tested several algebraic models to predict turbulent transport around a wing-body junction flow at the center of a horse-shoe vortex. In this comparison all models performed poorly in predicting this complex flow. Hence, in the present work an experiment is setup to provide a highly three dimensional flow to obtain low uncertainty measurements of mean flow velocities, Reynolds stresses and the triple velocity correlations to serve as a test case for improving modeling efforts of triple velocity correlations. Flow velocities are measured using Laser Doppler Velocimetry (LDV) techniques at a number of locations. Also spectral measurements are made at selected stations. The layout of the dissertation is given in the next section.

\subsection{Dissertation Organization}

The dissertation is organized as follows. In the first chapter an introduction and literature survey is presented as well as the motivation for this work. In chapter two the experimental facility is described along with the instrumentation and data acquisition systems used to acquire the measurements. A description of side wall boundary layer control procedure and final setup is also given in chapter two.

In chapter three mean flow data at different stream-wise planes are presented. In chapter four LDV measurements are presented. The measurements include mean flow velcoity components, all six components of the Reynolds Turbulent stresses, triple velocity product correlations and Power and Cross-Spectra measurements. In chapter five the results are discussed and some features of the experiment are highlighted and interpreted.

In chapter six different models for turbulent diffusion and velocity-pressure gradient correlations are compared to experimental data with suggestions for ways to improve modeling. Finally conclusions are presented in chapter seven. Appendices are added at the end that contain the wavy wall test section geometry data in Appendix A, boundary layer suction slots data in Appendix B, matching procedure and uncertainties in LDV measurements in Appendix $C$ and finally a simple numerical procedure used to calculate the velocity field inside the test section to help interpret the data in Appendix D. 


\section{Chapter 2}

\section{Experimental Setup and Instrumentation}

\subsection{Experimental Facility}

The test facility used in this research is the Virginia Tech Aerospace and Ocean Engineering Department Boundary Layer Wind Tunnel. It is a custom made open-circuit wind tunnel powered by a $19 \mathrm{~kW}$ centrifugal blower. The air blower withdraws ambient air through a rectangular intake that is equipped with a set of 8 air filters to prevent dust particles from entering the wind tunnel. After passing through the blower the air passes through a variable shutter to set the flow speed, then it enters into a plenum which is followed by a honeycomb section to remove large swirls in the mean flow, and seven screens to make the flow more uniform and reduce the intensity of remaining turbulence. Downstream of the screens the air passes through a $4: 1$ contraction to accelerate the flow to the test speed and further reduce turbulence intensities remaining.

The wind tunnel test section is composed of two main parts; one part is common to all experiments conducted in this facility and a second one that can be modified according to each experiment. The total length of the test section is $7.62 \mathrm{~m}$. The common part extends for about $2.34 \mathrm{~m}$ starting from the contraction exit and is $0.91 \mathrm{~m}$ in width. A $0.63 \mathrm{~cm}$ trip is located at the inlet to the test section in order to initiate the turbulent boundary layer. The ceiling height of this part varies along its length reaching a minimum of $0.25 \mathrm{~m}$ at about $1.41 \mathrm{~m}$ from the contraction exit. Downstream of this minimum the height increases to a value of $0.267 \mathrm{~m}$. The second part of the test section is usually rectangular in shape with parallel flat side walls and flat or tapered ceiling.

However in this experiment wavy sidewalls were used. The flow qualities with flat parallel side walls test section were studied extensively by Ahn [1] and Devenport and Simpson [11]. When the free stream speed is $27 \mathrm{~m} / \mathrm{s}$ the potential core of the flow is uniform to within $0.5 \%$ in the span-wise direction and $1.0 \%$ in the vertical direction and has a turbulence intensity of $0.1 \%$, Byun [4]; Ma and Simpson [29]. More information can be 
found on the department's website ${ }^{1}$

\subsection{Wavy Wall Test Section Geometry}

The test section is designed in a way such that an alternating sign span-wise pressure gradient is formed at the centerline of the test section. This is done by forming both side walls in the shape of sine waves that are in phase. In order for the pressure gradient to be felt at the centerline the wave amplitude was chosen to be $a=3$ " and the wave length equal to $\lambda=24$ ". Given the large side wall amplitude, boundary layer control is used to prevent the flow from separation over the side walls. The test section is length $L=16^{\prime}$ long and width $W_{x=0}=36.25$ " at the entrance.

Each side wall is composed of an upstream and downstream part, as well as the ceiling. The upstream side walls form a contraction in the test section's width, this contraction is $2^{\prime}$ long and the width is reduced by 4" at the end of contraction. Downstream of the contraction the side walls are curved over a $1^{\prime}$ to take on the shape of sine waves of amplitude $a=3$ " and wavelength of $\lambda=24$ ". Each side wall (upstream plus downstream part) has six full sine waves. The side walls are made of perforated aluminum sheets so that boundary layer suction can be applied at any given location along the test section length. The aluminum sheets used have $1 / 8$ " diameter staggered holes and the centers were 3/32" apart. Attached to the side walls are suction cabinets where the air withdrawn from the wavy channel exits the test section. Each cabinet is fitted with a gate valve to control the amount of air exiting the test section. There are a total of 16 suction cabinets attached to the side walls 8 cabinets on each side. One cabinet is attached to each wave. Also a cabinet is attached to the flat contracting part of the side wall on each side. The test section ceiling is made of 0.5 " Plexiglas that is cut in a way such that it has the same shape as the plan view of the test section. In order to compensate for the amount of air withdrawn from the test section, the ceiling is kept flat but having a negative gradient of 0.013 to attempt to have constant stream-wise pressure. The height of the ceiling at the entrance of the test section is 10.5 " and at the exit the height is reduced to 8". A plan view of the test section geometry is shown in Figure 2.1 and a side view schematic of the whole wind tunnel configuration is shown in Figure 2.2. Also a picture of the test section is shown in Figure 2.3. Detailed listing of the test section geometry is presented in Appendix A as well as uncertainty in measuring the geometry.

\footnotetext{
${ }^{1}$ http://www.aoe.vt.edu/research/facilities/bllab.php/
} 


\subsection{Measuring Instruments and locations}

Different instruments were used to obtain mean flow data as well as turbulent fluctuations. A brief description of each instrument is given below.

\subsubsection{Total Pressure Rake (16 tubes)}

Profiles of total pressure were made using a home-made compact probe rake consisting of 16,1/16" diameter parallel tubes soldered together, shown in Figure 2.4. Profiles were taken at the wave peaks of the test section at different streamwise locations in order to investigate the development of the flow over the wave peaks and whether the boundary layer is growing over the peaks or not. A total of 10 measurements were made using this rake, the locations and x-coordinates of each measurement are shown in Figure 2.9. Static pressure measurements were taken at the same location in order to calculate the local speed and obtain velocity profiles near the side wall at the mid-height point between the floor and the local test section ceiling. A home-made probe holder assembly was constructed to hold both the 16-tube rake and the Pitot-tube together so that they can be traversed vertically and in the span-wise direction to switch probes and to measure span-wise variation of pressure respectively. The assembly is shown in Figure 2.5 .

\subsubsection{Pitot-Static Tube}

A Pitot-static tube was used to measure the total and static pressure at different locations in the test section. Total and static pressures were measured along the centerline. While static pressures were measured at the wave peaks. The probe was rotated along the vertical axis until a maximum reading was obtained. A Dwyer Probe model 160 was used coupled with a Dwyer digital manometer model 474-FM mark III to calculate the pressure differential in inches of water. The manometer had an uncertainty of \pm 0.005 inches of water.

\subsubsection{Scannivalve system}

Floor surface pressures measurements were made using an 8 " diameter plate which had 426 pressure taps spanning an area of $2 " \times 8$ ". These pressure taps were connected to a Scannivalve system model CTLR2P/S2-S6 manufactured by Scanivalve Corp. A Setra 239 pressure transducer with a 15 " of water range was used to measure the floor pressure. A 286 IBM PC with a DT2801 A/D board for acquiring data was connected to the Scannivalve system to acquire data. The plate was used to measure the surface pressure 
at three different locations where the LDV profiles were taken to account for the pressure gradients while calculating the friction velocity $u_{\tau}$. The locations were the pressure measurements were taken are shown in Figure 2.1.

\subsubsection{Seven-Hole Pitot tube}

A seven-hole Pitot tube shown in Figure 2.6 was used to obtain the mean flow velocities and directions at different planes along the tunnel centerline. This probe was first used and documented by Wenger [70]. Pisterman [50] used the same probe to investigate the flow field around an axisymmetric bump flow. The same system setup, data acquisition and reduction as of Pisterman is used in this work. The probe calibration was done by Aeroprobe corporation ${ }^{2}$. The seven-hole probe has seven holes to detect the direction and magnitude of the flow velocity. One hole is at the center of the probe while the other six are distributed circumferentially around the center one. The probe has a conical tip with a half angle of $30^{\circ}$. The uncertainties in measuring flow angles and velocity magnitude are presented in Chapter 3 . The seven-hole probe is connected to seven differential pressure transducers through seven Tygon tubes with an internal diameter of $1 / 16$ ". The transducers, XPCL04DTC, have a range of \pm 2.5 " of water. Since the total pressure inside the test section was larger than the transducer's maximum pressure, reference pressure ports were connected to the free stream total pressure.

\subsubsection{Laser Doppler Velocimetery}

Two 3-velocity component LDV probes were used to measure the mean flow velocities as well as Reynolds Stress and Turbulent Transport tensors at some selected positions. Both probes shared the same optical table equipment, however the setup differed slightly. A brief description of each is given below.

\section{Optical Table}

The optical table contained two laser sources, mirrors, beam splitting cubes, polarization rotators, Bragg cells, laser to fiber couplers and fiber optics. In the present study beams with different colors were used; purple $(476.5 \mathrm{~nm})$, blue $(488.0 \mathrm{~nm})$ and green $(514.5 \mathrm{~nm})$ beams. The laser beams were supplied by INNOVA $90-5 \mathrm{C}$ argon-ion laser for both systems.

\footnotetext{
${ }^{2}$ http://www.aeroprobe.com/
} 


\section{Short System LDV Probe}

The Short System LDV probe is a fiber-optic argon-ion laser system developed by Ölçmen [42]. This system uses five laser beams, two blue beams $(488.0 \mathrm{~nm})$ and three green beams $(514.5 \mathrm{~nm})$. In order to resolve direction ambiguity the beams were frequency shifted using Bragg cells, IntraAction Acousto-optics AOM 405. The blue beams have a Bragg cell frequency shift of $0 \mathrm{MHz}$ and $40 \mathrm{MHz}$ while the green breams have a frequency shift of $0 \mathrm{MHz}, 30 \mathrm{MHz}$ and $50 \mathrm{MHz}$.

This probe was designed so that in the region $y \leq 40 \mathrm{~mm}$ the effective volume is $30 \mu \mathrm{m}$, hence it would be capable of resolving the rapid variations occurring in the velocity fields. Different researchers were able to use this probe as close as $50 \mu \mathrm{m}$ away from the wall, [42], [4].

LDV measurements very close to the wall, $y^{+} \leq 10$ are affected by Gradient Broadening effects. For the Short System LDV this is not significant for first and second moments due to small measurement volume size. However, for third and higher order correlations this is more significant. Hence, Gradient Broadening corrections were applied to all measurements.

\section{CompLDV Probe}

The CompLDV probe was developed by Lowe [25]. This system was designed to use up to 12 beams to measure velocity fluctuations as well as velocity-acceleration correlations and velocity gradients, however only six beams were used during the present work.

This probe used three colors, purple $(476.5 \mathrm{~nm})$, blue $(488.0 \mathrm{~nm})$ and green $(514.5 \mathrm{~nm})$. The probe consisted of three different heads that are adjusted independently. Each head receives two laser beams from the optics table through fiber optics. One beam of each pair is frequency shifted by the Bragg cells. The frequencies used are $40 \mathrm{M} \mathrm{Hz}, 60 \mathrm{M} \mathrm{Hz}$ and $80 \mathrm{MHz}$ for the purple, green and blue respectively. The receiving lens is located at the center of the three heads and attached to the green beams' head. The measurement volume of this system is approximately $120 \mu \mathrm{m}$. Since it has a larger measurement volume this system was used away from the wall $(y \geq 120 \mu m)$. The probe assembly is shown in Figure 2.7

\subsubsection{Seeding System}

In order to measure flow velocities using the LDV probes the flow had to be seeded. Di-Octal Phthalate (DOP) was used as a seeding fluid. 
In order to reduce the size of the seeding particles, the DOP is mixed with air under high pressure. The mixture is then heated up by passing through heated pipes. The mixture is then allowed to settle in a closed container then it is injected into the wind tunnel. The mixture is injected at the beginning of the test section through a row of tubes attached to the tunnel's floor.

The aerosol generator was originally designed by Echols and Young [13] and described by Ölçmen [42]. The seeding particles size distribution was measured by a TSIAerodynamic article Sizer and the geometric mean diameter, $\bar{d}_{g}$, of particles was calculated to be $0.956 \mu \mathrm{m}$ and the geometric standard deviation, $\sigma_{g}$, was $1.51 \mu \mathrm{m}$.

\subsubsection{Data Acquisition and Processing}

The light signals collected by the receiving lens of both probes is passed through three separate photomultiplier tubes, Hamamatsu model R4124, which converts all light signals into electrical signals. The signals are then amplified using three Sonoma Instrument 315 amplifiers. The blue and purple signal were merged together into one signal and the green light formed another signal. Both signals are then digitized using Strategic-Test model UF.258 high-speed digitizer board, which had an 8-bit resolution and $250 \mathrm{MS} / \mathrm{s}$ sampling rate. Data sampling was done in multiple short durations, $0.54 \mathrm{sec}$ durations, and a total of approximately $30 \mathrm{kSamples}$ were obtained for each data point. For mean flow and turbulences statistics, burst detection was used for triggering. The acquired data was then stored on multiple hard-drives for later processing.

Signal processing was done electronically using in-house codes developed by Lowe, [25]. The codes included different modules for burst recognition, dual-burst separation and frequency processing, [26]. The burst recognition is done by fitting the root-meansquare (RMS) of the signal to a Guassian distribution. The frequency spectrum is obtained for each valid burst and the Doppler frequency is calculated for each light signal. A threepoint Gaussian fit is used around each peak.

Finally, after determining the Doppler frequency for all light signals, the velocity component in each optical measurement direction is calculated by multiplying the associated frequency by the fringe spacing in that direction. Knowing the velocity components in the optical axes, a coordinate transformation is done to transform the velocity components into tunnel coordinates. Histograms of all three velocity components are constructed in order to remove noise in measured data by rejecting unphysical points in the skirts of the distribution. This is done by smoothing the histogram using built-in function in MATLAB, "csaps", that uses a smoothing parameter. If the smoothing parameter is set to 0 a least-squares straight line fit is performed, on the other hand if the smoothing parameter is set to 1 , a cubic spline fit is performed and the original data is recovered. The smoothing parameter was always set to 0.98 or higher. After smoothing the histogram the location where the smoothed histogram first reaches a lower threshold. This thresh- 
old was usually taken to be 1-3 samples. Then the original non-smoothed histogram is clipped at the identified limits. This approach was tested with a Poisson distribution with added random noise and yielded excellent results. Applying this approach to measured data resulted in very smooth statistics especially third-order moments. Using the clipped original histogram, mean flow velocities, turbulent Reynolds Stresses, third and fourth moments were calculated.

\subsection{Boundary Layer Control}

In order to achieve the required flow field, boundary layer control had to be implemented. Since the waves on the side wall had a large amplitude to wavelength ratio $2 a / \lambda=0.25$, separation occurred down-stream of each wave peak without boundary layer control. Initially boundary layer control was implemented by having the side walls made of perforated aluminum sheets starting at $x=2^{\prime}$ along the tunnel axis. The sheets were covered with clear adhesive plastic tape that covered the perforated sheets except where suction is allowed. The aluminum sheets had 1/16" diameter staggered holes and a total of $22 \%$ of open area. Boundary layer suction was implemented using a suction fan that had a flow rate of $3000 \mathrm{cfm}$ at $14 " \mathrm{H}_{2} \mathrm{O}$ pressure difference. The first suction trials had most of the area downstream of the wave's peaks open for suction. However, these trials were not successful in eliminating separation. It was decided then to use larger holes for suction to reduce losses and increase suction rate. The aluminum sheets were replaced by similar aluminum sheets that had larger holes, $1 / 8$ " diameter, and $40 \%$ total open area. The holes' centers were placed at $3 / 32$ ". Figure 2.8 shows a picture of the inlet side wall taken from upstream of the test section inlet through the wind tunnel's glass side wall. The perforated aluminum sheets covering the side walls can be seen in this picture.

During the second trial several suction patterns were tried to eliminate separation. It was possible to eliminate separation but at a very low free stream speed, $50 \mathrm{fps}$. This was due to limited suction capability such that the flow speed had to be reduced so that the required flow to be withdrawn out of the test section is the same as the fan capacity. Having realized this problem, we had to find another way to implement suction.

It was decided to raise the tunnel's internal pressure so that the flow was ejected from inside the tunnel to the outside through the holes under the effect of the pressure difference from the atmospheric pressure. This was done by inserting a set of back pressure screens with different grid densities at the downstream end of the test section in order to increase the flow resistance and hence increase the total pressure inside the tunnel. A pressure rise of $7.2 " \mathrm{H}_{2} \mathrm{O}$ above the ambient pressure was achieved. This helped increase the amount of side wall suction. However, the flow was still separated in parts of the test section.

The failure of the second trial led us to try using vortex generators to energize the 
boundary layer close to the surface of the side walls. It was also noted that the boundary layer at the end of the contraction at the beginning of the test section was too big. There was no suction applied along both sides of the contraction. Therefore it was decided that suction should be applied at the middle of the flat sides of the contraction at the entrance to the wavy wall test section. One row of vortex generators pairs was installed 4" upstream of each peak and another one about the same distance downstream. After applying this setup it was noticed that the vortex generators weren't effective and that the flow separated after going over the peak. It was also noticed that the flow stayed attached over the first wave when the vortex generators were taken off and suction was applied at the middle of the flat sides of the contraction and using few suction slots along the first wave. Hence, it was decided to remove all of the vortex generators and locate where suction slots are need along each wave.

The locations where suction had to be applied were determined by trial and error. Starting at the end of the contraction, rows of 2" long tufts were attached to the side walls in order to know where separation occurs. Once the tufts start moving randomly this indicated separation, a slot was then cut upstream of that row. Slots were cut vertically along the whole height of the test section and their width varied according to the amount of suction needed. This process was continued on both side walls of the test section going in the downstream direction until the end of the test section was reached. The final locations of the suction slots are shown in Figure 2.1. The stream-wise coordinates, width of each slot as well as the number of holes are given in Appendix B. The suction flow rates coming out of each cabinet were measured using a Lambda Square Venturi- meter tube model 2300 with a throat diameter of 2.6" and inlet diameter of 5.8". The measured volume flow rates from each cabinet are shown in Figure 2.10 and listed in Table 2.1.

In order to make sure that all or most of the boundary layer flow along the side walls was either minimized or removed, the velocity profiles at the local mid height of each wave was measured. This was done by measuring the total and static pressure profiles normal to the side wall. The total pressure was measured using the pressure rake which was connected to the Scannivalve system while the static pressure was measured using an ordinary Pitot-Static tube. The pressure rake and the Pitot-Static tube were attached to the same probe holder but they were separated with a fixed distance in the vertical direction. The total pressure rake was aligned to the mid point at each wave then a measurement was taken; then the probe was displaced in the horizontal direction to complete the profile. Afterward the probe was moved vertically and the Pitot-Static tube was aligned to the mid point and the static pressure was measured at selected points. Since the static and total pressures were not measured at the same normal distances from the wall, the static pressure measurements were curve-fit and evaluated at the same locations as the total pressure. Then the flow speed is calculated from the dynamic pressure.

Due to the complexity of the test section's shape, the velocity profiles normal to the side wall were measured at few selected stream-wise locations which are shown in Figure 2.9. A plot of the normalized velocity profiles at different streamwise locations for the 
left and right side walls are shown in Figures 2.11 and 2.12 respectively. It is clear from these plots that the boundary layer thickness is less than $0.30 \mathrm{in}$ for all peaks shown. The locations normal to the side wall of velocity profile maxima is given in Table 2.2. Figure 2.13 shows the $C_{p}$ distributions at the side wall peaks for both side walls. These pressure coefficients were obtained from the first rake port measurement. This distribution can be utilized for numerical flow simulation.

For any attached viscous flow over a curved surface the velocity profile reaches a maximum at the edge of the boundary layer then goes to zero at the wall. In the absence of a boundary layer the maximum velocity occurs at the surface. This fact was used to judge if the side wall boundary layer was removed effectively or not. It is clear that a value close to unity occurs within at most $0.3 \mathrm{in}$ away from the wall.

\begin{tabular}{lcccccccc}
\hline Cabinet\# & 1 & 2 & 3 & 4 & 5 & 6 & 7 & 8 \\
\hline Right Wall & 163 & 356 & 582 & 402 & 365 & 350 & 347 & 254 \\
\hline Left Wall & 178 & 234 & 460 & 446 & 317 & 383 & 308 & 286 \\
\hline
\end{tabular}

Table 2.1: Boundary Layer Suction Volume Flow Rate in $f t^{3} / \mathrm{min}$.

\begin{tabular}{cccc}
$X_{\text {Right Wall }}[f t]$ & $Z_{U_{\max }}[$ in. $]$ & $X_{\text {Left Wall }}[\mathrm{ft}]$ & $Z_{U_{\max }}[$ in. $]$ \\
\hline 2 & 0.196 & 3 & 0.156 \\
6 & 0.094 & 5 & 0.031 \\
10 & 0.288 & 9 & 0.031 \\
12 & 0.125 & 11 & 0.031 \\
14 & 0.188 & 13 & 0.031 \\
\hline
\end{tabular}

Table 2.2: Location of Side Wall Velocity Profiles Maxima 


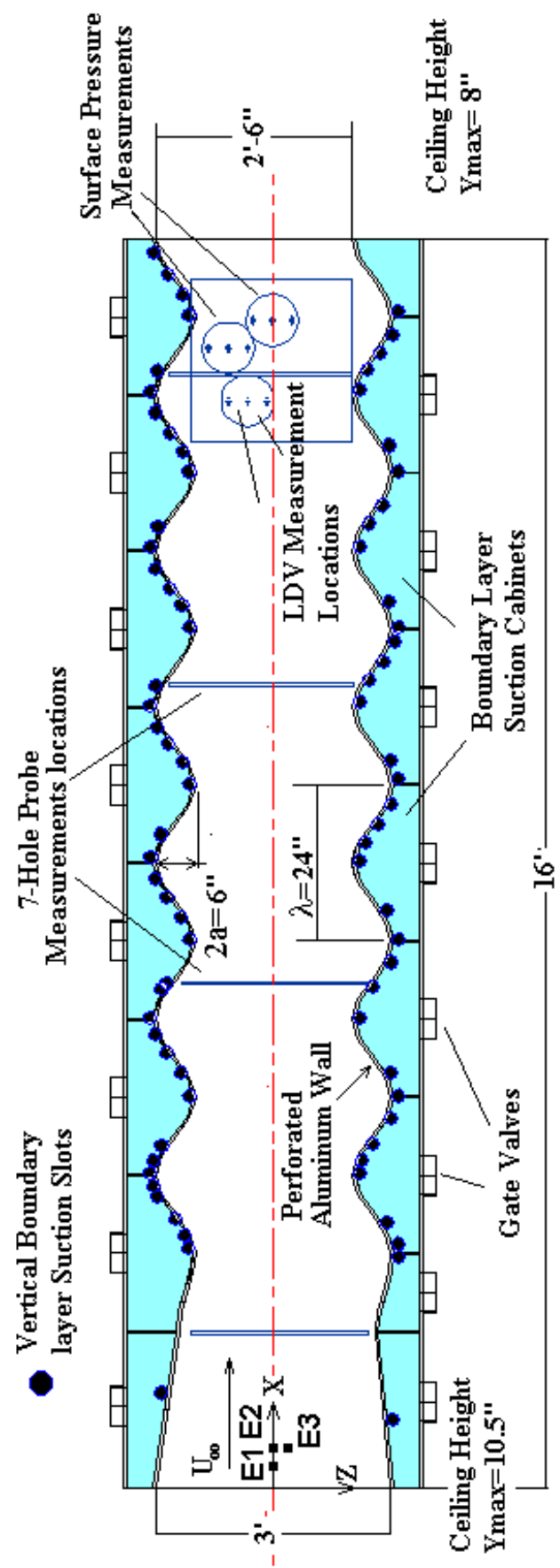

Figure 2.1: Wavy Wall Plan View 


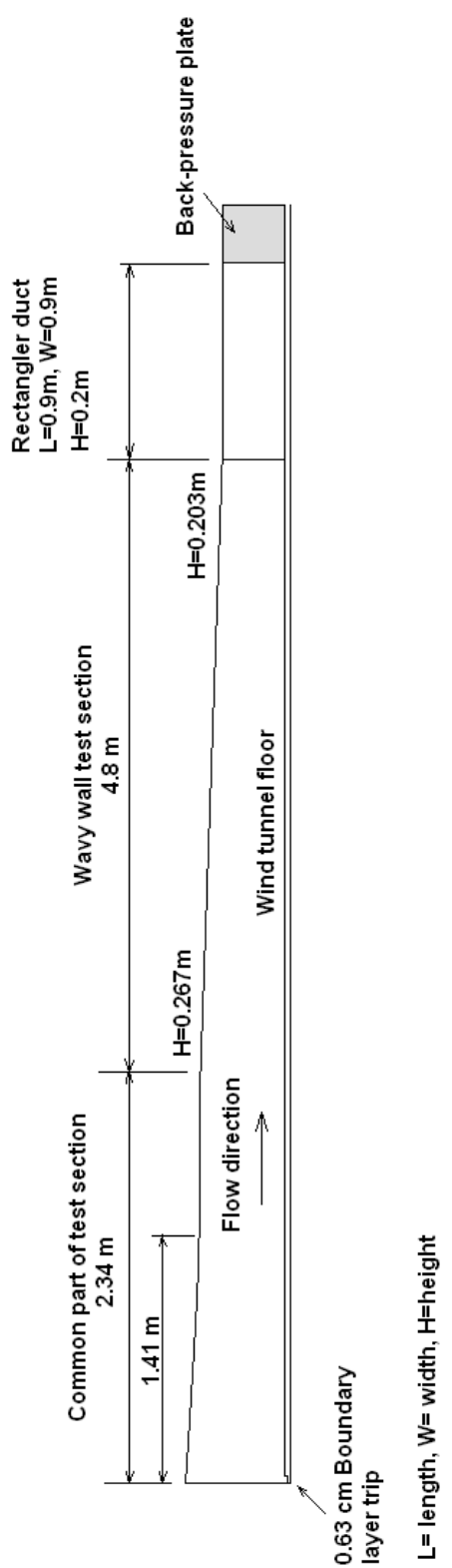

Figure 2.2: Wind Tunnel Side View Schematic 


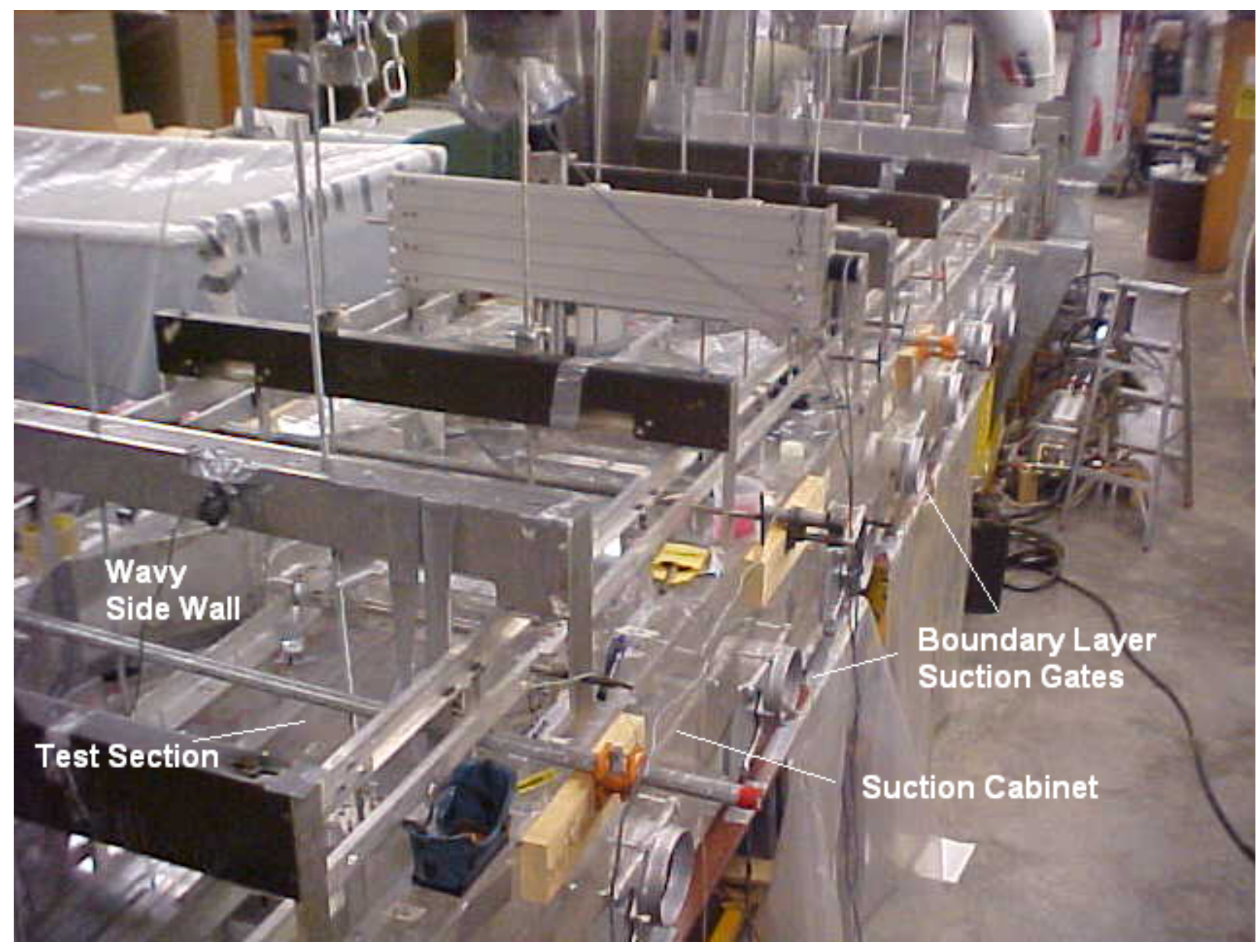

Figure 2.3: Wavy Wall Right Side Wall 


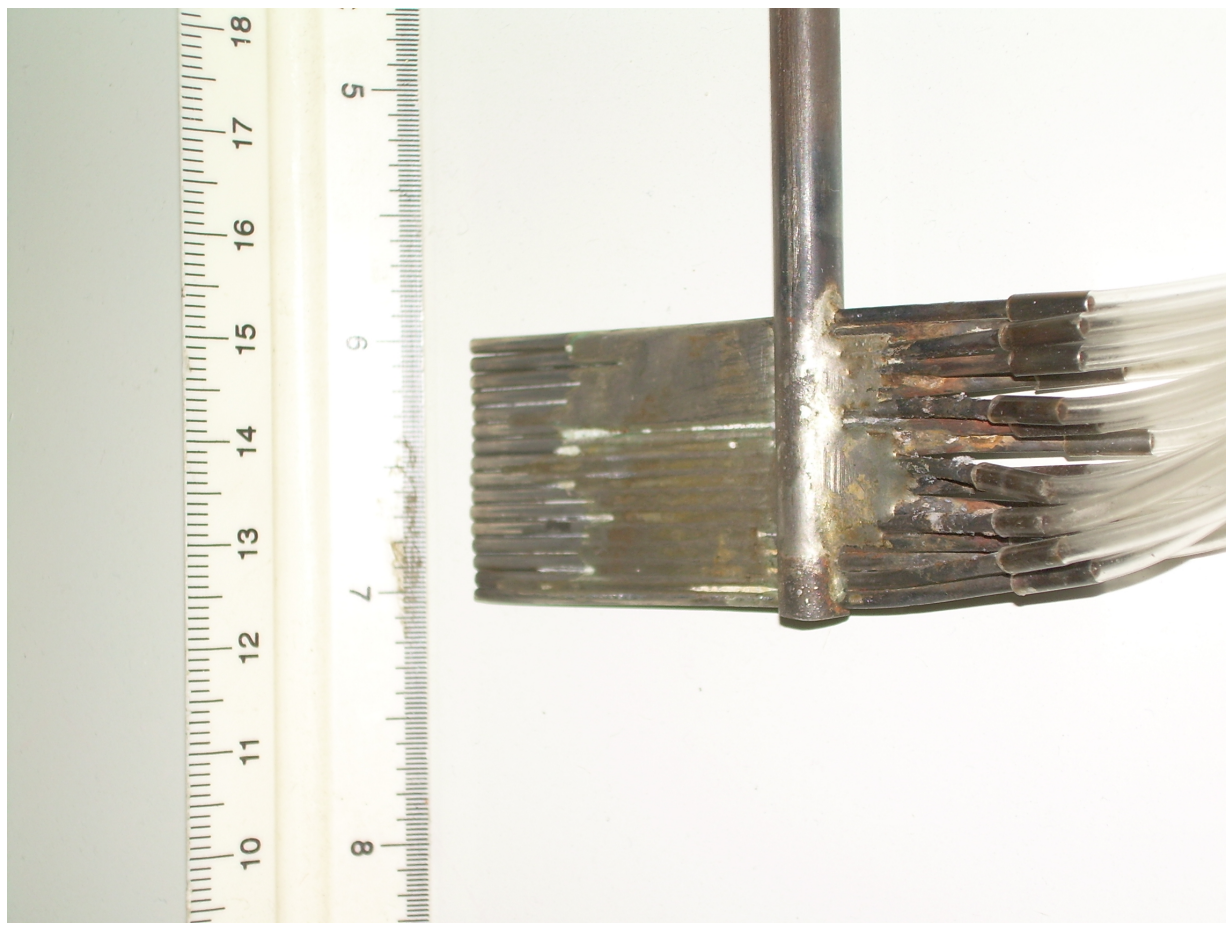

Figure 2.4: 16 hole Total Pressure Rake

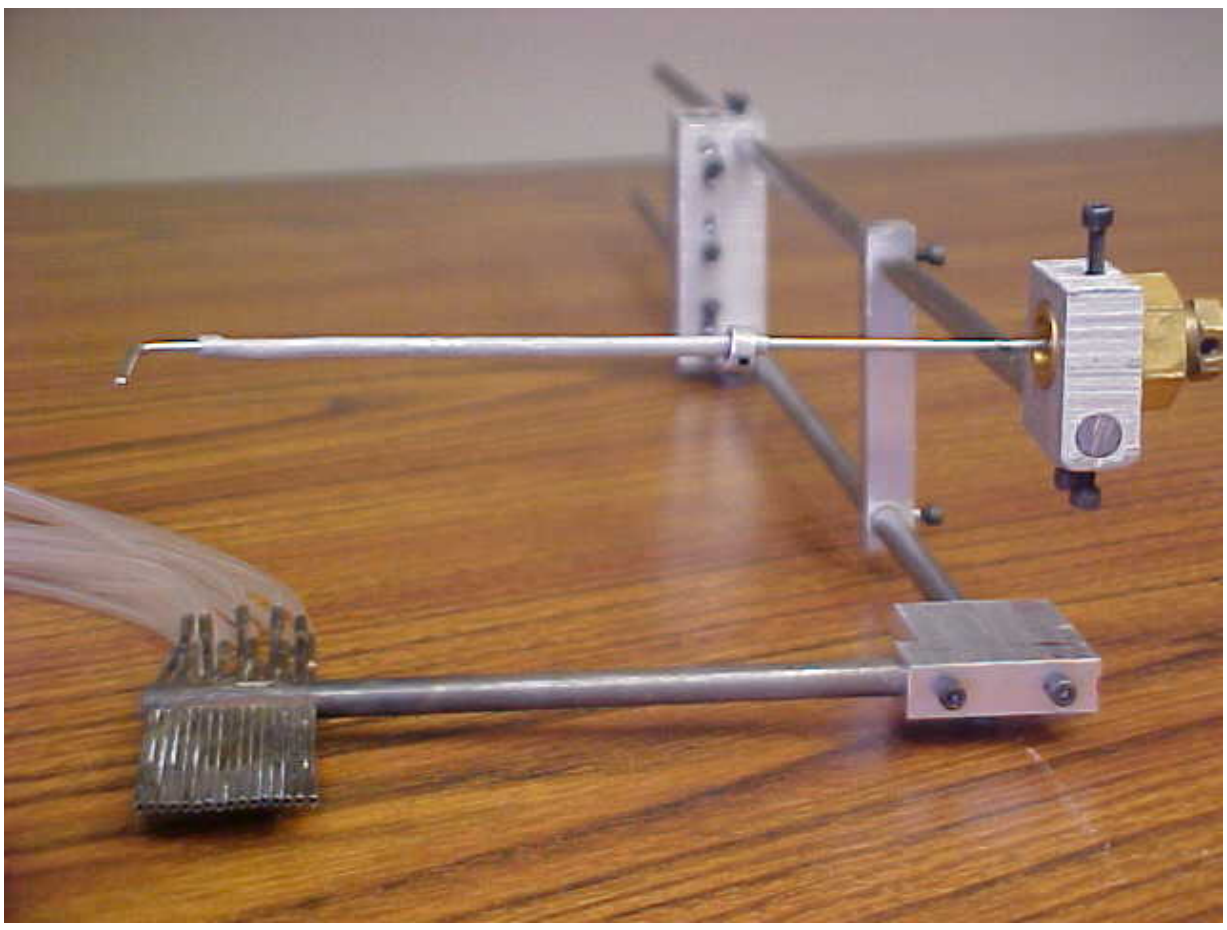

Figure 2.5: Rake-Pitot Tube assembly 


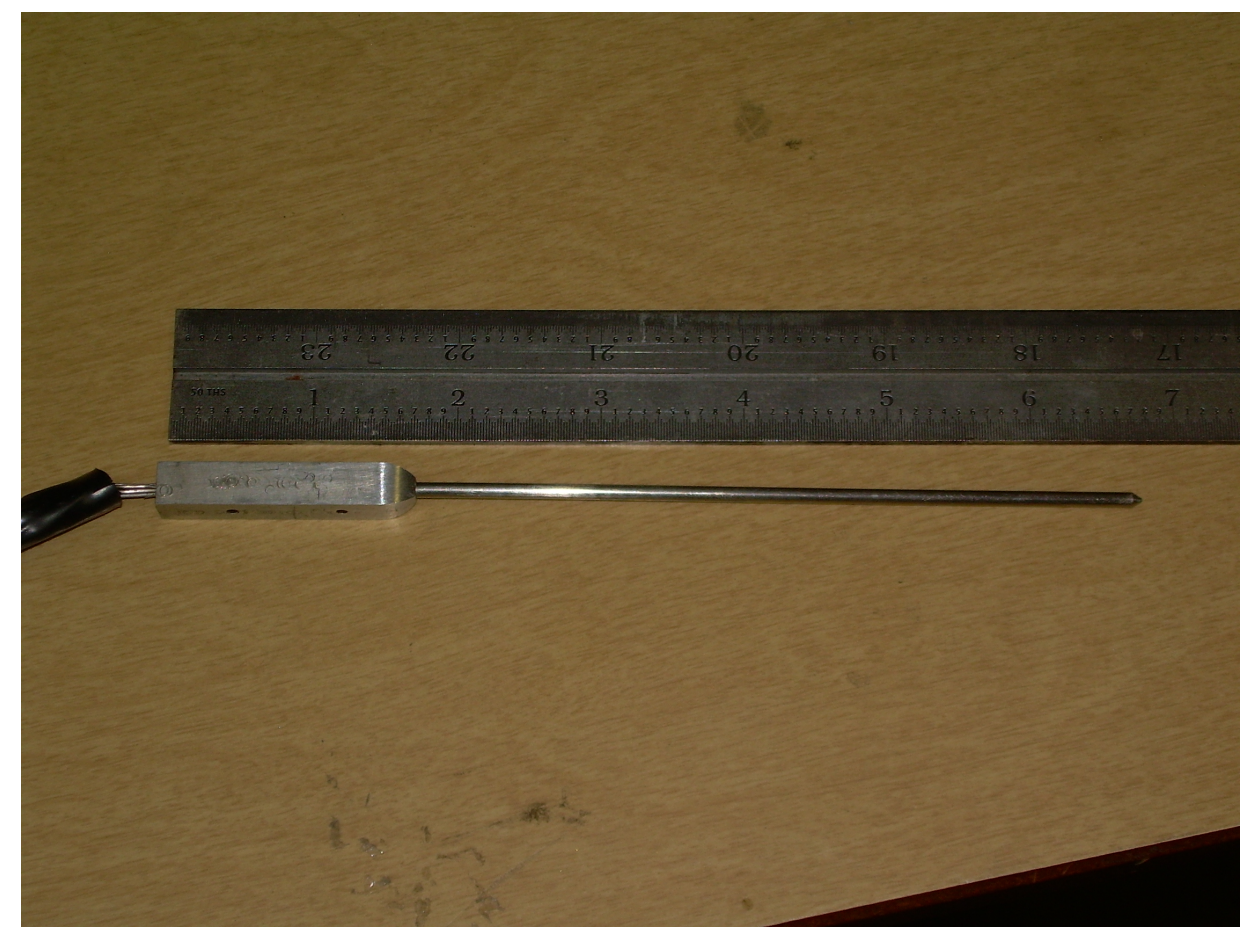

Figure 2.6: Seven-Hole Probe

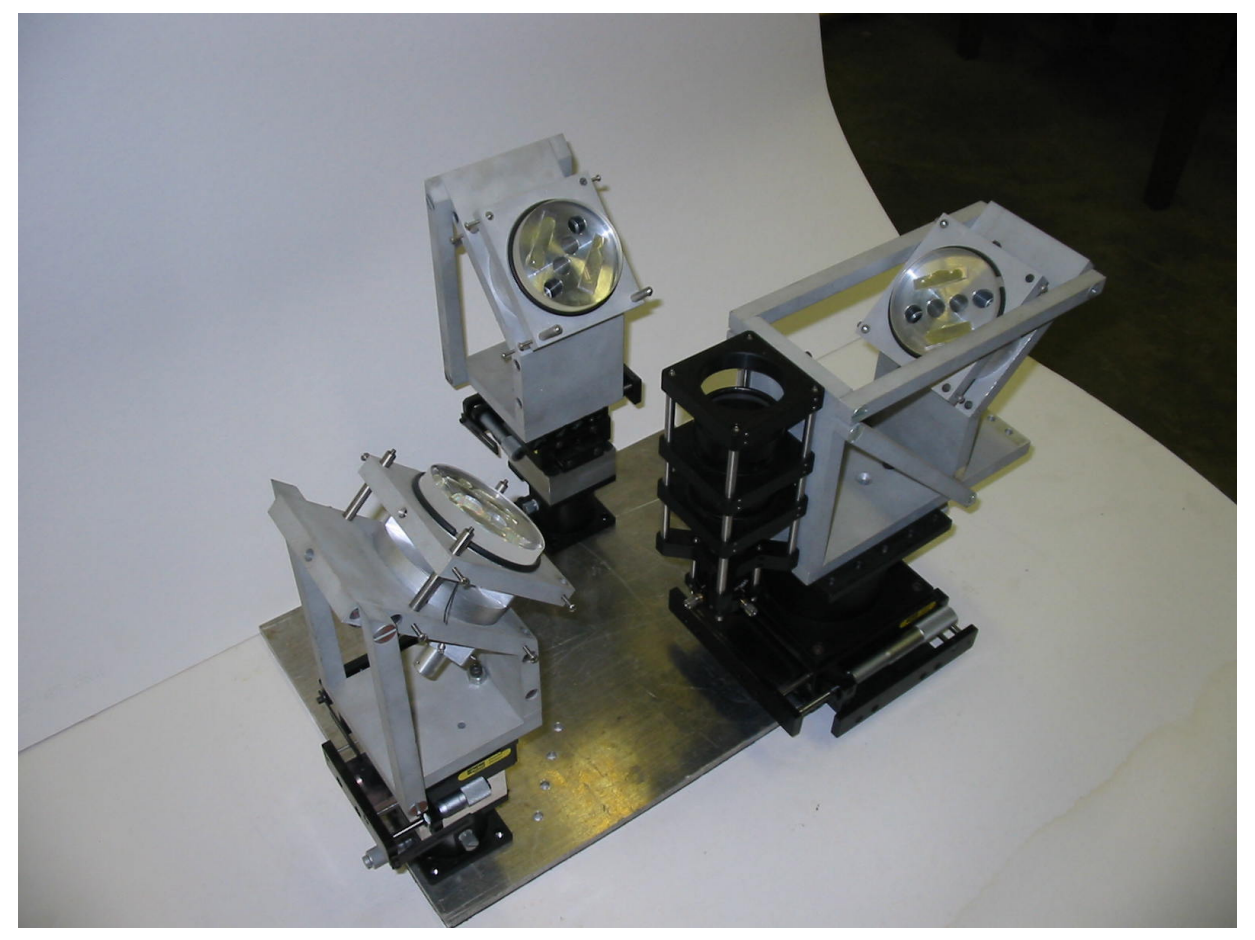

Figure 2.7: CompLDV Probe Head Assembly 


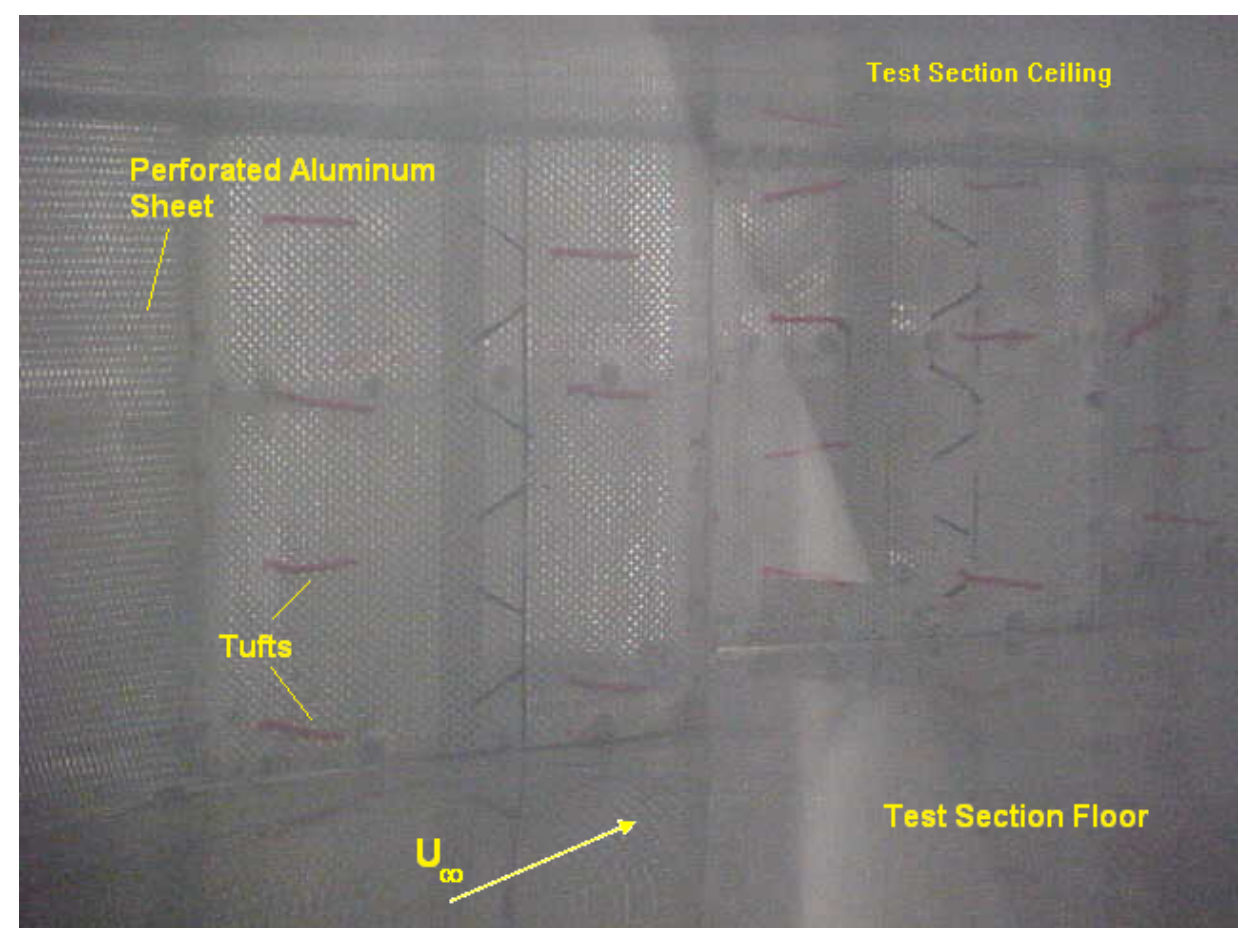

Figure 2.8: Wavy Wall Test section Inlet Side Wall showing tufts attached to the side wall 


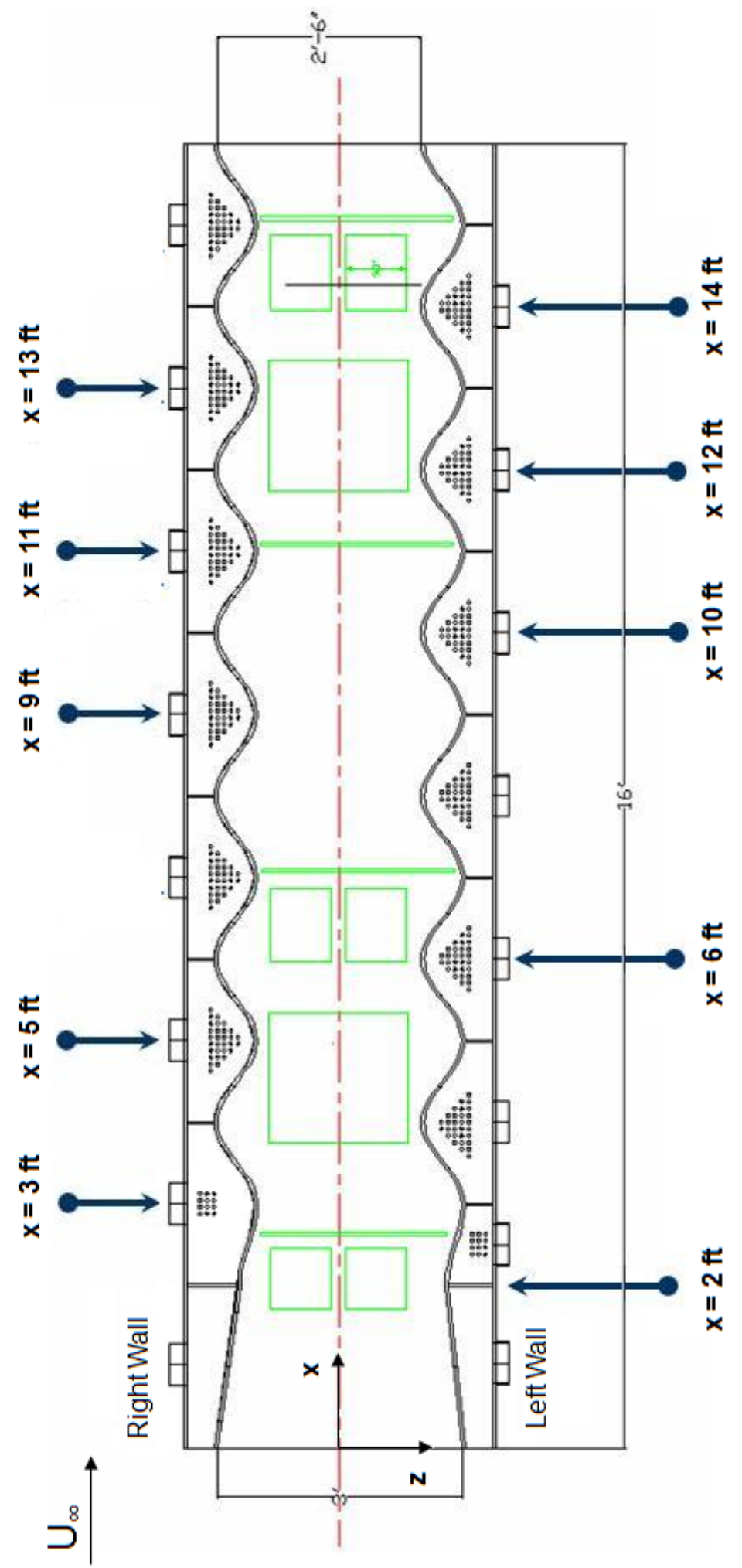

Figure 2.9: Side Wall Boundary Layer Measurement Locations 


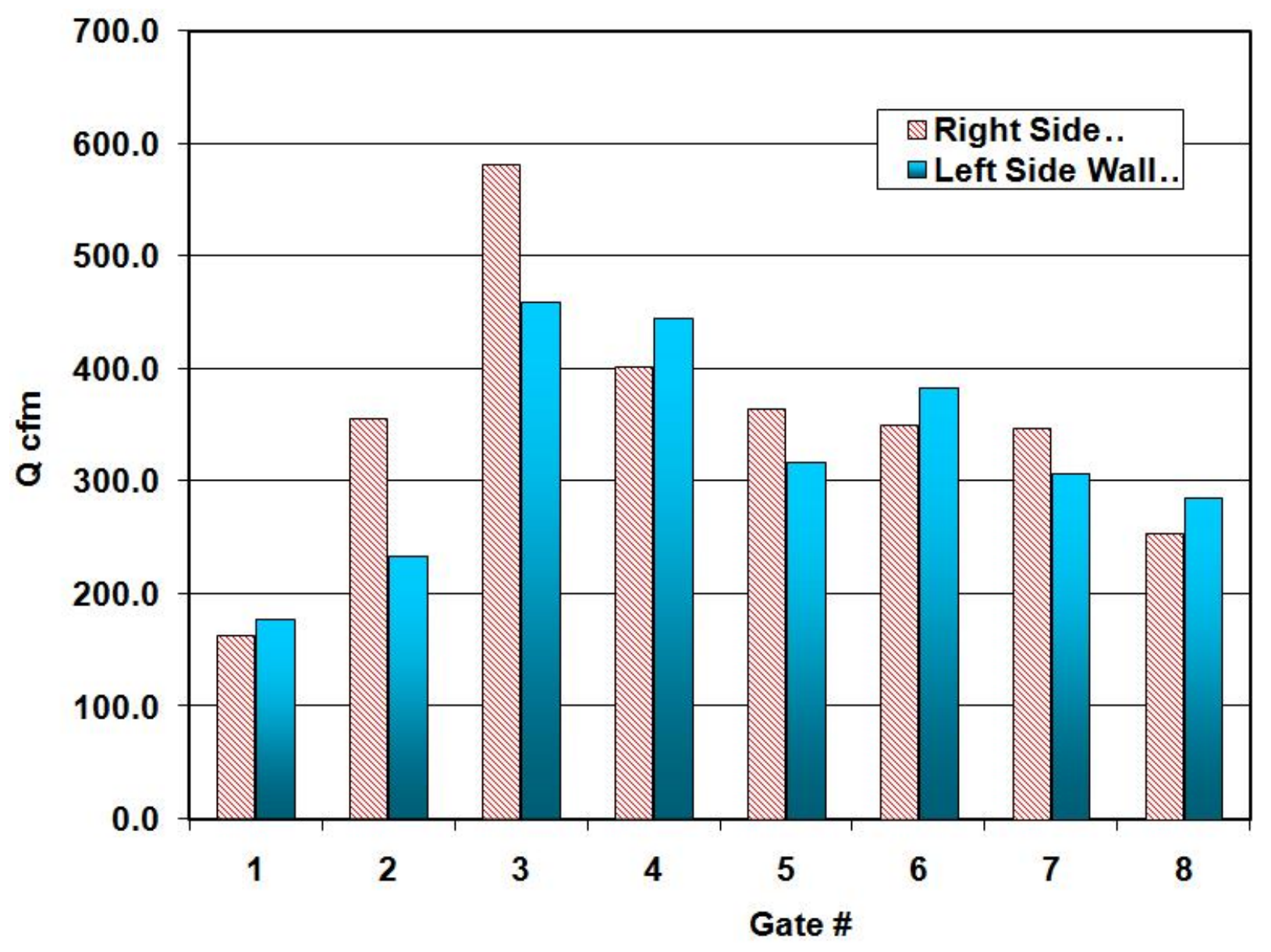

Figure 2.10: Side Wall Suctoin Flow Rates 


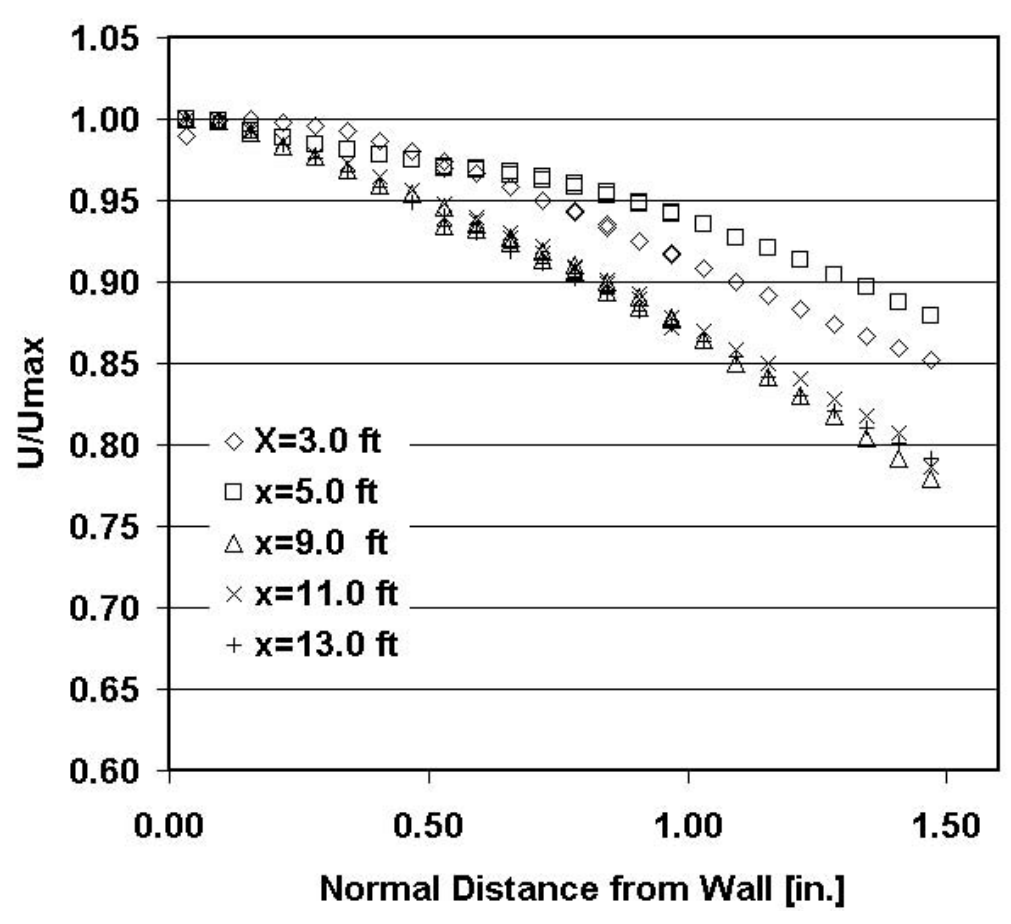

Figure 2.11: Right side wall normalized velocity profiles vs normal distance

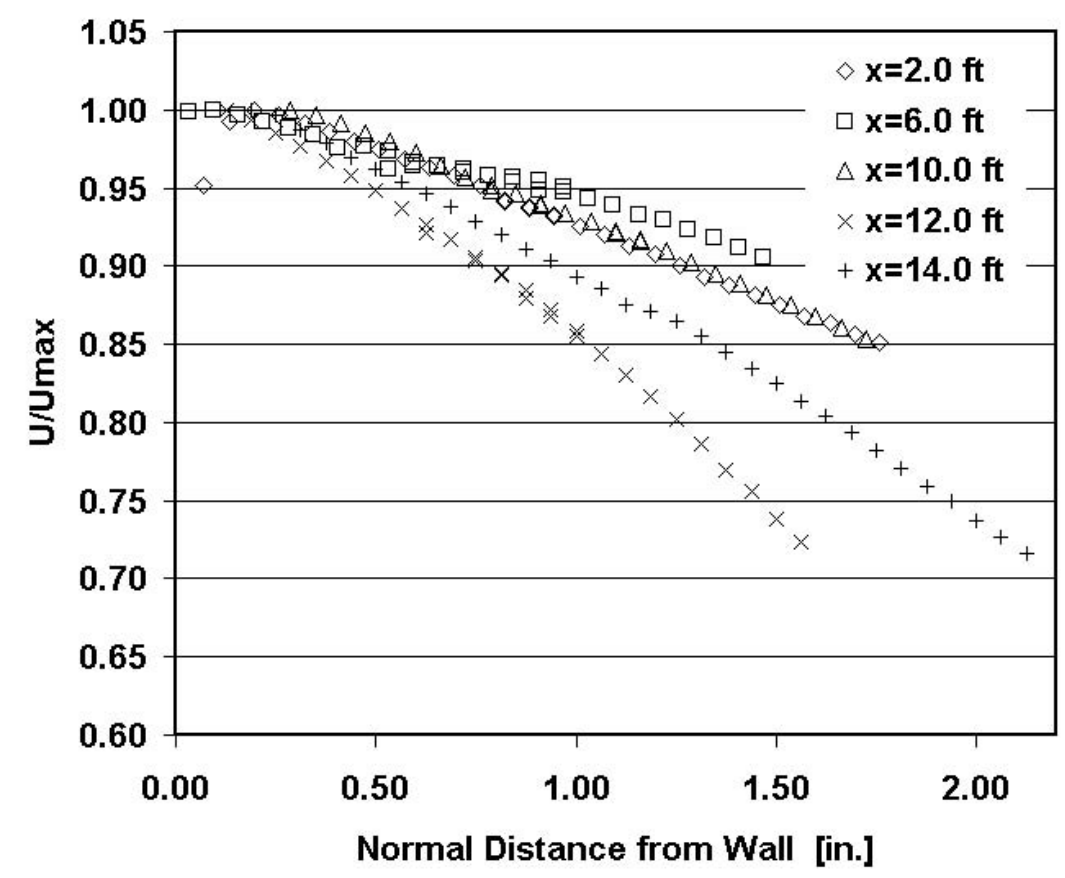

Figure 2.12: Left side wall normalized velocity profiles vs normal distance 


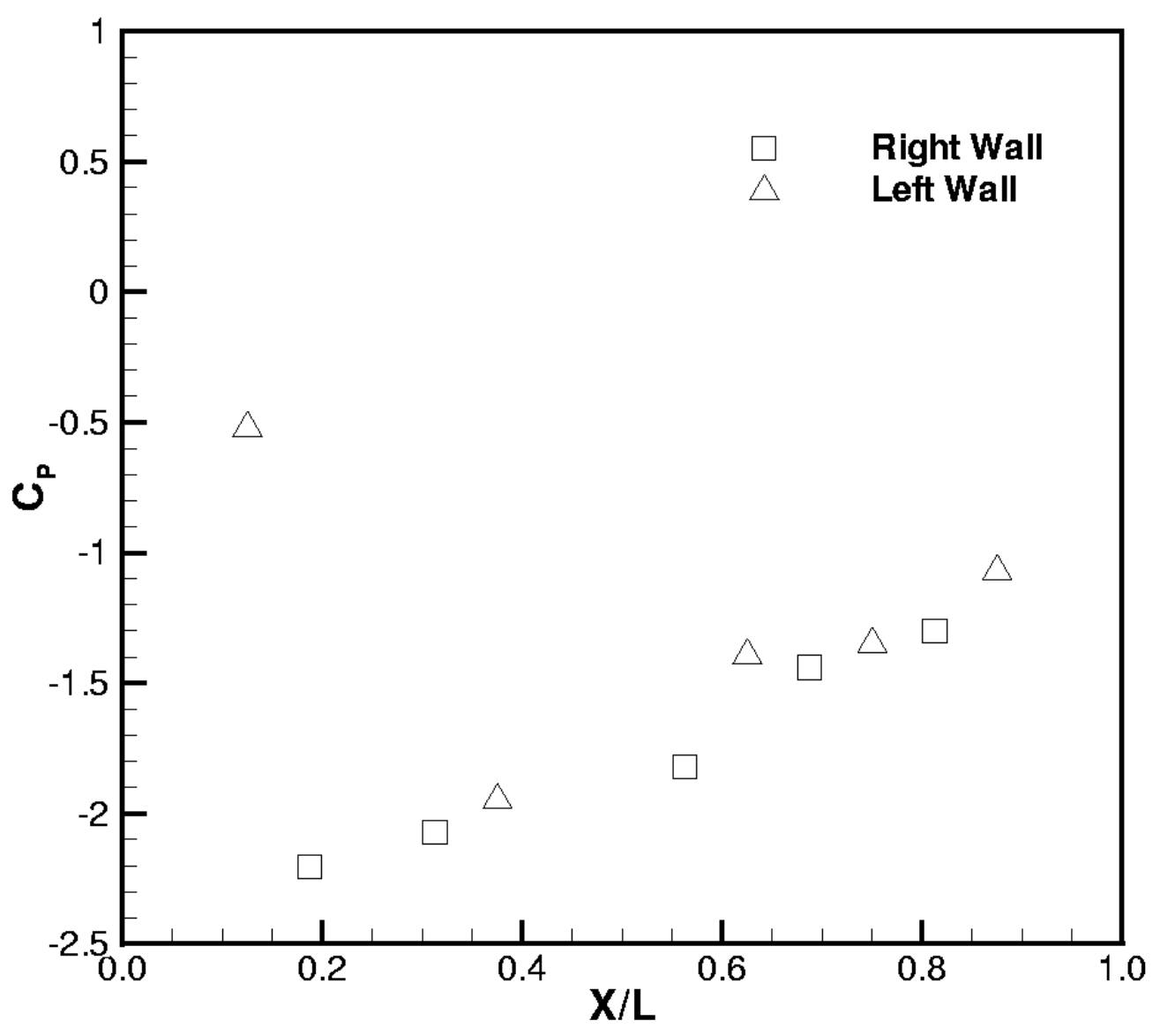

Figure 2.13: $C_{p}$ distribution along the side wall peaks 


\section{Chapter 3}

\section{Mean Flow Development}

\subsection{Measurement Locations}

In order to document the flow development in the wavy wall test section and to ensure that there are no flow separation, mean flow velocities were measured using the 7hole probe at different planes parallel to the $y-z$ plane at different stream-wise locations. A total of 6 planes were measured along the length of the test section at $x / L=0.00,0.13$, $0.39,0.64,0.79$ and 0.90 . In this chapter the mean flow velocities along with centerline profiles at different locations are presented.

\subsection{Seven-Hole Probe Alignment}

For a seven-hole probe to measure flow direction and velocity component correctly, the probe must be used to measure a uniform flow with a known direction after being installed in place in the test section where a measurement is to be taken. This is essential so that any misalignment with the tunnel centerline can be determined. For experiments with simple geometries this is usually not a problem. However in the case of the wavy wall, this was impossible since the side walls geometry altered the direction of the uniform incoming flow. Another method for checking misalignment had to be used. This was done by using the wind tunnel's centerline as a reference direction. A line was drawn on the tunnel's floor along the tunnel's center. Then the probe was traversed to the centerline and placed on the tunnel floor and aligned with the mark on the floor visually. Then the distances between the centerline and two distant points on the probe were measured with an uncertainty of $1 / 16 "$ " This resulted in an uncertainty in yaw angle of $\pm 0.72^{\circ}$. In order to account for pitch angle misalignment, the probe was traversed vertically and the height of the probe from the floor was measured at two different points on the probe us- 
ing guage blocks with a precision of $0.01 "$. The calculated uncertainty in pitch angle was $\pm 0.2^{\circ}$.

\subsection{Uncertainty Estimates}

In order to confirm the level of uncertainties in aligning the probe and to estimate the uncertainty in measuring the velocity magnitudes, two velocity profiles were taken at the centerline of the entrance plane, $x / L=0$. To estimate the 20:1 odds uncertainty in the flow angles and velocity magnitude, the standard deviation is estimated using Chauvenet's criterion, Ölçmen [43] and Byun [4], was used,

$$
\frac{d_{\max }}{\sigma}=1.15
$$

Where, $d_{\max }$ is half the mean difference between the two measurements over the entire profile and $\sigma$ is the estimated standard deviation. The 20:1 odds uncertainty is defined as $\pm 1.96 \sigma$. The estimated overall uncertainties for the pitch, yaw angles $(\alpha, \beta)$ and the velocity components are presented in Table 3.1. These estimates agree with the estimates performed by Wenger [70].

\begin{tabular}{cccccc} 
& $\alpha\left[^{\circ}\right]$ & $\beta\left[^{\circ}\right]$ & $U[\mathrm{~m} / \mathrm{s}]$ & $V[\mathrm{~m} / \mathrm{s}]$ & $W[\mathrm{~m} / \mathrm{s}]$ \\
\hline$\delta(\cdot)$ & 0.29 & 0.29 & 0.18 & 0.14 & 0.13 \\
\hline
\end{tabular}

Table 3.1: Seven-Hole Probe Uncertainties

\subsection{Mean Flow Velocity}

In this section the mean flow velocities are discussed. The local ceiling maximum heights at stations where seven-hole probe measurements were made are listed in Table 3.3. The free-stream conditions for each plane measurements are shown in Table 3.2. Since the measurements were made over an extended period of time, the atmospheric conditions varied which influenced the free stream flow conditions to some extent.

- $x / L=0.00$

Figure 3.1 shows contours of U-velocity component at $x / L=0.00$. The $y$ and $z$ coordinates are normalized by the maximum height and width at the entrance 
respectively. Due to the limited space inside the test section, due to the contraction at the entrance and due to the ceiling structure, the entire entrance plane was not measured. The ranges covered in the vertical and spanwise directions were $0.03<y / y_{\max }<0.74$ and $-0.35<z / W_{x=0}<0.4$ respectively.

The U-velocity component contours show a uniform flow entering the test section with a uniform boundary layer thickness. The boundary layer thickness, $\delta_{99}$, is approximately equal to $34[\mathrm{~mm}]$. The $\mathrm{V}$ and $\mathrm{W}$ velocity components are also shown in Figure 3.1 as a superimposed vector plot. The magnitudes of the $\mathrm{V}$ and $\mathrm{W}$ velocity components are less than $1[\mathrm{~m} / \mathrm{s}]$ and the velocity vectors don't seem to have a bulk directed motion.

One observation that was made while measuring the mean flow using the SevenHole probe is that there was a noticeable amount of air exiting the test section from the slots in the test section ceiling that were used to traverse the probe. These slots were kept clear so that the traverse can move freely. The amount of outflow from these slots is estimated to be less than $3 \%$ of the amount of flow rate entering the test section. However, all slots were covered when not in use.

- $x / L=0.13$

This plane is at the end of the straight contracting side walls. At this location also, the flow starts going around the sine wave shaped side walls. At the right side wall, negative z-coordinates see Figure 2.1. the flow encounters the start of the first sine wave, while at the left side wall, positive $z$-coordinate, the flow is at the peak of a half sine wave. The bulk flow motion is directed into the positive z-direction. Figure 3.2, shows the U-velocity component contours at $x / L=0.13$. Also shown in the same figure the in-plane velocity vectors.

The left wall is located at $z / W_{x=0}=0.44$. It is clear from Figure 3.2 that the boundary layer is almost removed from the left wall by the effect of the suction slots. However, there is a pocket of retarded flow at the side wall mid-height. This is due to some protrusions in the side wall surface. Moreover, the floor boundary layer is almost the same height but is not as uniform as the inlet plane. The floor boundary layer near the left side wall is removed out by the suction slots leading to a decrease in floor boundary layer height as the left side wall is approached. The bulk directed motion can also be seen from the in-plane velocity vectors where the vectors are directed toward the left wall.

The U-velocity component is almost uniform at the center of the plane, however it increases dramatically as the left wall is approached. An increase of about $17 \%$. The large magnitude of the $\mathrm{W}$-velocity component is attributed to the geometry of the side walls whereas the magnitude of the V-velocity component can be attributed to air exiting the test section from the ceiling slots.

- $x / L=0.39$

This plane is at the peak of the second full wave close to the left wall, positive z- 
coordinates. The side wall is located at $z / W_{x=0}=0.358$. Figure 3.3 shows the Uvelocity components as well as in plane velocity vectors. The velocity vectors show a strong directed motion in the positive $\mathrm{z}$-coordinate direction. Also from the $\mathrm{U}$ contours the boundary layer is seen to be stretched in the spanwise direction due to suction effects. The boundary layer height is minimum around the centerline and larger closer to the side walls. This may be due to the fact that before reaching the second wave peak the flow encounters an adverse pressure gradient after going around the first wave peak.

In Figure 3.3, the in-plane vector plots show the effect of suction on the span-wise velocity profile close to the tunnel floor. Close to the side wall $z / W_{x=0}=0.2-0.3$ the flow increases its magnitude in the span-wise direction. Away from the side wall $z / W_{x=0}=0.15$ the span-wise velocity component decreases significantly.

- $x / L=0.64$

This plane is shown in Figure 3.4. The left side wall is located at $z / W_{x=0}=0.351$. The first column of measurement is 1 " away from the left side wall. The $U$-velocity component contours show an almost uniform value close to the side wall. It also shows that the velocity magnitude increases as the side wall is approached. Along the tunnel centerline, the boundary layer grows significantly, reaching about $40 \%$ of the channel height. At this location there still exists some potential flow core at the channel's center. The effects of the side wall on the mean flow can be seen close to the tunnel centerline by noticing the vertical contours lines of the $U$-velocity component at $z / W_{x=0}=0.1$.

The in plane velocity vectors also show the effects of the side wall as it turns the flow. Close to the wall the velocity vectors are almost horizontal, meaning that the spanwise component $(W \approx 12 \mathrm{~m} / \mathrm{s})$ is much greater than the vertical component. The uniform magnitude of the velocity vectors close to the side wall can also be noticed in Figure 3.4. Away from the side wall, the spanwise component decreases and it has the same order as the vertical component.

- $x / L=0.79$

This plane is located just upstream of the fifth wave peak on the right side wall, negative $z$-coordinates. The measurements are closer to the right wall than to the left side wall. Figure 3.5 shows the $U$-velocity component contours and in plane velocity vectors.

Since the measurements are closer to the right wall for this plane, the contour patterns are mirror imaged around the vertical axes. Figure 3.5 shows almost constant $U$-velocity component close to the right wall. It also shows some potential core left at the channel's center.

The in plane velocity vectors show a directed motion in the positive z-direction. The magnitude of spanwise and vertical velocity components are of the same order 
of magnitude. However, the vertical component tends to increase at the tunnel's center and decrease closer to the side walls.

- $x / L=0.90$

This is the most downstream measured plane and is located upstream of the last wave on the right side wall and downstream of the last wave on the left side wall.

Contour and in plane velocity vector plots are shown in Figure 3.6. The contour lines have the same pattern as the plane $x / L=0.64$ but with different magnitudes. Moreover, the floor and ceiling boundary layers have merged together and there is no free-stream left at the tunnel's centerline.

Near the left side wall, the velocity contours are vertical and the in plane velocity vectors are almost horizontal. At the tunnel's centerline the vertical and spanwise components are of the same order of magnitude.

- Summary

In order to have a good view of the global flow development, the $U$-velocity component contour plots at five measurement planes are shown in Figure 3.7. The side walls plan view is also shown in Figure 3.7 in order to relate flow development to the test section geometry.

In summary, the flow enters the wavy wall test section with a uniform flow in the $\mathrm{x}$-direction. The flow undergoes acceleration in the contraction and the boundary layer thickness is almost constant. Afterward the flow is turned by the effect of side walls and the floor boundary layer thickness grows.

Close to the side walls the velocity is almost constant in the vertical direction but varies in the spanwise direction. At the center of the tunnel, the potential core is present up to about $x / L=0.79$ afterwords the flow becomes developed and the floor and ceiling boundary layers merge together.

\subsection{Centerline Flow Development}

In the previous section mean flow velocities at different streamwise locations were presented. In this section mean flow velocities and pressure coefficient at the channel's midheight along the tunnel's centerline will be presented. These quantities are extracted from the plane measurements.

Figure 3.8 shows the velocity components and pressure coefficient at the channel's midheight versus distance along the tunnel's centerline. The $U$-velocity component increases along the centerline up to about $x / L=0.35$ then decreases until the end of test section. On the other hand the $V$ and $W$ velocity components are almost zero at the entrance to the test section. However, they increase in the convergent region at the test 
section inlet until they reach almost a constant value at about $x / L=0.35$. It should be noted here that the plane measurements where taken at integral number of wavelengths, this means that if there were variations in the $W$-velocity component sign it won't be shown here unfortunately.

Also shown in Figure 3.8 the pressure coefficient along at the channel's midheight along the centerline. The pressure coefficient shows variations similar to a sine wave. However, the variations only make one sine wave shape.

Finally, the floor boundary layer thickness development is also extracted from the plane measurements. The boundary layer thickness, $\delta$, is taken to be the distance from the floor to the location where the velocity parallel to the floor, $Q_{e}$, reaches $99 \%$ of the edge or maximum velocity for that location.

Table 3.4 lists the edge speed as a fraction of the free stream speed, $Q_{e} / U_{\infty}$, as well as boundary layer thickness, $\delta_{99}$, boundary layer as a fraction of the channel height, $\delta_{99} / y_{\max }$, and the Reynolds' number, $R e_{\delta_{99}}$, based on edge velocity and boundary layer thickness. Figure 3.9 shows the boundary layer thickness development versus $x / L$.

\begin{tabular}{cccccc}
\hline$x / L$ & $P_{a}[\mathrm{~Pa}]$ & $T_{a}\left[{ }^{\circ} \mathrm{K}\right]$ & $P_{\infty}[\mathrm{Pa}]$ & $T_{\infty}\left[{ }^{\circ} \mathrm{K}\right]$ & $U_{\infty}[\mathrm{m} / \mathrm{s}]$ \\
\hline 0.00 & 94590 & 297.00 & 95962 & 297.00 & 25.23 \\
\hline 0.13 & 94810 & 297.15 & 96144 & 297.65 & 25.42 \\
\hline 0.39 & 94795 & 298.15 & 96134 & 298.15 & 25.26 \\
\hline 0.64 & 93925 & 297.55 & 95323 & 298.15 & 25.16 \\
\hline 0.79 & 95015 & 298.00 & 96407 & 298.00 & 25.26 \\
\hline 0.90 & 94319 & 298.30 & 95714 & 298.65 & 25.15 \\
\hline
\end{tabular}

Table 3.2: Mean Flow Free Stream Conditions

\begin{tabular}{ccccccc}
\hline$x / L$ & 0.00 & 0.13 & 0.39 & 0.64 & 0.79 & 0.90 \\
\hline$Y_{\max }$ in. & 10.5 & 10.17 & 9.72 & 9.22 & 8.92 & 8.70 \\
\hline
\end{tabular}

Table 3.3: Maximum test section ceiling height at seven-hole probe measurement planes

\begin{tabular}{ccccc}
\hline$x / L$ & $Q_{e} / U_{\infty}$ & $\delta_{99} / y_{\max }$ & $\delta_{99}[\mathrm{~mm}]$ & $R e_{\delta_{99}}$ \\
\hline 0.00 & 1.140 & 0.1305 & 33.58 & $5.139 E+04$ \\
\hline 0.13 & 1.159 & 0.1373 & 34.22 & $5.265 E+04$ \\
\hline 0.39 & 1.234 & 0.2331 & 54.41 & $8.294 E+04$ \\
\hline 0.64 & 1.088 & 0.4716 & 102.82 & $1.548 E+05$ \\
\hline 0.79 & 1.091 & 0.4871 & 101.74 & $1.557 E+05$ \\
\hline 0.90 & 0.929 & 0.5505 & 111.27 & $1.676 E+05$ \\
\hline
\end{tabular}

Table 3.4: Centerline Mean Edge Velocity and Boundary Layer Thickness 


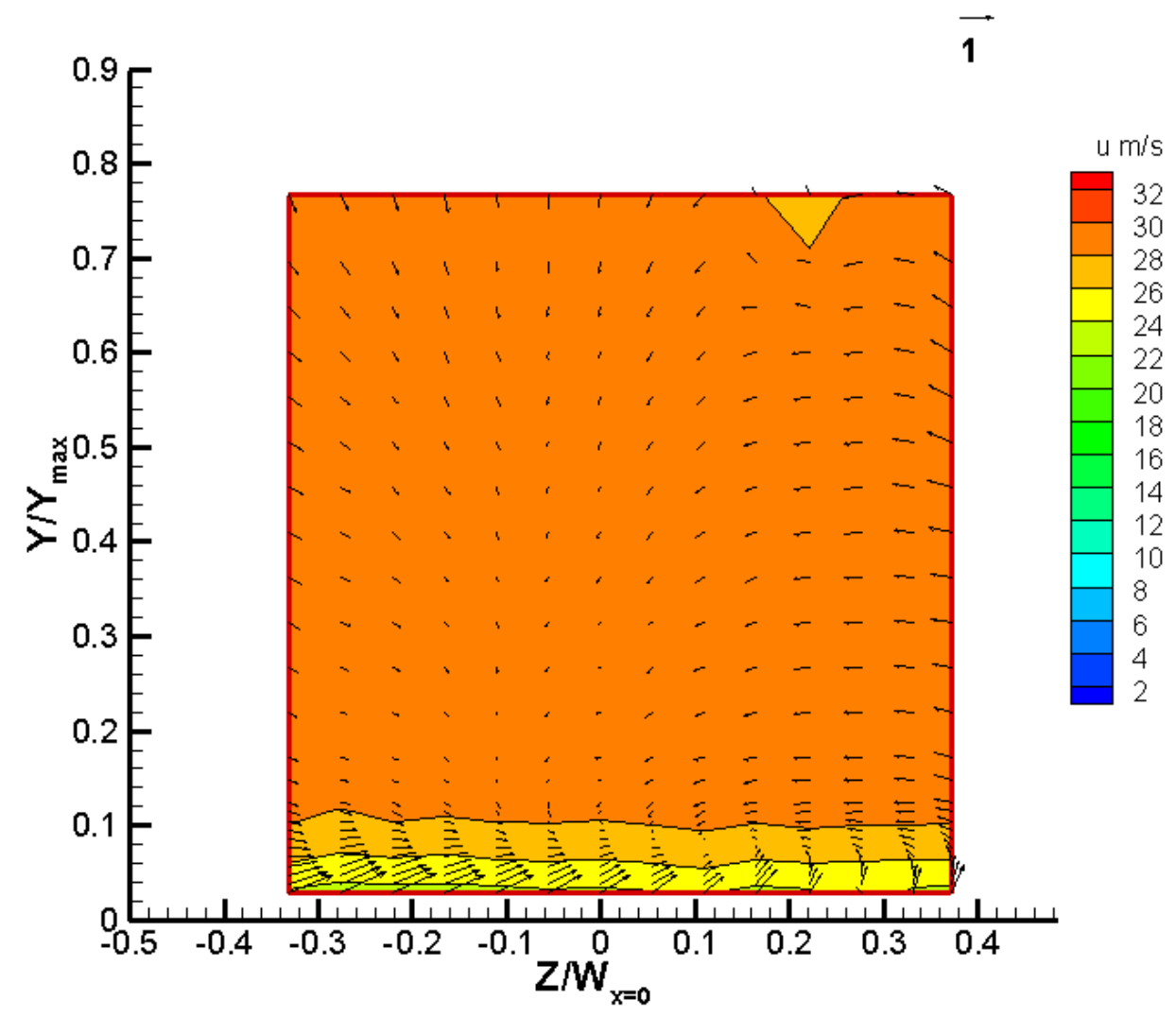

Figure 3.1: U-velocity component contours at $x / L=0.00, Y_{\max }=10.5 \mathrm{in}$. 


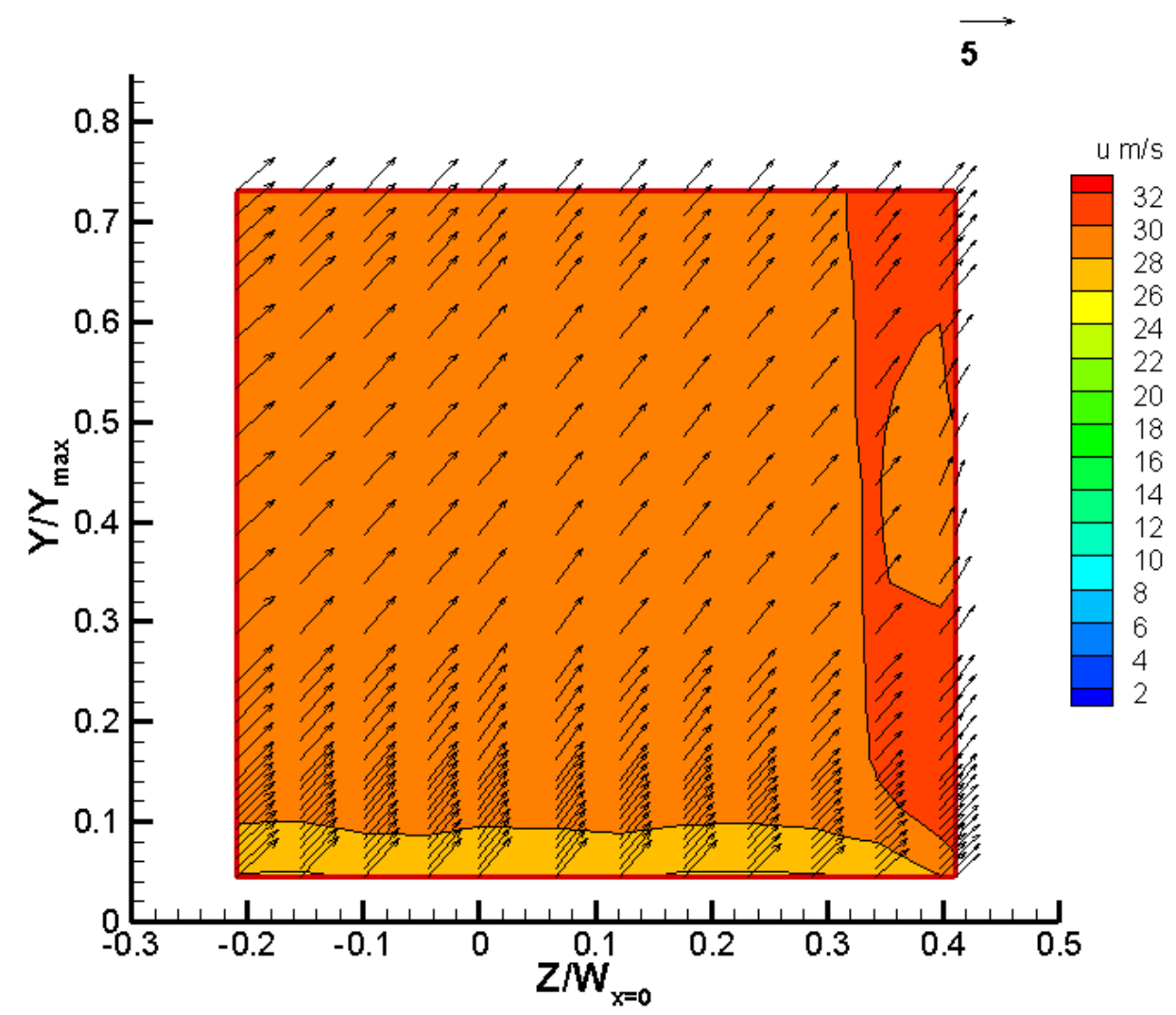

Figure 3.2: U-velocity component contours at $x / L=0.13, Y_{\max }=10.17 \mathrm{in}$. 


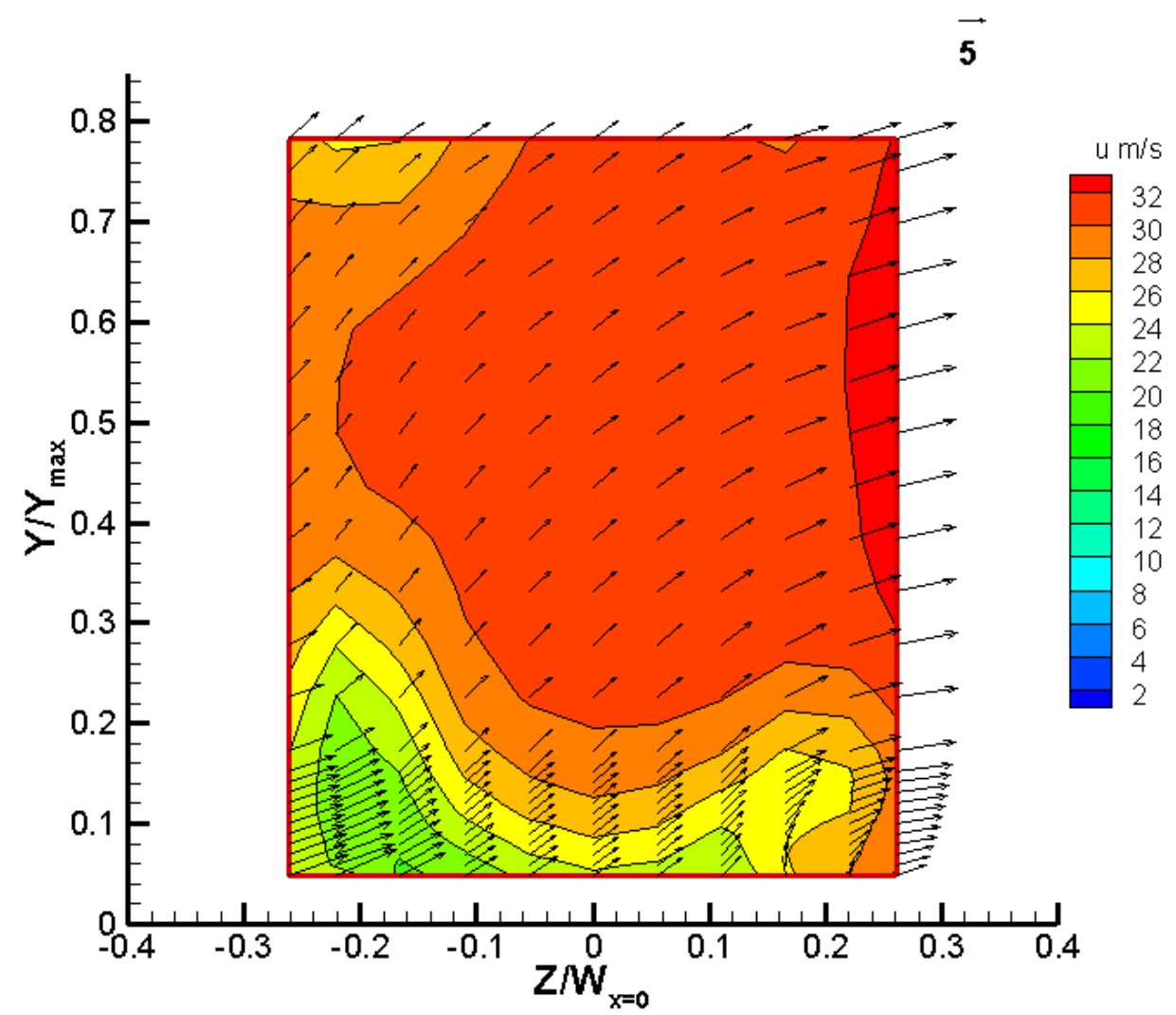

Figure 3.3: U-velocity component contours at $x / L=0.39, Y_{\max }=9.72 \mathrm{in}$. 


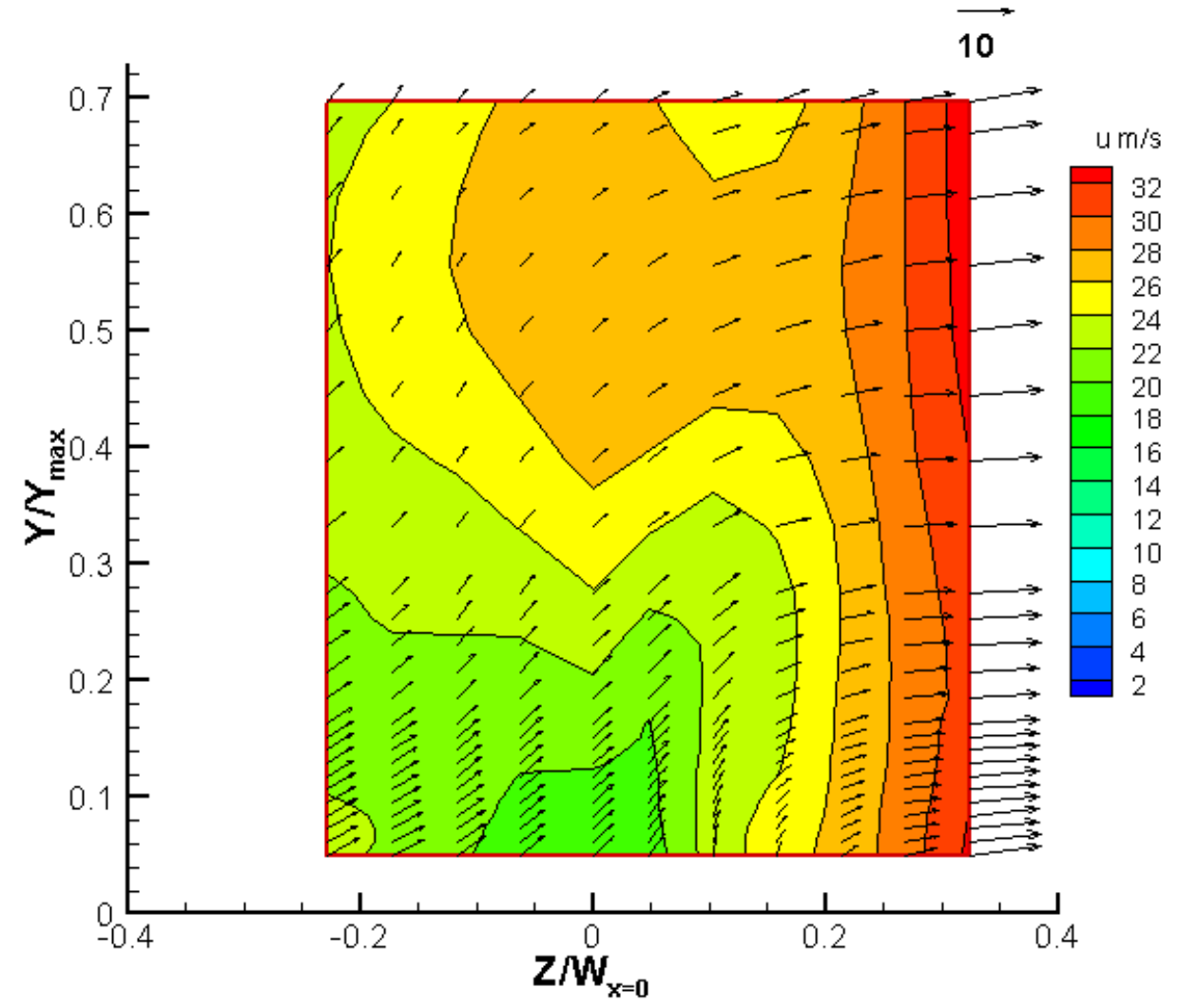

Figure 3.4: U-velocity component contours at $x / L=0.64, Y_{\max }=9.22 \mathrm{in}$. 


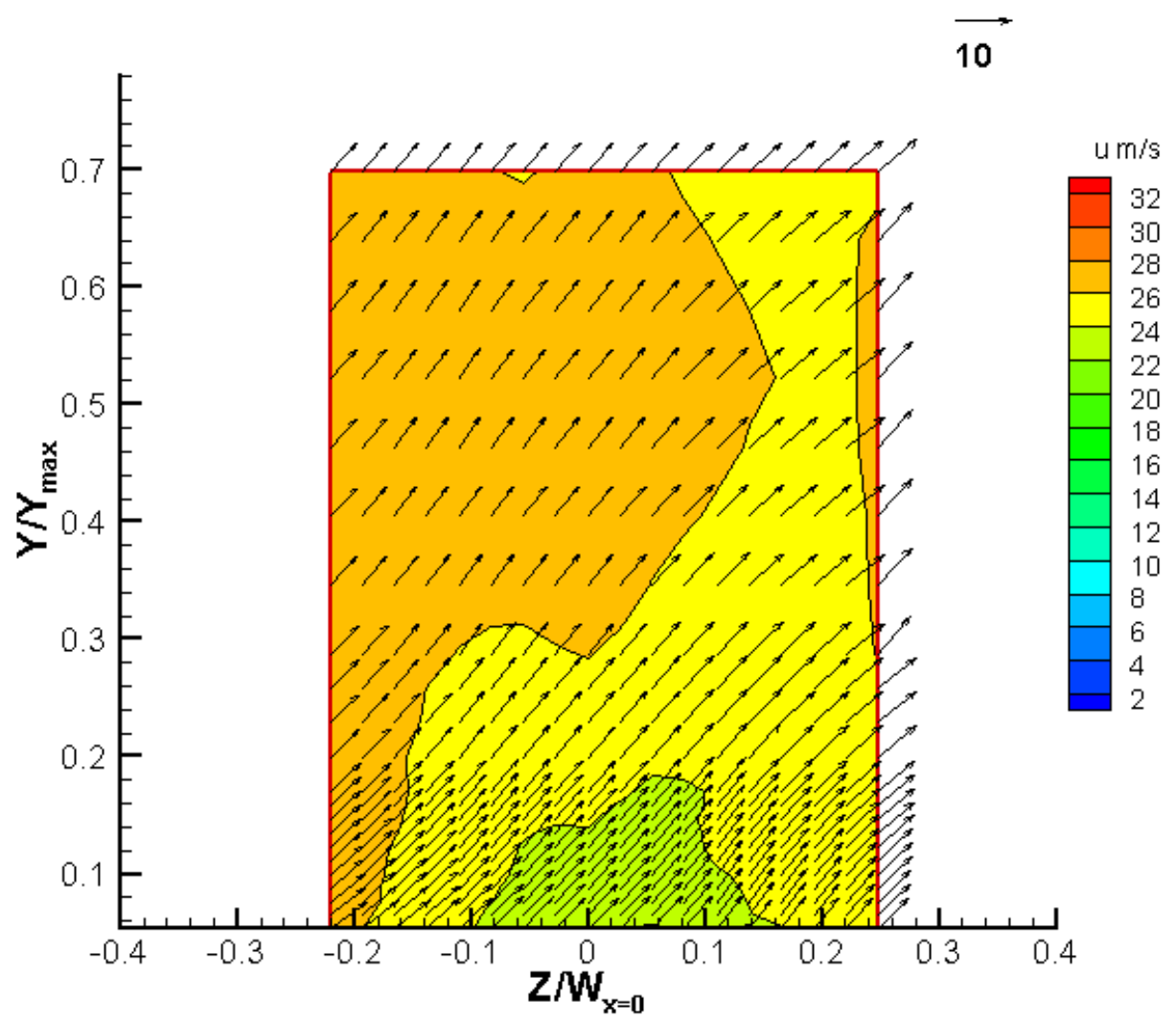

Figure 3.5: U-velocity component contours at $x / L=0.79, Y_{\max }=8.92 \mathrm{in}$. 


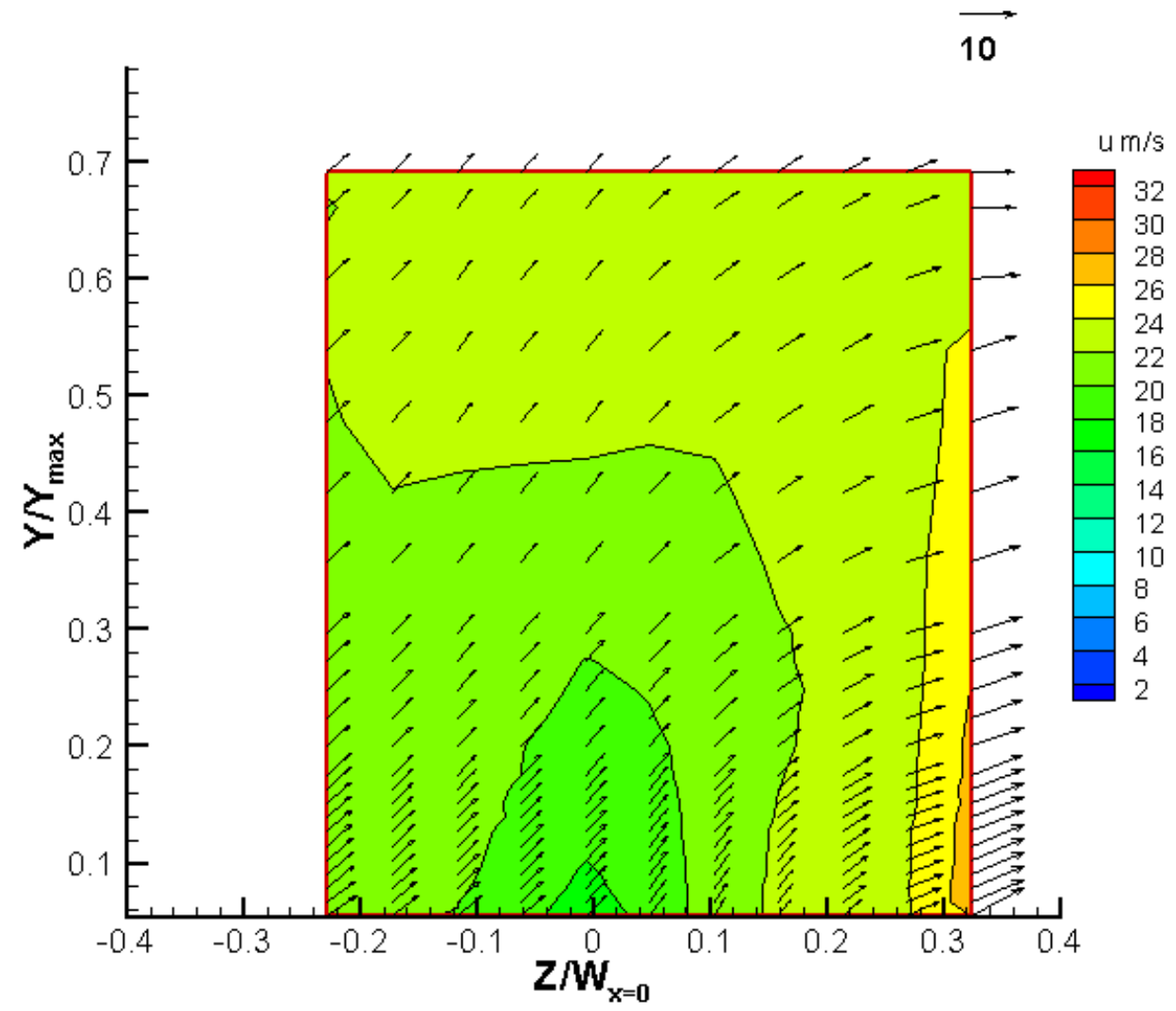

Figure 3.6: U-velocity component contours at $x / L=0.90, Y_{\max }=8.70 \mathrm{in}$. 


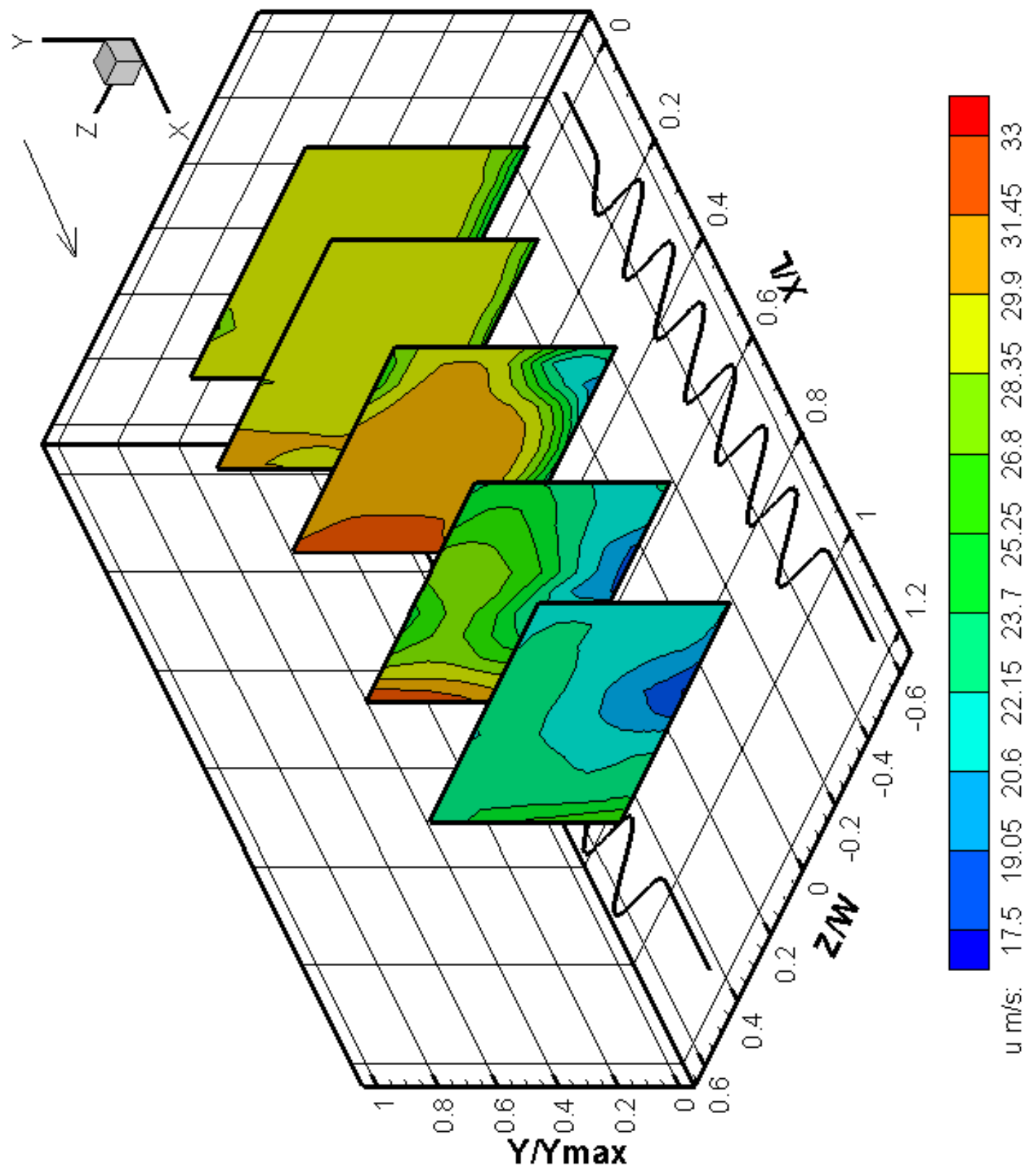

Figure 3.7: U-velocity component contours at $5 \mathrm{x} / \mathrm{L}$ stations 


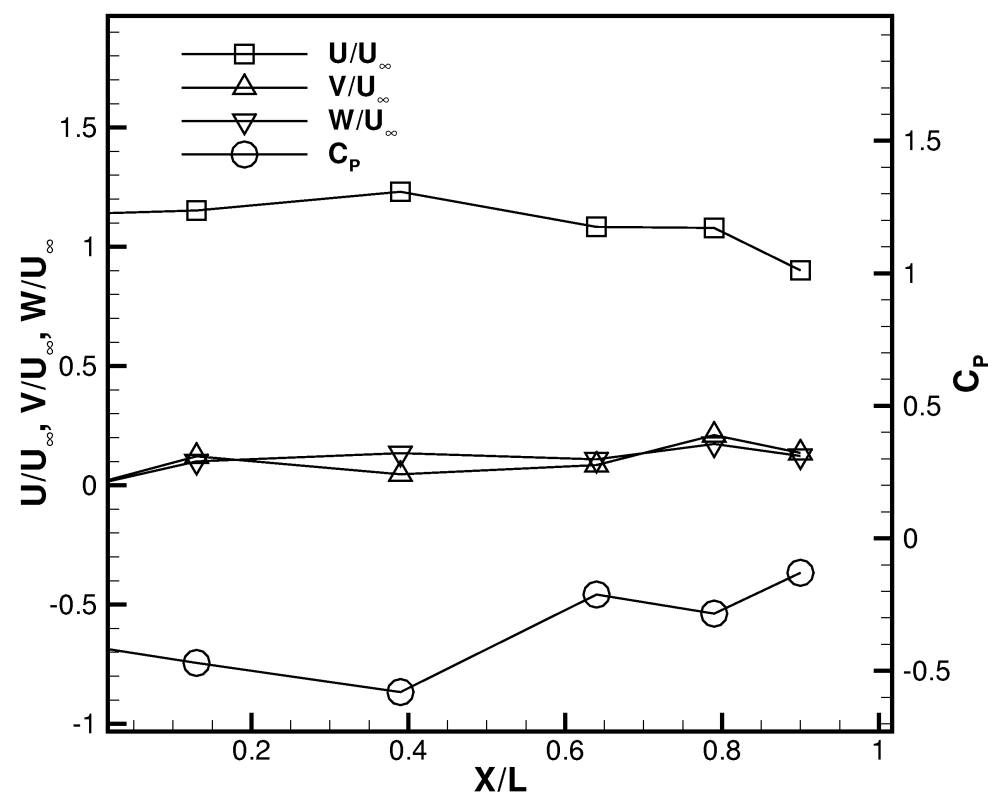

Figure 3.8: Centerline $\frac{U}{U_{\infty}}, \frac{V}{U_{\infty}}, \frac{W}{U_{\infty}}, C_{P}$

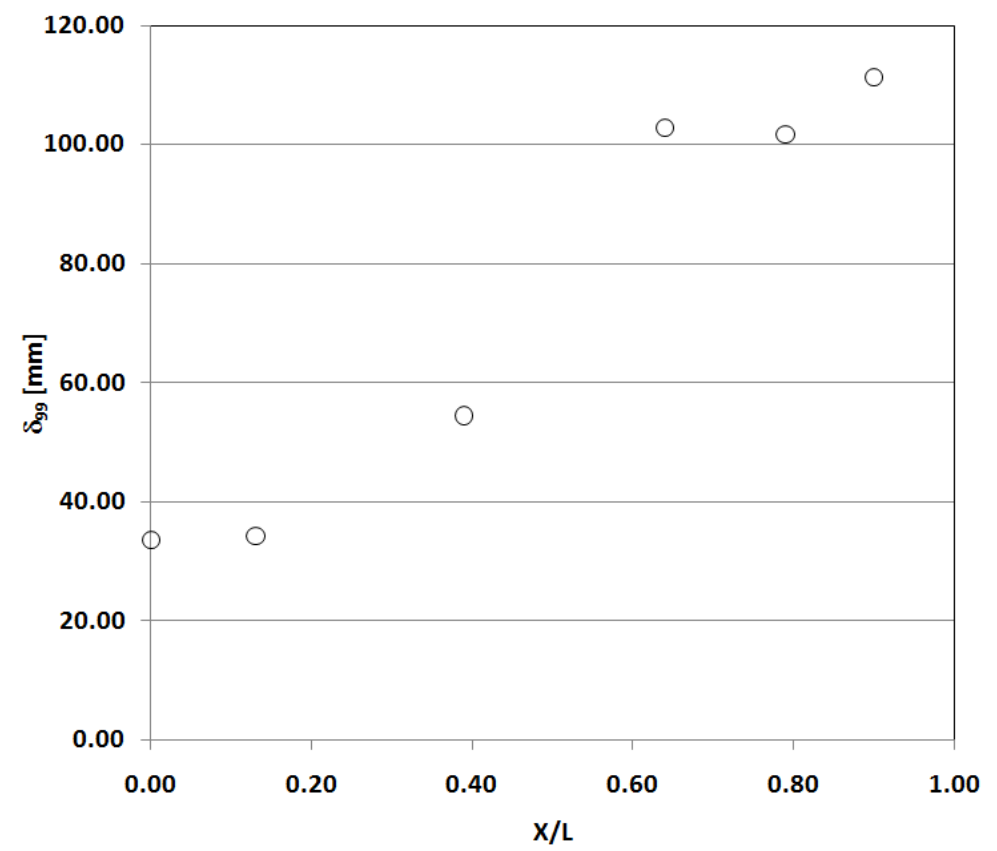

Figure 3.9: Boundary Layer Thickness along tunnel centerline 


\section{Chapter 4}

\section{LDV Measurement Results}

\subsection{Measurement Locations}

LDV measurements were taken at 30 locations at the last wave cycle in the test section. Two more profiles were also performed at the test section entrance to determine the properties of the incoming flow boundary layer. All LDV measurement locations are marked in Figure 2.1. An enlarged view of the measurement locations at the tunnel's downstream end is shown in Figure 4.1 .

The measurement locations consist of 3 clusters of five point stencils and a grid of $5 \times 3$ points in the $x$ and $z$ directions, respectively. For each cluster, the stream-wise and spanwise distances were set to be $1 \mathrm{in}$. This value was chosen based on a trial measurement with a $0.5 \mathrm{in}$ separation in the span-wise direction and $1 \mathrm{in}$ in the stream-wise direction. These trials showed little variation in the span wise direction so it was decided to use 1 in in both directions. For the $5 \times 3$ grid, the grid step size in the $x$ direction was also 1 in while the step in the $z$ direction was $0.79 \mathrm{in}$ between the first and second row and $1 \mathrm{in}$ between the second and third row. On the other hand, the measurements were limited to these locations due to the fact that the LDV system did not have optical access because of the tunnel's structure under the test section's floor. This setup was anticipated to give a good view of the flow changes across the test section and to enable estimation of convection, production, turbulent diffusion and viscous diffusion terms in the Reynolds stress transport equations.

All 30 profiles were measured using the Short system and the CompLDV probes with an overlap length between both profiles in order to match the two set of measurements. All profiles were corrected for velocity-gradient broadening and normalized by wall variables $u_{\tau}$ and $\nu / u_{\tau}$. Friction velocity estimation, $u_{\tau}$, velocity-gradient broadening corrections and matching procedure are discussed in the following sections. 
All profiles are presented in tunnel coordinates, with $x$-axis in the downstream direction as shown in Figure 2.1, the $y$-axis normal to the test section floor and the $z$-axis is defined according to a right handed coordinate system.

\subsection{Velocity-Gradient Broadening}

Velocity-Gradient broadening effects are due to the fact that the LDV measurement volume has a finite size and in flow-fields with strong gradients can influence the measured turbulence quantities. Hence corrections must be applied to each measured quantity. However, from practice, the only quantities affected are the normal stresses, specifically the stream-wise stress $\overline{u^{2}}$ and the triple-velocity correlation in the stream-wise direction $\overline{u^{3}}$. The corrections of Fischer [15] were used. The effective measurement volume of the short system reported by Olcmen [41] and Byun [4] was $30 \mu \mathrm{m}$ and $50 \mu \mathrm{m}$ respectively. However, in this study it is was estimated to be $80 \mu m$ while the CompLDV probe had an effective measurement volume of $120 \mu \mathrm{m}$. The scaling of DeGraff and Eaton [10] can be used to confirm that the correct value of friction velocity is used to scale the profiles. This scaling was used and is shown in Figure 4.2e, which shows excellent agreement with DNS data of Spalart [53].

\subsection{Skin Friction velocity $u_{\tau}$}

The skin friction velocity defined in Equation 4.1. was estimated using the asymptotic behavior of the mean flow velocity as dictated by the momentum equation. The near wall behavior, $y^{+} \leq 9$, can be modeled using equation 4.2

$$
\begin{array}{r}
u_{\tau}=\sqrt{\frac{\tau}{\rho}} \\
Q=\frac{u_{\tau}}{\nu} y+\frac{1}{2 \mu} \frac{\partial P}{\partial s} y^{2}+C \cdot y^{4}
\end{array}
$$

Where $Q$ is the velocity magnitude parallel to the wall, $Q=\sqrt{U^{2}+W^{2}}$, $\frac{\partial P}{\partial s}$ is the surface pressure gradient in the flow direction and $C$ is a constant related to the shear stress profile. Surface pressure measurements were made using pressure taps on a surface mounted flat plate. The same plate was used and documented by Tian [69]. The pressure taps were connected to the Scannivalve system. The pressure data were interpolated linearly over a cartesian grid and the pressure gradients were evaluated at each profile location. The level of pressure gradients were small so that the inclusion of the pressure term resulted in a change in $u_{\tau}$ less than $2 \%$. 
In general, for all Short system profiles, at least 4 or 5 points were present in the viscous sublayer that were used to fit the measurements to equation 4.2. In addition to determining the friction velocity and the constant $C$ in equation 4.2 , a shift in the $y$ coordinates, $\delta y$, is also determined. This is done by minimizing the sum of the square of the residual between the left and right sides of the equation. The y-shifts for the Short system profiles were less than $60 \mu \mathrm{m}$ for all profiles.

The details of matching the Short system and CompLDV profiles are presented in Appendix C. Table 4.1 shows the tunnel coordinates of all profiles as well as the flow conditions, pressure gradients, skin-friction velocity using Short System LDV probe, $y$ shift and the correlation coefficient, $R$, for the fit to Equation 4.2 .

It can be seen from table 4.1 that the estimated friction velocity for different clusters showed some scatter. This led to some differences in the non-dimensional quantities. In order to obtain better collapse between profiles, the friction velocity used is obtained by averaging the wall shear stress instead of the friction velocity. This way there is less uncertainty in estimating the friction velocity. All profiles in each cluster are nondimensionalized using the cluster CompLDV average friction-velocity. Table C.1 lists the friction velocity for each profiles for both probes and the average friction velocity for each cluster.

\subsection{Entrance Profiles}

Two LDV profiles were taken at the entrance to the test section in the straight convergent side walls part using the Short LDV system. The measured LDV profiles didn't reach the free-stream location. Hence the mean flow measurements obtained by the seven-hole probe at the entrance, $x=0$, were scaled using $u_{\tau}$ estimated from short system profiles and fitted to the LDV measurement in order to obtain an estimate of the boundary layer thickness as well as momentum thickness. From the measured mean $U$ profiles, the incoming boundary layer thickness, $\delta_{99}$ is about $39 \mathrm{~mm}$ and the Reynolds number based on momentum thickness is $R e_{\theta}=7200$.

Plots of mean velocity, Reynolds stresses and triple products are shown in Figure 6.15 . The measured profiles are in very good agreement with DNS data. Moreover the Reynolds normal streamwise stress scaled using the scaling proposed by DeGraff and Eaton [10] is shown in Figure 4.2e. From this figure it is shown that this scaling is good up to $y^{+} \approx 700$ for this flow condition. 


\subsection{Down-stream Profiles}

In this section LDV measurements at the down-stream locations will be discussed. Profiles of measured quantities will be discussed at each measurement cluster.

\subsubsection{P08 Cluster}

Profiles P06, P08 and P10 are along the stream-wise direction, while profiles P07, P08 and $P 09$ are along the span-wise direction as shown in Figure 4.1. The center profile, $P 08$, in this cluster is located at $x / L=0.871$ and $Z / W_{x=0}=-0.158$. The cluster is at the stream-wise end of the fifth wave on the right side wall.

\section{P08: Mean Stream-wise and Span-wise Velocities, $U^{+}, W^{+}$}

Mean flow profiles of stream-wise and span-wise velocity components are shown in Figure $4.3 \mathrm{a}$. In general, all profiles show a viscous sub-layer and a semi-log layer. All profiles collapse onto a single profile which is close to the two-dimensional boundary layer profile. For a two dimensional boundary layer the flow velocity in the semi-logarithmic layer is given by Equation 4.3, [52]. Also shown in the figure, the stream-wise velocity profile for a turbulent boundary layer DNS calculations by Spalart, [53]. The $U$-velocity profiles become constant at about $y^{+}=10^{3}$, however the speed seems to increase slowly to a higher value. The span-wise velocity component increases in the stream-wise direction.

$$
U=\frac{1}{\kappa} \ln y^{+}+B
$$

The span-wise flow velocity is positive, $W^{+}$, near the wall and increases in the streamwise direction and is almost constant in the span-wise direction. This is due to the fact that this cluster is at the beginning of the last wave on the right side wall, hence, the skewing effects of the new wave is felt more in the downstream direction. At $y^{+}=700$, the span-wise velocity component changes sign and becomes negative. This is due to the mean flow moving over the fifth wave downstream of the previous peak which is toward the negative $w$-direction.

\section{P08: Reynolds' Normal Stresses, ${\overline{u^{2}}}^{+}, \bar{v}^{2}$ and ${\overline{w^{2}}}^{+}$}

Plots of the normal Reynolds stress in the stream-wise direction are shown in Figure $4.3 \mathrm{~b}$ for all profiles along with DNS data of Spalart, [53], for a two-dimensional turbulent boundary layer which are presented as a reference. The plot shows a much higher values of stress than in the two-dimensional turbulent boundary layer case and instead 
of one peak, there is a secondary peak. The primary peak occurs at nearly $y^{+}=14$ while for the two-dimensional case the peak value occurs at $y^{+}=14.4$. The peak value ranges between 12-13. The second peak is visible in all profiles between $y^{+}=300$ and 500 . The second peak value is higher than the primary one and ranges from 12 to 14. Fernholz,[14], also reported the presence of a second peak at high Reynolds number. All profiles however collapse near the wall into a single curve approaching the DNS data.

At the maximum measured height the stresses didn't reach zero value. Measurements at higher distances from the wall, could not be obtained since the LDV probe was blocked by the tunnel structure under the test section's floor.

The normal to the wall and span-wise normal stresses are shown in Figure $4.3 \mathrm{c}$. The normal stresses increase slightly in the downstream direction, while the increase in the span-wise direction is larger. Again, near the wall the stresses collapse on top of one another and they approach the two-dimensional turbulent boundary layer DNS calculations. However, the magnitude of maximum stress is much higher than the twodimensional case. Furthermore, both stresses reach a value of about 6.5 around $y^{+}=3000$ which is the same magnitude of the stream-wise normal stress. This suggests that the turbulence has reached near isotropic state at that height.

P08: Reynolds' Shear Stresses, $\overline{u v}+, \overline{u w}^{+}$and $\overline{v w}$

The negative of Reynolds shear stresses, $\overline{u v}$ and $\overline{v w}$ are shown in Figure $4.3 \mathrm{~d}$. The differences in both stresses between profiles are very small. Below $y^{+}=20$ the $\overline{u v^{+}}$stress collapses with DNS data. Above this height the shear stress decreases to a minimum value of -2 at $y^{+}=366$. Between $y^{+}=800$ and 1300 there is another extremum that is aligned with the extremum in the $\overline{v w}$ stress.

The $\overline{v w^{+}}$stress behavior is the same for all profiles, reaching a maximum of 1.9 at $y^{+}=1518$. At this maximum value, the $\overline{v w^{+}}$stress is actually larger than the $\overline{u v}+$ stress which results in a very large magnitude of shear angle, $\gamma_{s}$.

Figure $4.3 \mathrm{e}$ shows the $\overline{u w^{+}}$stress. There are two maxima at $y^{+}=11$ and $y^{+}=530$ approximately. The shear stress increases in the span-wise direction while it is almost unchanged in the stream-wise direction. At $y^{+}=530$ the stress starts decreasing to a negative value with almost the same slope as the $\overline{u v^{+}}$slope. Profile P07 is lower than other profiles to some extent. Again, at the maximum measured height all shear stresses are roughly equal in magnitude.

\section{P08:Correlation Coefficient, $\rho_{u v}$}

Figure $4.3 \mathrm{f}$ shows the correlation coefficient, $\rho_{u v}$, as defined by Equation 4.4 along with values from DNS data.

$$
\rho_{u v}=\frac{-\overline{u v}}{\sqrt{\bar{u}^{2}} \cdot \sqrt{v^{2}}}
$$


Measured values are lower than DNS data, which is usually the case in three-dimensional flow fields. The correlation coefficients for the shown profiles don't collapse near the wall, however they have the same trend. The correlation coefficient for all profiles reaches a value of about 0.3 at $y^{+}=345$, then it decreases to a minimum value of about 1.5.

\section{P08: 1/S Parameter}

The $1 / S$ parameter as defined by Equation 4.5 is shown in Figure 4.3g. DNS data are also shown for comparison.

$$
S^{-1}=\frac{\sqrt{(-\overline{u v})^{2}+(-\overline{v w})^{2}}}{\overline{v^{2}}}
$$

This parameter collapses all profiles' data in the semi-log region and in the outer region. Near the wall there is no collapse. Also the value of $S^{-1}$ approaches the two-dimensional value of 0.7 in the semi-log region. This suggests that this function approaches a universal behavior in the semi-log and outer region for two and three-dimensional flows. According to Olcmen and Simpson [41] this is true as long as there are no stream-wise vortices.

Profiles P08 and P09 experience lower values near the wall than the rest of the profiles. This might suggest that the alignment for these profiles is not as accurate as the other ones.

\section{P08: Turbulent Kinetic Energy $T K E$}

The turbulent kinetic energy, $T K E$ as given by Equation 4.6, is shown in Figure 4.3h with corresponding DNS data.

$$
T K E=\frac{{\overline{u^{2}}}^{+}+{\overline{v^{2}}}^{+}+{\overline{w^{2}}}^{+}}{2}
$$

The level of turbulent kinetic energy is so much larger than the two-dimensional boundary layer value. This might be due to the larger production due to larger shear stresses in the stream-wise and span-wise directions, which is observed and explained in Chapter 5. Near the wall all profiles collapse and approach the two dimensional values. At $y^{+}=20$ all profiles reach different relatively constant values of $T K E$ and at $y^{+}=42$ the energy increases more rapidly up to a maximum value between $y^{+}=200$ and 400 . There is also a local minimum at about $y^{+}=1000$.

\section{P08: Turbulent Diffusion Velocity $\vec{V}_{q}$}


Turbulent diffusion vector non-dimensionalized by $u_{\tau}$ and defined by Equation 4.7 , is shown in Figures $4.3 \mathrm{i}$ to $4.3 \mathrm{k}$ for $V_{q x}, V_{q y}$ and $V_{q z}$ respectively.

$$
\vec{V}_{q}^{+}=\frac{{\overline{u_{l}^{2} u}}^{+} \cdot \hat{i}+{\overline{u_{l}^{2} v}}^{+} \cdot \hat{j}+{\overline{u_{l}^{2} w}}^{+} \cdot \hat{k}}{\left[{\overline{u^{2}}}^{+}+{\overline{v^{2}}}^{+}+{\overline{w^{2}}}^{+}\right]}
$$

The turbulent diffusion in the $x$-direction for profiles P06, P07 and P10 almost collapse. On the other hand, profiles P07, P08 and P09 increase in value in the positive $z$-direction. Profiles of the three velocity components show an extremum around $y^{+}=7$ with almost a constant magnitude for all profiles. Another extremum is also shown around $y^{+}=550$ for the $x$ and $y$-velocity components while for the $z$-component the extremum is at $y^{+}=900$. In the region $30 \leq y^{+} \leq 150$, the turbulent velocity is almost constant with $V_{q x}^{+}=0.45$ while $V_{q y}^{+}$and $V_{q z}^{+}$are almost zero. This region corresponds to the semi-log region. The positive peak in the $V_{q x}^{+}$near the wall is due to sweeping motions with positive $u$-fluctuations and negative $v$-fluctuations. In the outer layer the negative peak is due to ejection motion creating negative $u$-fluctuations correlated with positive $v$-fluctuations. The peaks in $V_{q y}^{+}$and $V_{q z}^{+}$can also be explained by sweeping and ejection events.

\section{P08: Townsend's Structural Parameter $A_{1}$}

Townsend's Structural parameter is given by Equation 4.8 and shown in Figure 4.31 . This parameter tests the correlation between the Reynolds shear stress and the normal stresses (turbulent kinetic energy). A typical maximum value for two-dimensional boundary layers is 0.15 . However, Olcmen and Simpson [41] showed that for three-dimensional flow fields the maximum value is usually lower than that. In addition, the level of turbulence in this experiment is higher than the two-dimensional boundary layer levels which further reduces this parameter.

$$
A_{1}=\frac{\sqrt{\overline{u v}^{2}+\overline{v w}^{2}}}{\overline{u^{2}}+\overline{v^{2}}+\overline{w^{2}}}
$$

Near the wall all profiles approach DNS data; however, in the semilog layer the profiles don't collapse to a single curve. All profiles increase up to a value of 0.08 and stay almost constant for the range $200 \leq y^{+} \leq 600$. Then all profiles increase in a similar manner.

\section{P08: Flow Angle $\gamma_{a}$, Shear Stress Angle $\gamma_{s}$ and Flow Gradient Angle $\gamma_{g}$}

The flow, shear stress and flow gradient angles are defined as follows,

$$
\gamma_{a}=\arctan \left(\frac{W}{U}\right)
$$




$$
\begin{gathered}
\gamma_{s}=\arctan \left(\frac{-\overline{v w}}{-\overline{u v}}\right) \\
\gamma_{g}=\arctan \left(\frac{\partial W / \partial y}{\partial U / \partial y}\right)
\end{gathered}
$$

Plots of flow angles, shear stress angles and flow gradient angles are shown in Figure $4.3 \mathrm{~m} 4.30$ respectively.

In a two dimensional flow the flow angle is zero, hence the flow angle is a measure of three-dimensionality of the flow field. At this station close to the wall the flow is moving in the positive $z$-direction. Close to the wall $y^{+} \leq 5$ the flow angle seems to be constant which is consistent with findings by Olcmen [41] and Byun [4]. All profiles in this cluster show a semi-logarithmic region between $10 \leq y^{+} \leq 100$. Near the wall, the flow angle magnitude increases along profiles $P 06, P 08$ and P10 up to $y^{+}=800$ due to pressure gradient effects as the wave peak at $x / L=0.94$ is approached. In the positive span-wise direction the flow angle also increases but with a lesser rate. For all profiles, the flow changes direction from positive to negative span-wise direction at about $y^{+}=1420$. This might be due to the fact that away from the wall the flow is still directed in the negative $z$-direction by the side wall turning effect from the previous wave cycle.

Shear angle profiles are shown in Figure 4.3n. For this cluster the shear angle is close to zero near the wall and decreases away from the wall. Also the profiles show a semi-logarithmic region; however, the variation is smaller than for the flow angle. A local minimum is shown at about $y^{+}=1420$, which is the same height where all profiles experience a reversal in the flow angle sign. The magnitude of the minimum doesn't change in the stream-wise direction while it increases in the span-wise direction.

Flow gradient angle profiles are shown in Figure 4.30. Near the wall the flow gradient angle tends to a constant value. Also in the range $5 \leq y^{+} \leq 30$ all profiles show a semi-logarithmic region. In the region $100 \leq y^{+} \leq 300$ the profiles experience a minimum followed by a maximum. At $y^{+}=1450$ there exists another minimum. Above this height the flow gradient increases to a positive value. Finally, an idea of the flow anisotropy can be obtained by plotting the difference between the shear and flow gradient angles. If the flow is isotropic, then both angles should be equal meaning that the shear stress component and the mean strain in the $x-z$ plane are in the same direction. From Figure 4.3p it can be seen that near the wall all profiles almost collapse to a one curve except profiles P08 and P09, which might be due to some misalignment of LDV. Also near the wall the magnitude of the difference decreases to a constant value in the semi-logarithmic region; then it reaches a maximum at $y^{+}=770$.

P08: Mixing Length $L_{m}$ and Turbulent Eddy viscosity $\nu_{T x}$ 
The mixing length (Cebeci [5], Olcmen [41]), as defined by Equation 4.12, is given in Figure 4.3q] All profiles are non-dimensionalized using wall variables.

$$
L_{m}^{+}=\frac{\left[(\overline{u v})^{2}+\left(\overline{v w^{+}}\right)^{2}\right]^{\frac{1}{4}}}{\sqrt{\left(\frac{\partial U^{+}}{\partial y^{+}}\right)^{2}+\left(\frac{\partial W^{+}}{\partial y^{+}}\right)^{2}}}
$$

All profiles collapse to one profile in the range $y^{+} \leq 1260$, with more variation for $y^{+} \geq 700$. Above this height each profile experiences a maximum value that differs from one profile to the other. If the mixing length is plotted versus $y^{+}$in a log-log plot, the profiles behaves almost linearly with a sharp change of slope at $y^{+}=80$. In the range $80 \leq y^{+} \leq 584$ the profiles also show a semi-logarithmic behavior. Above $y^{+}=584$ all profiles don't collapse and their behavior is non-linear, however all profiles still have the same trend. Figure $4.3 \mathrm{q}$ also shows the length scale for two-dimensional boundary layer from DNS calculations by Spalart, [53]. The length scale for the two-dimensional boundary layer collapses with measured data up to $y^{+}=100$ approximately.

The turbulent eddy viscosity as defined by Equation 4.13 is shown in Figure $4.3 \mathrm{~s}$.

$$
\frac{\nu_{T x}}{\nu}=\frac{-\overline{u v^{+}}}{\frac{\partial U^{+}}{\partial y^{+}}}
$$

Shown in the figure are the turbulent eddy viscosity in the stream-wise direction, $\nu_{T x}$, normalized by the kinematic viscosity, $\nu$. The behavior of eddy viscosity is similar to the mixing length. All profiles collapse to a single curve below $y^{+}=400$. The slope near the wall, $y^{+} \leq 80$, is not constant and it decreases away from the wall. In the range $80 \leq y^{+} \leq 620$ the variation is non-linear then the profiles reach a maximum value of 1000 to 1300 at about $y^{+}=1500$. DNS data are also shown. Again, the measured data collapse onto DNS data up to $y^{+}=100$ approximately.

\subsubsection{P13 Cluster}

Profiles $P 11, P 13$ and $P 15$ are aligned in the stream-wise direction, while profiles P12, $P 13$ and P14 are aligned in the span-wise direction as shown in Figure 4.1. The center profile, $P 13$, in this cluster is located at $x / L=0.871$ and $Z / W_{x=0}=-0.0173$. This cluster is at the same stream-wise location as cluster $P 08$. This cluster is close to the centerline of the test section.

\section{P13: Mean Stream-wise and Span-wise Velocities, $U^{+}, W^{+}$}

Mean flow velocities, $U$ and $W$, are shown in Figure 4.4a, Again the profiles show a viscous sublayer and a semi-logarithmic region between $y^{+}=30$ and $y^{+}=200$. The 
$U$ velocity profiles don't change much from one location to the other. Also the boundary layer thickness is maximum at the center of the tunnel, hence measurements were not made out to the free stream. The $W$ velocity component show a similar behavior from one location to the other. All profiles have a negative value close to the wall. The $W$ velocity component decreases away from the wall for all profiles up to $y^{+}=300$ where the slope reaches zero, then the slope increases toward the end of profiles. As the flow proceeds downstream the magnitude of the span-wise velocity decreases as the flow starts turning in the same way as the side walls. In the span-wise direction the $W$ profiles don't show any difference between profiles $P 12, P 13$ and P14; this is expected since all profiles are close to the tunnel centerline and very close to the stream-wise location of the side wall peak.

\section{P13: Reynolds' Normal Stresses, ${\overline{u^{2}}}^{+}, \bar{v}^{+}$and ${\overline{w^{2}}}^{+}$}

Figure $4.4 \mathrm{~b}$ shows the stream-wise Reynolds' normal stress. All profiles collapse very well to a single curve up to the peak value. The peak value of the normal stress is almost twice the peak value for a two dimensional boundary layer as given by DNS data of Spalart [53]. The location of the peaks occur around $y^{+}=14.9$. All profiles show another peak at $y^{+}=474$. The second peak value range from 10 to 12.9. Between $y^{+}=74$ up to the maximum height reached the stresses increase with a very small rate. Due to the thickening boundary layer the stresses didn't reach a zero value up to the measured distance.

The normal to the wall and span-wise normal Reynolds' stresses are shown in Figure $4.4 \mathrm{c}$ along with DNS data. Near the wall all stresses approach the two-dimensional boundary layer behavior. However away from the wall the magnitude of both stresses are much higher than the two-dimensional ones. The normal to the wall normal stress changes slightly which might be due to uncertainty the matching procedure. Profiles for the span-wise normal stress collapses to a single profile up to $y^{+}=25$ approximately, however profile P13 is slightly lower than the others. Both stresses tend to reach the same values at the maximum height achieved.

\section{P13: Reynolds' Shear Stresses, $\overline{u v}^{+}, \overline{u w}^{+}$and $\overline{v w}$}

The Reynolds' shear stresses $\overline{u v}^{+}$and $\overline{v w}^{+}$are shown in Figure $4.4 \mathrm{~d}$ along with DNS data of Spalart [53]. Below $y^{+}=11$ all $\overline{u v}^{+}$stress profiles collapse together on top of the DNS profile. However away from the wall the shear stresses increase rapidly up to the maximum height of measurements. The $\overline{u v^{+}}$reaches a value of unity at about $y^{+}=60$. The $v w^{+}$stress has a similar behavior. Since the flow in this region is close to a two dimensional flow, the magnitude of the $\overline{v w}$ is very small close to the wall but increases away from the wall. 
The $\overline{u w^{+}}$stress is shown in Figure 4.4e. Near the wall the stress is negative and reaches a minimum at $y^{+}=10$ then increases linearly up to the maximum measured height. The trend is not clear at this location, since the profiles show some scatter due to relatively small value of stress.

\section{P13: Correlation Coefficient, $\rho_{u v}$}

Figure $4.4 \mathrm{f}$ shows the correlation coefficient along with values from DNS data. Similar to $P 08$ cluster the measured values are much lower than the DNS data. However the correlation coefficient profiles have the same behavior as the DNS data increasing semilogarithmically with a peak at $y^{+}=15$ of 0.30 . Afterwards the coefficient decreases to a minimum at $y^{+}=86$ then increases with distance from the wall to values above 0.30 at the maximum height measured.

\section{P13: 1/S Parameter}

Figure $4.4 \mathrm{~g}$ shows the 1/S parameter. All profiles don't collapse to a single curve below $y^{+}=60$. Above that height all profiles collapse to a single curve. For a twodimensional boundary layer, the value of $1 / \mathrm{S}$ is 0.7 in the semi-logarithmic region, however for this cluster the 1/S parameter doesn't show a constant value but instead it is decreasing semi-logarithmically, in contrast to the two-dimensional behavior and to cluster $P 08$.

\section{P13: Turbulent Kinetic Energy $T K E$}

The turbulent kinetic energy is shown in Figure 4.4h with DNS data for a two-dimensional boundary layer. Again, the level of kinetic energy is much higher than the two dimensional boundary layer case. The TKE increases to a value of 9 at $y^{+}=15.7$ then it stays almost constant up to $y^{+}=74$ and then increase again. This behavior suggest that the boundary layer history increases the turbulence level by convection and diffusion downstream.

\section{P13:Turbulent Diffusion Velocity $\vec{V}_{q}$}

Figures $4.4 \mathrm{i} 4.4 \mathrm{k}$ show the turbulent diffusion velocity components in the $x, y$ and $z$ directions, respectively. The diffusion velocity in the stream-wise direction has a peak at $y^{+}=6$ with a value of 2.6 then decreases to a minimum at $y^{+}=35$; the values of the minimum ranges from 0.6 to 1.0 . Above this height the stream-wise diffusion velocity is almost constant. 
Figure 4.4j]shows the normal to the wall turbulent diffusion velocity component. All profiles collapse to a single curve near the wall up to $y^{+}=32$, approximately. All profiles show a negative peak with a value of -0.12 at $y^{+}=6$. In the range $32 \leq y^{+} \leq 116$ the diffusion velocity is positive and close to zero, then above that height the velocity decreases. There isn't a clear trend in the profiles due to some scatter in the data.

Figure $4.4 \mathrm{k}$ shows the span-wise turbulent diffusion velocity component. All profiles don't collapse, however the trend is the same for all curves. In general the diffusion velocity is positive and it increases to a maximum value at about $y^{+}=85$ then decreases towards zero. Values increase in the span-wise direction but almost constant in the stream-wise direction.

\section{P13: Townsend's Structural Parameter $A_{1}$}

Townsend's structural parameter is shown in Figure 4.41. At this cluster, $A_{1}$ increases semi-logarithmically near the wall up to $y^{+}=35$. In the same region all profiles collapse to one curve, in contrast with $P 08$ cluster which doesn't show as good a collapse. Figure 4.41 also shows that the $A_{1}$ parameter has a value less than DNS data for a twodimensional boundary layer. However, near the wall the measured profiles approach the DNS data.

\section{P13: Flow Angle $\gamma_{a}$, Shear Stress angle $\gamma_{s}$ and Flow Gradient Angle $\gamma_{g}$}

Flow angles are shown in Figure $4.4 \mathrm{~m}$. All profiles show a semi-logarithmic region corresponding to the semi-logarithmic region in the velocity profile. The flow angle changes sign near the wall in the stream-wise direction, signifying changes in the flow direction caused by surface pressure gradients. Away from the wall, the flow angle decreases into negative values as the flow in the outer layer is less sensitive to the local floor pressure gradient. A minimum value is reached in the range $200 \leq y^{+} \leq 400$ then the flow angle increase up to the maximum height measured.

The shear stress angle is shown in Figure $4.4 \mathrm{n}$. First thing to note is the discontinuity in the curves. This is due to the fact that the curves are composed of two data sets obtained by different probes. Data above $y^{+}=7$ were obtained by the CompLDV and the data below this height were obtained from the Short system. For the CompLDV data, the probe alignment was not accurate enough which led to some unphysical spikes very close to the wall in all quantities involving the $v$-velocity component fluctuations.

Near the wall, $y^{+} \leq 30$, the shear stress angle doesn't show significant changes from one profile to the next since at this cluster the flow is very close to being two dimensional and that in this range the $\overline{u v}$ and $\overline{v w}$ shear stresses almost collapse. Above this height all profiles show an increase in the shear stress angle. In the stream-wise direction the shear 
angle profiles don't change, while in the span-wise direction the shear stress angle profile slope increase.

Flow gradient angle profiles are shown in Figure 4.40. Again there is no significant change among the profiles very close to the wall up to $y^{+}=30$. The flow gradient angle is very close to zero near the wall and it has a negative sign. This means that the mean strain rate lags the shear stress, in contrast with P08 cluster, where the shear stress was lagging the mean strain. Profiles in the stream-wise direction experience a minimum at $y^{+}=100$ and a maximum with a positive value at $y^{+}=565$. This change in the sign of the mean rate of strain is due to the flow changing direction away from the wall, near the wall the flow angle is positive while away form the wall is negative.

Figure 4.4p shows the difference between the shear stress angle and the flow gradient angle. Near the wall, there is a very large positive difference between both angles. The positive value means that the turbulent shear stress is leading the mean rate of strain vector. Near the wall the maximum difference is obtained reaching about $55^{\circ}$. In the loglayer range the difference drops to about $5^{\circ}$ and is almost constant, then increases in the span-wise direction.

\section{P13: Mixing Length $L_{m}$ and Turbulent Eddy viscosity $\nu_{T x}$}

Figures $4.4 \mathrm{q}$ and $4.4 \mathrm{~s}$ show the mixing length and turbulent eddy viscosity, respectively. An enlarged view of near wall region is also shown in Figures $4.4 \mathrm{r}$ and $4.4 \mathrm{t}$ for the mixing length and eddy-viscosity, respectively. Both quantities collapse to a single curve for the entire range of measurement. In the range $y^{+} \geq 500$ both quantities show a semi-logarithmic behavior.

\subsubsection{P18 Cluster}

Profiles $P 16 p, P 16, P 18, P 18_{1}$ and $P 18_{2}$ are aligned in the span-wise direction as shown in Figure 4.1. The center profile, $P 18$, in this cluster is located at $x / L=0.912$ and $z / W_{x=0}=-0.192$. The distance between each two profiles is $1.0 \mathrm{in}$ in the stream-wise direction. This cluster is close to a side wall peak which is located at $x / L=0.9375$ and $z / W_{x=0}=-0.331$.

\section{P18: Mean Stream-wise and Span-wise Velocities, $U^{+}, W^{+}$}

Figure 4.5 a shows the mean stream-wise velocity components. All $U$-velocity profiles show a viscous sub-layer and some what of a log-layer with different slopes. The $U$ velocity profile becomes fuller going downstream as the side wall peak is approached. 
Also going downstream the slope in the log-layer region changes and at $P 18_{2}$ the loglayer region becomes very small.

The $W$-velocity components also shown in Figure 4.5a. All profiles show the same trend with a maximum value that increases going downstream and ranges between 4.5 and 5.0. The location of the maximum shifts away from the wall in the downstream direction from $y^{+}=87$ to $y^{+}=167$. All profiles show a semi-logarithmic behavior that starts after the maximum value is reached. This region starts at the end of the semilogarithmic region in the stream-wise velocity profile as also pointed out by Olcmen [41].

\section{P18: Reynolds' Normal Stresses, ${\overline{u^{2}}}^{+}, \bar{v}^{+}$and ${\overline{w^{2}}}^{+}$}

The stream-wise Reynolds' normal stresses, ${\overline{u^{2}}}^{+}$, is shown in Figure $4.5 \mathrm{~b}$. All profiles show a similar trend. Near the wall, $y^{+} \leq 20$, all profiles follow the two-dimensional boundary layer behavior with values that are higher than the two-dimensional values. The peak value is located around $y^{+}=14$ for all profiles which is the same location for the two-dimensional case. At about $y^{+}=500$, all profiles show a second peak that is lower in value than the first peak. The magnitude of the peak decreases going downstream and the location shifts away from the wall. The second peak tends to smooth out at locations down-stream, forming a plateau. This plateau region is followed by anther decrease in the normal stress and a second plateau region is formed.

The normal to the wall and span-wise normal Reynolds' stresses are shown in Figure 4.5c. Near the wall, profiles of both stresses collapse up to $y^{+}=25$. The normal to the wall normal stress shows values less than the two-dimensional case below $y^{+}=200$, higher than that the stress increases steadily. At $y^{+}=1392$ the slope increases sharply and the stress levels increase.

The span-wise normal stress increases up to $y^{+}=20$, stays relatively constant, and then increases again until it reaches a maximum at $y^{+}=700$ for all profiles. Above this height the stress decrease until it reaches almost the same values as the normal to the wall normal stress.

A unique feature of this cluster is that all normal stresses reach a state of equal magnitude at the maximum height measurements were obtained. Also in the range $1345 \leq y^{+} \leq 5200$ the span-wise normal stress is actually higher than the stream-wise normal stress. This might be due to the flow approaching the side wall peak where a very strong favorable pressure gradient exists which accelerates the flow and reduces the turbulence levels.

P18: Reynolds' Shear Stresses, $\overline{u v}+, \overline{u w}^{+}$and $\overline{v w}$

The $\overline{u v}^{+}$and $\overline{v w^{+}}$stresses are shown in Figure $4.5 \mathrm{~d}$. Below $y^{+}=30$ all profiles collapse to single curve. The $\overline{u v^{+}}$shear stress show a plateau between $y^{+}=30$ to $y^{+}=150$ 
with a value of -0.5 . All profiles have a minimum with a variable magnitude. The location of the minimum also shifts upward going in the free-stream direction. At $y^{+}=2100$ all profile show a local maximum of about -0.17 , then all profiles decrease again.

The $\overline{v w^{+}}$stress, also shown in Figure $4.5 \mathrm{~d}$ is self similar below $y^{+}=30$. At this height all profiles show a minimum with a value of -0.3 . The stress then increases with semilogarithmic behavior up to $y^{+}=533$. In the range $533 \leq y^{+} \leq 1359$ the stress is almost constant at 0.37 , then it undergoes anther maximum at $y^{+}=2367$.

The $\overline{u w^{+}}$stress is shown in Figure 4.5e Profiles collapse below $y^{+}=14.1$, where all profiles experience a peak value between 2.0 and 2.5. All profiles experience two local minimums and a local maximum at $y^{+}=550, y^{+}=4234$ and $y^{+}=1624$ respectively.

Another unique feature of this cluster is that the shear stresses also reach a state at the maximum height achieved where all shear stresses almost have the same values but with different signs. Moreover, the $\overline{v w}$ stress, which is usually the smallest in magnitude of all three stresses, is the largest stress in this case.

\section{P18: Correlation Coefficient, $\rho_{u v}$}

The correlation coefficient, $\rho_{u v}$, is shown in Figure 4.5f. A similar trend to that of cluster $P 08$ and $P 13$ can be seen for this cluster. The correlation coefficient reaches a very low value at the maximum height measured, even though the normal stresses are still significantly higher than zero. This is due to the rapid decrease in shear stress as the test section's mid-height is approached which is located at about $y^{+}=5800$, based on $u_{\tau}$ calculated at $P 18$.

\section{P18: 1/S Parameter}

The $S^{-1}$ parameter is shown in Figure $4.5 \mathrm{~g}$. Above $y^{+}=20$ all profiles almost collapse to a single curve. Near the wall and below $y^{+}=10$, measured profiles don't collapse and they also don't follow DNS data. This is due to measurement uncertainties. Above this height all profiles follow DNS data decreasing with the same slope. In the range $150 \leq y^{+} \leq 478$ the $S^{-1}$ is almost constant with a value of about 0.5 which is lower than the two-dimensional value of 0.7 . Above $y^{+}=478$ the $S^{-1}$ parameter decreases semi-logarithmically.

\section{P18: Turbulent Kinetic Energy $T K E$}

The turbulent kinetic energy is show in Figure $4.5 \mathrm{~h}$. Again the measurements follow the DNS data trend near the wall up to $y^{+}=14$. The turbulence levels are higher than those experienced in the two-dimensional channel flow. The TKE profiles have the same 
trend as found in cluster P08, where there is a second peak in the TKE profile. The first peak is located at $y^{+}=14$ for all profiles and has a value between 5.0 and 6.5 . The second peak is located between $y^{+}=400$ and 600 and it's value ranges from 4.5 to 5.5 . The value of the second peak decreases monotonically going downstream. This can be attributed again to the presence of a favorable pressure gradient that accelerates the flow as the side wall peak is approached.

\section{P18:Turbulent Diffusion Velocity $\vec{V}_{q}$}

The turbulent diffusion velocity components $V_{q x}, V_{q y}$ and $V_{q z}$ are shown in Figures 4.5i, $4.5 \mathrm{j}$ and $4.5 \mathrm{k}$ respectively. The $x$-component of the turbulent diffusion vector experience multiple maxima at $y^{+}=4,140$ and 3900 and two minima at $y^{+}=30$ and 950. The stream-wise diffusion velocity has a positive sign near the wall indicating the dominance of sweeping motion near the wall. In the range $14 \leq y^{+} \leq 50$ and $488 \leq y^{+} \leq 2227$ the diffusion velocity is negative indicating dominant ejection motion.

The turbulent diffusion velocity in the vertical direction, $V_{q y}$ shows a collapse of profiles at these locations within measurement uncertainties. All profiles seem to follow the same trend and values. The diffusion velocity component changes sign five times over the entire profile with three minima at $y^{+}=7,300$ and 3500 and two maxima at $y^{+}=50$ and 1000. Near the wall the diffusion velocity is negative which again indicates the dominance of sweeping motion. In the log-layer range the diffusion velocity is mostly positive. Away from the wall the velocity component tends to shift towards a positive value.

Figure $4.5 \mathrm{k}$ shows profiles of $V_{q z}$. The diffusion velocity is positive near the wall indicating a preferred positive direction of motion in the $z$-direction. This is due to the skewing effect due to the approaching side wall. Away from the wall, the velocity is negative which is due to the direction of motion upstream of this cluster.

\section{P18: Townsend's Structural Parameter $A_{1}$}

Figure 4.51 shows Townsend's structural parameter. For this cluster this parameter works well in collapsing most of the data in contrast with P08. The significance is that the ratio of shear stress in a plane parallel to the tunnel centerline to twice the kinetic energy doesn't change from one profile to the other. All profiles show two peaks, at $y^{+}=70$ and $y^{+}=800$. Again all values are much lower than two-dimensional values.

\section{P18: Flow Angle $\gamma_{a}$, Shear Stress angle $\gamma_{s}$ and Flow Gradient Angle $\gamma_{g}$}

Figure $4.5 \mathrm{~m}$ shows flow angle profiles. At the wall the flow angle decreases in the stream-wise direction. This is due to the fact that the side wall starts to curve in the 
negative $z$-direction, which results in reduction in pressure which in turn would curve the surface streamlines toward the wall. Away from the wall in the range $y^{+} \leq 145$ for profiles P16p and P16 the flow angle decreases and for profiles $18_{1}$ and $P 18_{2}$ the flow angle increases while for profile $P 18$ the flow angle is almost constant. This is due to a change in sign for the span-wise pressure gradient close to profile $P 18$. Upstream of $P 18$ the span-wise pressure gradient is negative while positive downstream. Above $y^{+}=145$ pressure gradient effects are negligible and all profiles have the same trend and decreases to zero.

Figure $4.5 \mathrm{n}$ shows the shear stress angle. The first few points of all profiles experience some scatter so they can't be interpreted. However close to the wall all profiles show an almost constant shear stress angle. The stress angle then decreases slowly until $y^{+}=$ 100 , approximately, then the decrease is semi-logarithmic with a more steeper slope. An interesting observation to note here, is that all angle profiles change sign at about the same height approximately which is the same height where all flow angles roughly have the same value at $y^{+}=150$. Also, all profiles show a plateau in the range $260 \leq y^{+} \leq 1000$ followed by a minimum at $y^{+}=2200$.

Figure 4.50 shows profiles of flow gradient angle. Near the wall and below $y^{+}=60$, profiles $P 16 p$ and $P 16$ while profiles $P 18_{1}$ and $P 18_{2}$ increase and $P 18$ is constant. All profiles then decrease semi-logarithmically in a similar behavior as the shear stress angle.

Profiles of the difference between shear stress angle and mean flow gradient angle are shown in Figure 4.5p. Near the wall the difference is almost constant in the viscous sub-layer and is equal approximately to $15^{\circ}$. Then the difference decreases up to $y^{+}=150$ then it increases again. This is the same height where the flow angle profiles show similar behavior. Also all profiles show a maximum at $y^{+}=704$ with different peak values. The difference then decreases to positive values.

\section{P18: Mixing Length $L_{m}$ and Turbulent Eddy viscosity $\nu_{T x}$}

Figures $4.5 \mathrm{q}$ and $4.5 \mathrm{~s}$ show the turbulent length scale, $L_{m}$, and the turbulent eddy viscosity, $\mu_{T x}$, respectively. An enlarged view of near wall region is also shown in Figures $4.5 \mathrm{r}$ and $4.5 \mathrm{t}$ for the mixing length and eddy-viscosity, respectively. Both quantities have the same behavior with all profiles collapsing up to $y^{+}=1000$. The length scale increases monotonically until it reaches $y^{+}=300$, then it increases rapidly. All profiles have a maximum at $y^{+}=3800$. The eddy viscosity profiles don't collapse as well as the mixing length profiles.

\subsubsection{P19 Cluster}

Profiles P19pp, P19p, P19, $P 19_{1}$ and $P 19_{2}$ are aligned in the span-wise direction as shown in Figure 4.1. The center profile, P19, in this cluster is located at $x / L=0.912$ 
and $z / W_{x=0}=-0.1714$. The distances between profiles is 1.0in in the stream-wise direction. The distance between this cluster and P18 cluster is $0.76 \mathrm{in}$ in the positive span-wise direction.

\section{P19: Mean Stream-wise and Span-wise Velocities, $U^{+}, W^{+}$}

Figure $4.6 \mathrm{a}$ shows the mean stream and span-wise velocity components. All profiles show a viscous sub-layer and a semi-logarithmic regions. However the slope of the semilogarithmic region is less than $0.41^{-1}$ and in general changes slightly from one profile to the other. No profiles show a free stream edge. The mean span-wise velocity component shows a similar trend as the $P 18$ cluster. The velocity profiles have a maximum that shifts away from the wall moving in the stream-wise direction. The magnitude of the peak is almost constant. Near the wall the profiles' slope which is approximately equal to the stream-wise vorticity component decreases going downstream, this is due to the change in sign of the local span-wise pressure gradient between clusters P19p and P19.

\section{P19: Reynolds' Normal Stresses, ${\overline{u^{2}}}^{+},{\overline{v^{2}}}^{+}$and ${\overline{w^{2}}}^{+}$}

Figure $4.6 \mathrm{~b}$ shows the stream-wise normal stress. Qualitatively the stream-wise normal stresses at this cluster have the same trend as the P18 cluster. The stress profiles have two peaks with the first at $y^{+}=14$ and the location of the second peak shifting away from the wall going downstream. The first peak has almost the same magnitude as the P18 cluster, however the second peak magnitude is higher than that observed in the previous cluster. This may be due to a higher value of local shear stress than that for P18 cluster.

The normal to the wall and span-wise normal stresses are shown in Figure $4.6 \mathrm{c}$. The normal stresses show similar behavior as those in the P18 cluster. However, similar to the stream-wise normal stress, the magnitudes of both stresses are higher than those in the previous P18 cluster. The normal to the wall stress shows magnitudes lower than the DNS data, which is due to three-dimensionality effects as pointed out by Moin [37]. Similarly, the span-wise normal stress follows the same trends from the previous cluster, but with higher magnitudes. For $y^{+} \geq 4000$ all normal stresses approach the same magnitude.

\section{P19: Reynolds' Shear Stresses, $\overline{u v}+, \overline{u w}^{+}$and $\overline{v w^{+}}$}

The $\overline{u v^{+}}$shear stress is shown in Figure 4.6d. All profiles collapse as the wall is approached. However, shear stress values near the wall are much smaller than those for two-dimensional boundary layer. For $y^{+} \geq 20$ all profiles start to depart, developing a maximum whose location shifts away from the wall with profile's stream-wise location. 
The magnitude of the peaks decrease with stream-wise location. This may be due to decrease in the shear stress production which is reduced due to decrease in vertical normal stress with stream-wise distance. Profiles at the downstream end of this cluster experience stronger three-dimensional effects than the upstream profiles since they are closer to the side wall peak as shown in Figure 4.1. Also in the same figure, the $\overline{v w}$ shear stress is shown. All profiles collapse near the wall up to $y^{+}=20$ where they start to depart. All profiles develop a minimum between $y^{+}=20$ to 50 where the magnitude of the $\overline{v w}^{+}$ stress is about $60 \%$ of the $\overline{u v}^{+}$stress. Above $y^{+}=50$ the stress increases away from the wall until it develops a plateau area with a positive sign at $y^{+}=350$. Further away from the wall a maximum is developed at $y^{+}=3000$, then the stress start decreasing again. Again as in the previous cluster, at the positive maxima the $\overline{v w}$ is greater than the $\overline{u v}^{+}$ shear stress.

The $\overline{u w^{+}}$stress is shown in Figure 4.6e. The same trend shown in the previous cluster is shown here. The $\overline{u w^{+}}$stress develops a large positive peak value at $y^{+}=13$. The peak value is about six times larger than the $\overline{u v}^{+}$shear stress. The $\overline{u w}$ stress then decreases to a negative value, developing two minimas and a maxima at $y^{+}=450,3000$ and 2000, respectively. The locations of the peaks differ slightly from one profile to the other. The location of the maxima is coincident with the location of the maxima in the $\overline{v w^{+}}$shear stress.

\section{P19:Correlation Coefficient, $\rho_{u v}$}

The correlation coefficient $\rho_{u v}$ is shown in Figure 4.6f All profiles show almost a constant value in the semi-logarithmic region with a value of about 0.3 . At $y^{+}=655$ there is a local maxima; above this height the correlation coefficient decreases to about $y^{+}=2500$ where it seems to have leveled off. This is due to the fact that the shear stress approaches a constant value at the highest measurement location and that the normal stresses are approaching equal values.

\section{P19: 1/S Parameter}

Figure $4.6 \mathrm{~g}$ shows the $S^{-1}$ parameter. All profiles collapse very well in the semilogarithmic and outer layers. In the semi-log layer the $S^{-1}$ parameter is about constant at about 0.6 . At $y^{+}=700$ all profiles decrease semi-logarithmically. This height is coincident with the minimum in the $\overline{u v}$ shear stress.

\section{P19: Turbulent Kinetic Energy $T K E$}

Turbulent kinetic energy is shown in Figure 4.6h. Near the wall all profiles collapse and have the same slope as the two-dimensional DNS data. Similar to the previous cluster there are two peaks. However, the second peak is as high as the primary one at $y^{+}=18$. The position of the second peak shifts away from the wall going downstream. 


\section{P19:Turbulent Diffusion Velocity $\vec{V}_{q}$}

The turbulent diffusion velocity components $V_{q x}, V_{q y}$ and $V_{q z}$ are shown in Figures 4.6i, 4.6j and 4.6k, respectively. All profiles collapse near the wall up to $y^{+}=15$. The trend is the same as for the previous cluster. Contributions to $V_{q x}$ are mainly from ${\overline{u^{3}}}^{+}$and ${\overline{u w^{2}}}^{+}$. The first positive peak and the last negative peak are associated with motions creating $u$ and $w$-fluctuations of same positive and negative signs, respectively. Between about $15 \leq y^{+} \leq 600$ the peaks are associated with motions creating $u$ and $w$-fluctuations of opposite signs. The ${\overline{u w^{2}}}^{+}$correlation contributes significantly to the turbulent diffusion velocity. This can be seen in the range $60 \leq y^{+} \leq 600$ where for a two-dimensional boundary layer, not shown here, the stream-wise diffusion velocity component is almost zero.

The vertical turbulent diffusion velocity component is shown in Figure 4.6j. The $\bar{u}^{2} v^{+}$ correlation is the major contributor to this diffusion velocity component. The effect of the $\bar{v}^{3}$ and $\overline{v w^{2}}$ correlations is to shift the diffusion velocity into positive values away from the wall. The first peak near the wall is due to sweep motion while the positive peaks are due to ejection motion.

Finally, the turbulent diffusion velocity in the span-wise direction is shown in Figure 4.6k. The diffusion velocity is mainly due to ${\overline{u^{2} w}}^{+}$and $\bar{w}^{3}$. Near the wall up to $y^{+}=15$, motions creating positive $u$ and $w$-fluctuations correlate while in the range $15 \leq y^{+} \leq 600$ motions creating opposite sign $u$ and $w$-fluctuations correlate. Above this height the same trend repeats but with a much lower values.

\section{P19: Townsend's Structural Parameter $A_{1}$}

Townsend's structural parameter is shown in Figure 4.61 All profiles collapse near the wall up to $y^{+}=30$. All profiles have two peaks at $y^{+}=70$ and 940 , approximately. The values of this parameter are much lower than two dimensional case which is typical for three-dimensional flows.

\section{P19: Flow Angle $\gamma_{a}$, Shear Stress angle $\gamma_{s}$ and Flow Gradient Angle $\gamma_{g}$}

The flow angle, shear angle and flow gradient angle are shown in Figures $4.6 \mathrm{~m}$, $4.6 \mathrm{n}$ and 4.60, respectively. All profiles show a semi-logarithmic region extending to $y^{+}=115$. The extent of this region changes for different profiles and is larger than for the previous cluster. Above this height there is a small difference between all profiles. At the wall, flow angles decrease in the downstream direction. The shear stress angle shows a little variation for this cluster. All profiles show two semi-logarithmic variations with different slopes in the ranges $8 \leq y^{+} \leq 60$ and $60 \leq y^{+} \leq 300$. The profiles in this cluster are 
very similar to those in the previous one even though both clusters have different flow angles. Flow gradient angles have the same trend as in the previous cluster but have different values. The near wall behavior of the flow gradient angle is similar to the flow angle which indicate that the mean strain rate responds quickly to mean flow changes in contrast with the shear stress angle. This is shown in Figure $4.6 \mathrm{p}$ where the difference between the shear stress angle and the flow gradient angle profiles is shown. At the wall the difference is close to $30^{\circ}$, discarding the first point due to scatter, and is about constant in the semi-log layer at about $20^{\circ}$. The profiles develop a maxima at about $y^{+}=650$ with different peak values then all profiles decrease to a negative value.

\section{P19: Mixing Length $L_{m}$ and Turbulent Eddy viscosity $\nu_{T x}$}

The mixing length is shown in Figure 4.6q. All profiles collapse to a single curve very close to the wall, $y^{+} \leq 50$. All profiles show two regions with constant slope when plotted in a log-log scale. The slopes change at $y^{+}=70$ approximately. Above $y^{+}=950$ all profiles increase steeply and show a peak with different values.

The eddy-viscosity is plotted in Figure $4.6 \mathrm{~s}$. The eddy viscosity shows a similar trend as the mixing length, however the profiles increase smoothly then decrease with no steep changes in slope.

Enlarged views of near wall region is shown in Figures $4.6 \mathrm{r}$ and $4.6 \mathrm{t}$ for the mixing length and eddy-viscosity, respectively.

\subsubsection{P20 Cluster}

Profiles P20pp, P20p, P20, $P 20_{1}$ and $P 20_{2}$ are aligned in the stream-wise direction as shown in Figure 4.1. The center profile, $P 20$, in this cluster is located at $x / L=0.912$ and $z / W_{x=0}=-0.144$. The distances between profiles is $1.0 \mathrm{in}$ in the stream-wise direction. The distance between this cluster and P19 cluster is $1.0 \mathrm{in}$ in the positive span-wise direction. The distance between profile $P 20_{1}$ and $P 20_{2}$ is $0.4 i n$. only in the stream-wise direction, due to probe interference with tunnel structure.

\section{P20: Mean Stream-wise and Span-wise Velocities, $U^{+}, W^{+}$}

Figure $4.7 \mathrm{a}$ shows the mean velocities in the stream and span-wise directions. For the stream-wise velocity component, all profiles show a viscous sub-layer region and a semi-logarithmic region. The slope of the semi-logarithmic region is almost constant. However the profiles show higher levels in the stream-wise direction. All profiles show the beginning of a wake-like region close to the last measurement point. The last two profiles show very similar values due to the fact that they are closer in distance than 
the other profiles. The span-wise velocity component shows the same shape as previous clusters with a reduced value of the peak value.

\section{P20: Reynolds' Normal Stresses, ${\overline{u^{2}}}^{+}, \bar{v}^{+}$and ${\overline{w^{2}}}^{+}$}

The stream-wise normal stress is shown in Figure 4.7b. All profiles show the same trend as the P18 and P19 clusters. All profiles show two peaks, with the largest peak at $y^{+}=15$ and the second is at $y^{+}=800$. The location of the second peak varies slightly for different profiles. Peak values are slightly higher than the previous two clusters. Figure $4.7 \mathrm{c}$ shows the normal to the wall and span-wise normal stresses. The normal to the wall normal stress profiles show a plateau region starting at $y^{+}=60$ that is present in all profiles in this cluster and the previous two clusters. At about $y^{+}=170$ the stress increases semi-logarithmically, reaching values close to the span-wise normal stress. The span-wise normal stress is also shown in Figure $4.7 \mathrm{c}$. The profiles show the same trend as previous clusters, approaching two-dimensional values close to the wall. Away from the wall the stress levels off at about $y^{+}=60$, which is the same height where a plateau exists in the $\overline{v^{2}}$ stress. The second peak that was present in the P18 and P19 clusters seems to have smoothed out in this cluster. The scaling for profile $P 20$ seems to be incorrect since it higher than other profiles. Also, for profile $\mathrm{P}_{2} \mathrm{O}_{2}$ no near wall data were obtained so the skin friction velocity was estimated by fitting the profile to DNS data. In general, profiles of all three stresses show higher turbulence levels than two-dimensional data and most of available three-dimensional attached boundary layer flows.

\section{P20: Reynolds' Shear Stresses, $\overline{u v}^{+}, \overline{u w}^{+}$and $\overline{v w}{ }^{+}$}

The shear stresses $\overline{u v}$ and $\overline{v w}^{+}$are shown in Figure $4.7 \mathrm{~d}$. Profiles of the $\overline{u v}^{+}$shear stress show a plateau area starting at approximately $y^{+}=40$ with a value of about -0.60 , then decreases to a minimum at about $y^{+}=700$. This is probably due to the plateau area in the ${\overline{v^{2}}}^{+}$normal stress. The $\overline{v w}$ shear stress is also shown in the same figure. All profiles show a negative sign close to the wall then changes sign in the range $75 \leq y^{+} \leq 230$. The negative peak close to the wall is smaller in magnitude than the previous two clusters. All profiles also show a slight change in slope at about $y^{+}=920$. The magnitude of this stress is higher than the $\overline{u v}^{+}$and $\overline{u w}$ shear stresses at the maximum height achieved. The $\overline{u w}^{+}$ stress shown in Figure 4.7e, has the same trend as the previous clusters, but with smaller peak magnitudes. Also the last peak at $y^{+}=1050$ has a positive value in contrast with the previous two clusters.

\section{P20:Correlation Coefficient, $\rho_{u v}$}

The correlation coefficient is shown in Figure 4.7f. Profiles $P 20 p, P 20$ and $P 20_{2}$ have almost constant $\rho_{u v}$ at 0.3 . Profiles $P 20 p p$ and $P 20_{1}$ show lower values close to the wall 
which might be due to mis-alignment of the LDV probe since the probe had to be moved multiple times to measure all profiles in this cluster. However, above $y^{+}=400$ all profiles more or less have the same values and trend.

\section{P20: 1/S Parameter}

Figure $4.7 \mathrm{~g}$ shows the $1 / S$ profiles. Near the wall the profiles don't collapse, since this parameter tends to an infinite value at the wall. However, above $y^{+}=70$ all profiles collapse well to a single profile. The value of this parameter is almost constant in the range $70 \leq y^{+} \leq 480$ at about 0.6. Above $y^{+}=480$ the parameter decreases semilogarithmically.

\section{P20: Turbulent Kinetic Energy $T K E$}

The turbulent kinetic energy is shown in Figure 4.7h. All profiles collapse to a single profile up to about $y^{+}=60$. Near the wall TKE profiles have the same slope as that of the two-dimensional profile, but at a higher turbulence level. All profiles show two peaks, the first is near $y^{+}=18$ while the second peak's location varies with position. Profile $P 20$ shows a higher second peak than all other profiles. This profile resembles the P19pp profile. However, the second peak in the P20 profile is higher and shifted away from the wall.

\section{P20:Turbulent Diffusion Velocity $\vec{V}_{q}$}

The stream-wise turbulent diffusion velocity component, $V_{q x}$, is shown in Figure 4.7i All profiles collapse very well to a single curve except in the range $15 \leq y^{+} \leq 145$. The diffusion velocity has a positive peak close to the wall and changes sign about four times away from the wall. This indicates that the direction of turbulence diffusion is changing away from the wall. Figure 4.7j shows the turbulent diffusion velocity vertical component. Similar to the previous two clusters the diffusion velocity normal to the wall is negative close to the wall and positive away from the wall with a change in sign twice at $y^{+}=20$ and 2000. Profiles don't collapse away from the wall maybe due to uncertainty in the data. The last two peaks are lower in value than those observed in the last cluster. Finally, Figure $4.7 \mathrm{k}$ shows the span-wise turbulent diffusion velocity component. All profiles collapse fairly to a single profile within measurement uncertainty. However, the shape of these profiles are similar to profiles in the P18 and P19 clusters. All profiles show a positive peak close to the wall and a change in sign around $y^{+}=300$. A negative peak is also present at about $y^{+}=1170$.

\section{P20: Townsend's Structural Parameter $A_{1}$}


Townsend's structural parameter is shown in Figure 4.71. Profiles don't collapse for this cluster, however they still exhibit a semi-logarithmic behavior near the wall with two plateau areas at $y^{+}=60$ and 500 . The maximum value is about 0.1 which is the same magnitude for all other clusters. The lack of collapse in this cluster is probably due to misalignment of the CompLDV probe as mentioned earlier.

\section{P20: Flow Angle $\gamma_{a}$, Shear Stress angle $\gamma_{s}$ and Flow Gradient Angle $\gamma_{g}$}

Figure $4.7 \mathrm{~m}$ shows the flow angles. The same trends observed in $P 18$ and $P 19$ clusters can be seen here. However, profiles P2Opp and $\mathrm{P}_{2} \mathrm{O}_{2}$ show much lower values than other profiles near the wall. Away from the wall, all profiles approach the same angle and slope. However, in the stream-wise and span-wise direction, flow angle profiles show consistent trends for all clusters. Figure $4.7 \mathrm{n}$ shows the shear stress angle. For this cluster the profiles don't collapse well along the height of the profiles. However, all profiles have the same trend as previous clusters.

In the buffer layer the angle is almost constant then in the semi-log layer it decreases semi-logarithmically. There is a small plateau area around $y^{+}=300$ where the angle is approximately $20^{\circ}$. The shear angle then decreases to a large negative value. The flow gradient angle is shown in Figure 4.70. The flow gradient angle profiles show very small difference between profiles. Comparing mean flow angles and mean flow gradient angles, it can be seen that the mean flow gradient angles follow closely the changes in the mean flow angles. On the other hand the shear stress angle does not respond as fast to changes in the mean flow, hence the shear stress angle is lagging the flow gradient angle. This is shown in Figure $4.7 \mathrm{p}$ which shows the difference between the shear stress angle and the flow gradient angle. The differences are almost constant or very slightly changing along the profiles up to $y^{+}=200$. At about $y^{+}=1000$ all profiles cross zero, meaning that at that height there is no lag between the two angles.

\section{P20: Mixing Length $L_{m}$ and Turbulent Eddy viscosity $\nu_{T x}$}

The mixing length is shown in Figure 4.7q, All profiles collapse to a single profile near the wall. Away from the wall at about $y^{+}=500$ all profiles start to depart. However, the differences are within $15 \%$. Up to the maximum height the mixing length is increasing. Figure $4.7 \mathrm{~s}$ shows the turbulent eddy viscosity. Similar trends to the mixing length can be seen in this figure, however the differences between profiles are more clear in the eddy viscosity profiles than the mixing length.

\subsubsection{P33 Cluster}

Profiles P31, P33, P35 are aligned in the stream-wise direction, while profiles P32, P33, and P34 are aligned in the spanwise direction as shown in Figure 4.1. The center 
profile, $P 33$, in this cluster is located at $x / L=0.935$ and $z / W_{x=0}=-0.059$. The distance between any profile and the center profile is 1.0in in the stream-wise and span-wise direction. The flow behavior in this cluster is very similar to that of $P 13$ cluster. However, there are some differences in flow angles, turbulent Reynolds shear stress angles and flow gradient angles.

\section{P33: Mean Stream-wise and Span-wise Velocities, $U^{+}, W^{+}$}

The stream-wise velocity profiles are shown in Figure $4.8 \mathrm{a}$. All profiles are very close to two-dimensional boundary layer profiles, showing a viscous sub-layer and a semilogarithmic region. All profiles collapse in the viscous layer and agree well with DNS data. In the semi-logarithmic region, the slope increases slightly in the stream-wise and span-wise directions. A wake region starts to develop at the tip of the profiles. Also all profiles show wider semi-logarithmic regions which suggest that the flow is becoming more and more developed towards the end of the test section. Also shown in Figure 4.8a is the span-wise velocity component. All profiles show inflexion points where the second derivative changes sign. This means that the stream-wise vorticity which is approximately equal to $\partial W / \partial y$ changes sign along profiles. This is due to change in the spanwise pressure gradient direction upstream of this location.

\section{P33: Reynolds' Normal Stresses, ${\overline{u^{2}}}^{+}, \bar{v}^{+}$and ${\overline{w^{2}}}^{+}$}

Figure $4.8 \mathrm{~b}$ shows the stream-wise normal stress profiles. All profiles collapse to a single profile in the viscous sublayer below $y^{+}=10$. The peak value is approximately located at $y^{+}=14$, which is the same as DNS data. However the peak value is much higher than the two-dimensional value. Above $y^{+}=100$ all profiles show a near plateau region where the stress doesn't change significantly. However the last two points in the profiles show a decrease in stress values except for profile P34 which is the furthest in the positive span-wise direction. The trends in this cluster is the same as those observed in P13. Profiles P13 and P34 are about $0.60 \mathrm{in}$. apart in the span-wise direction. Figure $4.8 \mathrm{c}$ shows the normal to the wall and span-wise Reynolds normal stresses. Profiles of the normal to the wall normal stress collapse very well near the wall and agree with DNS data up to $y^{+}=40$. The vertical stress has the same trend as the two-dimensional profile up to about $y^{+}=80$; above that the stress increases monotonically with a small plateau area around $y^{+}=100$. The span-wise normal stress profiles shown in the same figure approach two-dimensional DNS data close to the wall. However, the level of stress is much higher than the two-dimensional case. In the semi-logarithmic region the spanwise normal stress decreases in the span-wise direction while is almost constant in the stream-wise direction. At the highest end of the profile the stress reaches values very close to the stream-wise normal stress. 
P33: Reynolds' Shear Stresses, $\overline{u v}^{+}, \overline{u w}^{+}$and $\overline{v w}$

The negative of $\overline{u v}$ and $\overline{v w}$ shear stresses are shown in figure $4.8 \mathrm{~d}$. Profiles of the $\overline{u v}{ }^{+}$shear stress collapse near the wall and agree well with DNS data up to $y^{+}=60$. Above this height the shear stress decreases to more negative values. Profiles P32 show a peak at $y^{+}=1245$. In general, the trends in the $\overline{u v}^{+}$shear stress profiles are similar to those observed at $P 13$. The $\overline{v w}$ shear stress also shows similar trends to those for the $P 13$ profile. However, in the outer layer the $\overline{v w}$ stress has a positive value while the $P 13$ profile show a negative value in the outer layer.

The $\overline{u w}$ shear stress is shown in Figure 4.8e. Profiles P31, P34 and P35 show very small stress values. On the other hand profiles $P 32$ and $P 33$ show a higher constant stress value. This might be due to the fact that the velocity histograms at this location experienced a lot of noise which might have affected the histogram clipping procedure. The $\overline{v w}^{+}$stresses in this cluster are similar to those in $P 13$ cluster, while the $\overline{u w}{ }^{+}$stresses look different from $P 13$ to some extent.

\section{P33:Correlation Coefficient, $\rho_{u v}$}

Figure 4.8f shows the correlation coefficient $\rho_{u v}$. The profiles show consistent trends with almost constant value of about 0.3 . The uncertainty in the correlation coefficient in the semi-logarithmic region is about \pm 0.12 ; hence, the differences between profiles are within measurement uncertainty.

\section{P33: 1/S Parameter}

The $1 / S$ parameter is shown in Figure $4.8 \mathrm{~g}$. Profiles show a very good collapse above $y^{+}=10$. In the outer layer the profiles reach a value of 0.55 which is lower than the two-dimensional case. At $y^{+}=40$ the slope changes significantly. Also in the range $10 \leq y^{+} \leq 40$, buffer-layer, profiles decrease in a semi-logarithmic fashion.

\section{P33: Turbulent Kinetic Energy $T K E$}

The turbulent kinetic energy is shown in Figure $4.8 \mathrm{~h}$. Near the wall all profiles collapse up to $y^{+}=10$. All profiles show a peak around $y^{+}=19$. The peak value for some of the profiles is about $80 \%$ greater than the two-dimensional vlaue. All profiles show a small dip in the TKE value at about $y^{+}=100$, followed by an increase in value to a value equal to or greater than the peak value. This trend is also seen at the P13 cluster.

\section{P33:Turbulent Diffusion Velocity $\vec{V}_{q}$}


Figure 4.8i shows the turbulent diffusion velocity component in the stream-wise direction. The collapse near the wall is not as good as P18, P19 and P20 clusters. However, the trend of all profiles is similar and is the same as $P 13$ cluster. The diffusion velocity in the stream-wise direction is positive near the wall due to sweep events. The near wall peak value is located roughly at $y^{+}=7$. All profiles show positive values for the entire profile which is the same trend in $P 13$ cluster. This indicates that there is a preferred direction for the transfer of turbulent kinetic energy. Profiles P32 and P33 show a smaller peak value whose location is further away from the wall. This might be an artifact due to the matching procedure between the two LDV probes used. Also these profiles show lower values in the outer layer. The vertical component of the turbulent diffusion velocity is shown in Figure 4.8j All profiles collapse very well near the wall up to $y^{+}=30$. The diffusion velocity component has a negative sign which is consistent with DNS data. This is due to the sweep events being the active motion near the wall. Above $y^{+}=20$ all profiles change sign. Another sign change is observed in between $y^{+}=150$ and 800 . In the range $30 \leq y^{+} \leq 80$ the turbulent diffusion velocity is almost constant. Figure $4.8 \mathrm{k}$ shows the span-wise turbulent diffusion velocity component. The profiles show more scatter than previous profiles. However, there is a trend in the profiles. The uncertainty in the span-wise turbulent velocity diffusion is \pm 0.05 . The profiles change signs twice, one very close to the wall and in the outer layer. Profile P33 shows some scatter in the data. Attempts have been made to remove the scatter by clipping multiple times but it seems that there were not enough data samples to obtain convergent histogram.

\section{P33: Townsend's Structural Parameter $A_{1}$}

Townsend's structural parameter is shown in Figure 4.81. Profiles collapse very well close to the wall up to about $y^{+}=165$. All profiles show a semi-logarithmic increase close to the wall up to $y^{+}=30$. Between $y^{+}=60$ and 185 the parameter is almost constant at about 0.65 . Higher than this range the parameter increases again semi-logarithmically. Towards the end of profiles a peak appears to form. Again, profile P34 shows some scatter near the wall.

\section{P33: Flow Angle $\gamma_{a}$, Shear Stress angle $\gamma_{s}$ and Flow Gradient Angle $\gamma_{g}$}

Figure $4.8 \mathrm{~m}$ shows the mean flow angle. Near the wall, the flow angle increases semilogarithmically up to $y^{+}=100$. Profiles P31, P32, P33 and P35 show bilateral, skewing where the flow angle near the wall has a different sign than the free stream flow angle. Profile P34 show changes in the slope sign, however, the flow angle remains negative. The first three points in P33 show a change in the flow angle equal to $3^{\circ}$ degrees over a distance of about two wall units. This seems to be unreasonable change at such low height. Hence, it is believed that this is due to uncertainty. The location of the peak values in the outer layer shifts away from the wall as the flow progresses downstream. 
The shear stress angle is shown in Figure $4.8 \mathrm{n}$. All profiles show an almost zero value near the wall. However, the data show some scatter. The uncertainty in the shear stress angle is about $\pm 4^{\circ}$. In general, all profiles don't show any region of collapse. The angle then increases to a positive value in the semi-logarithmic region, then changes sign above $y^{+}=100$. This shows the fact that the shear stress angle is lagging the flow mean angle, since in the near wall region pressure changes affect the mean flow directly. However, the shear stresses don't respond as fast as the mean flow. Figure 4.80 shows the mean flow gradient angle. It is clear that the mean strain rate direction adjust to changes in the mean flow as the mean flow gradient angle is almost the same as the mean flow angles very close to the wall in the viscous sub-layer. In the semi-logarithmic region the flow gradient angles are larger than the mean flow angles. All profiles show a peak value around $y^{+}=145$. The flow gradient angles then change sign at $y^{+}=315$. To show the lag between the shear stress angle and the mean flow gradient angle, the difference between the two angles are shown in Figure $4.8 \mathrm{p}$. In the viscous sub-layer, the difference is almost constant and equal to about $3^{\circ}$. The difference then changes sign and decreases semilogarithmically in the range $30 \leq y^{+} \leq 210$. The difference then reaches a minimum in the range $220 \leq y^{+} \leq 400$. The difference increases up to the last point in the profiles.

\section{P33: Mixing Length $L_{m}$ and Turbulent Eddy viscosity $\nu_{T x}$}

The mixing length is shown in Figure 4.8q, All profiles collapse to a single profile near the wall. Away from the wall at about $y^{+}=600$, all profiles start to depart slightly. However, the differences are within $15 \%$. Up to the maximum height the mixing length is increasing. As observed in previous clusters all profile collapse when plotted in a log-log scale. Figure $4.8 \mathrm{~s}$ shows the turbulent eddy viscosity. Similar trends to the mixing length can be seen in this figure, however the differences between profiles are more clear in the eddy viscosity profiles than the mixing length.

\subsection{Spectral Measurements}

Spectral measurements were obtained at few selected locations. Five profiles were acquired at profiles $P 08, P 18, P 19 p, P 19$ and $P 19_{1}$. The one-dimensional Power and Cross Spectra of the Reynolds Normal and Shear Stresses are shown in Figures 4.94 .12 for Profiles $P 18, P 19 p, P 19$ and $P 19_{1}$. Data. Also shown in these figures lines of slopes equal to $-1,-5 / 3$ and $-7 / 3$ for comparison. Details of the procedure used to estimate spectra can be found in Lowe, [25].

Two methods were available to estimate the turbulent kinetic energy dissipation rate depending on the shape of the spectrum. For spectra showing a $-5 / 3$ slope the dissipation rate is estimated using Equation 4.14.Hinze, [19]. 


$$
\begin{gathered}
\frac{E_{11}}{\nu}=C\left(\epsilon^{+}\right)^{2 / 3}\left(\frac{2 \pi}{U^{+}}\right)\left(\frac{f \nu}{u_{\tau}^{2}}\right)^{-5 / 3} \\
{\overline{u_{i} u_{j}}}^{+}=\int_{0}^{\infty} \frac{E_{i j}}{\nu} d\left(\frac{f \nu}{u_{\tau}^{2}}\right)
\end{gathered}
$$

where, $E_{i j}$ is the one-dimensional power spectrum such that the Reynolds stresses are given by Equation 4.15, $U$ is the local velocity component, $f$ is the frequency and $C$ is a constant equal to 0.49, Pope [52]. The second method is the Tchen theory which was used for profiles not showing a $-5 / 3$ region. Tchen's high mean vorticity model, (Hinze, [19], and Saripali and Simpson, [56]), relates the spectral density to the turbulent dissipation by Equation 4.16 .

$$
\epsilon^{+}=\frac{3}{2} \alpha\left(\frac{f \nu}{u_{\tau}^{2}}\right) \frac{E_{11}}{\nu} \overline{u^{2}}+\frac{\partial U^{+}}{\partial y^{+}}
$$

Where $\alpha$ is a constant equal to 0.8 to match two-dimensional boundary layer results. This method was used for all profiles except $P 08$. For this profile both methods were attempted to estimate the dissipation but no fit was possible. For each profile, the slope of the power spectrum on a log-log scale is matched to either $-5 / 3$ if the $-5 / 3$ law is used and to -1 if Tchen's theory is used.

The estimated dissipation rate from spectral measurements were compared with estimates from balancing the turbulent kinetic energy transport equation using Lumley's model for the pressure diffusion term. Figures $4.13 \mathrm{a}-4.13 \mathrm{~d}$ shows dissipation rate estimates for the above profiles. The first point in each profiles is roughly at $y^{+}=15$.

In general, estimates from spectral measurements agree very well with estimates from the turbulence kinetic energy transport equation balance except for very close to the wall. In the log-layer and towards the outer layer the dissipation rate balances the turbulence kinetic energy production. Also shown in the figures are the DNS calculation of turbulence production and dissipation rate in a fully developed channel flow by Iwamoto and Kasagi [20]. The spectral measurement estimate are estimates made using Equation 4.16, while TKE transport equation estimates are estimates obtained by balancing the terms in the kinetic energy transport equation with the dissipation rate. The agreement with the DNS data is very good above $y^{+}=100$. Near the wall DNS data has higher level of turbulence kinetic energy production and dissipation rate due to two dimensionality. Also, measured near wall data suffered from low data samples. 
Table 4.1: Profiles' Coordinates, estimated Pressure gradients, y-Shift and Friction Velocities

\begin{tabular}{|c|c|c|c|c|c|c|c|c|c|}
\hline Profile & $X / L$ & $Z / W_{x=0}$ & $\begin{array}{c}\delta y \\
{[\mu m]}\end{array}$ & $\begin{array}{c}u_{\tau} \\
{[\mathrm{m} / \mathrm{s}]}\end{array}$ & $\begin{array}{c}P_{\infty} \\
{\left[N / m^{2}\right]}\end{array}$ & $\begin{array}{c}T_{\infty} \\
{\left[{ }^{\circ} C\right]}\end{array}$ & $\begin{array}{l}\partial P / \partial x \\
{\left[N / m^{3}\right]}\end{array}$ & $\begin{array}{l}\partial P / \partial z \\
{\left[N / m^{3}\right]}\end{array}$ & $R$ \\
\hline$P 06$ & 0.866 & -0.158 & 25 & 0.77 & 95874 & 25.5 & 138.8 & -218.6 & 0.9971 \\
\hline$P 07$ & 0.871 & -0.182 & 43 & 0.69 & 95714 & 25.8 & 127.5 & -241.7 & 0.9983 \\
\hline$P 08$ & 0.871 & -0.158 & 11 & 0.75 & 95599 & 25.8 & 125.0 & -240.0 & 0.9998 \\
\hline P09 & 0.871 & -0.134 & 52 & 0.71 & 95594 & 27.0 & 123.3 & -176.6 & 0.9991 \\
\hline$P 10$ & 0.877 & -0.158 & 21 & 0.69 & 95864 & 25.5 & 103.5 & -219.1 & 0.9991 \\
\hline$P 11$ & 0.866 & -0.017 & 42 & 0.62 & 95919 & 26.8 & 40.2 & -90.0 & 0.9992 \\
\hline$P 12$ & 0.871 & -0.045 & 28 & 0.61 & 95919 & 26.8 & 68.5 & -96.5 & 0.9938 \\
\hline$P 13$ & 0.871 & -0.017 & 58 & 0.56 & 95919 & 26.8 & 63.2 & -137.1 & 0.9872 \\
\hline$P 14$ & 0.871 & 0.010 & 22 & 0.67 & 95919 & 26.8 & 50.7 & -155.0 & 0.9950 \\
\hline$P 15$ & 0.877 & -0.017 & 42 & 0.67 & 95919 & 26.8 & 94.3 & -97.0 & 0.9963 \\
\hline$P 16 p$ & 0.902 & -0.192 & 33 & 0.78 & 95314 & 26.3 & -349.2 & -178.6 & 0.9986 \\
\hline$P 16$ & 0.907 & -0.192 & 22 & 0.90 & 95429 & 25.5 & -354.5 & -64.7 & 0.9999 \\
\hline$P 18$ & 0.912 & -0.192 & 31 & 0.96 & 95284 & 27.0 & -359.6 & 56.2 & 0.9987 \\
\hline$P 18_{1}$ & 0.917 & -0.192 & 6 & 0.90 & 95214 & 26.5 & -372.6 & 194.5 & 0.9990 \\
\hline$P 18_{2}$ & 0.923 & -0.192 & 66 & 0.93 & 95181 & 25.5 & -377.2 & 338.3 & 0.9718 \\
\hline$P 19 p p$ & 0.902 & -0.171 & 48 & 0.79 & 95491 & 26.0 & -260.1 & -158.6 & 0.9746 \\
\hline$P 19 p$ & 0.907 & -0.171 & 22 & 0.83 & 95989 & 24.8 & -268.5 & -56.3 & 0.9962 \\
\hline P19 & 0.912 & -0.171 & 34 & 0.91 & 95914 & 25.3 & -268.4 & 42.8 & 0.9696 \\
\hline$P 19_{1}$ & 0.917 & -0.171 & 56 & 0.93 & 95939 & 25.0 & -269.9 & 150.3 & 0.9737 \\
\hline$P 19_{2}$ & 0.923 & -0.171 & 18 & 0.97 & 95954 & 25.8 & -274.4 & 264.9 & 0.9783 \\
\hline$P 20 p p$ & 0.902 & -0.144 & 8 & 0.91 & 95079 & 25.5 & -138.6 & -220.3 & 0.9986 \\
\hline$P 20 p$ & 0.907 & -0.144 & 29 & 0.70 & 95679 & 25.3 & -139.0 & -97.8 & 0.9984 \\
\hline$P 20$ & 0.912 & -0.144 & 43 & 0.83 & 95884 & 26.0 & -145.0 & 17.8 & 0.9919 \\
\hline$P 20_{1}$ & 0.917 & -0.144 & 23 & 0.83 & 96069 & 25.0 & -139.1 & 130.7 & 0.9839 \\
\hline $\mathrm{P} 2 \mathrm{O}_{2}$ & 0.920 & -0.144 & 20 & 0.87 & 95869 & 25.0 & -139.1 & 130.7 & 0.9839 \\
\hline P31 & 0.929 & -0.059 & 11 & 0.74 & 95689 & 26.0 & -81.2 & 187.0 & 0.9984 \\
\hline P32 & 0.935 & -0.087 & 25 & 0.80 & 96034 & 24.8 & -61.6 & 136.2 & 0.9993 \\
\hline P33 & 0.935 & -0.059 & 53 & 0.71 & 96021 & 24.5 & -71.0 & 186.0 & 0.9993 \\
\hline P34 & 0.935 & -0.032 & 16 & 0.66 & 95749 & 25.3 & -50.3 & 197.0 & 0.9991 \\
\hline P35 & 0.940 & -0.059 & 33 & 0.71 & 95579 & 25.8 & -59.1 & 189.2 & 0.9995 \\
\hline E01 & 0.046 & 0.000 & 57 & 0.94 & 94884 & 25.0 & 0.0 & 0.0 & 0.9960 \\
\hline E03 & 0.056 & 0.028 & 0 & 0.94 & 96006 & 25.3 & 0.0 & 0.0 & 0.9965 \\
\hline
\end{tabular}



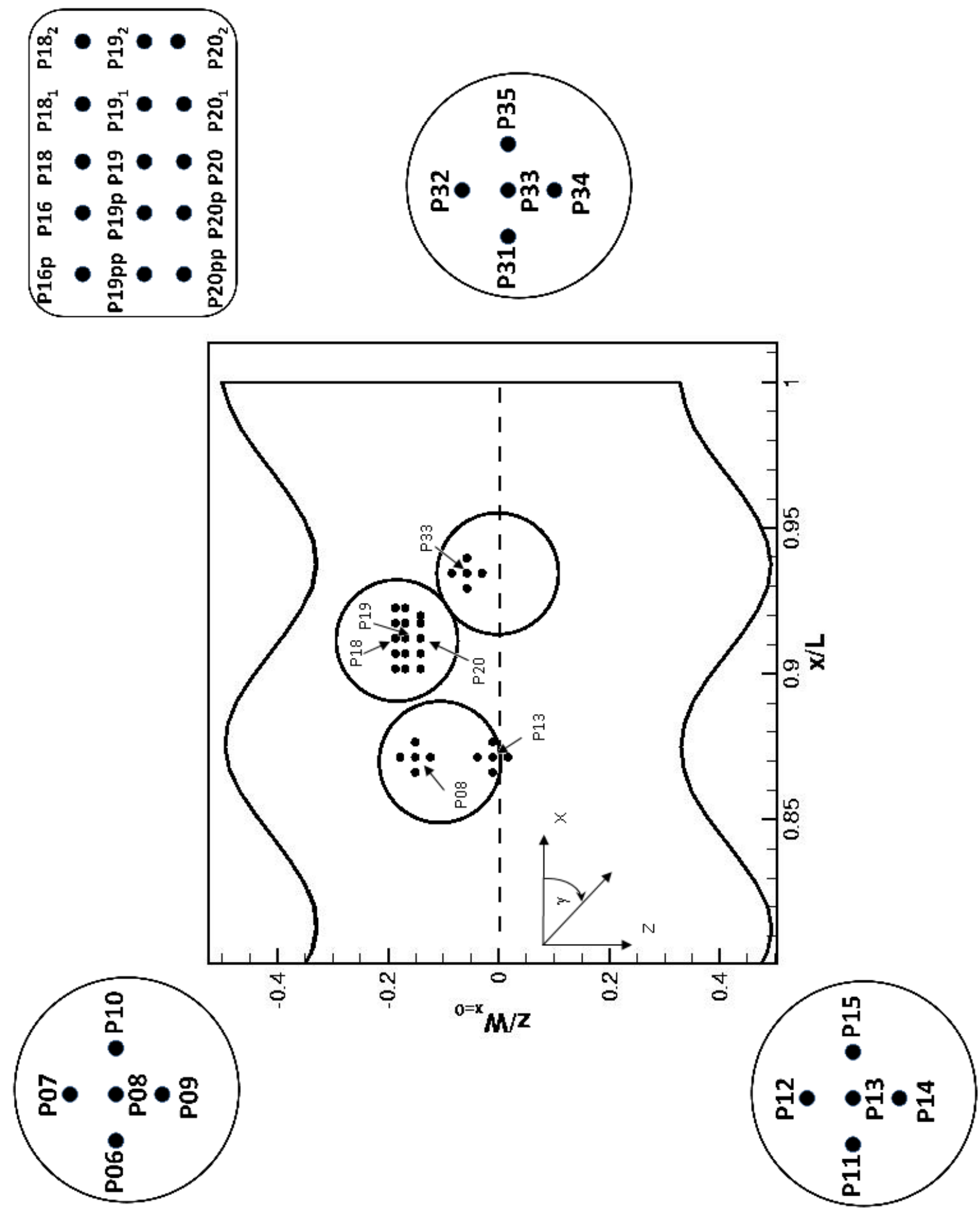

Figure 4.1: LDV Measurement Locations 


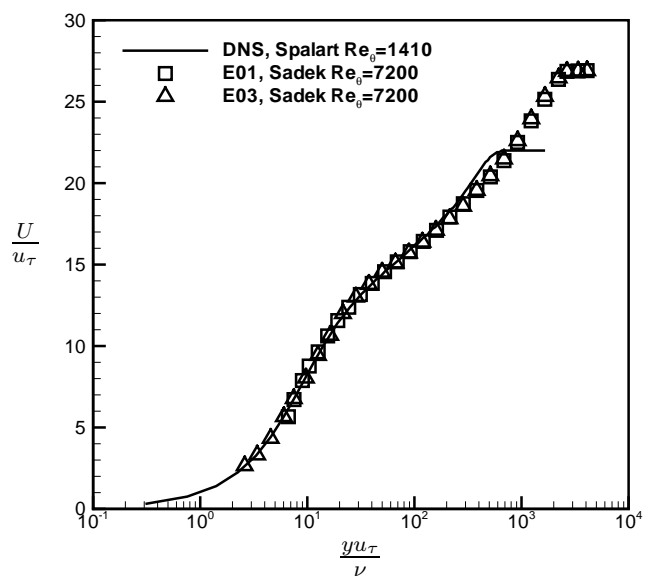

(a) Mean Streamwise Velocity

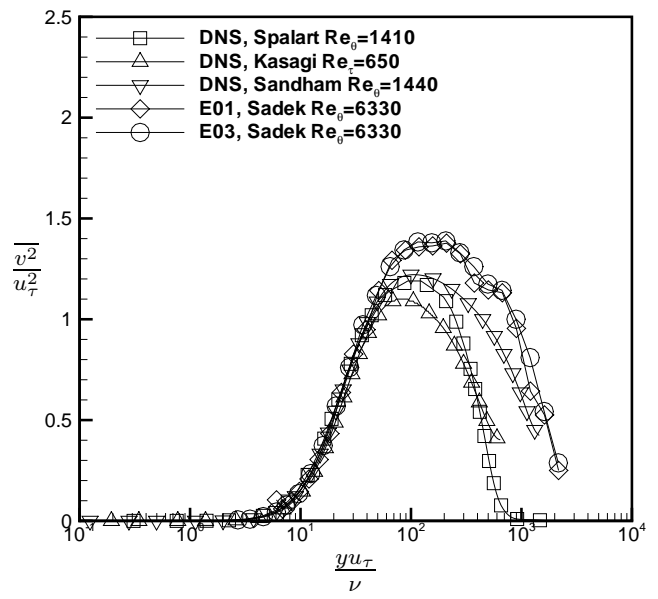

(c) Normalized Longitudinal Normal Stress

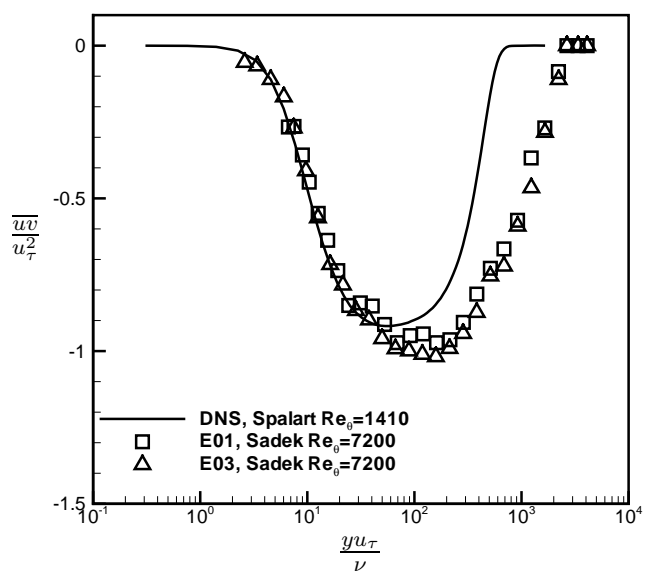

(e) Reynolds Shear Stress $\overline{u v}+$

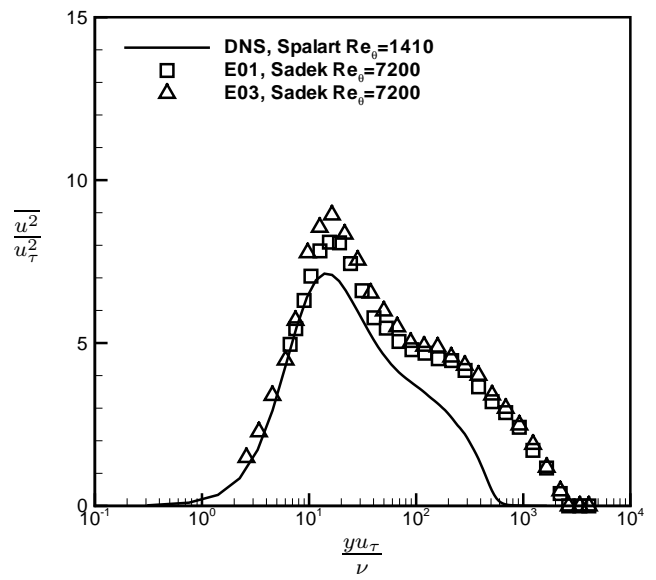

(b) Normalized Streamwise Normal Stress

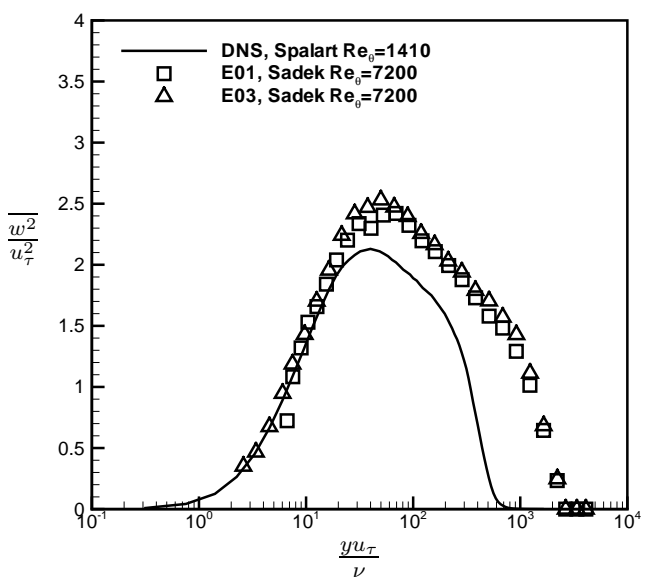

(d) Normalized Spanwise Normal Stress

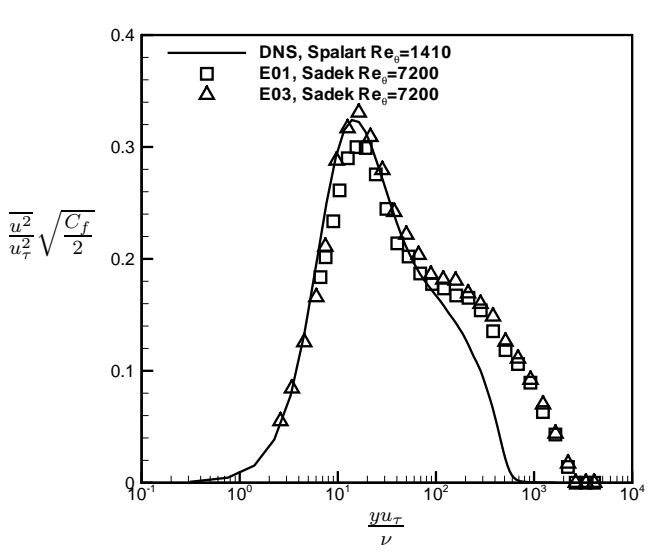

(f) Normalized Streamwise Normal Stress using $u_{\tau}$ and $U_{e}$

Figure 4.2: Entrance Profiles Comparison with DNS data 


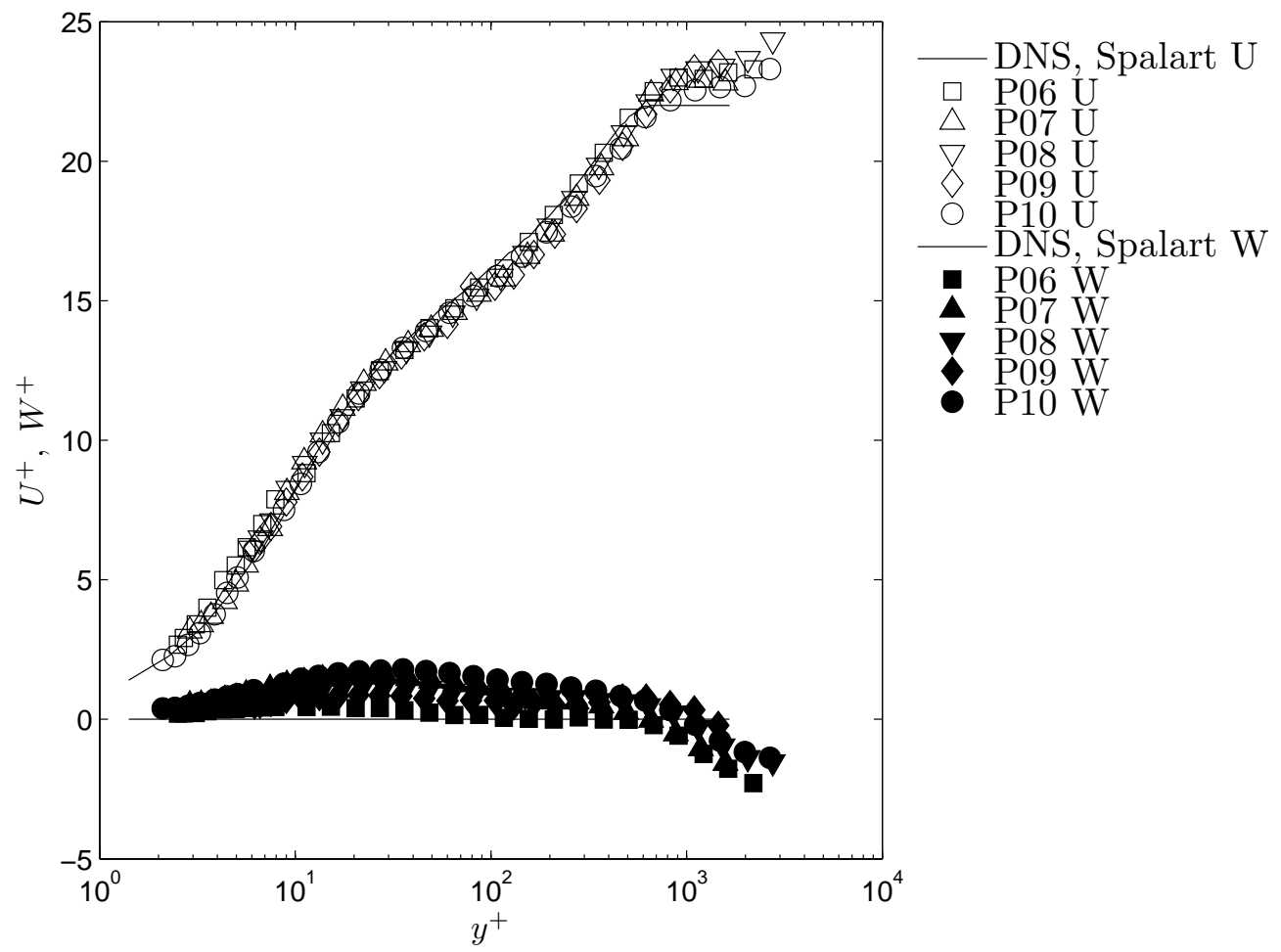

(a) Mean Stream-wise and Span-wise Velocities, $U^{+}, W^{+}$

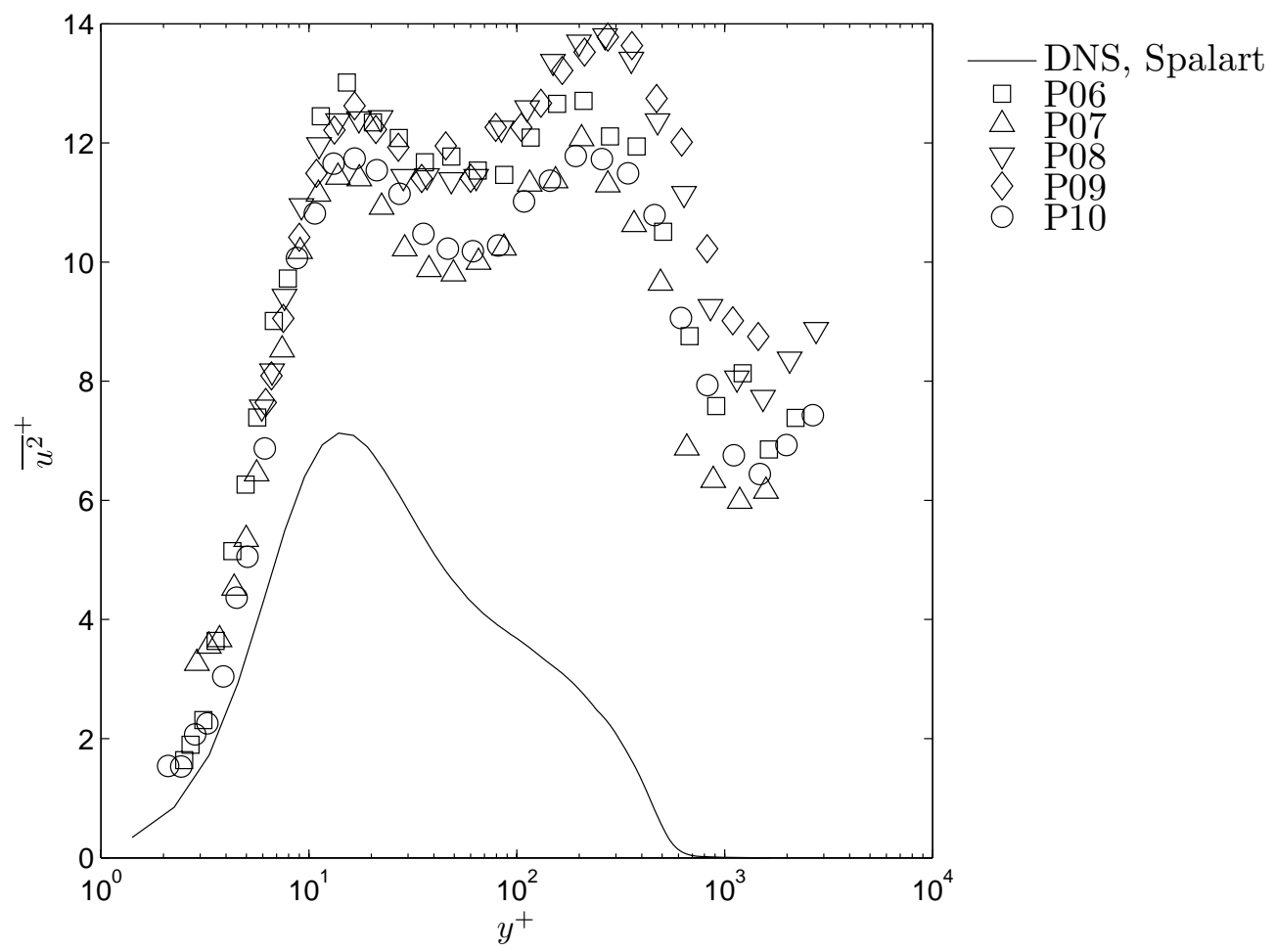

(b) Stream-wise Reynolds' Normal Stress, ${\overline{u^{2}}}^{+}$

Figure 4.3: P08 Cluster 


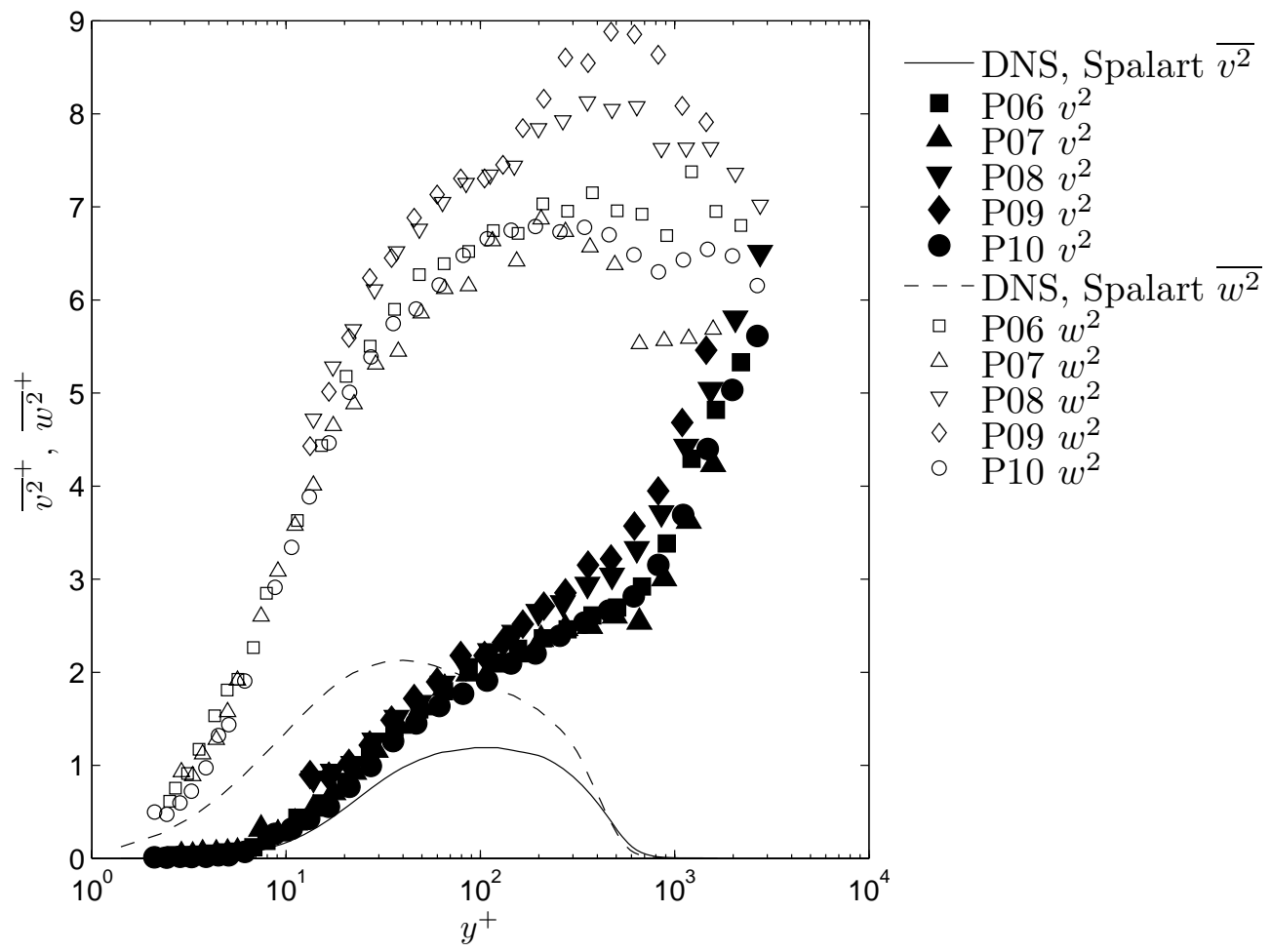

(c) Longitudinal and Span-wise Normal Reynolds' Stresses, ${\overline{v^{2}}}^{+}$and ${\overline{w^{2}}}^{+}$

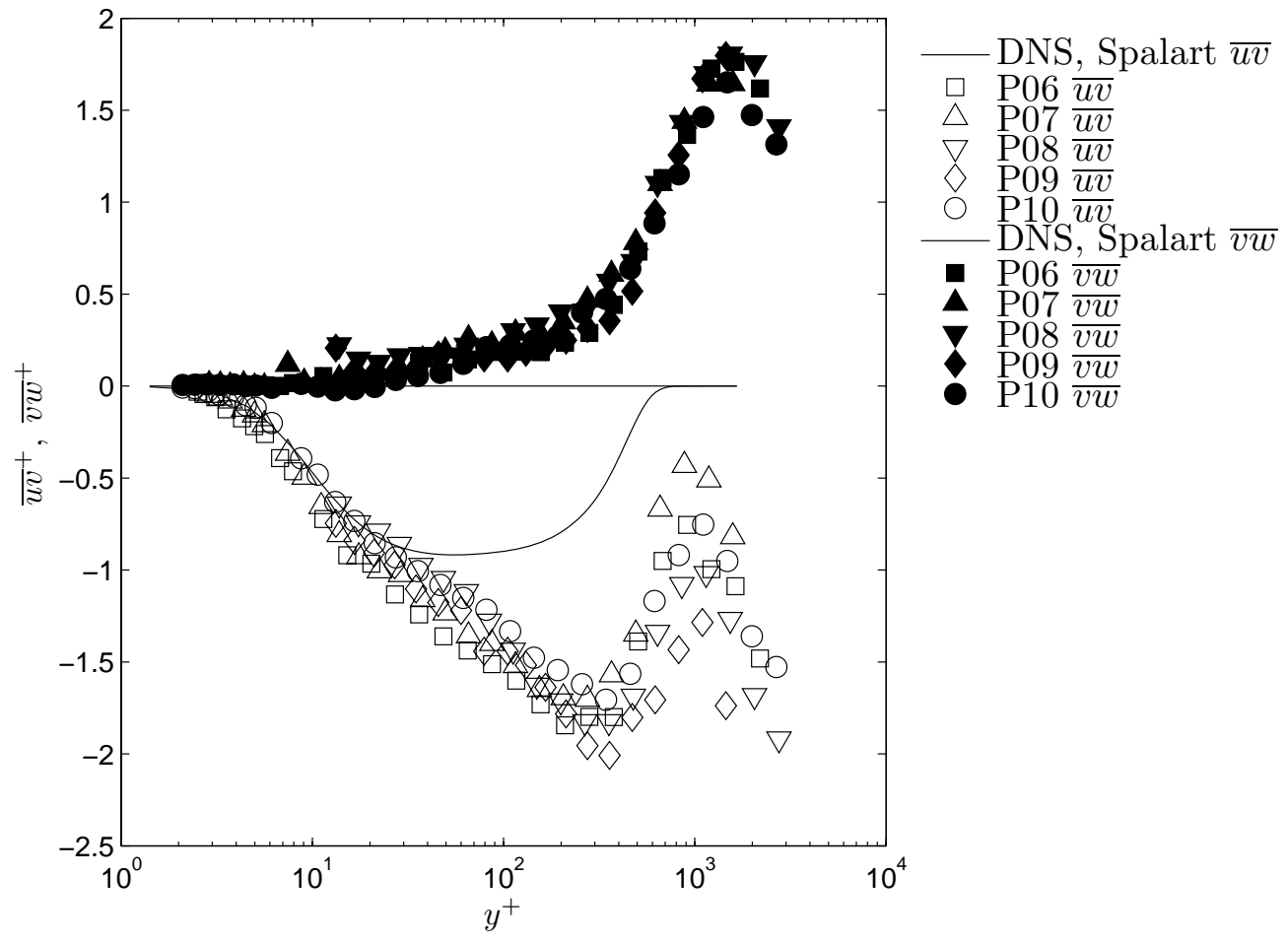

(d) Reynolds' Shear Stresses, $\overline{u v}^{+}$and $\overline{v w}^{+}$

Figure 4.3: Continued, P08 Cluster 


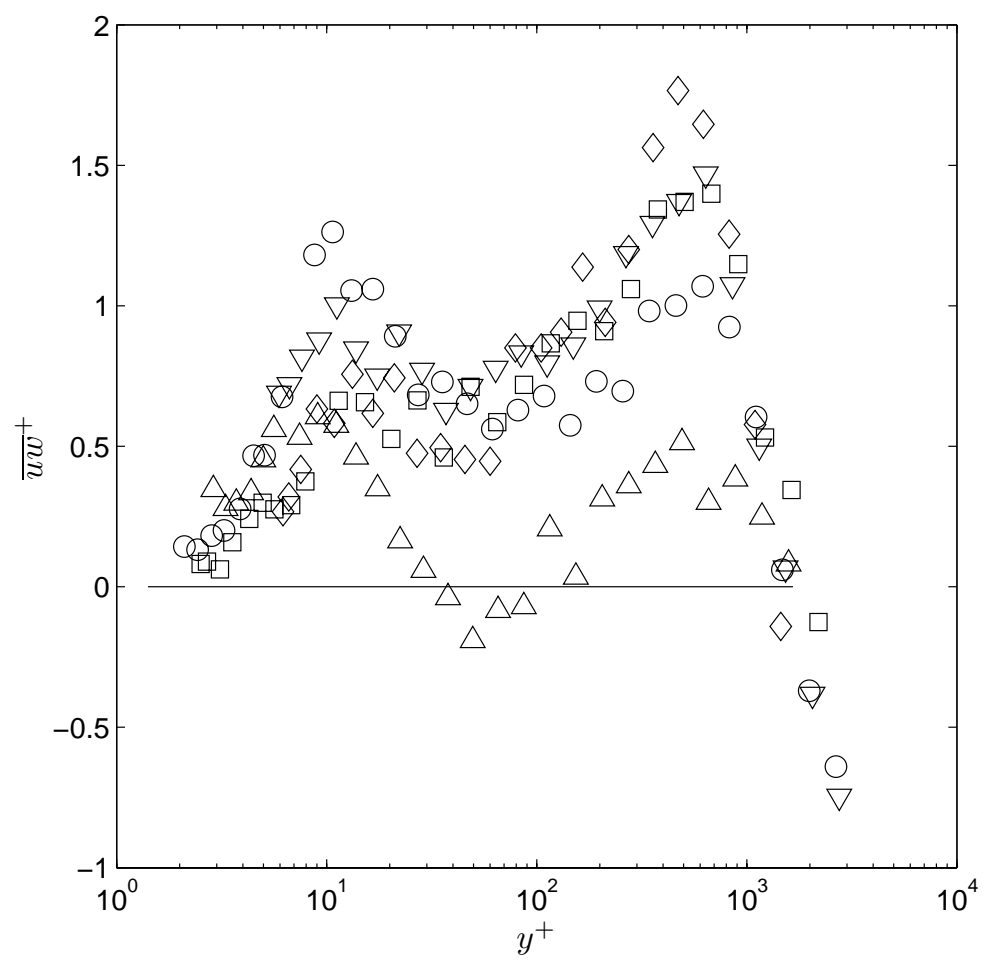

$\begin{array}{ll} & \text { DNS, Spalart } \\ \square & \mathrm{P} 06 \\ \triangle & \mathrm{P} 07 \\ \nabla & \mathrm{P} 08 \\ \diamond & \mathrm{P} 09 \\ \bigcirc & \mathrm{P} 10\end{array}$

(e) Reynolds' Shear Stress, $\overline{u w}{ }^{+}$

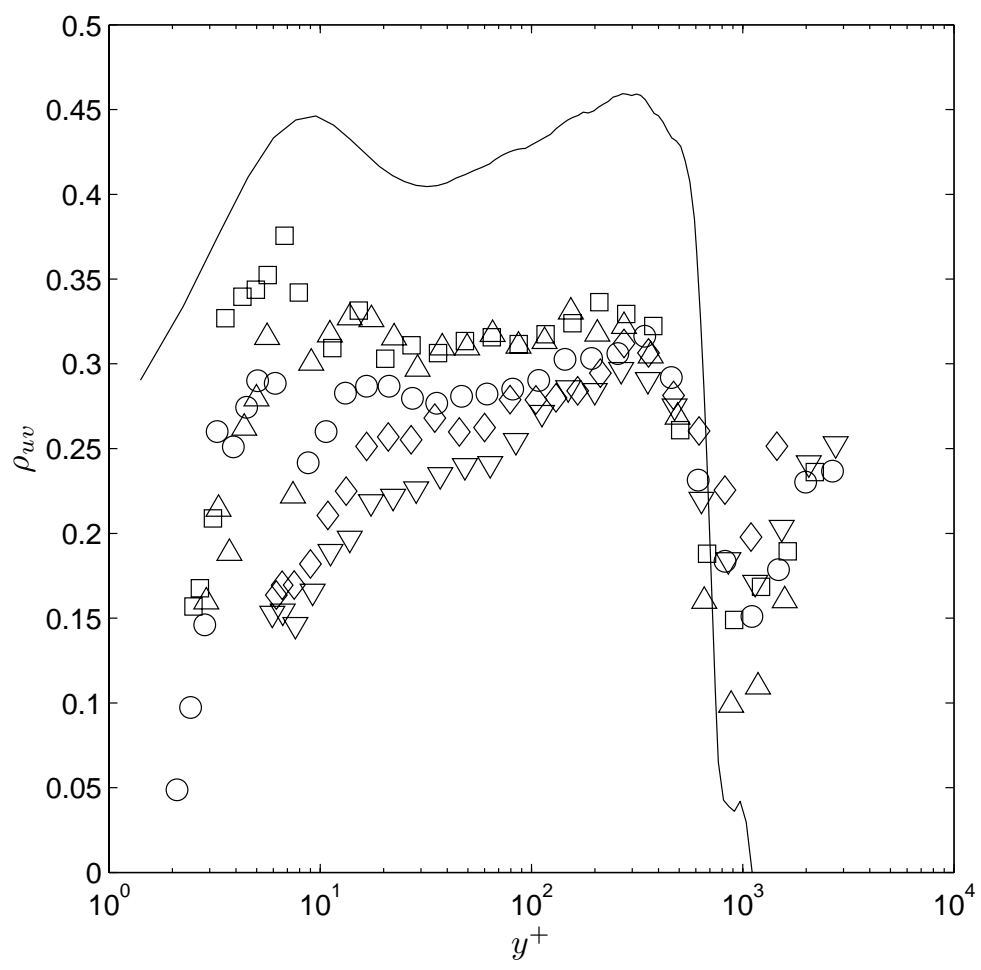

DNS, Spalart

$\square$ P06

$\triangle \mathrm{P} 07$

$\nabla \mathrm{P} 08$

$\diamond \mathrm{P} 09$

(f) Correlation Coefficient $\rho_{u v}$

Figure 4.3: Continued, P08 Cluster 


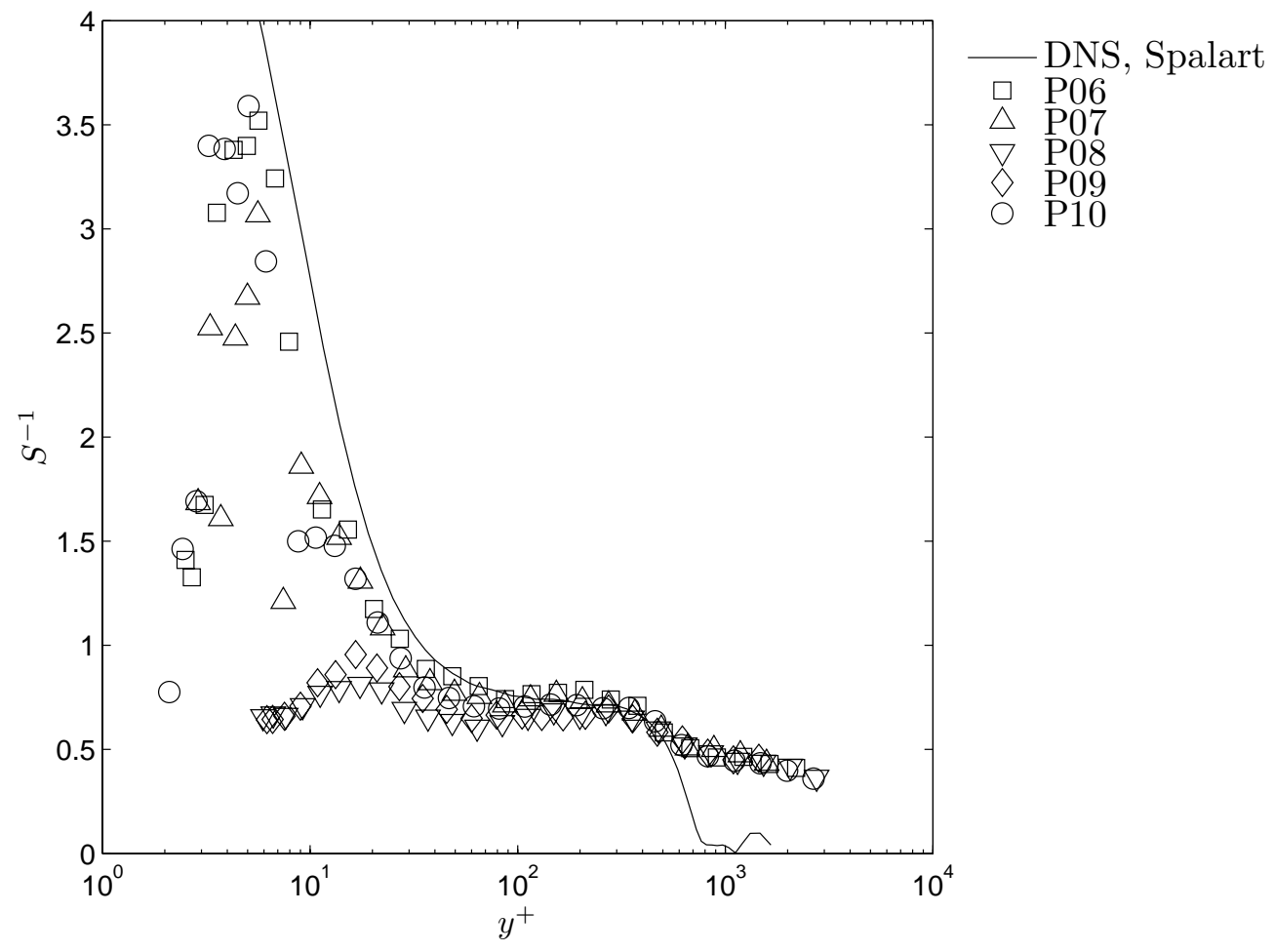

(g) $S^{-1}$ Parameter

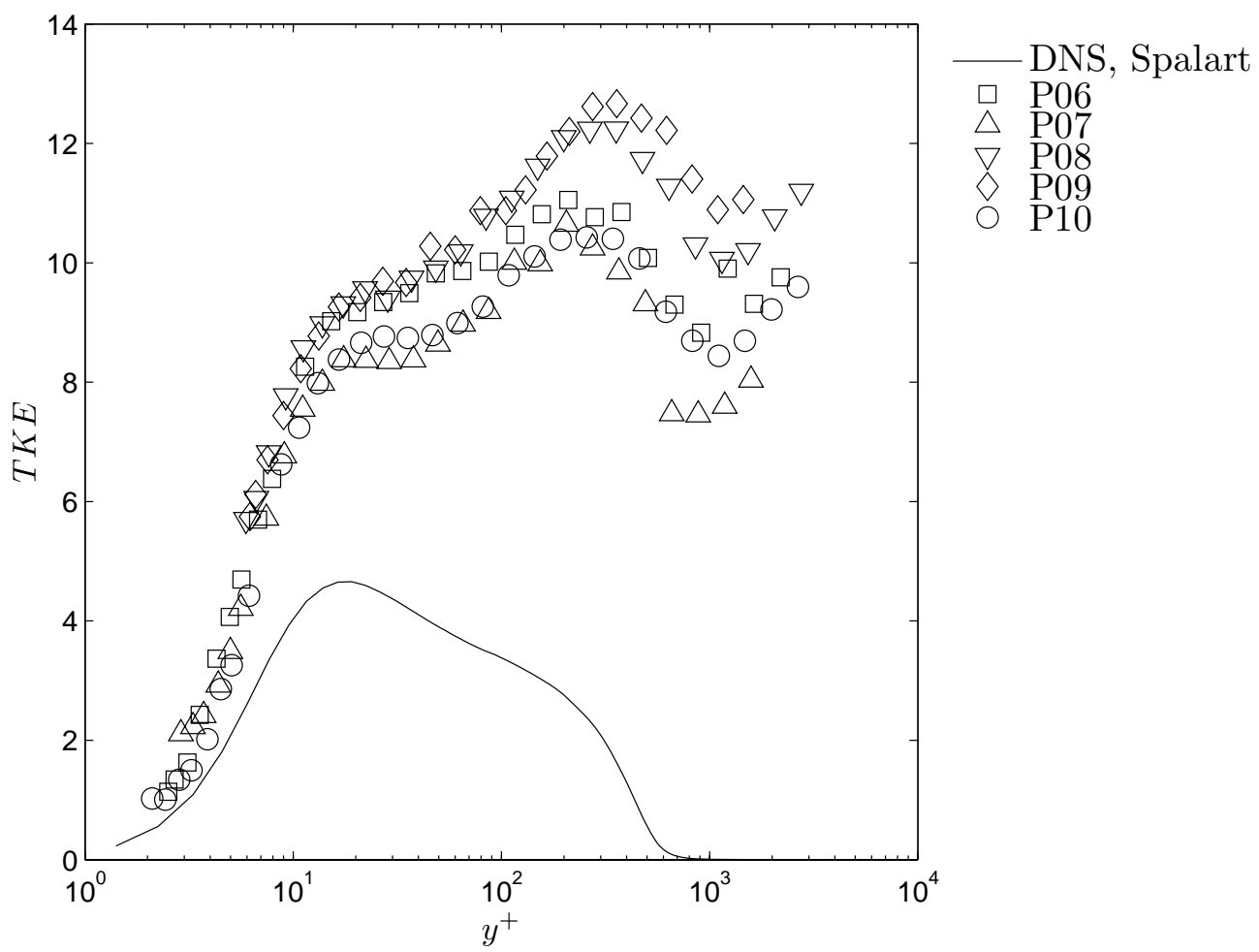

(h) Turbulent Kinetic Energy, TKE

Figure 4.3: Continued, P08 Cluster 


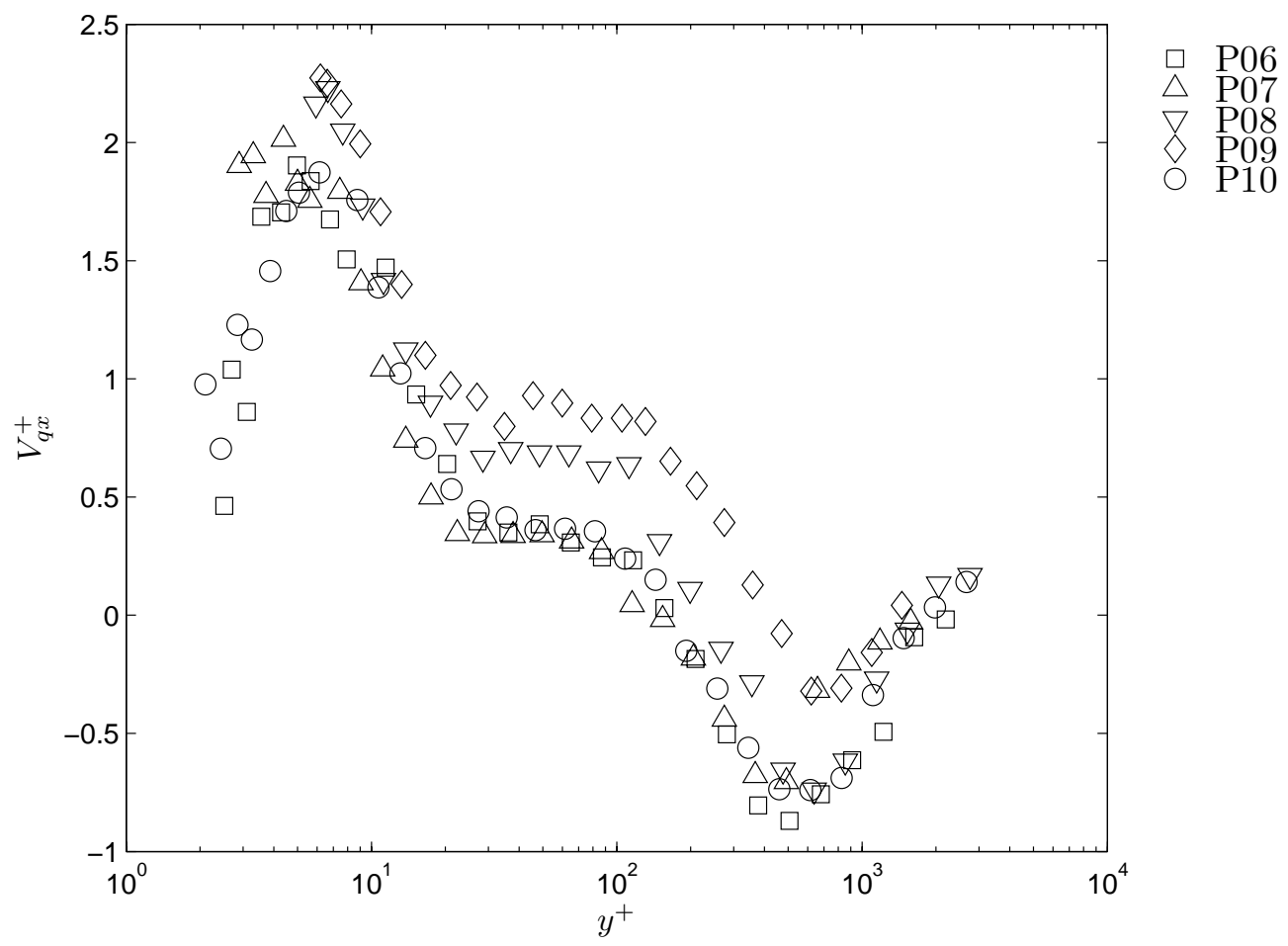

(i) Stream-wise Turbulent Diffusion Velocity component, $V_{q x}$, normalized on $u_{\tau}$

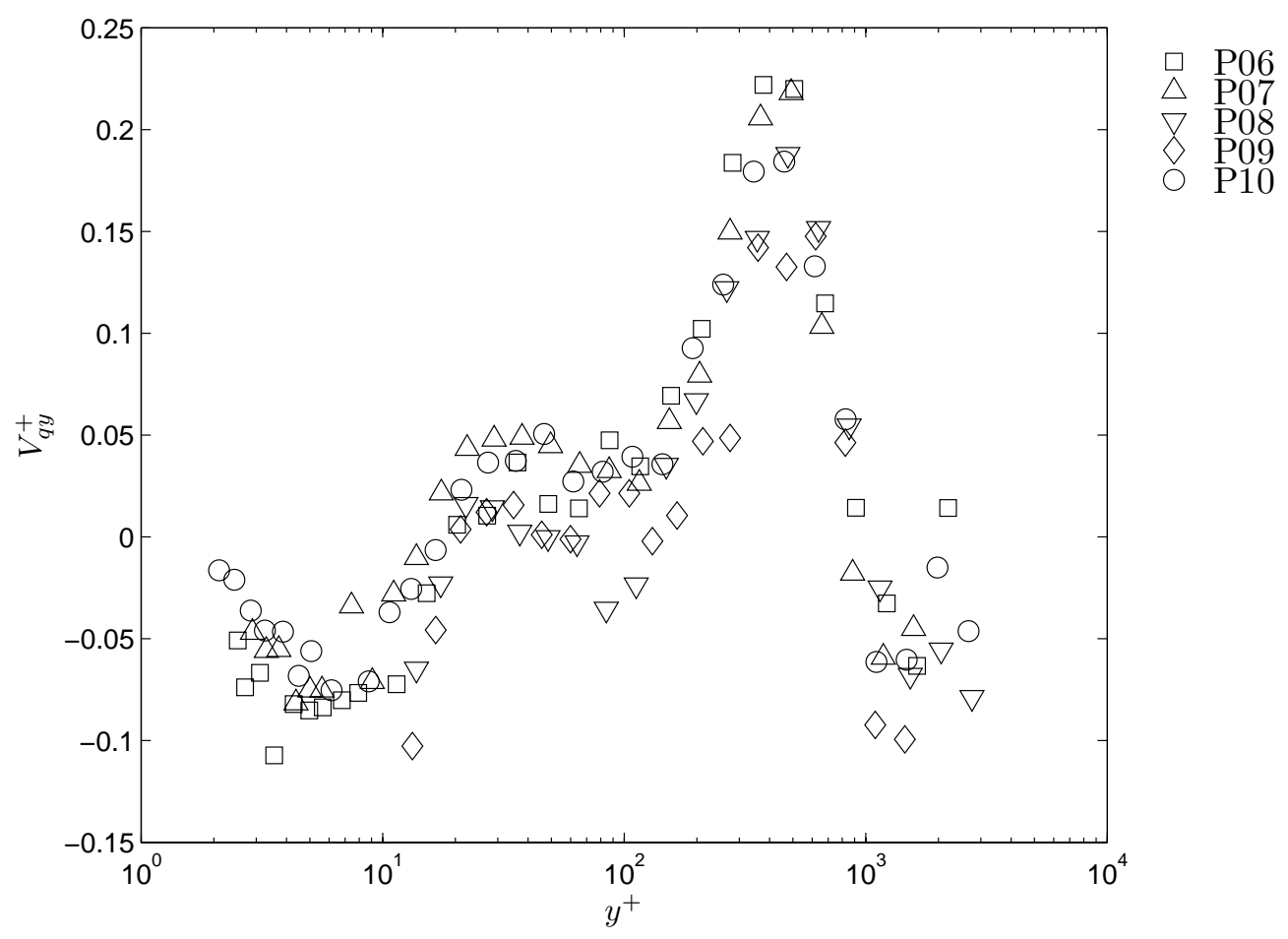

(j) Wall-Normal Turbulent Diffusion Velocity component, $V_{q y}$, normalized on $u_{\tau}$

Figure 4.3: Continued, $P 08$ Cluster 


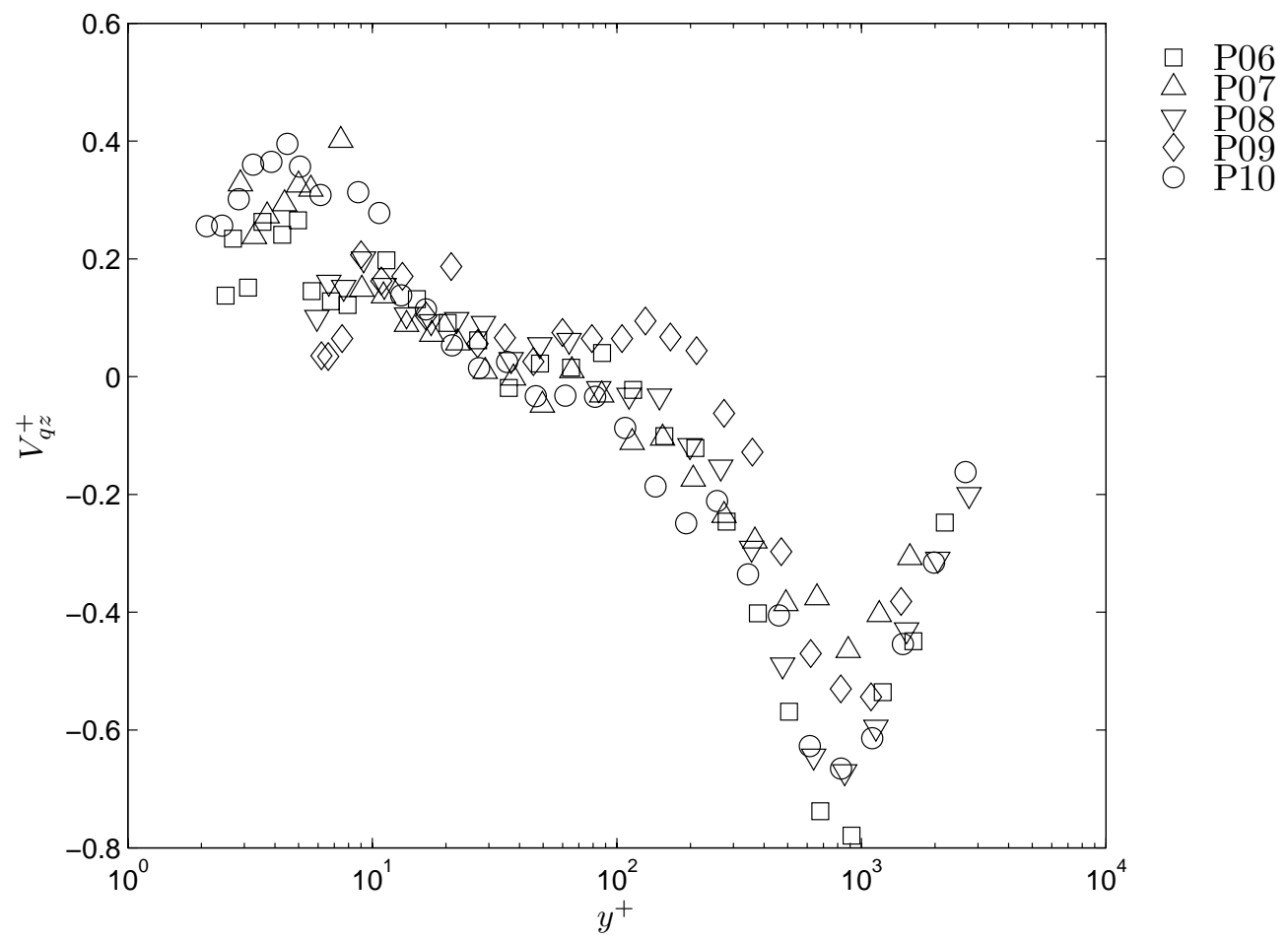

(k) Span-wise Turbulent Diffusion Velocity component, $V_{q z}$, normalized on $u_{\tau}$

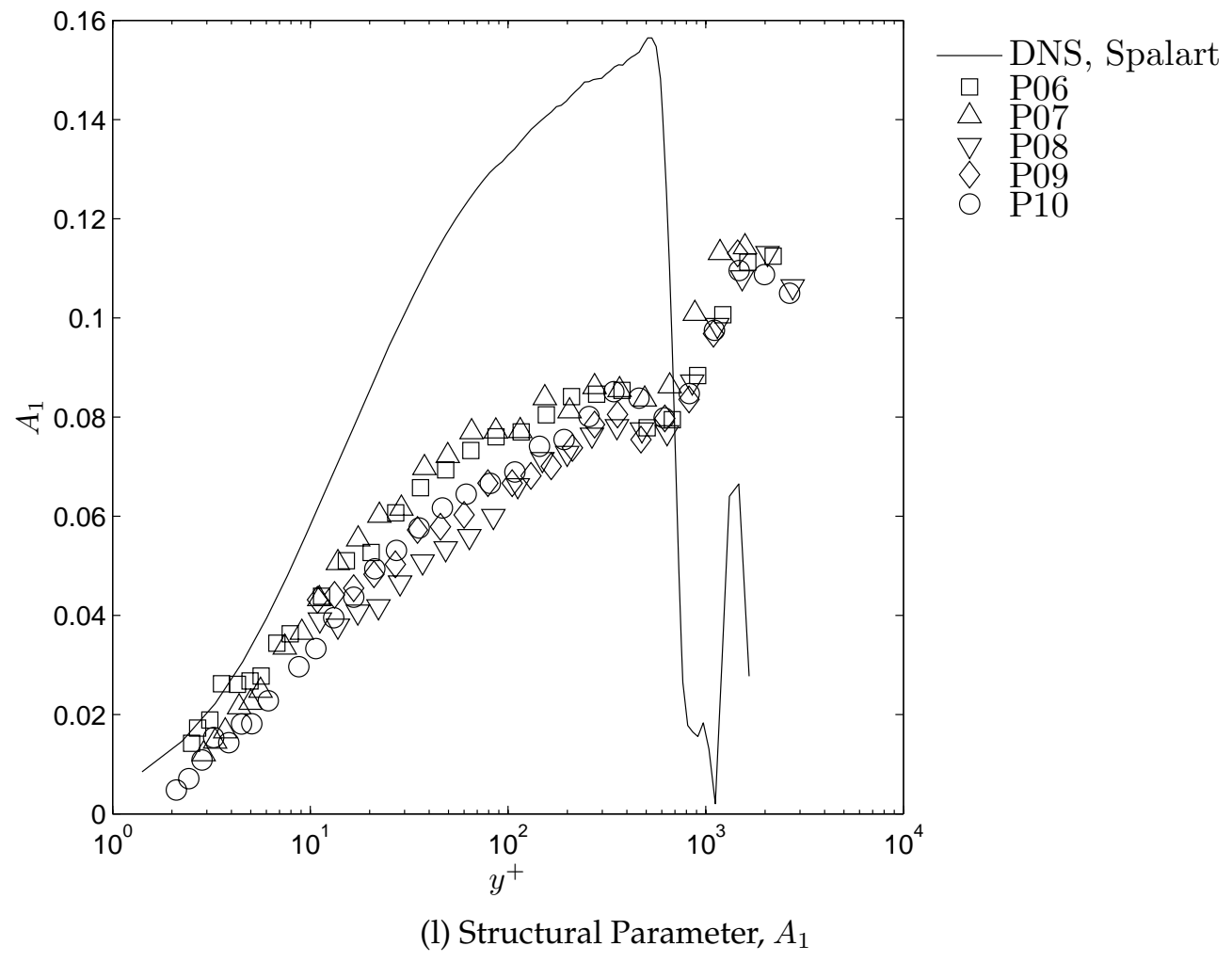

Figure 4.3: Continued, P08 Cluster 


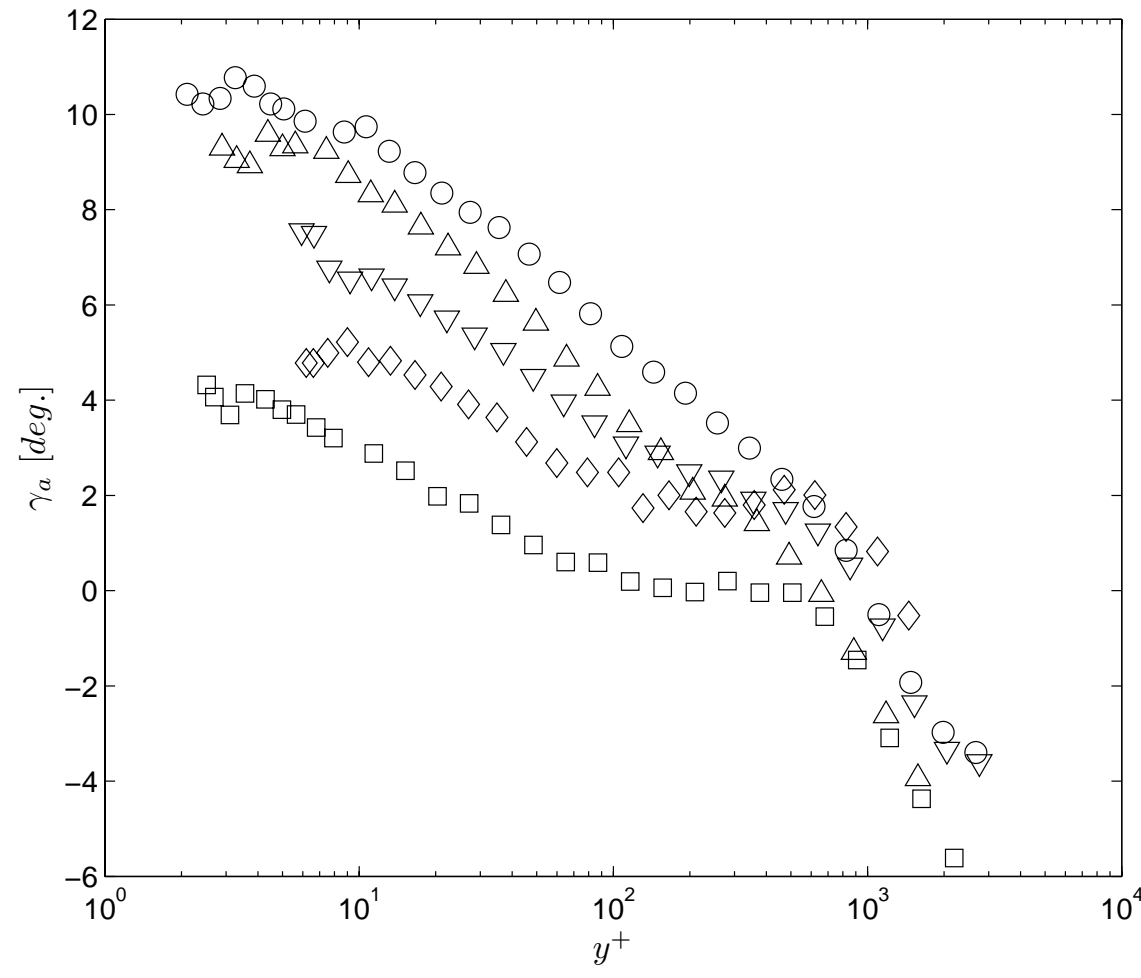

$\begin{array}{ll}\square & \text { P06 } \\ \triangle & \text { P07 } \\ \nabla & \text { P08 } \\ \searrow & \text { P09 } \\ \bigcirc & \text { P10 }\end{array}$

(m) Flow Angle, $\gamma_{a}$

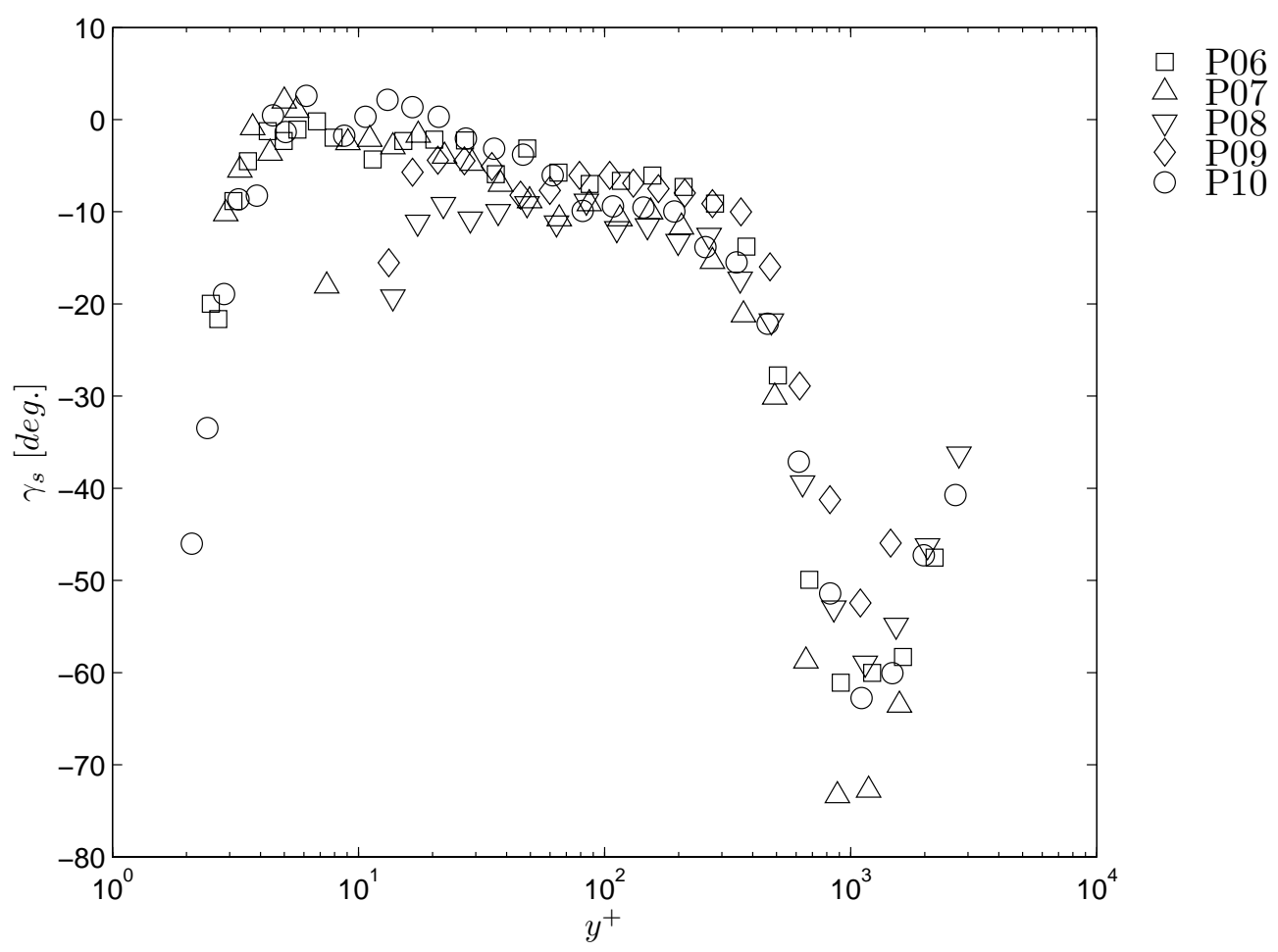

(n) Shear Stress Angle $\gamma_{s}$

Figure 4.3: Continued, P08 Cluster 


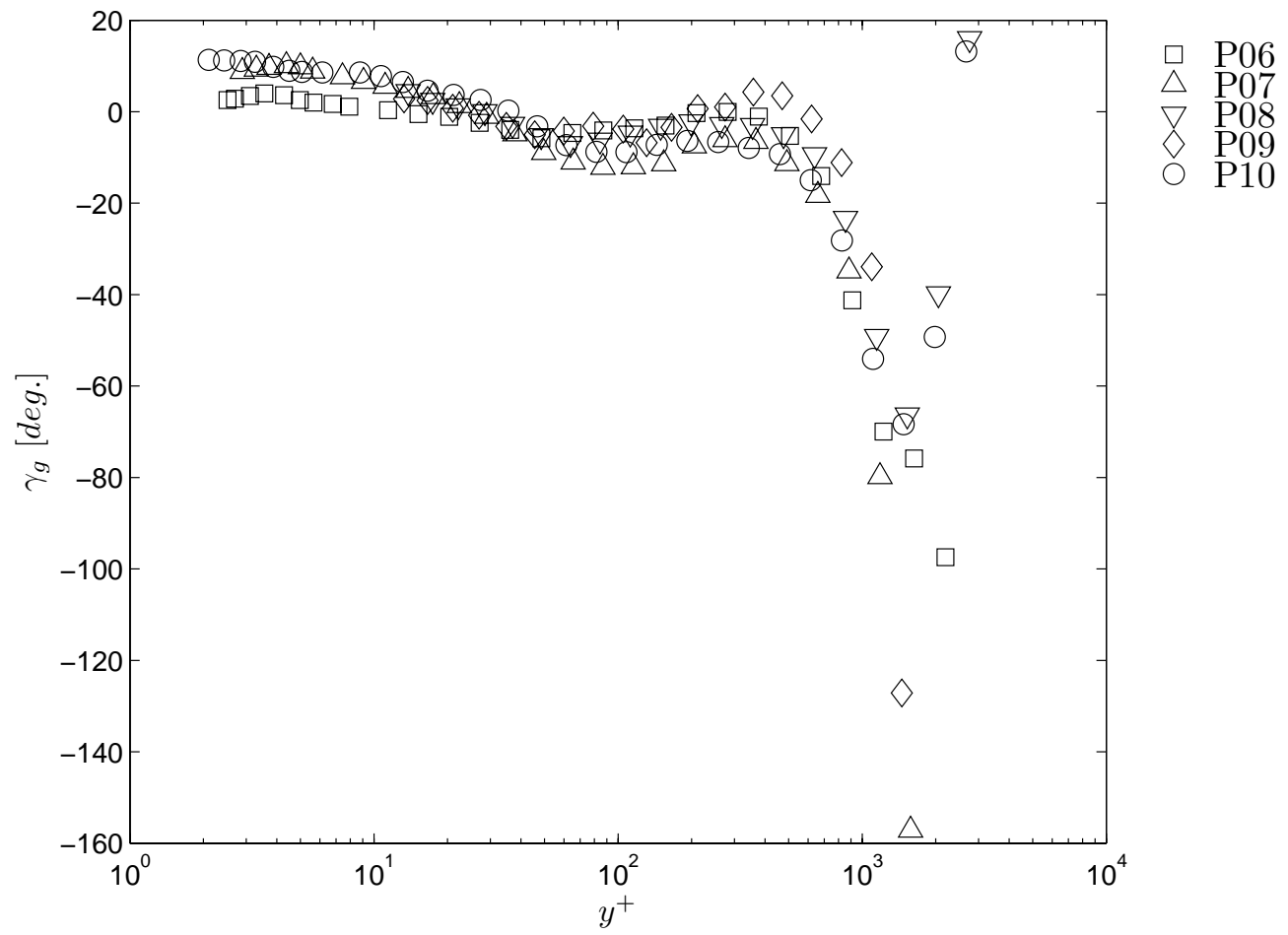

(o) Flow Gradient Angle $\gamma_{g}$

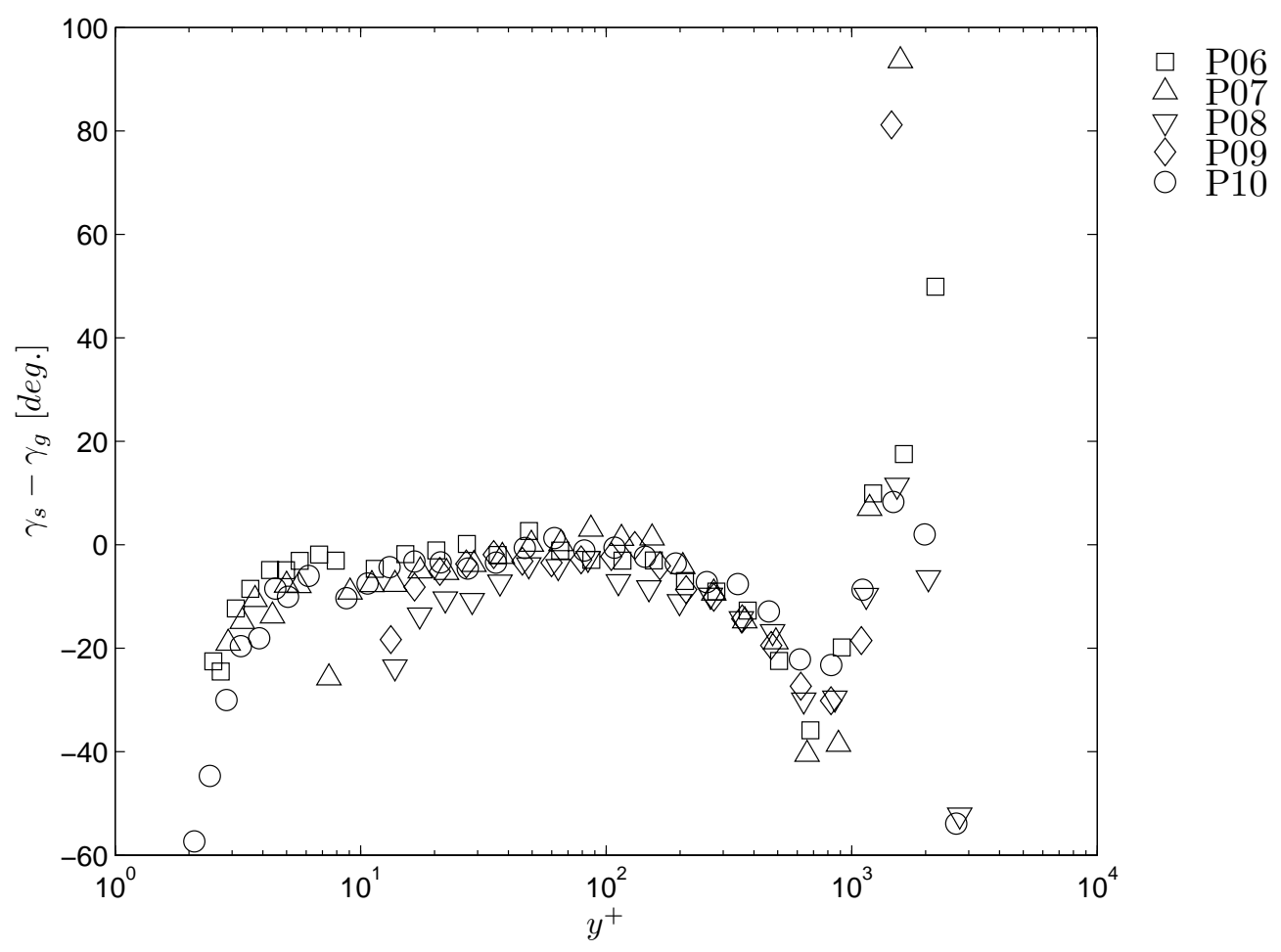

(p) Difference between Shear Angle and Flow Gradient Angles, $\gamma_{s}-\gamma_{g}$

Figure 4.3: Continued, P08 Cluster 


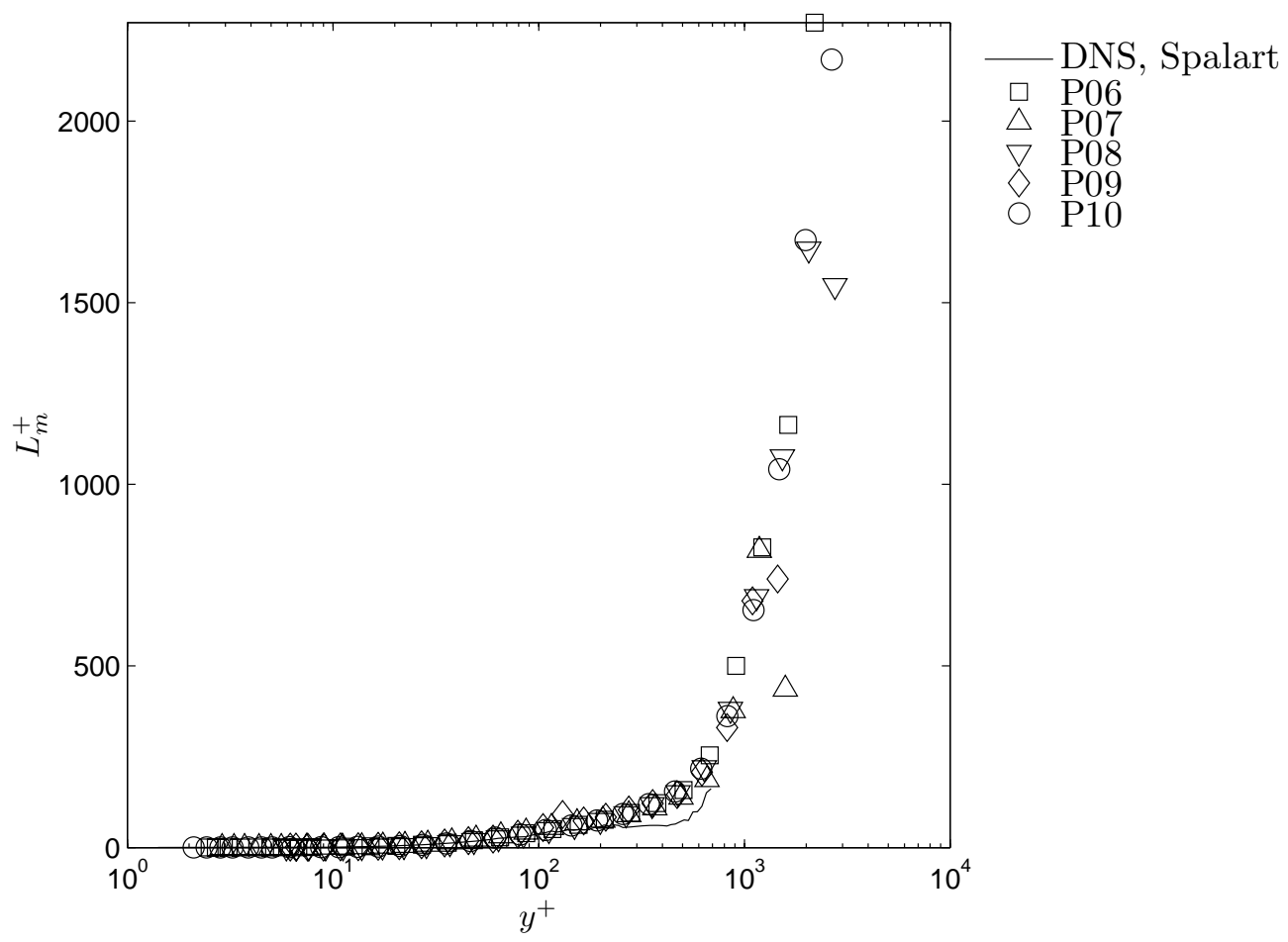

(q) Mixing Length $L_{m}^{+}$

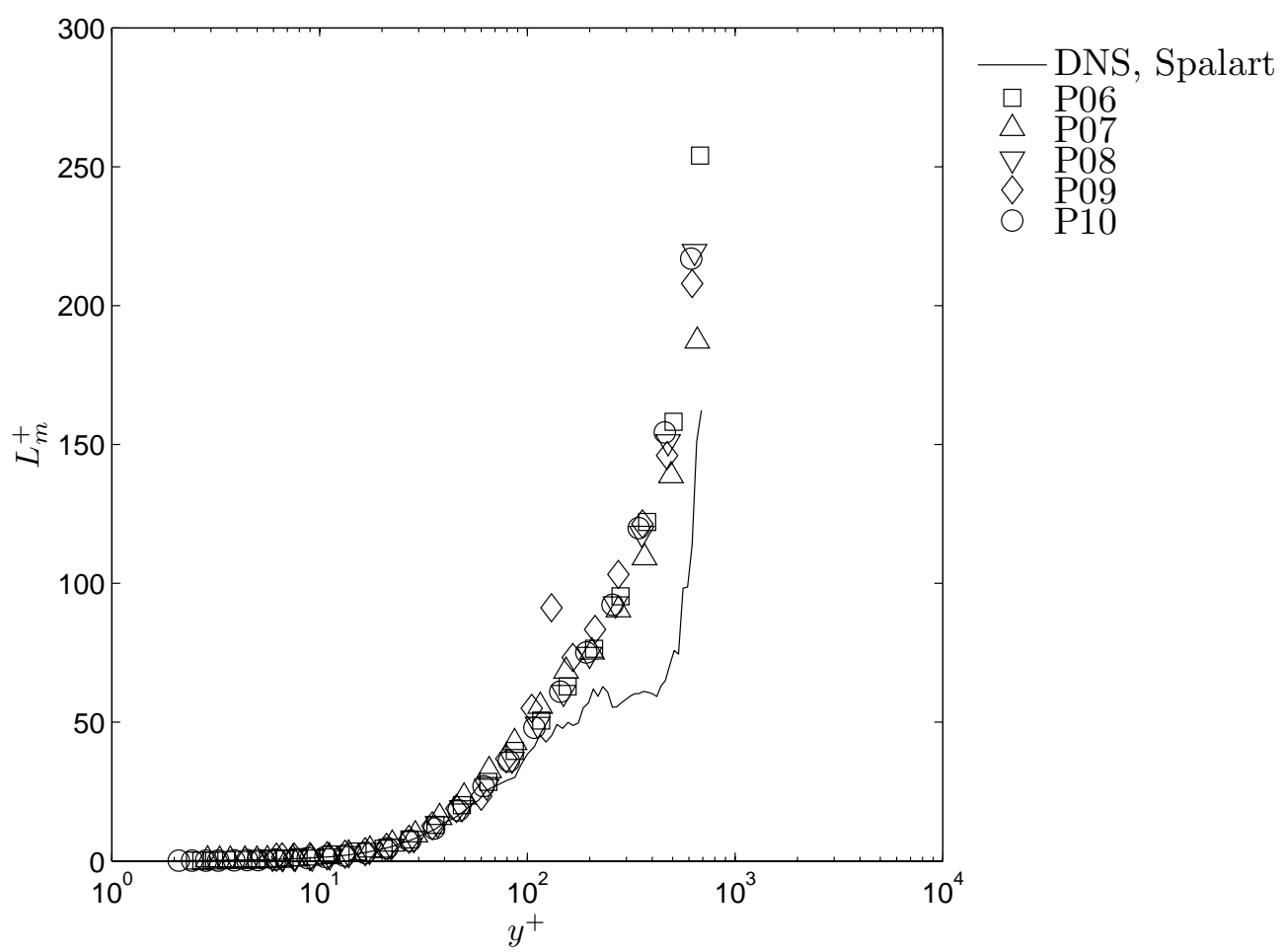

(r) Near Wall Mixing Length $L_{m}^{+}$

Figure 4.3: Continued, P08 Cluster 


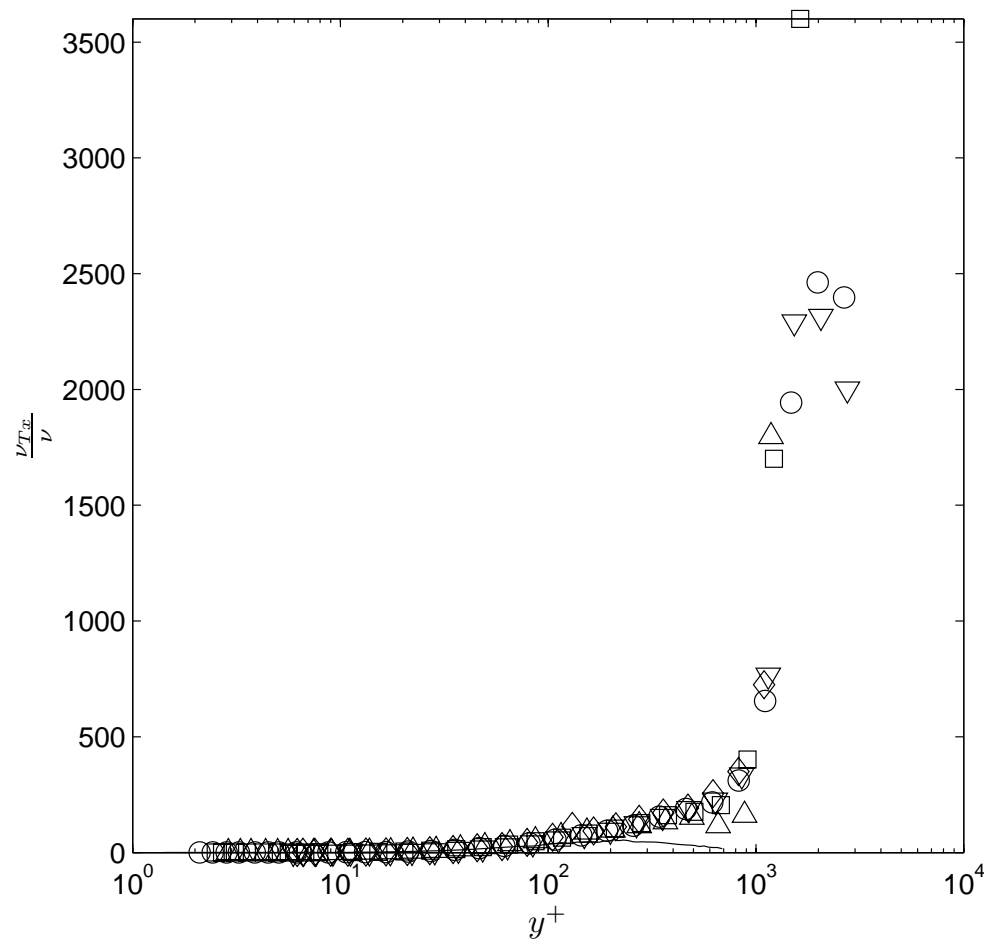

$$
\begin{array}{ll} 
& \text { DNS, Spalart } \\
\square & \mathrm{P} 06 \\
\triangle & \mathrm{P} 07 \\
\nabla & \mathrm{P} 08 \\
\diamond & \mathrm{P} 09 \\
\bigcirc & \mathrm{P} 10
\end{array}
$$

(s) Turbulent Eddy Viscosity $\nu_{T x}$

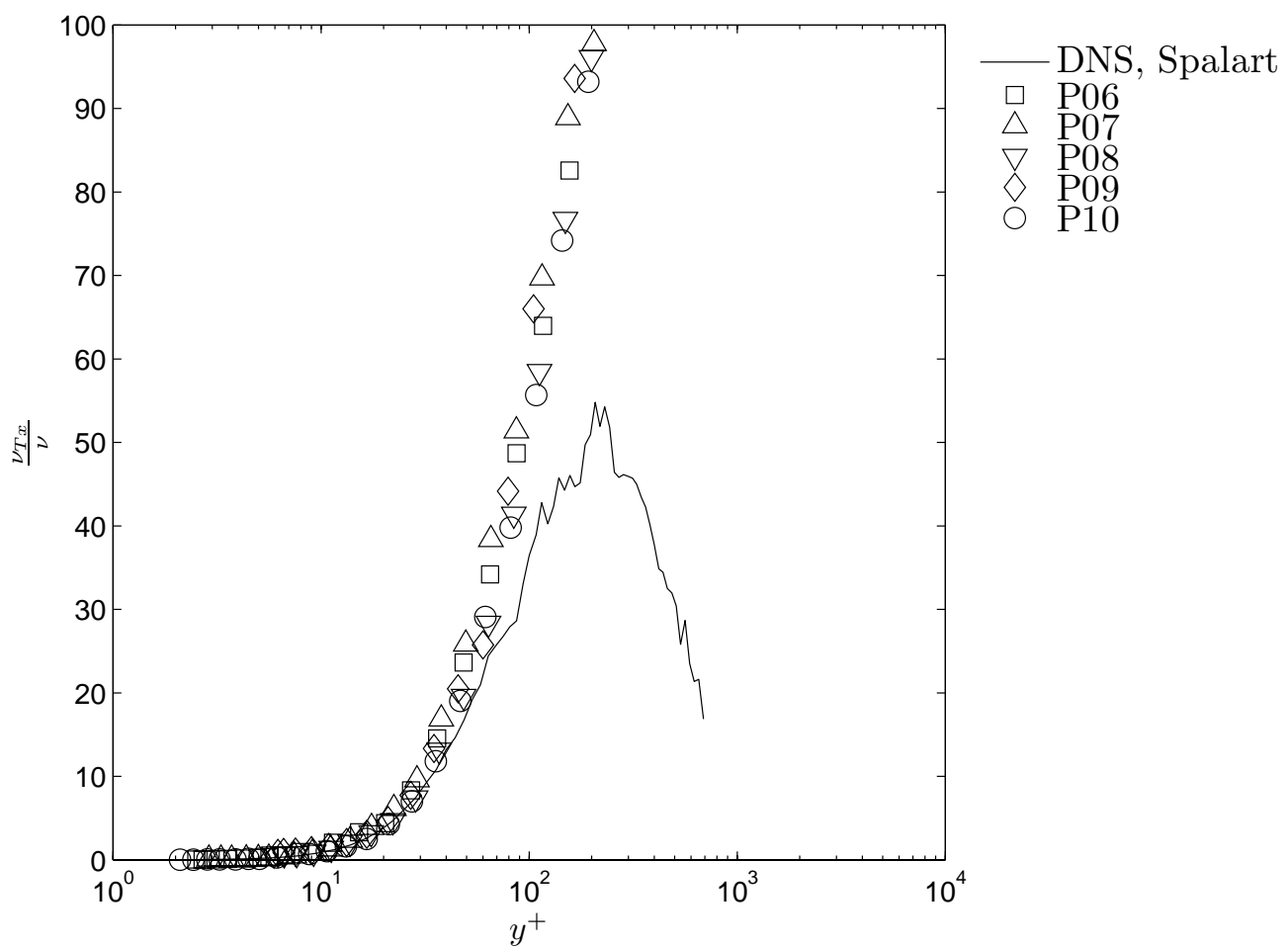

(t) Near Wall Turbulent Eddy Viscosity $\nu_{T x}$

Figure 4.3: Concluded, P08 Cluster 


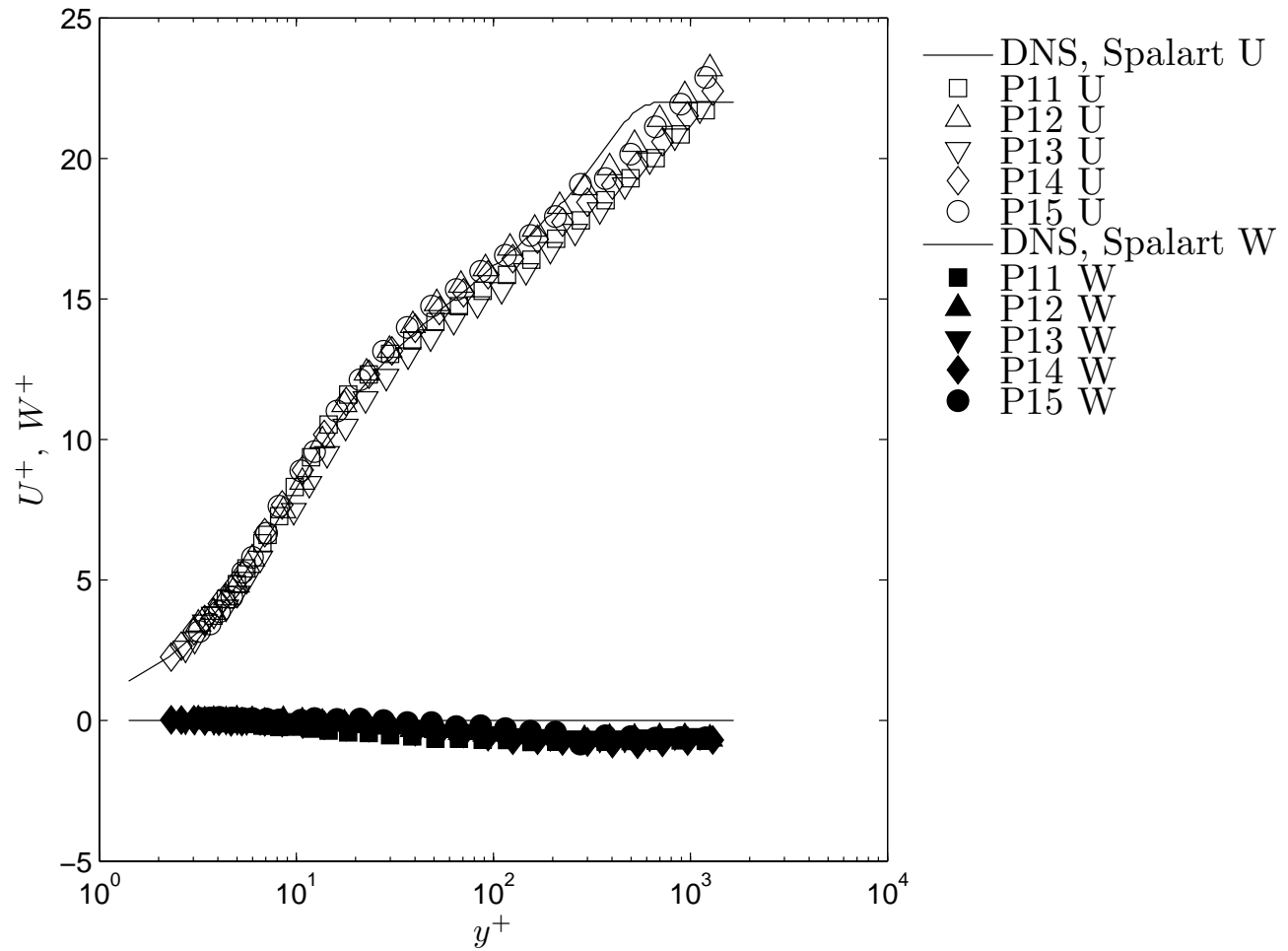

(a) Mean Stream-wise and Span-wise Velocities, $U^{+}, W^{+}$

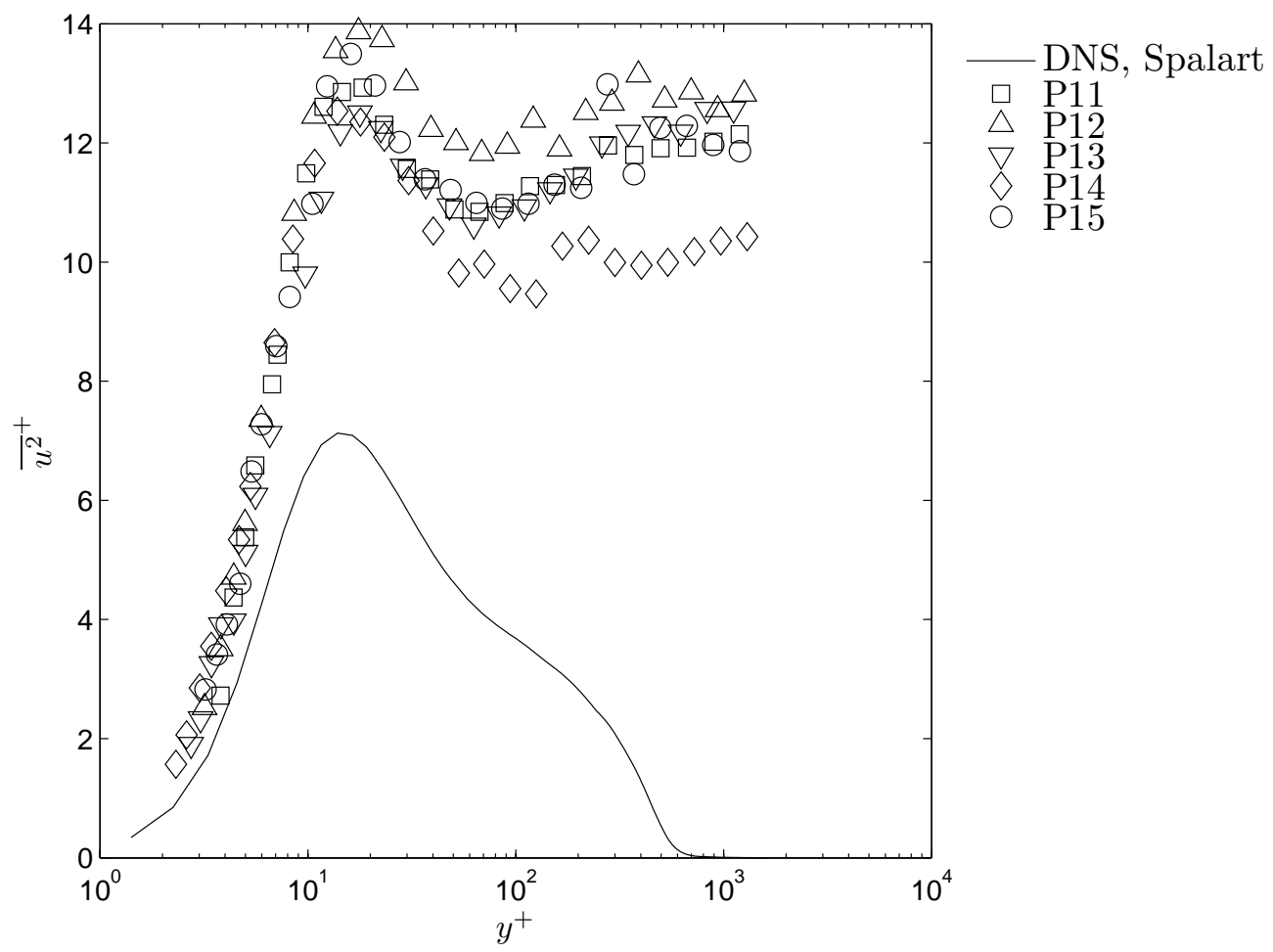

(b) Stream-wise Reynolds' Normal Stress, ${\overline{u^{2}}}^{+}$

Figure 4.4: P13 Cluster 


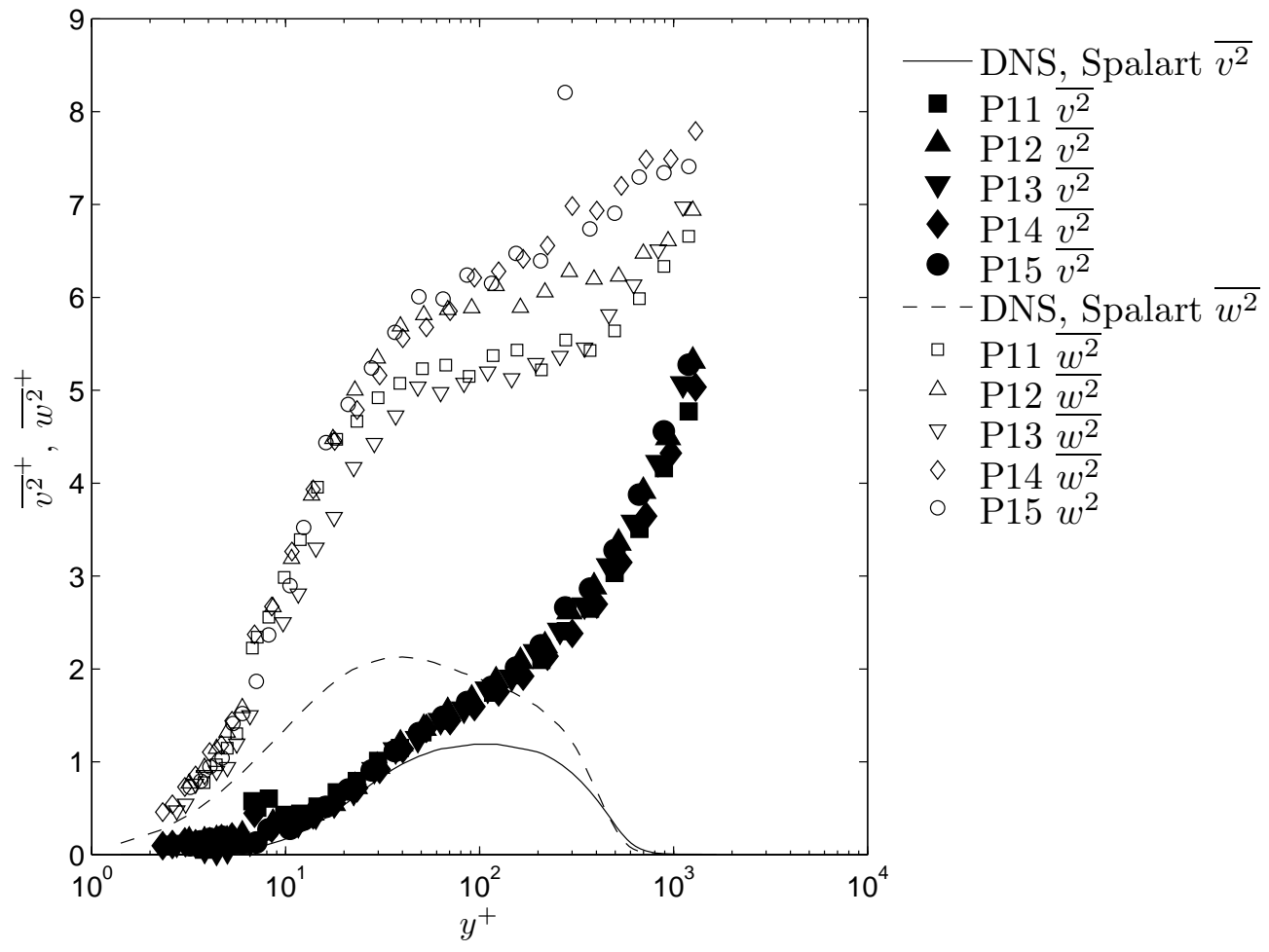

(c) Longitudinal and Span-wise Normal Reynolds' Stresses, ${\overline{v^{2}}}^{+}$and ${\overline{w^{2}}}^{+}$

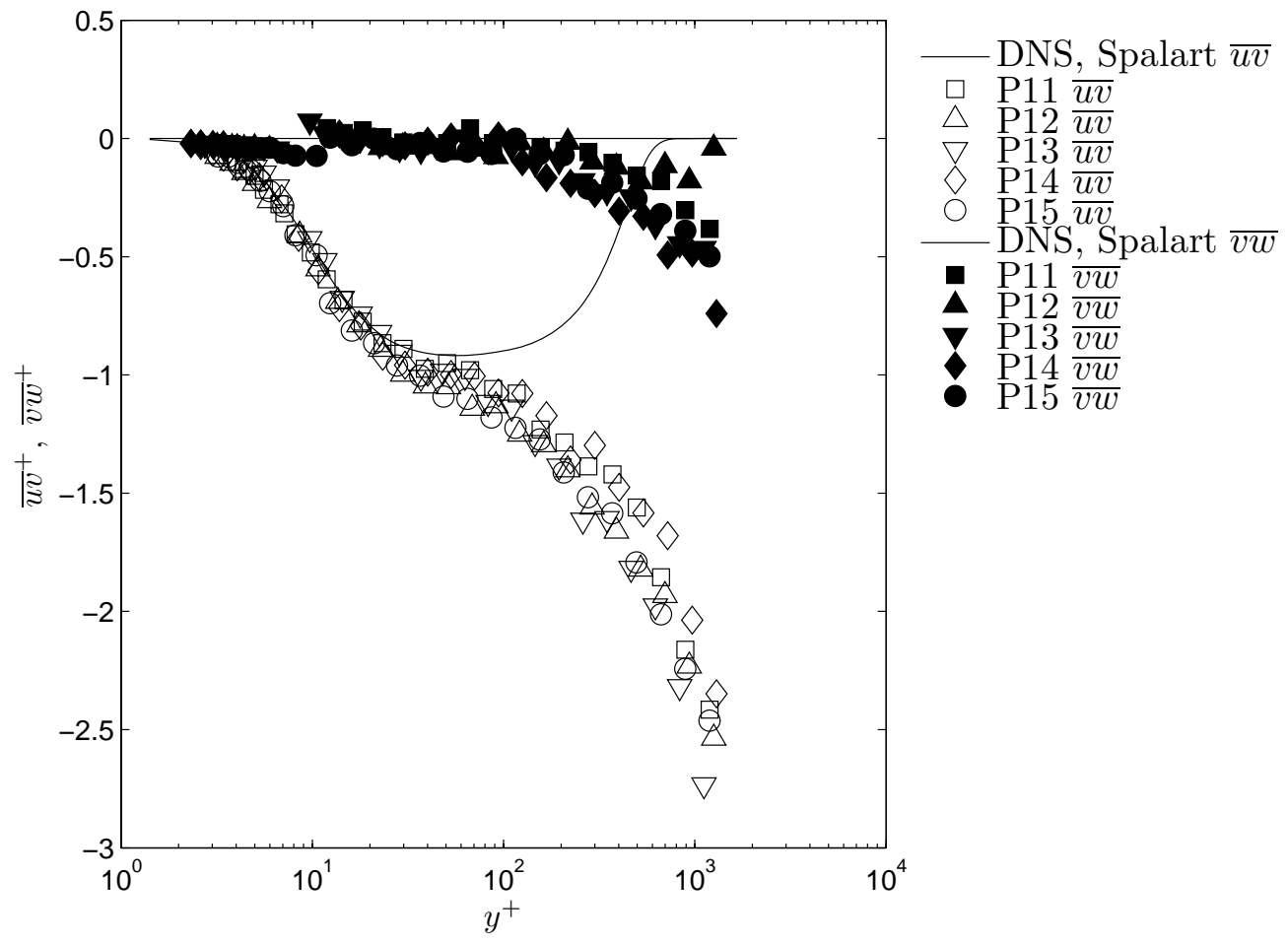

(d) Reynolds' Shear Stresses, $\overline{u v}^{+}$and $\overline{v w}^{+}$

Figure 4.4: Continued, $P 13$ Cluster 


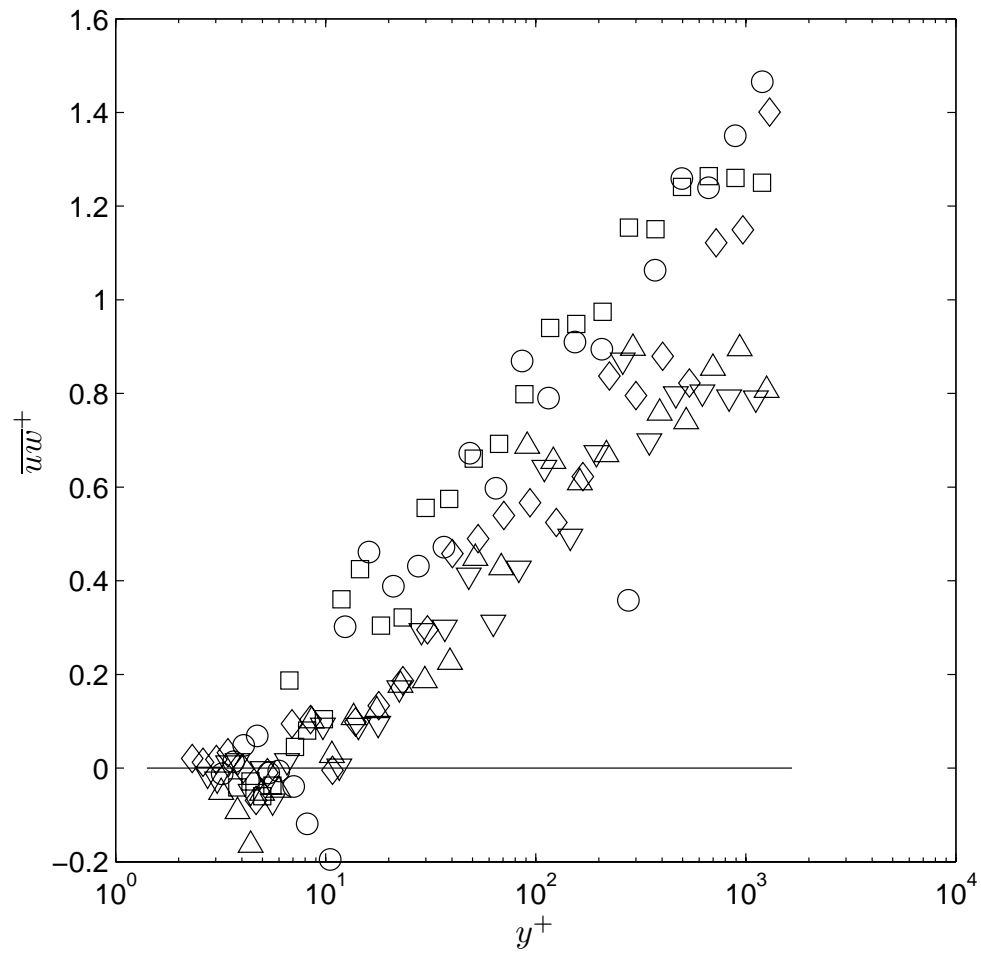

$\begin{array}{ll} & \text { DNS, Spalart } \\ \square & \mathrm{P} 11 \\ \triangle & \mathrm{P} 12 \\ \nabla & \mathrm{P} 13 \\ \diamond & \mathrm{P} 14 \\ \bigcirc & \mathrm{P} 15\end{array}$

(e) Reynolds' Shear Stress, $\overline{u w}^{+}$

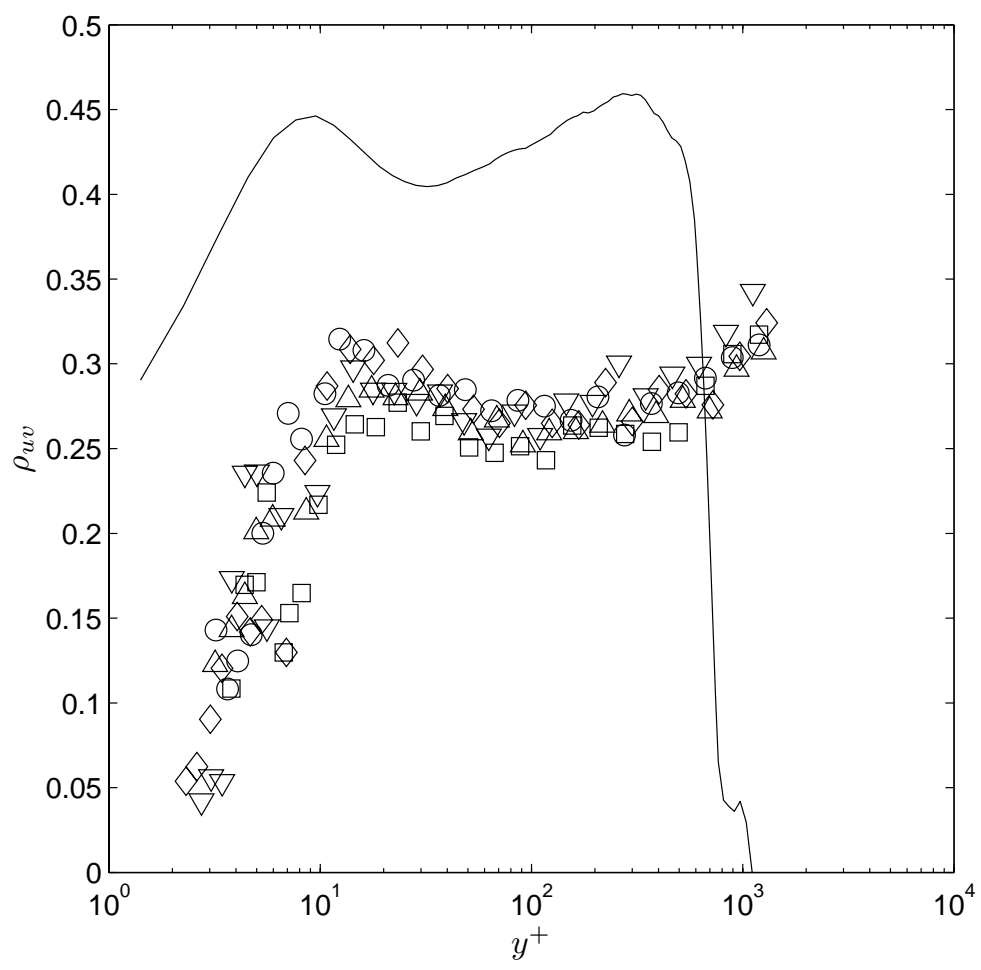

DNS, Spalart

$\square$ P11

$\triangle \mathrm{P} 12$

$\nabla \mathrm{P} 13$

$\checkmark \mathrm{P} 14$

(f) Correlation Coefficient $\rho_{u v}$

Figure 4.4: Continued, P13 Cluster 


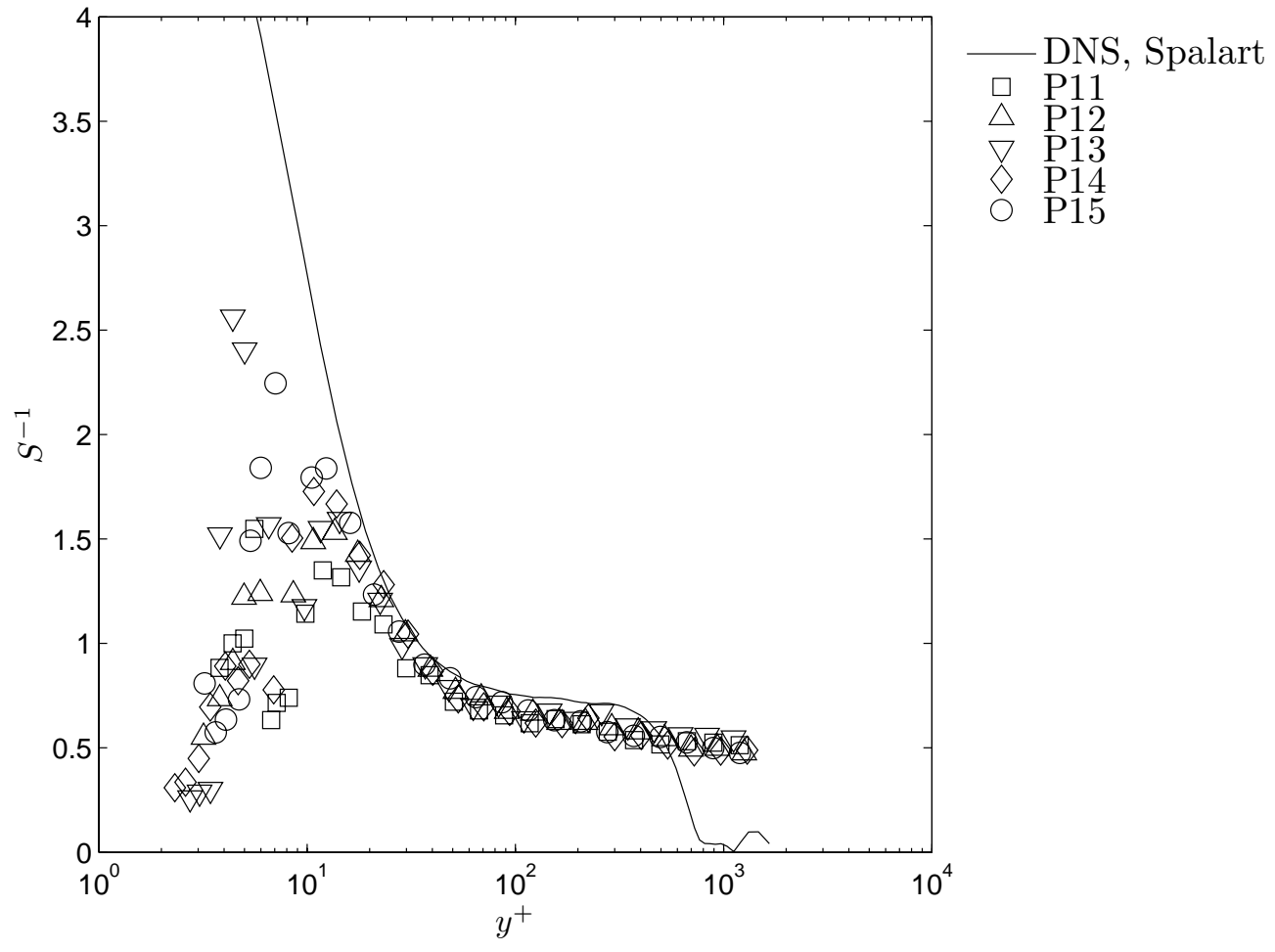

(g) $S^{-1}$ Parameter

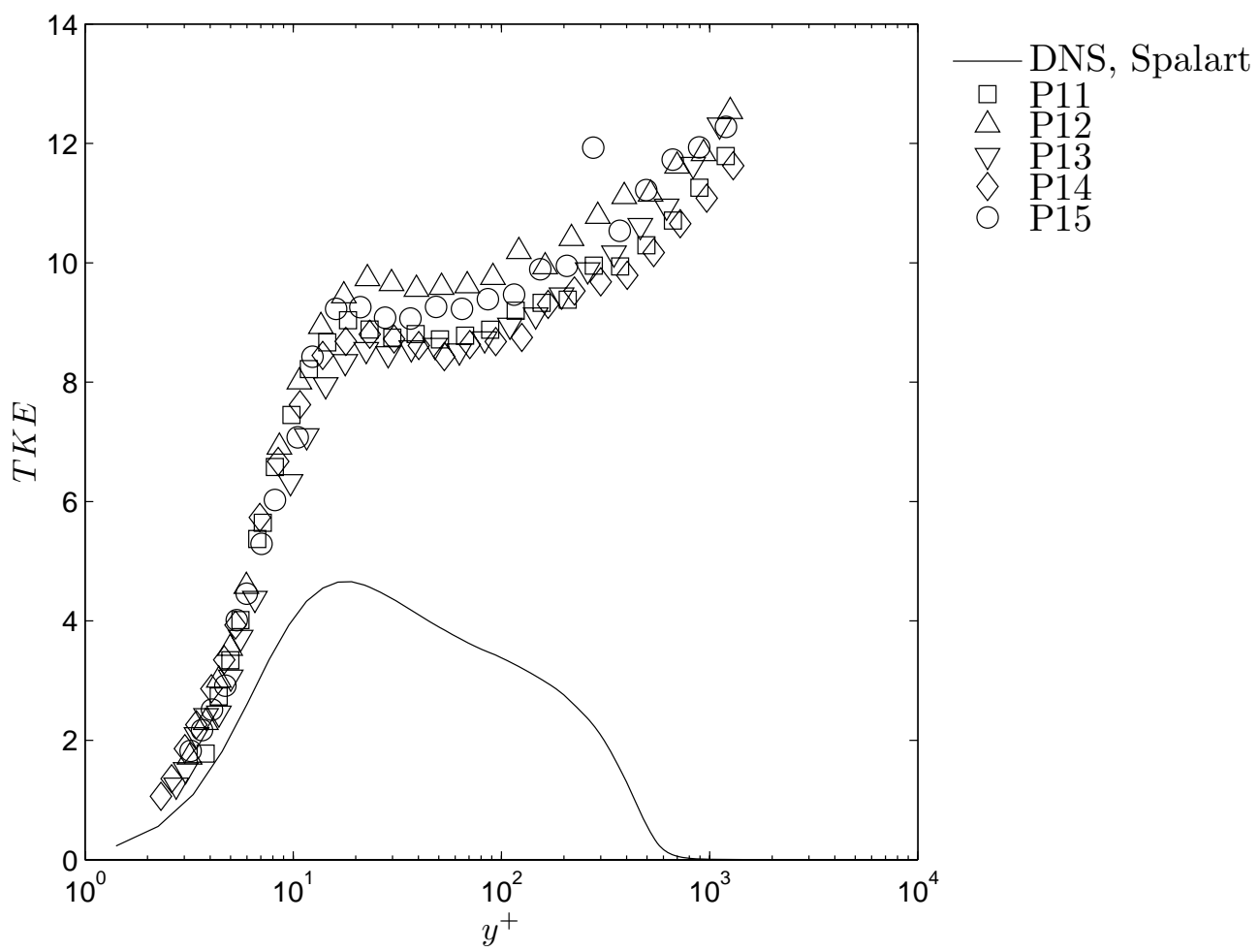

(h) Turbulent Kinetic Energy, $k$

Figure 4.4: Continued, P13 Cluster 


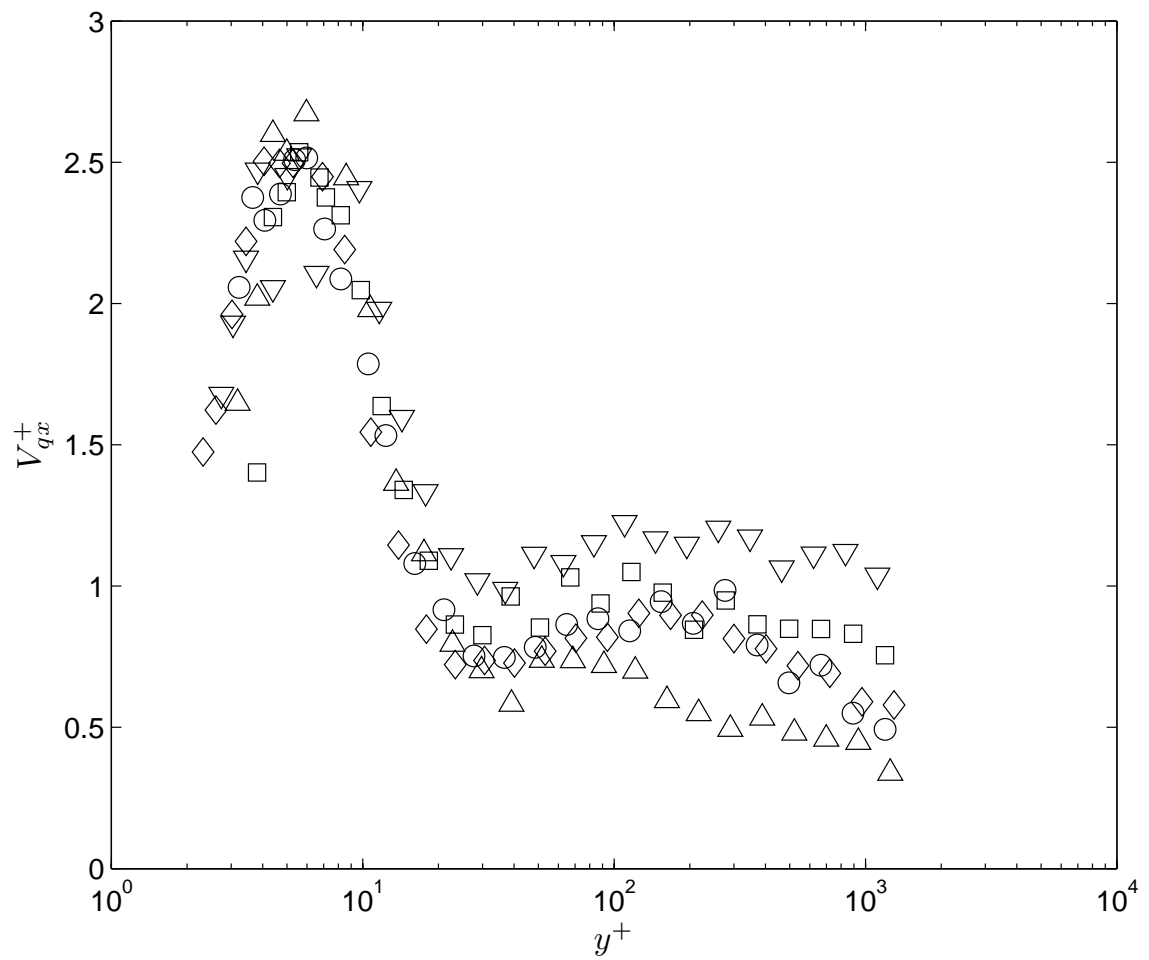

$\begin{array}{ll}\square & \mathrm{P} 11 \\ \triangle & \mathrm{P} 12 \\ \searrow & \mathrm{P} 13 \\ \diamond & \mathrm{P} 14 \\ \bigcirc & \mathrm{P} 15\end{array}$

(i) Stream-wise Turbulent Diffusion Velocity component, $V_{q x}$, normalized on $u_{\tau}$

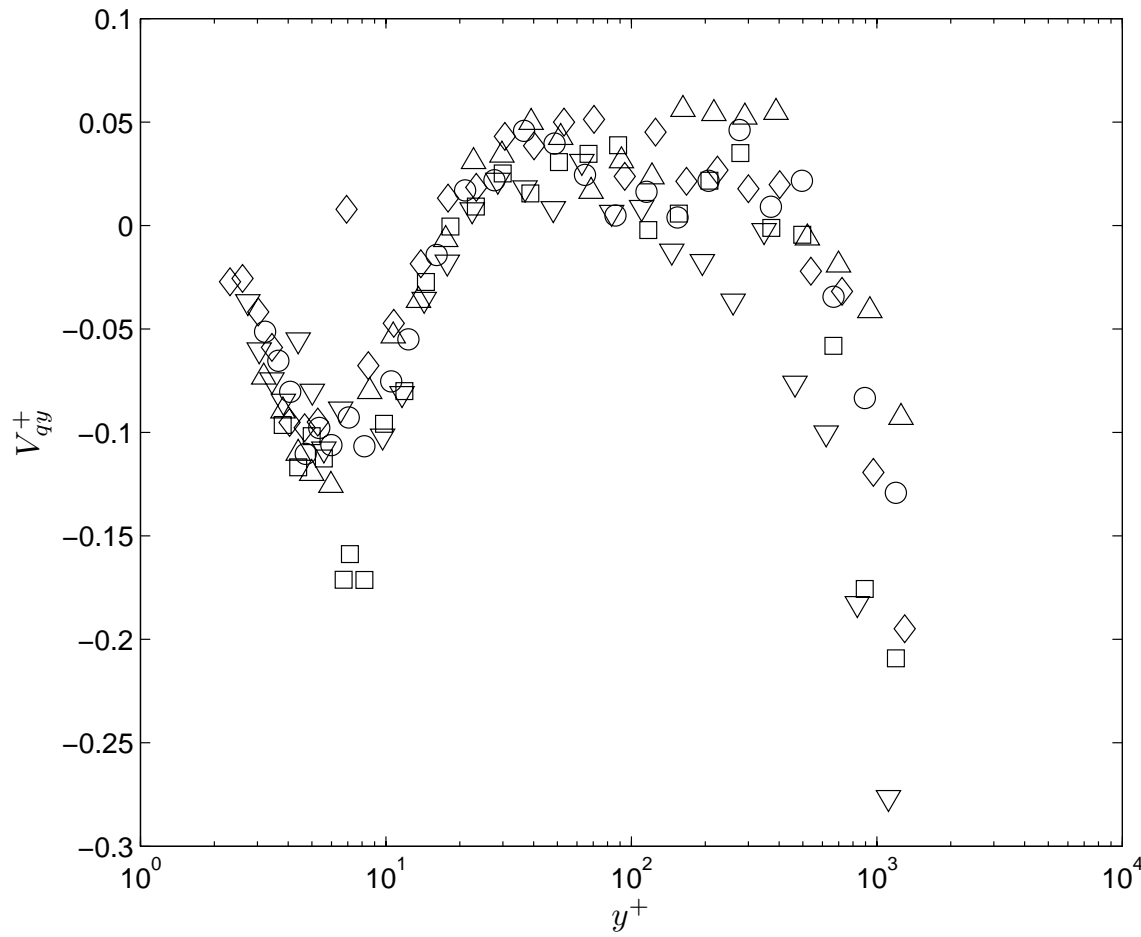

$\begin{array}{ll}\square & \mathrm{P} 11 \\ \triangle & \mathrm{P} 12 \\ \nabla & \mathrm{P} 13 \\ \searrow & \mathrm{P} 14 \\ \bigcirc & \mathrm{P} 15\end{array}$

(j) Wall-Normal Turbulent Diffusion Velocity component, $V_{q y}$, normalized on $u_{\tau}$

Figure 4.4: Continued, P13 Cluster 


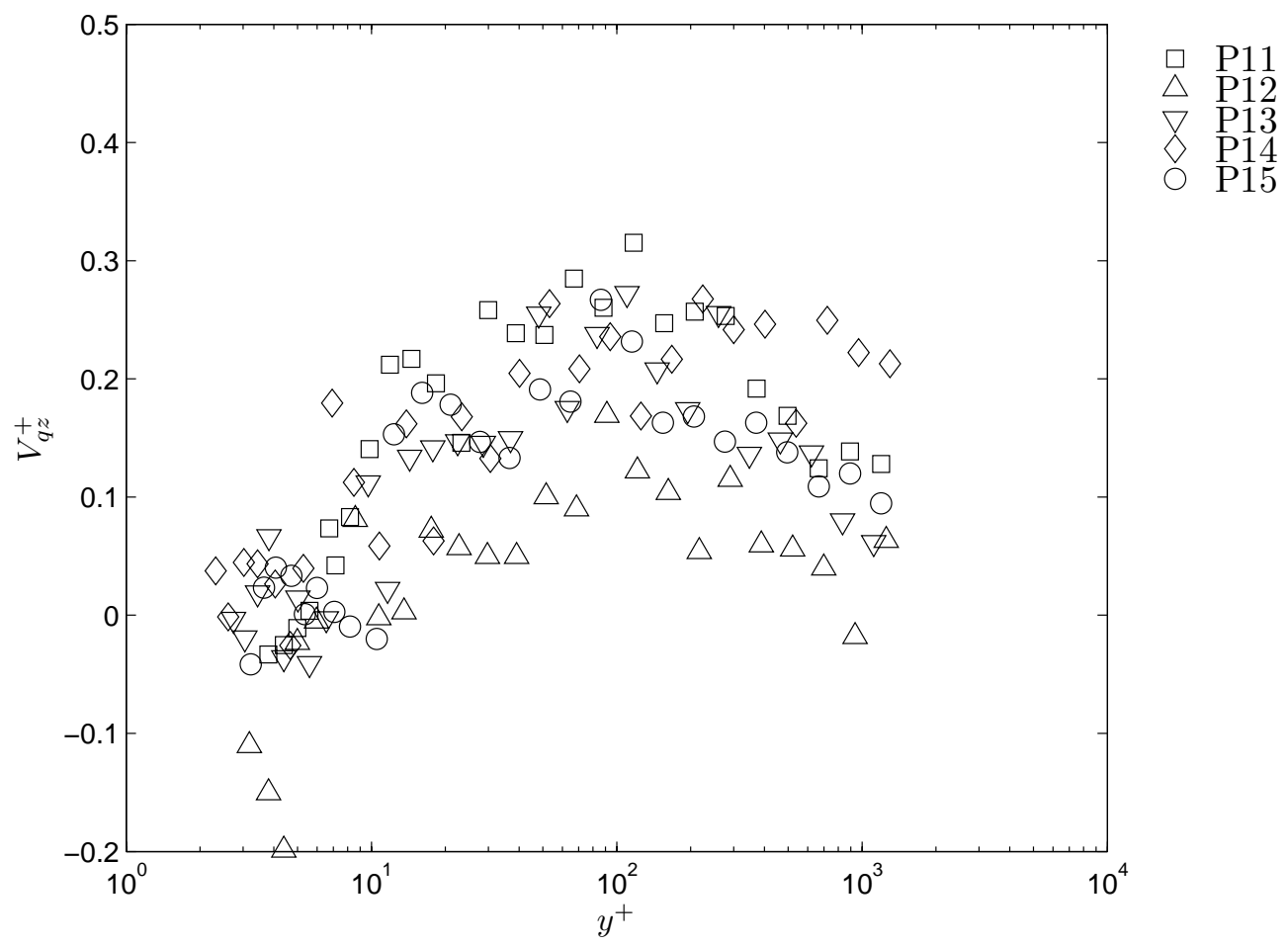

(k) Span-wise Turbulent Diffusion Velocity component, $V_{q z}$, normalized on $u_{\tau}$

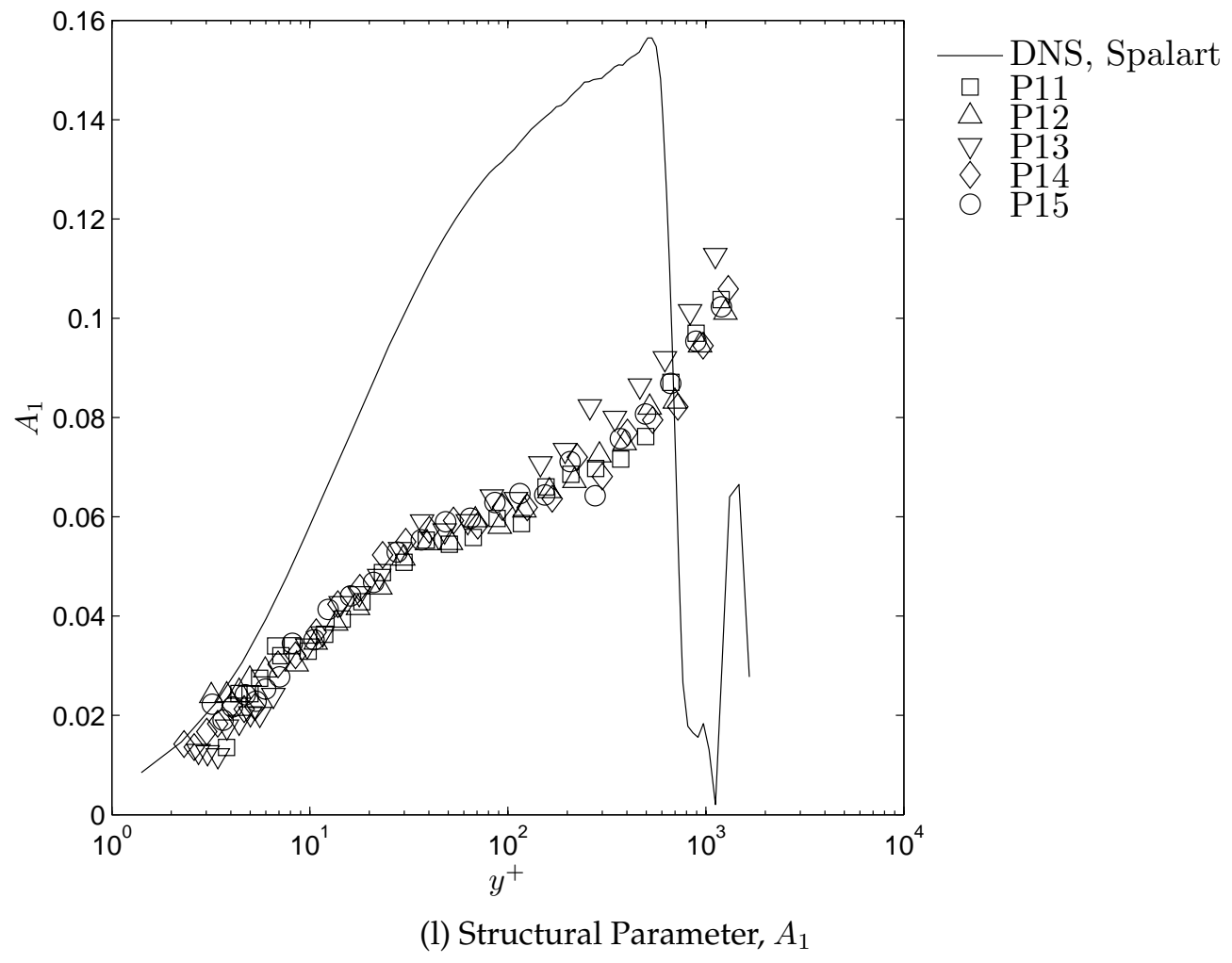

Figure 4.4: Continued, P13 Cluster 


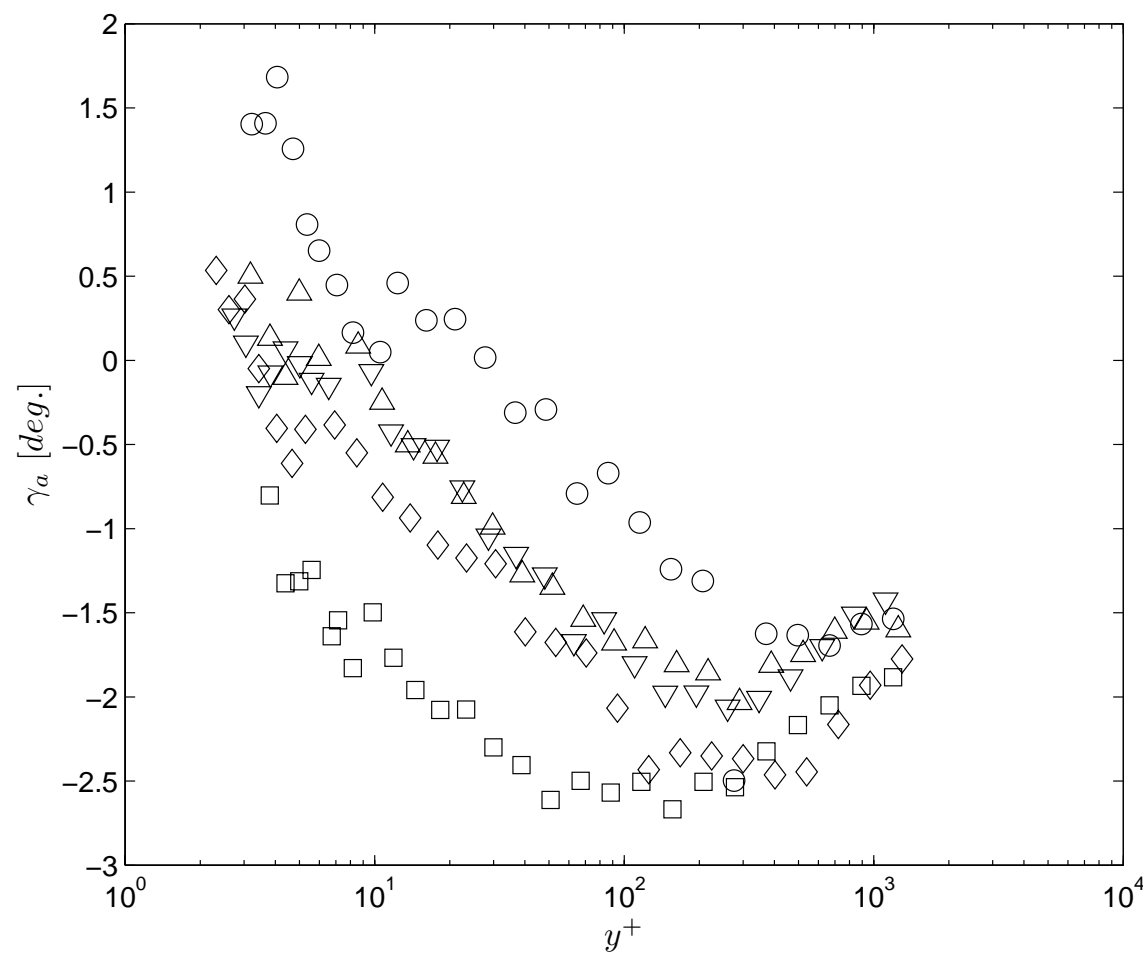

$\begin{array}{ll}\square & \mathrm{P} 11 \\ \triangle & \mathrm{P} 12 \\ \nabla & \mathrm{P} 13 \\ \searrow & \mathrm{P} 14 \\ \mathrm{O} & \mathrm{P} 15\end{array}$

(m) Flow Angle, $\gamma_{a}$

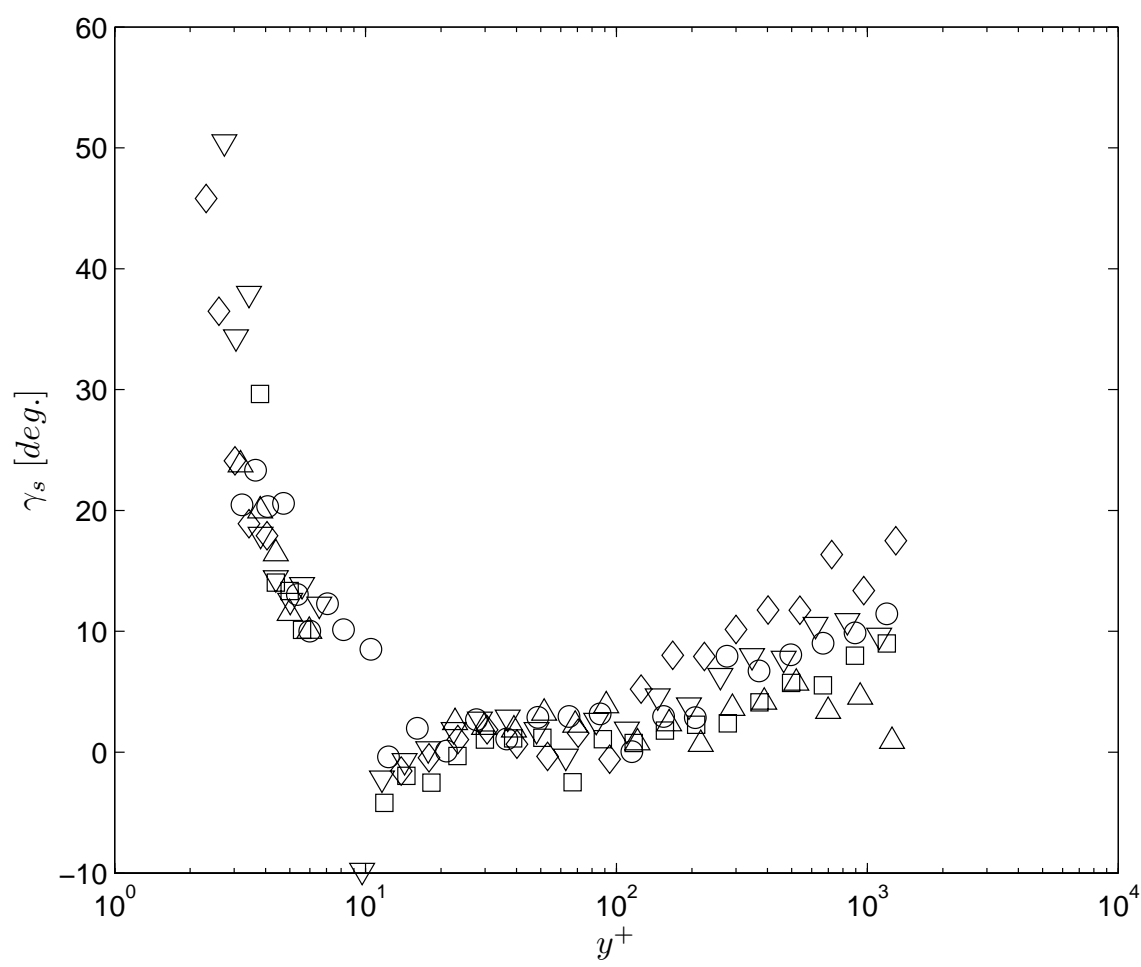

$\begin{array}{ll}\square & \mathrm{P} 11 \\ \triangle & \mathrm{P} 12 \\ \nabla & \mathrm{P} 13 \\ \searrow & \mathrm{P} 14 \\ \bigcirc & \mathrm{P} 15\end{array}$

(n) Shear Stress Angle $\gamma_{s}$

Figure 4.4: Continued, P13 Cluster 


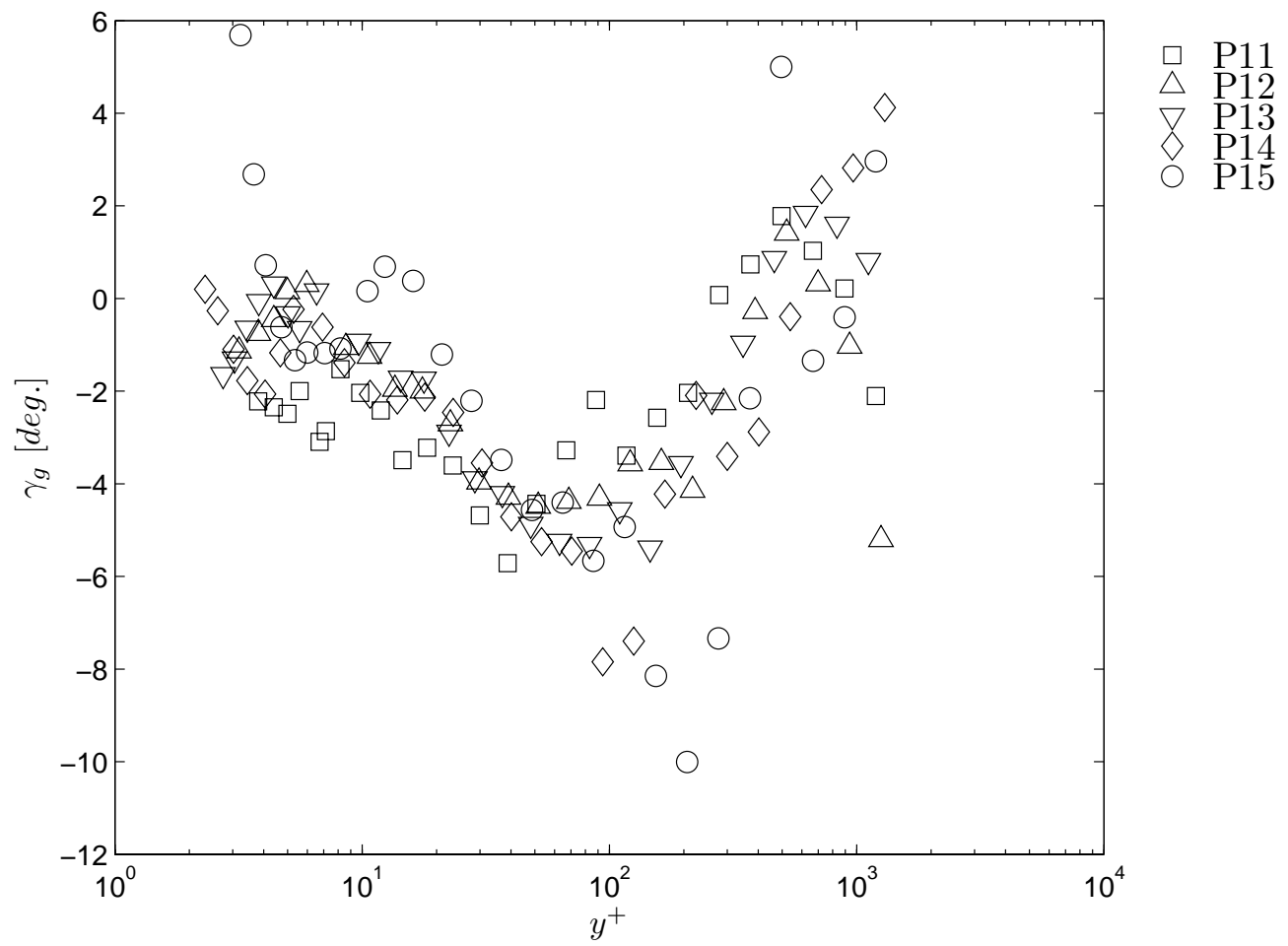

(o) Flow Gradient Angle, $\gamma_{g}$

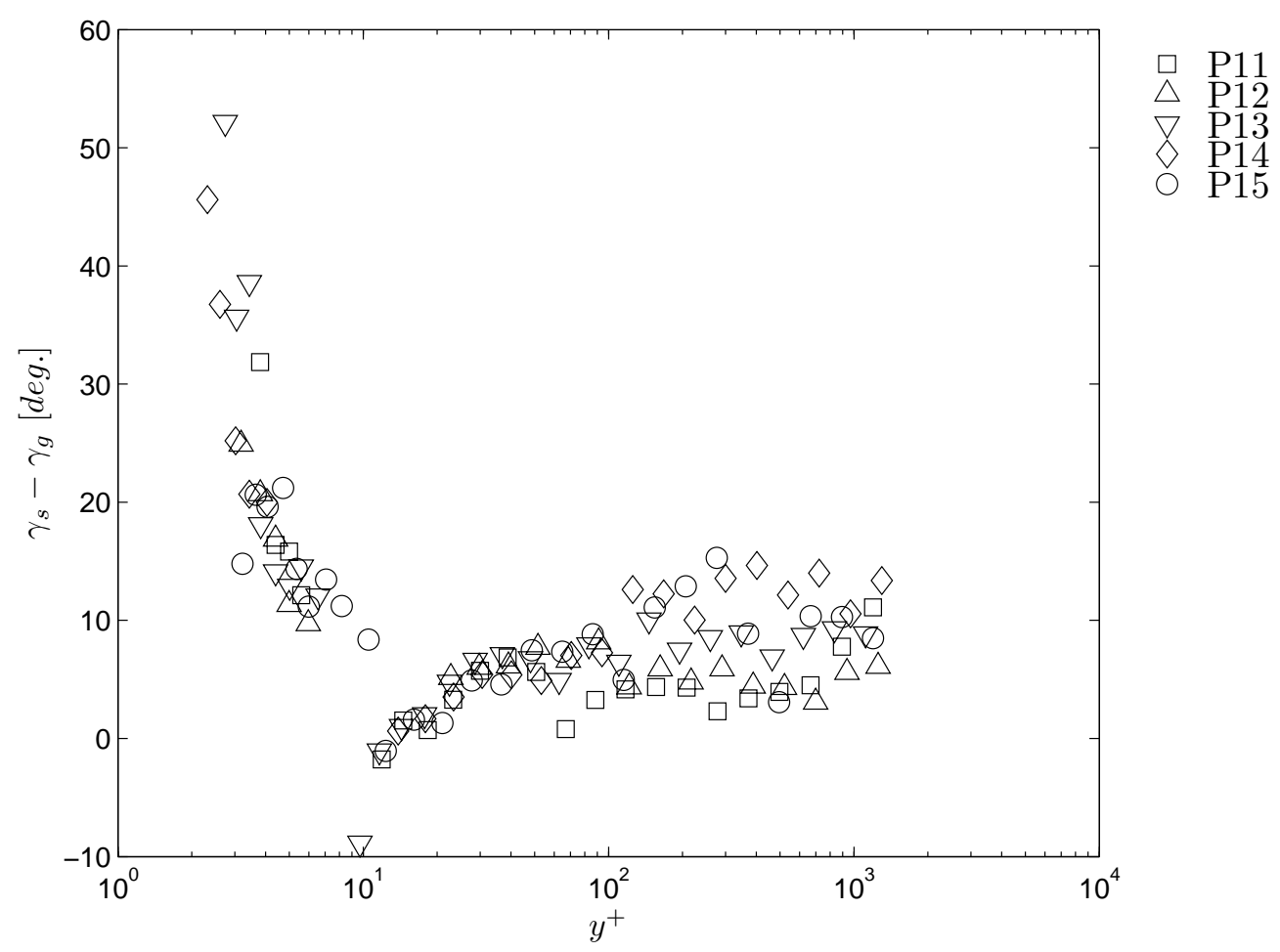

(p) Difference between Shear Angle and Flow Gradient Angles, $\gamma_{s}-\gamma_{g}$

Figure 4.4: Continued, P13 Cluster 


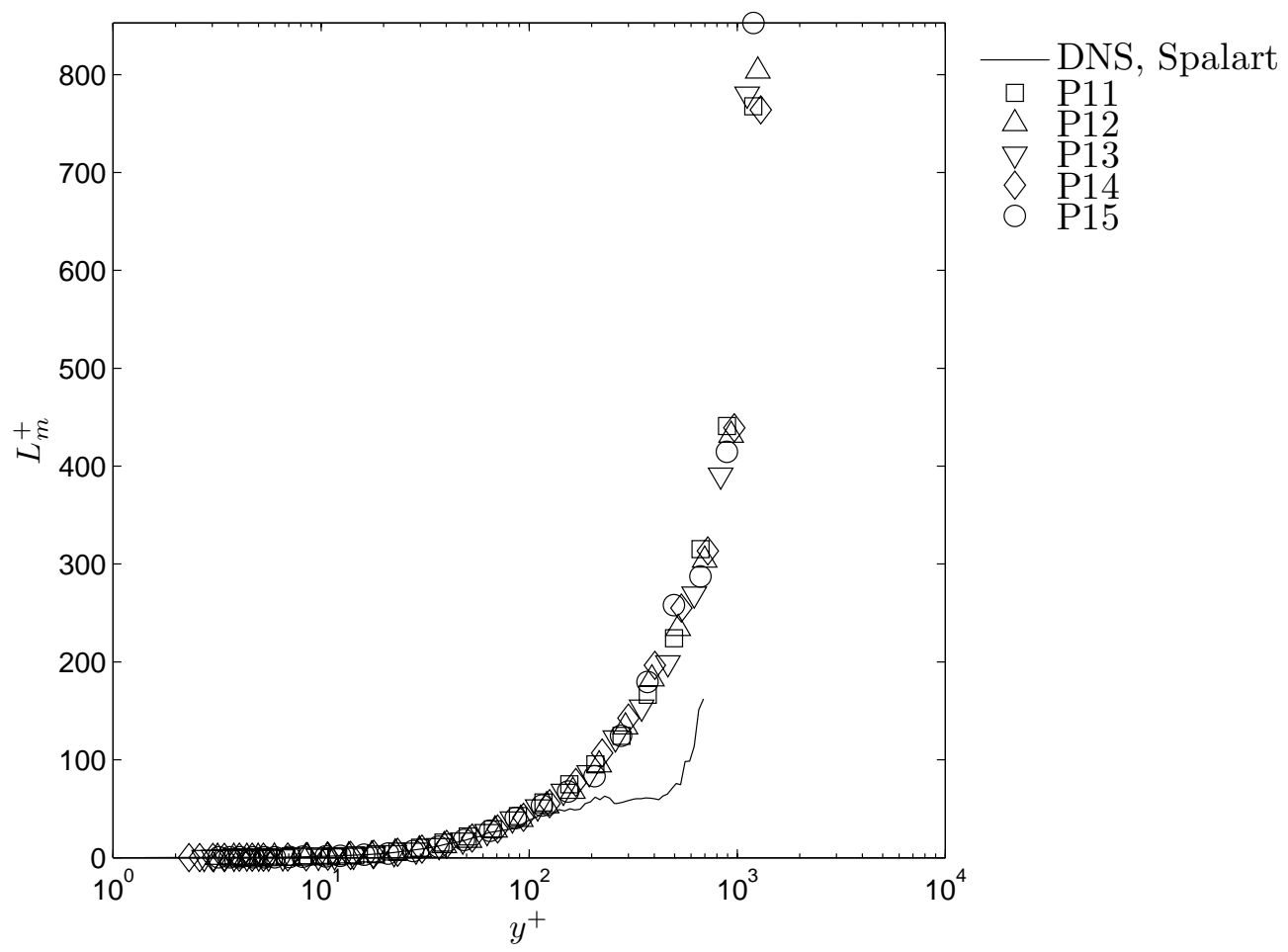

(q) Mixing Length $L_{m}^{+}$

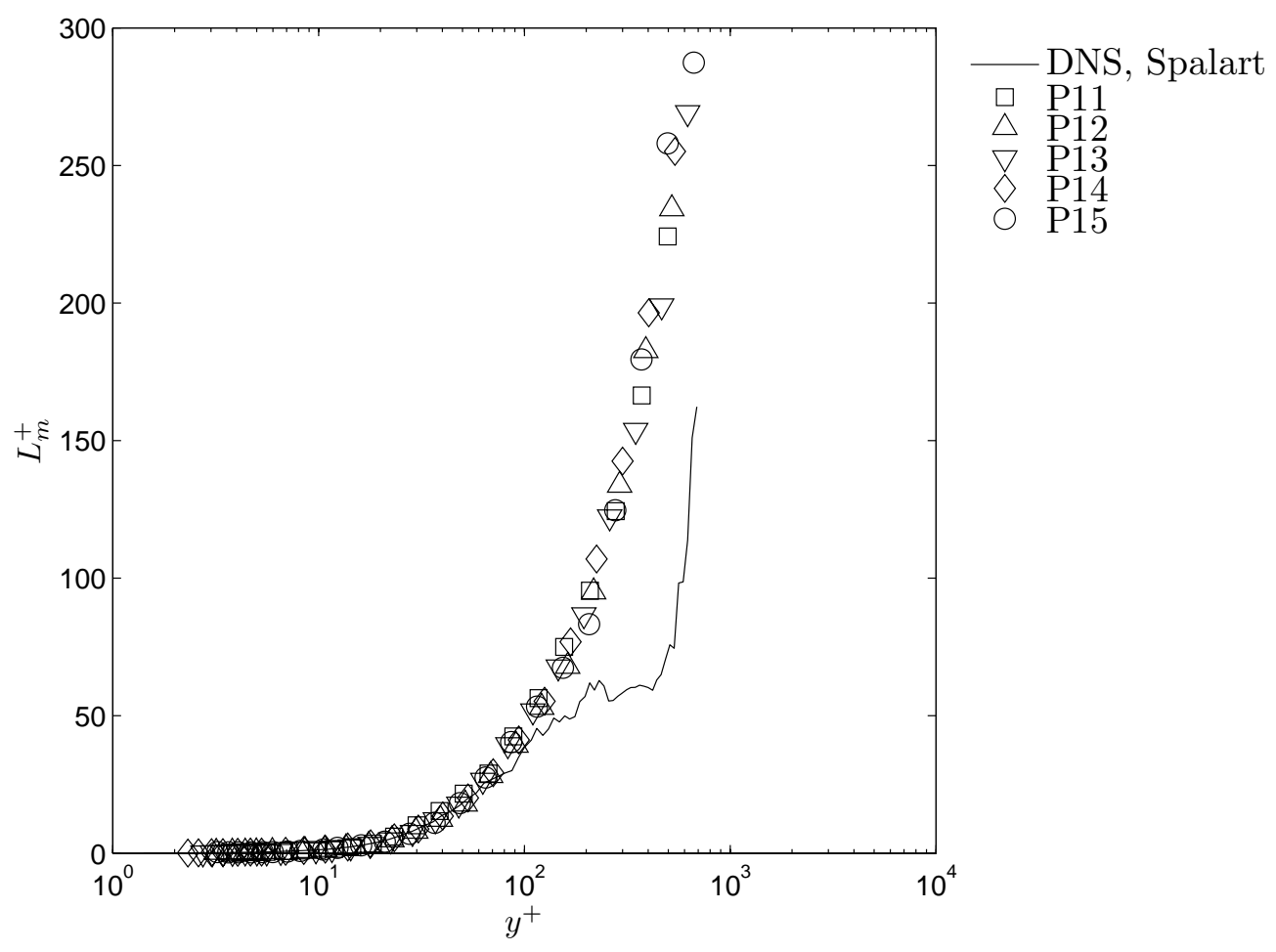

(r) Near Wall Mixing Length $L_{m}^{+}$

Figure 4.4: Continued, P13 Cluster 


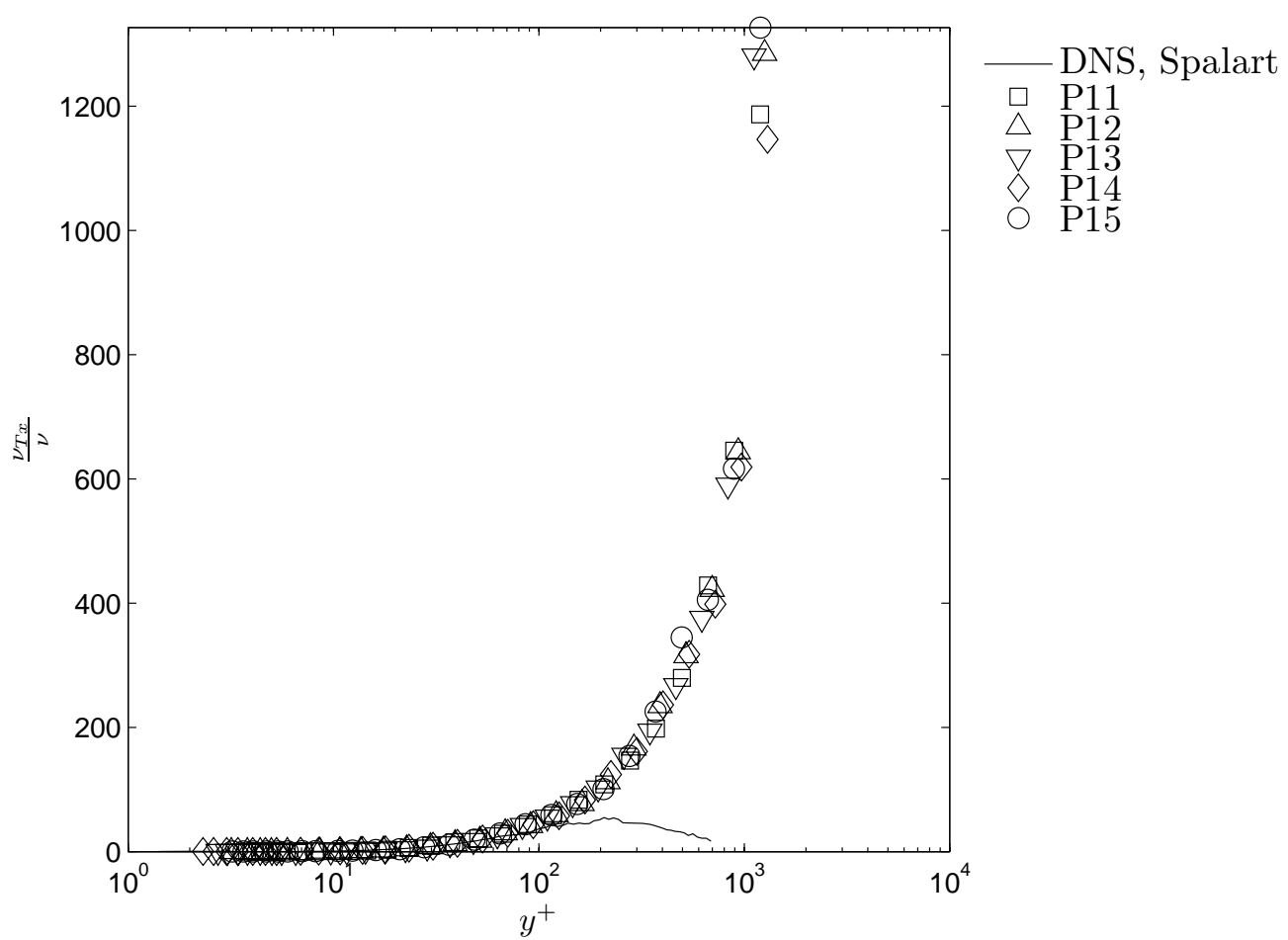

(s) Turbulent Eddy Viscosity $\nu_{T x}$

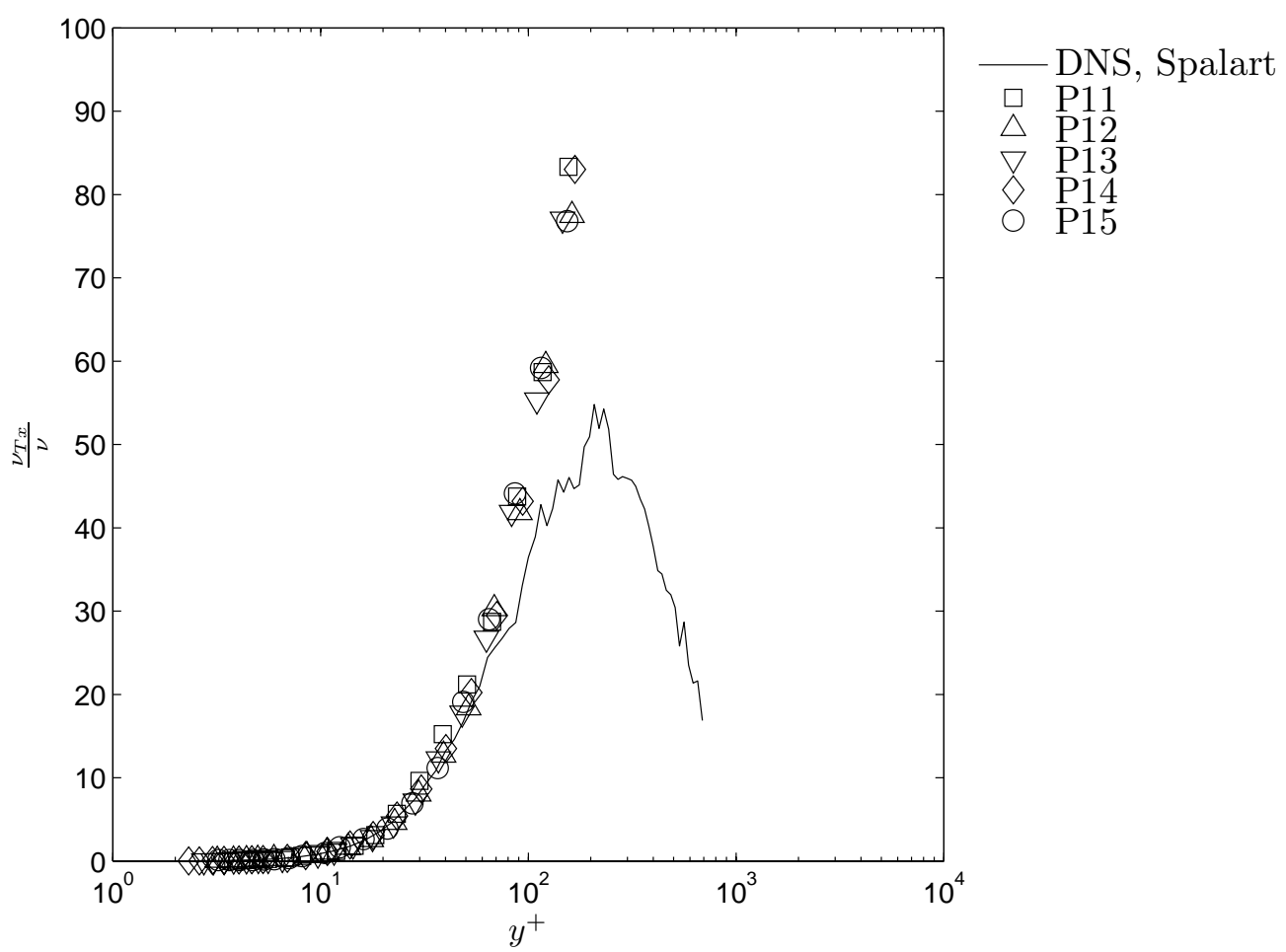

(t) Near Wall Turbulent Eddy Viscosity $\nu_{T x}$

Figure 4.4: Concluded, P13 Cluster 


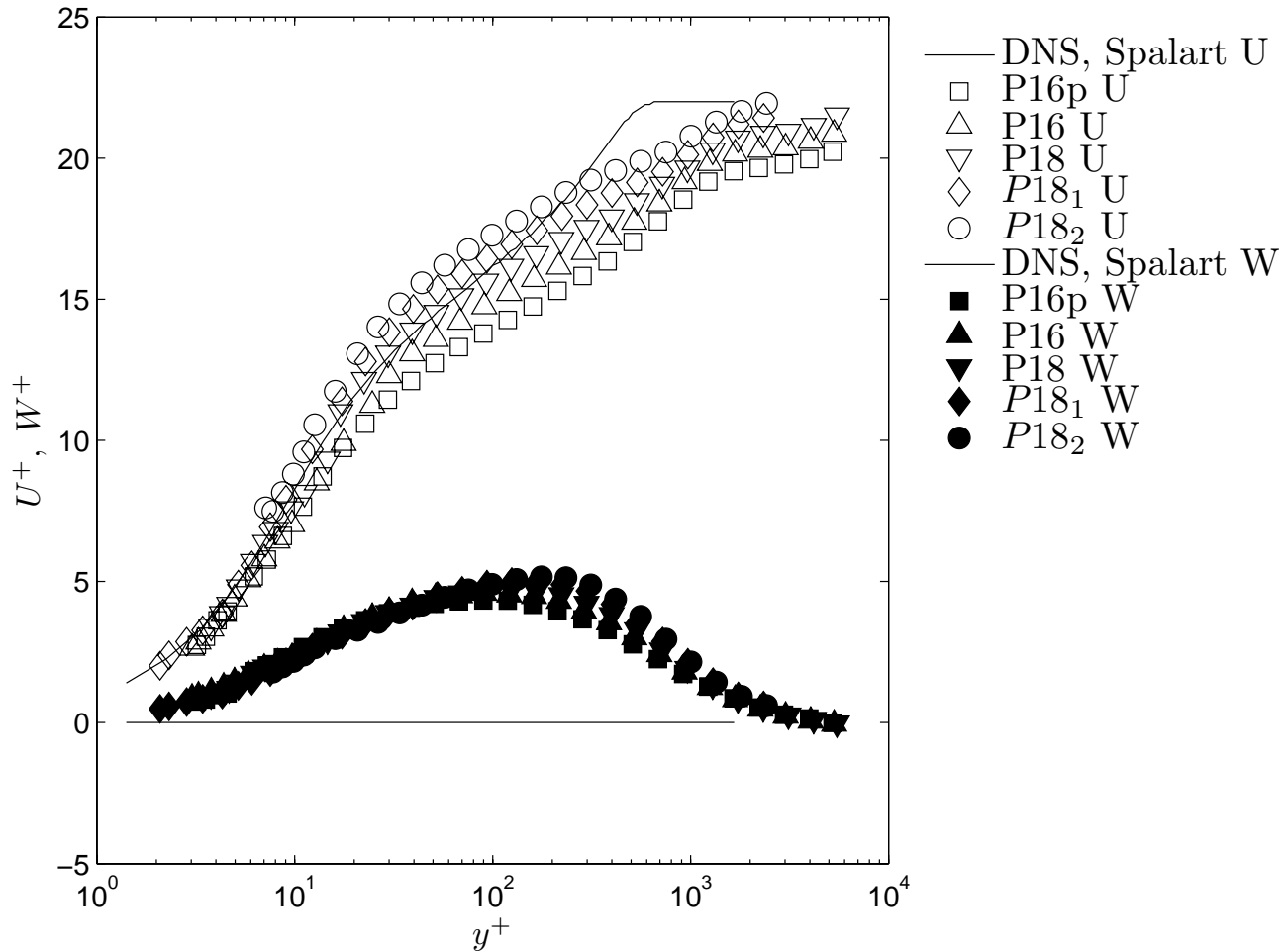

(a) Mean Stream-wise and Span-wise Velocities, $U^{+}, W^{+}$

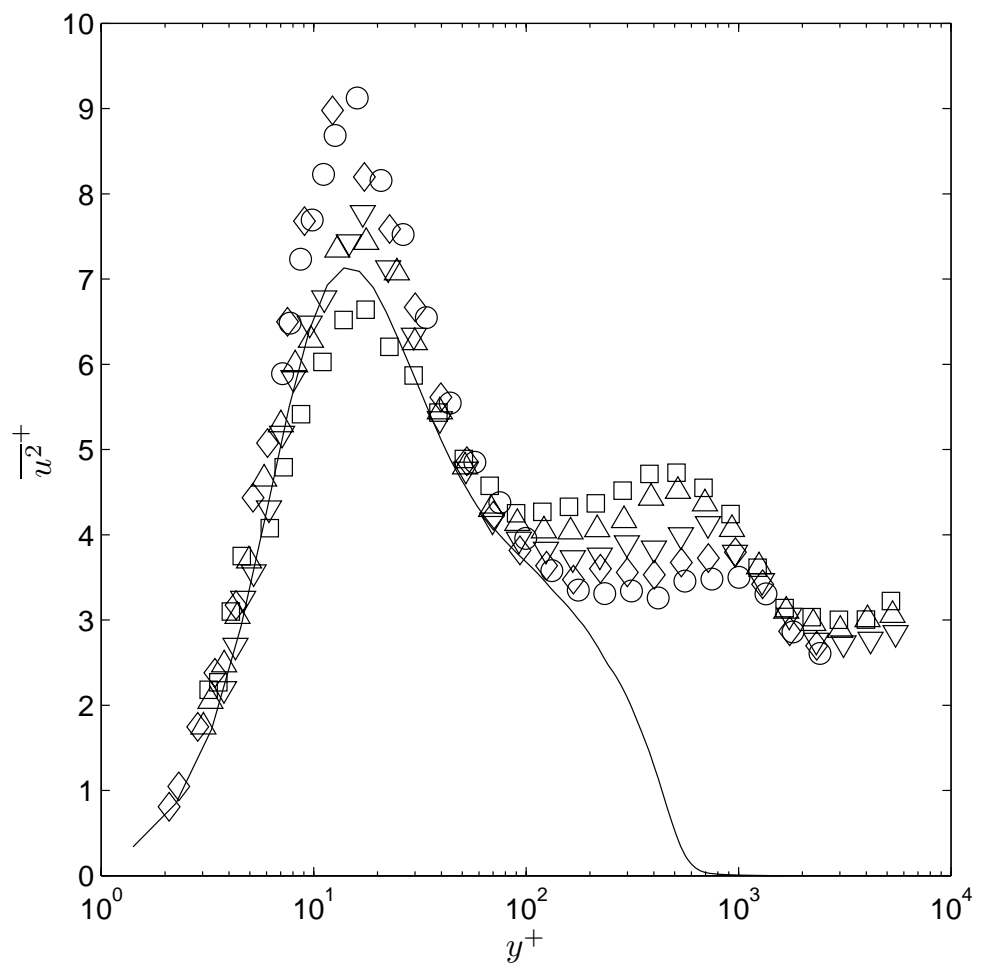

$\begin{array}{ll} & \text { DNS, Spalart } \\ \square & \text { P16p } \\ \triangle & \mathrm{P} 16 \\ \nabla & \mathrm{P} 18 \\ \diamond & P 18_{1} \\ \bigcirc & P 18_{2}\end{array}$

(b) Stream-wise Reynolds' Normal Stress, ${\overline{u^{2}}}^{+}$

Figure 4.5: P18 Cluster 


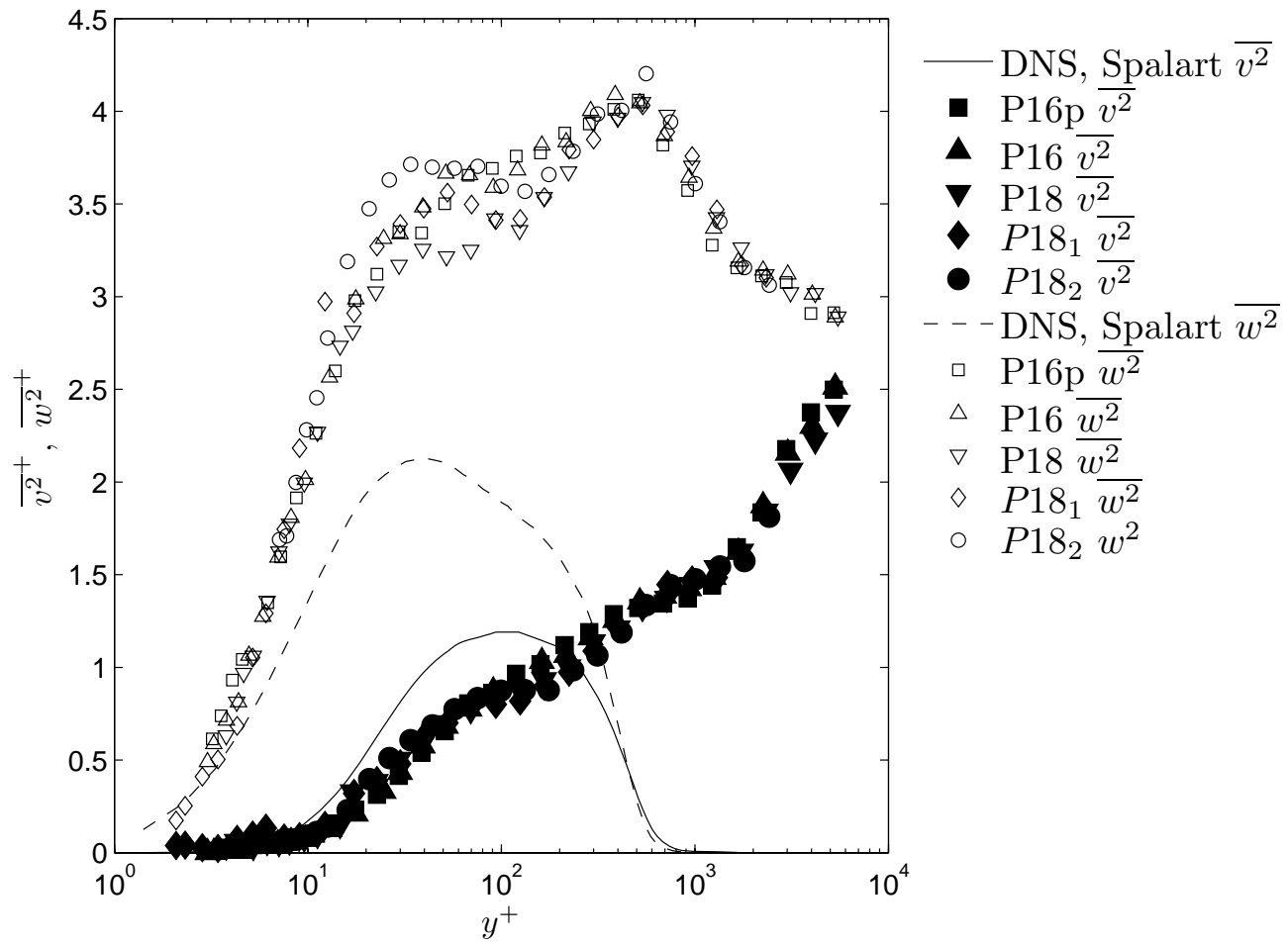

(c) Longitudinal and Span-wise Normal Reynolds' Stresses, ${\overline{v^{2}}}^{+}$and ${\overline{w^{2}}}^{+}$

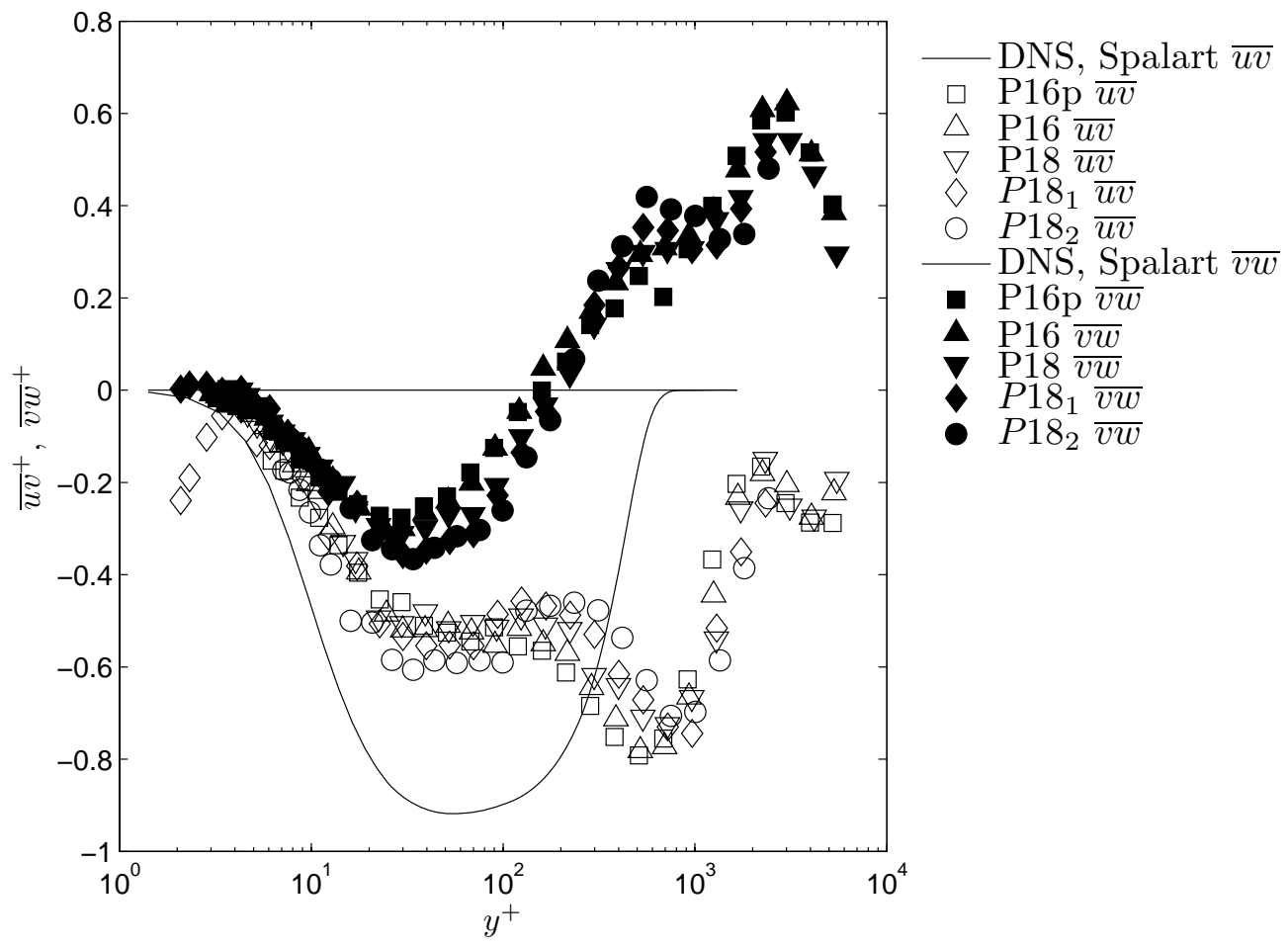

(d) Reynolds' Shear Stresses, $\overline{u v}^{+}$and $\overline{v w}{ }^{+}$

Figure 4.5: Continued, $P 18$ Cluster 


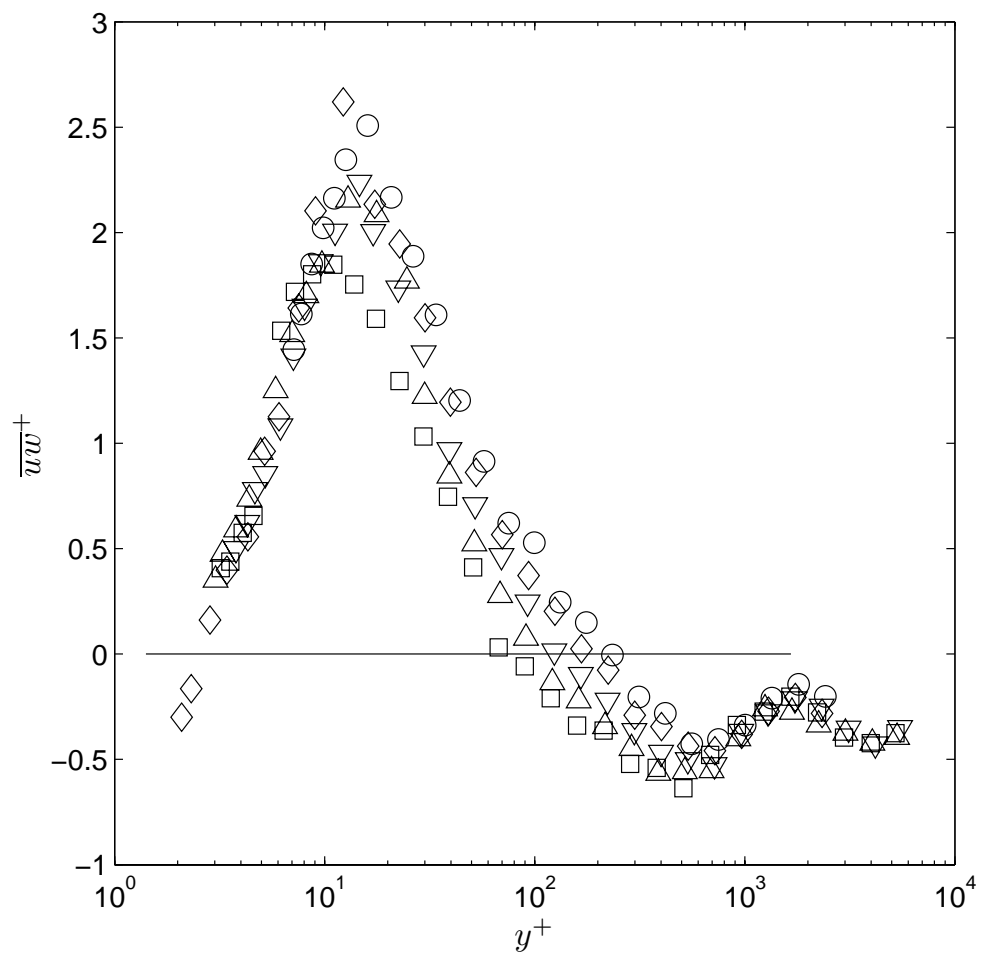
DNS, Spalart
$\square$ P16p
$\triangle \mathrm{P} 16$
$\nabla \mathrm{P} 18$
$\diamond P 18_{1}$
○ $\mathrm{P} 18_{2}$

(e) Reynolds' Shear Stress, $\overline{u w}^{+}$

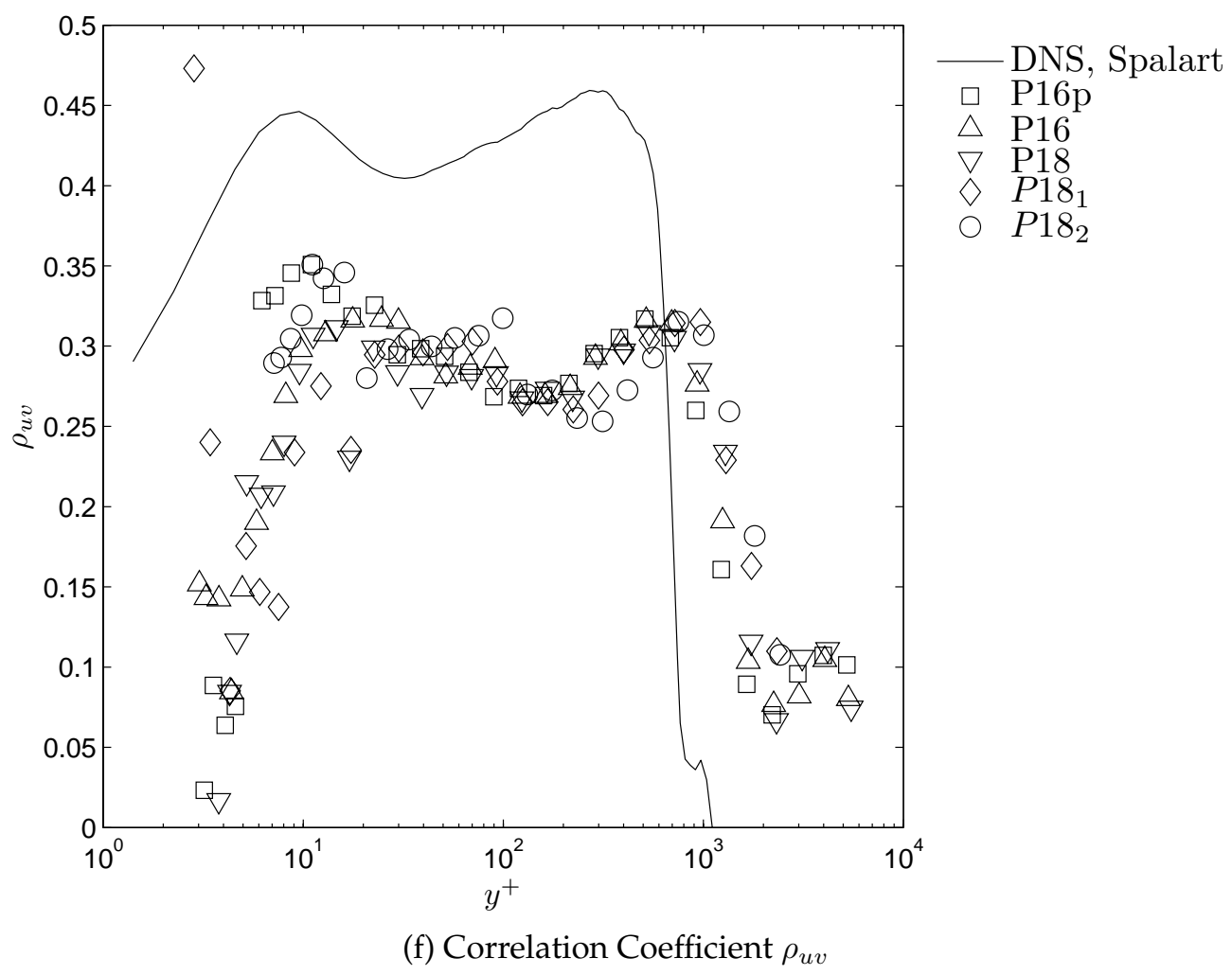

Figure 4.5: Continued, P18 Cluster 


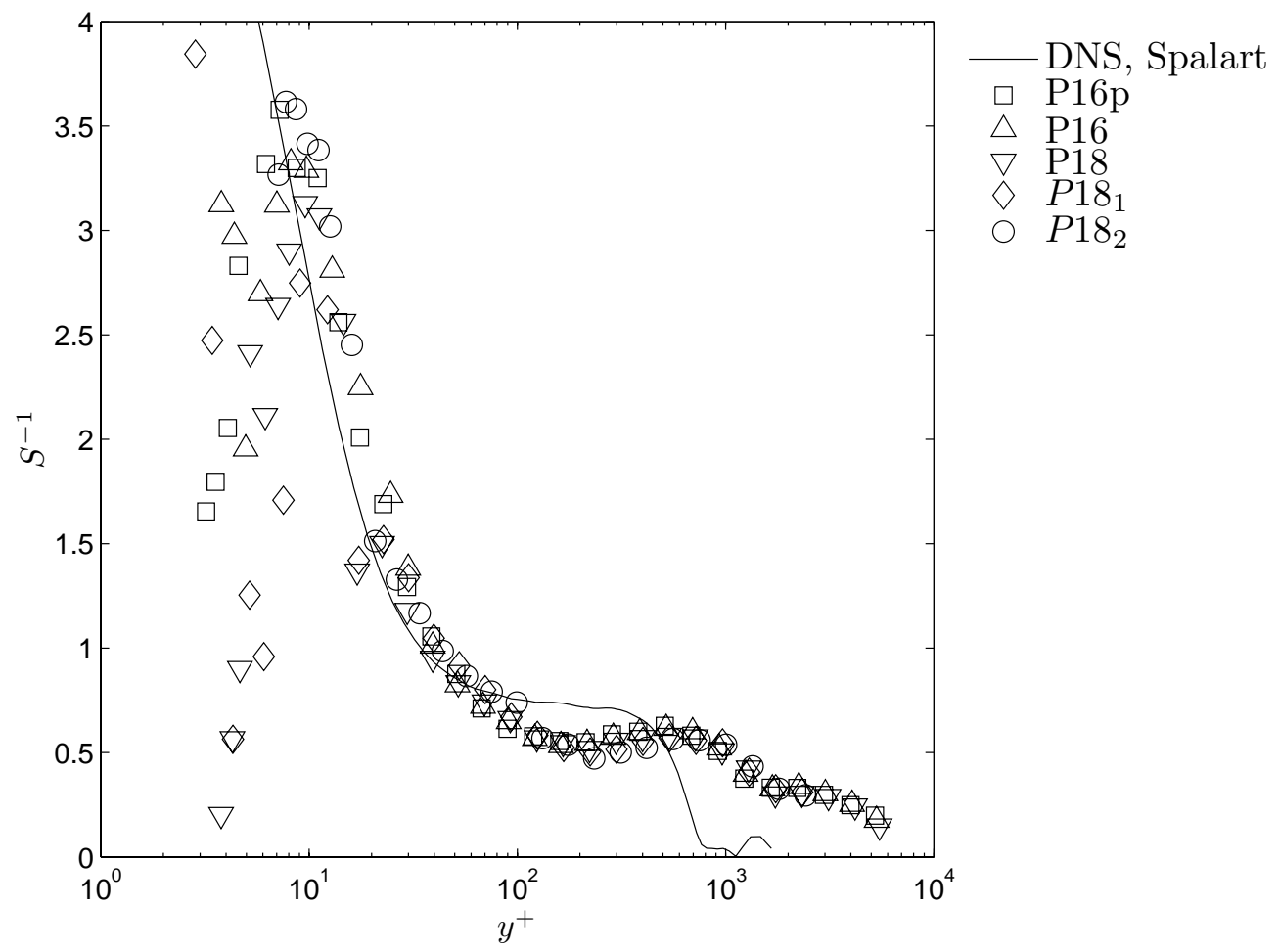

(g) $S^{-1}$ Parameter

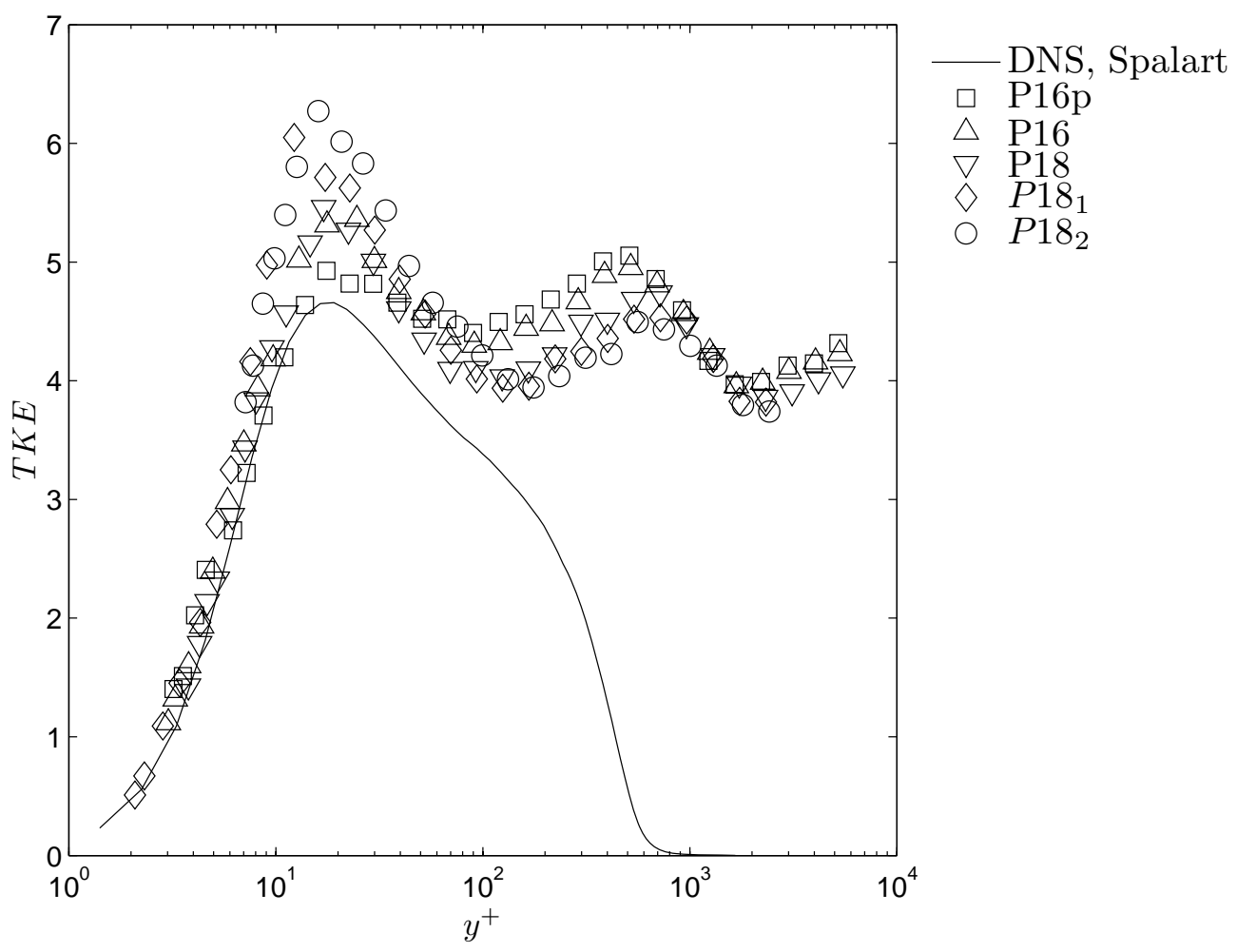

(h) Turbulent Kinetic Energy, TKE

Figure 4.5: Continued, P18 Cluster 


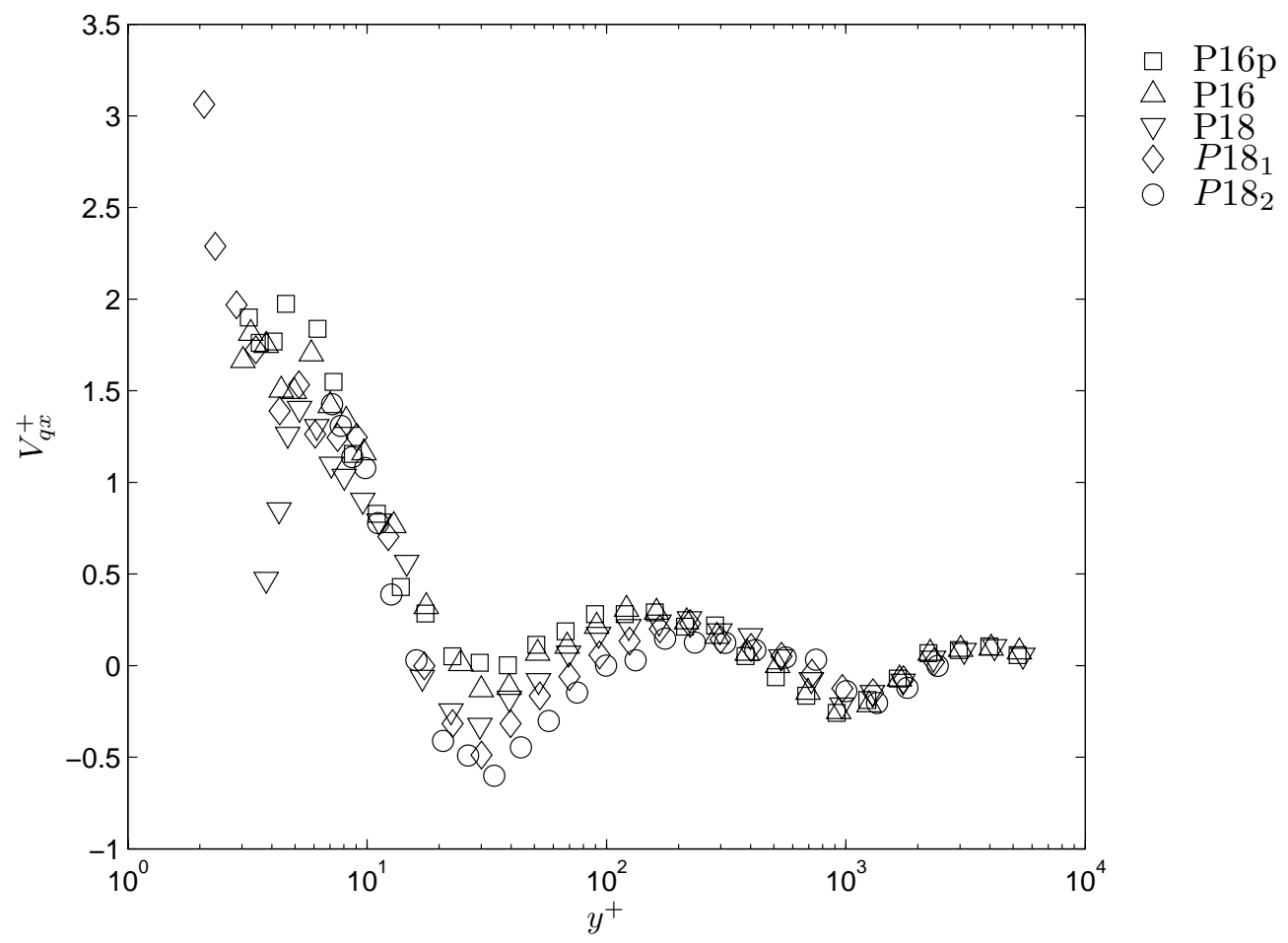

(i) Stream-wise Turbulent Diffusion Velocity component, $V_{q x}$, normalized on $u_{\tau}$

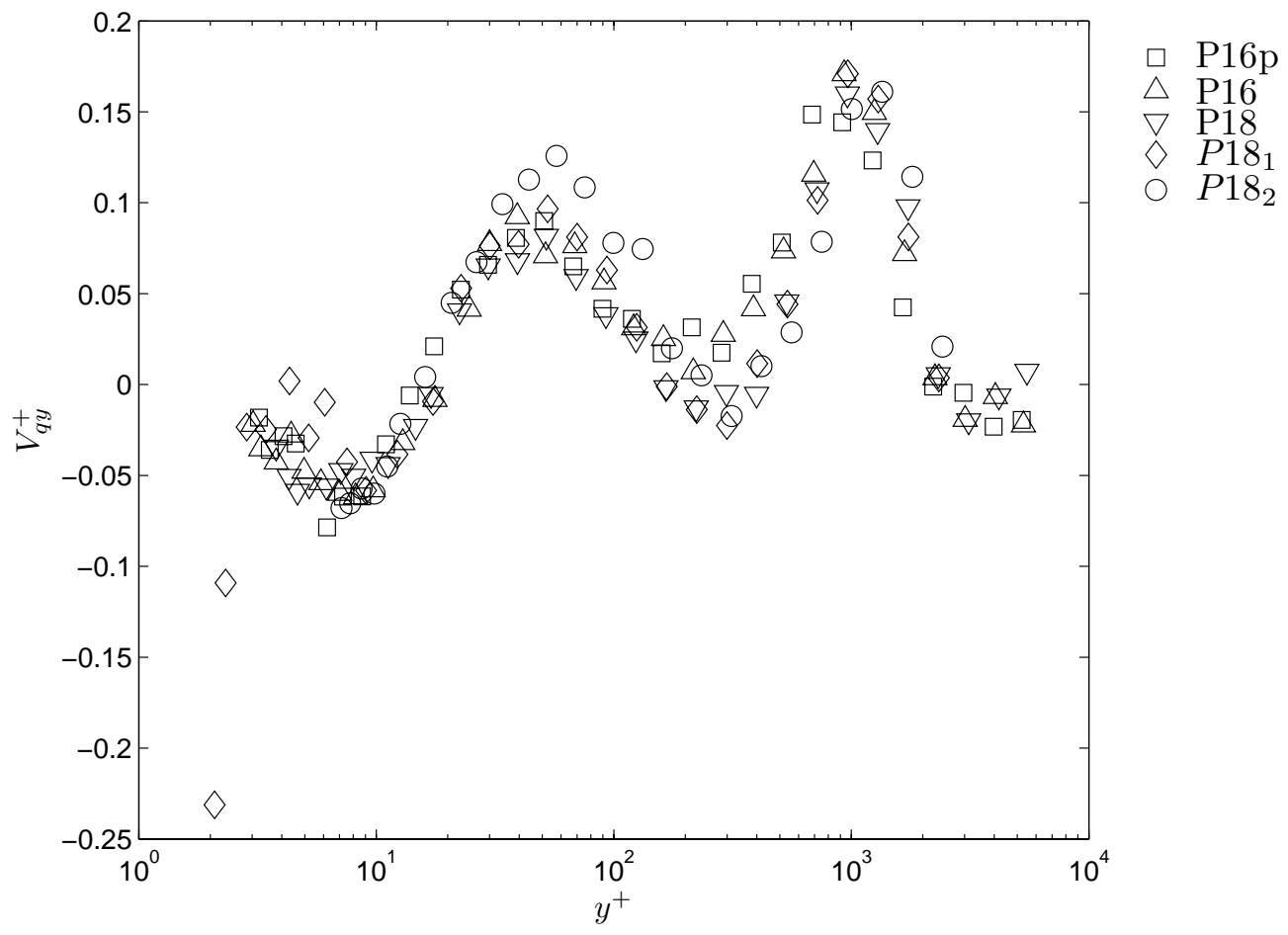

(j) Wall-Normal Turbulent Diffusion Velocity component, $V_{q y}$, normalized on $u_{\tau}$

Figure 4.5: Continued, $P 18$ Cluster 


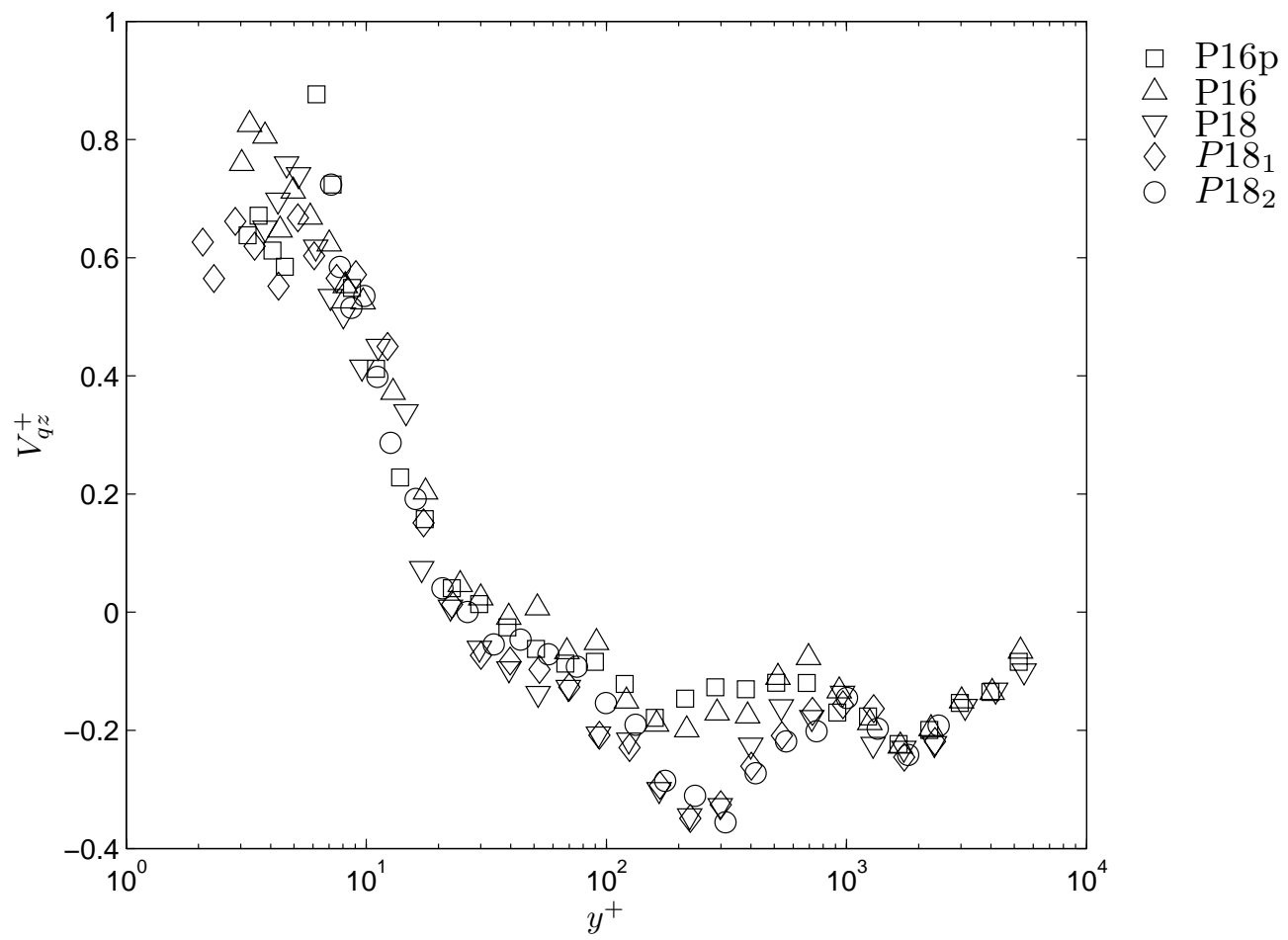

(k) Span-wise Turbulent Diffusion Velocity component, $V_{q z}$, normalized on $u_{\tau}$

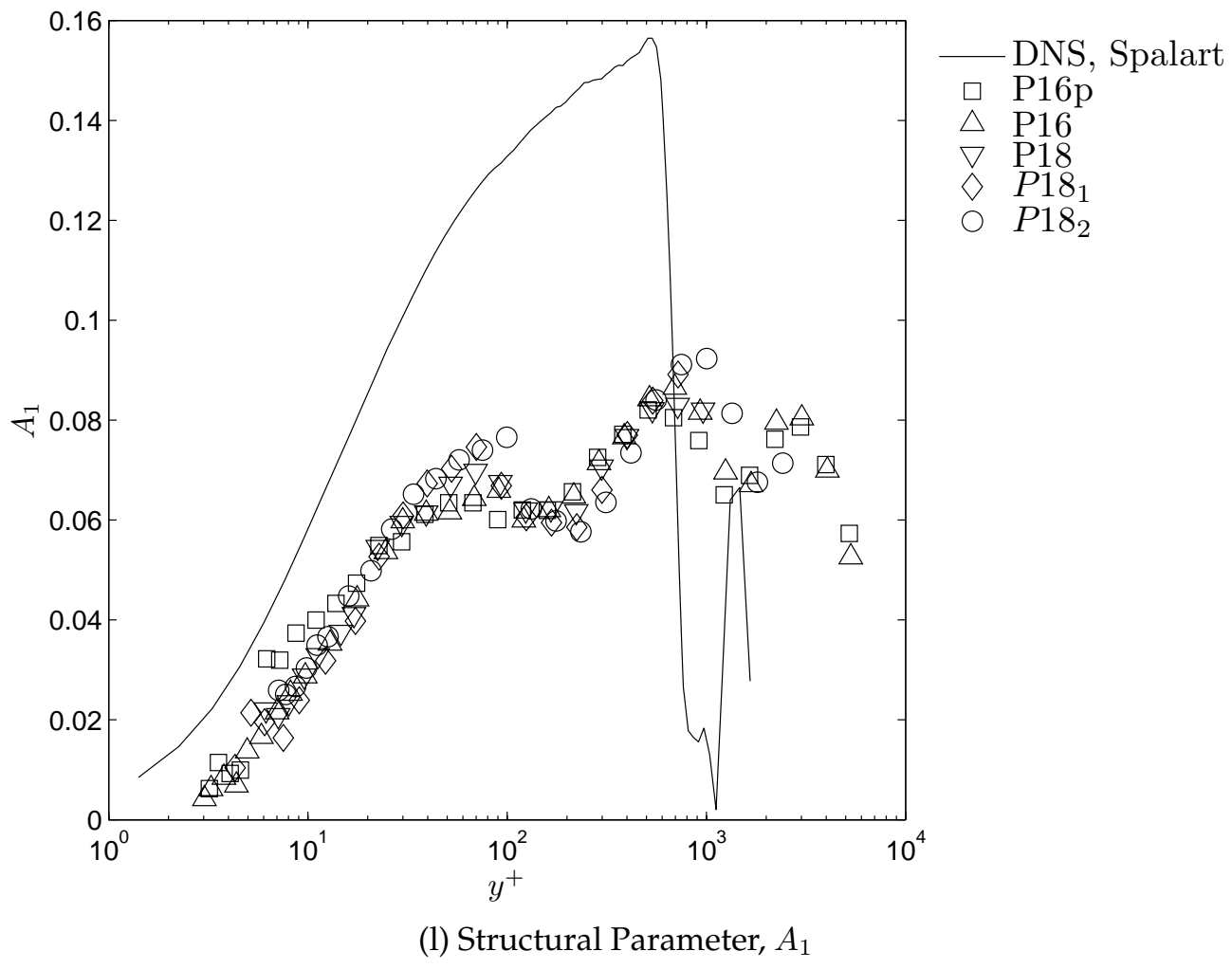

Figure 4.5: Continued, $P 18$ Cluster 


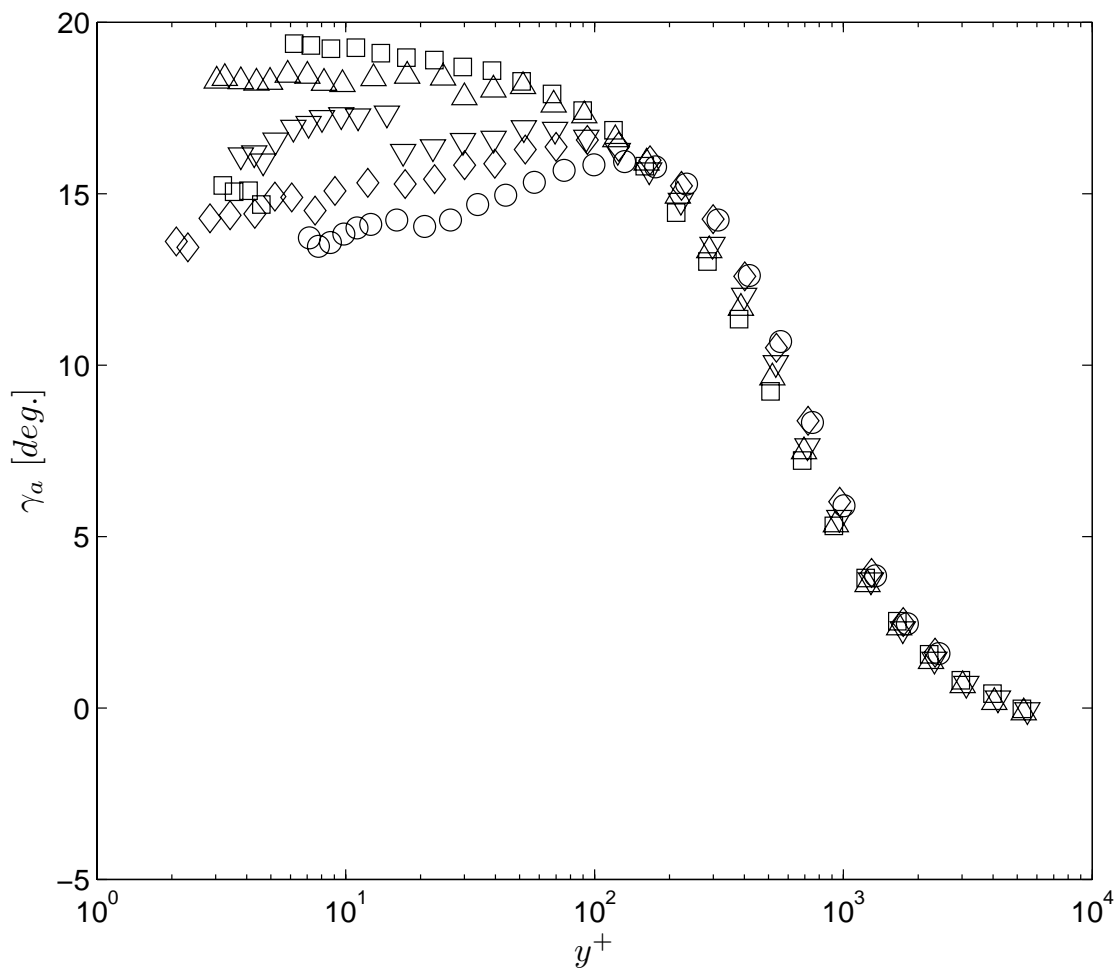

$\begin{array}{ll}\square & \text { P16p } \\ \triangle & \text { P16 } \\ \nabla & \text { P18 } \\ \diamond & P 18_{1} \\ \bigcirc & P 18_{2}\end{array}$

(m) Flow Angle, $\gamma_{a}$

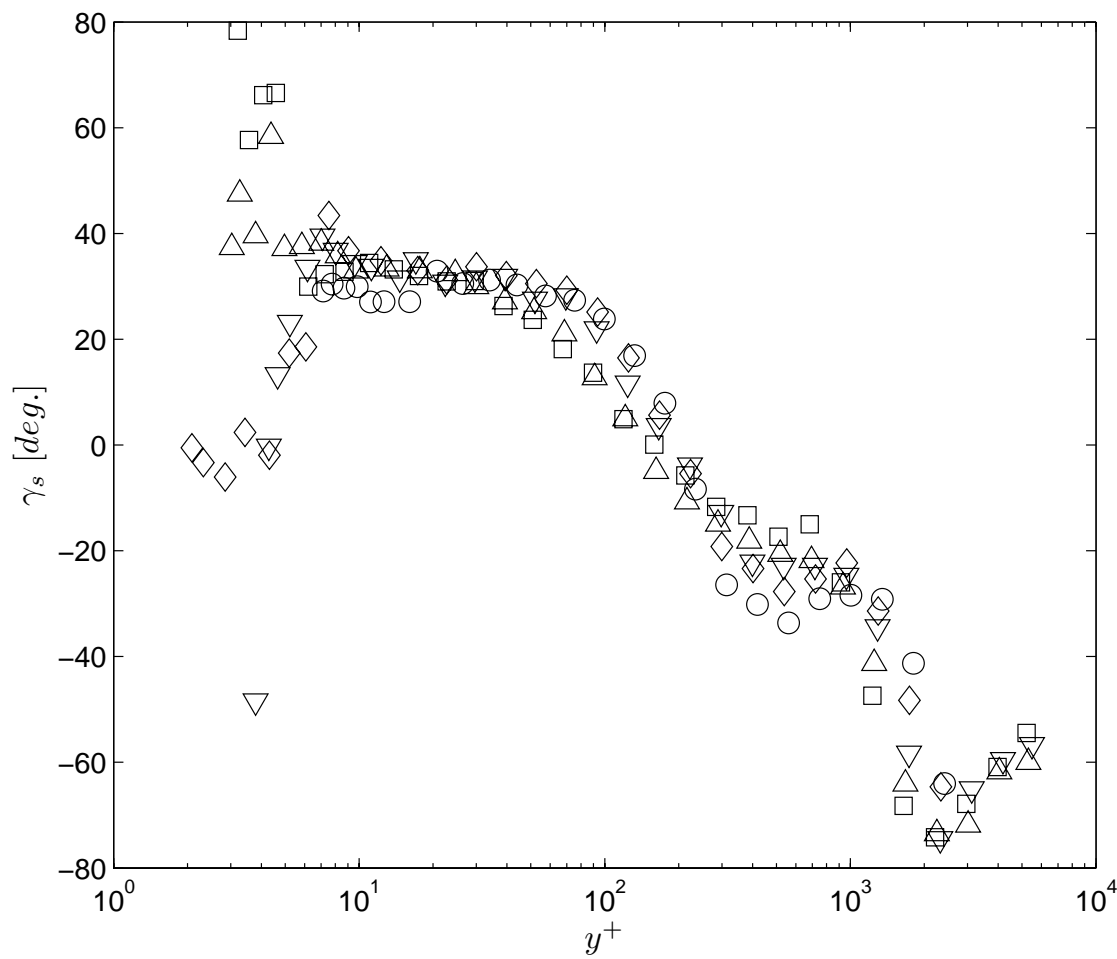

$\begin{array}{ll}\square & \mathrm{P} 16 \mathrm{p} \\ \triangle & \mathrm{P} 16 \\ \nabla & \mathrm{P} 18 \\ \diamond & P 18_{1} \\ \bigcirc & P 18_{2}\end{array}$

(n) Shear Stress Angle $\gamma_{s}$

Figure 4.5: Continued, $P 18$ Cluster 


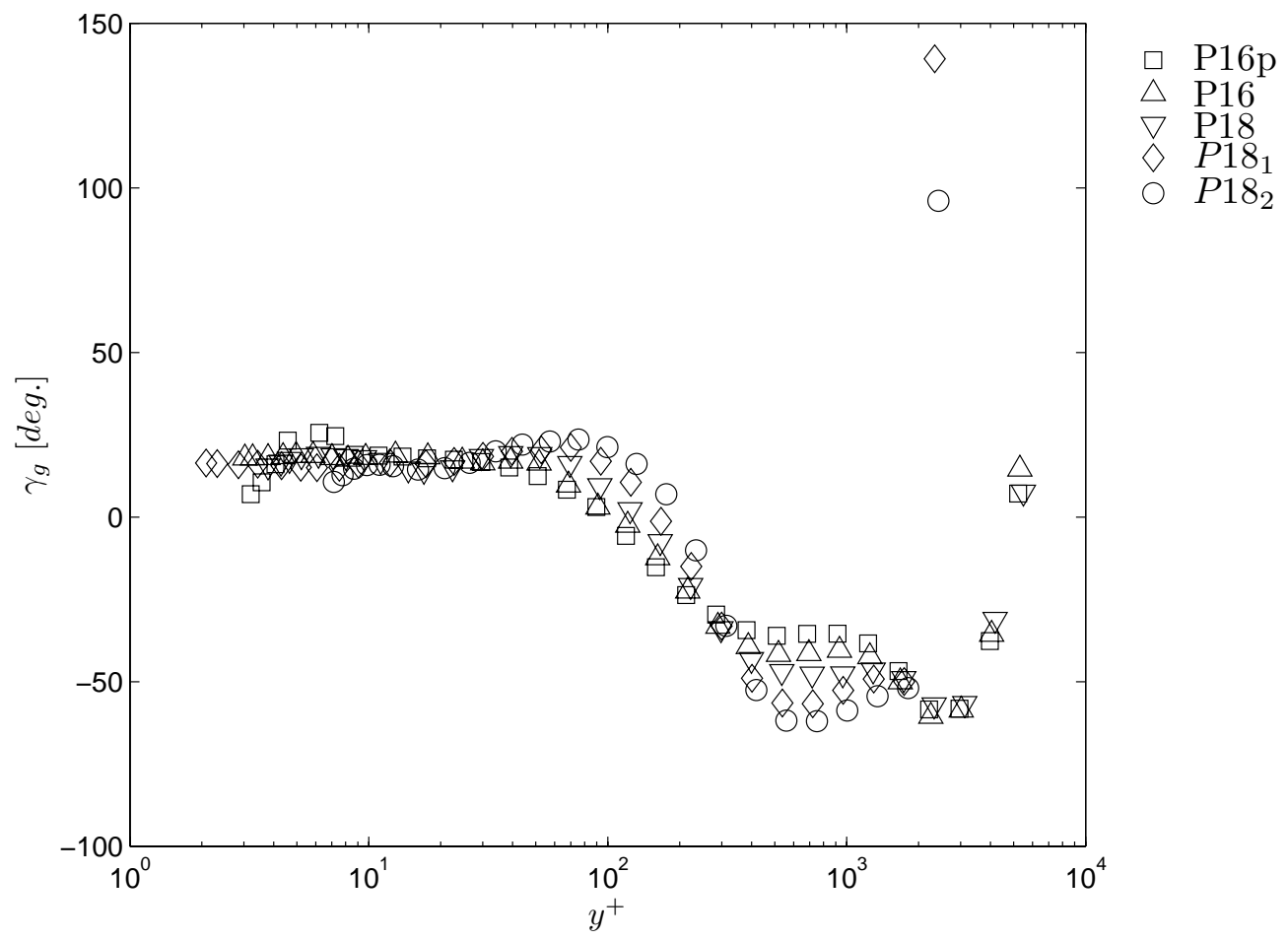

(o) Flow Gradient Angle $\gamma_{g}$

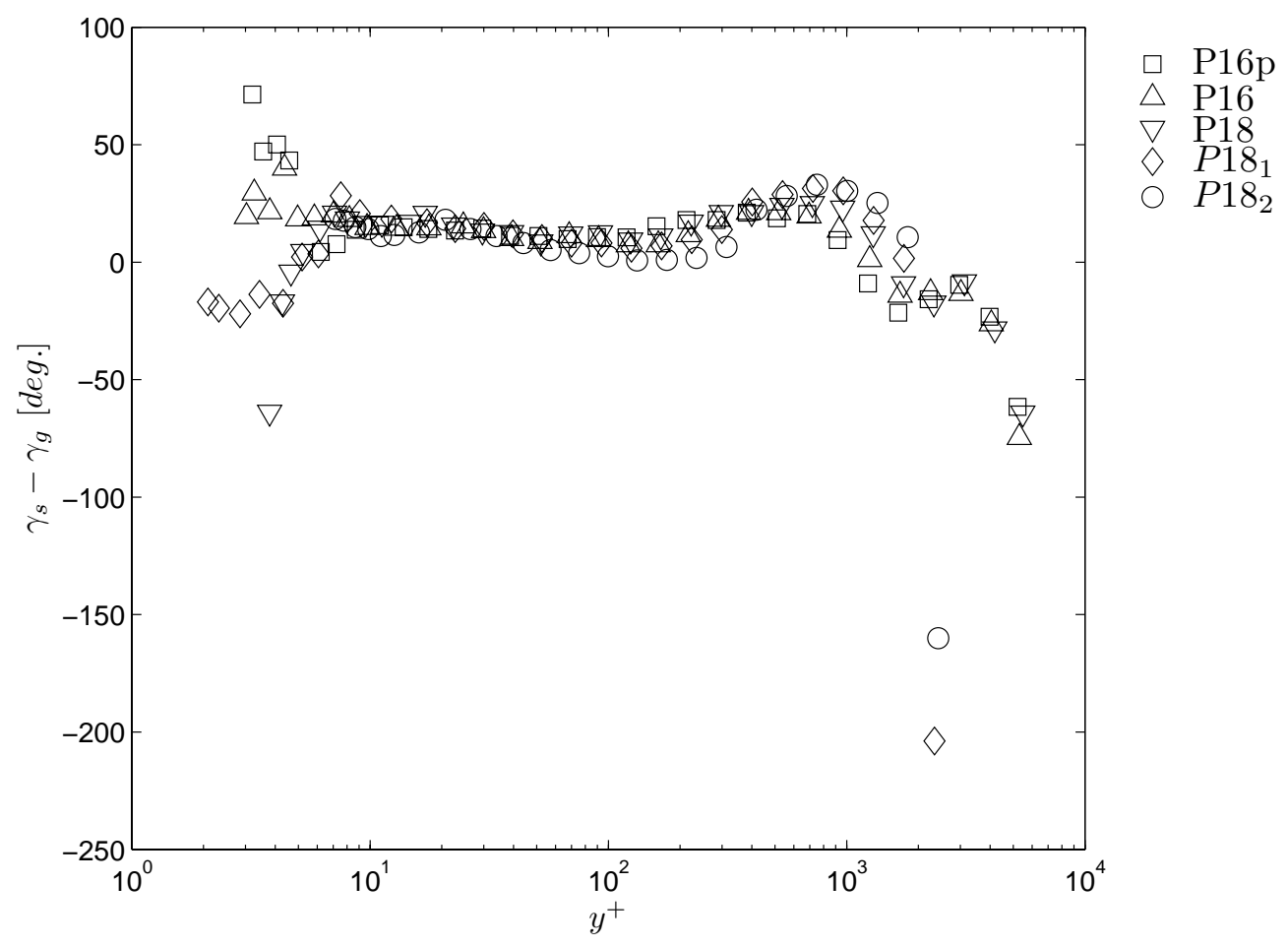

(p) Difference between Shear Angle and Flow Gradient Angles, $\gamma_{s}-\gamma_{g}$

Figure 4.5: Continued, $P 18$ Cluster 


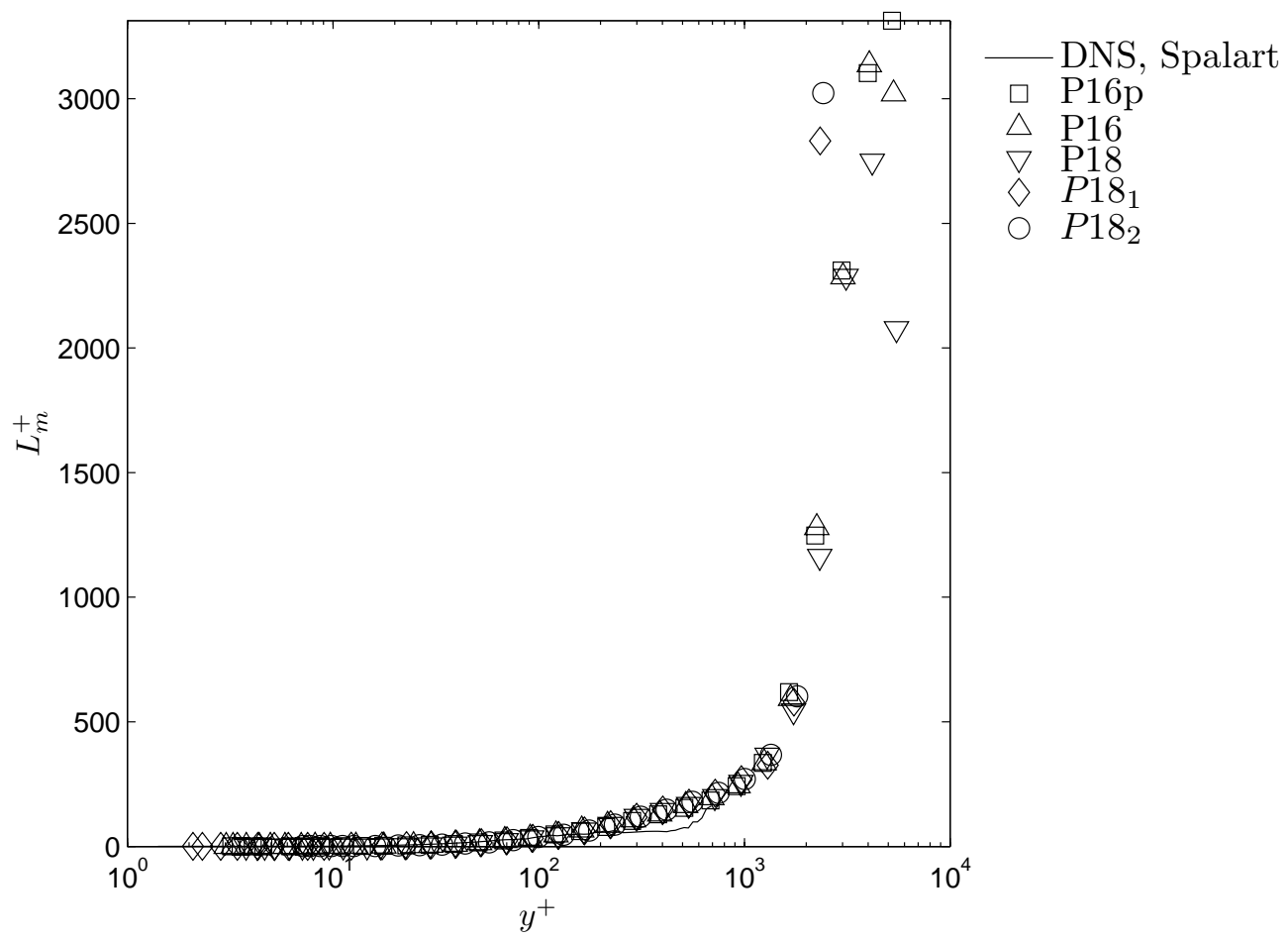

(q) Mixing Length $L_{m}^{+}$

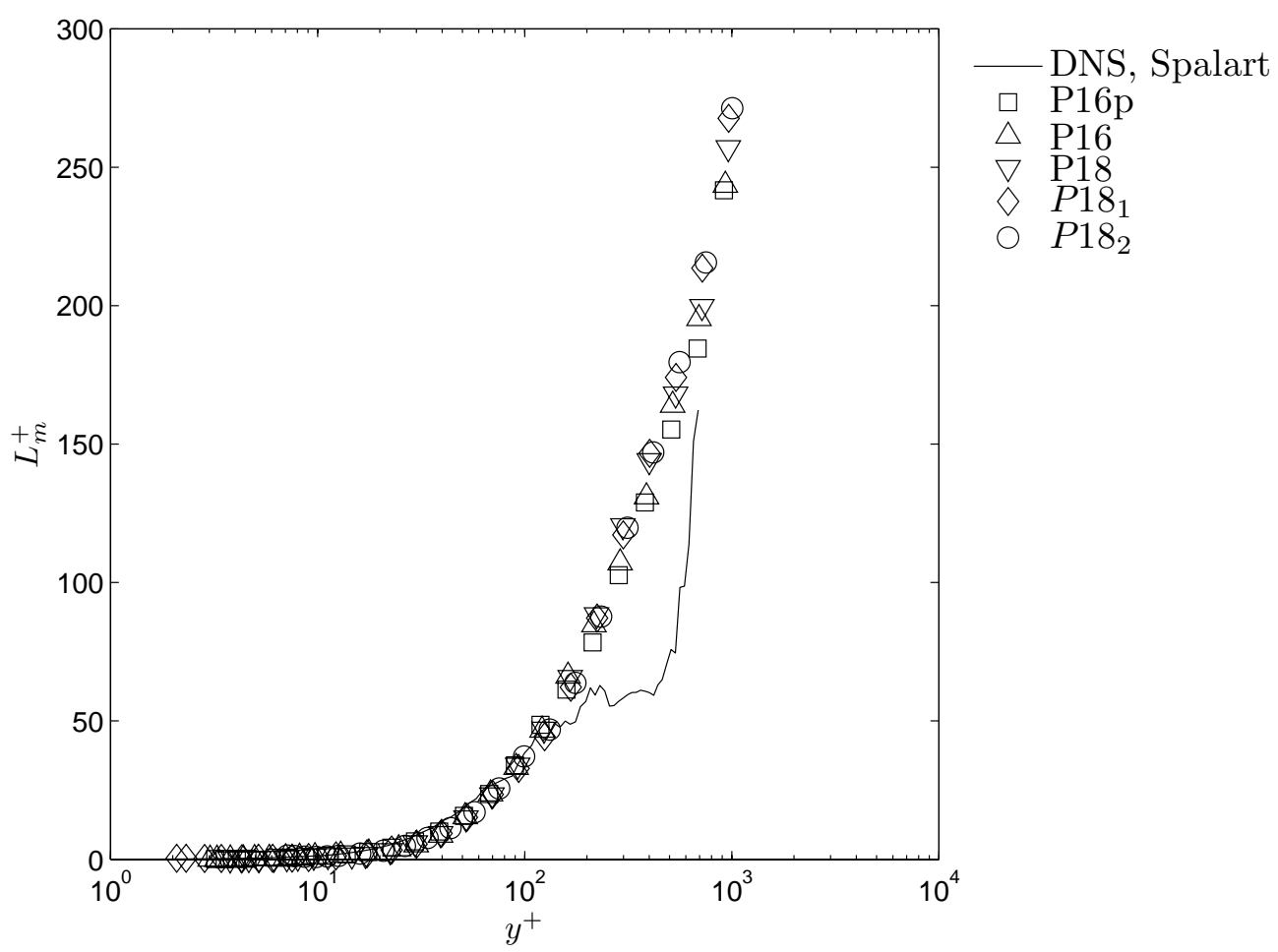

(r) Near Wall Mixing Length $L_{m}^{+}$

Figure 4.5: Continued, $P 18$ Cluster 


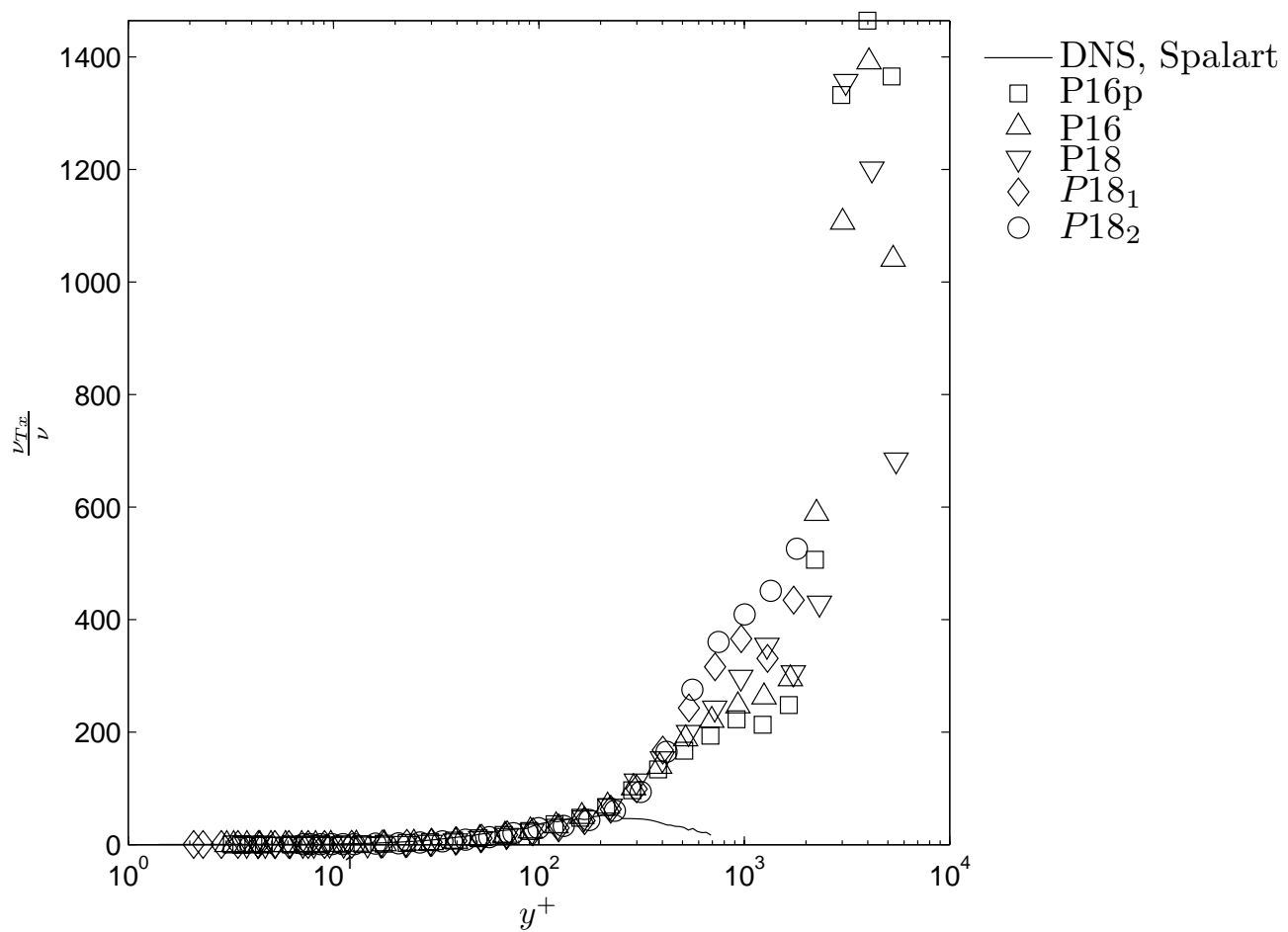

(s) Turbulent Eddy Viscosity $\nu_{T x}$

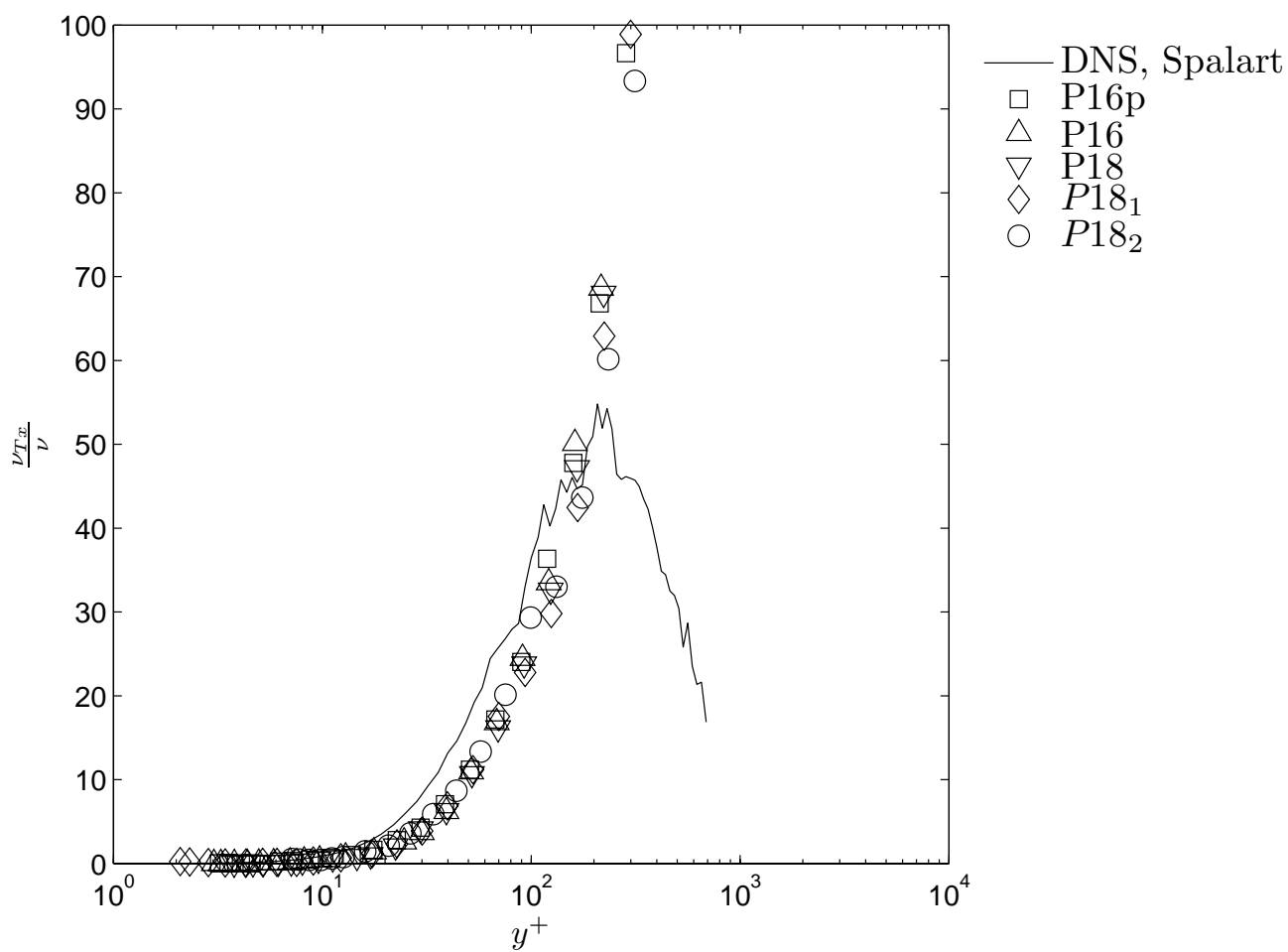

(t) Near Wall Turbulent Eddy Viscosity $\nu_{T x}$

Figure 4.5: Concluded, $P 18$ Cluster 


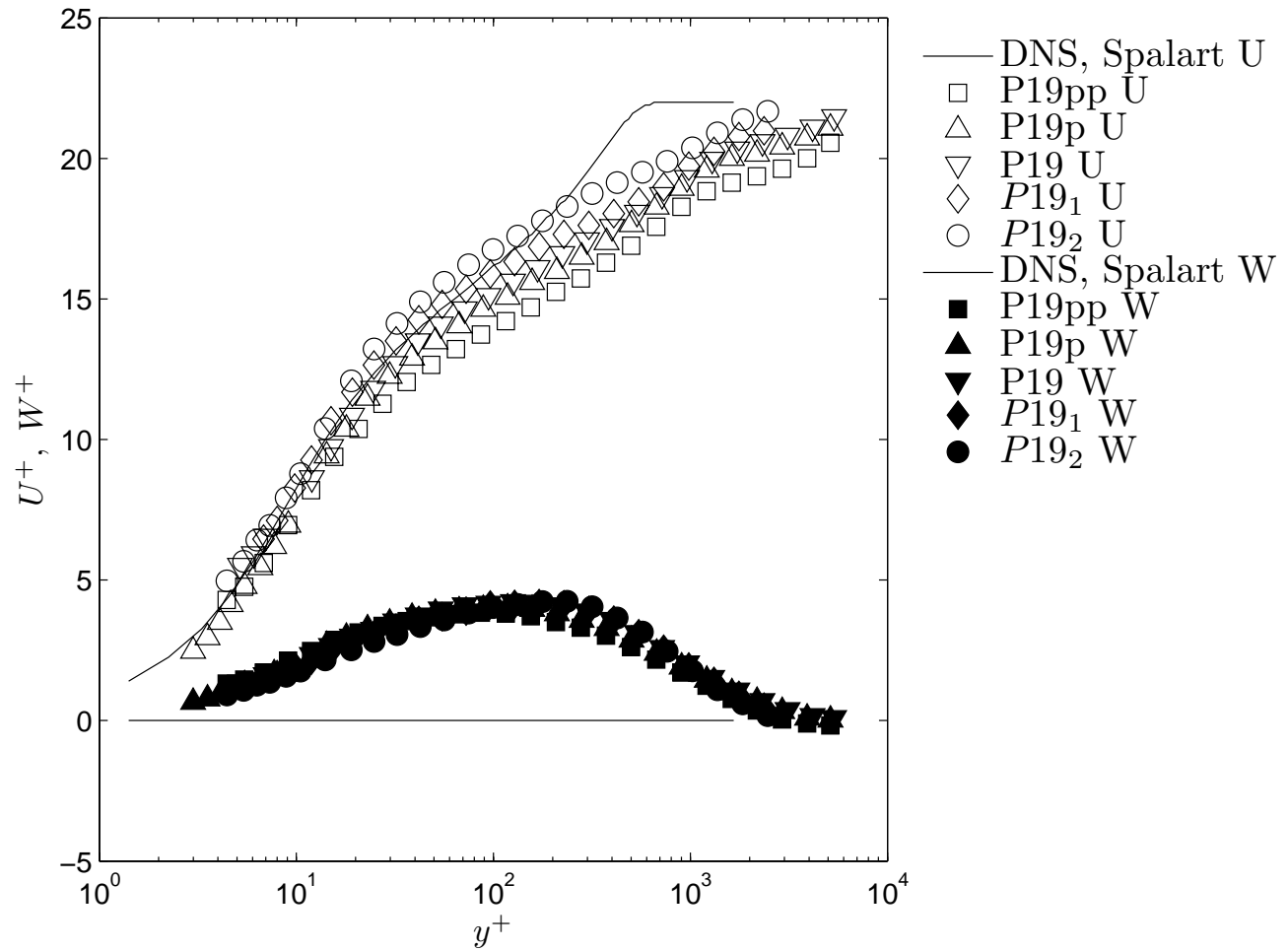

(a) Mean Stream-wise and Span-wise Velocities, $U^{+}, W^{+}$

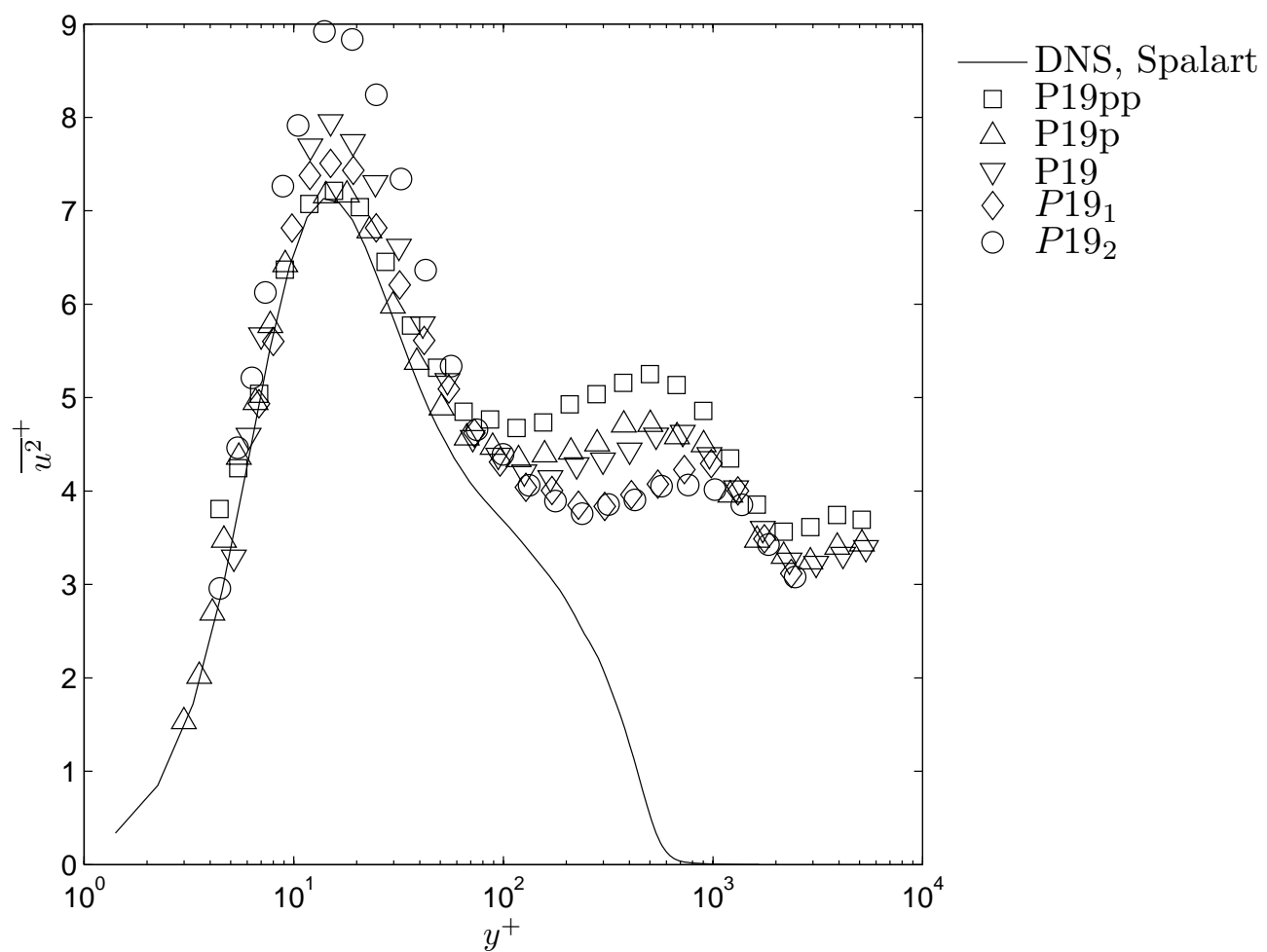

(b) Stream-wise Reynolds' Normal Stress, ${\overline{u^{2}}}^{+}$

Figure 4.6: P19 Cluster 


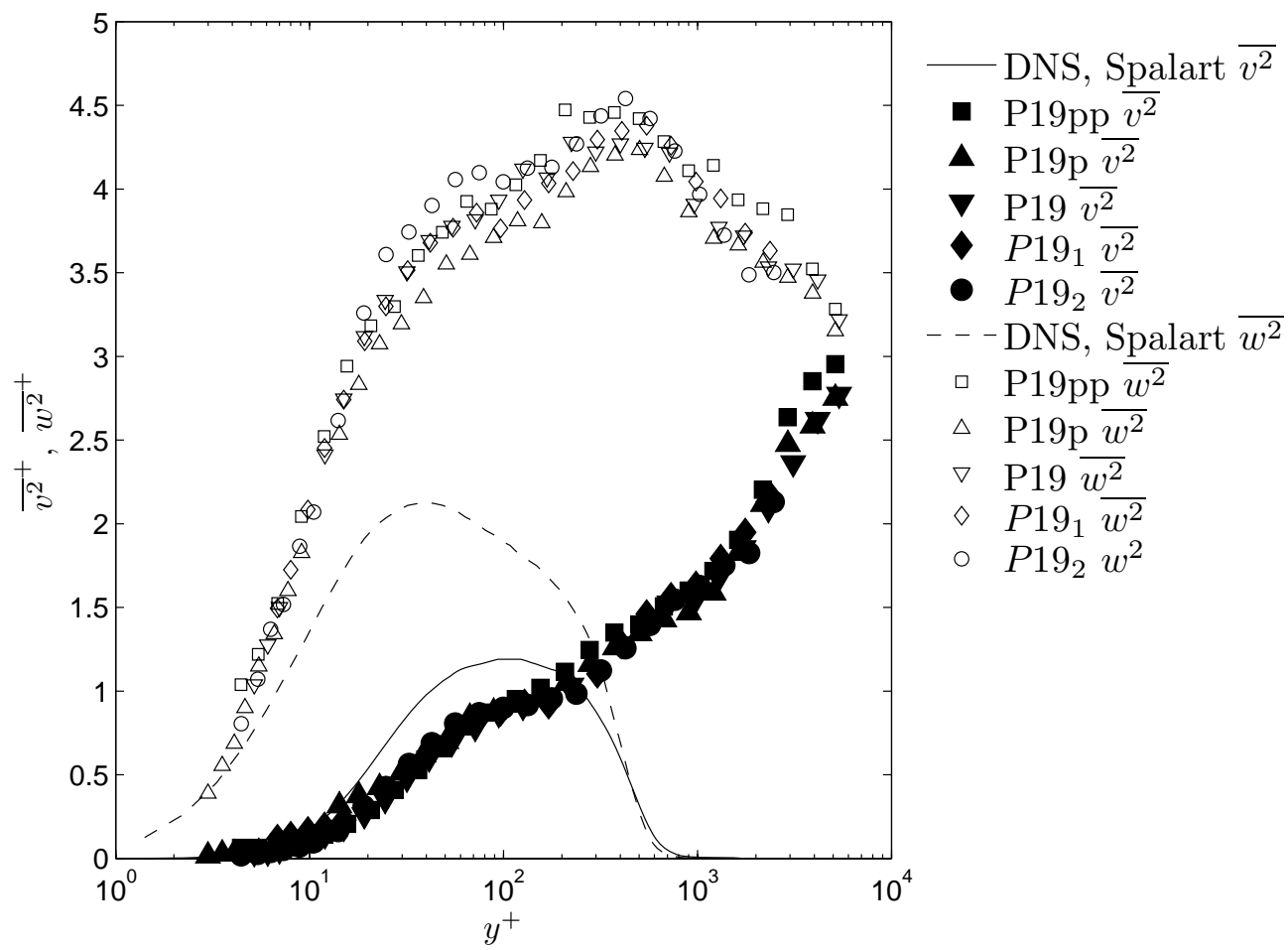

(c) Longitudinal and Span-wise Normal Reynolds' Stresses, ${\overline{v^{2}}}^{+}$and ${\overline{w^{2}}}^{+}$

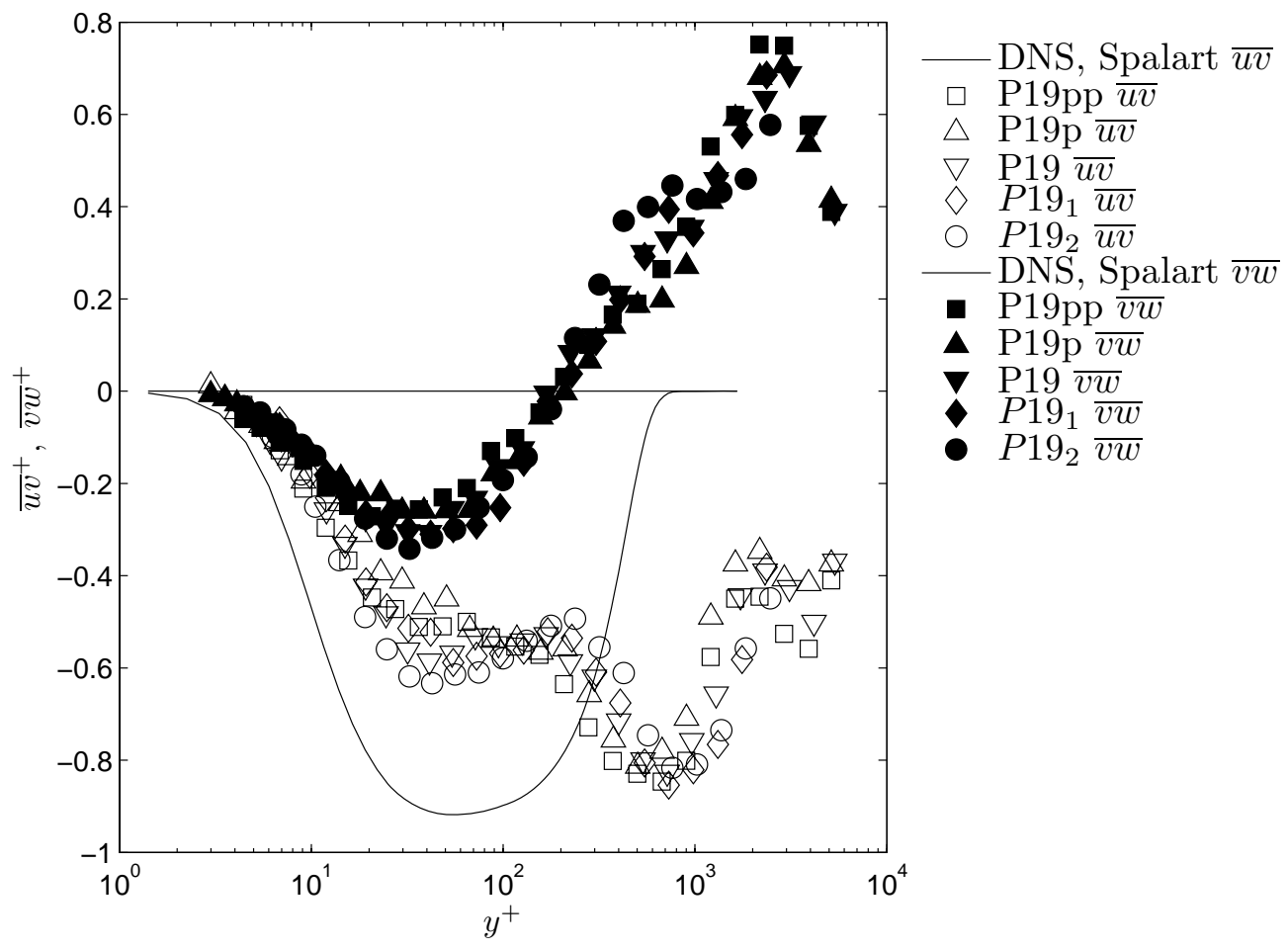

(d) Reynolds' Shear Stresses, $\overline{u v}^{+}$and $\overline{v w}{ }^{+}$

Figure 4.6: Continued, P19 Cluster 


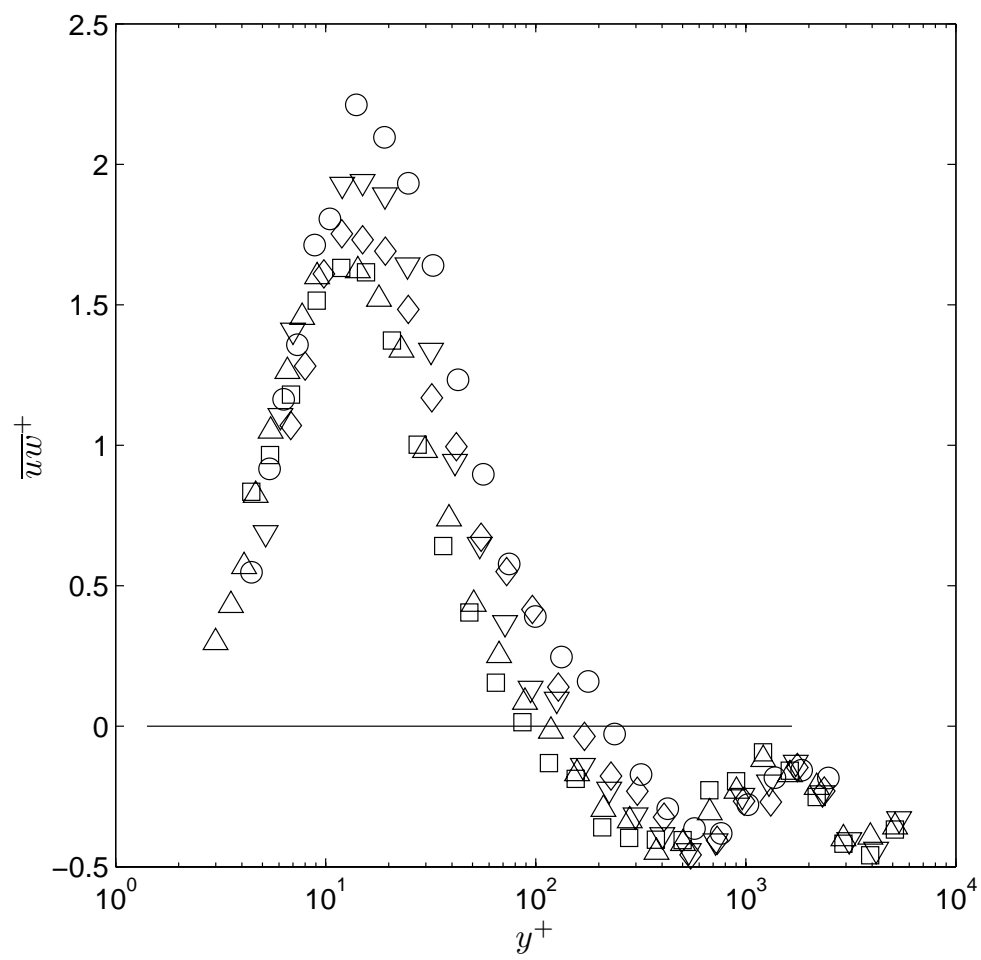

$\begin{array}{ll} & \text { DNS, Spalart } \\ \square & \text { P19pp } \\ \triangle & \text { P19p } \\ \nabla & \text { P19 } \\ \diamond & P 19_{1} \\ \bigcirc & P 19_{2}\end{array}$

(e) Reynolds' Shear Stress, $\overline{u w}^{+}$

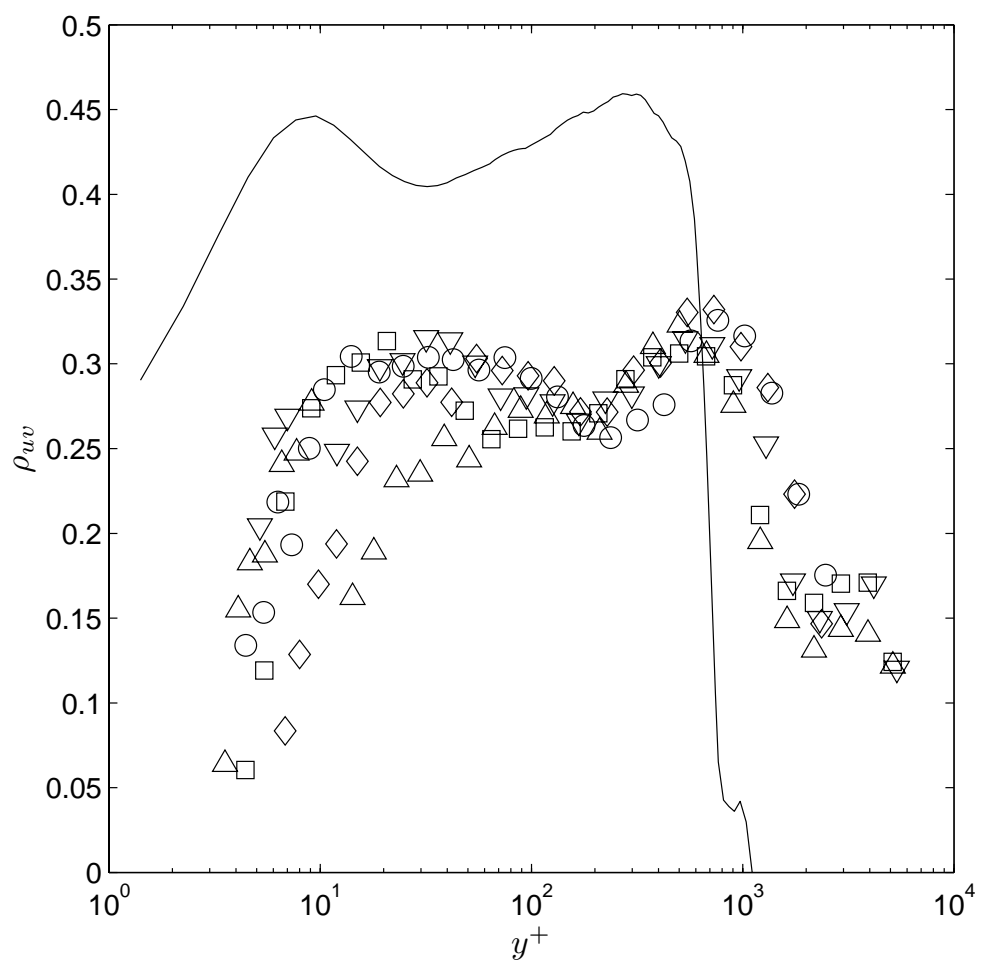

DNS, Spalart

$\square$ P19pp

$\triangle \mathrm{P} 19 \mathrm{p}$

$\nabla \mathrm{P} 19$

$\diamond P 19_{1}$

○ $P 19_{2}$

(f) Correlation Coefficient $\rho_{u v}$

Figure 4.6: Continued, P19 Cluster 


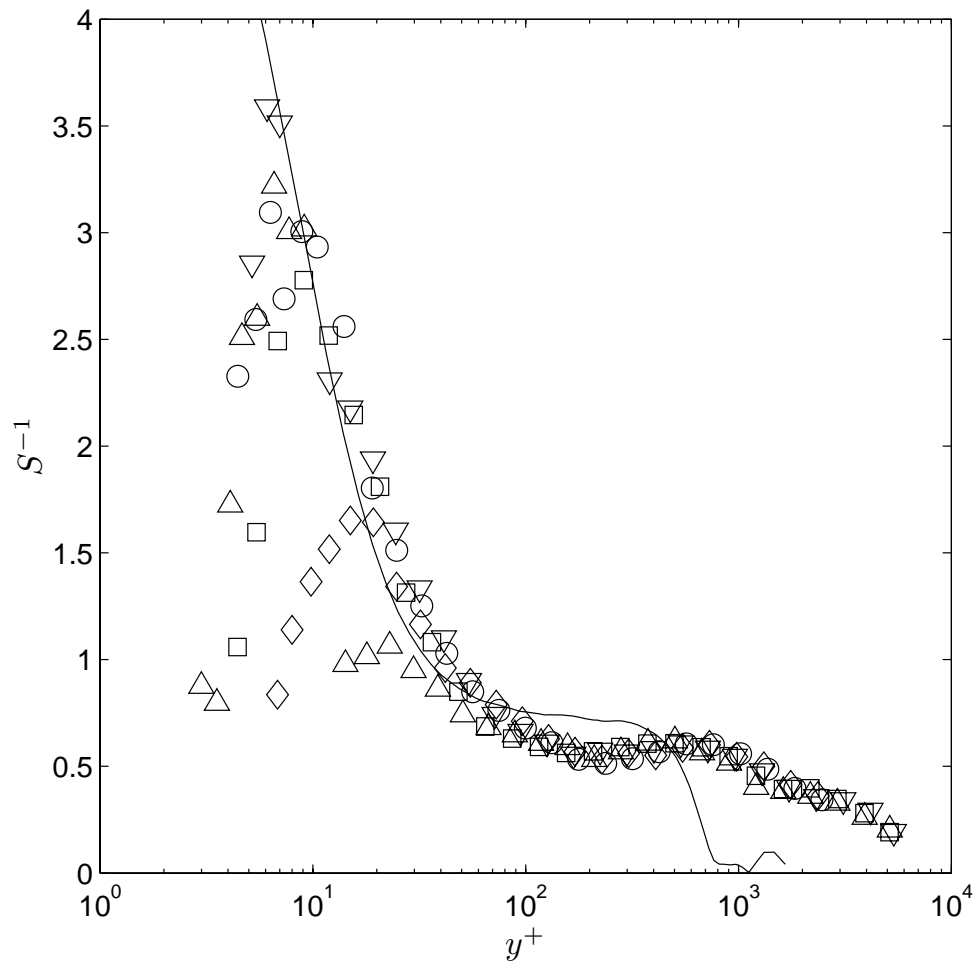

$\begin{array}{ll} & \text { DNS, Spalart } \\ \square & \text { P19pp } \\ \triangle & \text { P19p } \\ \nabla & \text { P19 } \\ \diamond & P 19_{1} \\ \bigcirc & P 19_{2}\end{array}$

(g) $S^{-1}$ Parameter

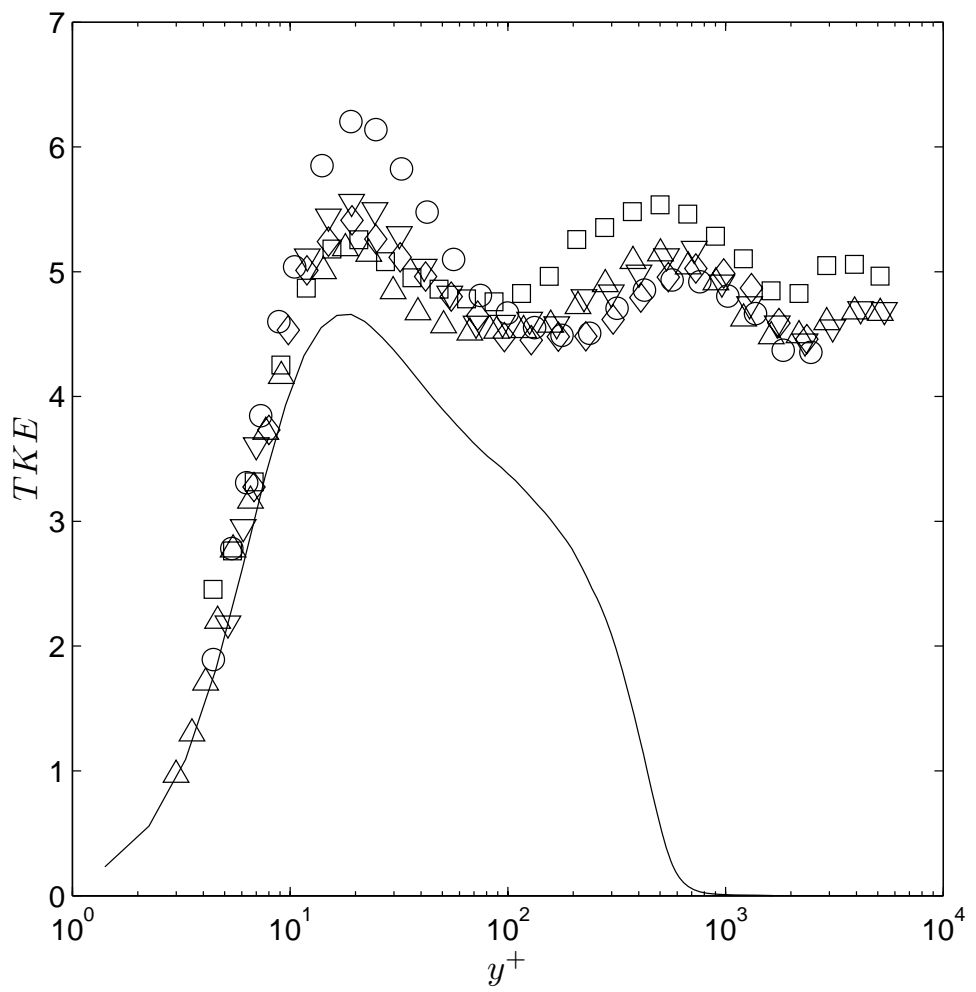

$\begin{array}{ll} & \text { DNS, Spalart } \\ \square & \text { P19pp } \\ \triangle & \text { P19p } \\ \nabla & \text { P19 } \\ \diamond & P 19_{1} \\ \bigcirc & P 19_{2}\end{array}$

(h) Turbulent Kinetic Energy, TKE

Figure 4.6: Continued, P19 Cluster 


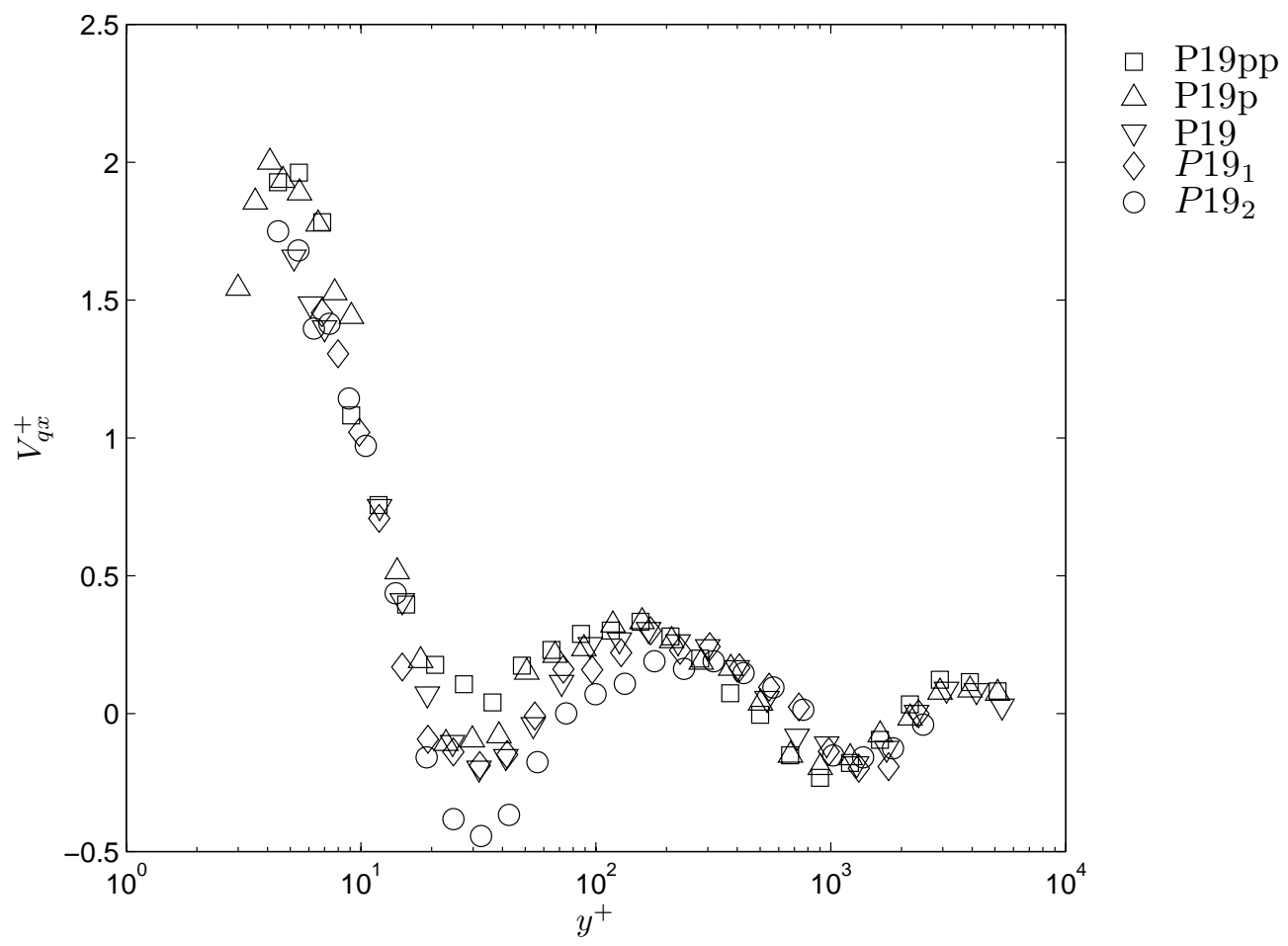

(i) Stream-wise Turbulent Diffusion Velocity component, $V_{q x}$, normalized on $u_{\tau}$

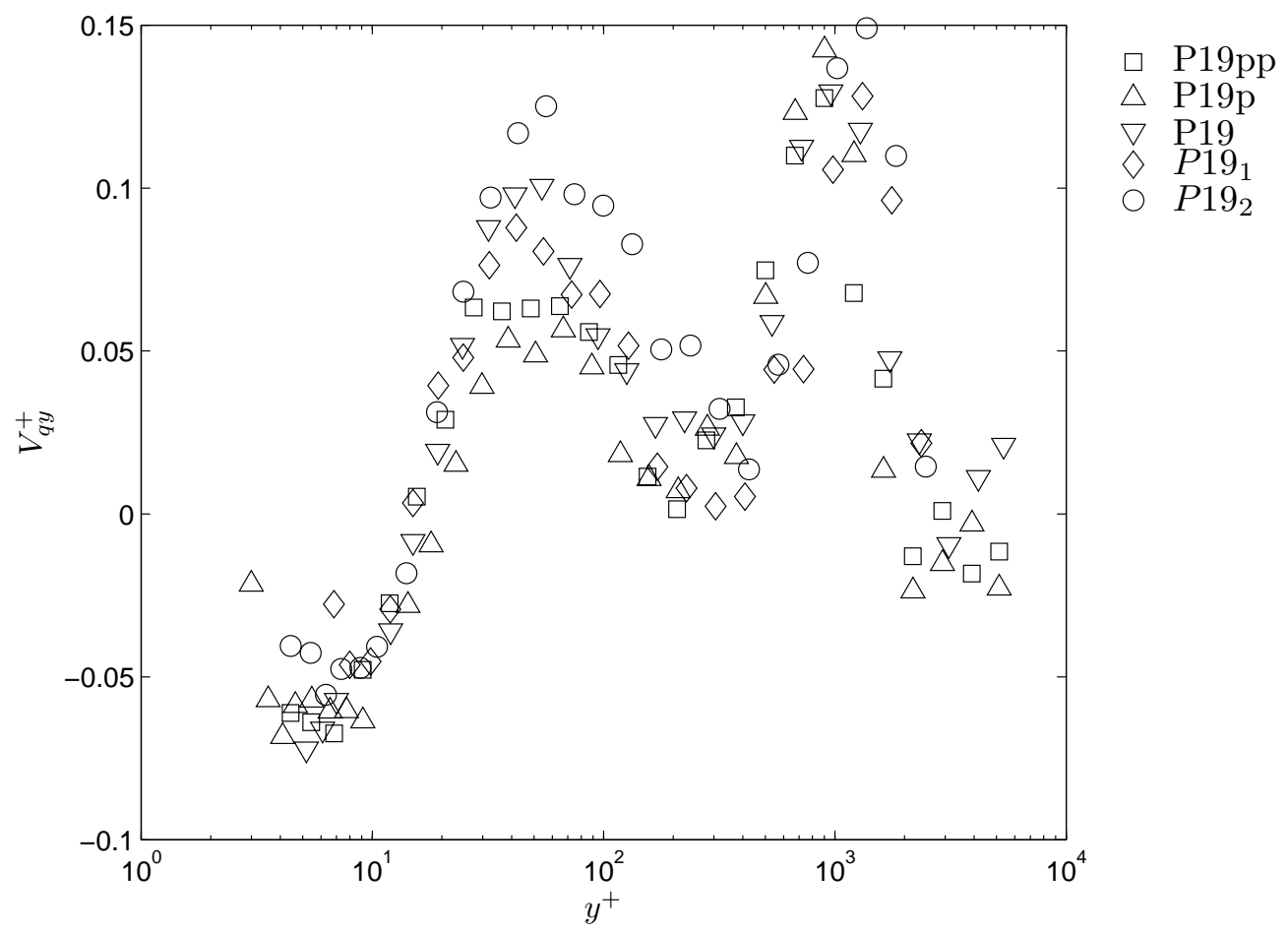

(j) Wall-Normal Turbulent Diffusion Velocity component, $V_{q y}$, normalized on $u_{\tau}$

Figure 4.6: Continued, P19 Cluster 


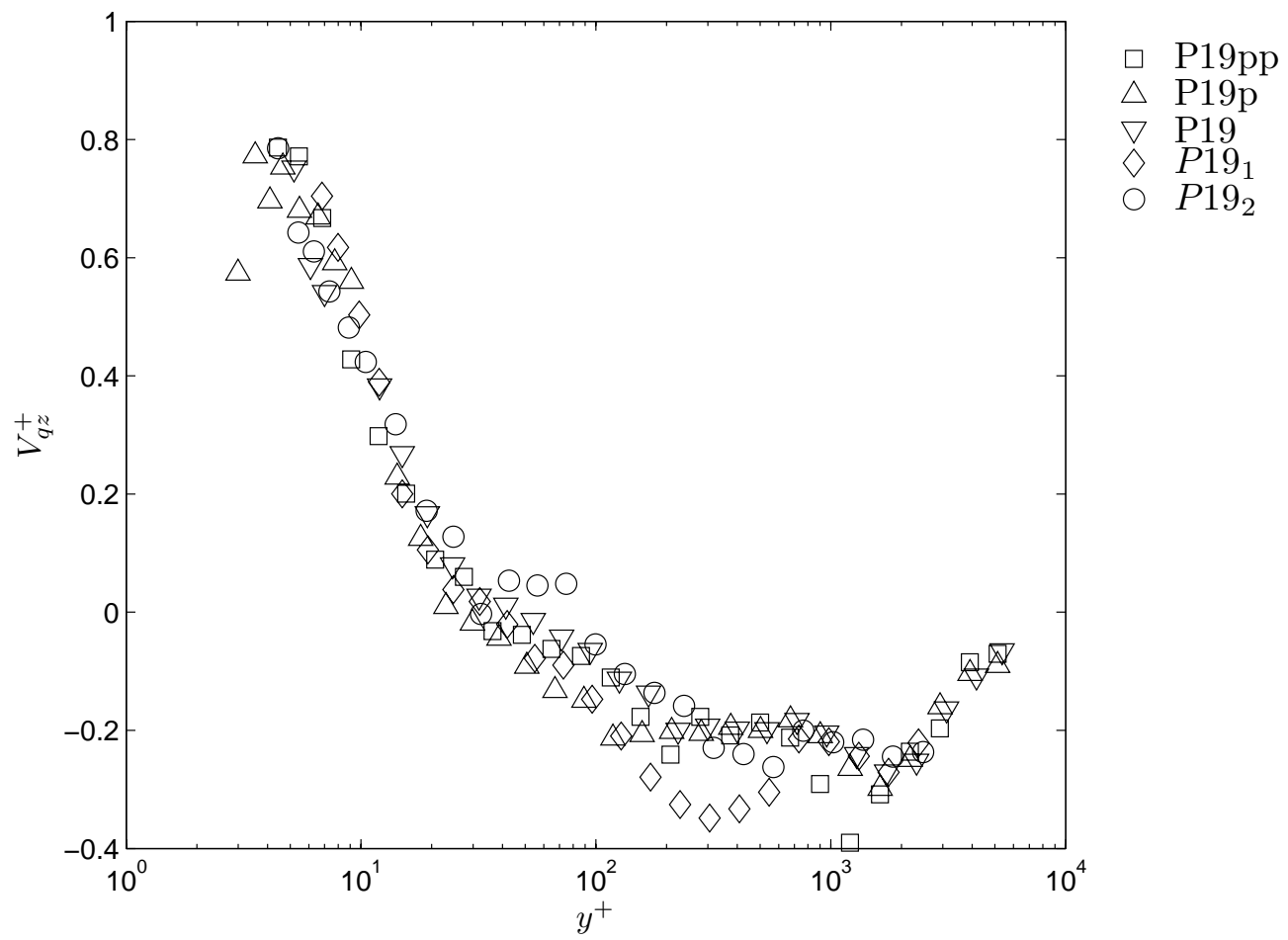

(k) Span-wise Turbulent Diffusion Velocity component, $V_{q z}$, normalized on $u_{\tau}$

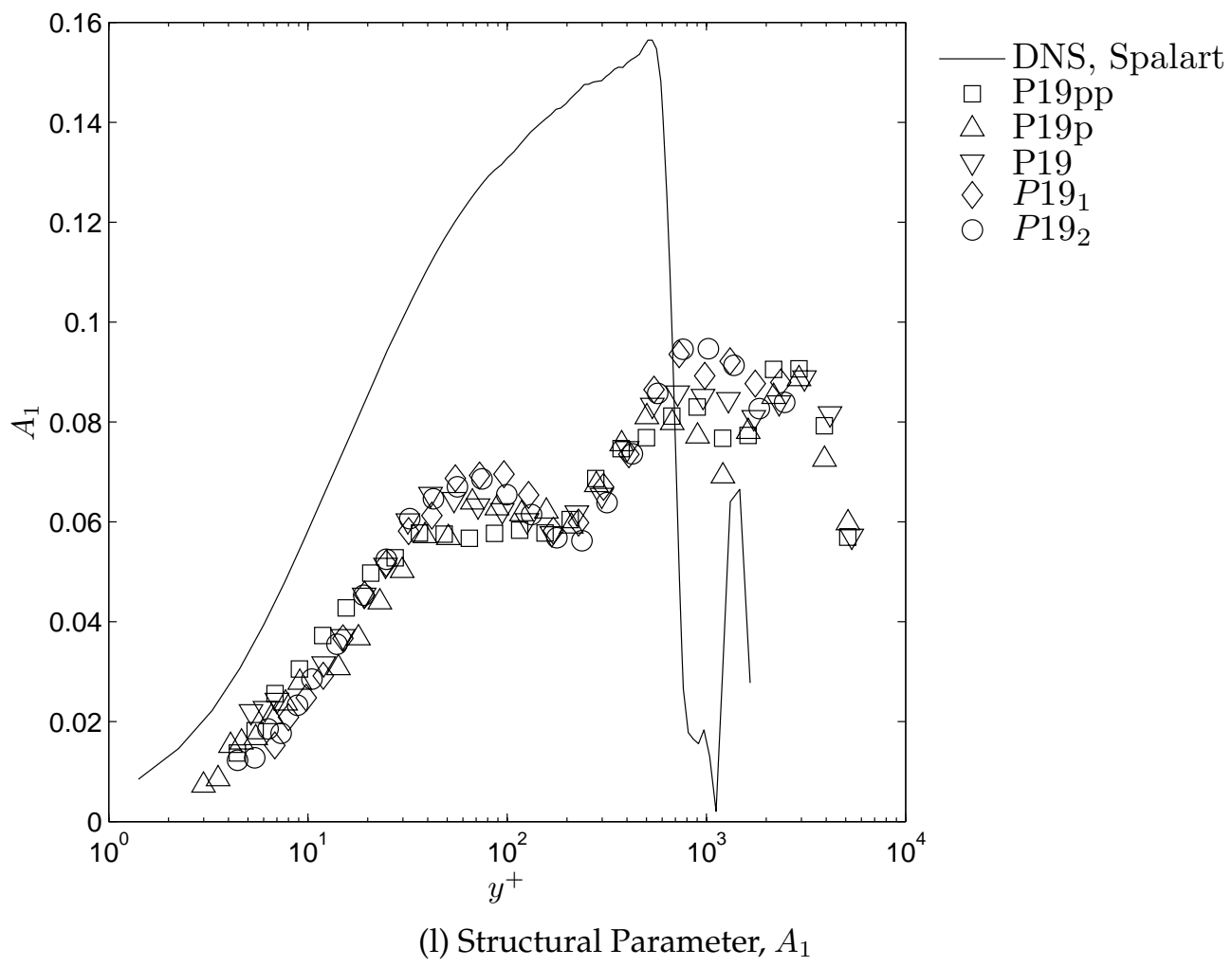

Figure 4.6: Continued, $P 19$ Cluster 


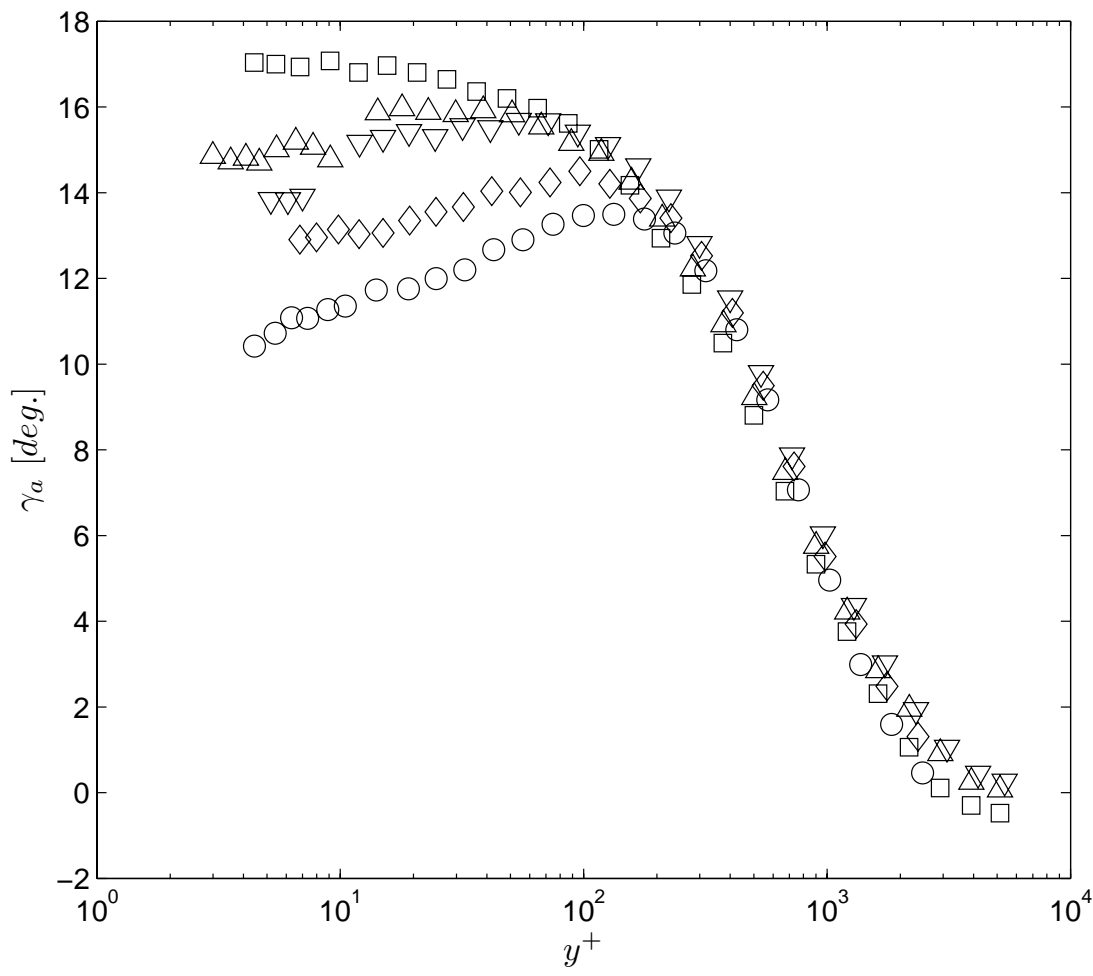

$\square \quad$ P19pp

$\triangle \mathrm{P} 19 \mathrm{p}$

$\nabla \quad \mathrm{P} 19$

$\diamond P 19_{1}$

○ $P 19_{2}$

(m) Flow Angle, $\gamma_{a}$

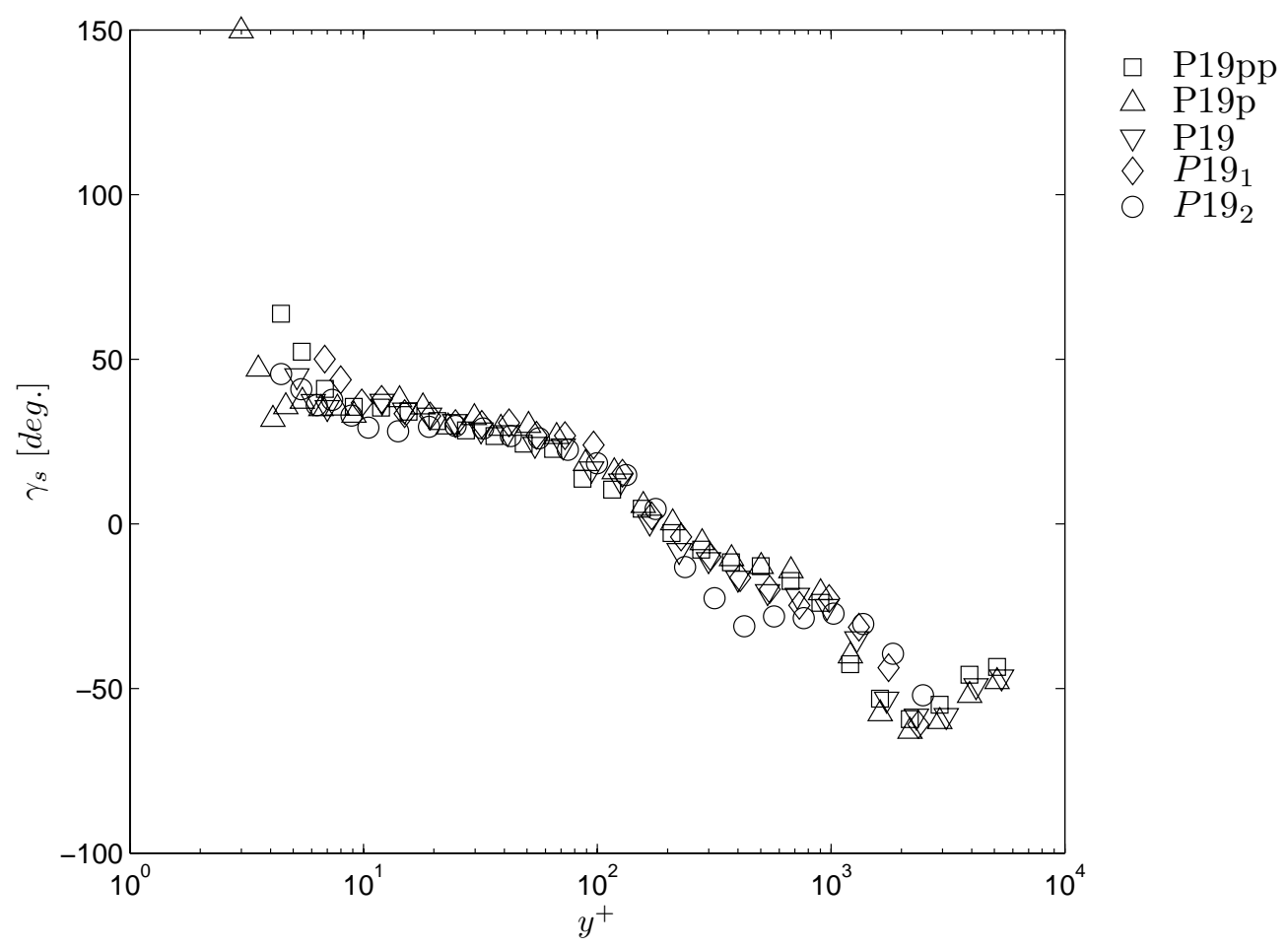

(n) Shear Stress Angle $\gamma_{s}$

Figure 4.6: Continued, P19 Cluster 


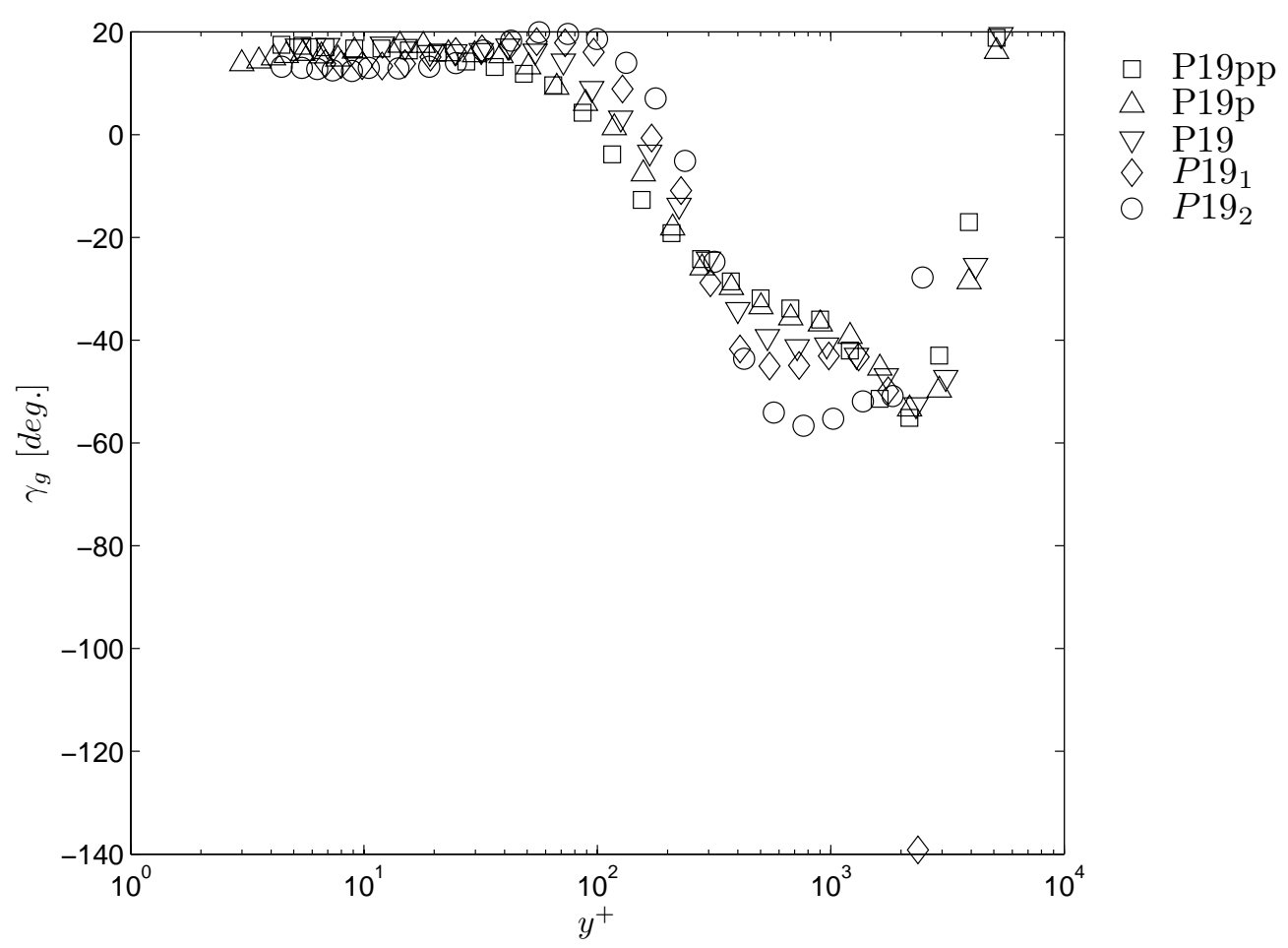

(o) Flow Gradient Angle $\gamma_{g}$

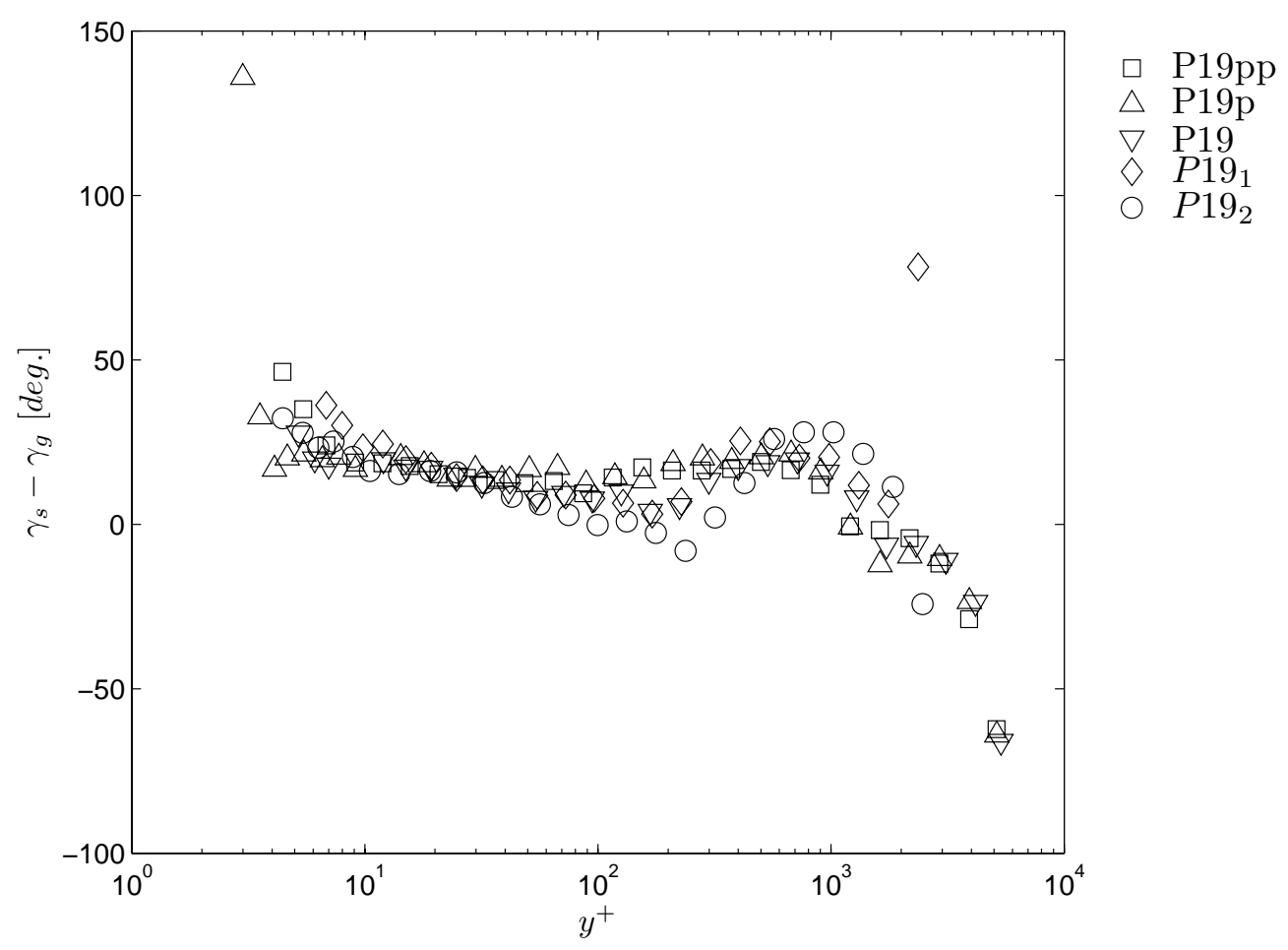

(p) Difference between Shear Angle and Flow Gradient Angles, $\gamma_{s}-\gamma_{g}$

Figure 4.6: Continued, P19 Cluster 


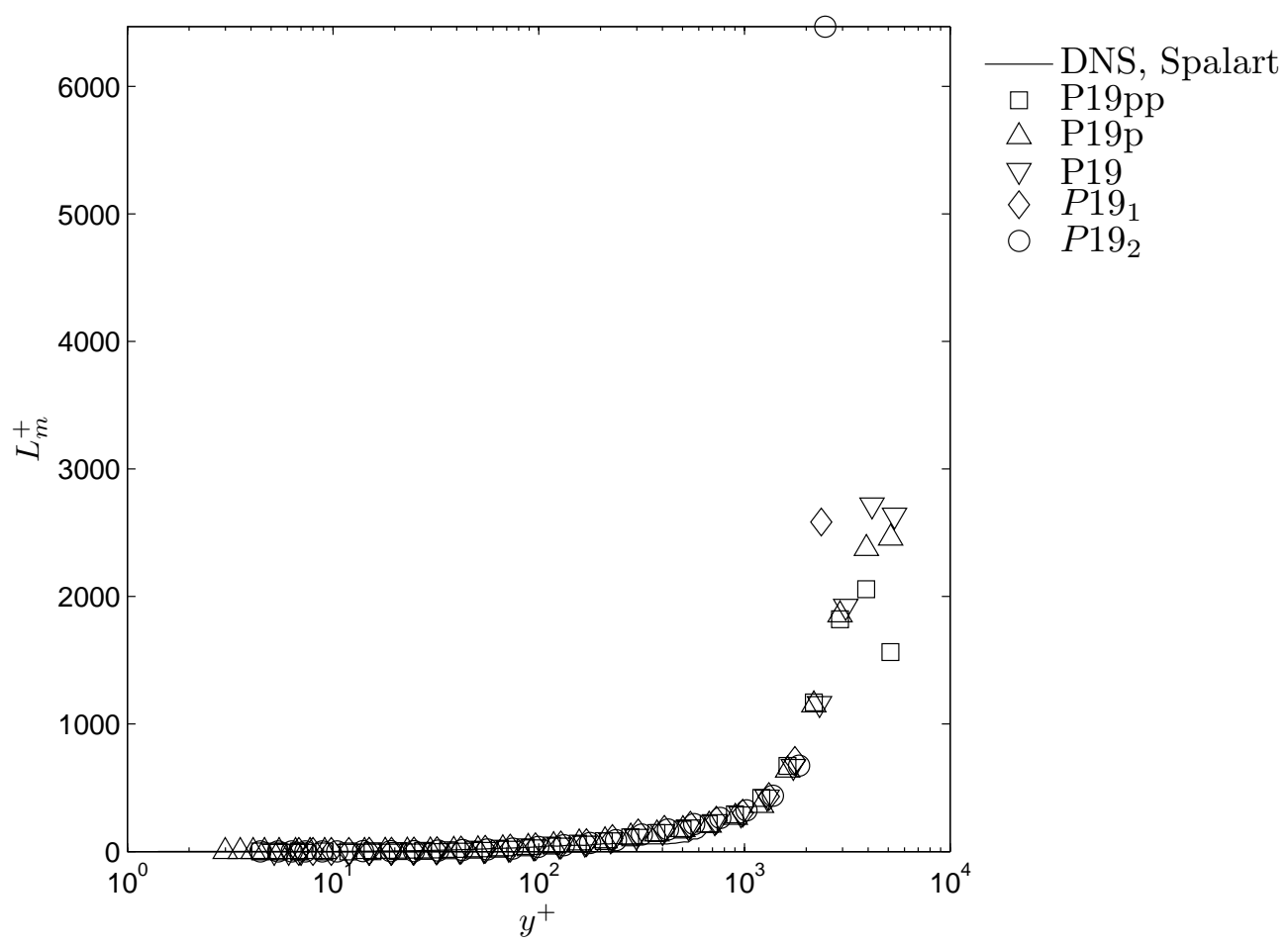

(q) Mixing Length $L_{m}^{+}$

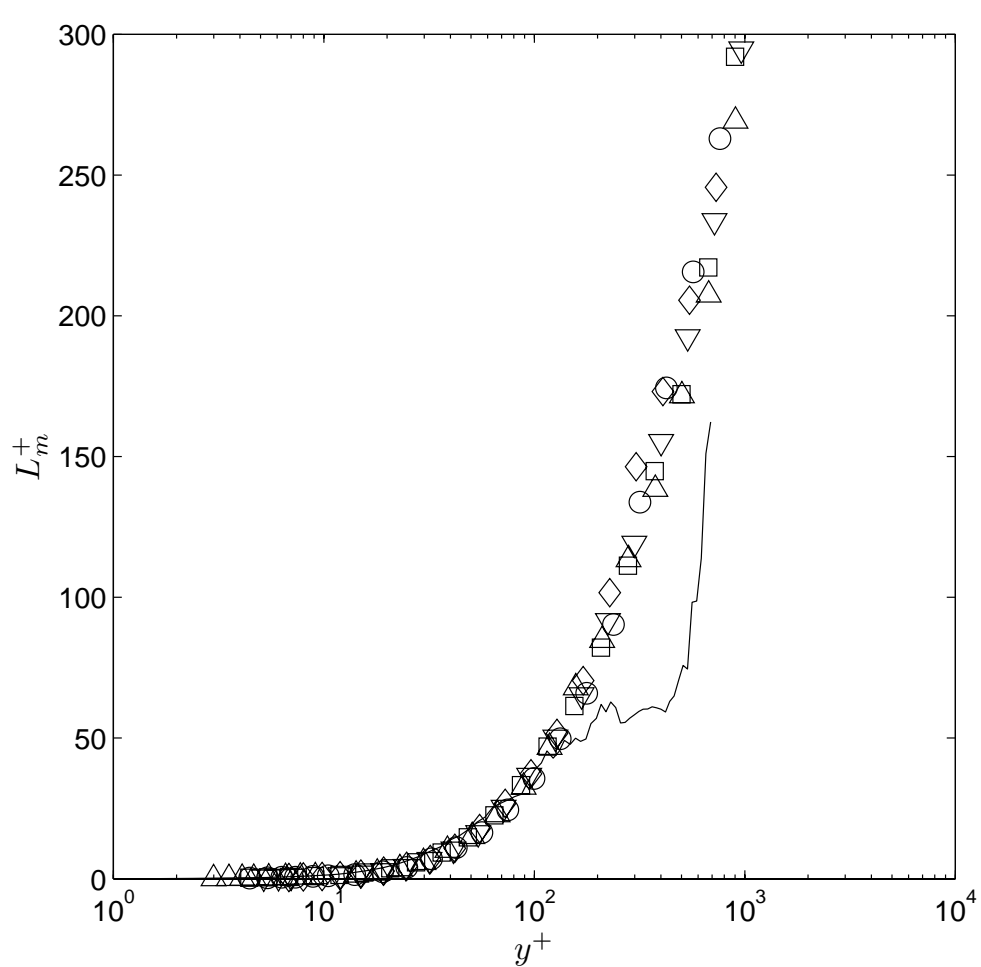

DNS, Spalart

$\square$ P19pp

$\triangle$ P19p

$\nabla \mathrm{P} 19$

$\diamond P 19_{1}$

○ $\mathrm{P} 19_{2}$

(r) Near Wall Mixing Length $L_{m}^{+}$

Figure 4.6: Continued, P19 Cluster 


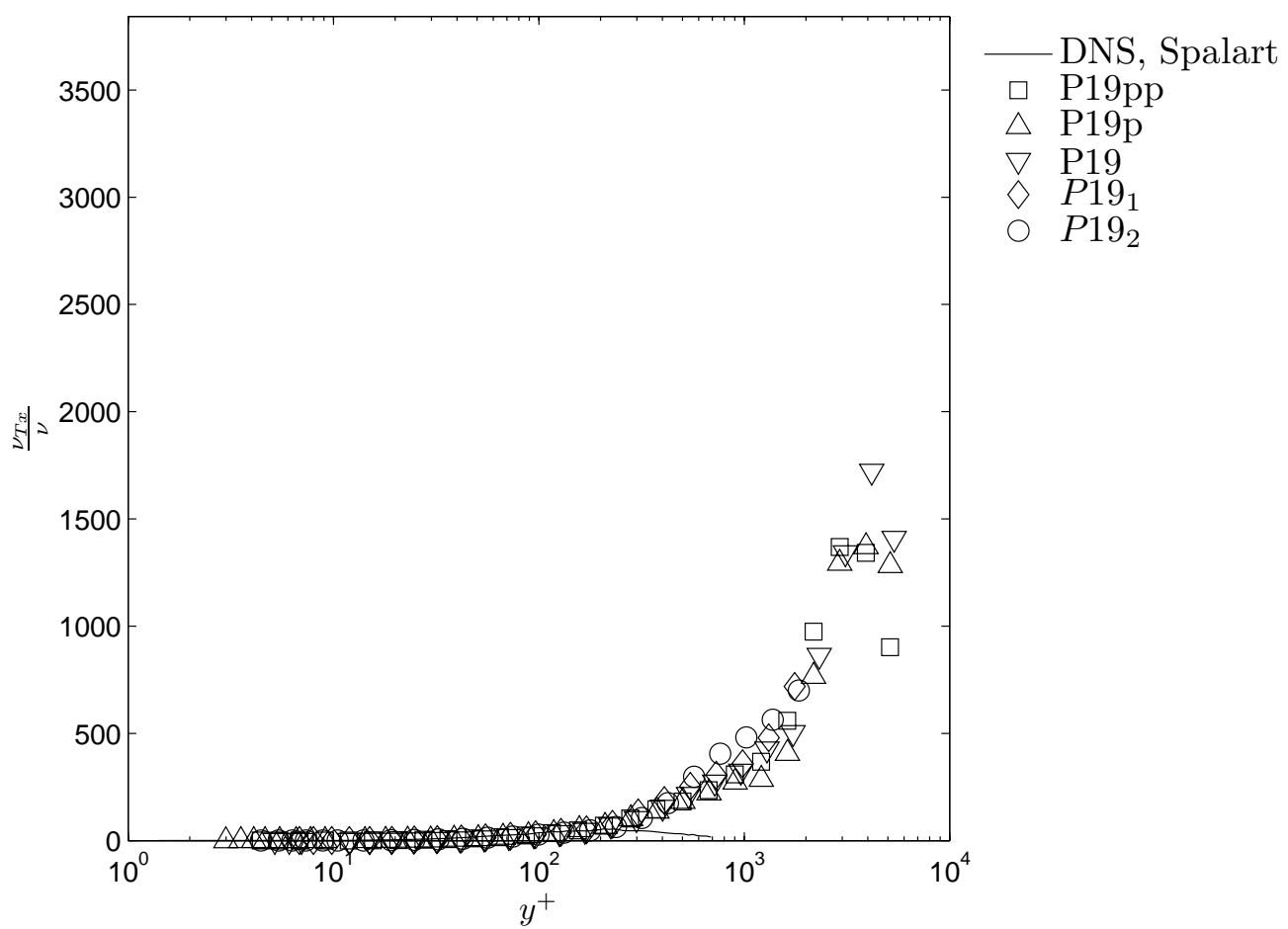

(s) Turbulent Eddy Viscosity $\nu_{T x}$

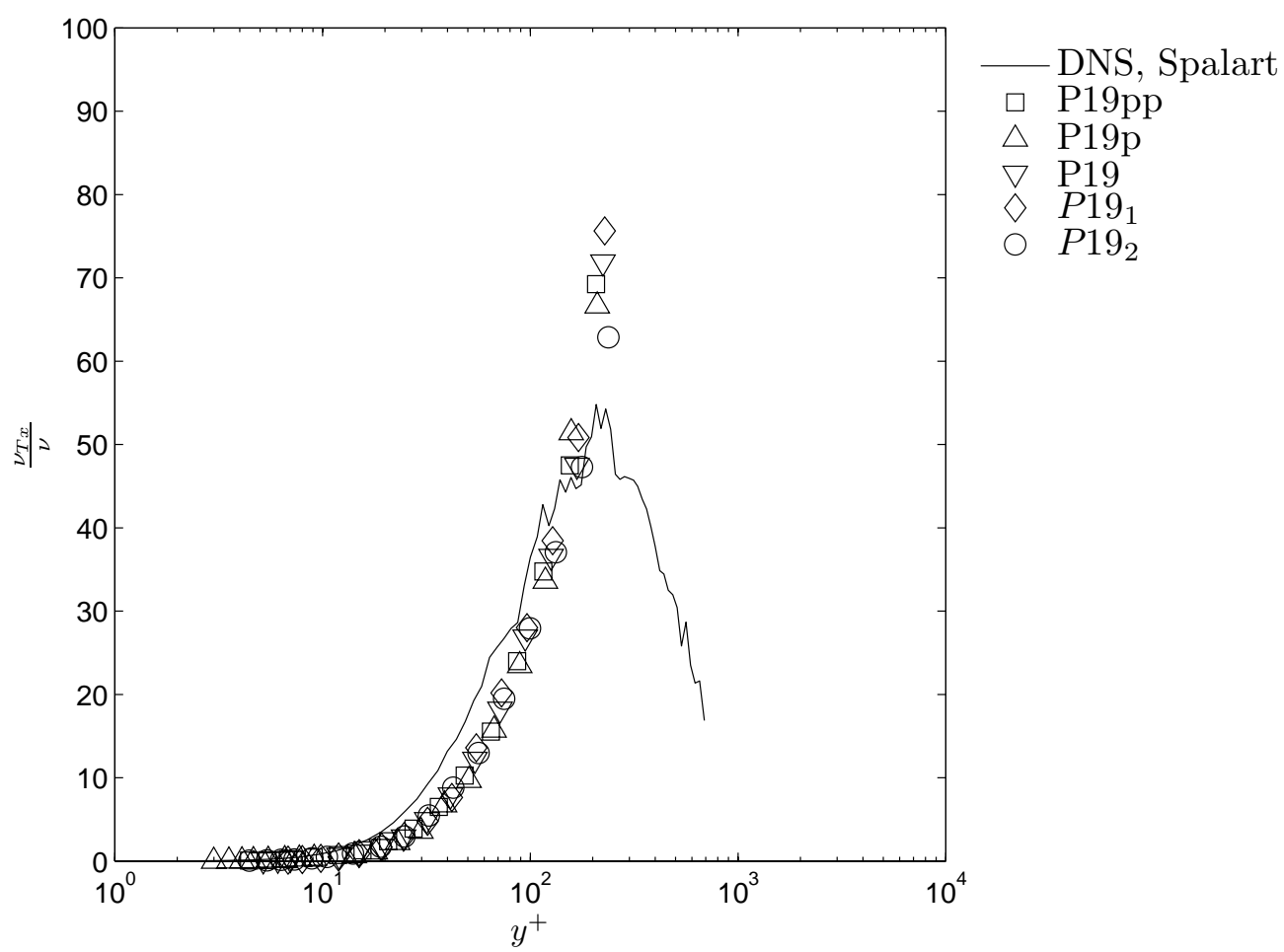

(t) Near Wall Turbulent Eddy Viscosity $\nu_{T x}$

Figure 4.6: Concluded, P19 Cluster 


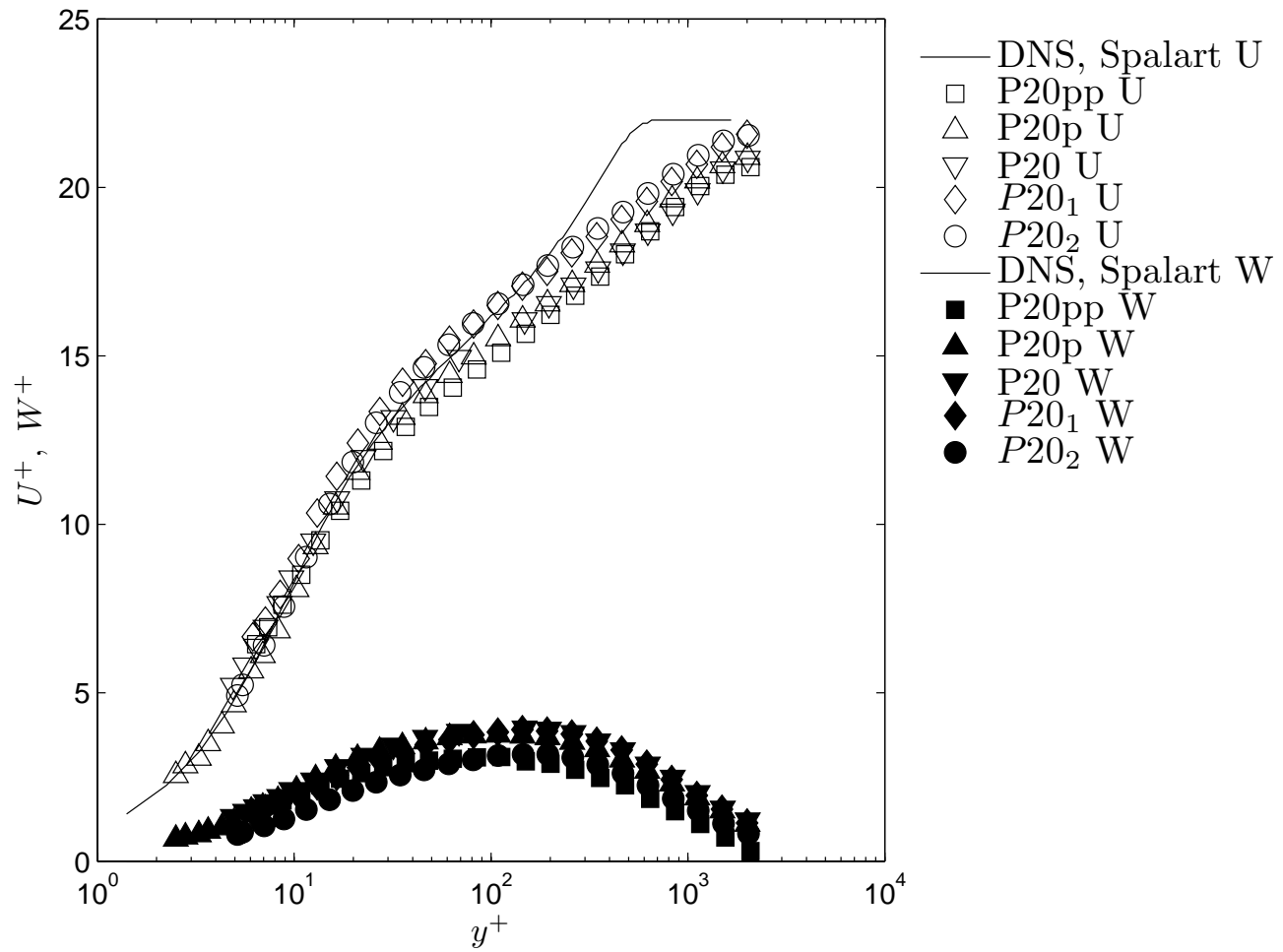

(a) Mean Stream-wise and Span-wise Velocities, $U^{+}, W^{+}$

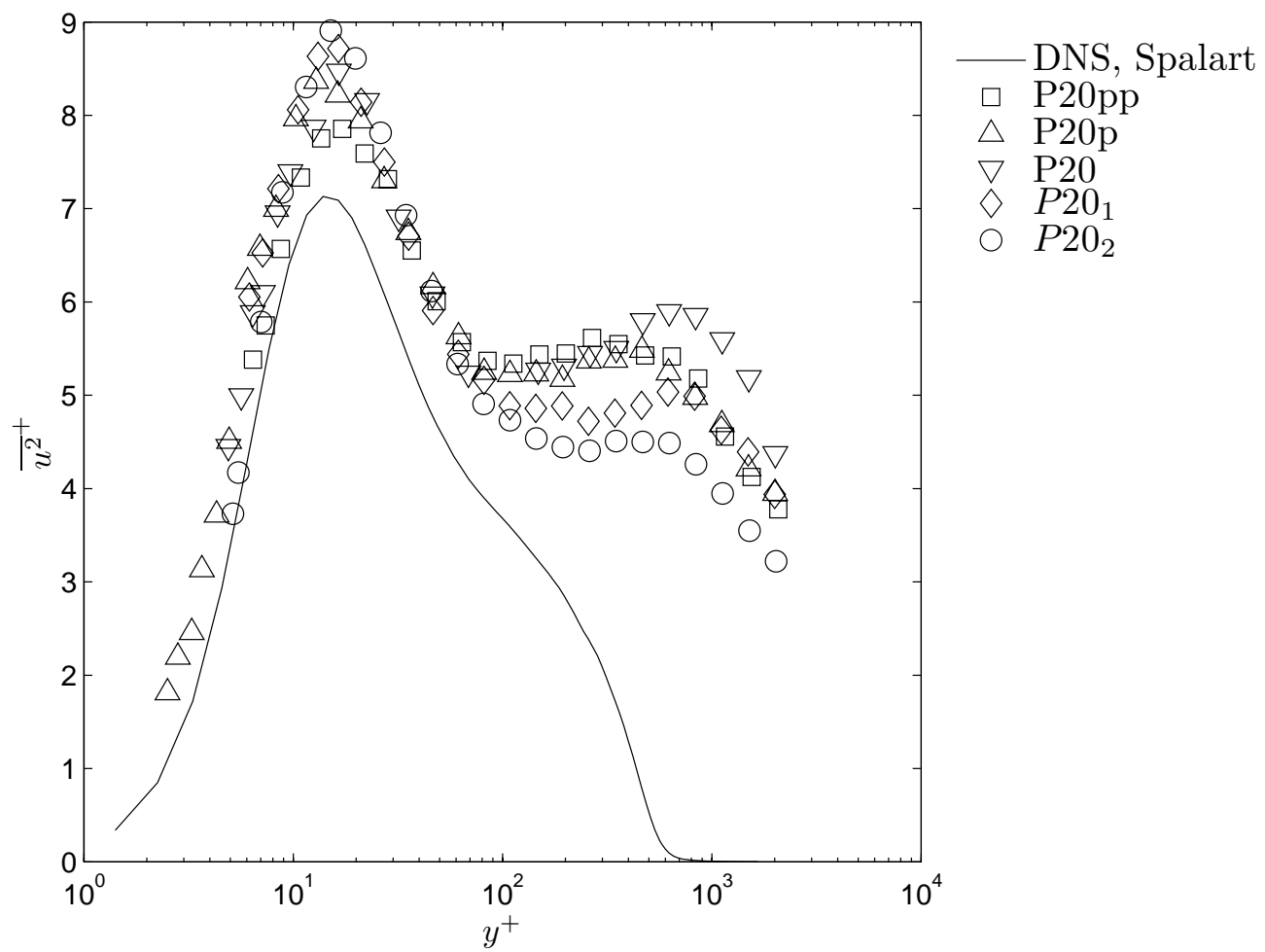

(b) Stream-wise Reynolds' Normal Stress, ${\overline{u^{2}}}^{+}$

Figure 4.7: P20 Cluster 


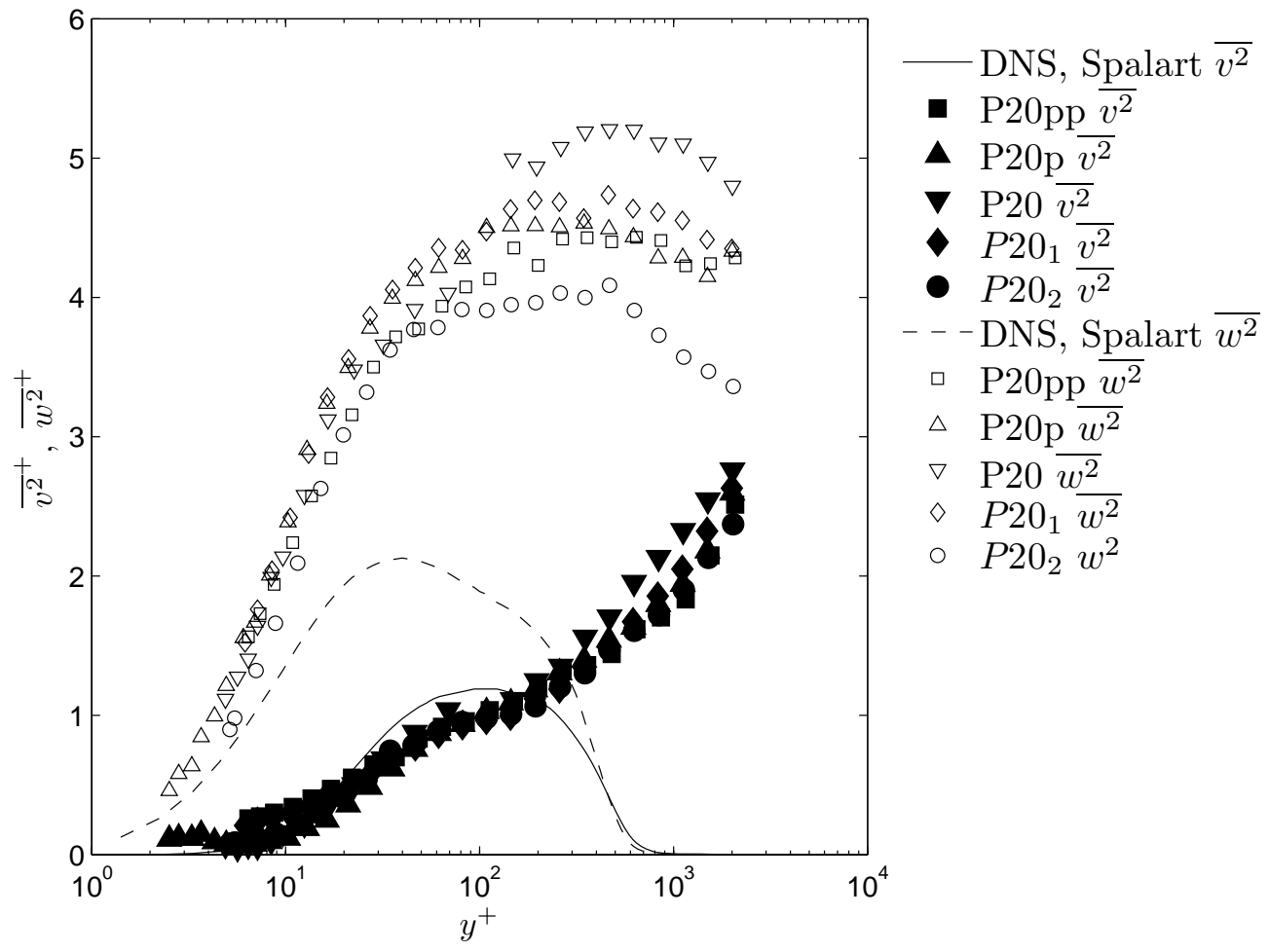

(c) Longitudinal and Span-wise Normal Reynolds' Stresses, ${\overline{v^{2}}}^{+}$and ${\overline{w^{2}}}^{+}$

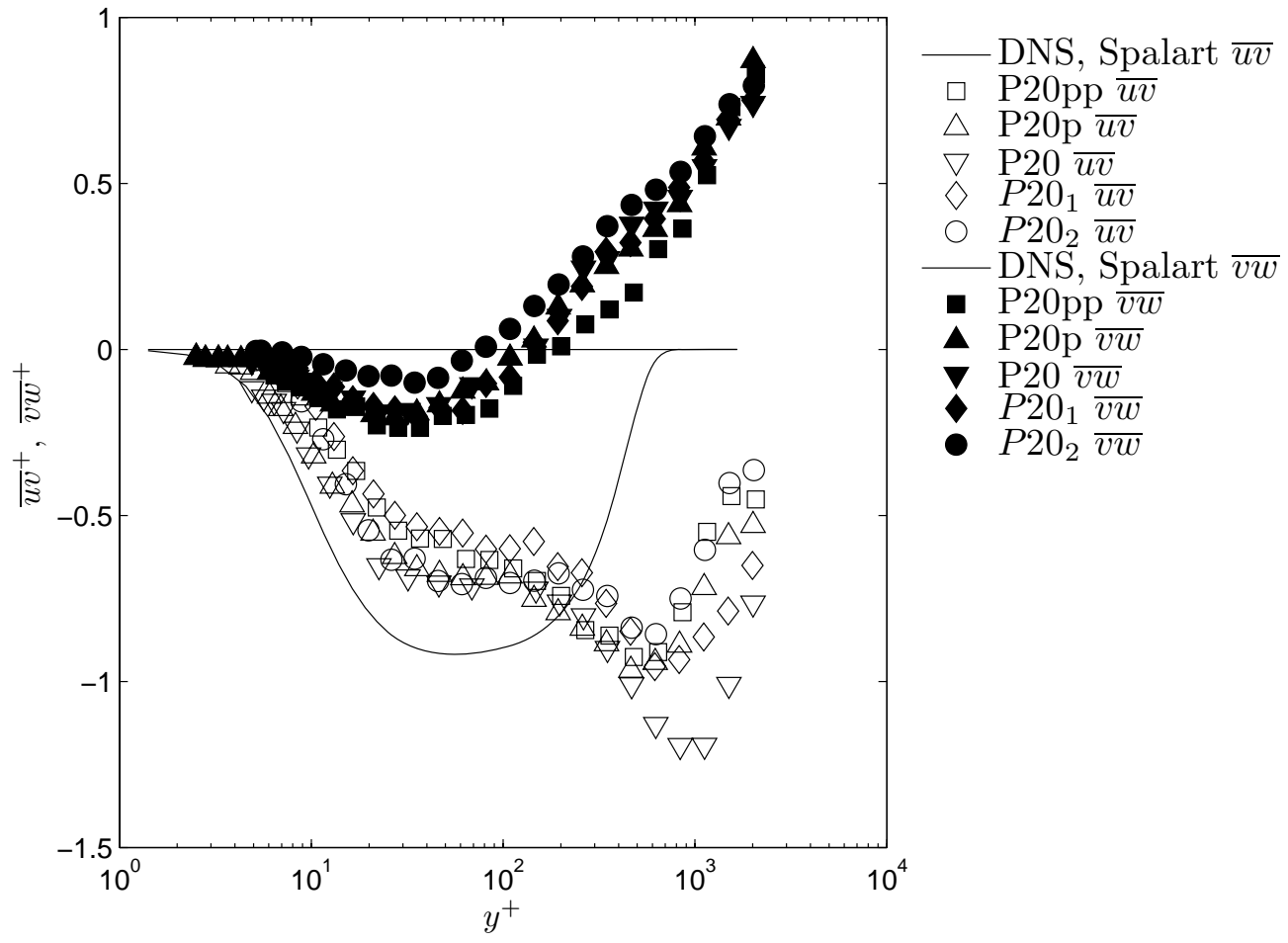

(d) Reynolds' Shear Stresses, $\overline{u v}^{+}$and $\overline{v w}^{+}$

Figure 4.7: Continued, P20 Cluster 


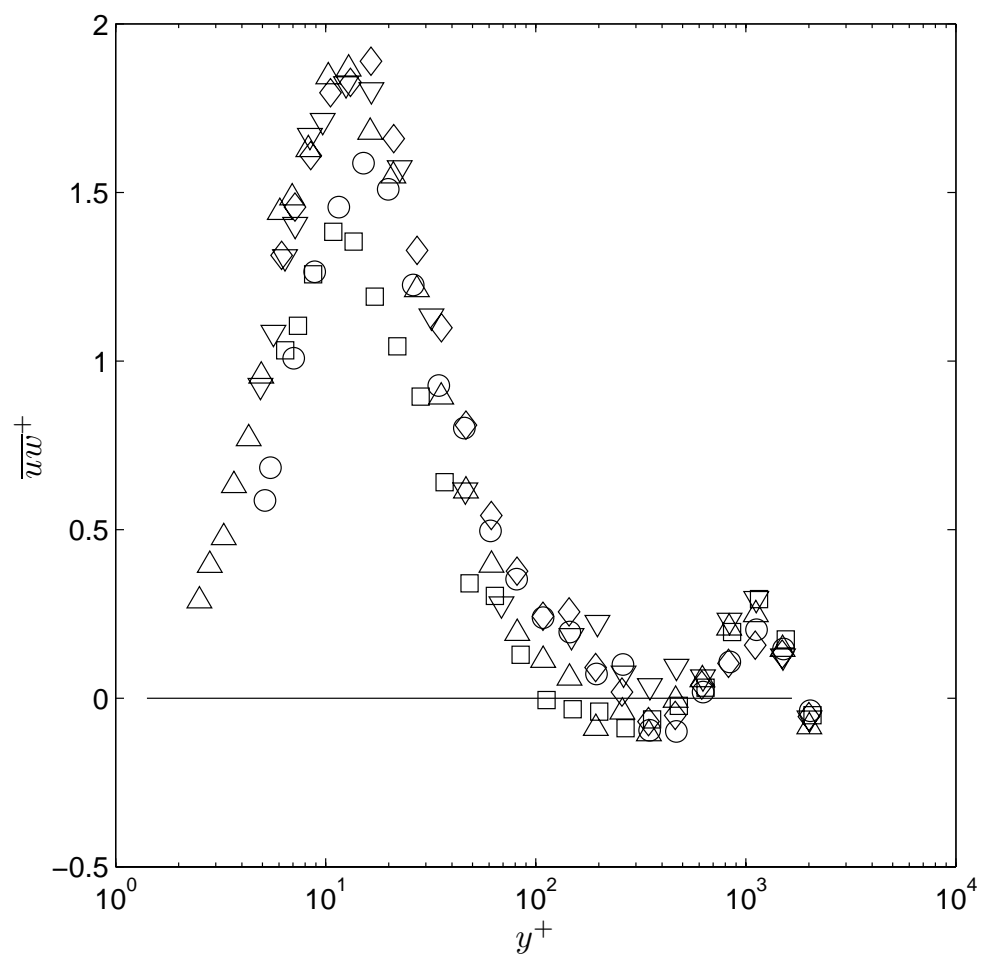

$\begin{array}{ll} & \text { DNS, Spalart } \\ \square & \text { P20pp } \\ \triangle & \text { P20p } \\ \nabla & \text { P20 } \\ \diamond & P 20_{1} \\ \bigcirc & P 20_{2}\end{array}$

(e) Reynolds' Shear Stress, $\overline{u w}^{+}$

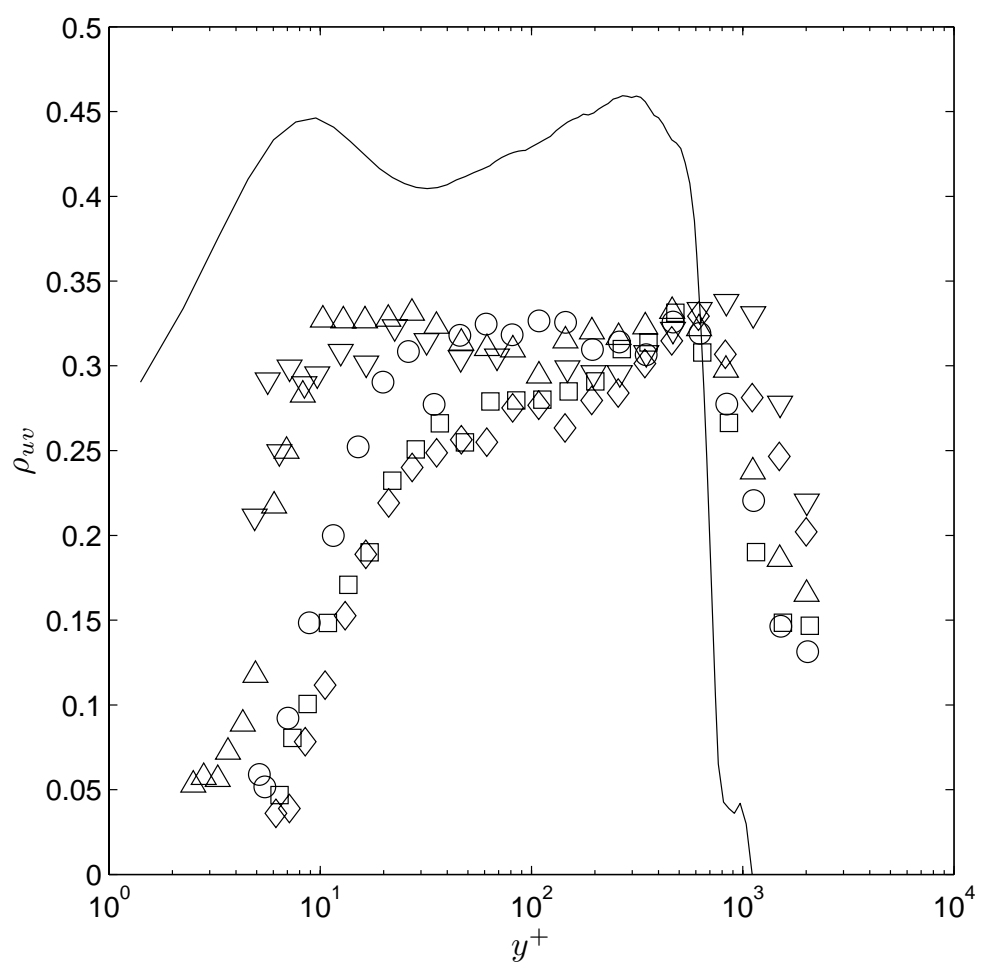

DNS, Spalart

$\square$ P20pp

$\triangle \mathrm{P} 20 \mathrm{p}$

$\nabla \mathrm{P} 20$

$\diamond P 20_{1}$

(f) Correlation Coefficient $\rho_{u v}$

Figure 4.7: Continued, P20 Cluster 


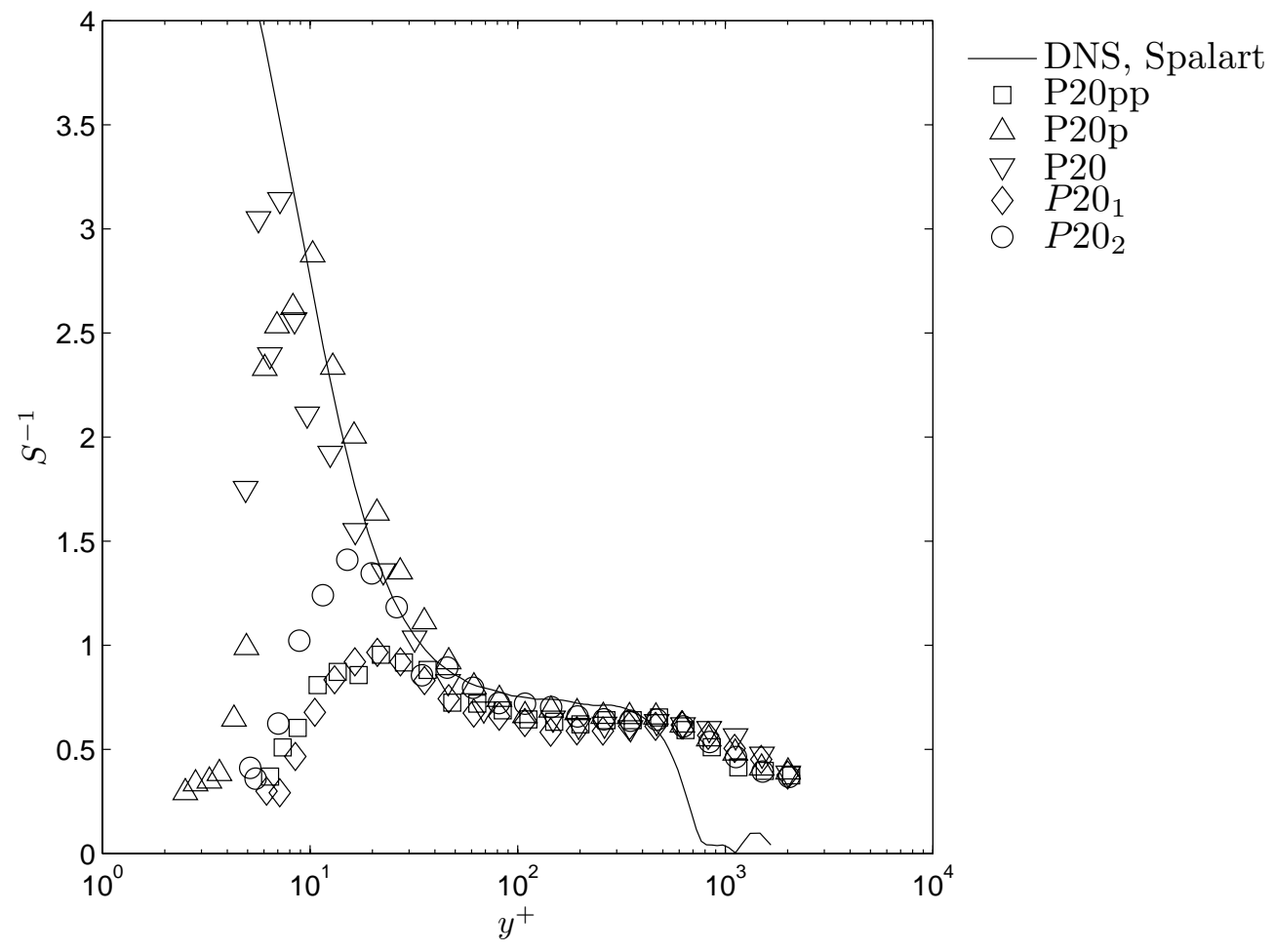

(g) $S^{-1}$ Parameter

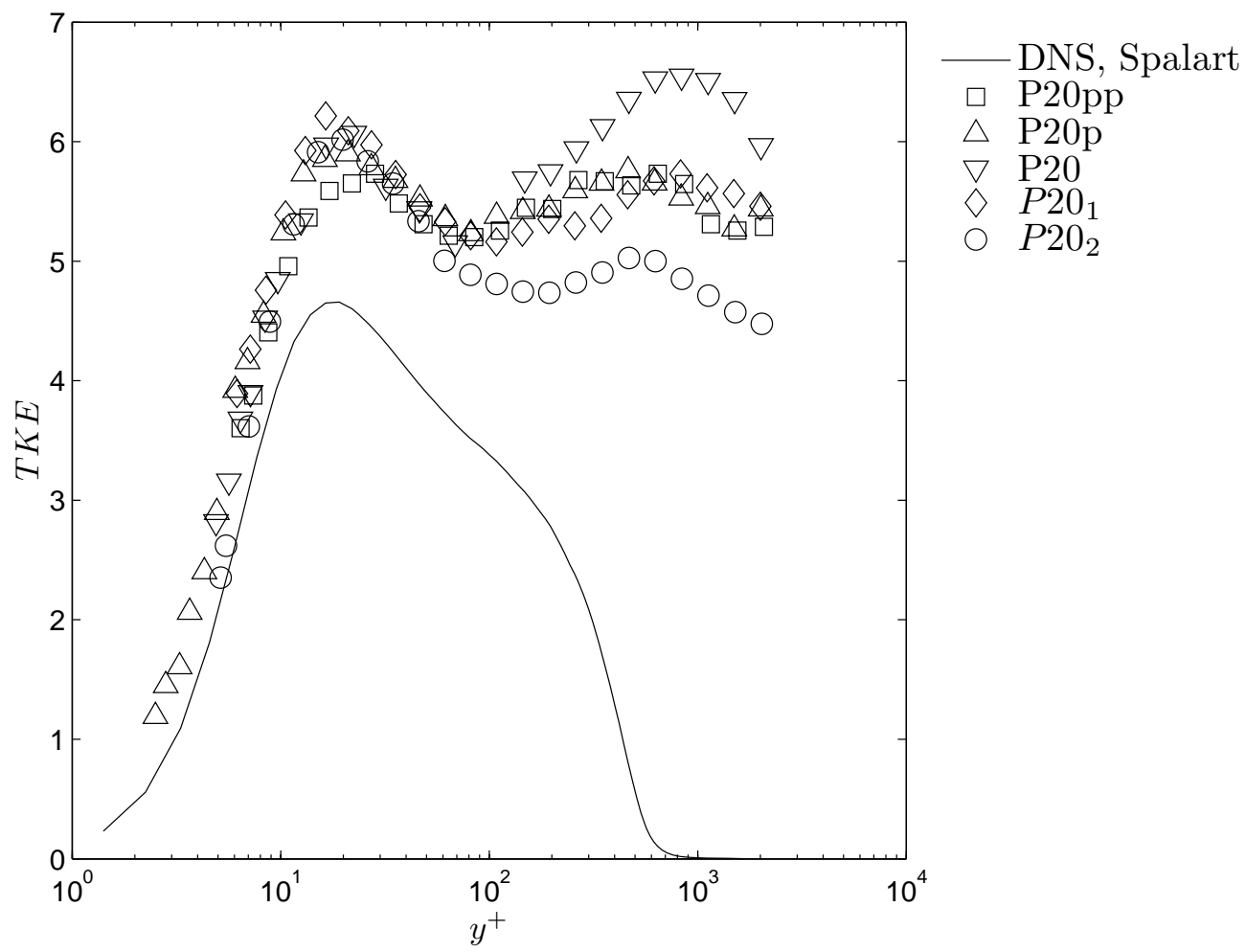

(h) Turbulent Kinetic Energy, TKE

Figure 4.7: Continued, P20 Cluster 


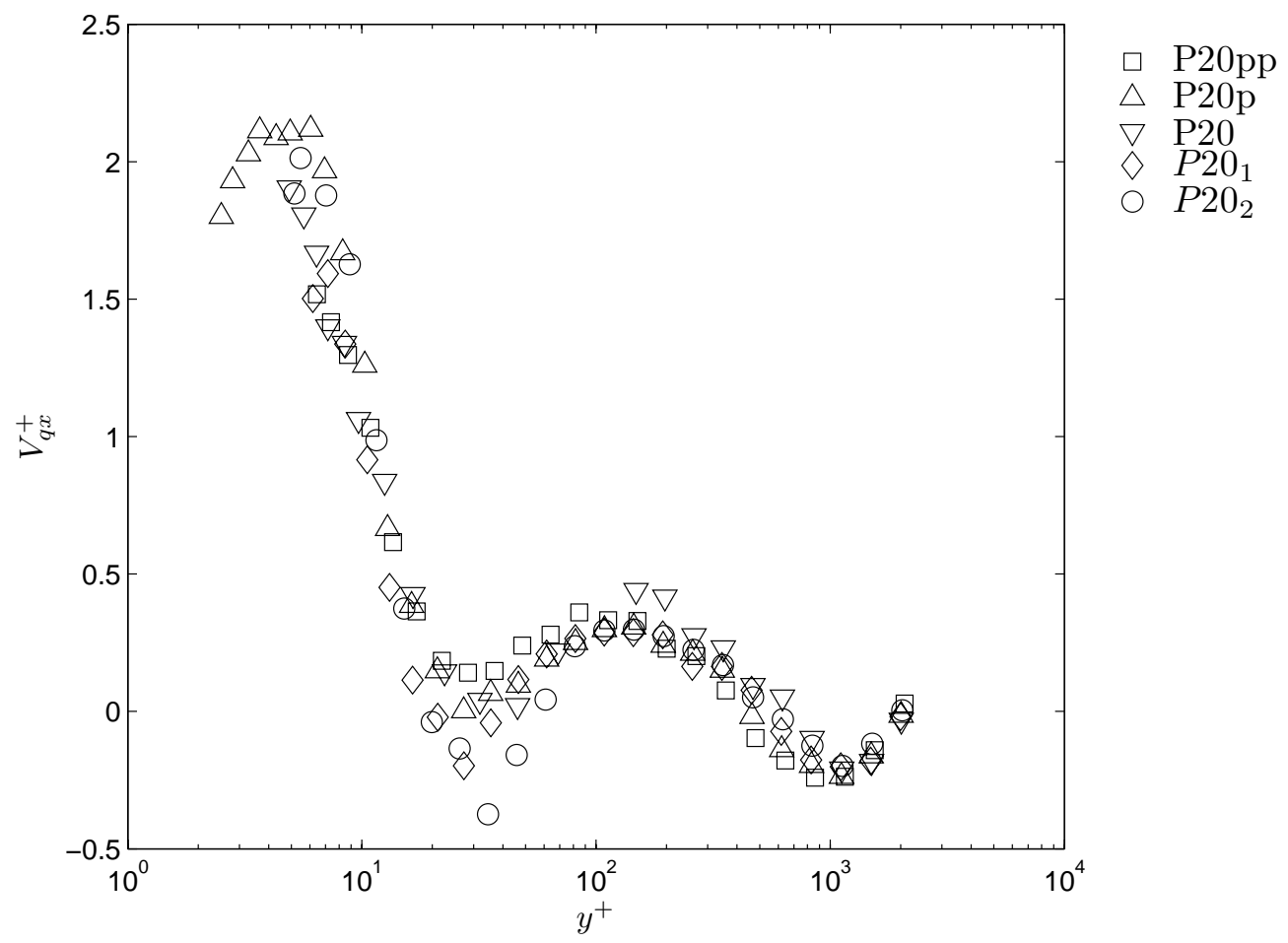

(i) Stream-wise Turbulent Diffusion Velocity component, $V_{q x}$, normalized on $u_{\tau}$

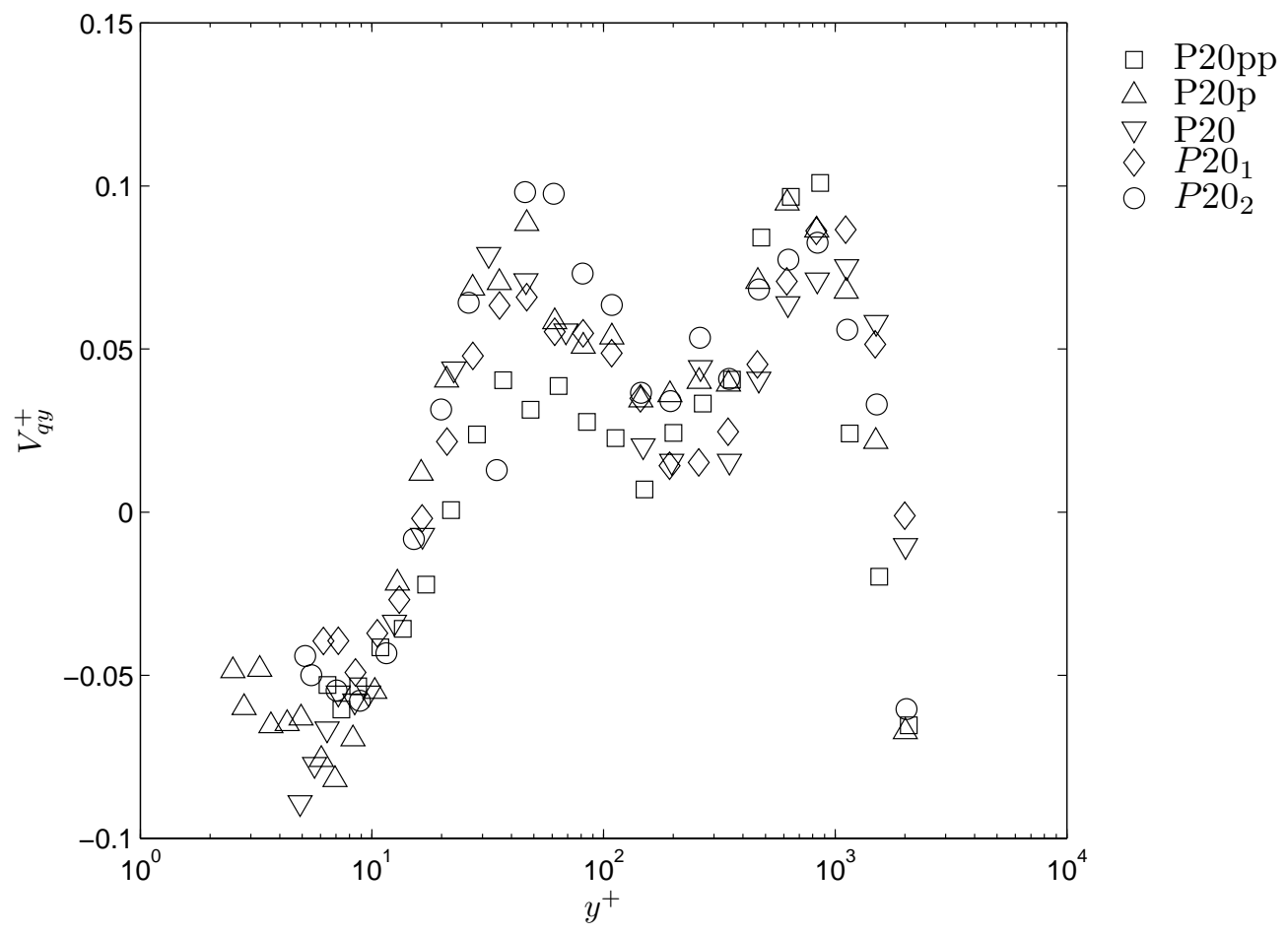

(j) Wall-Normal Turbulent Diffusion Velocity component, $V_{q y}$, normalized on $u_{\tau}$

Figure 4.7: Continued, P20 Cluster 


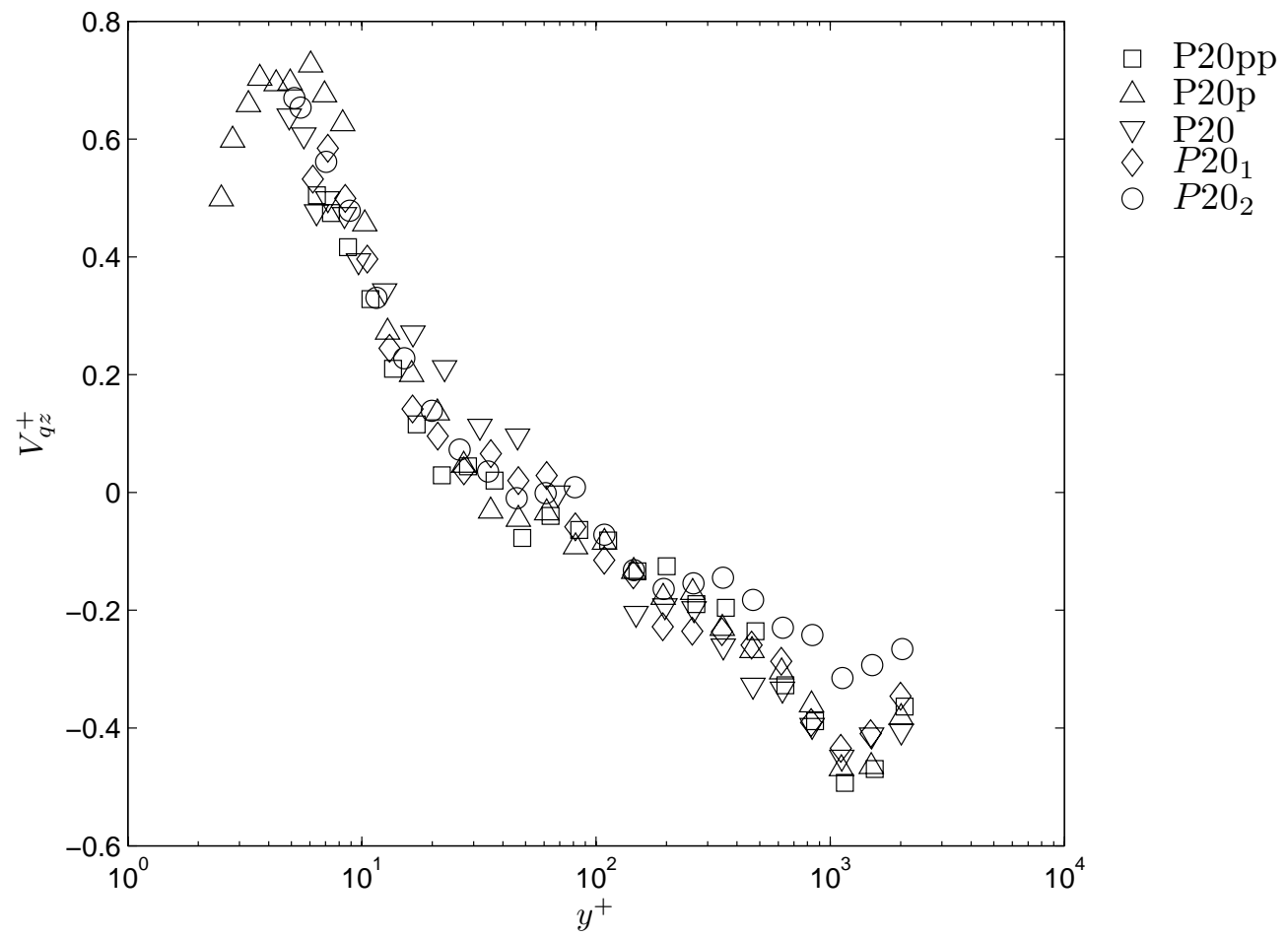

(k) Span-wise Turbulent Diffusion Velocity component, $V_{q z}$, normalized on $u_{\tau}$

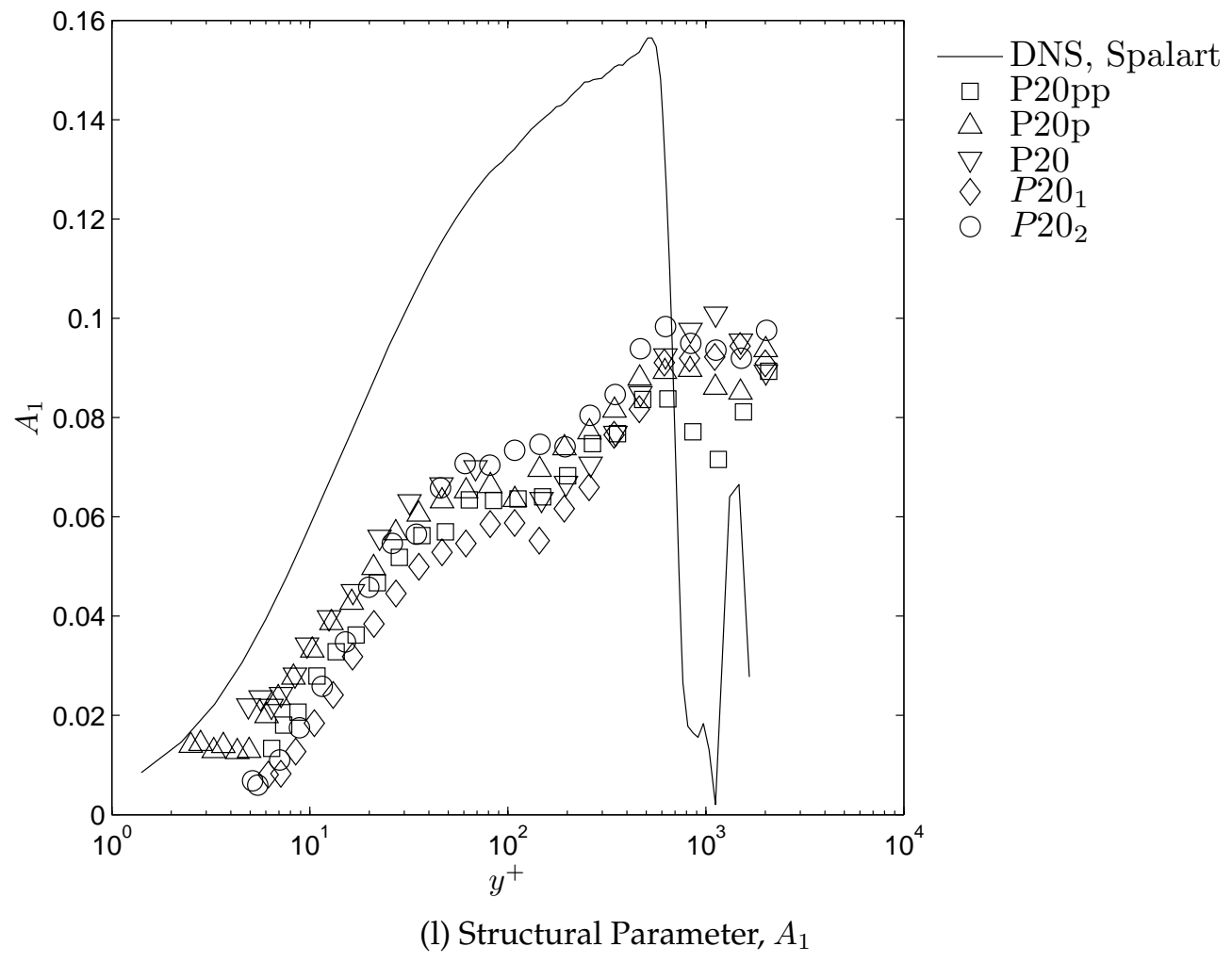

Figure 4.7: Continued, $P 20$ Cluster 


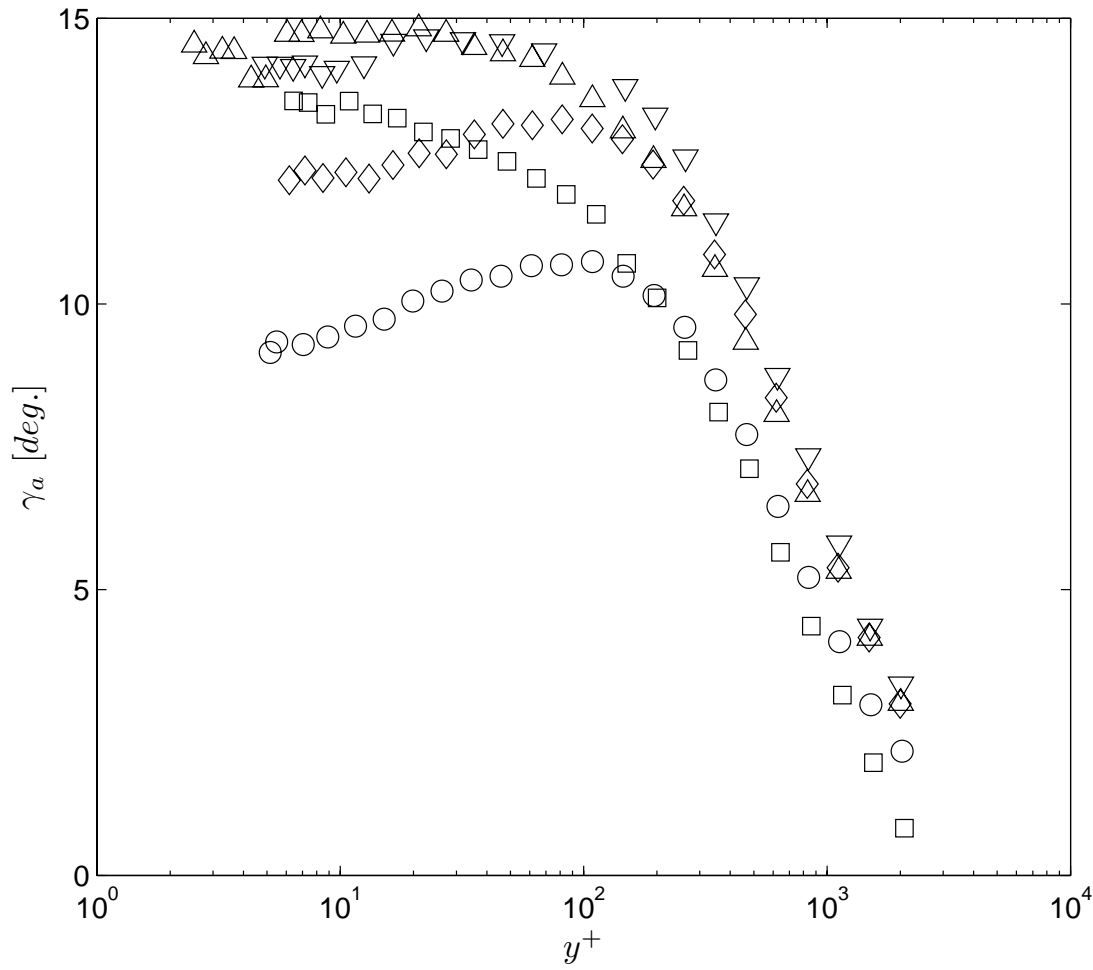

$\begin{array}{ll}\square & \text { P20pp } \\ \triangle & \text { P20p } \\ \nabla & \text { P20 } \\ \diamond & P 20_{1} \\ \diamond & P 20_{2}\end{array}$

(m) Flow Angle, $\gamma_{a}$

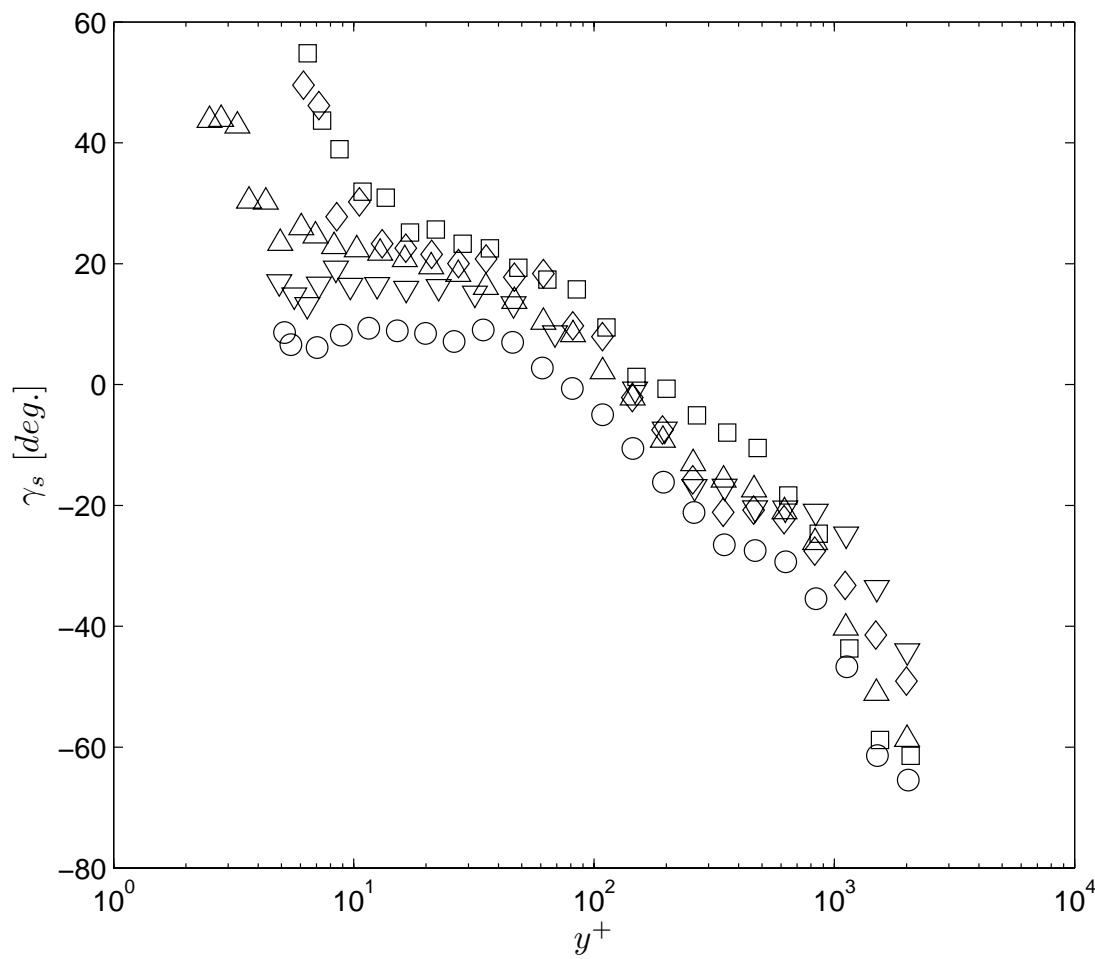

$\begin{array}{ll}\square & \text { P20pp } \\ \triangle & \text { P20p } \\ \nabla & \text { P20 } \\ \diamond & P 20_{1} \\ \bigcirc & P 20_{2}\end{array}$

(n) Shear Stress Angle $\gamma_{s}$

Figure 4.7: Continued, P20 Cluster 


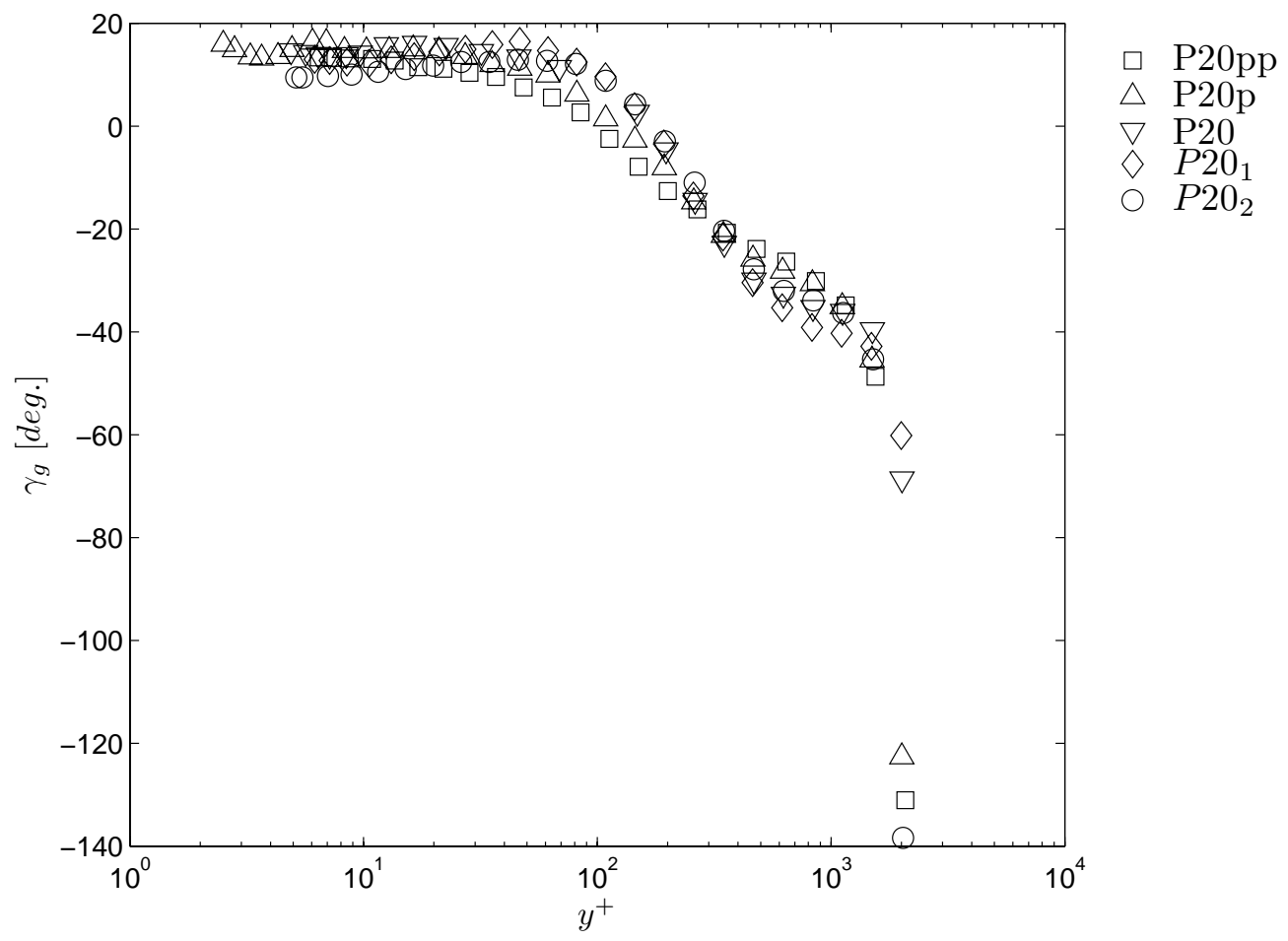

(o) Flow Gradient Angle $\gamma_{g}$

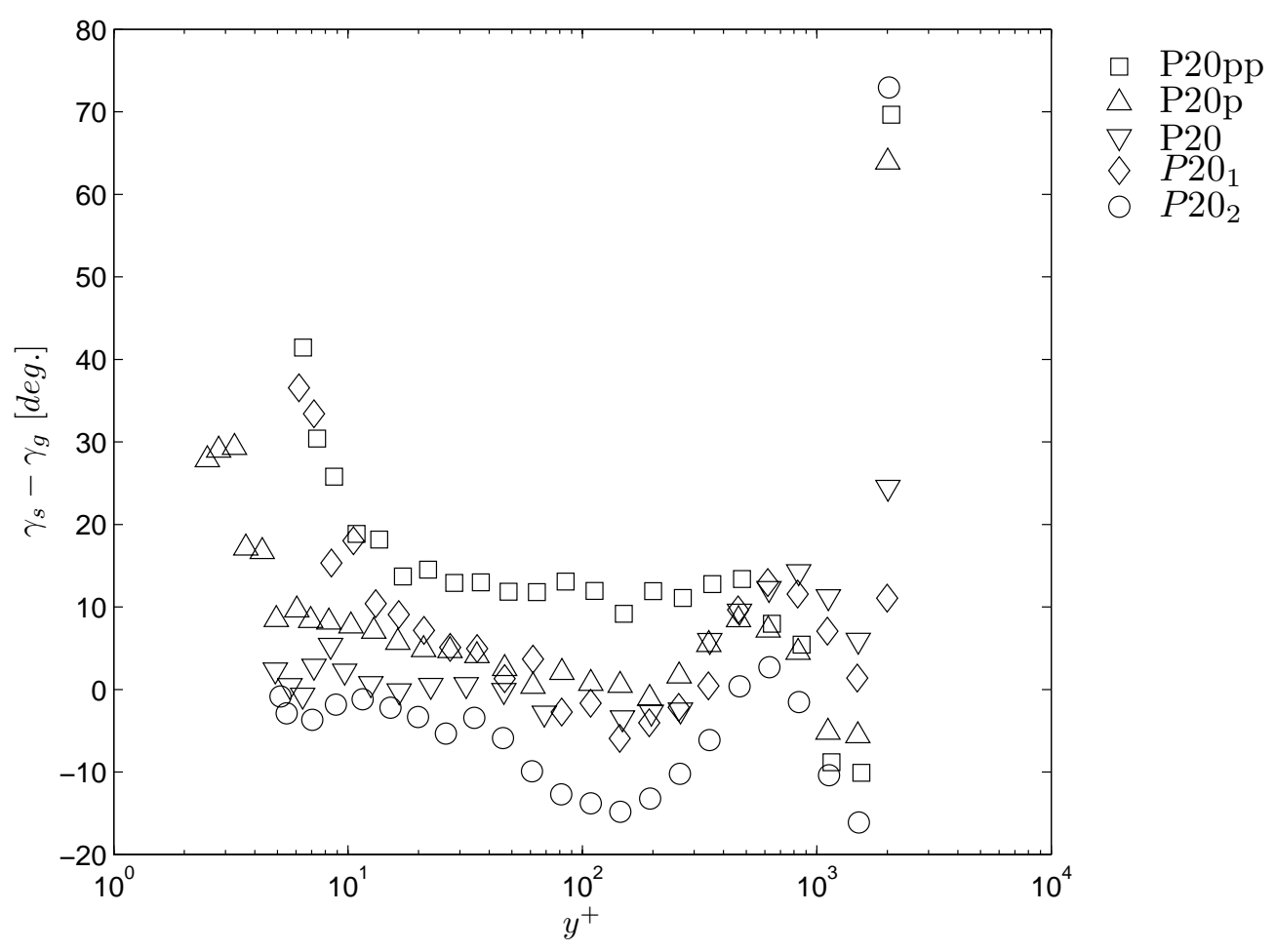

(p) Difference between Shear Angle and Flow Gradient Angles, $\gamma_{s}-\gamma_{g}$

Figure 4.7: Continued, P20 Cluster 


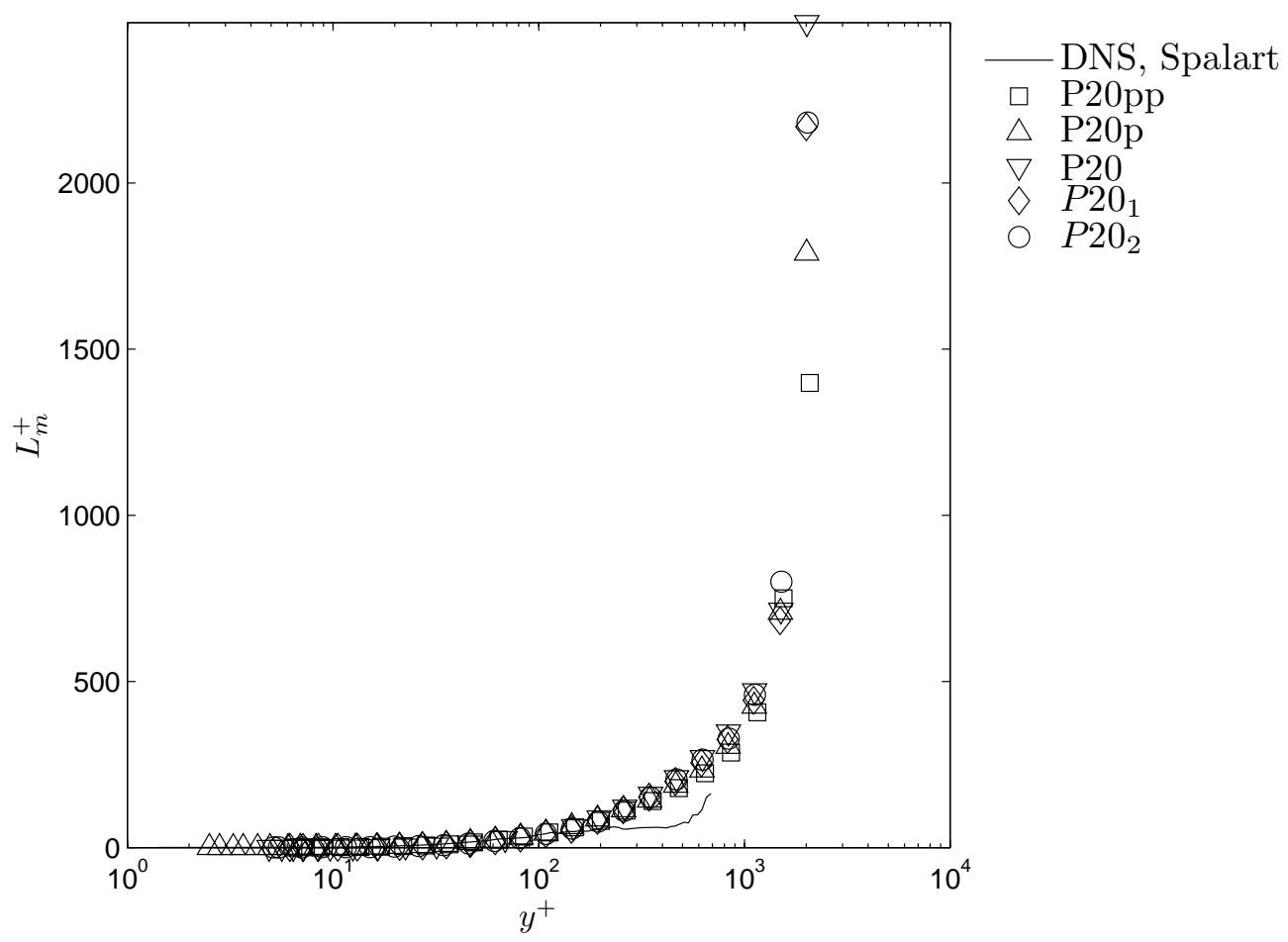

(q) Mixing Length $L_{m}^{+}$

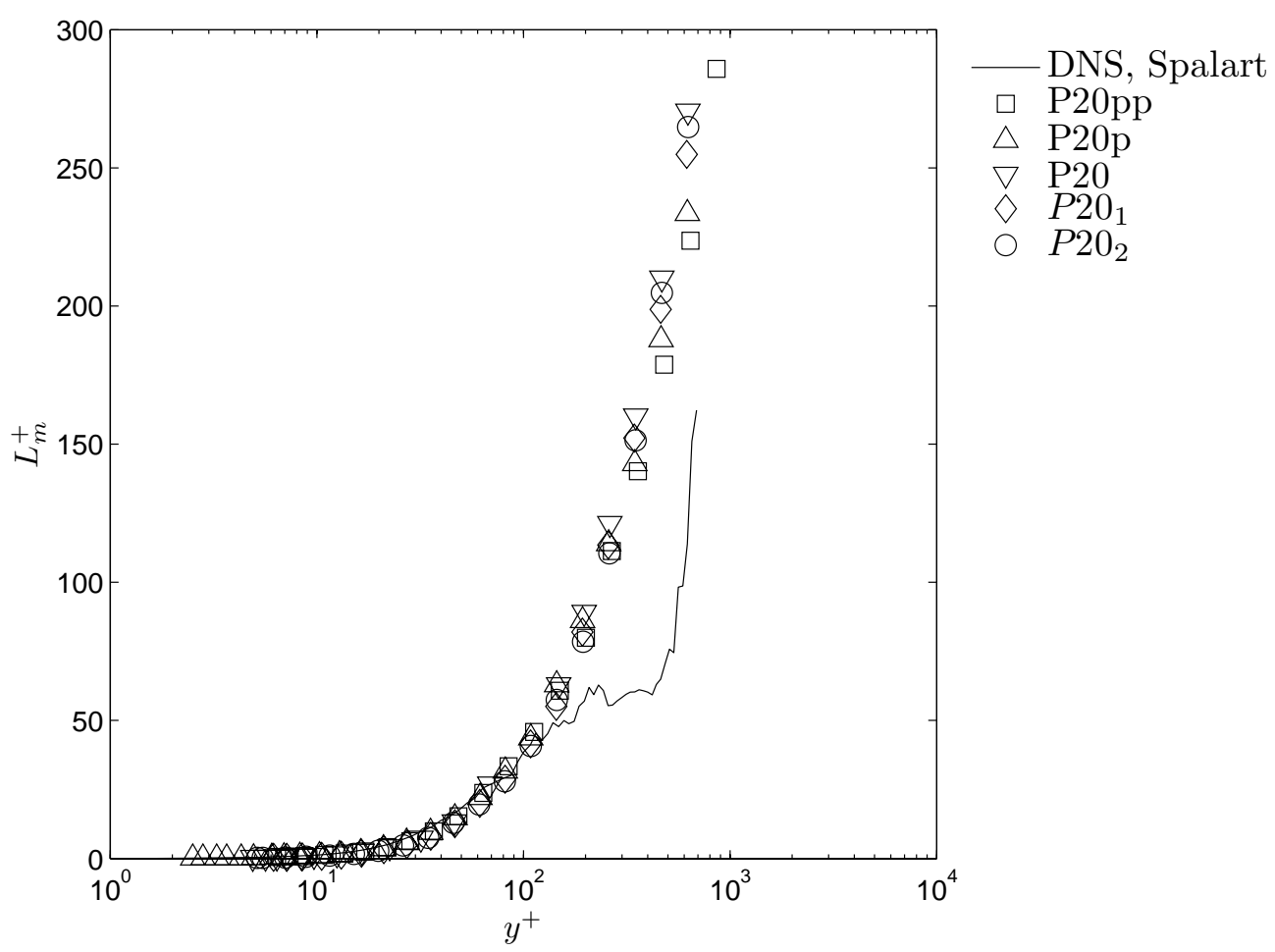

(r) Near Wall Mixing Length $L_{m}^{+}$

Figure 4.7: Continued, P20 Cluster 


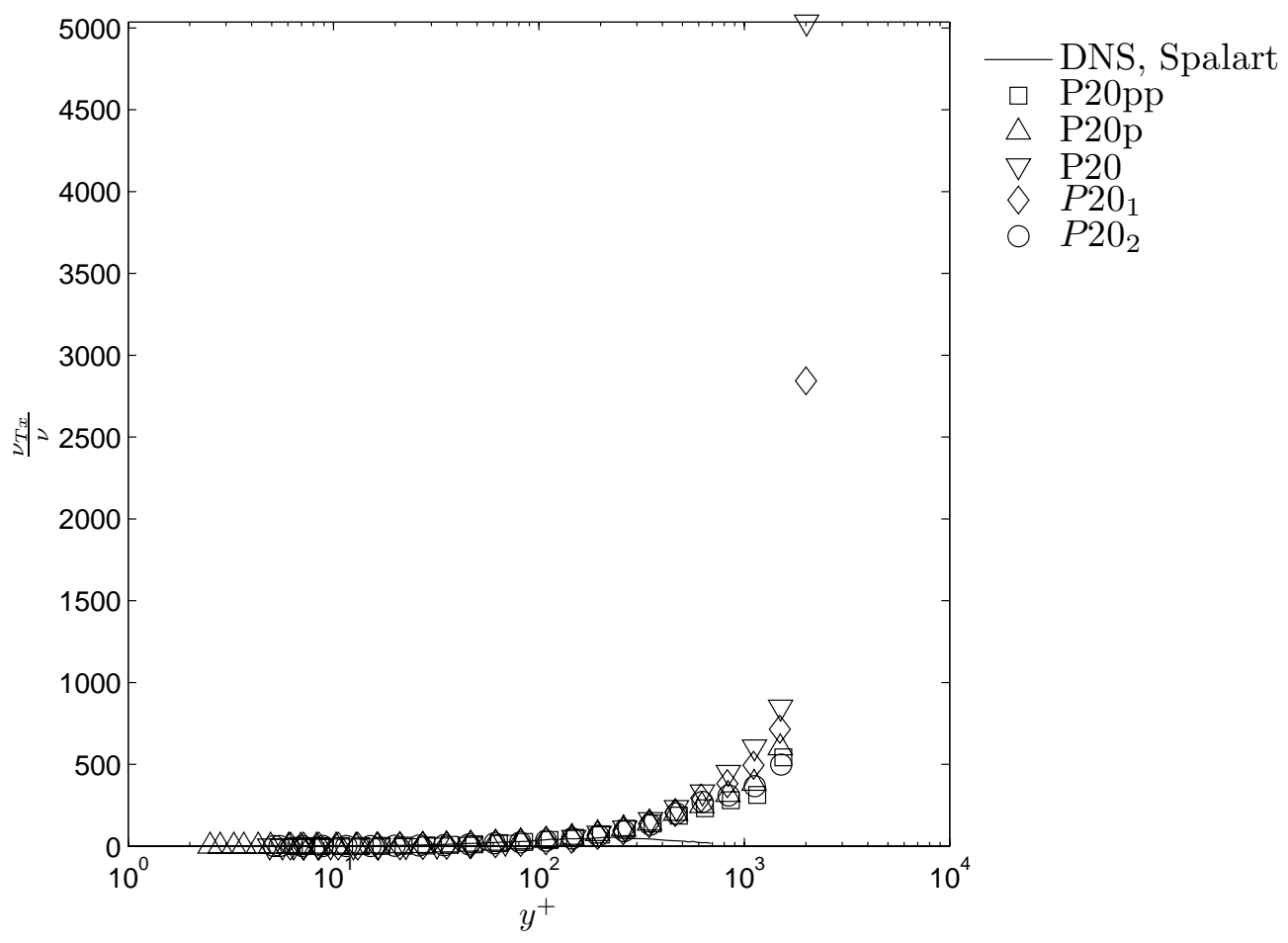

(s) Near Wall Turbulent Eddy Viscosity $\nu_{T x}$

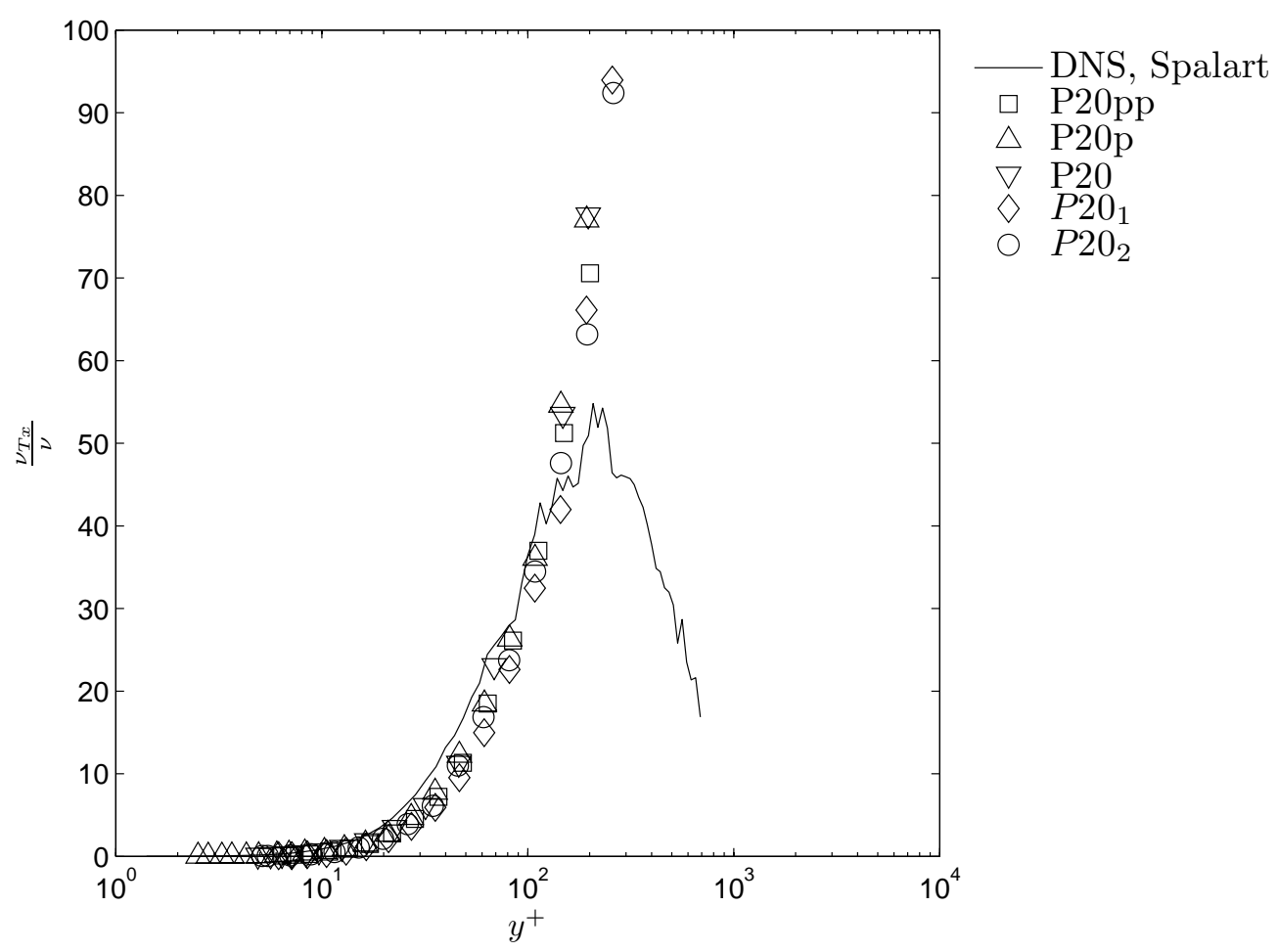

(t) Turbulent Eddy Viscosity $\nu_{T x}$

Figure 4.7: Concluded, P20 Cluster 


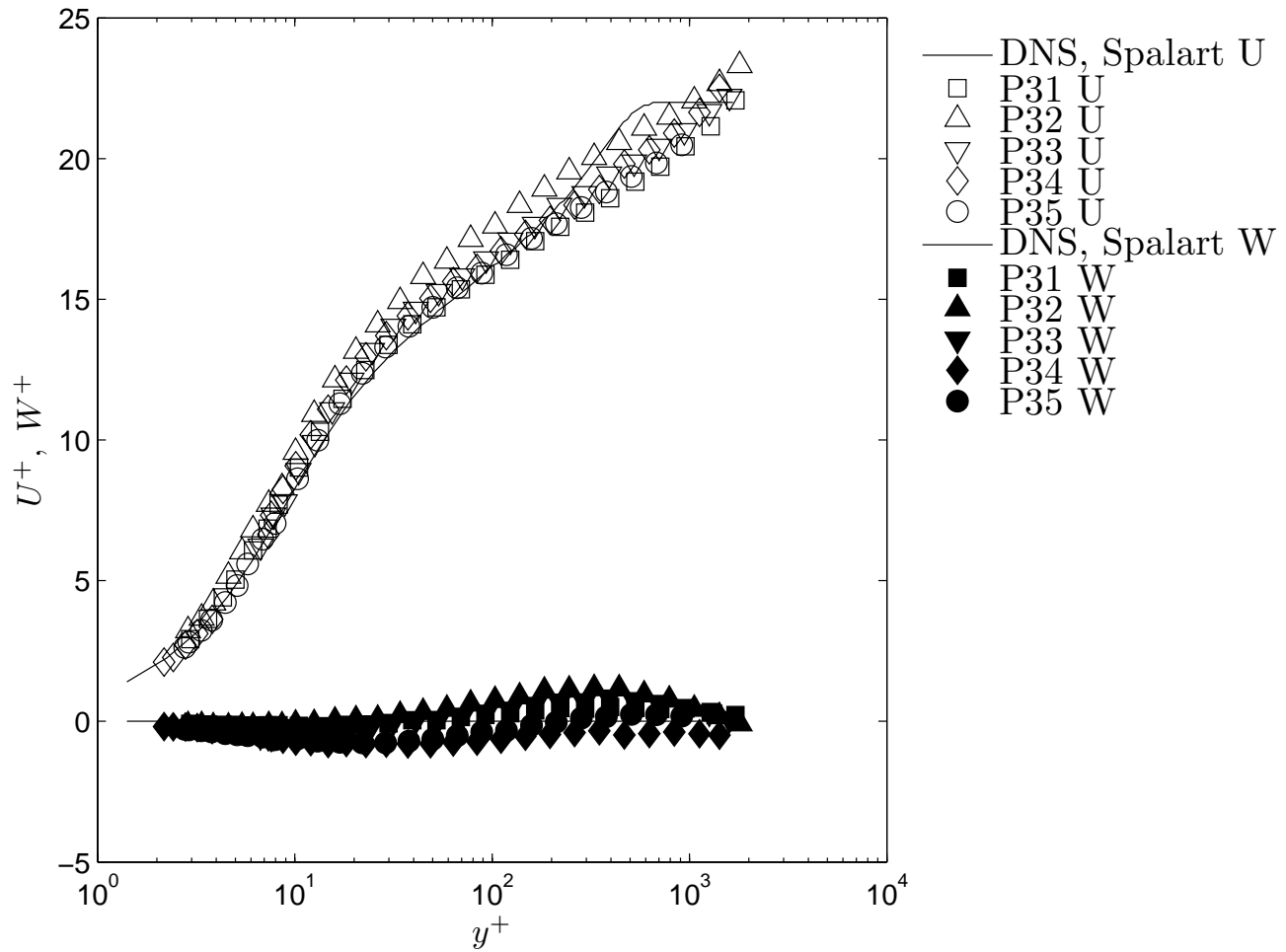

(a) Mean Stream-wise and Span-wise Velocities, $U^{+}, W^{+}$

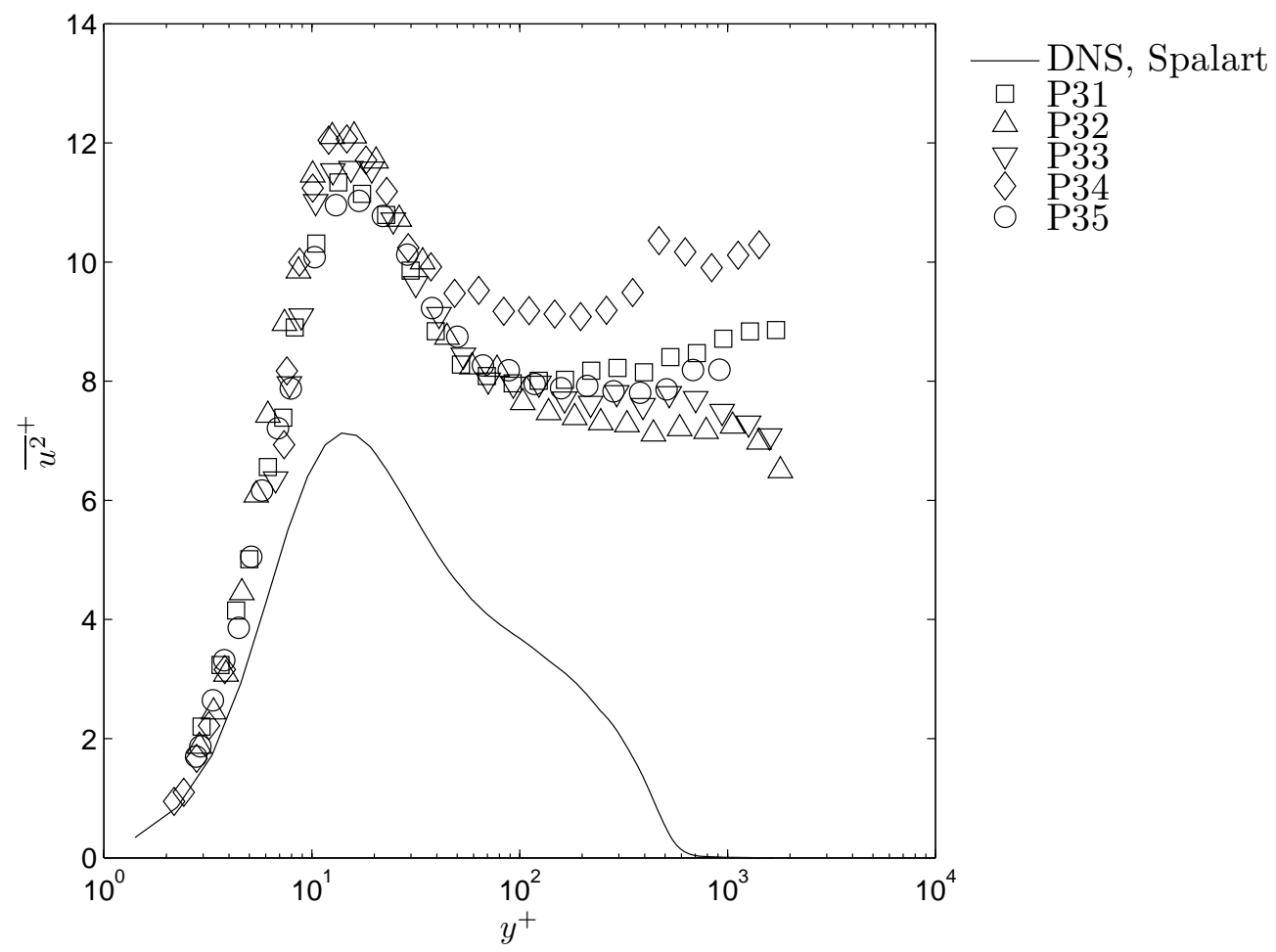

(b) Stream-wise Reynolds' Normal Stress, ${\overline{u^{2}}}^{+}$

Figure 4.8: P33 Cluster 


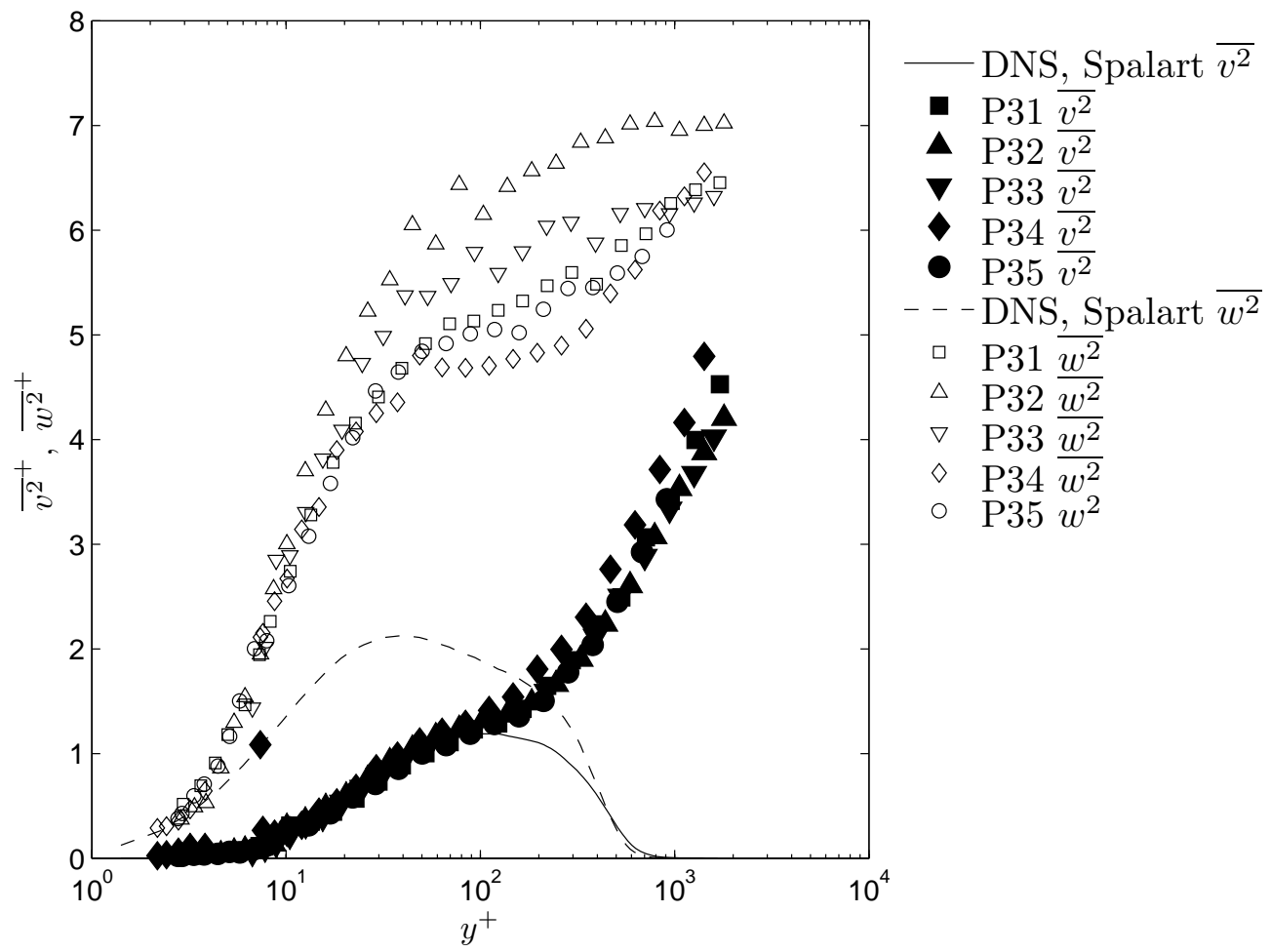

(c) Longitudinal and Span-wise Normal Reynolds' Stresses, ${\overline{v^{2}}}^{+}$and ${\overline{w^{2}}}^{+}$

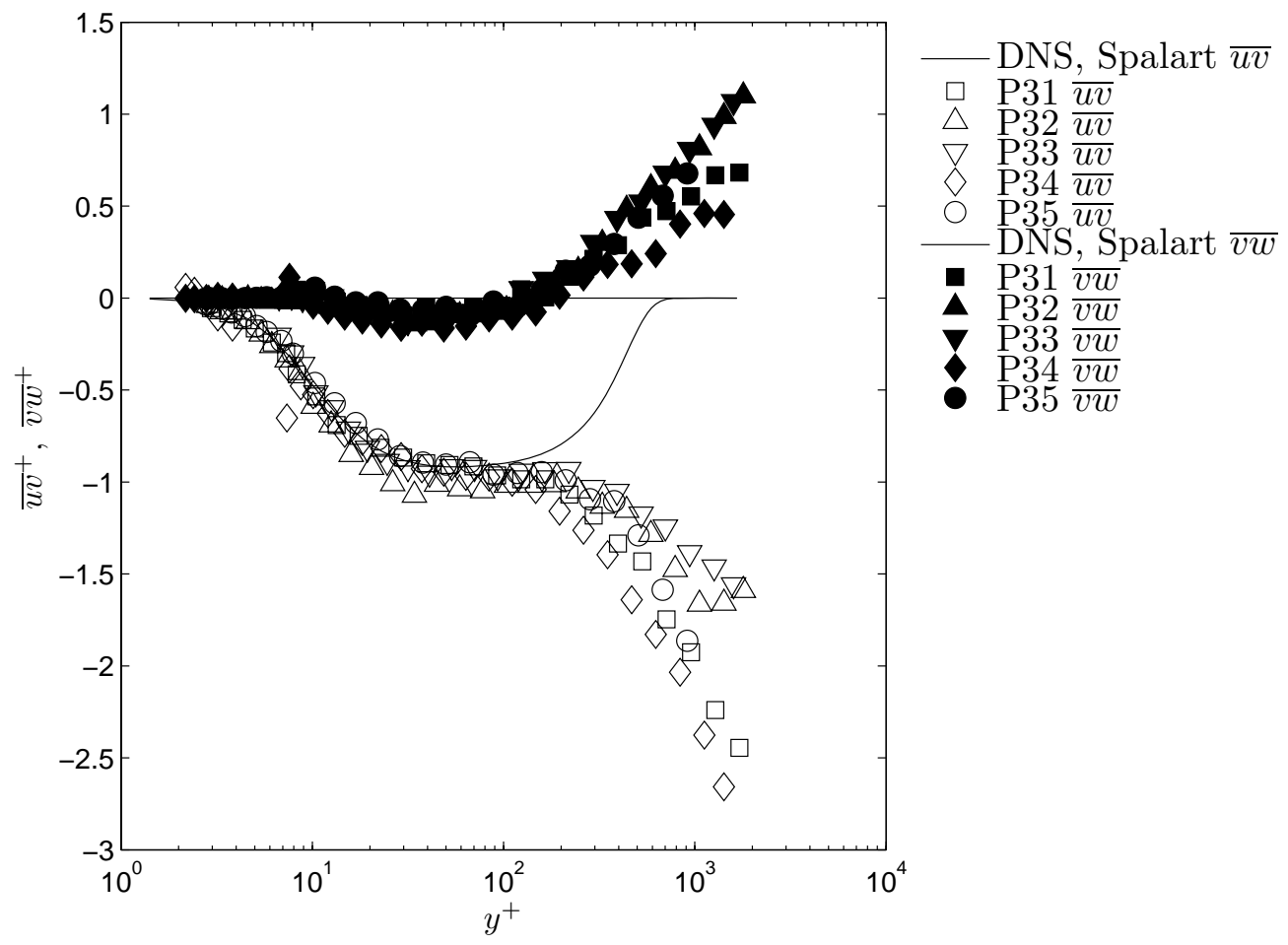

(d) Reynolds' Shear Stresses, $\overline{u v}^{+}$and $\overline{v w}^{+}$

Figure 4.8: Continued, P33 Cluster 

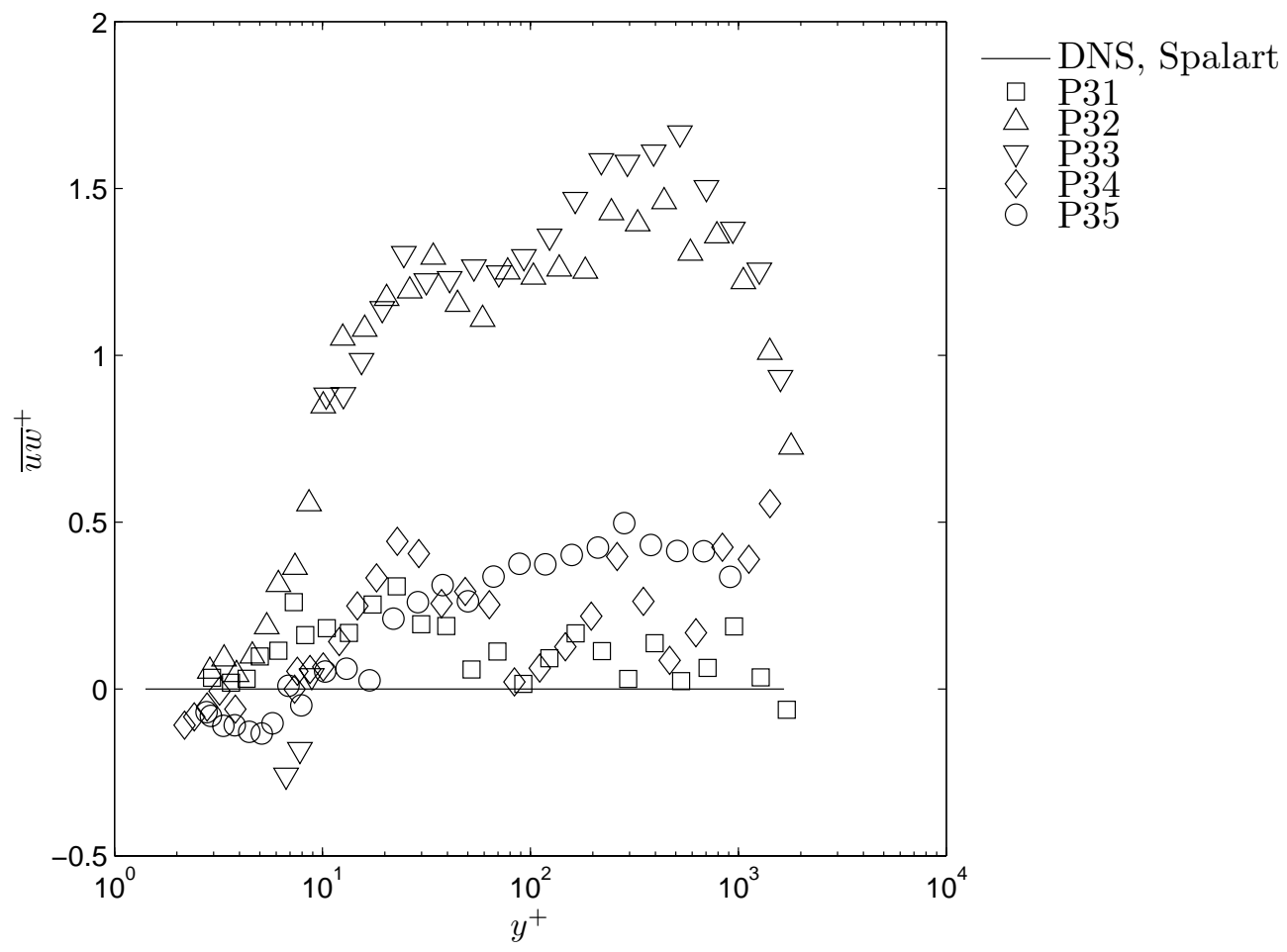

(e) Reynolds' Shear Stress, $\overline{u w}^{+}$

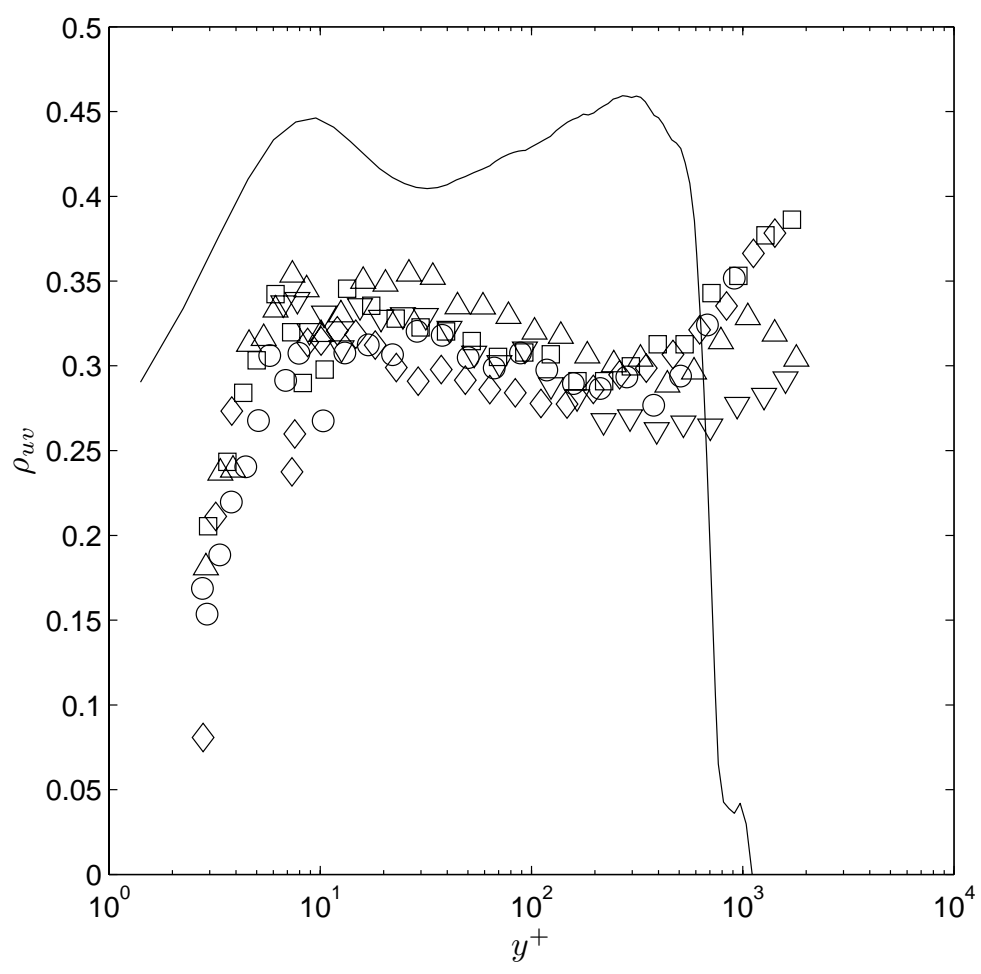

DNS, Spalart

ㅁ P31

$\triangle \mathrm{P} 32$

$\nabla$ P33

$\begin{array}{ll}\vee & \text { P34 } \\ \bigcirc & \text { P35 }\end{array}$

(f) Correlation Coefficient $\rho_{u v}$

Figure 4.8: Continued, P33 Cluster 


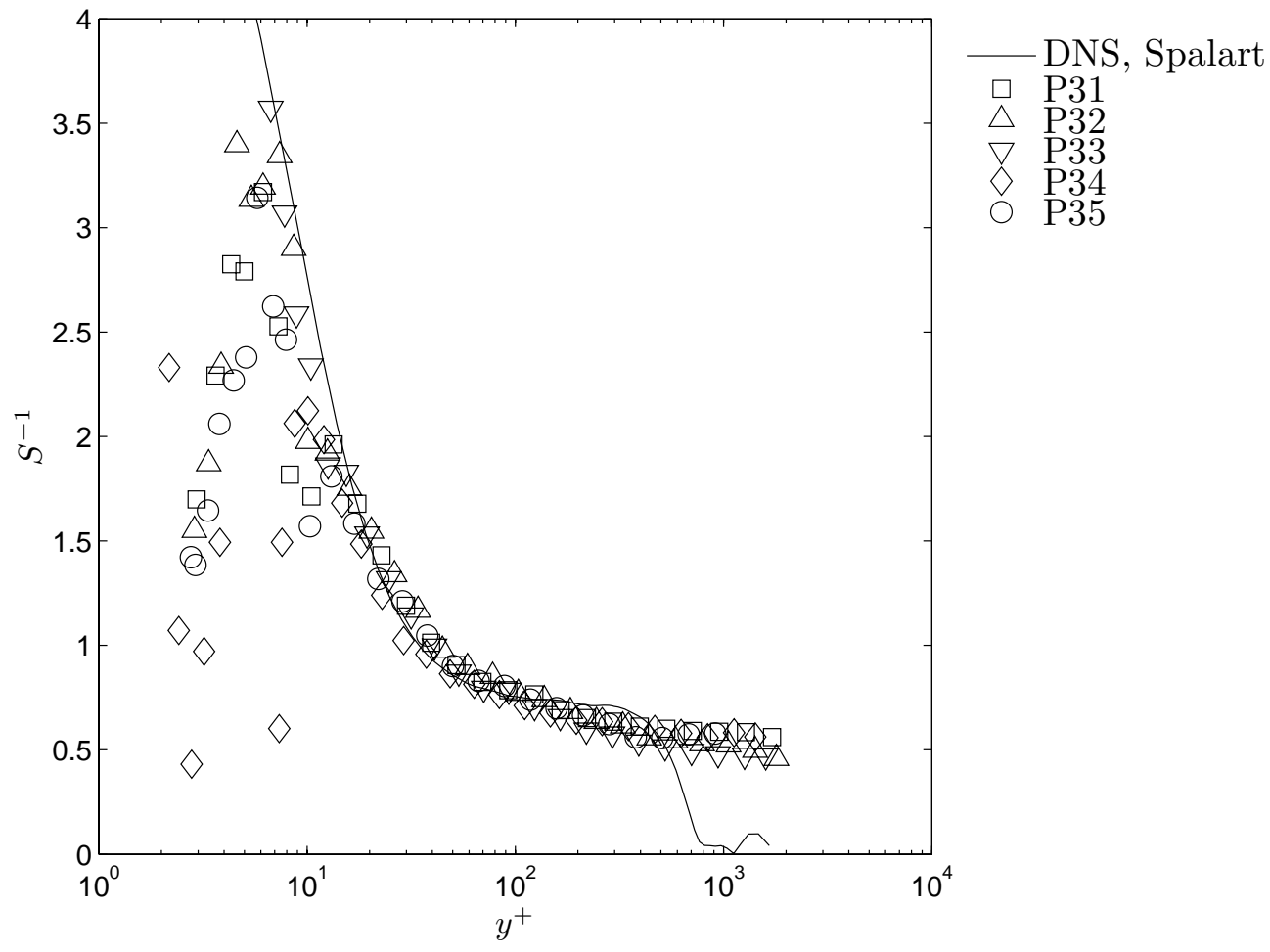

(g) $S^{-1}$ Parameter

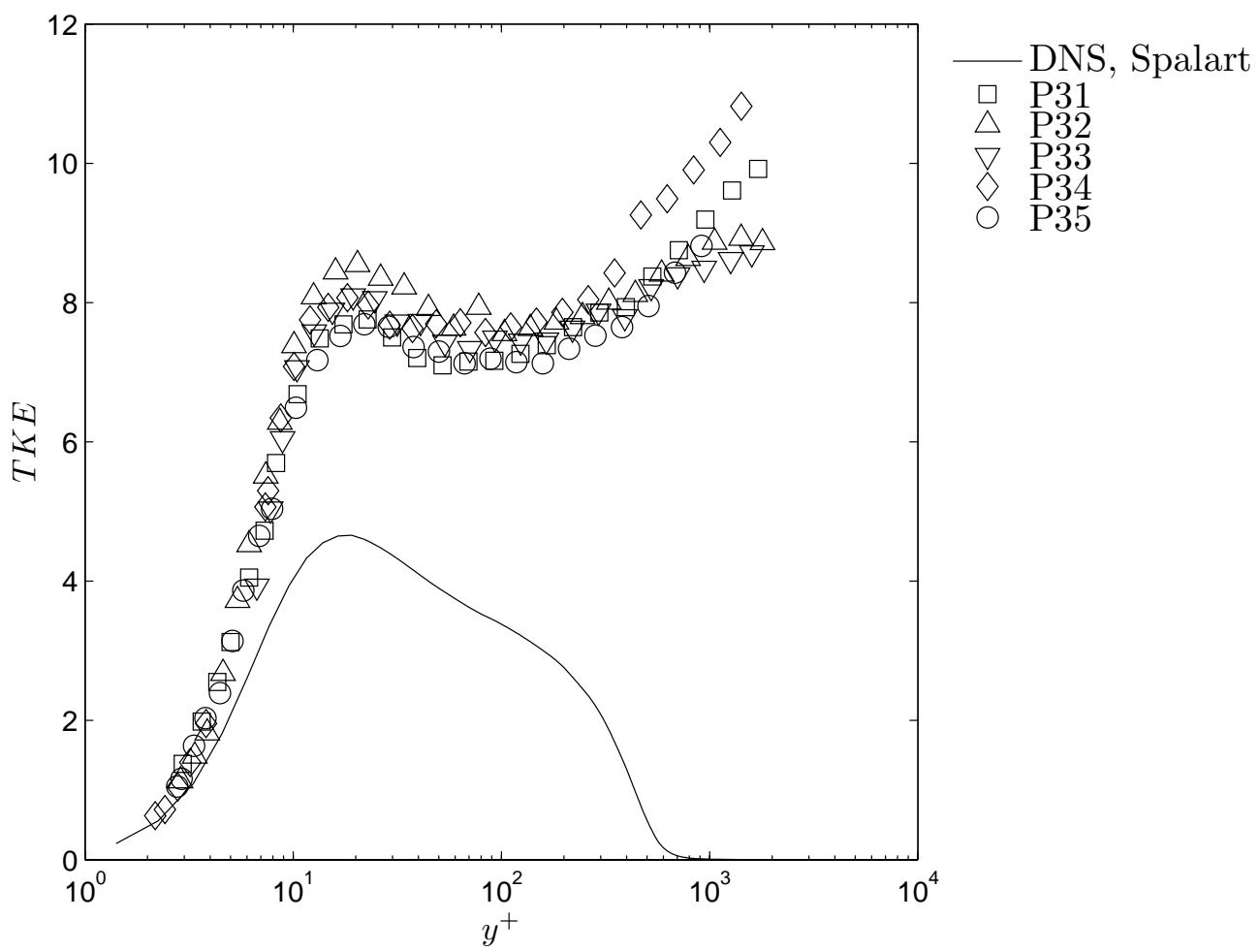

(h) Turbulent Kinetic Energy, TKE

Figure 4.8: Continued, P33 Cluster 


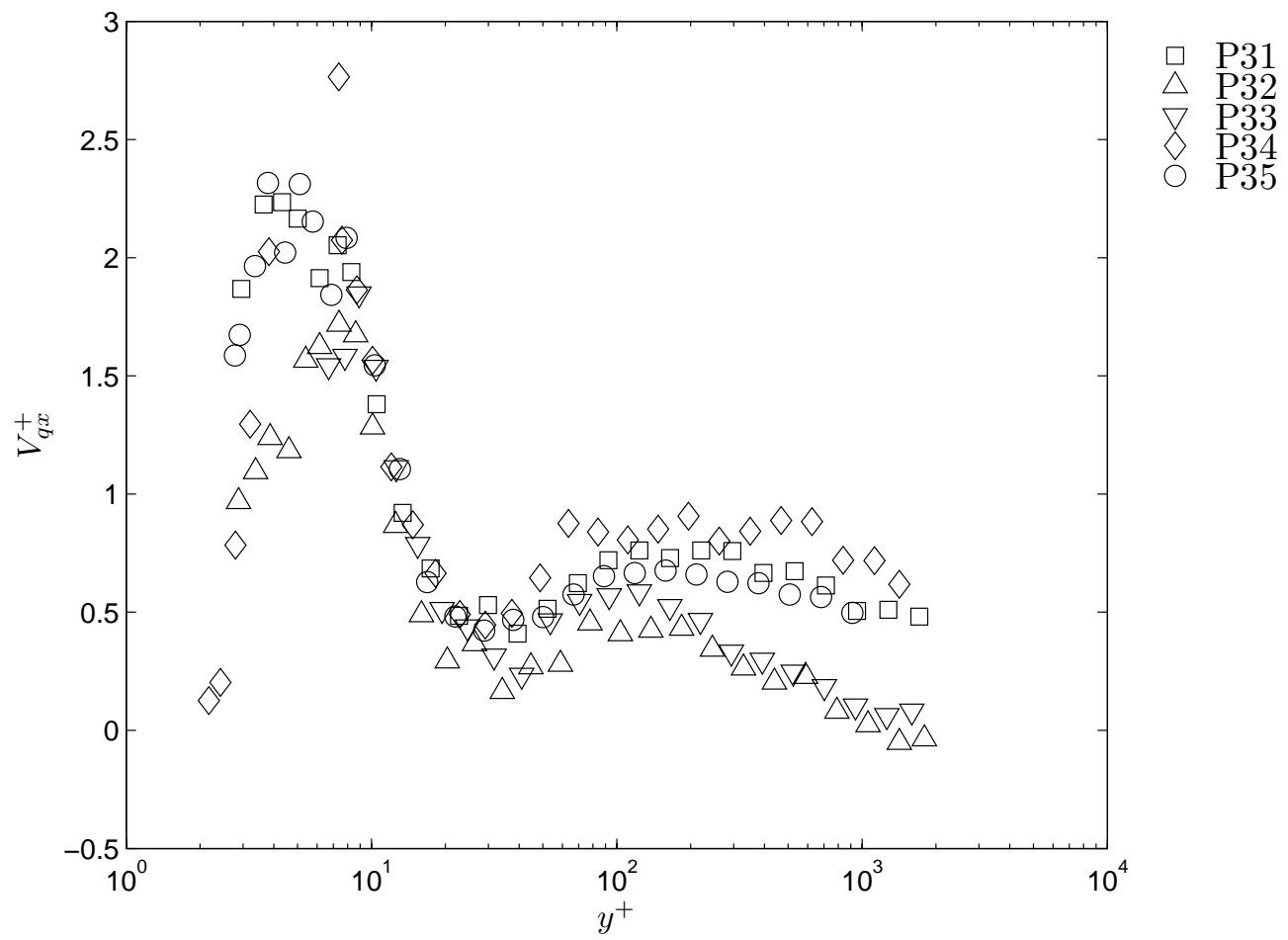

(i) Stream-wise Turbulent Diffusion Velocity component, $V_{q x}$, normalized on $u_{\tau}$

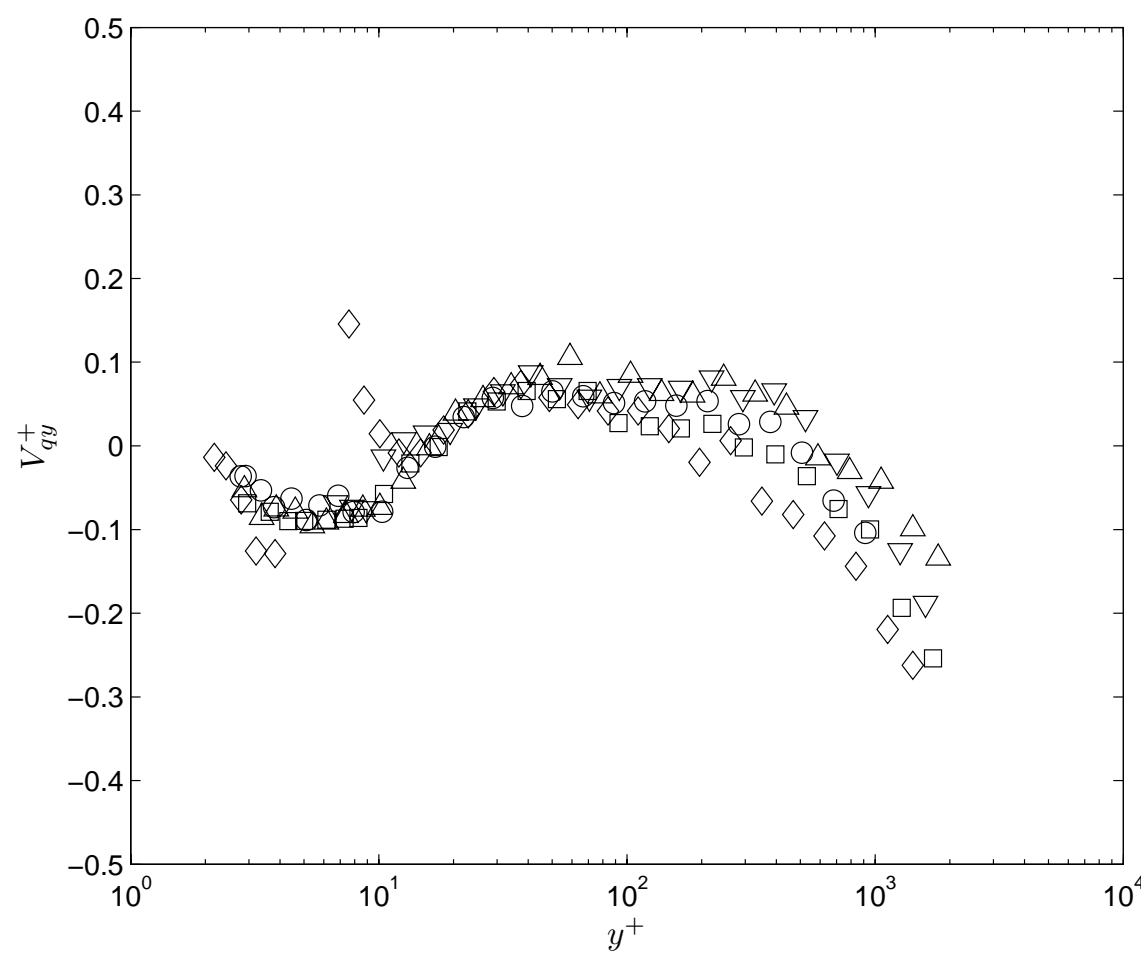

$\begin{array}{ll}\square & \text { P31 } \\ \triangle & \text { P32 } \\ \nabla & \text { P33 } \\ \searrow & \text { P34 } \\ \bigcirc & \text { P35 }\end{array}$

(j) Wall-Normal Turbulent Diffusion Velocity component, $V_{q y}$, normalized on $u_{\tau}$

Figure 4.8: Continued, P33 Cluster 


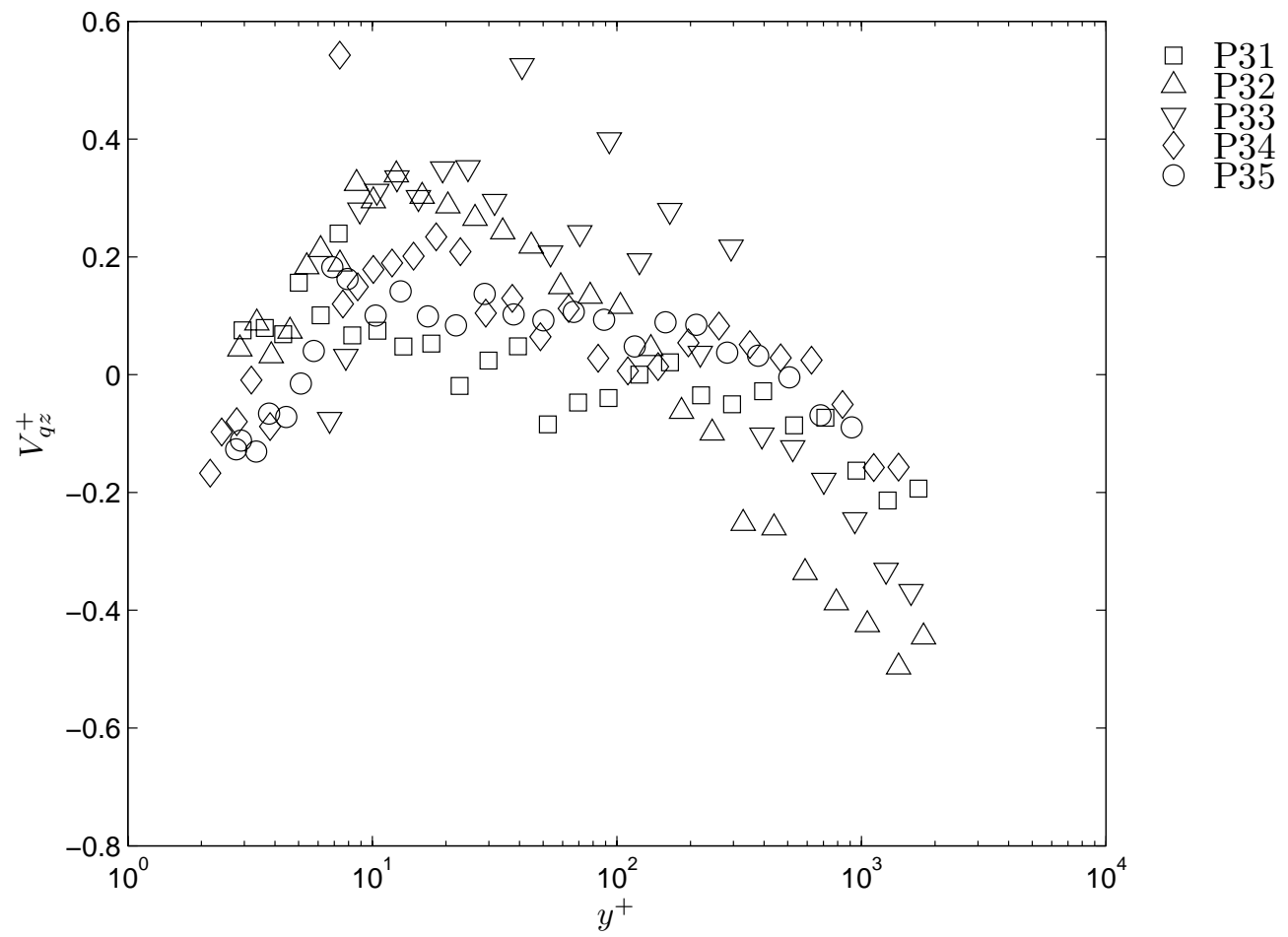

(k) Span-wise Turbulent Diffusion Velocity component, $V_{q z}$, normalized on $u_{\tau}$

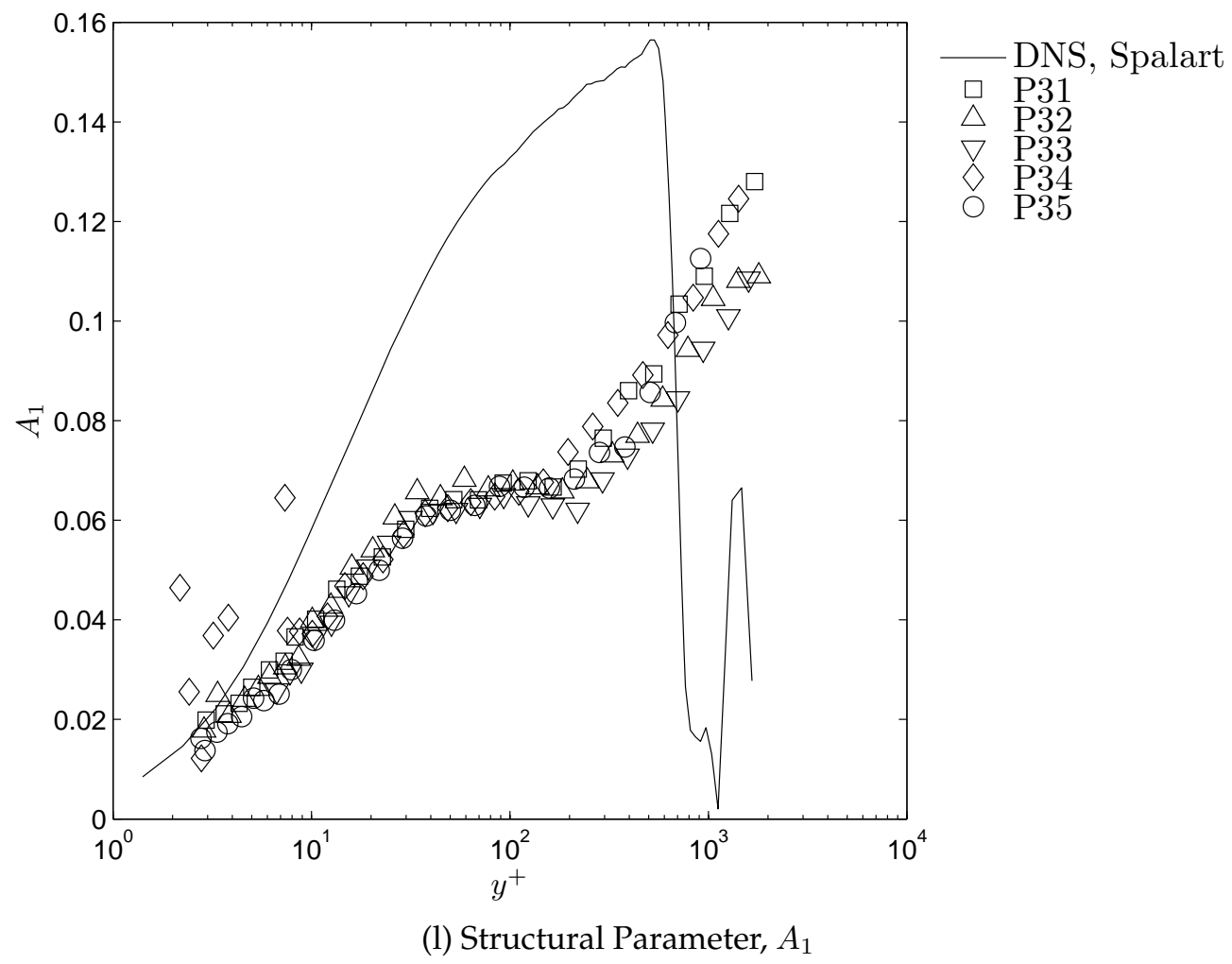

Figure 4.8: Continued, P33 Cluster 


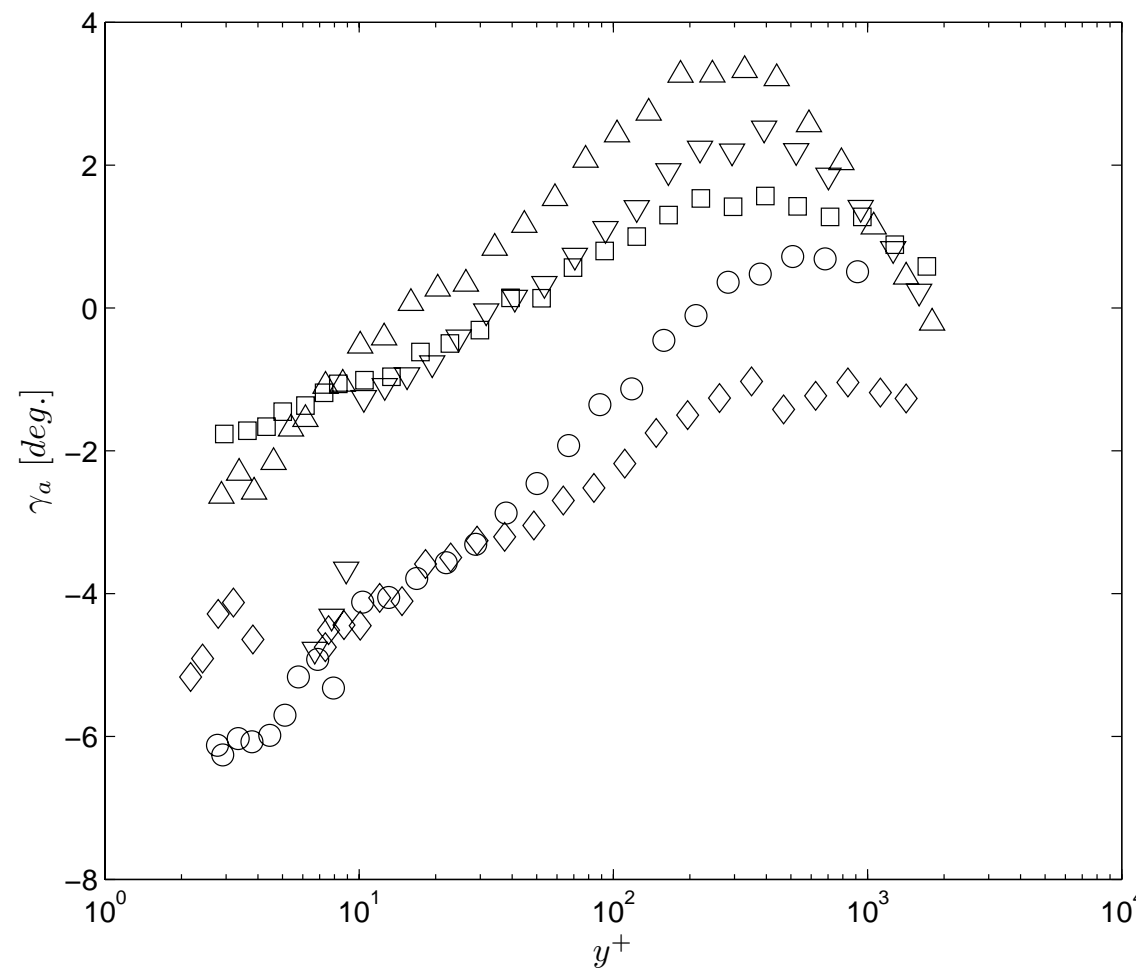

$\begin{array}{ll}\square & \text { P31 } \\ \triangle & \text { P32 } \\ \nabla & \text { P33 } \\ \searrow & \text { P34 } \\ \bigcirc & \text { P35 }\end{array}$

(m) Flow Angle, $\gamma_{a}$

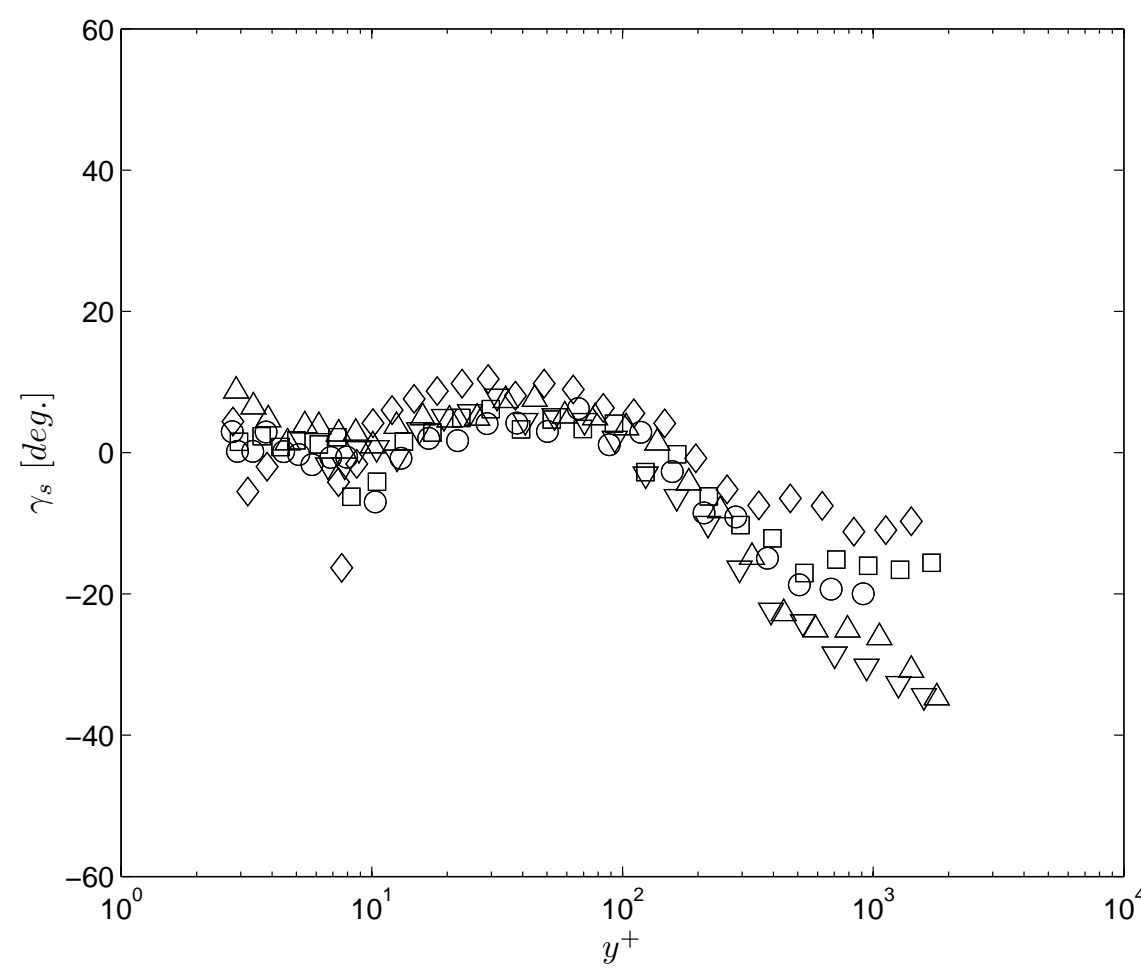

$\begin{array}{ll}\square & \text { P31 } \\ \triangle & \text { P32 } \\ \nabla & \text { P33 } \\ \searrow & \text { P34 } \\ \text { ○ } & \text { P35 }\end{array}$

(n) Shear Stress Angle $\gamma_{s}$

Figure 4.8: Continued, P33 Cluster 


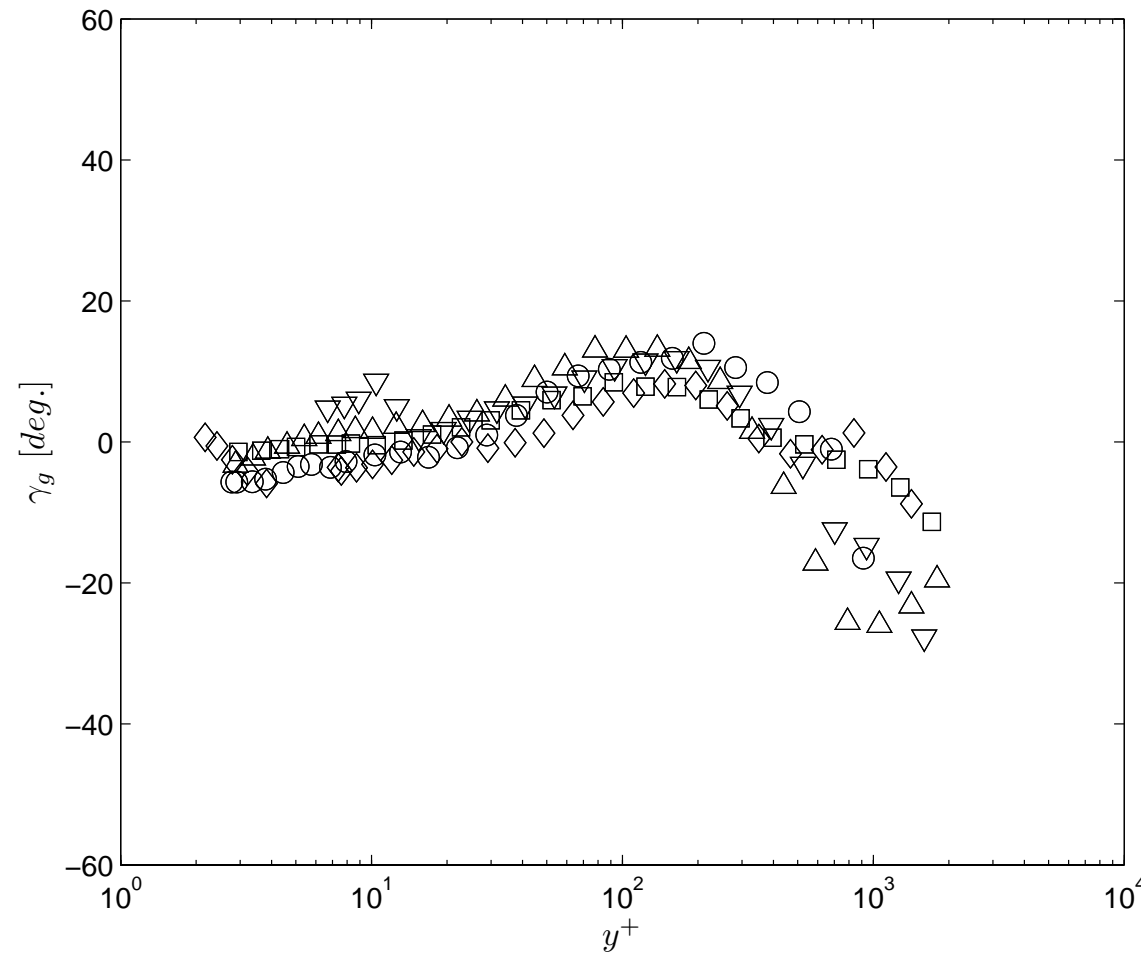

$\begin{array}{ll}\square & \text { P31 } \\ \triangle & \text { P32 } \\ \nabla & \text { P33 } \\ \searrow & \text { P34 } \\ \bigcirc & \text { P35 }\end{array}$

(o) Flow Gradient Angle $\gamma_{g}$

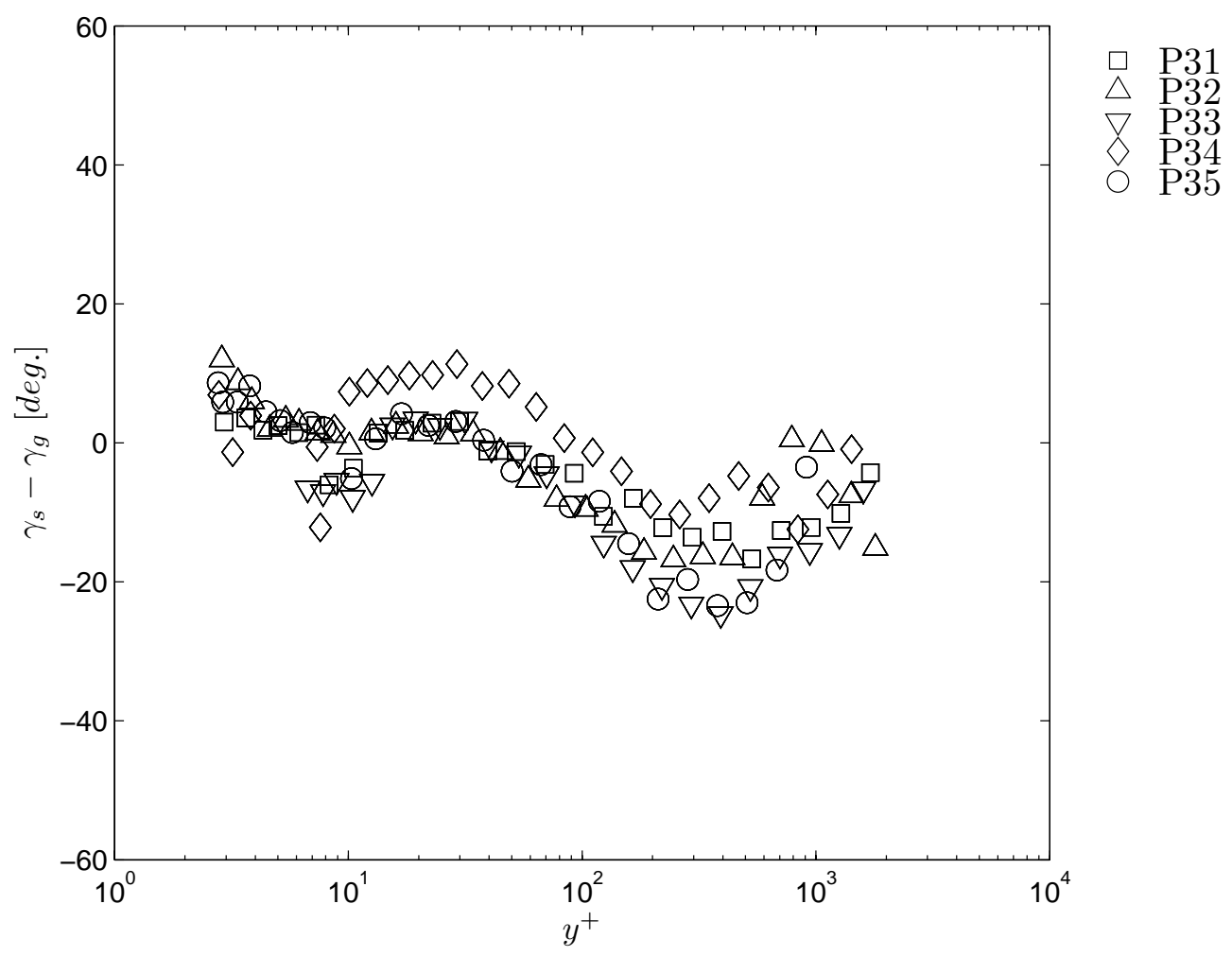

(p) Difference between Shear Angle and Flow Gradient Angles, $\gamma_{s}-\gamma_{g}$

Figure 4.8: Continued, P33 Cluster 


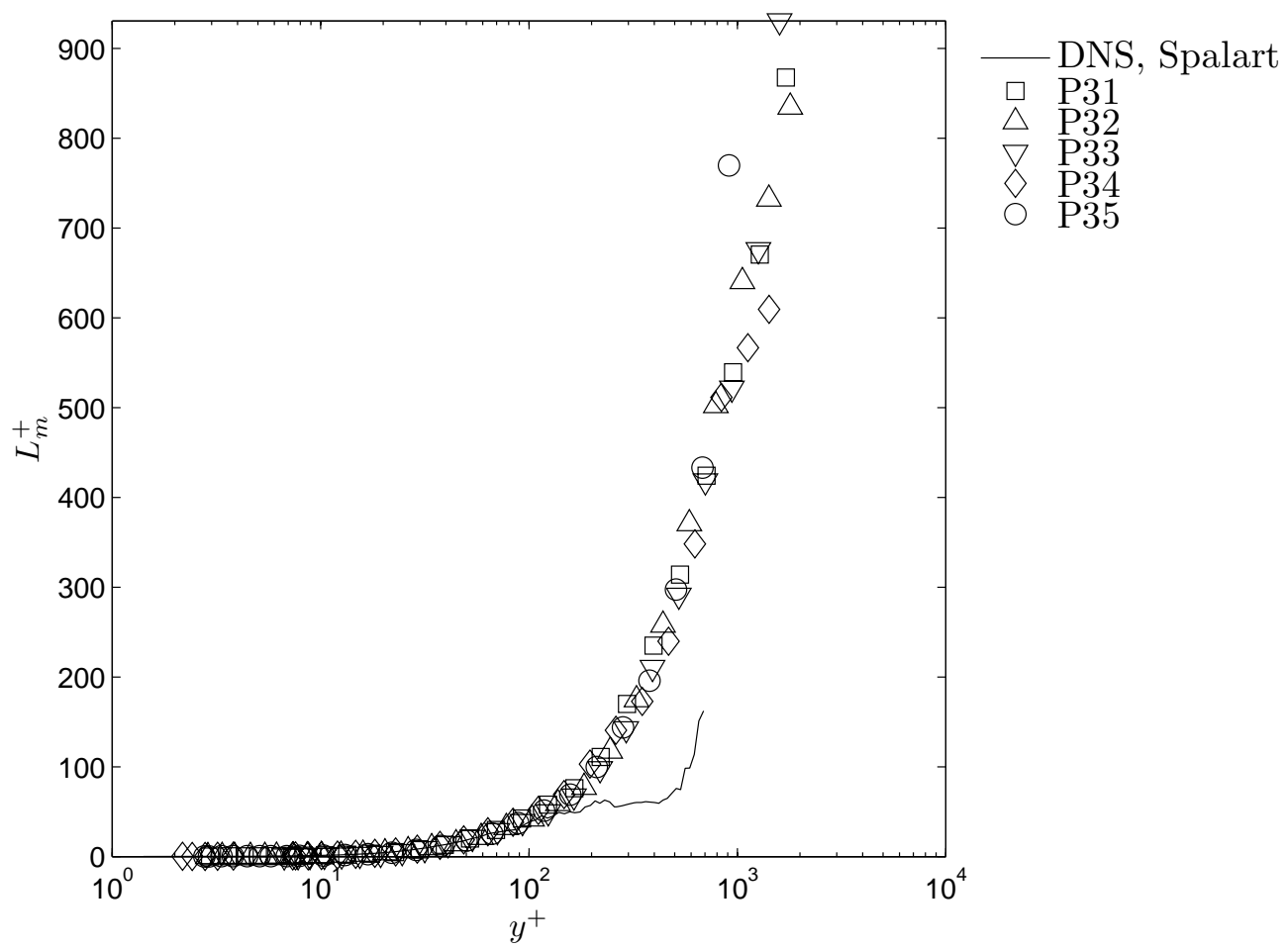

(q) Mixing Length $L_{m}^{+}$

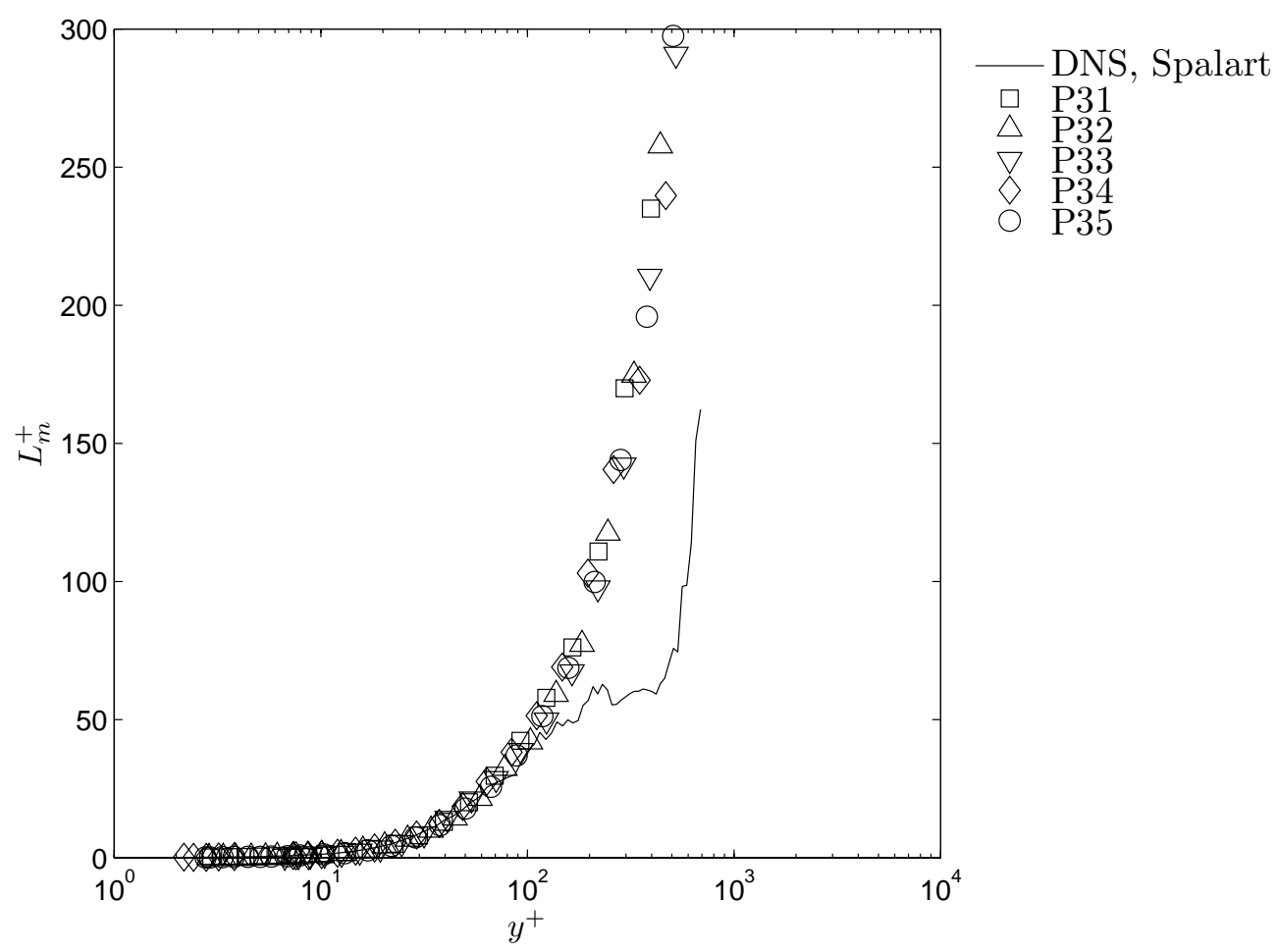

(r) Near Wall Mixing Length $L_{m}^{+}$

Figure 4.8: Continued, P33 Cluster 


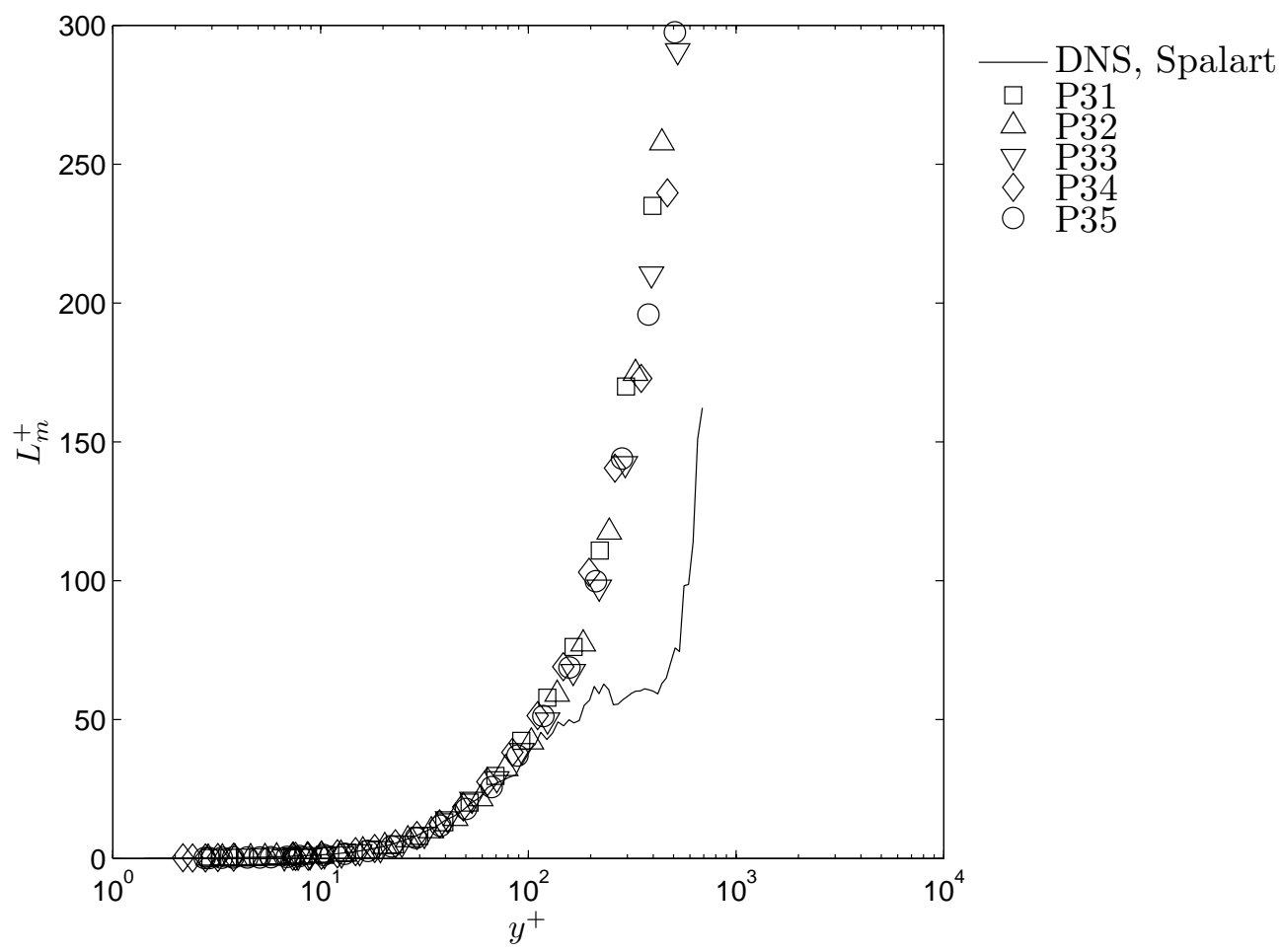

(s) Turbulent Eddy Viscosity $\nu_{T x}$

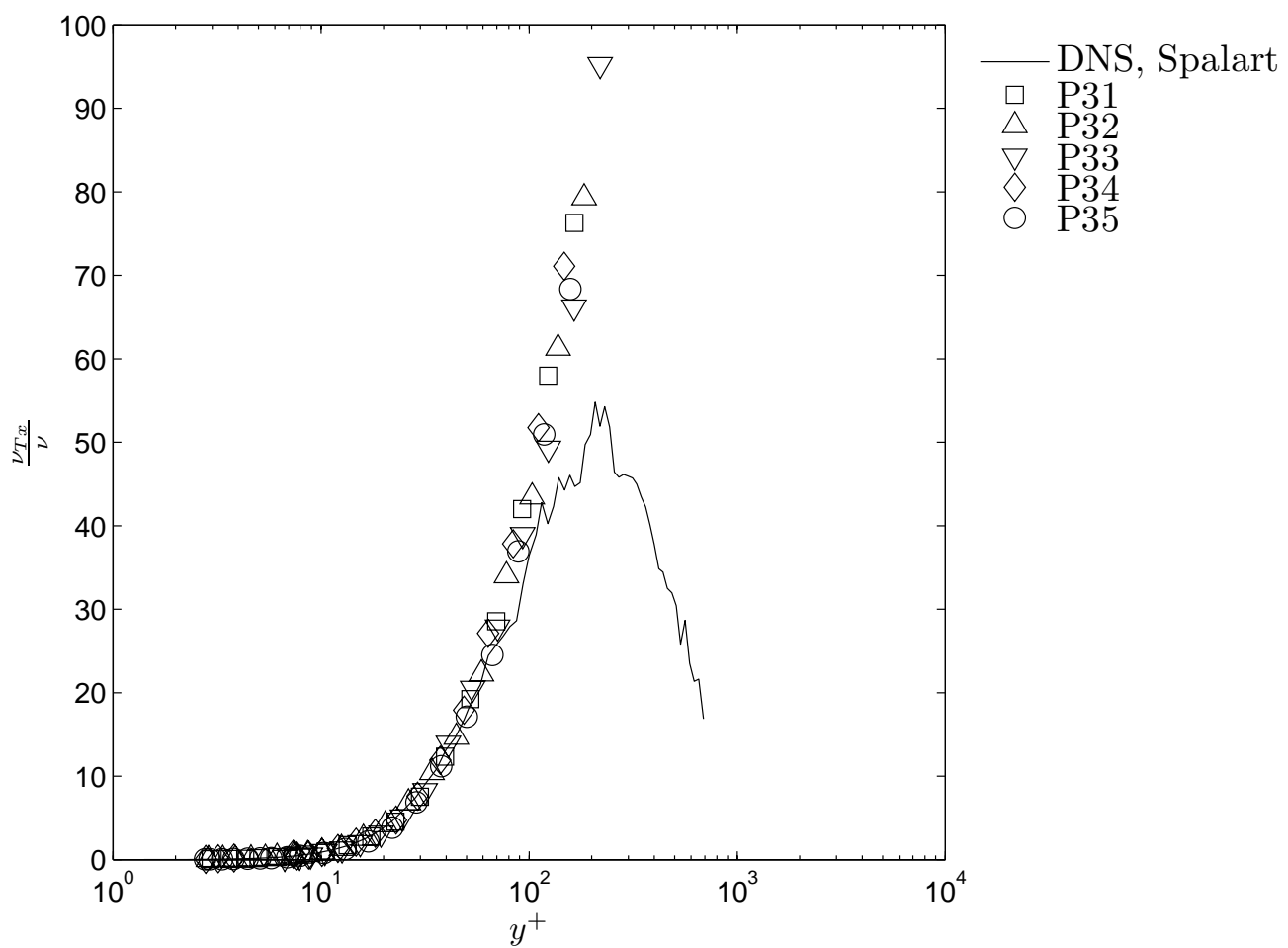

(t) Near Wall Turbulent Eddy Viscosity $\nu_{T x}$

Figure 4.8: Concluded, P33 Cluster 

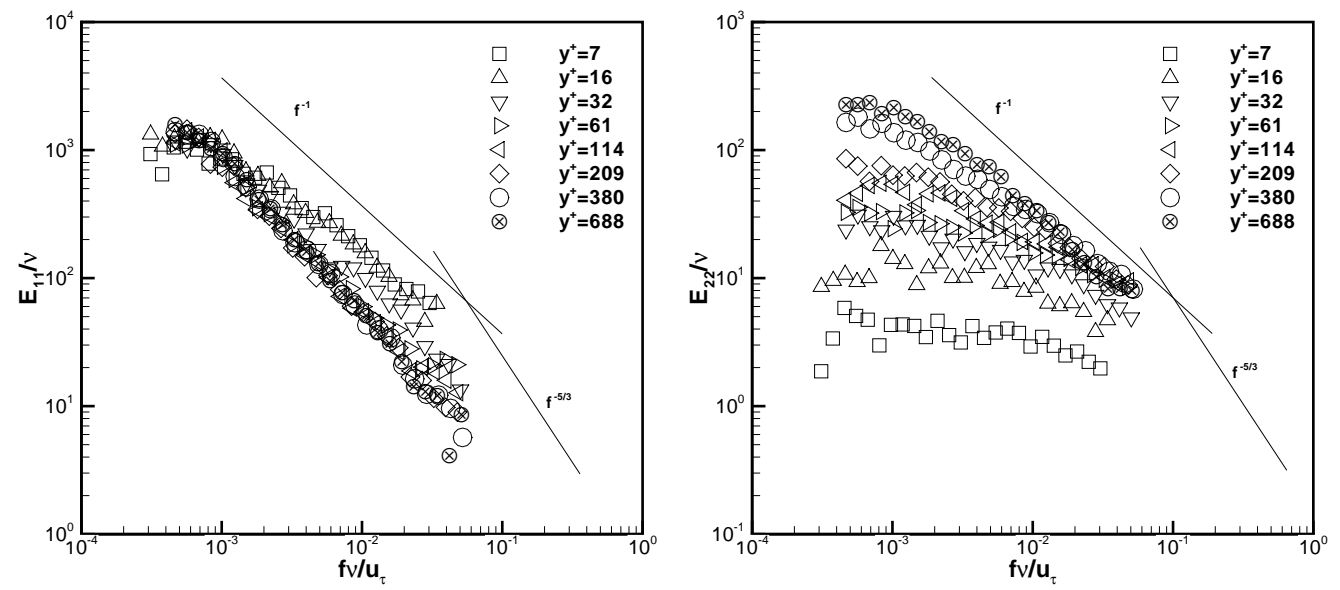

(a) ${\overline{u^{2}}}^{+}$Normal Stress Power Spectra, $E_{11}$

(b) ${\overline{v^{2}}}^{+}$Normal Stress Power Spectra, $E_{22}$
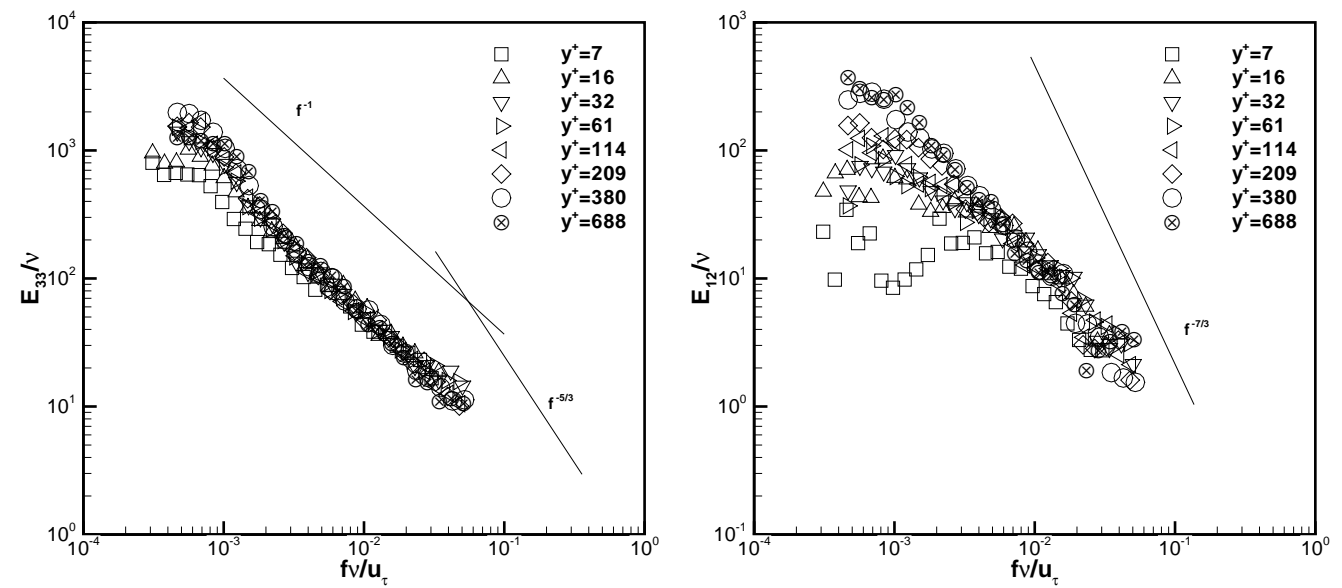

(c) ${\overline{w^{2}}}^{+}$Normal Stress Power Spectra, $E_{33}$

(d) $\overline{u v}+$ Shear Stress Cross Spectra, $E_{12}$

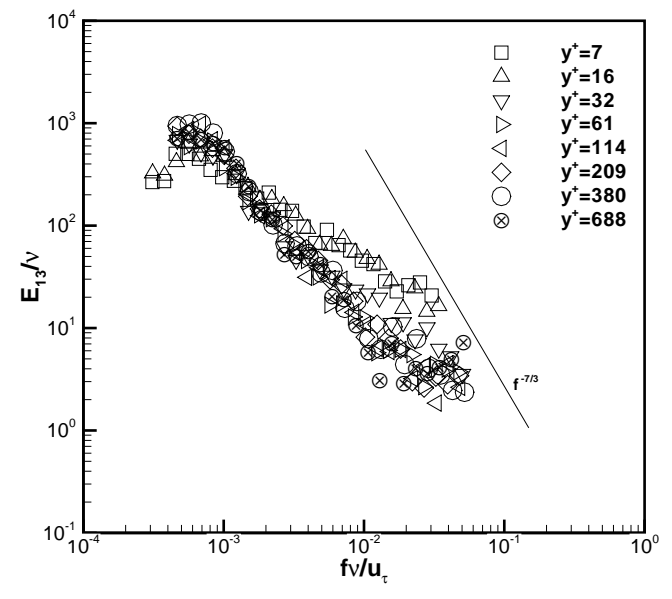

(e) $\overline{u w}+$ Shear Stress Cross Spectra, $E_{13}$

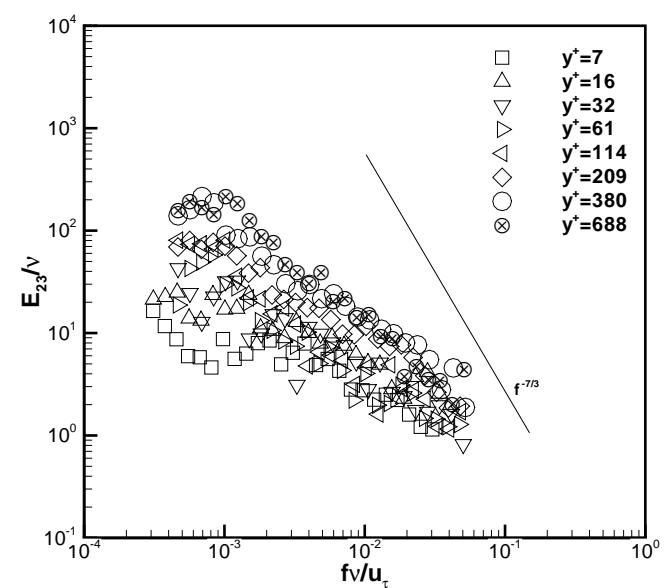

(f) $\overline{v w}{ }^{+}$Shear Stress Cross Spectra, $E_{23}$

Figure 4.9: One-Dimensional Spectra of Reynolds Stresses at Profile P18 

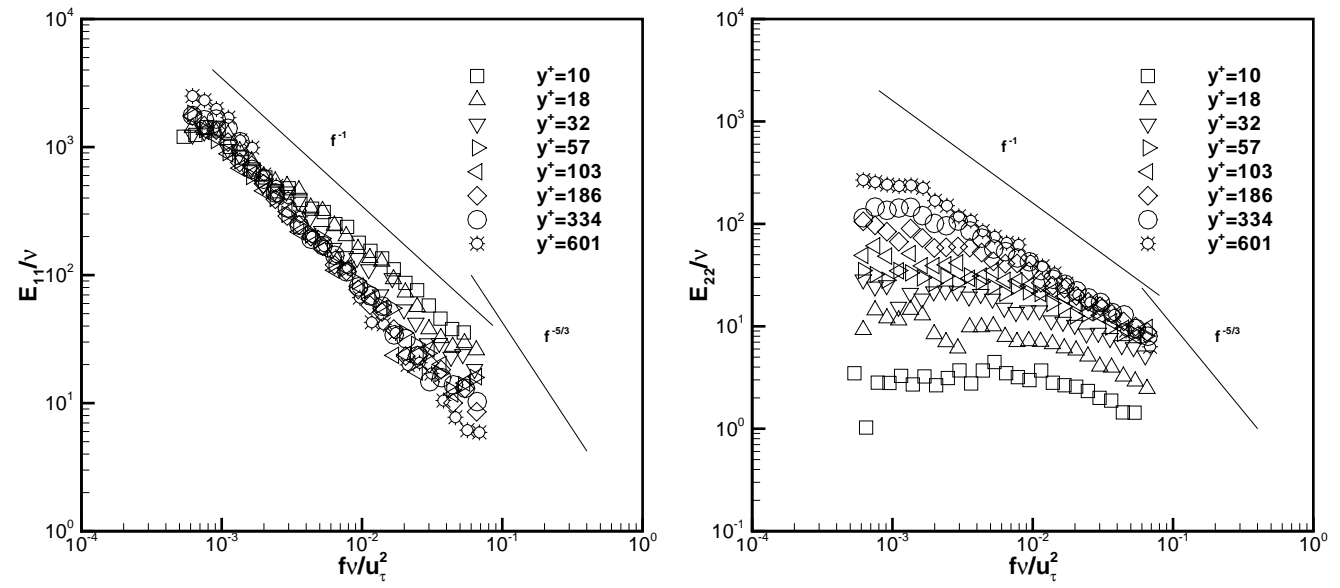

(a) $\bar{u}^{2}$ Normal Stress Power Spectra, $E_{11}$

(b) ${\overline{v^{2}}}^{+}$Normal Stress Power Spectra, $E_{22}$
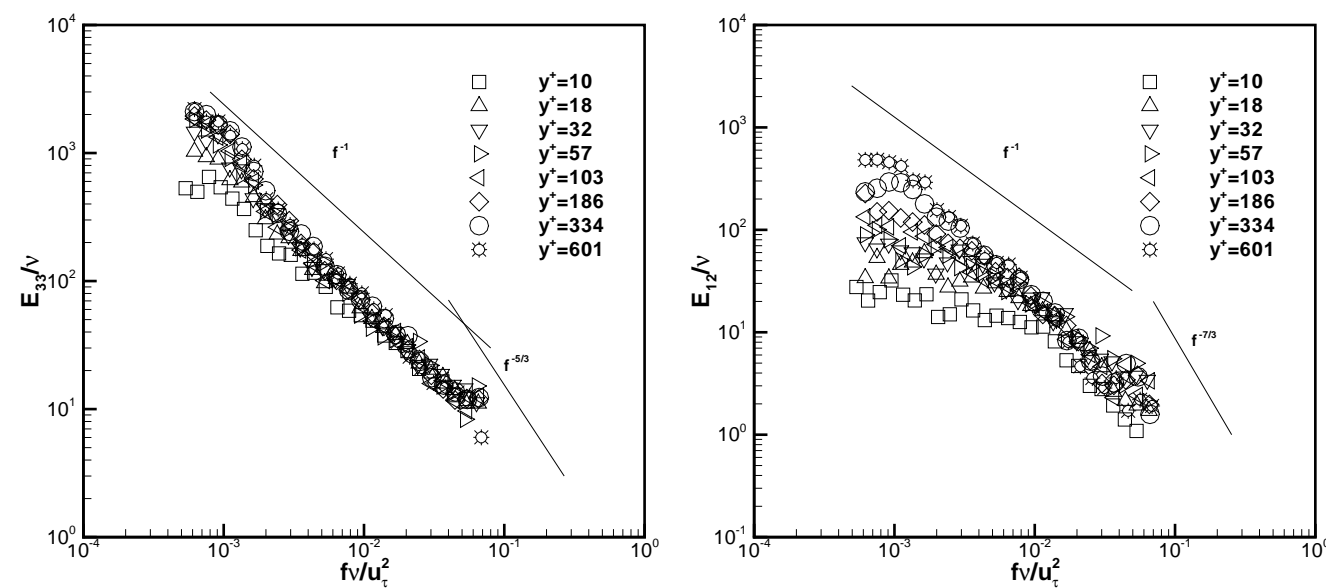

(c) ${\overline{w^{2}}}^{+}$Normal Stress Power Spectra, $E_{33}$

(d) $\overline{u v}^{+}$Shear Stress Cross Spectra, $E_{12}$

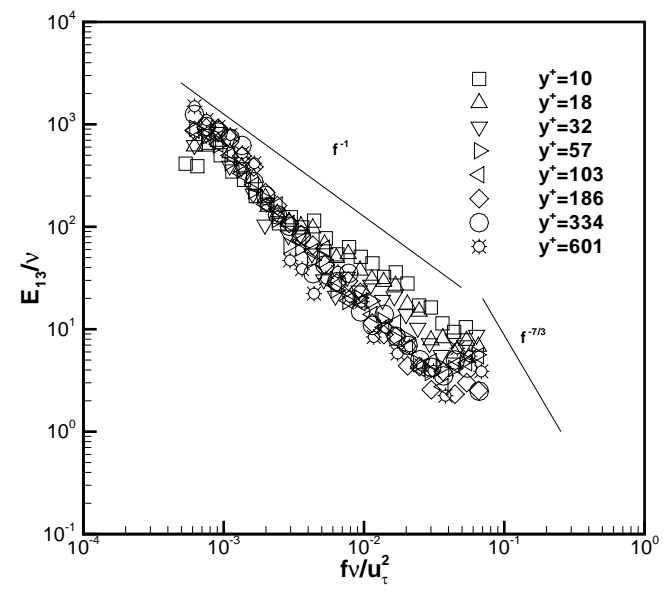

(e) $\overline{u w}+$ Shear Stress Cross Spectra, $E_{13}$

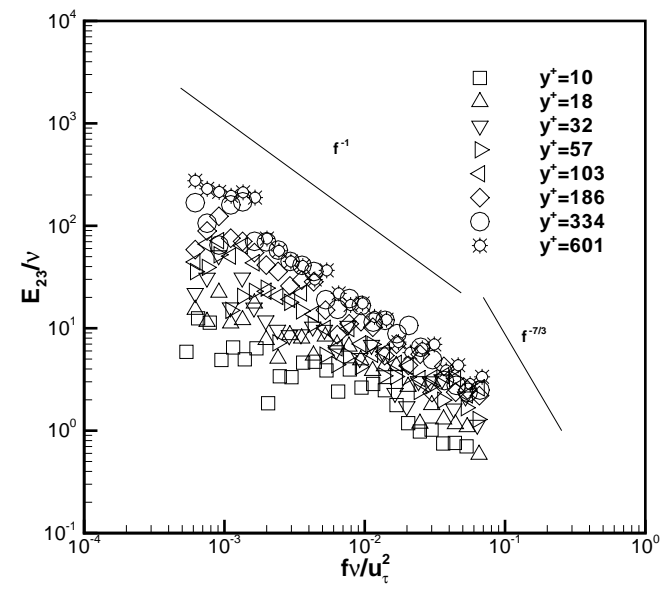

(f) $\overline{v w}^{+}$Shear Stress Cross Spectra, $E_{23}$

Figure 4.10: One-Dimensional Spectra of Reynolds Stresses at Profile P19p 

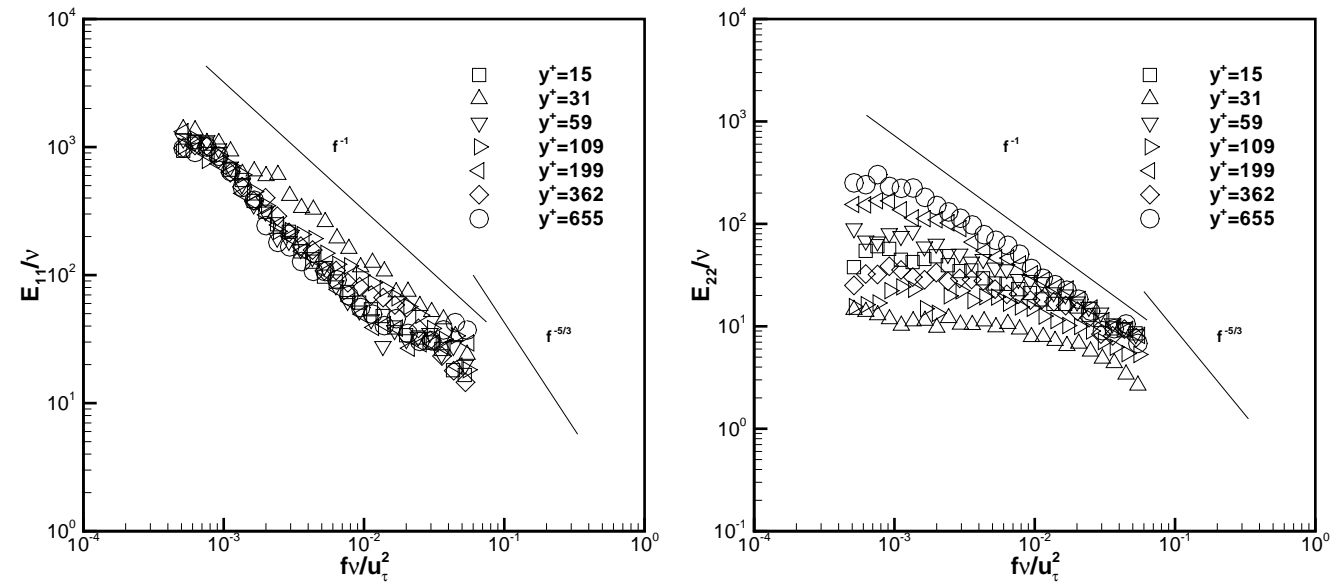

(a) ${\overline{u^{2}}}^{+}$Normal Stress Power Spectra, $E_{11}$

(b) ${\overline{v^{2}}}^{+}$Normal Stress Power Spectra, $E_{22}$
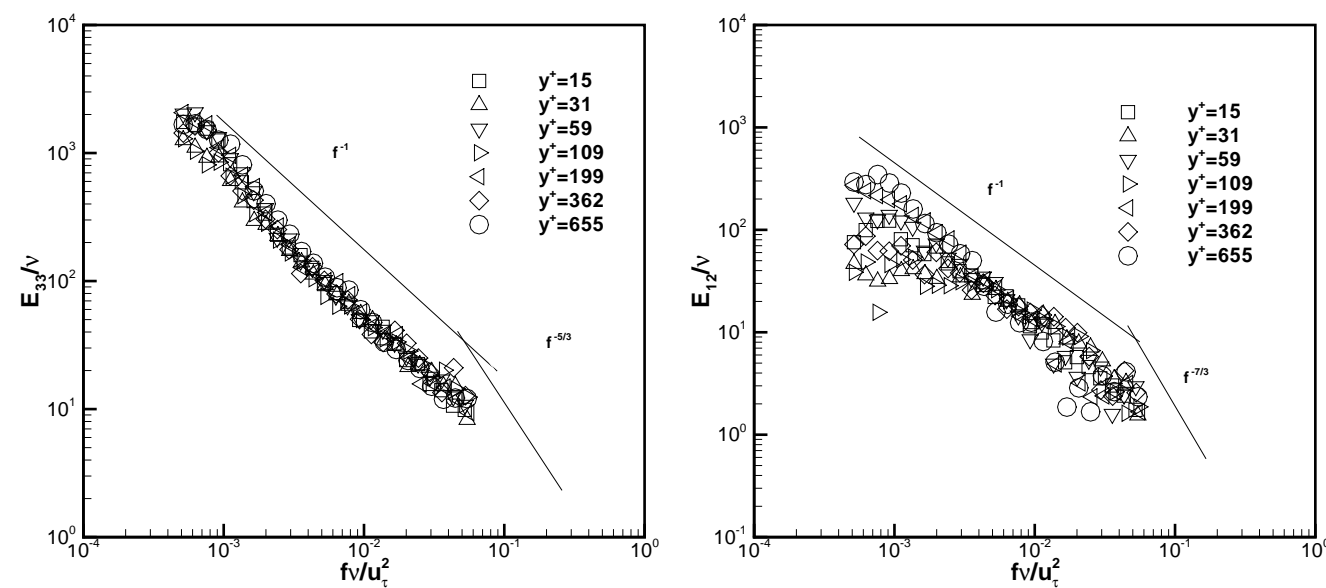

(c) ${\overline{w^{2}}}^{+}$Normal Stress Power Spectra, $E_{33}$

(d) $\overline{u v}+$ Shear Stress Cross Spectra, $E_{12}$

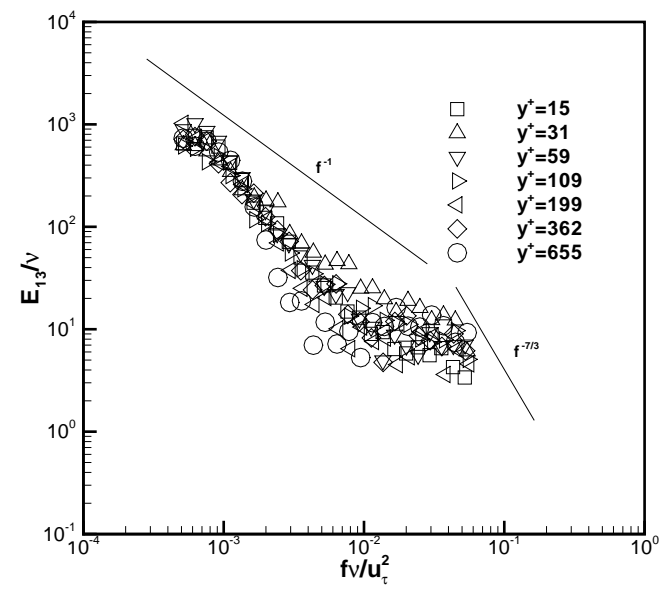

(e) $\overline{u w}{ }^{+}$Shear Stress Cross Spectra, $E_{13}$

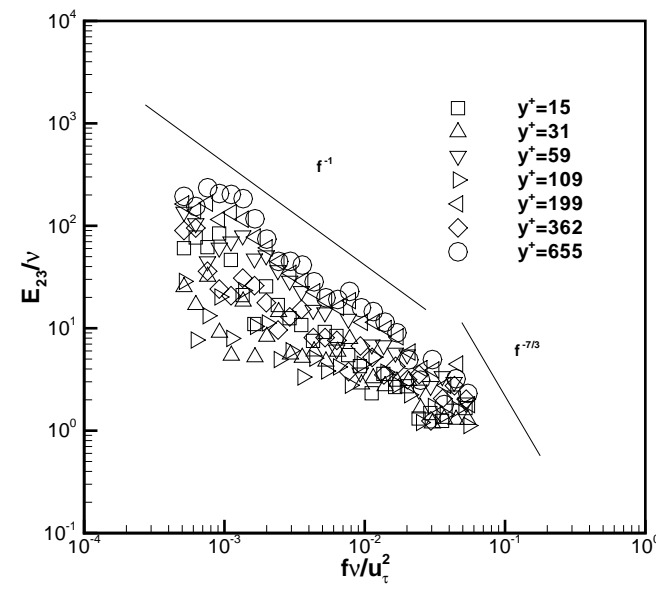

(f) $\overline{v w^{+}}$Shear Stress Cross Spectra, $E_{23}$

Figure 4.11: One-Dimensional Spectra of Reynolds Stresses at Profile P19 

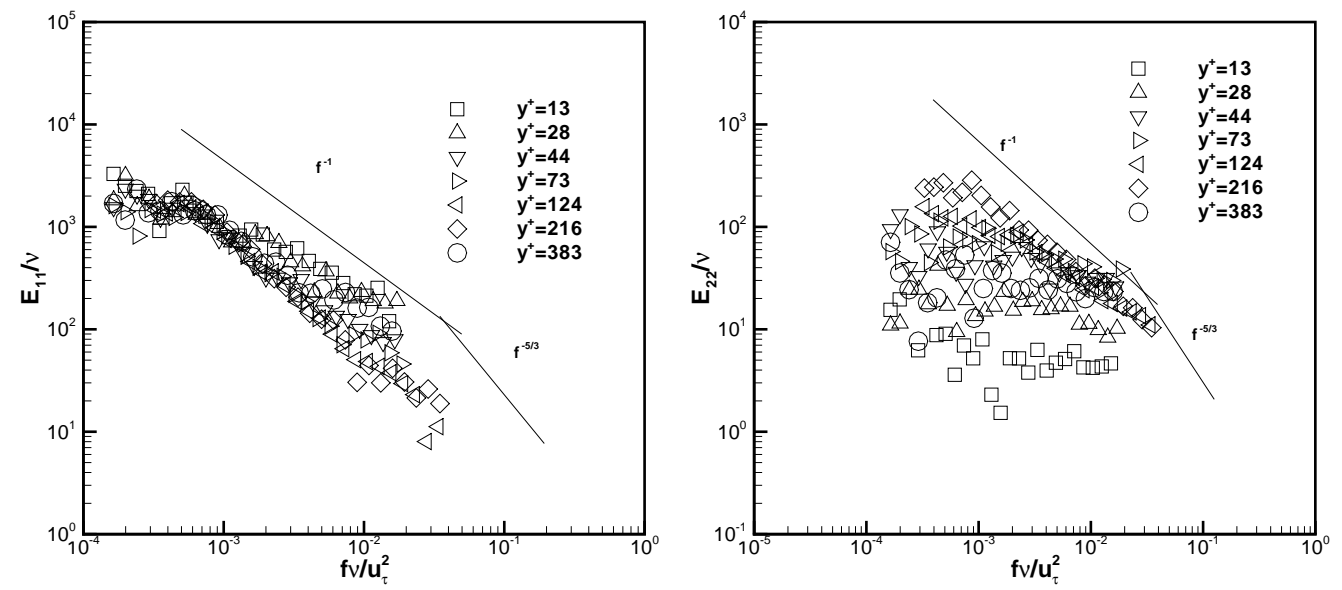

(a) $\bar{u}^{2}$ Normal Stress Power Spectra, $E_{11}$

(b) ${\overline{v^{2}}}^{+}$Normal Stress Power Spectra, $E_{22}$
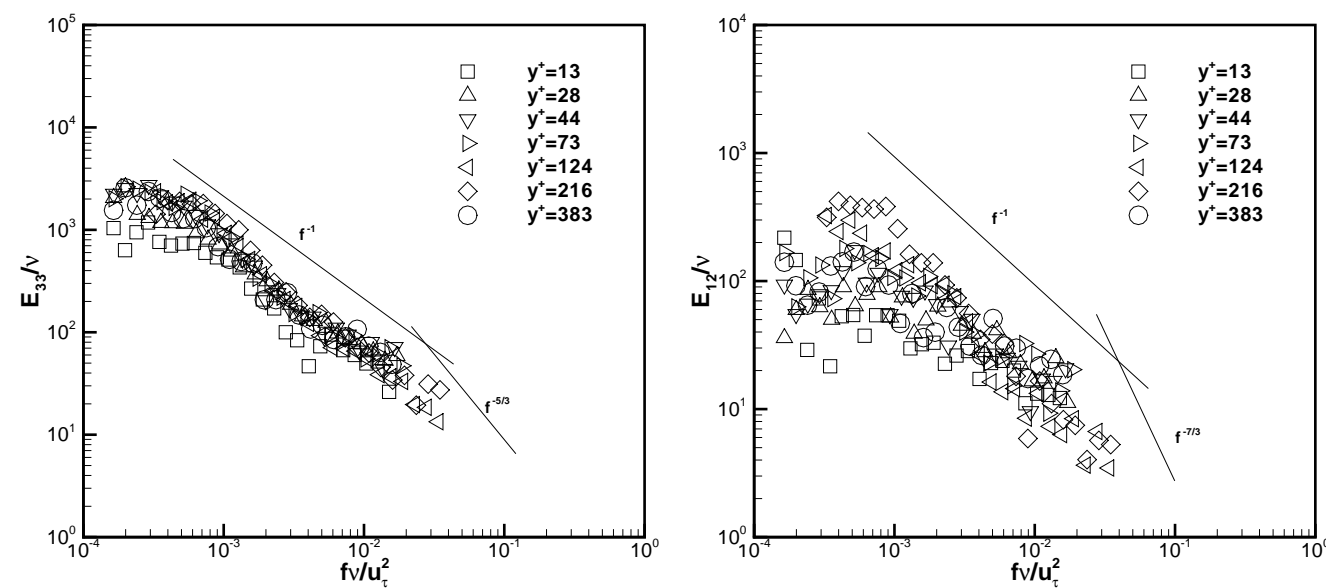

(c) ${\overline{w^{2}}}^{+}$Normal Stress Power Spectra, $E_{33}$

(d) $\overline{u v}^{+}$Shear Stress Cross Spectra, $E_{12}$

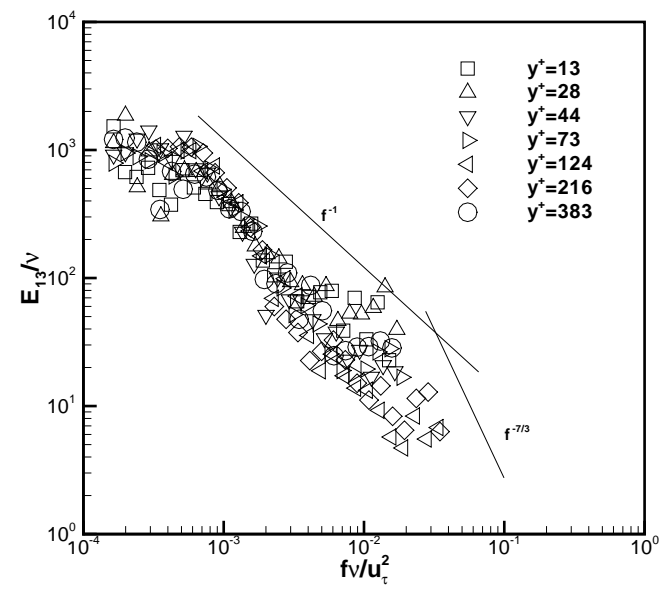

(e) $\overline{u w}^{+}$Shear Stress Cross Spectra, $E_{13}$

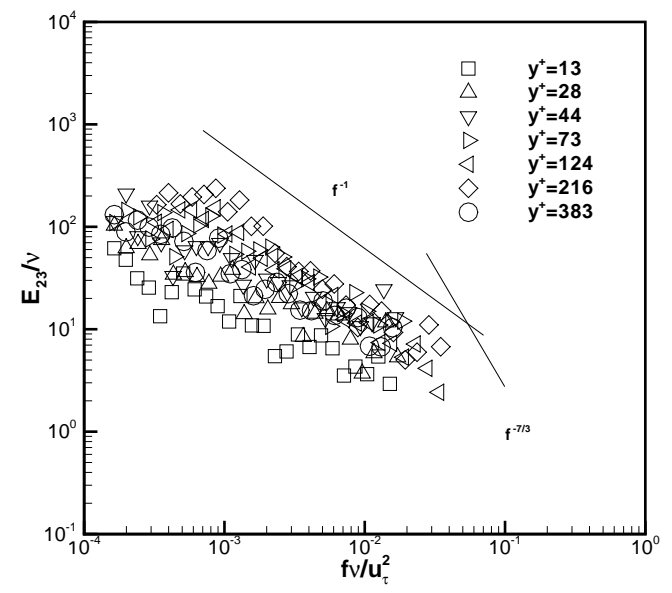

(f) $\overline{v w}^{+}$Shear Stress Cross Spectra, $E_{23}$

Figure 4.12: One-Dimensional Spectra of Reynolds Stresses at Profile $P 19_{1}$ 


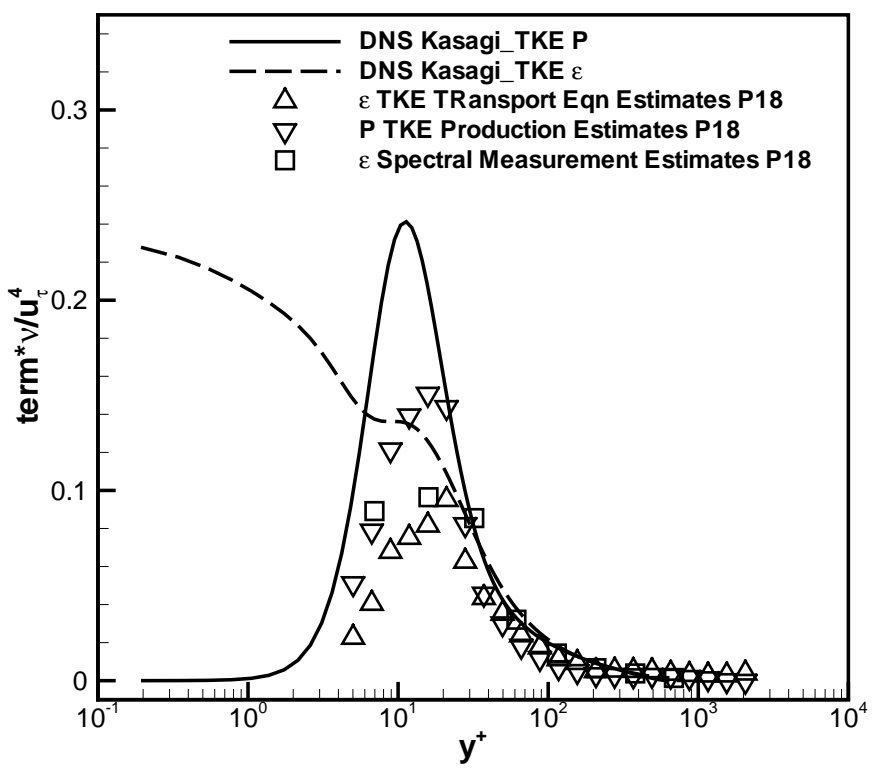

(a) $P 18$, Turbulence Kinetic Energy Dissipation Rate $\epsilon^{+}$

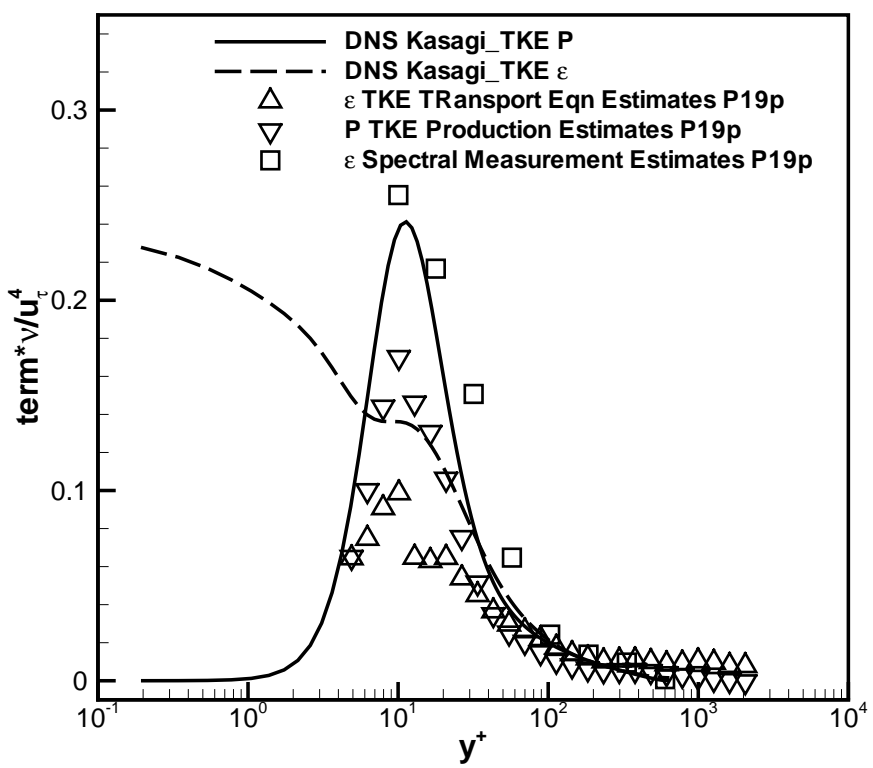

(b) $P 19 p$, Turbulence Kinetic Energy Dissipation Rate $\epsilon^{+}$

Figure 4.13: $\epsilon^{+}$Estimates 


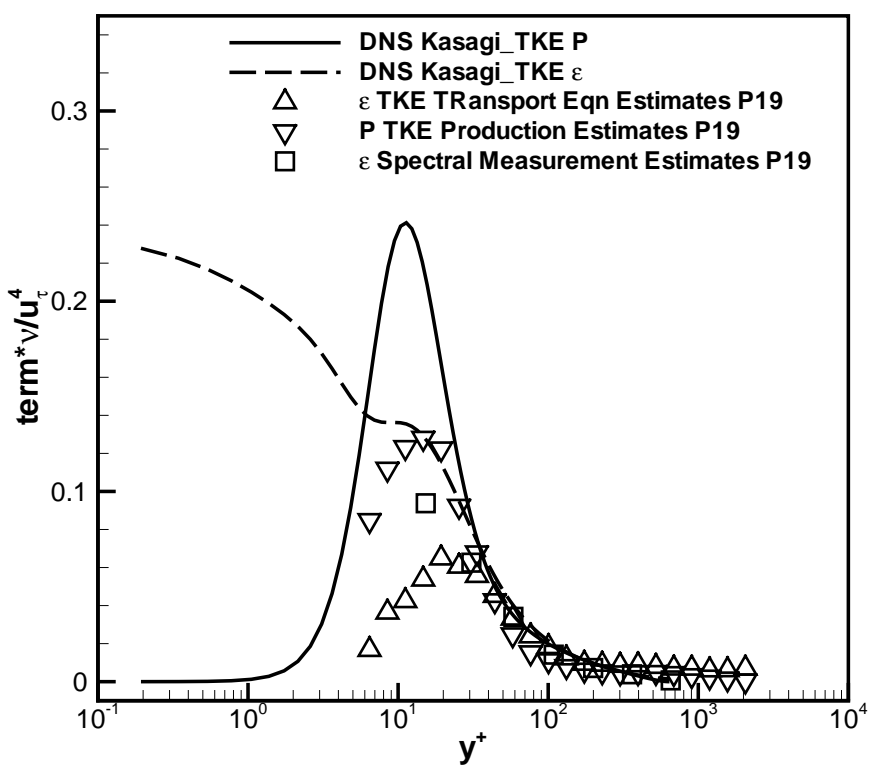

(c) P19, Turbulence Kinetic Energy Dissipation Rate $\epsilon^{+}$

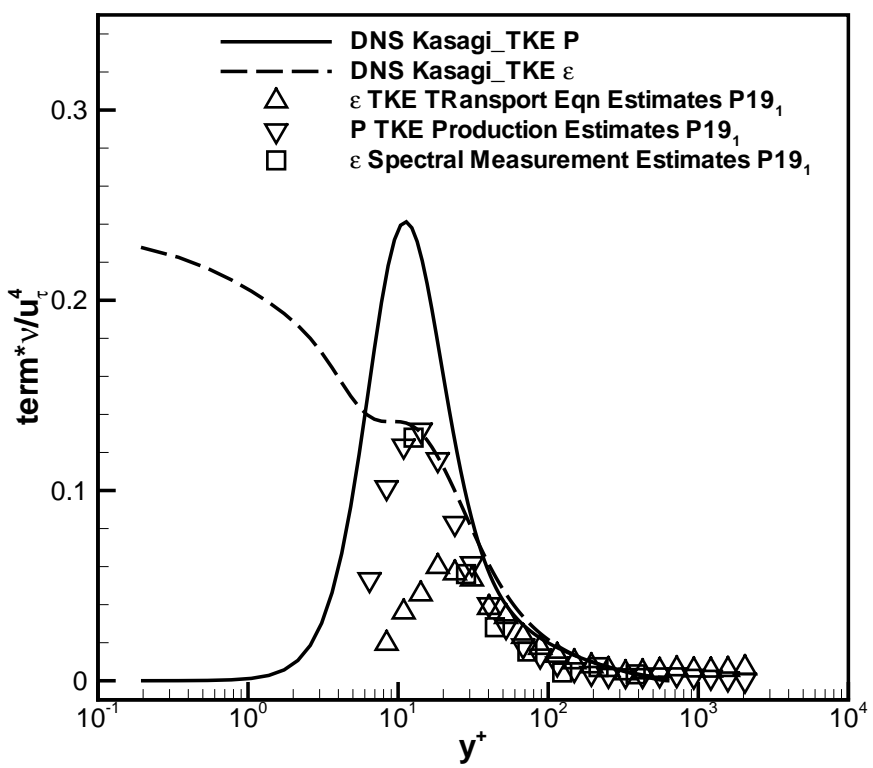

(d) $P 19_{1}$, Turbulence Kinetic Energy Dissipation Rate $\epsilon^{+}$

Figure 4.13: $\epsilon^{+}$Estimates 


\section{Chapter 5}

\section{Discussion}

\subsection{Features of Interest}

The data acquired in this experiment possess very distinctive features, some of which are not present in typical three-dimensional attached boundary layer flows. In this chapter those features will be highlighted and an attempt to explain them will be made. The most distinctive features are:

- Very high Turbulence intensities in the outer layer, $\dot{u} / U_{\infty} \approx 0.15$

- Low normal, ${\overline{v^{2}}}^{+}$, and shear, $\overline{u v}^{+}$, stresses near the wall

- Profiles of turbulent quantities show multiple peaks

- In some locations (P18, P19 and P20) all normal stresses reaches a state close to isotropy near the outer region, while the shear stresses are non-zero

- At the centerline, all stresses behave close to those for a 2-D boundary layer; however, in the outer layer turbulence levels reach very high values

- The mixing length shows a linear behavior in a log-log scale with regions of different approximately constant slopes

In the next section the flow field results are discussed.

\subsection{Discussion}

In order to gain an understanding of the flow development, Reynolds' Stress transport budget is performed and the transport equations are examined along with mean flow 
data acquired using the seven-hole probe and the calculated potential flow field.

\subsubsection{Reynolds' Stress Transport Budget}

Examining the Reynolds Transport equations gives an insight into the development of turbulence quantities. The Reynolds stress transport budget shows the importance of each term in the governing equations. The transport equations are given by Equation 5.1 .

$$
\underbrace{\frac{\partial \overline{u_{i} u_{j}}}{\partial t}}_{L_{i j}}+\underbrace{U_{l} \frac{\partial \overline{u_{i} u_{j}}}{\partial x_{l}}}_{C_{i j}}=\underbrace{-\overline{u_{i} u_{l}} \frac{\partial U_{j}}{\partial x_{l}}-\overline{u_{j} u_{l}} \frac{\partial U_{i}}{\partial x_{l}}}_{P_{i j}}-\underbrace{\overline{u_{i} \frac{\partial p}{\partial x_{j}}}-\overline{u_{j} \frac{\partial p}{\partial x_{i}}}}_{\Pi_{i j}}+\underbrace{\nu \frac{\partial^{2} \overline{u_{i} u_{j}}}{\partial x_{l}^{2}}}_{D^{v}}-\underbrace{\frac{\partial \overline{u_{i} u_{j} u_{k}}}{\partial x_{k}}}_{T_{i j}}-\epsilon_{i j}
$$

Where,

- $L_{i j} \quad$ is the local time rate of change

- $C_{i j} \quad$ is the convective transport

- $P_{i j} \quad$ is the stress production

- $\Pi_{i j} \quad$ is the velocity-pressure gradient correlation

- $D_{i j}^{v} \quad$ is the viscous diffusion

- $T_{i j}$ is the turbulent diffusion

- $\epsilon_{i j}$ is the turbulent dissipation

All terms in the stress transport equations are calculated from measured data except the velocity-pressure gradient correlation. Hence the difference between the turbulent dissipation and the velocity-pressure gradient correlation is balanced by all other terms in the equation. Figures $5.1 \mathrm{a}-5.7 \mathrm{f}$ show the stress budgets of all stresses for profiles $P 07, P 13, P 18$, $P 19 p, P 19, P 20$ and P33. Other profiles show similar trend as those presented here. Terms in Equation 5.1 that could be calculated from measured data are plotted versus the wall normal distance. The difference between the turbulent dissipation and velocity-pressure gradient correlation is also plotted as a single curve. The DNS data by Iwamoto [20], database for fully developed channel flow, are also shown in the figures for comparison.

In general, profiles $P 07, P 13$ and P33 show similar behavior close to that of a twodimensional boundary layer. However, all three profiles show higher normal and shear stress production, ${\overline{u^{2}}}^{+}$and $\overline{u v}^{+}$, respectively. Further, the production in the outer layer has large values compared to two-dimensional flows. Terms in the transport budgets for 
${\overline{v^{2}}}^{+}, \overline{u w}^{+}$and $\overline{v w^{+}}$stresses are about two orders of magnitude smaller than the ${\overline{u^{2}}}^{+}$and $\overline{u v}^{+}$stresses.

For profiles P18, P19, P19p and P20 shown in Figures 5.3a-5.6f, respectively, the production of $\bar{u}^{2}, \bar{v}^{2}$ and $\overline{u v}$ stresses show a reduction in value compared to twodimensional boundary layer. This observation is also made by Moin [37], Sendstand and Moin [58], Olcmen and Simpson [43]. Sendstand and Moin [58] pointed out that this is due to three-dimensional effects which reduces the production of ${\overline{v^{2}}}^{+}$, which in turn reduce the production of $\overline{u v}$ stress which ultimately reduces the production of $\bar{u}^{2}$ stress. However the level of normal stress production, ${\overline{u^{2}}}^{+}$, are higher than those reported by Olcmen [43] at station 5 .

On the other hand, production of ${\overline{w^{2}}}^{+}, \overline{u w}^{+}$and $\overline{v w^{+}}$are non-zero. Furthermore, the level of production of $\bar{w}^{2}, \overline{u w}^{+}$and $\overline{v w}^{+}$stresses are on the same order of magnitude of the $\overline{u v}$ stress production. The production of $\overline{v w}$ stress in the outer layer is at the same level as $\overline{u v}$ stress production, which explains why both stresses have the same value in the outer layer and in some parts the $\overline{v w}$ stress is larger than $\overline{u v}$. The high level of production is due to large Reynolds stress normal to the wall, $\bar{v}^{2}$, and mean span-wise velocity gradients, $\partial W / \partial y$. This is explained more in section 5.2.2.

The production of $\overline{u w^{+}}$and $\overline{v w}$ stresses change sign at about $y^{+}=150$. Also another important feature is that the main terms in the transport equations for $\overline{u w^{+}}$and $\overline{v w}^{+}$stresses are the production and the difference between turbulent dissipation and velocity-pressure gradient correlation, all other terms are almost negligible except in the inner layer where the viscous and turbulent diffusion contribute some to the transport equations. For the ${\overline{w^{2}}}^{+}$, all terms seem to be contributing to the transport equations.

To gain more insight into what is causing the high production rates of stresses, the stress production terms are broken up into their terms involving the Reynolds stresses and the mean strain rate. Since the mean flow undergoes changes in direction due to the presence of side walls that create stream-wise and span-wise pressure gradients which in turn affect the vorticity flux at the tunnel's floor, it would be useful to link changes in turbulence production to the mean vorticity vector. The production terms, $P_{i j}$, in the transport equations will be expressed in terms of mean vorticity components.

To achieve this, the mean strain rates are replaced by the components of the mean rotation tensor, $R_{i j}$, given by Equation 5.2. The rotation rate tensor is related to the mean vorticity vector, $\Omega_{i}$, through Equation 5.3, Tennekes and Lumley [68]. The stress production in terms of the rotation rate tensor is given by Equation 5.4 .

$$
R_{i j}=\frac{1}{2}\left(\frac{\partial U_{i}}{\partial x_{j}}-\frac{\partial U_{j}}{\partial x_{i}}\right)
$$




$$
\begin{gathered}
R_{i j}=-\frac{1}{2} \epsilon_{i j k} \Omega_{k} \\
P_{i j}=-\overline{u_{j} u_{l}} \frac{\partial U_{i}}{\partial x_{l}}-\overline{u_{i} u_{l}} \frac{\partial U_{j}}{\partial x_{l}}=-2\left(\overline{u_{j} u_{l}} R_{i l}+\overline{u_{i} u_{l}} R_{j l}\right)-\overline{u_{j} u_{l}} \frac{\partial U_{l}}{\partial x_{i}}-\overline{u_{i} u_{l}} \frac{\partial U_{l}}{\partial x_{j}}
\end{gathered}
$$

The components of the rotation rate tensor are given in matrix form in Equation 5.5. Since the rotation rate tensor is skew-symmetric, hence it has only three independent components which are the components of the vorticity vector as indicated by Equation 5.2

$$
R_{i j}=\frac{1}{2}\left(\begin{array}{ccc}
0 & -\Omega_{z} & \Omega_{y} \\
\Omega_{z} & 0 & -\Omega_{x} \\
-\Omega_{y} & \Omega_{x} & 0
\end{array}\right)
$$

Hence the production terms for all Reynolds stresses are expressed individually in Equation 5.6 in terms of vorticity vector components. In addition to the stress transport equations, the mean vorticity equation as derived by Tennekes and Lumley [68] is given by Equation 5.7. The terms on the left hand side of Equation 5.7 are the time rate of change and the convective rate of change of mean vorticity, respectively. The first term on the right hand side is the vortex stretching-tilting, present only in three-dimensional flows, is responsible for stretching and tilting the vorticity vector by the effect of mean flow strain. The second term is the viscous diffusion term and the last two terms are analogous to the Reynolds stress terms in the Navier-Stokes equations. The first of which is the stretching of fluctuating vorticity due to fluctuating velocity gradient and the last term is the turbulent transport of fluctuating vorticity.

$$
\begin{gathered}
P_{11}=-\underbrace{2\left[\overline{u w}\left(\Omega_{y}+\frac{\partial W}{\partial x}\right)\right]}_{I}+\underbrace{2\left[\overline{u v}\left(\Omega_{z}-\frac{\partial V}{\partial x}\right)\right]}_{I I}-\underbrace{2 \overline{u^{2}} \frac{\partial U}{\partial x}}_{I I I} \\
P_{22}=-2\left[\overline{u v}\left(\Omega_{z}+\frac{\partial U}{\partial y}\right)\right]+2\left[\overline{v w}\left(\Omega_{x}-\frac{\partial W}{\partial y}\right)\right]-2 \overline{v^{2}} \frac{\partial V}{\partial y} \\
P_{33}=-2\left[\overline{v w}\left(\Omega_{x}+\frac{\partial V}{\partial z}\right)\right]+2\left[\overline{u w}\left(\Omega_{y}-\frac{\partial U}{\partial z}\right)\right]-2 \overline{w^{2}} \frac{\partial W}{\partial z} \\
P_{12}=-\underbrace{\left[\overline{u^{2}}\left(\Omega_{z}+\frac{\partial U}{\partial y}\right)-\overline{v^{2}}\left(\Omega_{z}-\frac{\partial V}{\partial x}\right)\right]}_{I I} \\
-\underbrace{\left[\overline{u w}\left(\Omega_{x}-\frac{\partial W}{\partial y}\right)+\overline{v w}\left(\Omega_{y}+\frac{\partial W}{\partial x}\right)\right]}_{I}-\underbrace{\left[\overline{u v}\left(\frac{\partial U}{\partial x}+\frac{\partial V}{\partial y}\right)\right]}_{I I I} \\
P_{13}=-\left[\overline{w^{2}}\left(\Omega_{y}+\frac{\partial W}{\partial x}\right)-\overline{u^{2}}\left(\Omega_{y}-\frac{\partial U}{\partial z}\right)\right]
\end{gathered}
$$




$$
\begin{gathered}
-\left[\overline{u v}\left(\Omega_{x}+\frac{\partial V}{\partial z}\right)-\overline{v w}\left(\Omega_{z}-\frac{\partial V}{\partial x}\right)\right]-\left[\overline{u w}\left(\frac{\partial U}{\partial x}+\frac{\partial W}{\partial z}\right)\right] \\
P_{23}=-\left[\overline{v^{2}}\left(\Omega_{x}+\frac{\partial V}{\partial z}\right)-\overline{w^{2}}\left(\Omega_{x}-\frac{\partial W}{\partial y}\right)\right] \\
+\left[\overline{u v}\left(\Omega_{y}-\frac{\partial U}{\partial z}\right)-\overline{u w}\left(\Omega_{z}+\frac{\partial U}{\partial y}\right)\right]-\left[\overline{v w}\left(\frac{\partial V}{\partial y}+\frac{\partial W}{\partial z}\right)\right] \\
\frac{\partial \Omega_{i}}{\partial t}+U_{l} \frac{\partial \Omega_{i}}{\partial x_{l}}=\Omega_{j} S_{i j}+\nu \frac{\partial^{2} \Omega_{i}}{\partial x_{l}^{2}}+\overline{\omega_{j} s_{i j}}-\overline{u_{l} \frac{\partial \omega_{i}}{\partial x_{l}}}
\end{gathered}
$$

For the normal stresses, Equation 5.6 shows that the stress production is due to two parts, the first, terms $I$ and $I I$, is the interaction between the shear stresses and the mean vorticity normal to the normal stress direction and the interaction between shear stress and mean shear strains, respectively. The second part, III, is due to the interaction between the individual normal stress and the mean normal strain in the direction of the same stress. On the other hand the production terms for the shear stresses are due to three parts. The first part, term $I$, is the interaction between normal stresses and the mean shear strain in the direction of the individual stress and mean vorticity. The second part, term II, is due to the interaction between the shear stresses and the mean normal strains in the direction of shear stress and mean vorticity. Finally the third term, III, is due to the interaction between the individual shear stress and the normal mean strains in the direction of velocities making up the turbulent shear stress. This form is much more complicated than the conventional form in terms of mean strain rate. However, it has the advantage of revealing the effects of introducing mean vorticity, shear strain or normal strain in the flow field on Reynolds stresses production. Examining these terms will be used to interpret the behavior of measured turbulent quantities. This is done in the next section.

\subsubsection{Stress Production}

Terms I, II and III in the stress production, Equation 5.6, are plotted for each stress component for each of the profiles P08,P13, P18, P19p, P19, P20 and P33 in Figures 5.8a5.14f. respectively. In general, only one term in the stress production is responsible for producing that stress except for profiles P13 and P33 in the $\overline{u w}$ and $\bar{w}^{2}$ equations. Furthermore, these dominant terms contain only one vorticity vector component except for the $\overline{u w}^{+}$stress. Profiles P13 and P33 are located around the centerline, hence, have the least three-dimensionality effects. This causes contributions from all three terms to be of equal importance. However, the level of these contributions are much lower than those of other profiles.

For the ${\overline{u^{2}}}^{+}$stress production,Figure 5.8a-5.14a, the main contribution comes from 
the interaction between the shear stress $\overline{u v}$ and the spanwise vorticity component, $\Omega_{z}=$ $V_{, x}-U_{, y}$, in term II.

For the ${\overline{v^{2}}}^{+}$stress,Figure 5.8c 5.14c, the production term is practically zero as in twodimensional boundary layers, hence the balance between turbulent diffusion, viscous diffusion, velocity-pressure gradient and dissipation is responsible for the transport of this stress.

For the ${\overline{w^{2}}}^{+}$stress,Figure 5.8e 5.14e the main contribution comes from term I, which is the interaction between the stream-wise vorticity component and the $\overline{v w}$ stress. There is some contribution to the production of $\overline{w^{2}}$ stress from term III which is the interaction between the normal stress $\bar{w}^{2}$ and the normal strain $\partial W / \partial z$; however, this term is much smaller than term I.

The $\overline{u v}$ shear stress production is shown in Figure $5.8 \mathrm{~d} .5 .14 \mathrm{~d}$. The main contribution is from the interaction between the span-wise vorticity and the normal stress ${\overline{v^{2}}}^{+}$. It is important to note that this term contains two terms, both involving the span-wise vorticity component. However the first term reduces to almost zero and the result is almost totally from the second parenthesis which is the interaction between the ${\overline{v^{2}}}^{+}$stress and the span-wise vorticity.

Figures $5.8 \mathrm{e}-5.14 \mathrm{e}$ show the $\overline{u w}^{+}$stress production terms. The main contribution is coming from terms II, which contain the stream-wise and span-wise vorticity components. Both components have the same order of magnitude, however the span-wise component contribution is larger.

Finally, Figure 5.8f $5.14 \mathrm{f}$ show the $\overline{v w}$ stress production terms. The main contribution is from term I. Similar to the $\overline{u v}+$ shear stress the main contribution to term $I$ is coming from the interaction between $\bar{v}^{2}$ stress and $\Omega_{x}$.

In summary, the span-wise vorticity component is the driving force of the ${\overline{u^{2}}}^{+}$and $\overline{u v}^{+}$stresses. While the stream-wise vorticity component is responsible for producing the $\overline{v w}^{+}$stress. Both vorticity components are responsible for producing the $\overline{u w}^{+}$shear stress; however, one acts as a source and the other as a sink depending on the flow field geometry. Finally, the mean vorticity vector doesn't seem to contribute to the normal stress in the normal to the wall direction, $\bar{v}^{2}$, hence the mechanism of amplifying the wall-normal stress is different from other stresses.

It is important to note that the above observations are true for attached boundary layer flows only. In other flow conditions, other terms in the stress production might become important. The other important note to make here after reviewing the stress production terms behavior is that, a comparison between Figures 5.8a $5.14 \mathrm{f}$ and profiles of Reynolds' stresses given in the previous chapter, shows that each stress profile follow very closely the changes in the major production term in their respective equation. However, there is some lag in the stress profile behavior as is expected and reported by different previous experiments. 
Given the above observations, examining the development of stream-wise and spanwise vorticity components will shed more light on why the turbulent stress profiles have such high values, multiple peaks and sign changes. This will be shown in the next section.

\subsubsection{Mean Vorticity Development}

The mean vorticity, as shown in the previous section, is the main driving force into generation of turbulent stresses. Hence, understanding of its development will lead to understanding of the development of turbulent stresses. Profiles of mean vorticity in planes normal to the stream-wise direction can be calculated from seven-hole probe data, as well as close to the wall from LDV measurements. The vorticity defined by Equation 5.8, is calculated exactly where LDV measurements are available using multiple profiles in a cluster of five profiles forming a cross.

$$
\vec{\Omega}=\nabla \times \vec{V}=\left(W_{, y}-V_{, z}\right) \cdot \hat{i}+\left(U_{, z}-W_{, x}\right) \cdot \hat{j}+\left(V_{, x}-U_{, y}\right) \cdot \hat{k}
$$

For seven-hole probe data, the mean vorticity components, $\Omega_{y}$ and $\Omega_{z}$, are calculated approximately, since the derivatives in the stream-wise direction were not available. However, this affects only the vertical vorticity component, $\Omega_{y}$, the most. This is not of any importance in this discussion, since the span-wise vorticity $V_{, x}<<U_{, y}$, hence the conclusions won't be affected significantly.

In the following discussion, the side wall at the negative span-wise half of the test section is labeled as the right side wall, while the wall at the positive span-wise half of the test section is labeled the left side wall. Also, the peaks and troughs of waves on the side walls corresponds to points of minimum and maximum absolute $z$-coordinates, respectively.

Contour plots of the development of mean span-wise and stream-wise vorticity at six different stream-wise stations are shown in Figures 5.15a $5.15 \mathrm{f}$. Span-wise vorticity, $\Omega_{z}$, is shown as colored contours while the stream-wise vorticity, $\Omega_{x}$, is shown as line contours with dashed lines denoting negative values. All values are normalized by inflow freestream reference velocity and test-section inlet height.

The flow enters the test section with vorticity mainly in the span-wise direction as shown in Figure 5.15a. As the flow proceeds downstream, Figure $5.15 \mathrm{~b}$ shows the vorticity at $x / L=0.13$, some stream-wise vorticity is developed on the left side wall. This is due to the fact that the suction cabinet was divided into two sections in the vertical direction, so there was about $0.5 \mathrm{in}$ in the vertical direction at the center of the suction cabinet that has reduced suction. However, this vorticity is sucked out by the boundary layer control slots downstream. 
Figure 5.15c shows the vorticity contours at $x / L=0.64$. In this figure we see two massive areas of stream-wise vorticity of opposite signs at $z / W_{x=0}= \pm 0.2$. Also at the same location there is a reduction in span-wise vorticity and actually the span-wise vorticity changes sign at these locations. At this plane the flow has passed over two peaks on both side walls. Vorticity generation at the wall is related to the surface tangential pressure gradients as pointed out by Morton [39] and given by Equation 5.9 derived from the Navier-Stokes equations.

$$
\begin{array}{r}
\nu \frac{\partial \Omega_{x}}{\partial y}=\frac{1}{\rho} \frac{\partial P}{\partial z} \\
\nu \frac{\partial \Omega_{z}}{\partial y}=-\frac{1}{\rho} \frac{\partial P}{\partial x}
\end{array}
$$

This is viewed as the flux density of vorticity, flow per unit area per unit time out of the surface, hence any surface pressure gradients will generate new vorticity at the wall. As a result, new vorticity is generated as the flow undergoes changes in pressure gradients by passing over the first two waves on the side walls. On the tunnel's floor, the span-wise pressure gradient in the troughs of the left side wall and at the peaks of the right side wall are positive, which will generate positive stream-wise vorticity, while the pressure gradients at the peaks of the left side wall and troughs of the right side wall are negative which will generate negative stream-wise vorticity. This vorticity is convected downstream and diffused away from the wall. This explains the presence of two concentrated regions of stream-wise vorticity of opposite signs at this plane.

Another feature to note is that the span-wise vorticity at $z / W_{x=0}= \pm 0.2$ is positive, which might be counter-intuitive. However, this might be due to the fact that the first two profiles in the plane close to the left side wall, $1 \mathrm{in}$, are affected by the side wall suction which makes the flow turn sharply in the positive span-wise direction while the third profile is affected by the pressure gradient which forces the flow into the negative span-wise direction, however as the height increases the effects of the pressure gradient diminishes and the inertia effects are dominant and the flow turns in the positive spanwise direction. Another factor which might lead to increase of span-wise vorticity is the tilting of stream-wise vorticity component into the span-wise direction by the action of the stretching/titling term, $\Omega_{x} \cdot S_{31}$, in Equation 5.7 .

Figure $5.15 \mathrm{~d}$ shows vorticity contours at $x / L=0.64$. As the flow proceeds downstream more vorticity is generated and a growth of stream-wise vorticity is visible in the entire section reaching the center of the test section. The stream-wise vorticity is positive in the positive half of the test section and negative in the negative half of the test section. Figures 5.15e 5.15f show the vorticity contours at $x / L=0.79$ and $x / L=0.90$, respectively. The span-wise and stream-wise vorticity are cross-diffused with vorticity from the ceiling boundary layer of opposite sign indicating a channel flow type situation at the end of the test section. 
In order to qualitatively assess the validity of the measurements, a three-dimensional potential flow solver is developed to calculate the pressure distribution as well as the stretching/titling term in the vorticity transport equation. The details of the flow solver are presented in Appendix D. The stream-wise and span-wise pressure coefficient gradients are shown in Figure 5.16, dashed lines indicate negative values. The pressure coefficient gradients in the span-wise direction at the left wall troughs and the right wall peaks are positive, further the area of positive span-wise pressure gradients along the left wall is much greater than the areas where there is a negative span-wise gradient. Hence, the stream-wise positive vorticity flux is much higher than the negative flux which leads to the formation of resultant positive stream-wise vorticity in the positive span-wise half of the test section. Similarly the same can be said about the right wall with negative streamwise vorticity being dominant in the negative span-wise half of the test section. This is the reason why seven-hole probe data shows positive stream-wise vorticity for positive $z$-coordinates and negative stream-wise vorticity for negative $z$-coordinates.

Also shown in Figure 5.16 are the stream-wise pressure coefficient gradients. The sign of these gradients change along each single wave, being negative upstream of the peaks and positive downstream of the peaks. This will lead to the generation of positive and negative vorticity flux, respectively, according to Equation 5.9. As a result the spanwise vorticity will be amplified and attenuated near the side walls depending on the relative position along the waves.

The strain rates $S_{11}=\partial U / \partial x, S_{13}=0.5(\partial U / \partial z+\partial W / \partial x)$ and $S_{33}=\partial W / \partial z$ are also shown in Figure 5.17. In three-dimensional flows these terms are responsible for amplifying or attenuating the vorticity by means of stretching and tilting as it is diffused away from the wall as given by Equation 5.7. The terms $S_{11}$ and $S_{33}$ are stretching terms while $S_{13}$ is a tilting term. Hence, in the stream-wise vorticity transport equation, $\Omega_{x} \cdot S_{11}$ enhances stream-wise vorticity when the flow is accelerating and attenuates it when the flow is decelerating. Also, the term $\Omega_{z} \cdot S_{13}$ enhances the stream-wise vorticity, for positive $S_{13}$ and negative $\Omega_{z}$, by tilting the vorticity vector into the stream-wise direction. As a result we can see that at the left wall troughs $S_{13}$ is negative and hence it amplifies the stream-wise vorticity. The same argument can be said about the right wall but with opposite signs.

The strain rates $S_{11}$ and $S_{33}$ alternate signs along the side walls, hence amplify and attenuate the stream-wise and span-wise vorticity components, respectively, depending on location relative to the side wall.

In summary, the flow enters the test section with only a span-wise vorticity component. As the flow proceeds downstream, stream-wise and span-wise surface pressure gradients are generated by the side walls. The stream-wise pressure gradient alternates signs along the test section and along the span-wise direction while the span-wise pressure gradient alternates sign in the stream-wise direction only. This pattern of alternating pressure gradients give rise to waves of stream-wise and spanwise vorticity. In the pos- 
itive span-wise half of the test section, the pressure gradients give rise to large positive stream-wise vorticity flux in the trough areas followed by smaller amounts of negative stream-wise vorticity flux at the wave peak. This is continued until the end of the test section. As the vorticity is convected downstream and diffused away from the wall, it interacts with new vorticity generated at a downstream location and depending on their signs will either enhance or attenuate each other. Towards the end of the tunnel the vorticity is diffused across the entire height of the test section. In the negative span-wise half of the test section, the same pattern occurs but with opposite signs; the pressure gradients will give rise to large negative vorticity flux in the troughs and smaller positive stream-wise vorticity flux at the peaks. This pattern continues until the end of the test section.

A schematic plot of the generation of stream-wise vorticity is shown in Figure 5.18. A mirror image of this schematic is developed on the ceiling of the test section. A schematic of the stream-wise vorticity in the $y-z$ plane is shown in Figure 5.19. Keeping this schematic in mind, features of measured quantities can be explained as follows:

\section{- High level of ${\overline{v^{2}}}^{+}$in the outer layer at the centerline}

This is probably because in the outer layer there is a significant amount of production which might be attributed to the flow rate sucked out of the test section that will create a large $\partial V / \partial y$ as shown in Figure $5.9 \mathrm{c}$. Also, the velocity-pressure gradient correlation has the same order of magnitude as the production as seen in Figure 5.2b. The increase in pressure fluctuations might be attributed to the side-wall boundary layer suction effects.

\section{- High level of turbulent stresses at the centerline}

This is because, as the spanwise vorticity is diffused into the outer layer it interacts with the produced stream-wise vorticity. Since the vorticity from the tunnel's floor and ceiling have different signs, their effect on the span-wise vorticity near the center height of the test section adds up as shown in Figure 5.19 and the net result is stretching of the span-wise vorticity which in turn will produce a higher shear stress $\overline{u v}$. Another factor is the very high level of $\bar{v}^{2}$ stress in the outer layer mentioned above.

\section{- Multiple peaks in the turbulent stress profiles}

The multiple peaks in the stress profile's present in clusters P08 and P18-P20 are due to waves of stream-wise vorticity that are being created at each wave peak and trough. Cluster P08 is located halfway between the last two peaks on the right wall at which the stream-wise pressure gradient, shown in Figure 5.16, changes sign from positive to negative. In the outer layer the span-wise vorticity is enhanced by the effect of mean rate of strain and by the stretching contribution by the stream-wise vorticity. This is also why there is a large value of negative span-wise mean velocity. On the other hand clusters P18-P20 show a reduction of shear stress as well as the 
normal stresses. This is due to the fact that upstream of these locations the streamwise pressure gradient is negative which will generate positive span-wise vorticity flux which leads to reduction in span-wise vorticity at these locations. For example, Figure $4.5 \mathrm{~d}$ shows the $\overline{u v}$ stress at cluster P18. Near the wall the shear stress is equal to 0.5 , half the value at cluster P08, while in the outer layer a peak of 1.0 is still being diffused away from the wall which was produced upstream of cluster P18. The same conclusions can be generalized for clusters P19 and P20.

\section{- Multiple peaks in the turbulent diffusion velocity $\overrightarrow{V q}$}

The different vorticity wave generated by the alternating pressure gradients can also be the cause for multiple peaks in the turbulent diffusion velocity. For example, the turbulent diffusion velocity component $V q_{x}$ for cluster $P 08$ shows a positive peak close to the wall and a negative peak away from the wall. Since this cluster is halfway between peaks, the stream-wise vorticity is changing signs from negative to positive, hence near the wall vorticity is positive while away from the wall it is negative. This means that near the wall the sweep motion is more dominant and it is associated with positive $w$-fluctuations while away from the wall the ejection motion is more dominant and it is associated with negative $w$-fluctuations. This results in positive ${\overline{u^{3}}}^{+},{\overline{u^{2} w}}^{+},{\overline{u w^{2}}}^{+},{\overline{u v^{2}}}^{+}$and negative ${\overline{u^{2} v}}^{+}$and $\overline{v w^{2}}$ near the wall and opposite signs away from the wall.

\section{- Almost Equal normal stresses in the Outer Layer}

All profiles show normal stresses reaching a state where all stresses are almost equal in the outer layer and for some profiles the span-wise normal stress exceeds the stream-wise normal stress. This is due to the alternating pressure gradients giving rise to alternating sign stream-wise vorticity which in term tilts and stretches the span-wise component hence exchanging turbulent energy between normal directions. Also the different signs of strong and weak vorticies will create a swirling motion of weak vorticity around strong vorticity which will make turbulent quantities of more equal values as it diffuses away from the wall. 


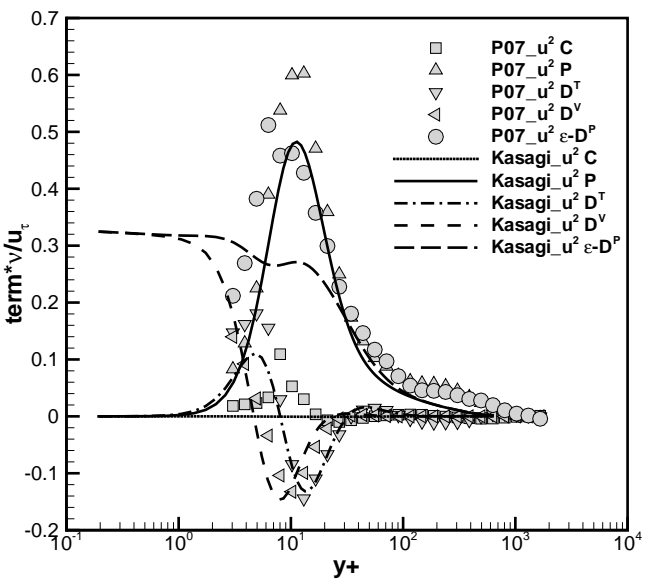

(a) $\overline{u^{2}}$ Stress Budget

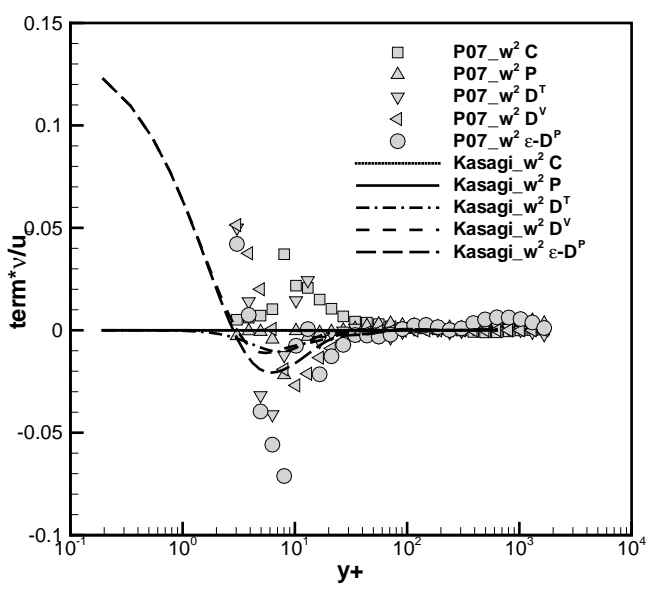

(c) $\overline{w^{2}}+$ Stress Budget

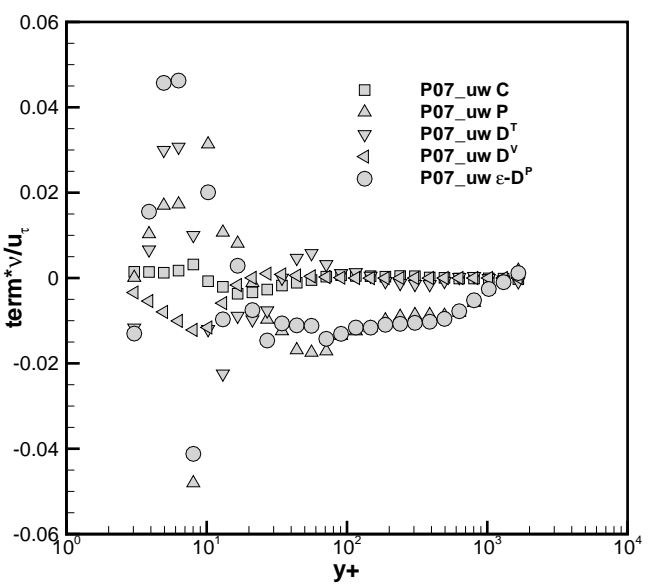

(e) $\overline{u w}+$ Stress Budget

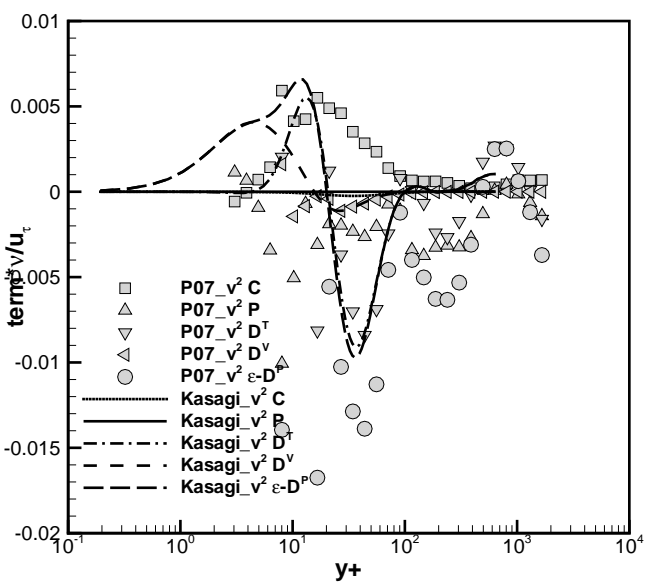

(b) $\bar{v}^{2}$ Stress Budget

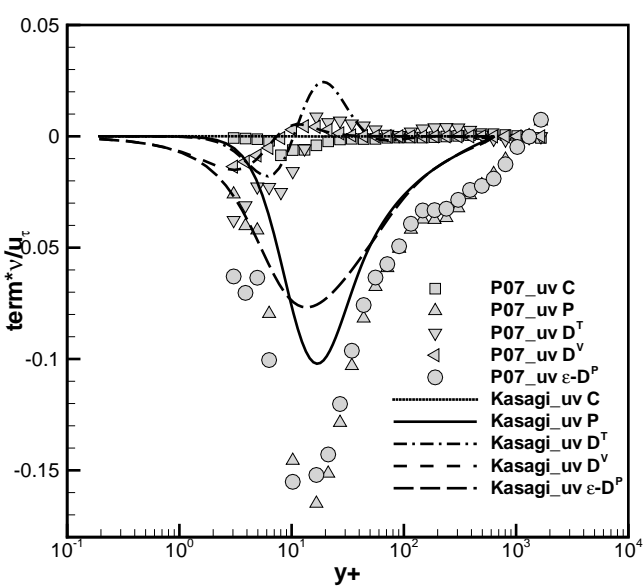

(d) $\overline{u v}+$ Stress Budget

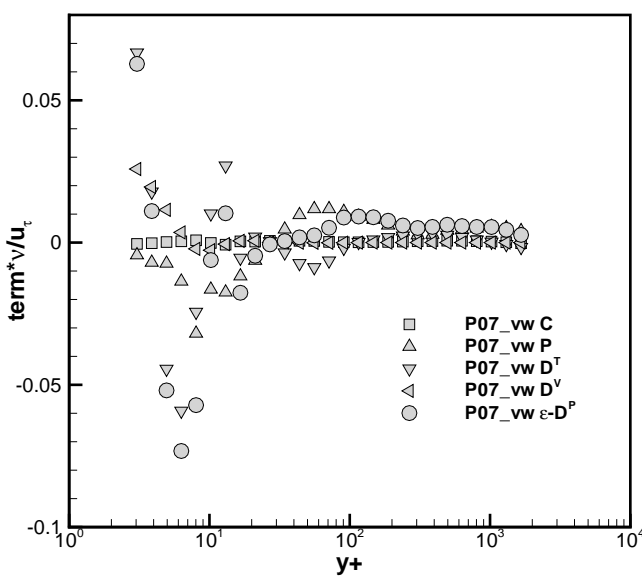

(f) $\overline{v w}{ }^{+}$Stress Budget

Figure 5.1: Reynolds Stresses Transport Budgets at P07 


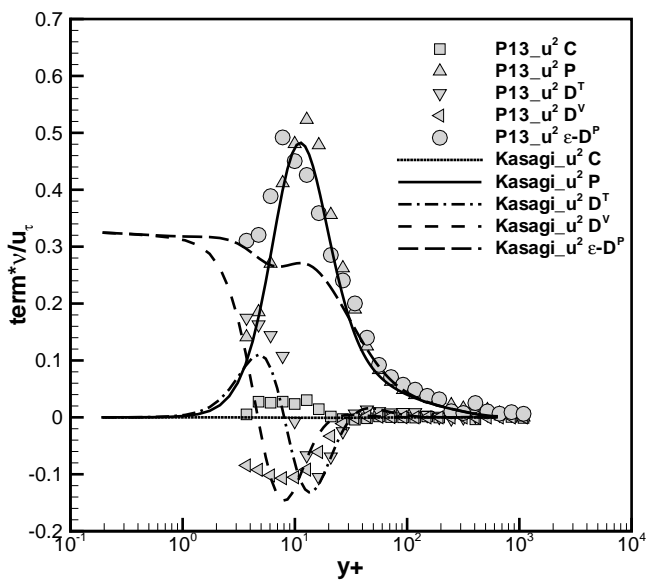

(a) $\bar{u}^{+}$Stress Budget

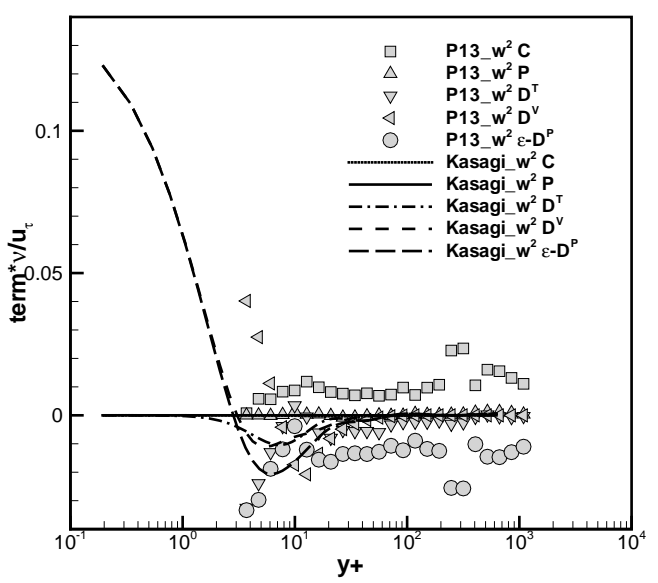

(c) $\overline{w^{2}}+$ Stress Budget

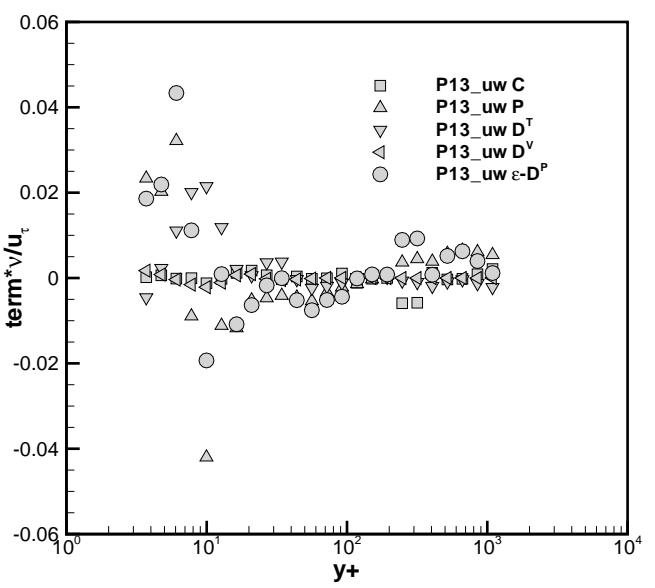

(e) $\overline{u w}+$ Stress Budget

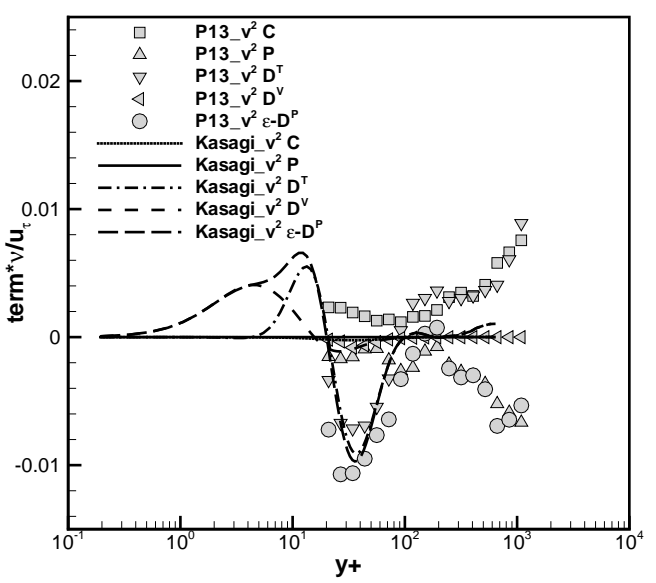

(b) $\bar{v}^{2}$ Stress Budget

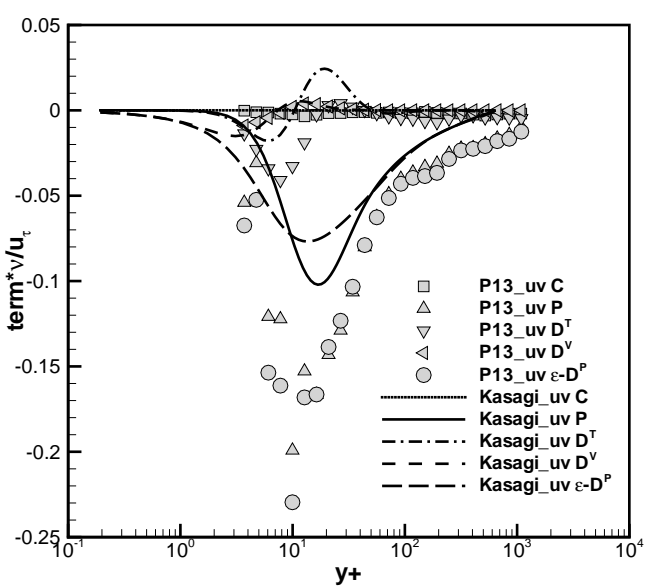

(d) $\overline{u v}^{+}$Stress Budget

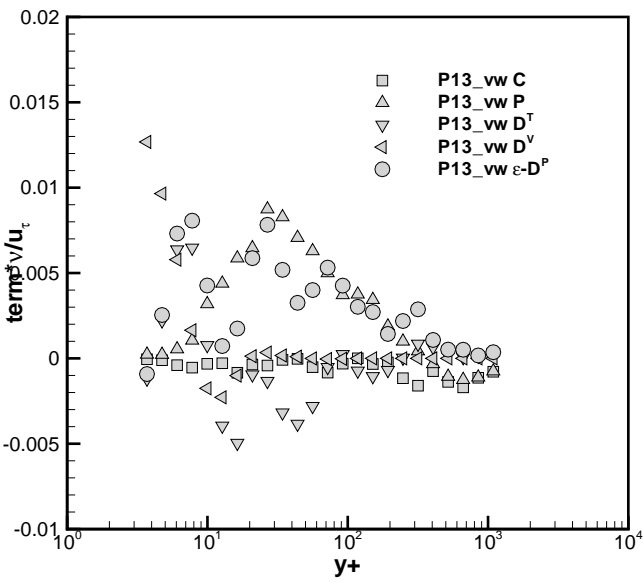

(f) $\overline{v w}{ }^{+}$Stress Budget

Figure 5.2: Reynolds Stresses Transport Budgets at P13 


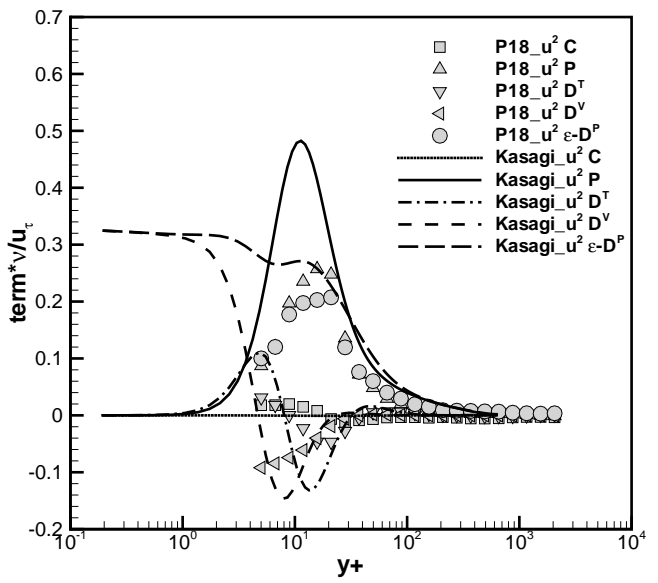

(a) $\bar{u}^{+}$Stress Budget

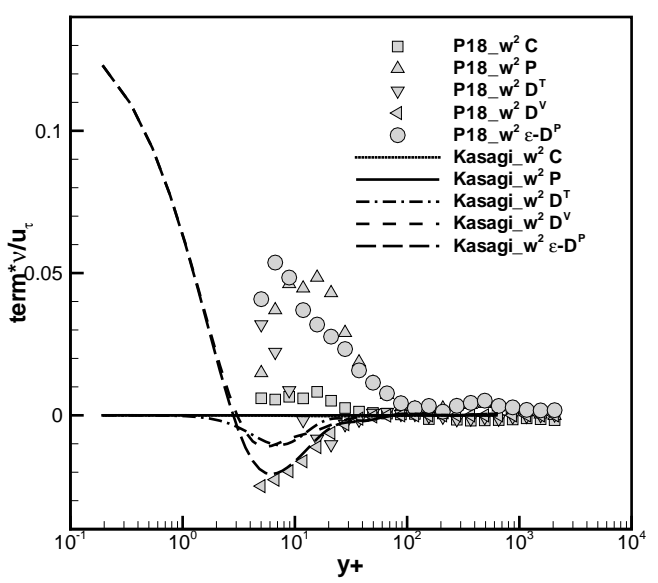

(c) $\overline{w^{2}}+$ Stress Budget

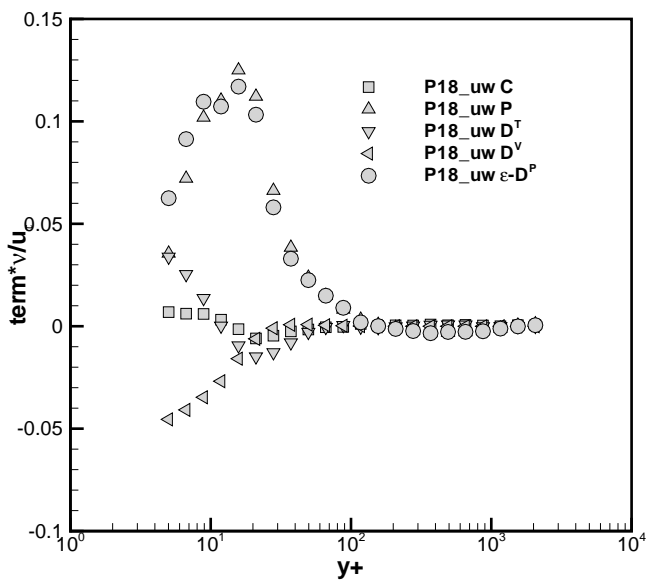

(e) $\overline{u w}+$ Stress Budget

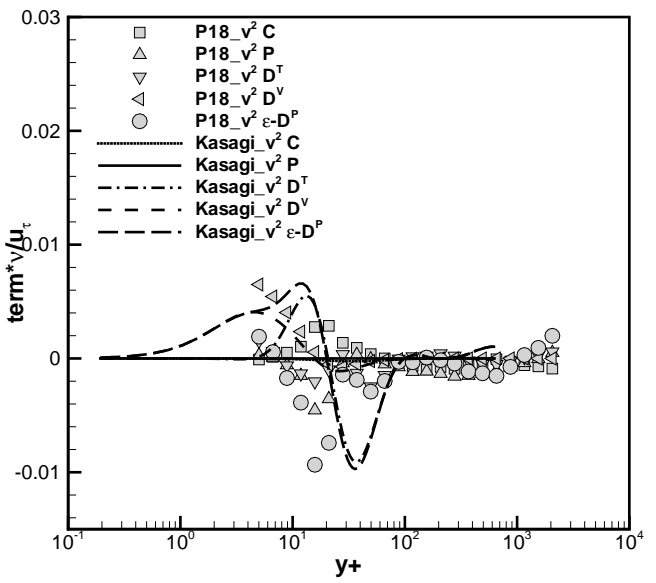

(b) ${\overline{v^{2}}}^{+}$Stress Budget

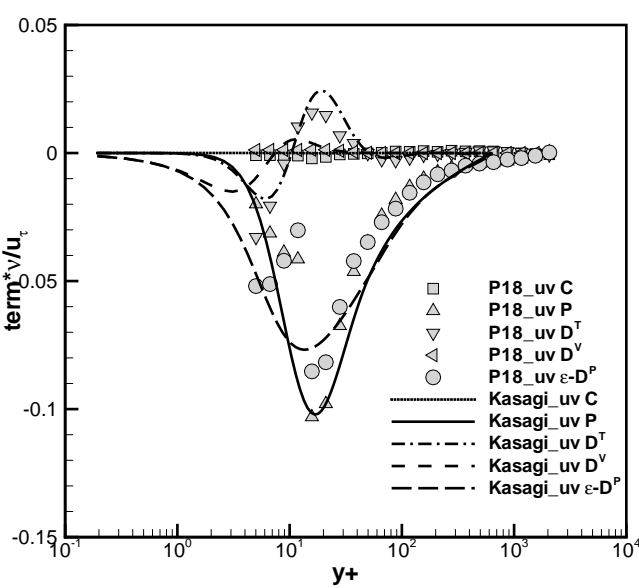

(d) $\overline{u v}+$ Stress Budget

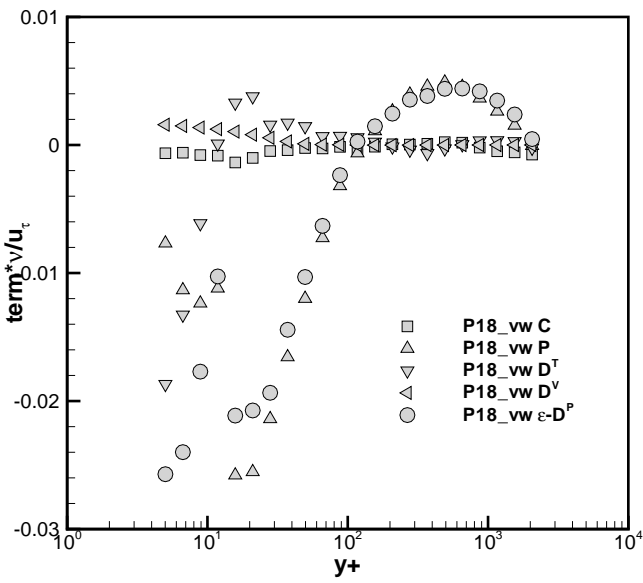

(f) $\overline{v w}{ }^{+}$Stress Budget

Figure 5.3: Reynolds Stresses Transport Budgets at P18 


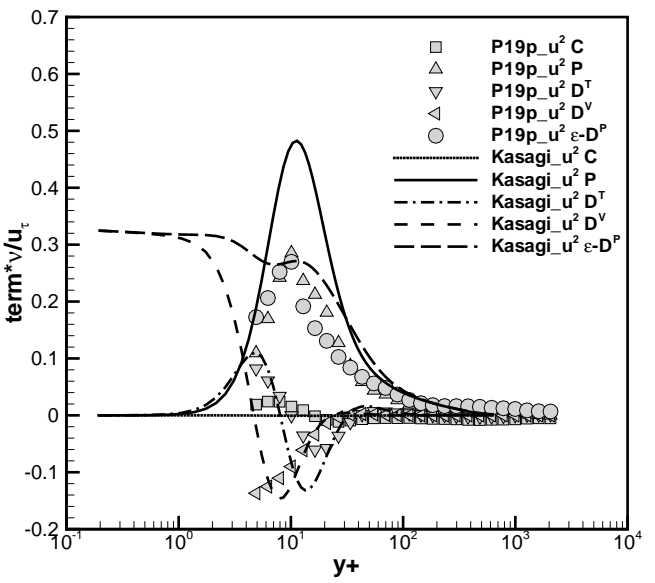

(a) $\overline{u^{2}}$ Stress Budget

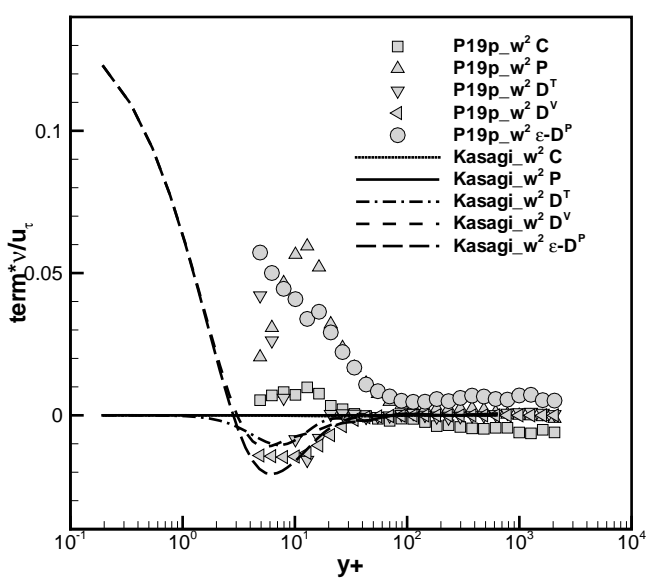

(c) $\overline{w^{2}}+$ Stress Budget

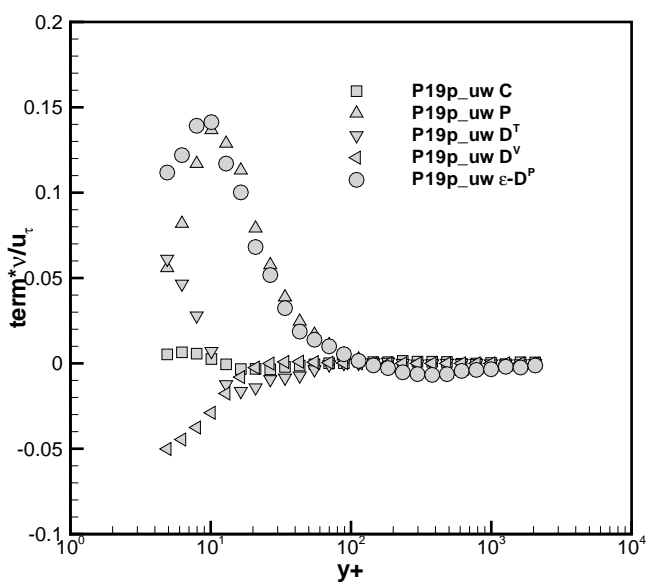

(e) $\overline{u w}^{+}$Stress Budget

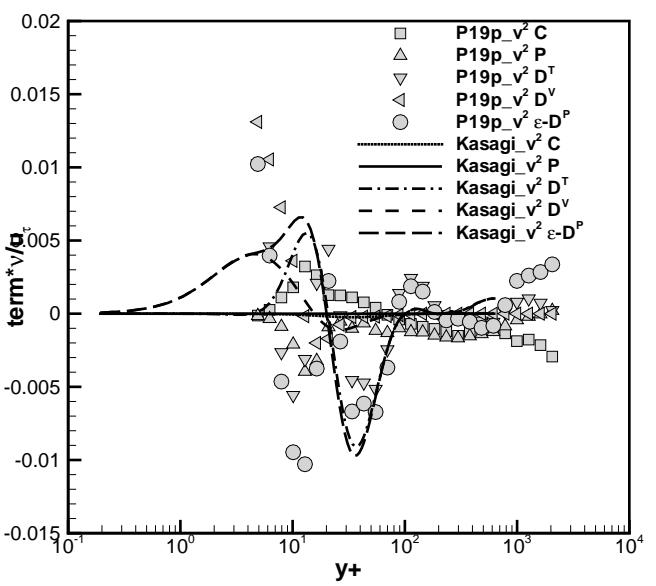

(b) $\bar{v}^{2}$ Stress Budget

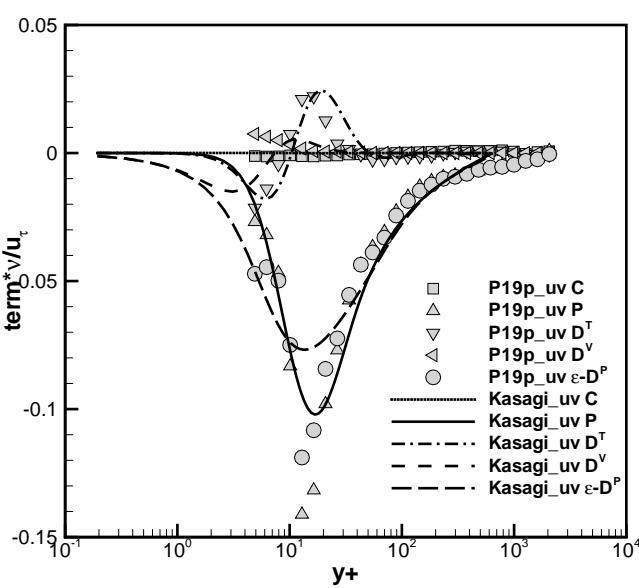

(d) $\overline{u v}^{+}$Stress Budget

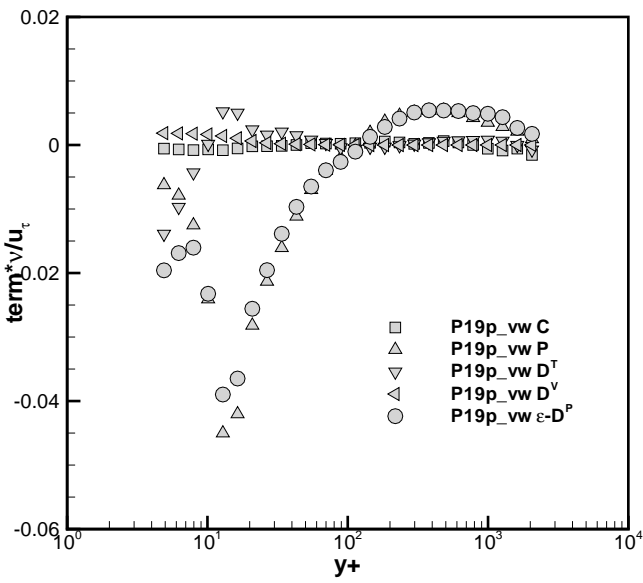

(f) $\overline{v w}{ }^{+}$Stress Budget

Figure 5.4: Reynolds Stresses Transport Budgets at P19p 


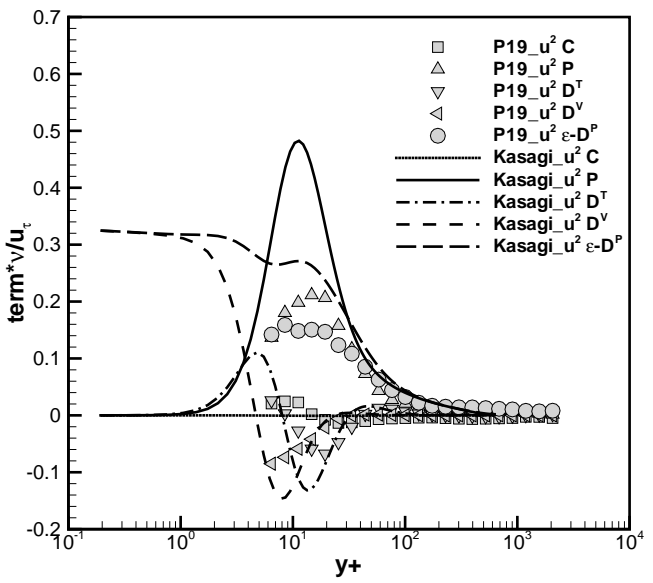

(a) $\bar{u}^{+}$Stress Budget

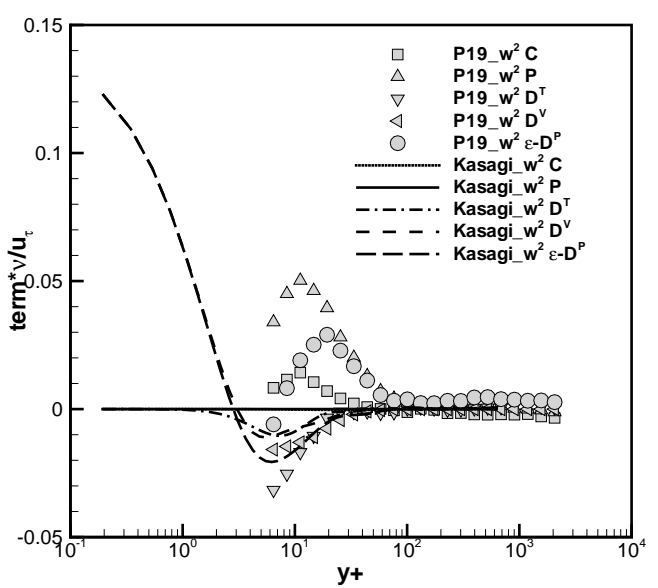

(c) $\overline{w^{2}}+$ Stress Budget

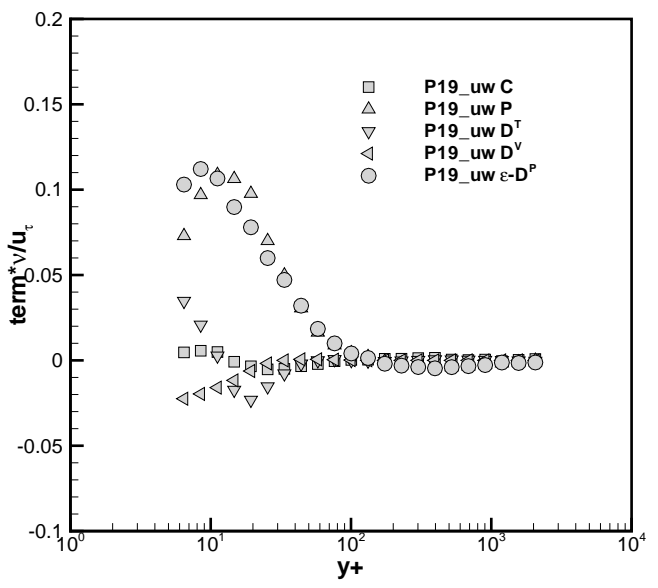

(e) $\overline{u w}^{+}$Stress Budget

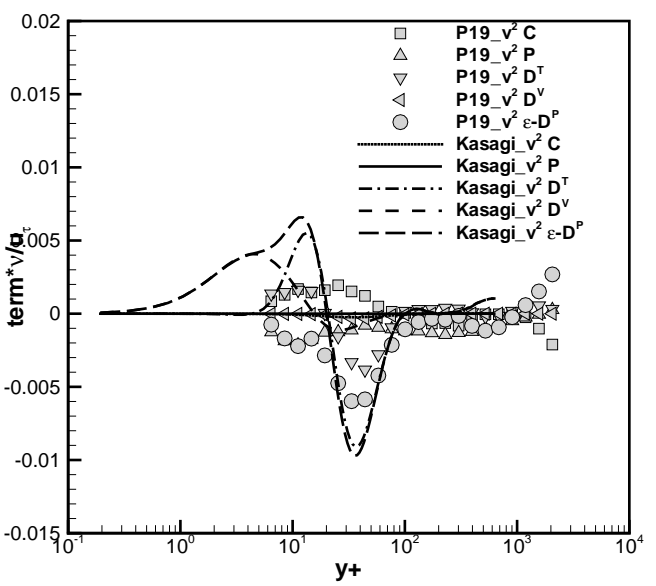

(b) $\bar{v}^{2}$ Stress Budget

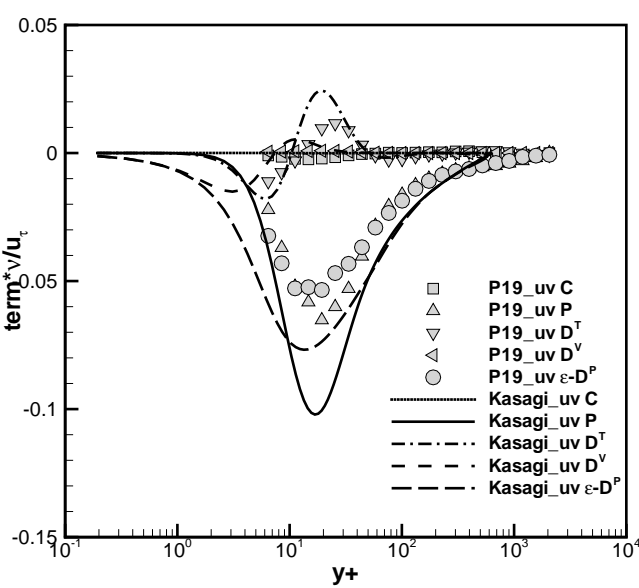

(d) $\overline{u v}+$ Stress Budget

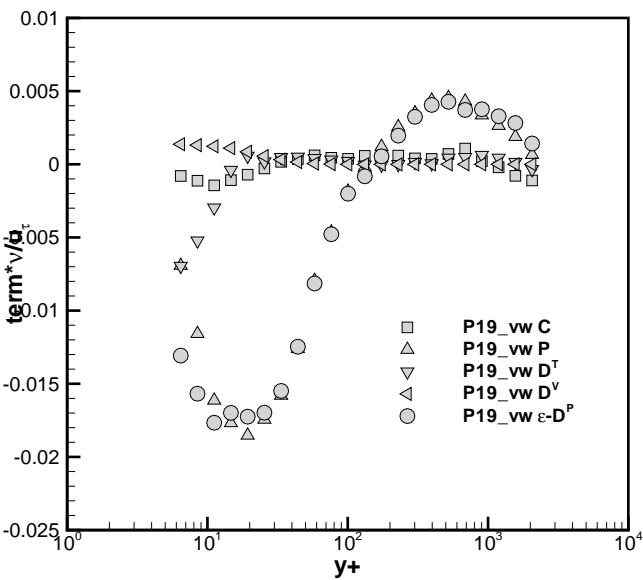

(f) $\overline{v w}{ }^{+}$Stress Budget

Figure 5.5: Reynolds Stresses Transport Budgets at P19 


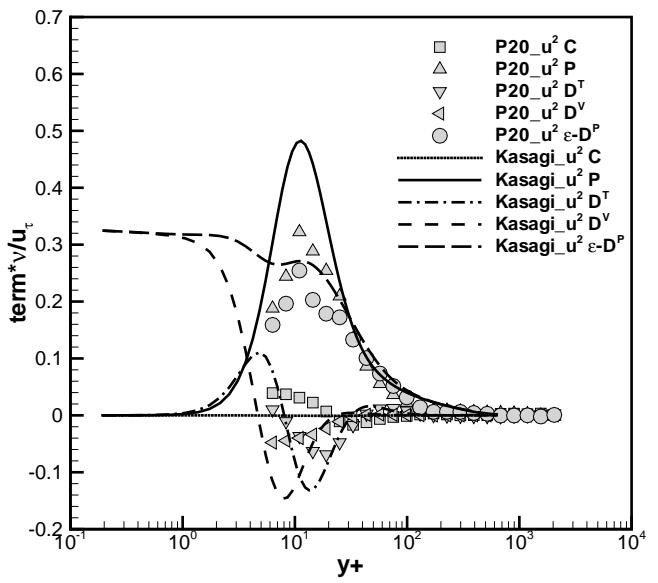

(a) $\bar{u}^{+}$Stress Budget

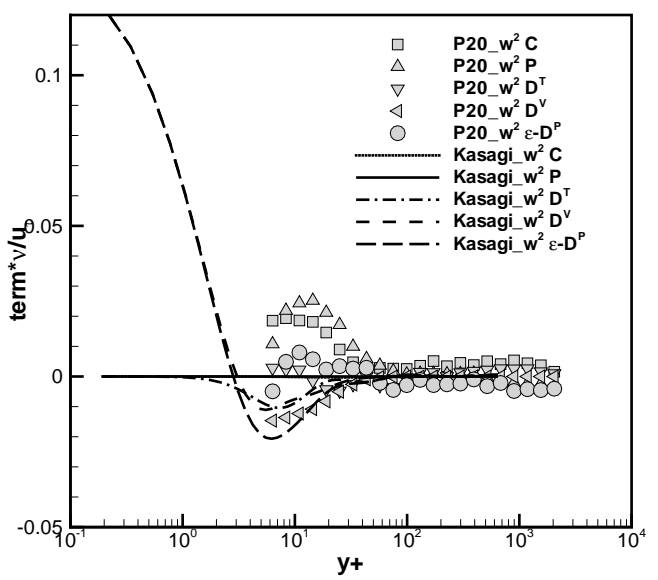

(c) $\overline{w^{2}}+$ Stress Budget

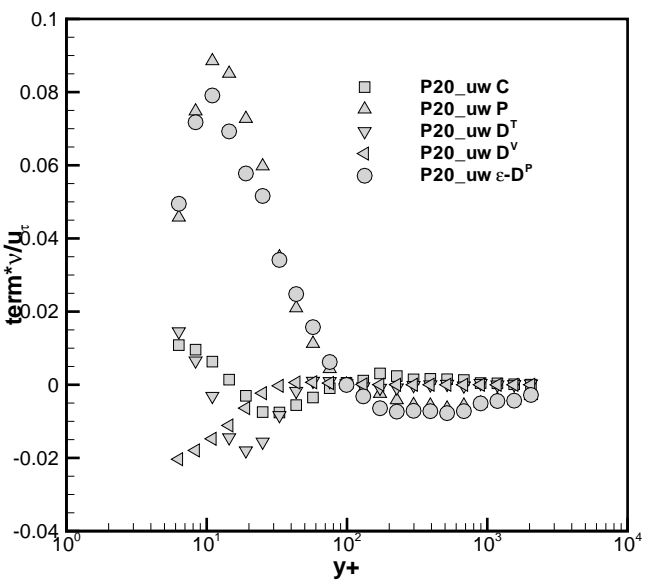

(e) $\overline{u w}^{+}$Stress Budget

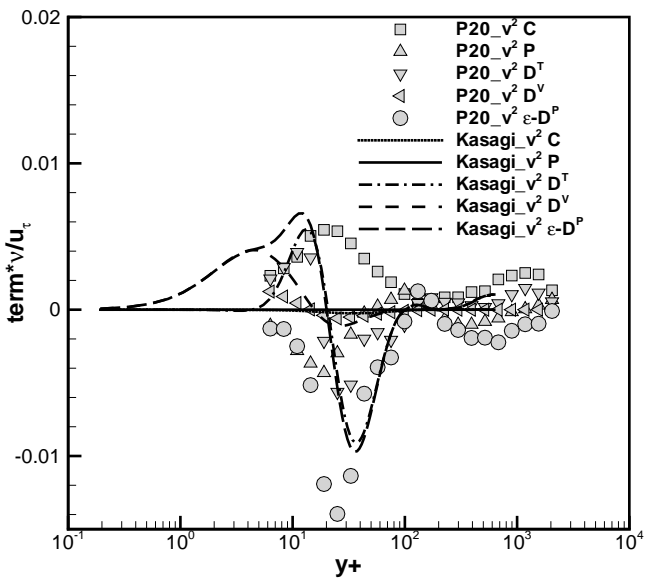

(b) $\bar{v}^{2}$ Stress Budget

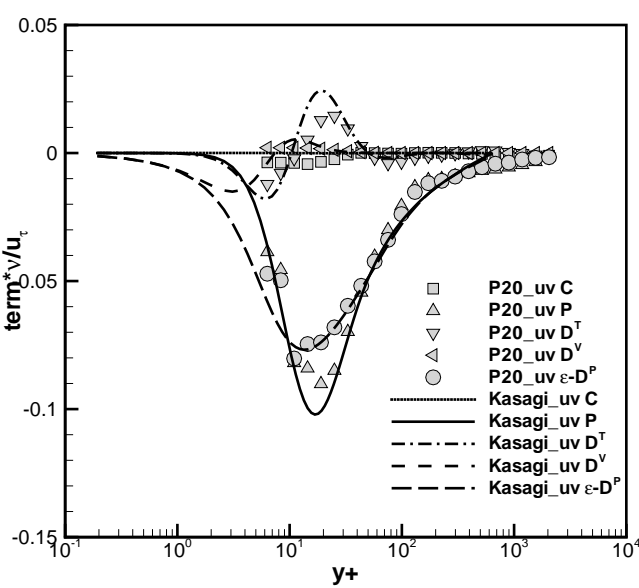

(d) $\overline{u v}+$ Stress Budget

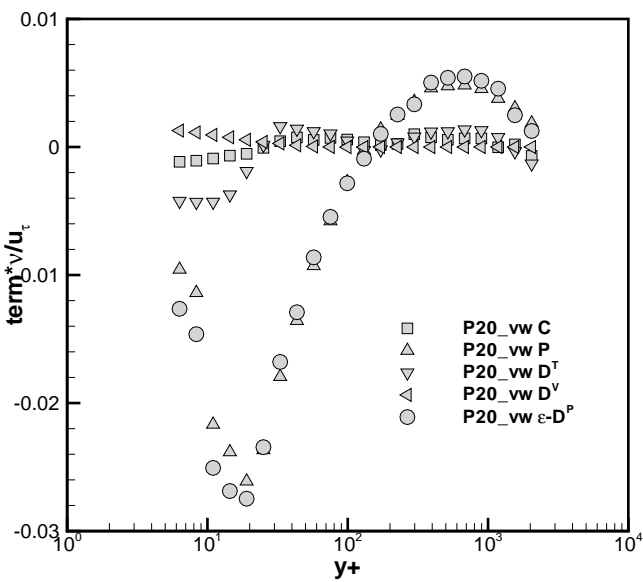

(f) $\overline{v w}{ }^{+}$Stress Budget

Figure 5.6: Reynolds Stresses Transport Budgets at P20 


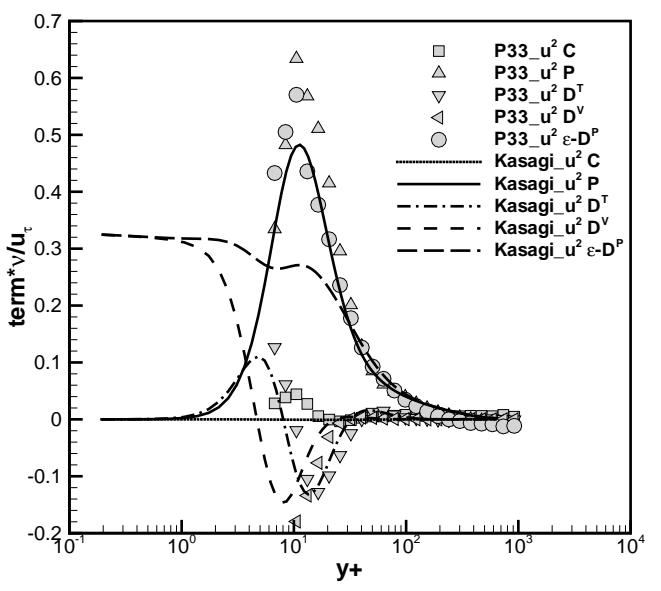

(a) $\bar{u}^{+}$Stress Budget

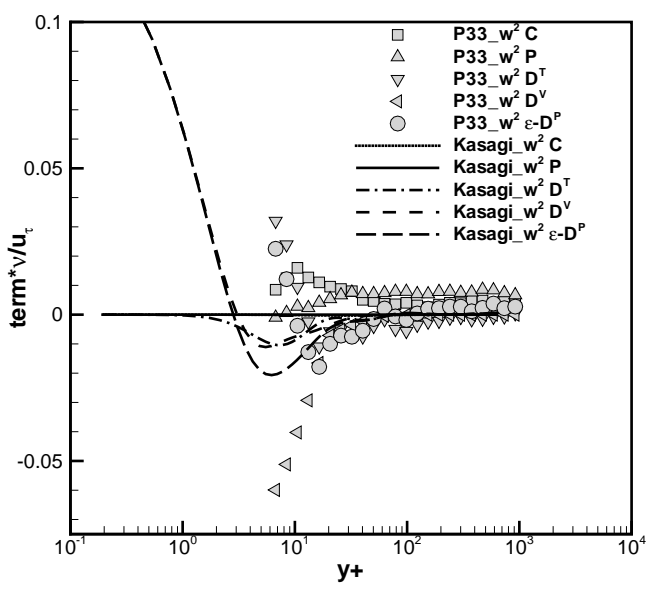

(c) $\overline{w^{2}}+$ Stress Budget

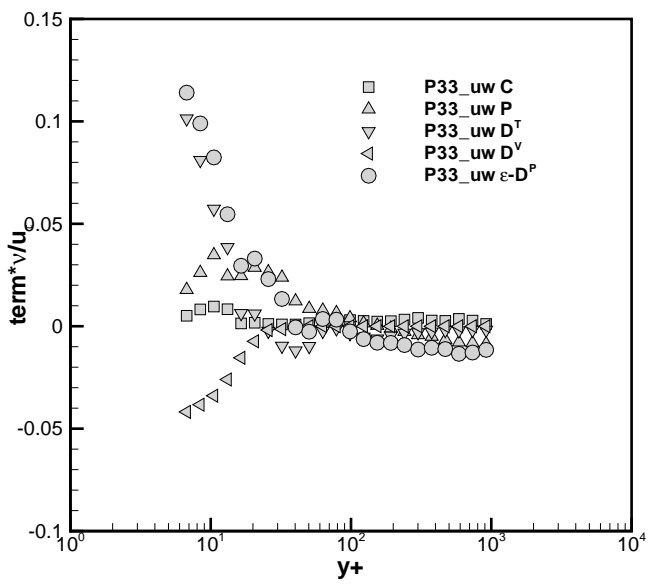

(e) $\overline{u w}+$ Stress Budget

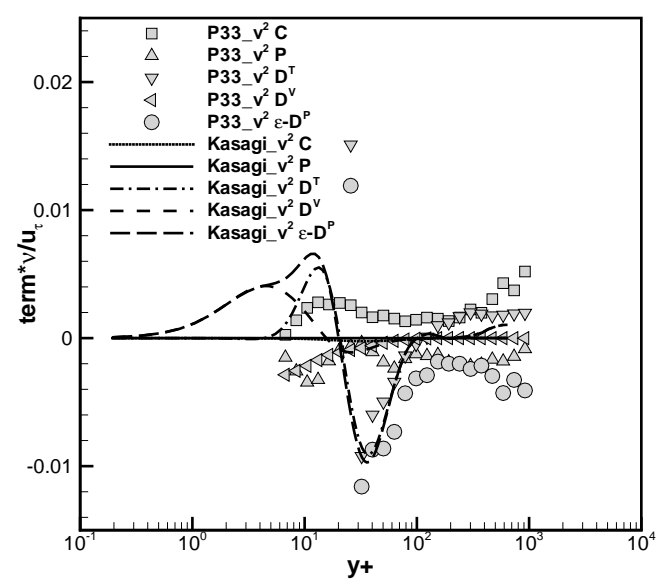

(b) $\bar{v}^{2}$ Stress Budget

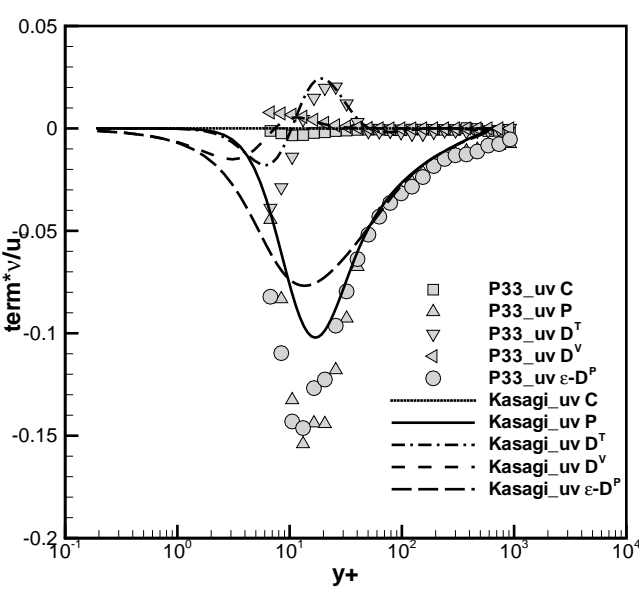

(d) $\overline{u v}+$ Stress Budget

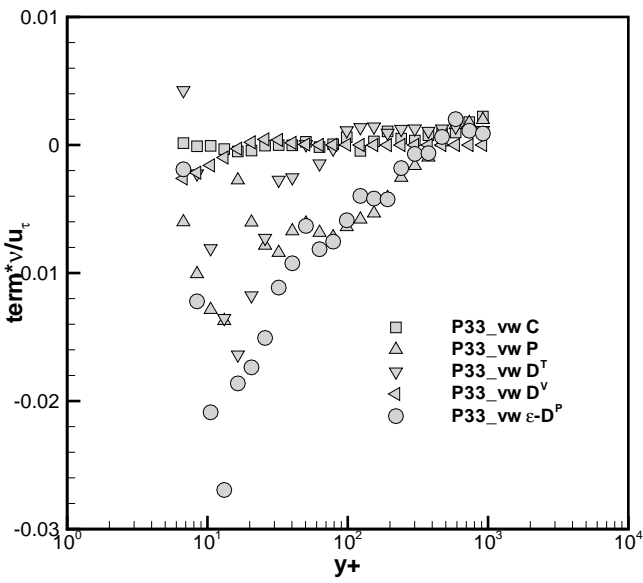

(f) $\overline{v w}{ }^{+}$Stress Budget

Figure 5.7: Reynolds Stresses Transport Budgets at P33 


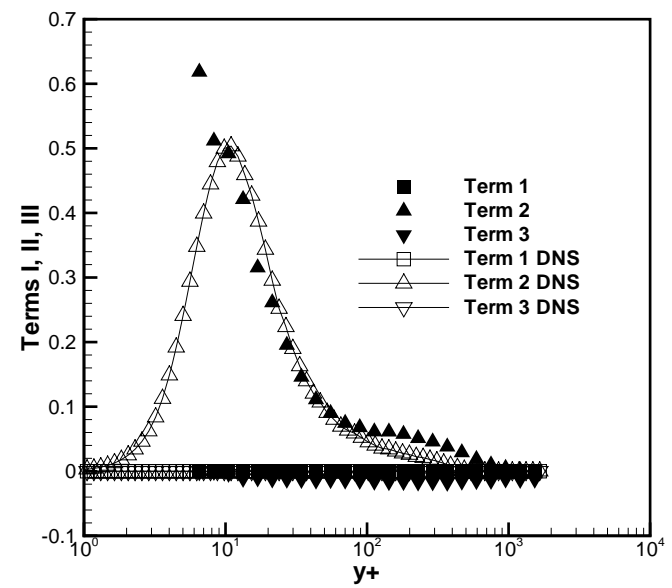

(a) ${\overline{u^{2}}}^{+}$Stress Production

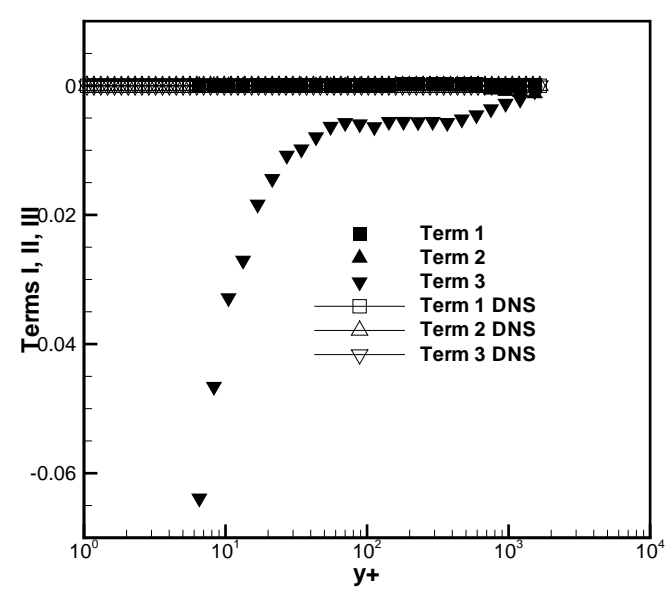

(c) ${\overline{v^{2}}}^{+}$Stress Production

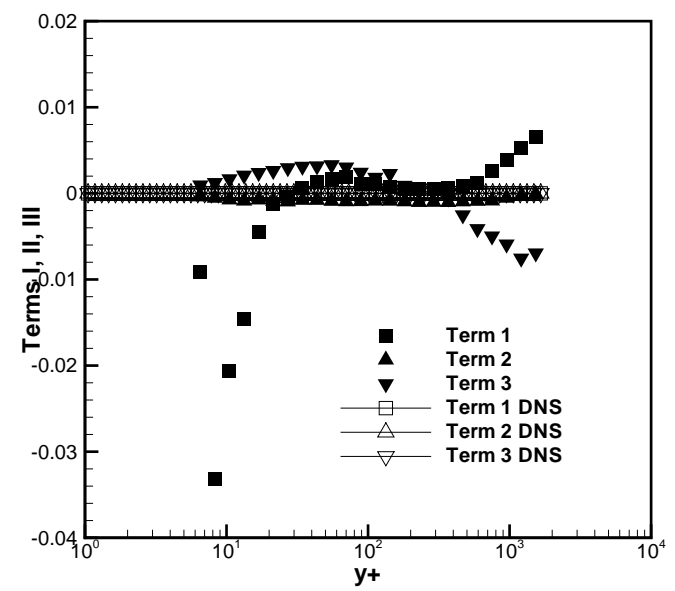

(e) $\bar{w}^{2}$ Stress Production

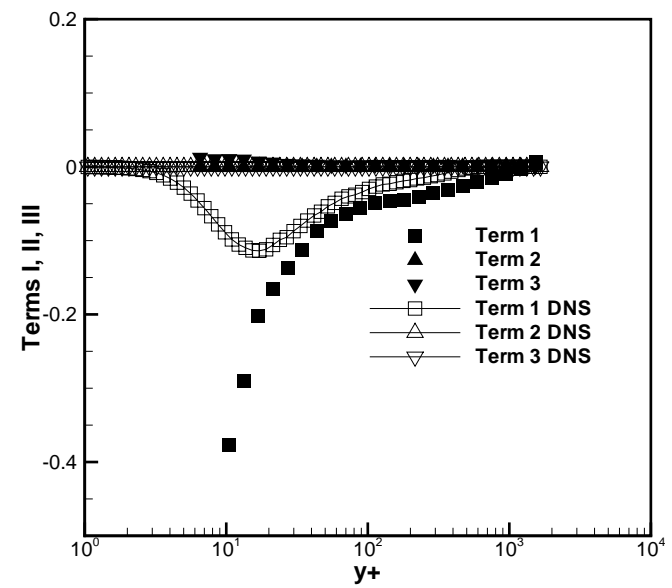

(b) $\overline{u v}+$ Stress Production

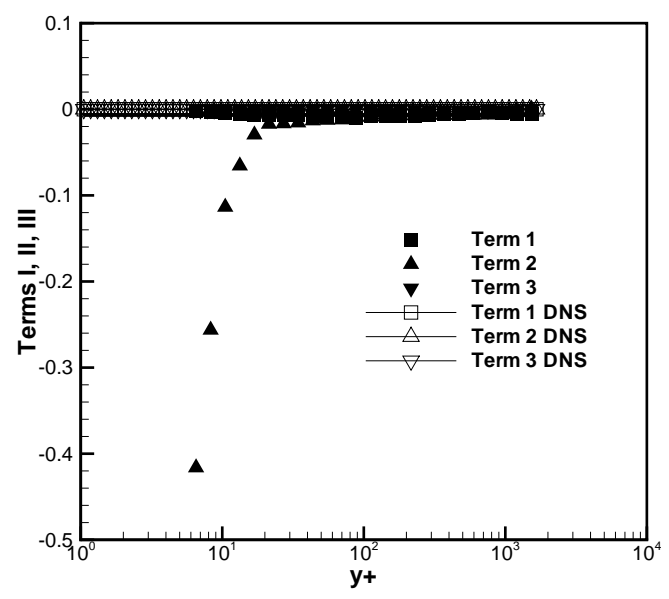

(d) $\overline{u w}^{+}$Stress Production

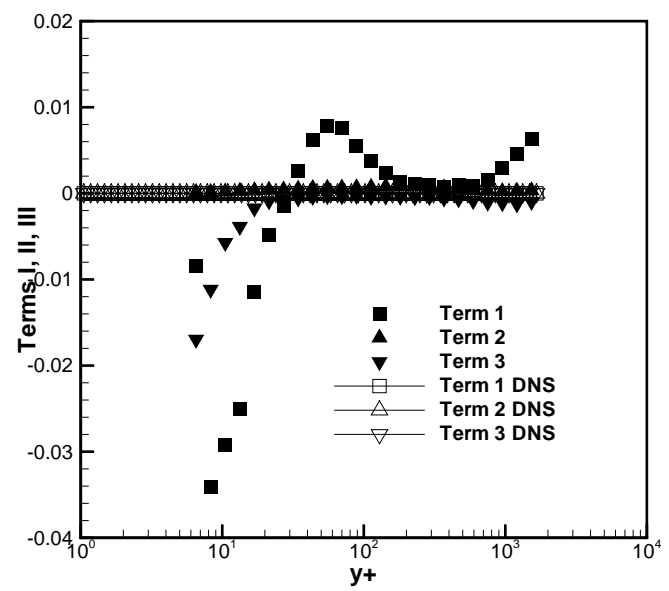

(f) $\overline{v w}^{+}$Stress Production

Figure 5.8: Reynolds Stresses Production at P08, DNS data by Iwamoto [20] 


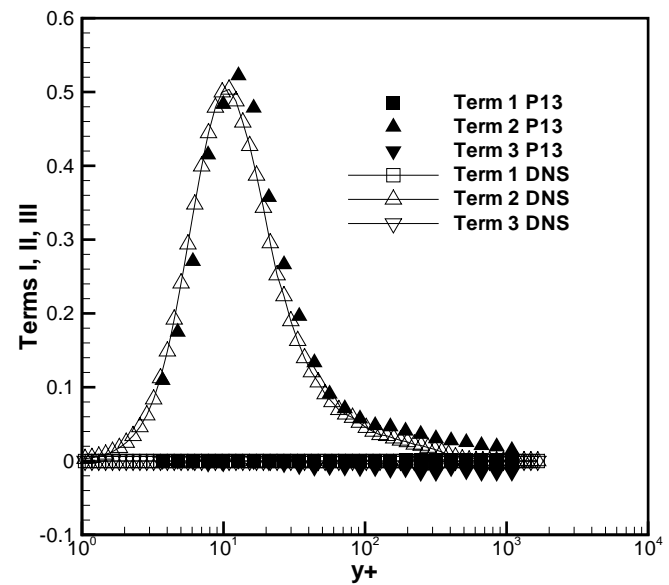

(a) $\bar{u}^{2}$ Stress Production

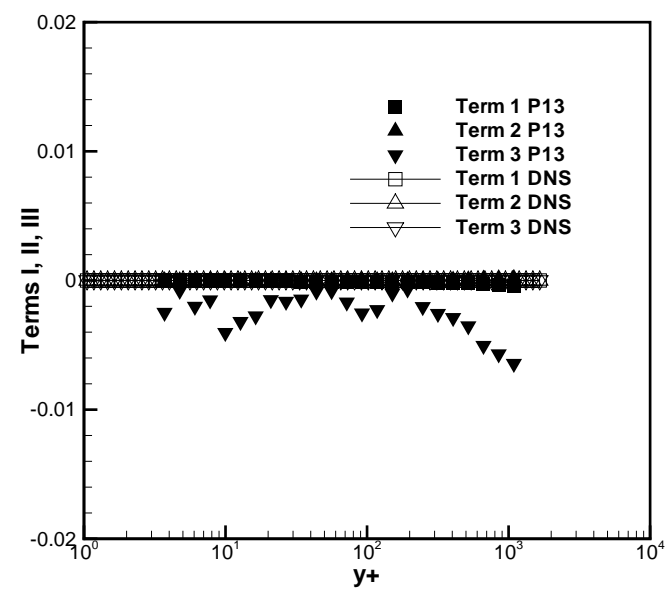

(c) ${\overline{v^{2}}}^{+}$Stress Production

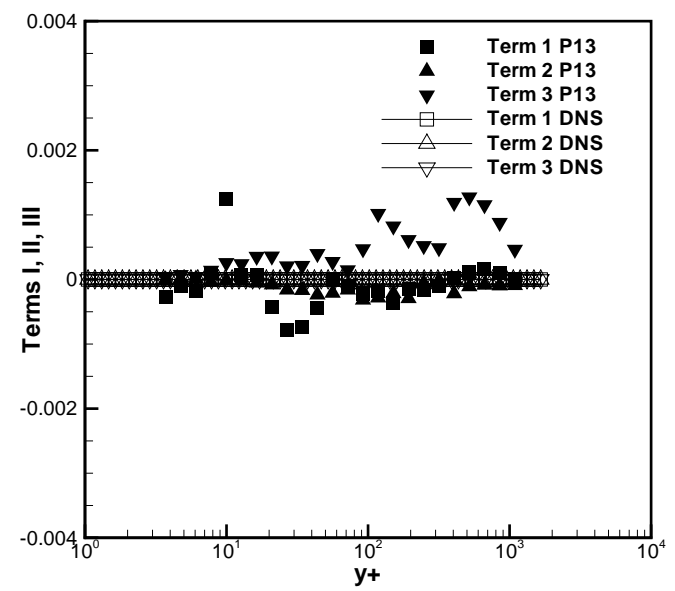

(e) $\bar{w}^{2}$ Stress Production

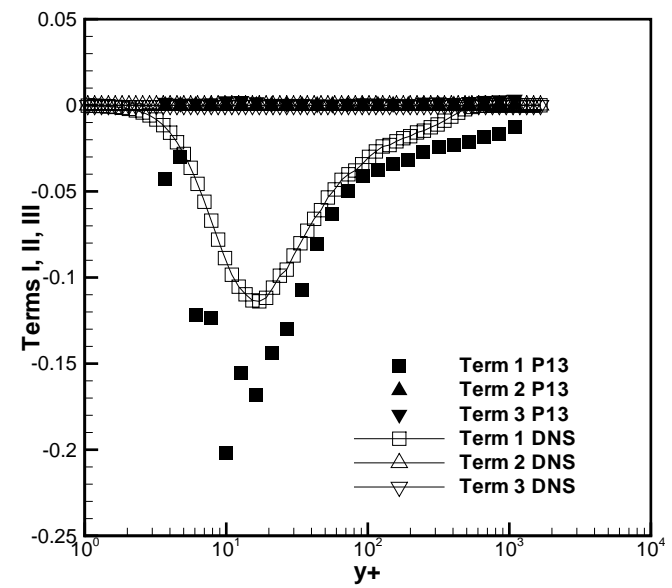

(b) $\overline{u v}+$ Stress Production

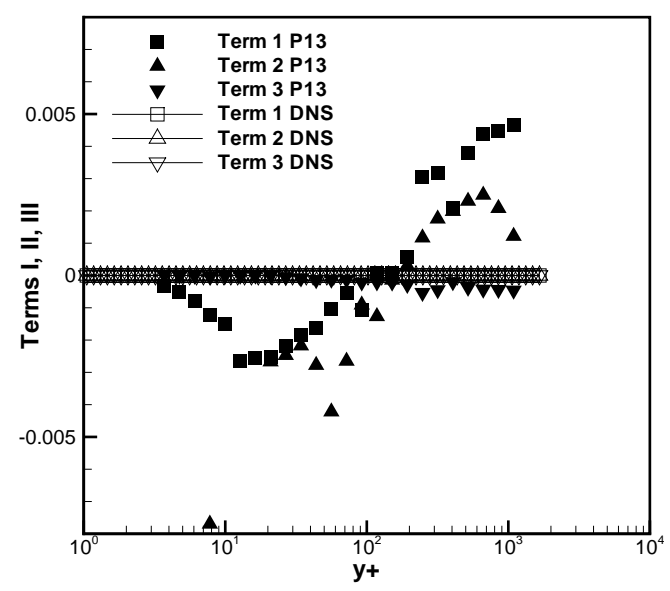

(d) $\overline{u w}^{+}$Stress Production

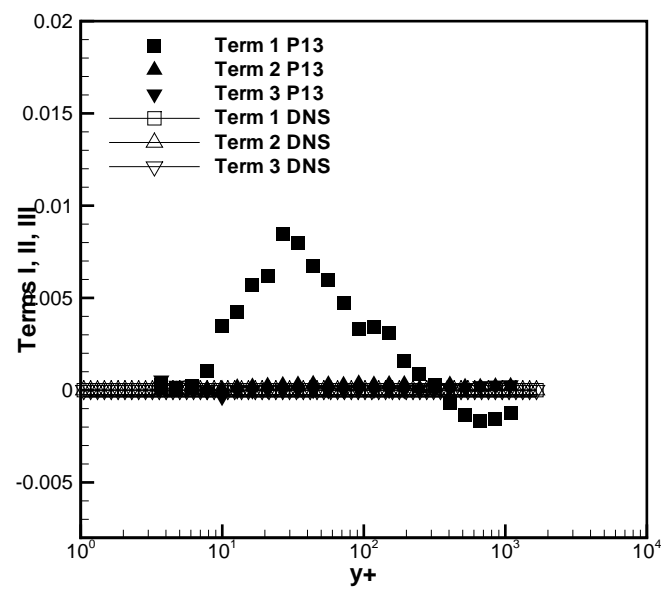

(f) $\overline{v w}^{+}$Stress Production

Figure 5.9: Reynolds Stresses Production at P13, DNS data by Iwamoto [20] 


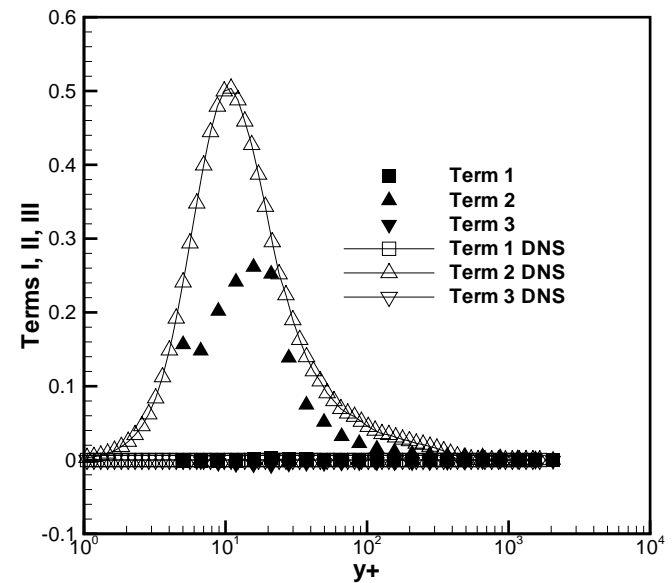

(a) $\bar{u}^{2}$ Stress Production

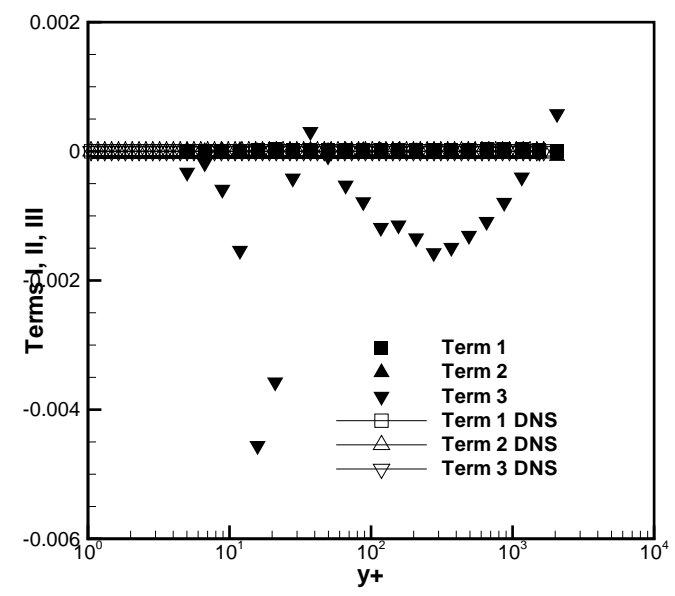

(c) ${\overline{v^{2}}}^{+}$Stress Production

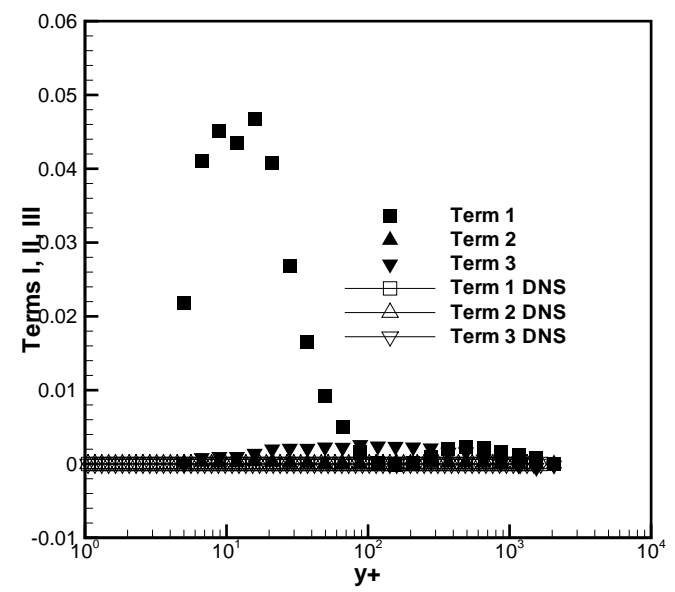

(e) ${\overline{w^{2}}}^{+}$Stress Production

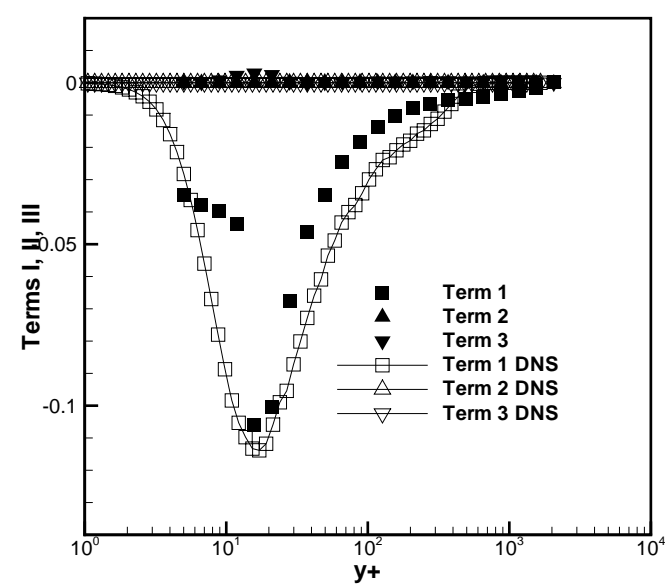

(b) $\overline{u v}+$ Stress Production

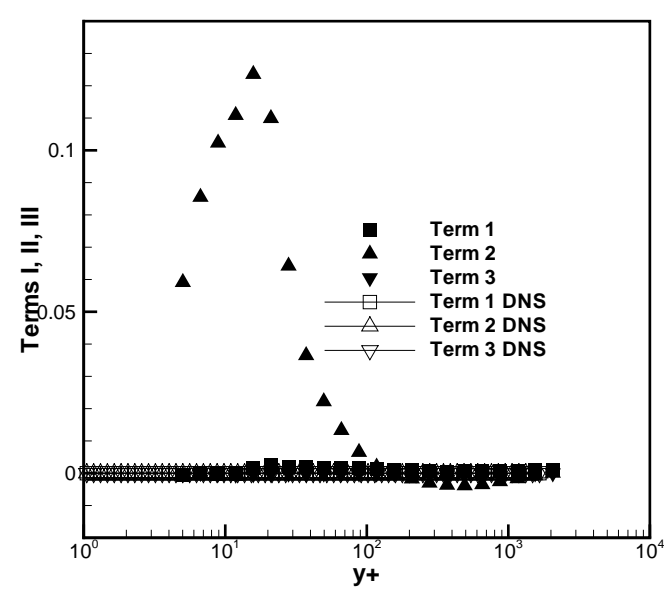

(d) $\overline{u w}^{+}$Stress Production

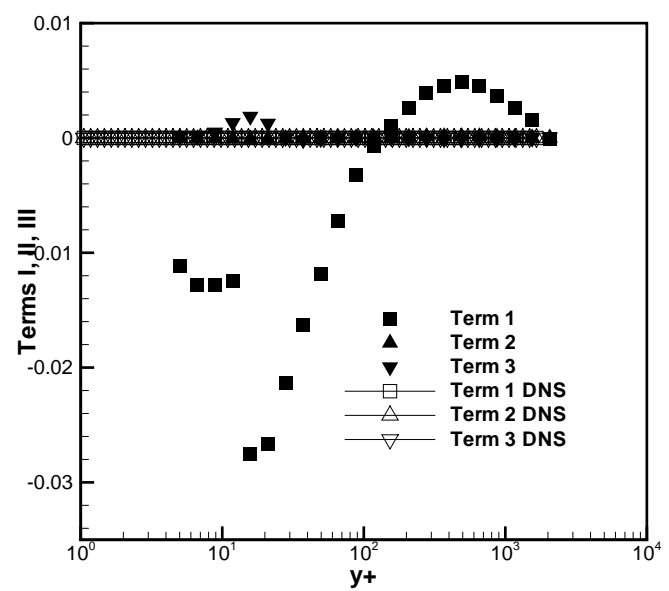

(f) $\overline{v w}$ Stress Production

Figure 5.10: Reynolds Stresses Production at P18, DNS data by Iwamoto [20] 


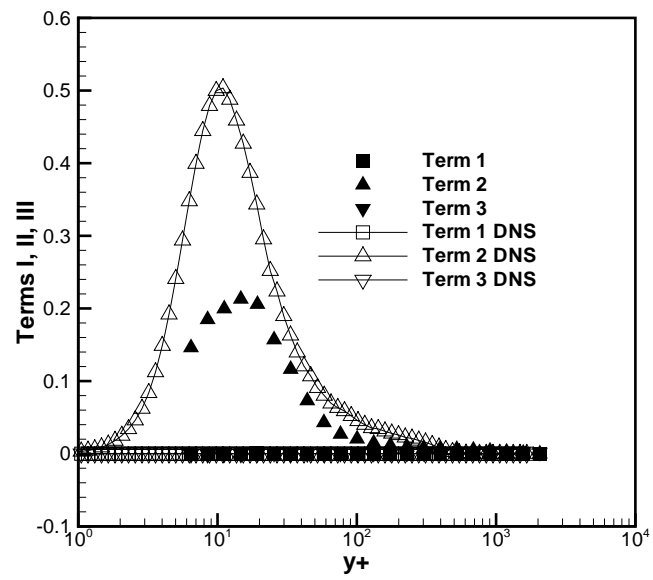

(a) ${\overline{u^{2}}}^{+}$Stress Production

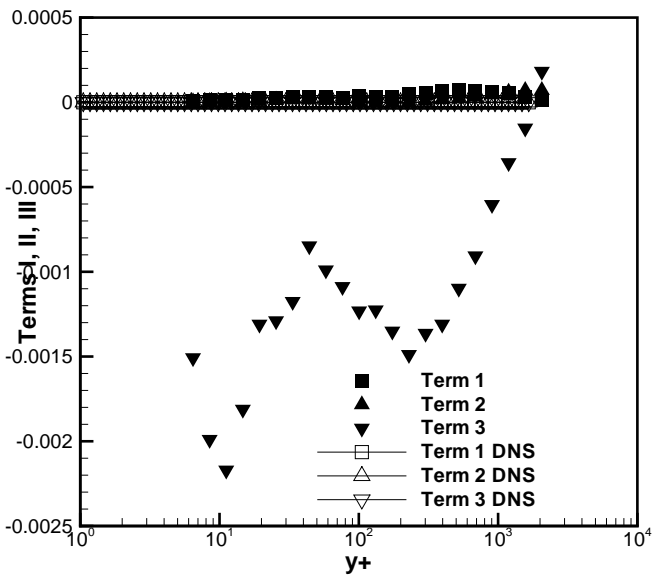

(c) $\overline{v^{2}}$ Stress Production

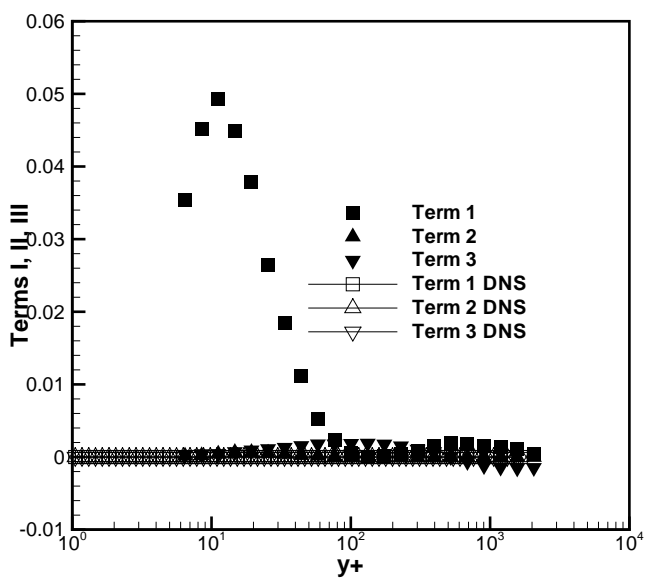

(e) $\bar{w}^{2}$ Stress Production

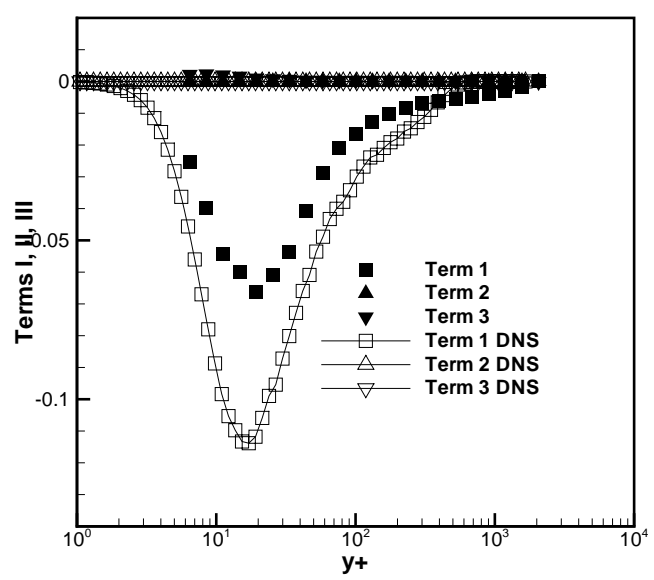

(b) $\overline{u v}+$ Stress Production

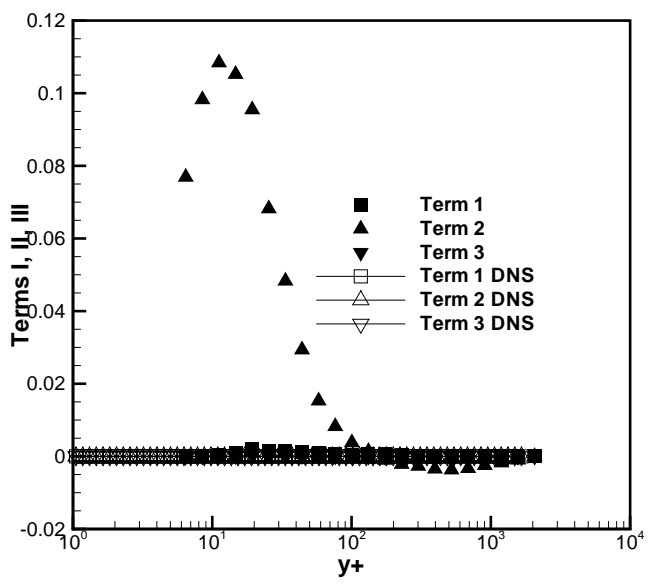

(d) $\overline{u w}^{+}$Stress Production

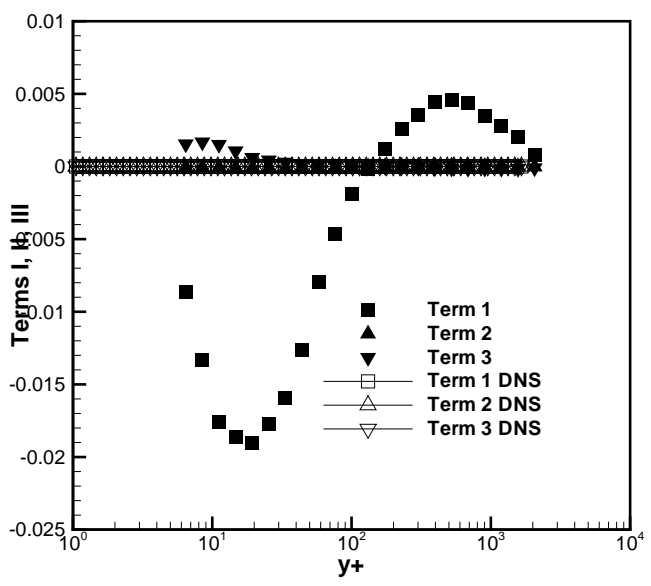

(f) $\overline{v w}^{+}$Stress Production

Figure 5.11: Reynolds Stresses Production at P19, DNS data by Iwamoto [20] 


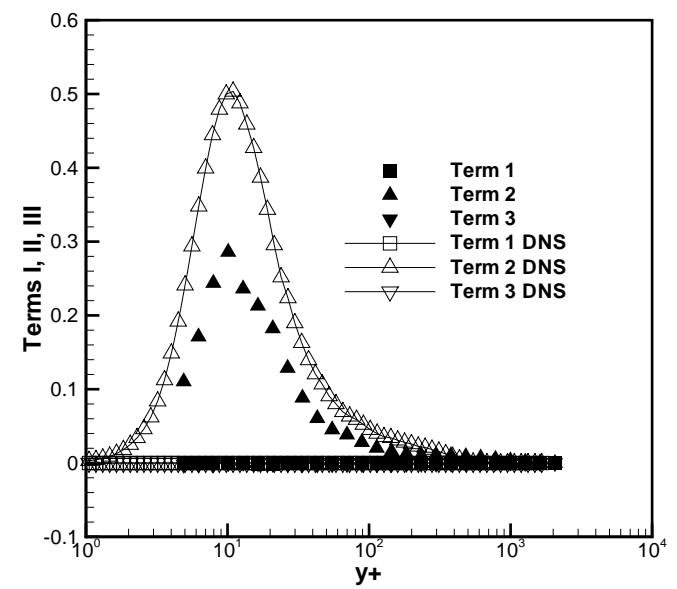

(a) ${\overline{u^{2}}}^{+}$Stress Production

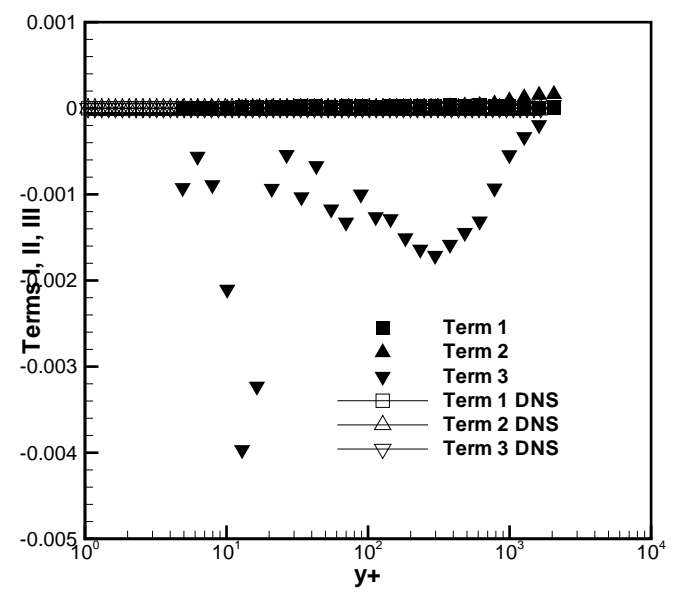

(c) $\overline{v^{2}}$ Stress Production

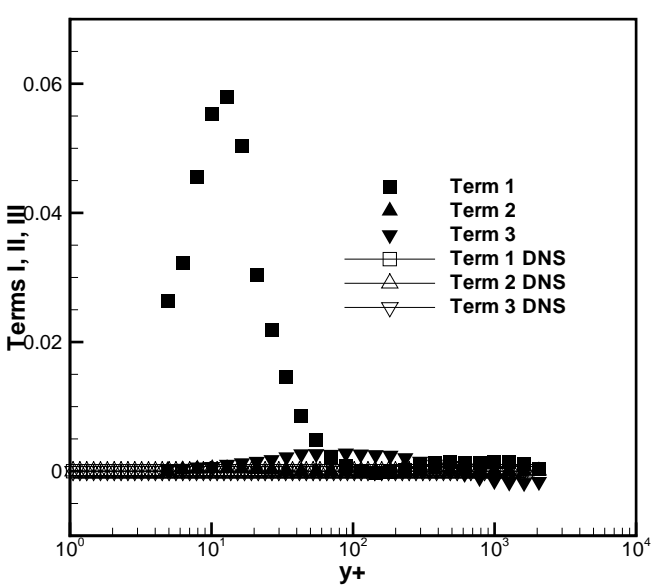

(e) ${\overline{w^{2}}}^{+}$Stress Production

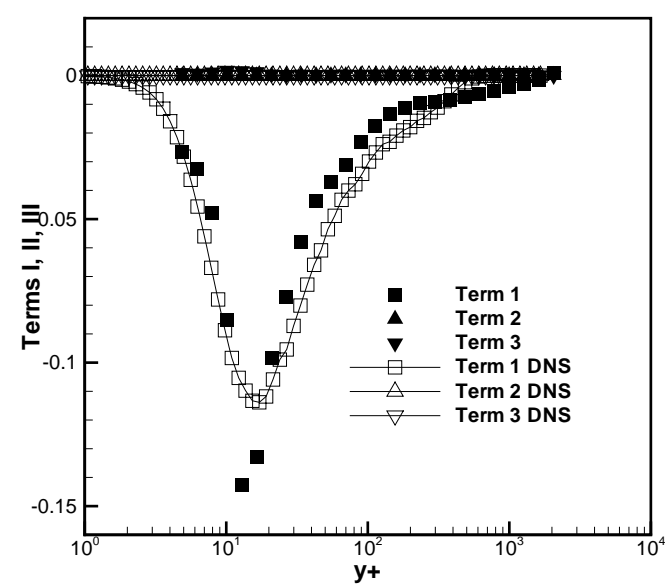

(b) $\overline{u v}+$ Stress Production

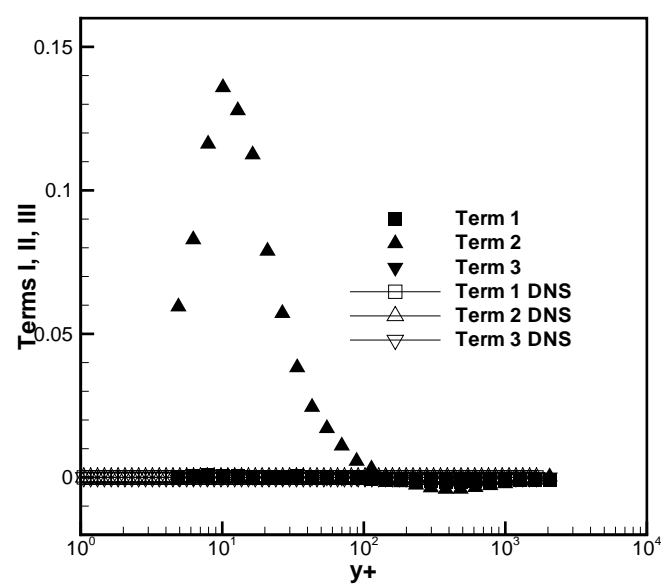

(d) $\overline{u w}^{+}$Stress Production

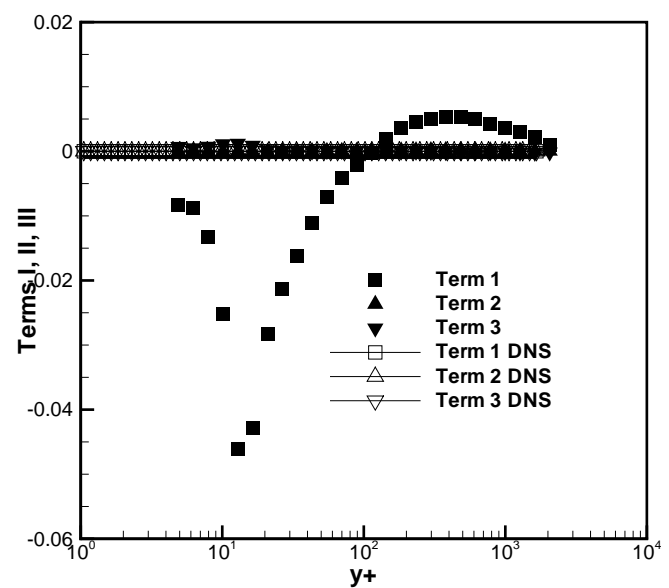

(f) $\overline{v w}^{+}$Stress Production

Figure 5.12: Reynolds Stresses Production at $P 19_{p}$, DNS data by Iwamoto [20] 


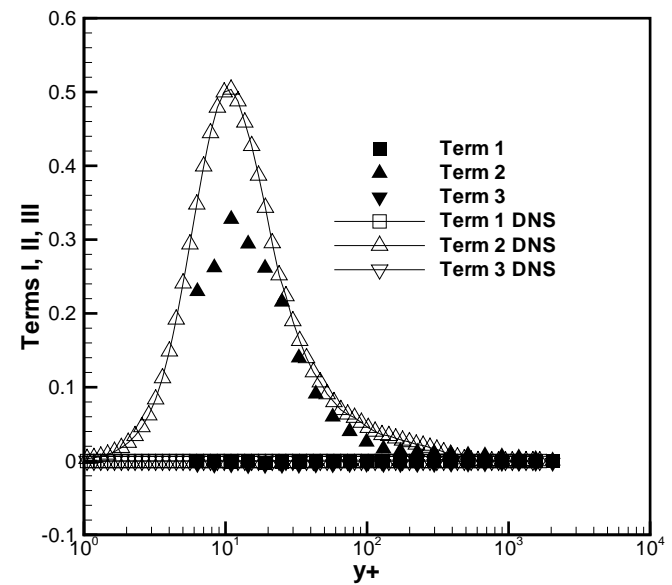

(a) $\bar{u}^{2}$ Stress Production

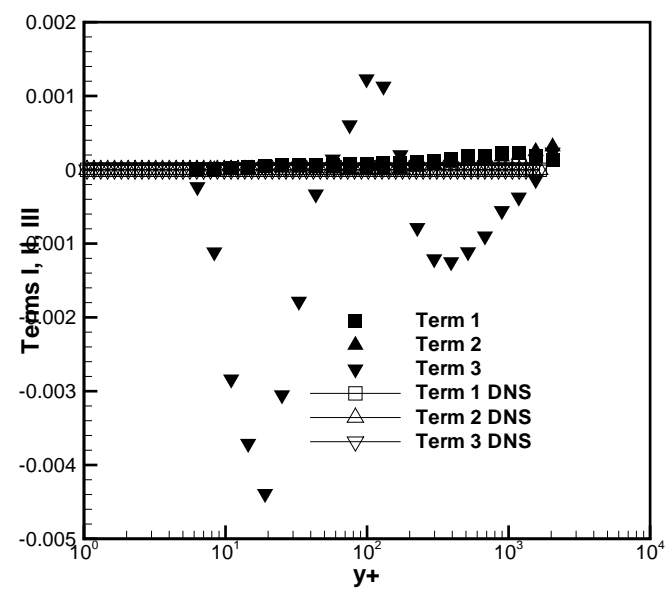

(c) ${\overline{v^{2}}}^{+}$Stress Production

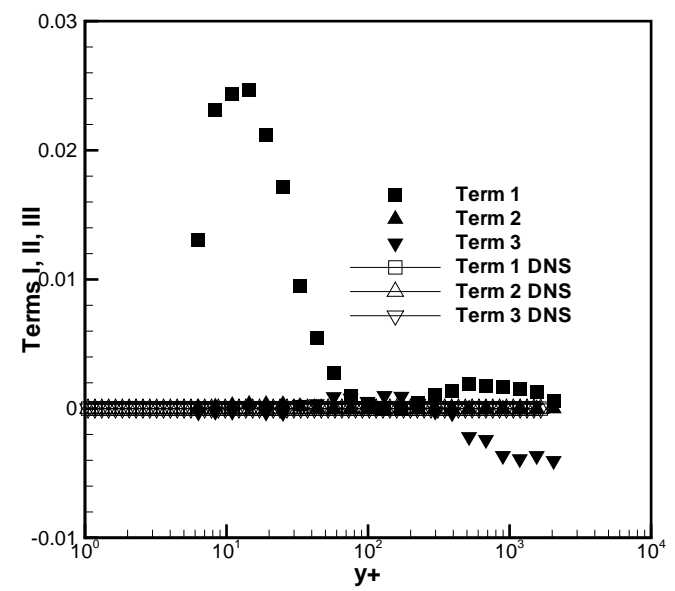

(e) ${\overline{w^{2}}}^{+}$Stress Production

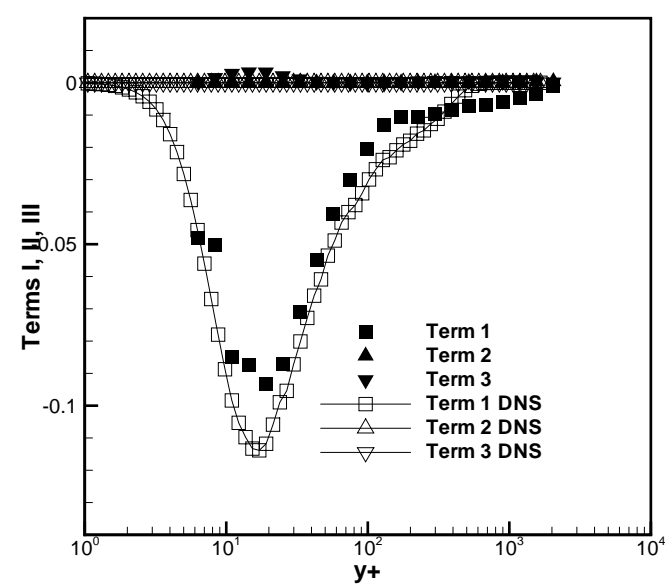

(b) $\overline{u v}+$ Stress Production

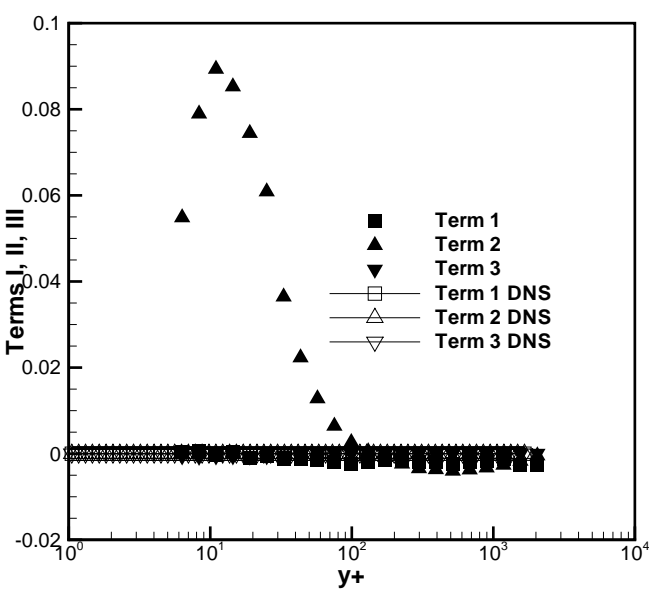

(d) $\overline{u w}^{+}$Stress Production

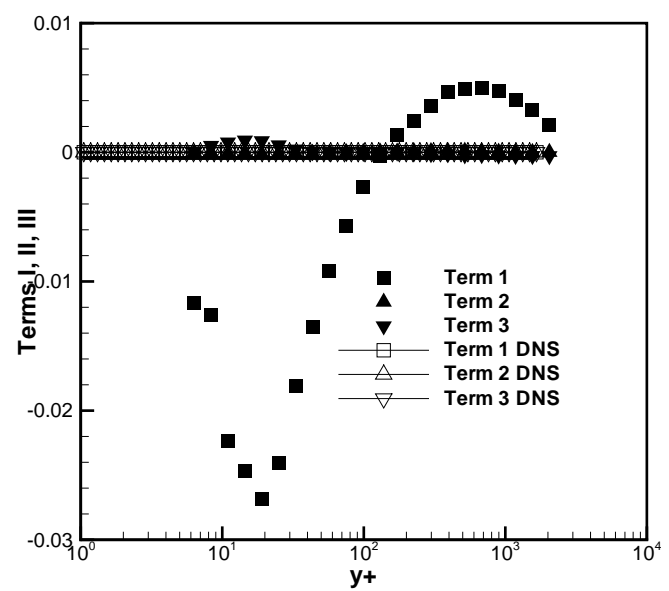

(f) $\overline{v w}^{+}$Stress Production

Figure 5.13: Reynolds Stresses Production at P20, DNS data by Iwamoto [20] 


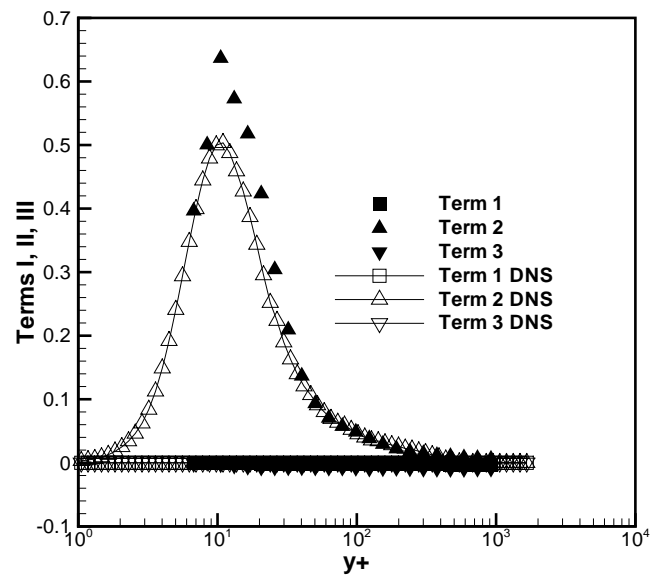

(a) ${\overline{u^{2}}}^{+}$Stress Production

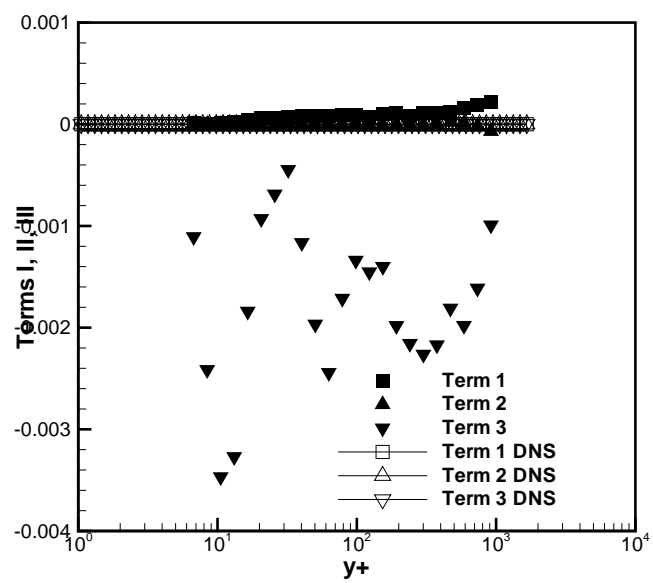

(c) $\overline{v^{2}}$ Stress Production

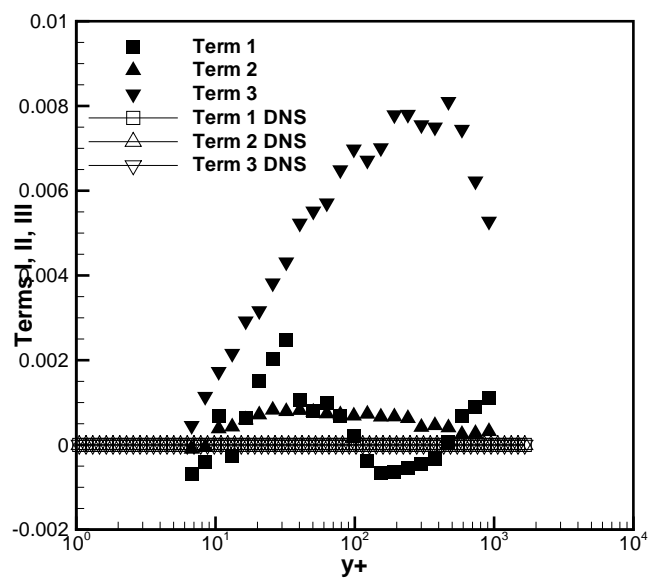

(e) ${\overline{w^{2}}}^{+}$Stress Production

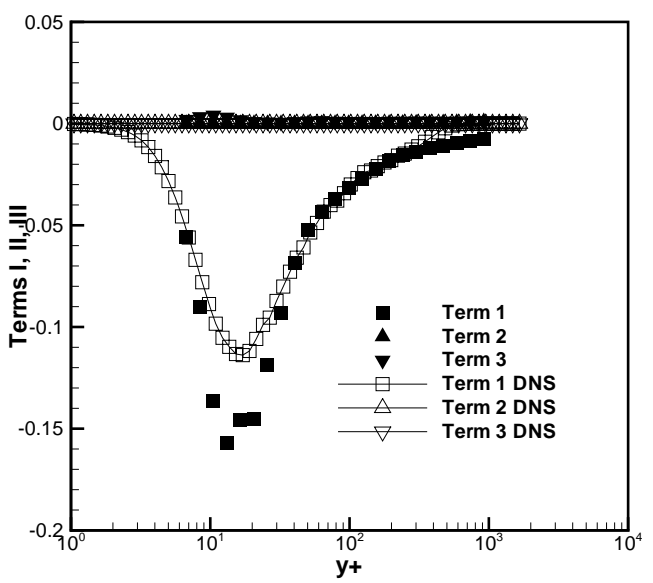

(b) $\overline{u v}^{+}$Stress Production

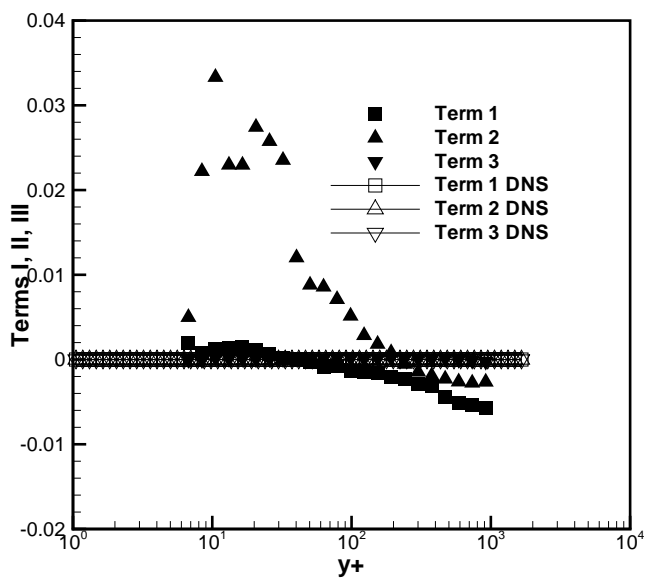

(d) $\overline{u w}^{+}$Stress Production

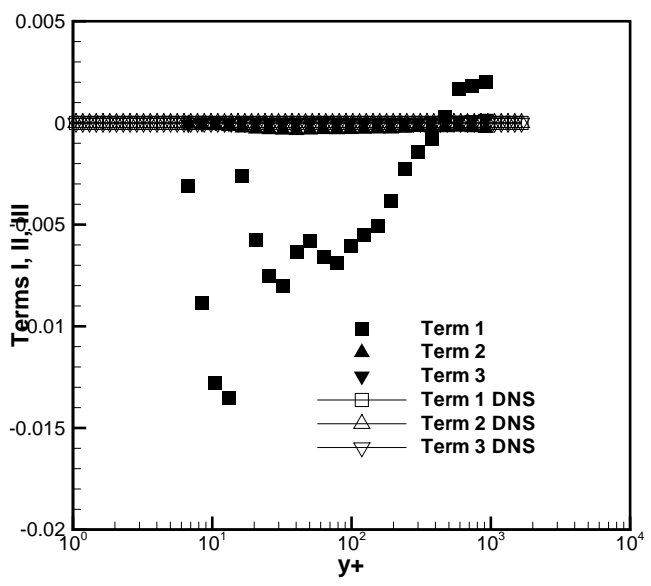

(f) $\overline{v w}^{+}$Stress Production

Figure 5.14: Reynolds Stresses Production at P33, DNS data by Iwamoto [20] 


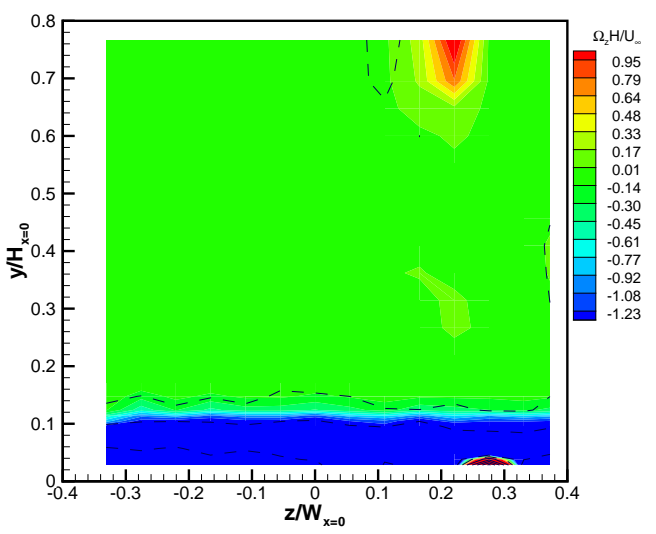

(a) $x / L=0.00$

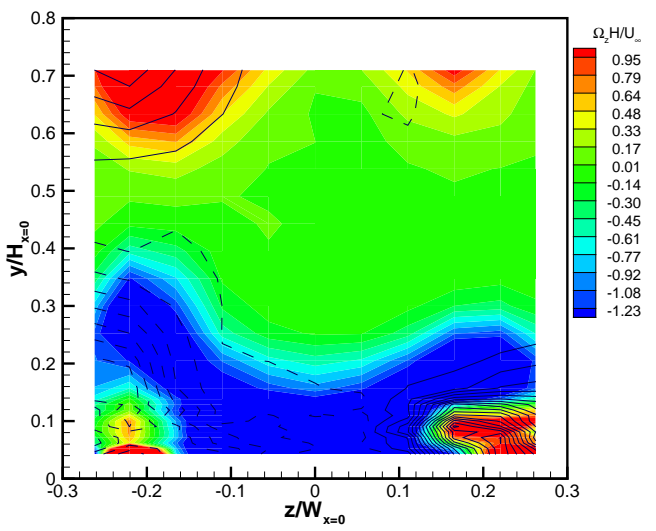

(c) $x / L=0.39$

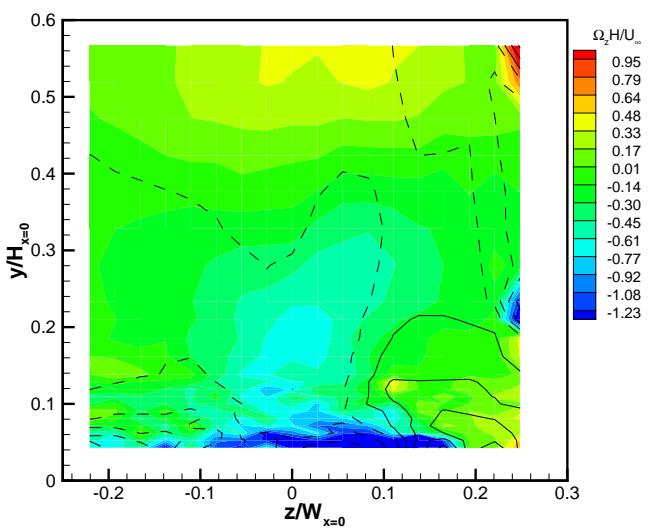

(e) $x / L=0.79$

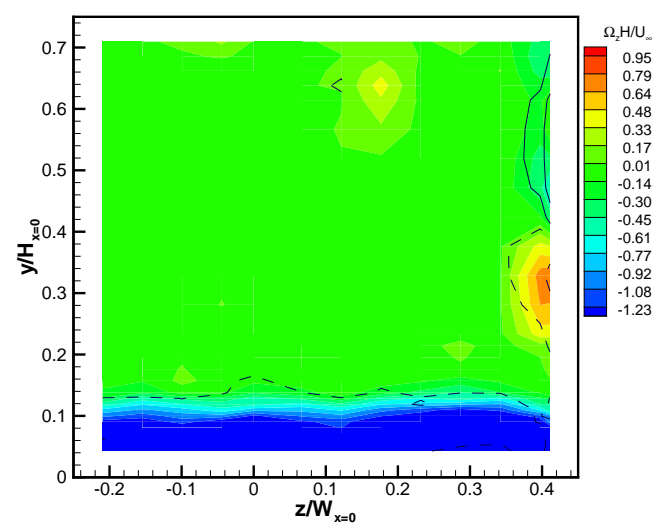

(b) $x / L=0.13$

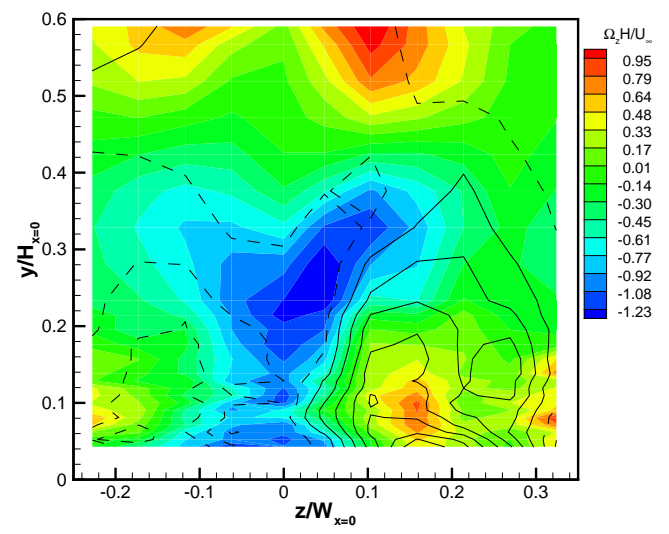

(d) $x / L=0.64$

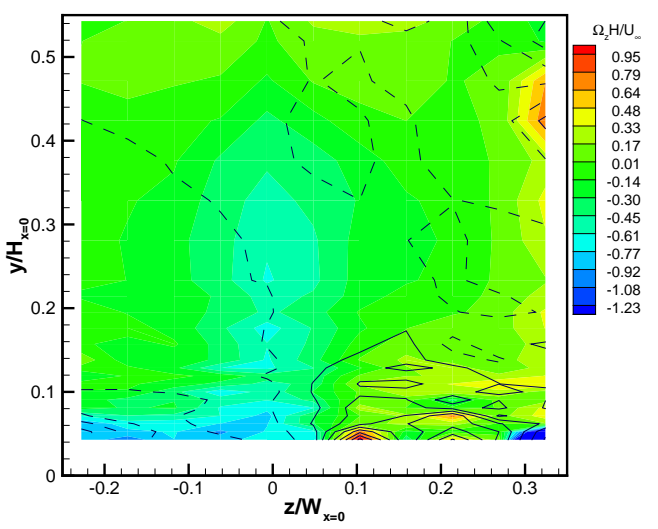

(f) $x / L=0.90$

Figure 5.15: Contours of Span-wise Mean Vorticity, $\frac{\Omega_{z} H}{U_{\infty}}$, shown as colored contours and Stream-wise Mean Vorticity, $\frac{\Omega_{x} H}{U_{\infty}}$, shown as line contours along different stream-wise stations. Dashed lines denote negative values. Step between each two lines is 0.1 

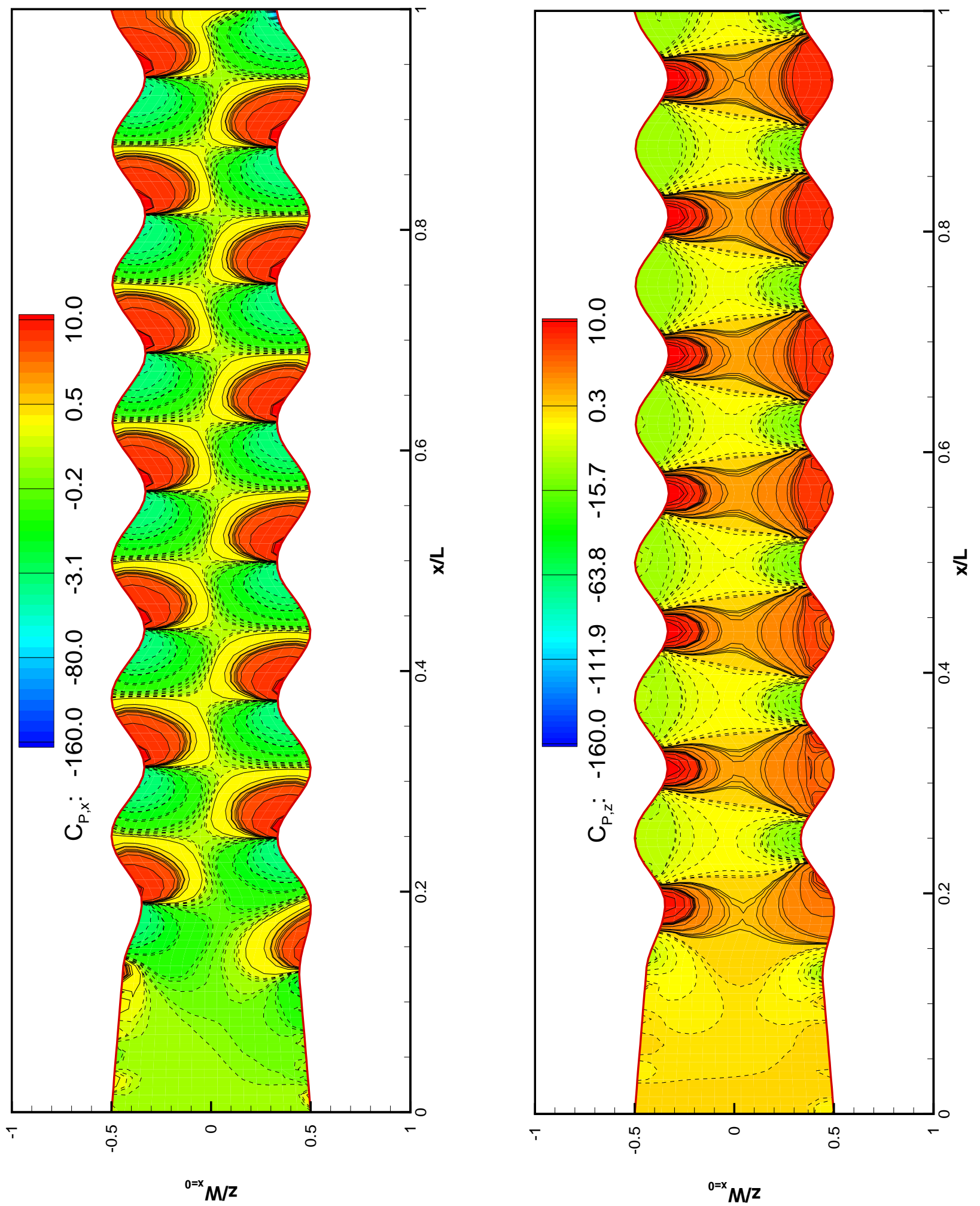

Figure 5.16: Stream-wise and Span-wise Coefficient of Pressure Gradients 


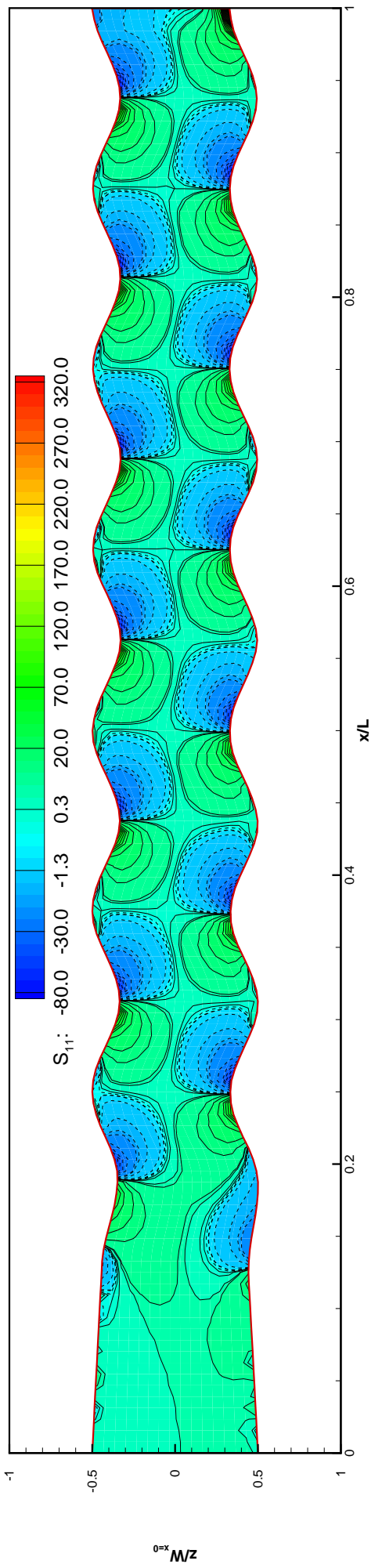

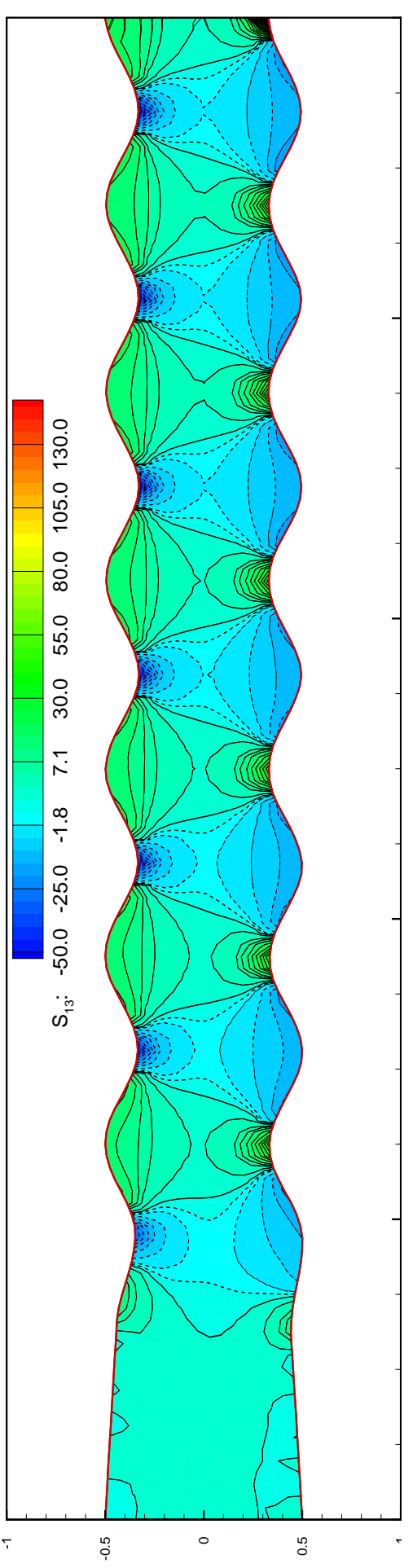

${ }^{0=x} M / \mathbf{z}$

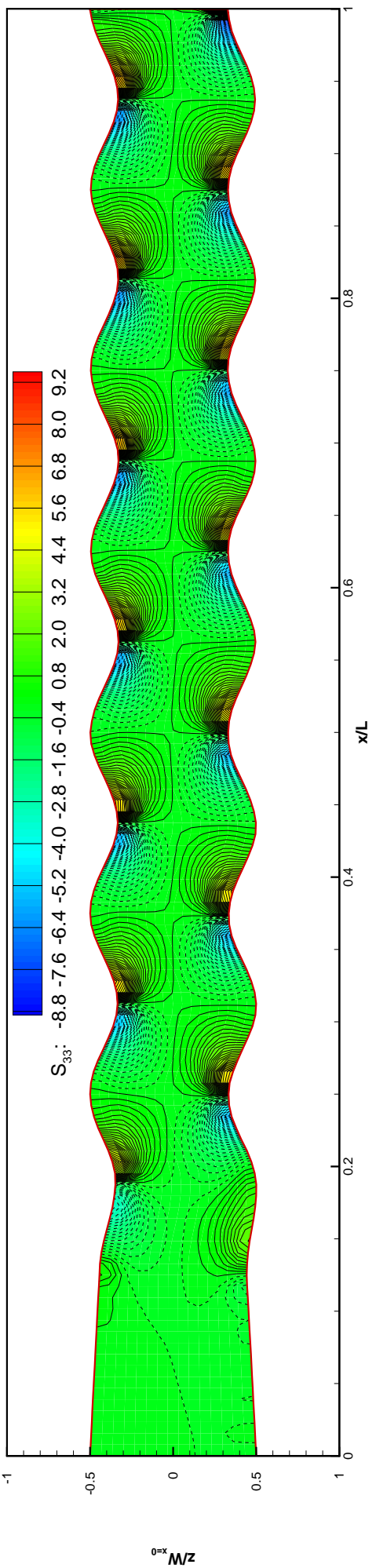

$\vec{x}$

Figure 5.17: Mean Rate of Strain, $S_{11}, S_{13}, S_{33}$ 

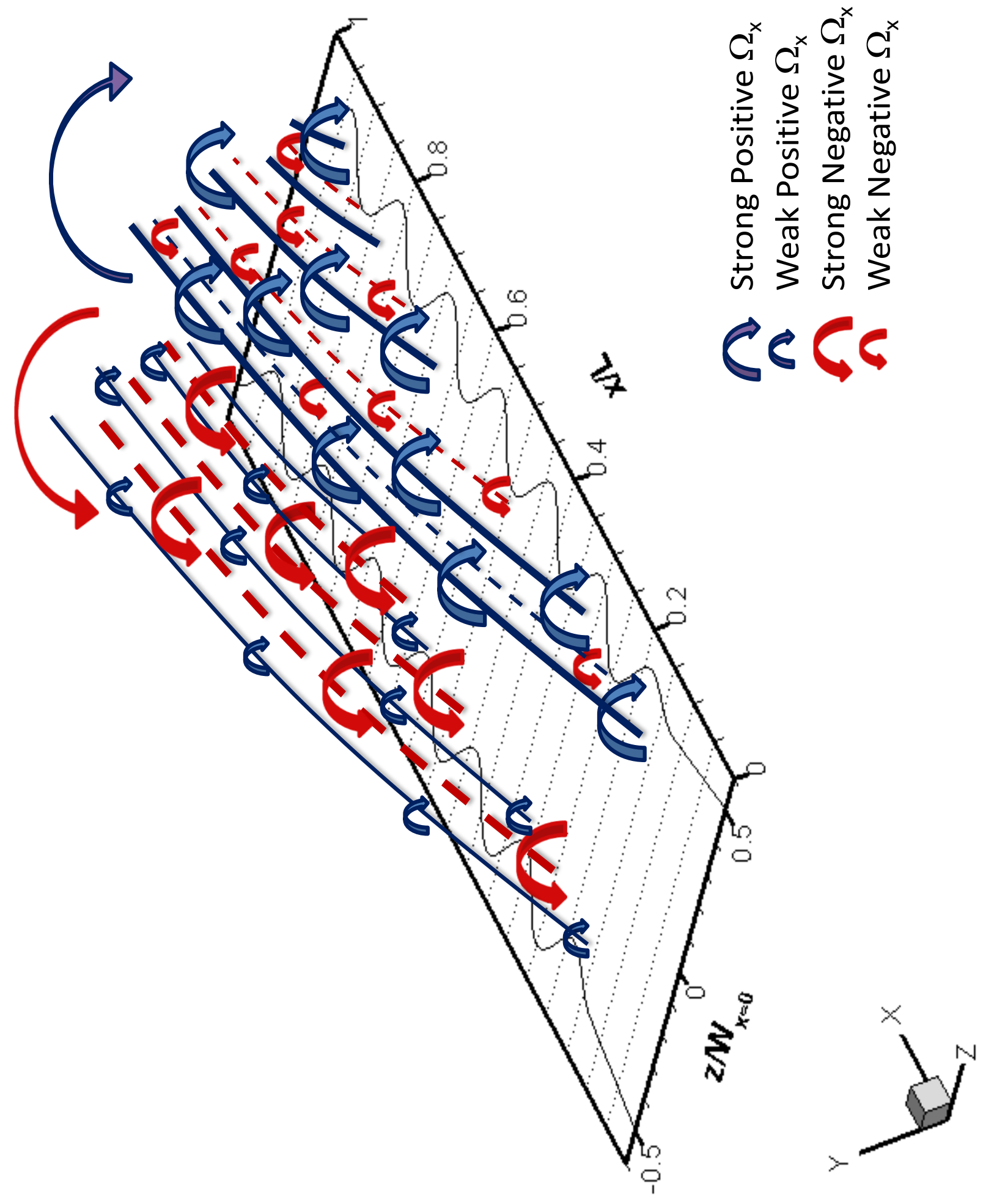

Figure 5.18: Stream-wise Vorticity development 


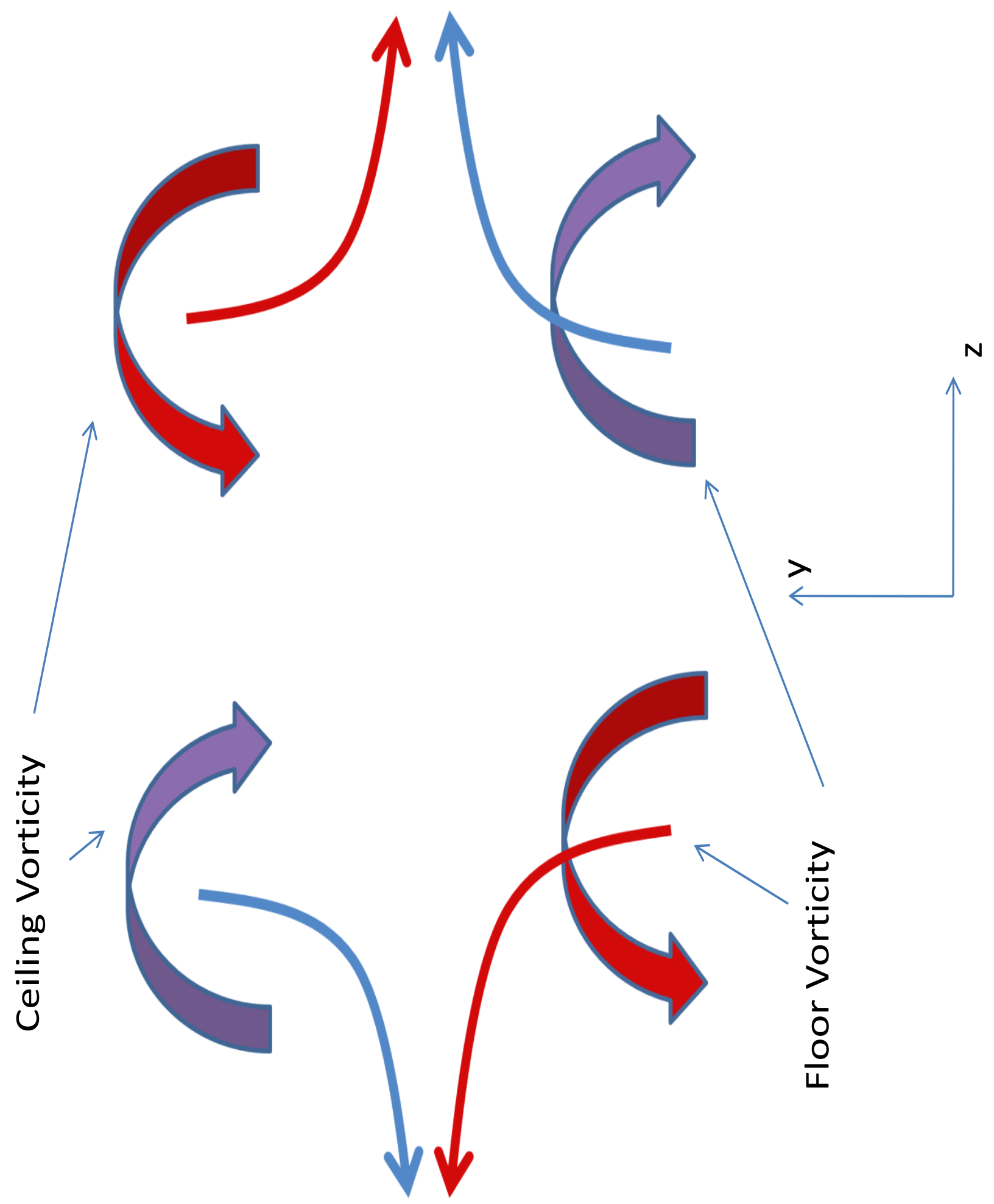

Figure 5.19: Stream-wise Vorticity interaction in $y$-z plane 


\section{Chapter 6}

\section{Second-Moment Closure Models}

\subsection{Turbulence Closure}

As mentioned in Chapter one, incompressible turbulent flow fields are governed by the Continuity and Navier-Stokes Equations, 1.1 and 1.2, respectively. The turbulent Reynolds stresses are calculated from the Reynolds Stress Transport Equations, 1.3. However, the Reynolds Stress Transport Equations contain three tensor correlations that need to be modeled. The modeling of these terms closes the set of equations required to calculate any flow field. The three tensors are the velocity pressure-gradient correlation, $\Pi_{i j}$, turbulent diffusion, $D_{i j}^{T}$, and dissipation rate, $\epsilon_{i j}$. The velocity pressure-gradient correlation is often split up into the pressure-strain correlation, $\phi_{i j}$, and pressure-diffusion correlation, $D_{i j}^{p}$.

In this chapter some selected models for turbulent diffusion will be examined and compared to measured values directly. All turbulent diffusion models require values for the turbulent kinetic energy dissipation rate, $\epsilon$. An estimate for the turbulent kinetic energy dissipation rate is obtained by used measured quantities to calculate all terms in the turbulent kinetic energy transport equation except the pressure-diffusion term and the dissipation rate term. For the pressure-diffusion correlation Lumley's pressure-diffusion model is used, and then the remaining term which is the dissipation rate is estimated by requiring that it balances all other terms calculated in the turbulent kinetic energy transport equation.

The elements of the pressure-strain correlation are also estimated from each Reynolds stress transport equation. This is done by calculating terms in the Reynolds stress transport equations using measured data and then requiring that the pressure-strain correlation balances all other terms in the equation. In order to do this, an estimate for the dissipation rate tensor is needed. Three models for the dissipation rate tensor are evaluated and only one is selected to estimate the pressure-strain tensor. All dissipation rate 
tensor models utilize the turbulent kinetic energy dissipation rate estimated as mentioned above. The extracted pressure-strain correlations are then compared with different models in the literature.

\subsection{Turbulent Diffusion Models}

Various diffusion models have been discussed in Chapter One. Most of the mentioned models have been examined by different researchers. Ölçmen and Simpson, [44], examined the Daly-Harlow, Mellor-Herring, Hanjalić and Launder and Lumley's diffusion models using data from their wing-body junction flow. In general, all models failed to capture the magnitude of the triple-velocity products suggesting that the length scale, $\overline{q^{2}} / \epsilon$, used by these models may not be correct.

Schwarz and Bradshaw [57] also studied turbulent diffusion models in a three dimensional turbulent boundary layer. They used a standard cross-hot-wire probe to measure the Reynolds stresses and triple products. According to their comparisons the turbulent diffusion models performed poorly. However, they argued that this correlation is not important since it is small in magnitude. This might be true for boundary layer flows but for wake flows or recirculating zones it becomes more dominant.

Kurbatskii and Poroseva,[22], tested the Daly-Harlow and Hanjalić-Launder models in a rotating pipe flow. None was capable of reproducing the experimental data for the entire flow for different swirl rates. They also proposed a differential model using the transport equations of the triple-velocity products. Their model gave good estimates; however for higher swirl and Reynolds Number the Hanjalić-Launder model showed more stable performance. The main condition for a diffusion model to work is being invariant with respect to coordinate transformation.

In addition to the models mentioned in Chapter One, Moore, [38], suggested a new diffusion model that is tensorially consistent. The new model has the same form as the Daly-Harlow but uses $q / \omega$ as a time scale instead of $k / \epsilon$ and a different velocity scale tensor than the Reynolds Stresses.

The models chosen to be tested here are the Hanjalić-Launder model since it is the most successful model to date, Moore's model since it hasn't been tested extensively yet, Mackinnon model and the Daly-Harlow model due to its popularity and simplicity. The models chosen are given as follows,

1. Hanjalić-Launder Model, [18]

$$
\overline{u_{i} u_{j} u_{k}}=-0.11 \frac{k}{\epsilon}\left[\overline{u_{i} u_{l}} \frac{\partial \overline{u_{j} u_{k}}}{\partial x_{l}}+\overline{u_{j} u_{l}} \frac{\partial \overline{u_{k} u_{i}}}{\partial x_{l}}+\overline{u_{k} u_{l}} \frac{\partial \overline{u_{i} u_{j}}}{\partial x_{l}}\right]
$$


This model was derived from the triple velocity product transport equations. It was intended for high Reynolds number flows away from boundaries such that viscous effects are negligible. It was also assumed that away from boundaries the flow would be isotropic. Fourth order moments were approximated using the quasinormality assumption of Millionshchikov, [35]. Finally, the convection terms were considered negligible and assuming the stress pressure gradient correlation to be proportional to the triple velocity product.

2. Mackinnon's Model, [30]

$$
\overline{u_{i} u_{j} u_{k}}=\left[C_{\phi 1} /\left(1+f^{*}\right)\right](k / \epsilon)\left[D P_{i j k}+\left(1-C_{\phi 2}\right) P_{i j k, 2}\right]
$$

Where, $C_{\phi 1}=0.6$ and $C_{\phi 2}=0.16$ are constants and

$$
\begin{aligned}
& f^{*}=\frac{\left(1-e^{0.615 b}\right) e^{b y^{*}}}{e^{0.615 b}\left(1-e^{b y^{*}}\right)} \\
& y^{*}=1.96\left(\frac{y}{k^{1.5} / \epsilon}\right) \text { and } b=-1.3 \\
& D P_{i j k}=-\left(\overline{u_{i} u_{l}} \frac{\partial \overline{j_{j} u_{k}}}{\partial x_{l}}+\overline{u_{j} u_{l}} \frac{\partial \overline{u_{k} u_{i}}}{\partial x_{l}}+\overline{u_{k} u_{l}} \frac{\partial \overline{u_{i} u_{j}}}{\partial x_{l}}\right) \\
& D P_{i j k, 2}=-\left(\overline{u_{i} u_{j} u_{l}} \frac{\partial U_{k}}{\partial x_{l}}+\overline{u_{j} u_{k} u_{l}} \frac{\partial U_{i}}{\partial x_{l}}+\overline{u_{k} u_{i} u_{k}} \frac{\partial U_{j}}{\partial x_{l}}\right)
\end{aligned}
$$

This model was developed to account for free stream turbulence and to correct the near wall behavior of the ${\overline{v^{2}}}^{+}$normal stress. The assumptions made to obtain this model are similar to those made by Hanjalić and Launder; however, the effect of mean flow gradients are taken into account. Furthermore, the length scale function is multiplied by an empirical function to account for free stream turbulence and wall proximity.

3. Moore's Model, [38]

$$
D_{i j}^{T}=-\frac{\partial \overline{u_{i} u_{j} u_{k}}}{\partial x_{k}}=\frac{\partial}{\partial x_{k}} c_{v q} \frac{q}{\omega} v_{k n} \frac{\partial \overline{u_{i} u_{j}}}{\partial x_{n}}
$$

Where, $c_{v q}=0.076$ and the velocity scale tensor, $v_{i j}$, is defined such that $v_{i k} v_{k j}=$ $\overline{u_{i} u_{j}}$. The velocity scale tensor $v_{i j}$ has the same eigen-vectors as the Reynolds stress tensor and its eigen-values are equal to the square root of the principal Reynolds normal stresses. In principal stress coordinates, this model can be viewed as based on a turbulent viscosity composed of an isotropic length scale and a velocity scale that is in the direction of diffusion, whose value is equal to the root mean square of the fluctuating velocity.

4. Daly-Harlow Model

$$
D_{i j}^{T}=\frac{\partial}{\partial x_{k}} c_{s} \overline{u_{k} u_{l}} \frac{\partial \overline{u_{i} u_{j}}}{\partial x_{k}}
$$

Where, $c_{s}=0.225$. This model was developed based on analogy between turbulent and viscous diffusion of a scalar quantity. 
Note that the last two models don't give an expression for the triple-velocity correlations but rather an expression for the turbulent diffusion tensor as it appears in the Reynolds Stress Transport equations. Hence, calculation of different elements in the triple velocity correlation tensor is not unique.

In the next section, the above models are used to calculate the triple velocity correlations and are compared to experimental values. In evaluating the gradients in each model only the normal to the wall gradients are considered, since the stream-wise and span-wise gradients are almost two orders of magnitude smaller than the normal to the wall gradients.

\subsubsection{Results}

Figures 6.1 6.4 show comparisons of different measured triple velocity correlations and different models for profiles $P 07, P 13, P 19 p p$ and $P 32$. Those profiles were chosen as representative for each cluster of profiles measured. Profile P07 shows almost twodimensional behavior near the wall, however, in the outer layer the span-wise velocity magnitude increases to a large value. Profile $P 13$ and $P 32$ show very high turbulence levels as well as turbulent transport levels. Profile P19pp, is among profiles showing the strongest three-dimensional behavior.

Figures $6.1 \mathrm{a}, 6.2 \mathrm{a}$, $6.3 \mathrm{a}$ and $6.4 \mathrm{a}$ show the $\overline{u^{3}}$ velocity correlation. In general, all models capture the right trend for all profiles except $P 13$, but they all fail to capture the right magnitude of the velocity correlation. For profile $P 13$ the measured values show positive correlation for the entire range while all the models show negative values at the beginning of the semi-logarithmic region. All models give very close estimates except for the Mackinnon model which shows very low values. This might be due to the fact that, the scaling function $k / \epsilon$ is multiplied by a damping function to account for wall effects on the pressure strain correlation.

Figures $6.1 \mathrm{~b}, 6.2 \mathrm{~b}, 6.3 \mathrm{~b}$ and $6.4 \mathrm{~b}$ show the $\overline{v^{3}}$ velocity triple product. In the inner layer, the $\overline{v^{3}}$ triple product remains very small with respect to $\overline{u^{3}}$ then starts to increase or decrease significantly in the outer layer above $y^{+}=200$. All models predict higher absolute values than the measured ones with different signs. Moreover, Moore's model doesn't seem to follow the right trend as the measured values.

Figures $6.1 \mathrm{c}, 6.2 \mathrm{c}, 6.3 \mathrm{c}$ and $6.4 \mathrm{c}$ show the $\overline{w^{3}}$ velocity triple product. It is clear from these figures that all models fail to reproduce the behavior of the $\overline{w^{3}}$ triple product; in addition, the magnitude from all model estimates is too low.

Figures $6.1 \mathrm{~d}$, $6.2 \mathrm{~d}, 6.3 \mathrm{~d}$ and $6.4 \mathrm{~d}$ show the $\overline{u^{2} v}$ velocity correlation. This is the most important triple velocity product since it represents the bulk of the turbulent diffusion. Fortunately, it is the best triple product predicted by the models. All models follow the same trend as the measured values. However, the difference in magnitude between mea- 
sured and modeled values increases away from the wall. Again, the Mackinnon model gives the poorest predictions. The Daly-Harlow and the Hanjalić-Launder models give the best predictions.

Figures 6.1e, 6.2e, 6.3e and $6.4 \mathrm{e}$ show the $\overline{v w^{2}}$ velocity triple product. This correlation represents the transport of the span-wise component of turbulence kinetic energy in the vertical direction. This correlation is typically zero in two dimensional boundary layers. Again the models fail to capture the behavior of this correlation. This might indicate that the models may be missing some physical ingredients to be able to predict three dimensional flow-fields.

Finally, figures 6.1f, 6.2f, $6.3 \mathrm{f}$ and $6.4 \mathrm{f}$ show the turbulence kinetic energy turbulent diffusion, $\overline{D_{i j}^{T}}$. The estimates for this quantity are much better than the estimates of the individual triple-velocity product. This is because of the fact that the models predict the $\overline{u^{2} v}$ triple product very well in terms of trend and magnitude, which is the main contributor to the turbulence kinetic energy turbulent diffusion term.

From the comparisons made above we can conclude the following,

- All models fail to model the correct magnitude of triple-velocity products. This was also pointed out by Ölçmen and Simpson, [44].

- All models fail to compute the correct trend for the $\overline{v^{3}}$ and $\overline{w^{3}}$.

- For profiles with high free stream turbulence (e.g. P13) all models fail to predict the right trend for $\overline{u^{3}}$

- The Daly-Harlow and Hanjalić-Launder models give the best overall estimates although their magnitudes are to some extent higher than the measured ones.

- Moore's model predictions are similar to Hanjalić-Launder model for $\overline{u^{2} v}$ in terms of trends, however the magnitude of the triple velocity products are much smaller than Hanjalić-Launder model.

- The Mackinnon model gives consistently poor predictions for all correlations and profiles.

- The Daly-Harlow and Hanjalić-Launder models consistently predict the turbulence kinetic energy turbulent diffusion fairly well for this flow.

- The fact that all models can't capture all triple-velocity products correctly and at the same time their estimates of the contribution towards the turbulence kinetic energy equation being close to measured values; suggests that, there are some physical components that are missing from these models. This means that in flow-fields that experience large gradients of all triple-velocity products these models will breakdown. 


\subsection{Dissipation Rate Tensor Anisotropy Models}

In this section a brief comparison is presented between three models for the Dissipation Rate anisotropy tensor. The models are due to Hallback et al.[17], Jakirlić and Hanjalić [21] and Perot and Natu [46]. All models require the estimation of turbulent kinetic energy dissipation rate. This is done by using measured quantities to calculate all terms in the turbulent kinetic energy transport equation except the pressure-diffusion term and the dissipation rate term. For the pressure-diffusion correlation Lumley's pressure-diffusion model is used, and then the remaining term which is the dissipation rate is estimated by requiring that it balances all other terms calculated in the turbulent kinetic energy transport equation.

Hallback et al., expressed the dissipation rate anisotropy, using the Cayley-Hamilton theorem, as a power series of the Reynolds stress anisotropy and the coefficients of the series contained the invariants of the stress anisotropy. The constants in the model were evaluated using the restrictions that the resulting tensor should be a traceless symmetric tensor and satisfies the homogeneous two-component turbulence limiting value. Hallback's model is given as follows,

$$
e_{i j}=\left[1+\alpha\left(\frac{1}{2} I I_{a}-\frac{2}{3}\right) a_{i j}-\alpha\left(a_{i k} a_{k j}-\frac{1}{3} I I_{a} \delta_{i j}\right)\right]
$$

Where, $\alpha=3 / 4, a_{i j}=\overline{u_{i} u_{j}} / 2 k-\delta_{i j} / 3$ and $I I_{a}=a_{i k} a_{k i}$.

Perot and Natu [46], derived an expression for the dissipation rate anisotropy by decomposing the fluctuating velocity components into two tensors, one has information about the magnitude of fluctuations and the other contains the temporal and spatial variation correlations. Their final expression is given as follows,

$$
\epsilon_{i j}=2 \nu\left(k_{, n}^{1 / 2}\right)^{2} \overline{u_{i} u_{j}}+\nu k_{, l}\left(\frac{\overline{u_{i} u_{j}}}{k}\right)+\frac{2}{3} \frac{\tilde{\epsilon} F k}{1+F} \delta_{i j}+\tilde{\epsilon} \frac{\overline{u_{i} u_{j}}}{1+F}+2 \nu \frac{F_{, n}^{1 / 2} \overline{u_{i} u_{j}} F_{, n}^{1 / 2}}{F} \delta_{i j}+C^{*} k S_{i j}
$$

Where, $F=\operatorname{det}\left[3 \overline{u_{i} u_{j}} / 2 k\right]$ and $C^{*}=0.18 F /(1+F)^{2}$.

Finally, Jakirlić and Hanjalić, [21], derived an expression for the dissipation rate tensor as equal to the homogeneous part of the dissipation rate tensor plus half the viscous diffusion tensor. They also expressed the anisotropy of the homogeneous part of dissipation rate as proportional to the Reynolds stress anisotropy. This form was shown to satisfy the wall limiting values exactly in an apriori DNS testing. The resulting expressions are as follows,

$$
\begin{array}{r}
\epsilon_{i j}=\epsilon_{i j}^{h}+\frac{1}{2} D_{i j}^{v} \\
\epsilon_{i j}^{h}=\left(1-f_{s}\right) \frac{2}{3} \epsilon^{h}+f_{s} \frac{\overline{u_{i} u_{j}}}{k} \epsilon^{h}
\end{array}
$$


Where, $f_{s}=1-\sqrt{A} E^{2}, A=1-\frac{9}{8}\left(a_{i k} a_{k i}-a_{i k} a_{k j} a_{j i}\right), E=1-\frac{9}{8}\left(e_{i k} e_{k i}-e_{i k} e_{k j} e_{j i}\right)$ and $e_{i j}=\epsilon_{i j} / 2 \epsilon-\delta_{i j} / 3$ is the dissipation rate anisotropy tensor.

In order to use this model an equation for the turbulent kinetic energy homogeneous dissipation rate part, $\epsilon^{h}$, must be solved. However, since the turbulent dissipation rate, $\epsilon$, is estimated from the turbulence kinetic energy transport equation, these estimates are inserted into Equation 6.8 and the homogeneous part, $\epsilon^{h}$, is estimated. Then, the homogeneous dissipation rate and the total dissipation rate tensors are estimated. The function, $f_{s}$, depends on the dissipation rate anisotropy tensor; hence, in order to evaluate the dissipation rate, $f_{s}$ must be calculated first. This is done by writing an expression for the dissipation rate anisotropy in terms of, $f_{s}$, and substituting in the definition of, $f_{s}$. The result is a sixth order algebraic equation in, $f_{s}$, which is solved numerically at each point in a profile. Once, $f_{s}$, is known the dissipation rate tensor can be calculated. Figure 6.5 shows the values of $f_{s}$ for multiple profiles.

The three models presented above were compared for different profiles. Figures 6.66.7 show the dissipation rate tensor component estimates for profiles $P 10$ and P35, respectively, along with the DNS data of Iwamoto and Kasagi, [20]. From these figures it can be pointed out that all models predict the trend correctly for $\epsilon_{11}, \epsilon_{22}, \epsilon_{33}$ and $\epsilon_{12}$.

In general, the Perot and Natu model, $(P N)$, and the Jakirlić and Hancalić, $(J H)$, model give similar results except for $\epsilon_{22}$ and $\epsilon_{12}$. The $(J H)$ model produces higher values of $\epsilon_{22}$. For $\epsilon_{12}$, the $(P N)$ model doesn't seem to give the right values for profile $P 10$, since it is giving a positive value and the DNS data and the other models show negative estimates. The Hallback and Johansson model, $(H J)$, gives lower estimates for $\epsilon_{12}$ and $\epsilon_{13}$ while its estimates of $\epsilon_{22}$ matches the $(J H)$ model.

Due to the fact that the differences between the three models are minor and that the Jakirlić and Hanjalić model gives the near wall behavior exactly, this model is chosen to be used to extract pressure-strain correlations from the Reynolds stress transport equations and compare it to different models. This is presented in the next section.

\subsection{Pressure-Strain Models}

The velocity-pressure gradient correlation, $\Pi_{i j}$, in equation 1.3 is usually split into the pressure-strain correlation tensor, $\phi_{i j}$, and the pressure-diffusion tensor, $D_{i j}^{P}$, such that,

$$
\underbrace{-\left(\overline{u_{i} \frac{\partial p}{\partial x_{j}}}+\overline{u_{j} \frac{\partial p}{\partial x_{i}}}\right)}_{\Pi_{i j}}=\underbrace{\left(p \overline{\frac{\partial u_{i}}{\partial x_{j}}}+\overline{p \frac{\partial u_{j}}{\partial x_{i}}}\right)}_{\phi_{i j}}-\underbrace{\left(\frac{\overline{\partial p u_{i}}}{\partial x_{j}}+\frac{\overline{\partial p u_{j}}}{\partial x_{i}}\right)}_{D_{i j}^{P}}
$$

In this section a comparison between the extracted pressure-strain correlations from the Reynolds stress transport equations and estimated values from different models is pre- 
sented.

Schwarz and Bradshaw, [57], tested four models for the pressure-strain correlation using experimental data, acquired using a standard cross hot-wire probe, in a threedimensional boundary layer. They concluded that the models were in good agreement with experimental data. However, the closest measurement point to the wall was at $y / \delta_{99}=0.1$, which corresponds to $y^{+} \approx 130$. At this distance away from the wall, most models are expected to agree to some extent with measured data.

Ölçmen and Simpson, [43], extracted pressure-strain correlations from measured data for flow around a wing-body junction using three-velocity component Laser Doppler Velocimetery. The measured profiles extended well into the viscous sublayer. The extracted values were compared with a number of pressure-strain models using isotropic and anisotropic estimates of the dissipation rate. They also examined the addition of wall corrections to the pressure-strain correlations. The comparisons showed poor performance for all models in the inner layer, $y^{+} \leq 25$, even with wall correction. Different models predicted different components better. For some models tested, the anisotropic dissipation rate approximation gave worse results than the the isotropic approximation.

To extract the pressure-strain correlation tensor, $\phi_{i j}$, an estimate of the dissipation rate tensor, $\epsilon_{i j}$ is required. The Jakirlić and Hanjalić model, highlighted in the previous section, is used. All other terms in the Reynolds stress transport equations, 1.3 , are evaluated from measured data except for the pressure-diffusion term, $D_{i j}^{P}$. This term is evaluated using Lumley's model, [27] and [52], shown in Equation 6.10. This is based on an analysis of nearly homogeneous turbulence.

$$
-\overline{p u_{i}}=\frac{1}{5} \overline{u_{i} u_{k}^{2}}
$$

Four models are compared to experimental data. The models are, the Launder, Reece and Rodi model, the Sarkar, Speziale and Gatski model, the Shih and Lumley model, the $\mathrm{Fu}$, Launder and Tselepidakis model. These are the most popular and widely used models in current flow simulation codes. The models are given below,

1. Launder, Reece and Rodi model, $(L R R)$ [23], [47]

$$
\phi_{i j}=-C_{1} \epsilon b_{i j}+C_{2} K\left(b_{i k} S_{k j}+S_{i k} b_{k j}-\frac{2}{3} b_{m n} S_{m n} \delta_{i j}\right)+C_{4} K\left(b_{i k} W_{k j}+W_{i k} b_{k j}\right)
$$

Where, $W_{i j}=0.5\left(\partial U_{i} / \partial x_{j}-\partial U_{j} / \partial x_{i}\right), C_{1}=3.0, C_{2}=0.8, C_{3}=1.75$ and $C_{4}=1.31$ and $K$ is the turbulence kinetic energy.

2. Speziale, Sarkar and Gatski model, $(S S G)$ [65], [47] 


$$
\begin{aligned}
\phi_{i j} & =-C_{1} \epsilon b_{i j}+\dot{C}_{1} \epsilon\left(b_{i k} b_{k j}-\frac{1}{3} b_{m n} b_{m n} \delta_{i j}\right)+C_{2} k S_{i j} \\
& +C_{3} K\left(b_{i k} S_{k j}+S_{i k} b_{k j}-\frac{2}{3} b_{m n} S_{m n} \delta_{i j}\right)+C_{4} K\left(b_{i k} W_{k j}+W_{i k} b_{k j}\right)
\end{aligned}
$$

Where, $C_{1}=3.4+1.8 \frac{P}{\epsilon}, \dot{C}_{1}=4.2, C_{2}=0.8-1.30\left(b_{m n} b_{m n}\right), C_{3}=1.25, C_{4}=0.40$ and $P$ and $\epsilon$ are the turbulence kinetic energy production and dissipation rate, respectively.

3. Shih and Lumley model, $(S H L)$ [59]

$$
\begin{aligned}
\phi_{i j} & =-\epsilon\left[\beta b_{i j}+\gamma\left(b_{i j}^{2}+\frac{2}{3} I I \delta_{i j}\right)\right] \\
& +K\left[0.8 S_{i j}+12 \alpha\left(b_{i k} S_{k j}+S_{i k} b_{k j}-\frac{2}{3} b_{m n} S_{m n} \delta_{i j}\right)\right] \\
& +K\left[\frac{4}{3}(2-7 \alpha)\left(b_{i k} W_{k j}+W_{i k} b_{k j}\right)\right] \\
& +0.8 K\left[\left(b_{i k}^{2} S_{k j}+S_{i k} b_{k j}^{2}-2 b_{i k} S_{k l} b_{l j}-3 b_{i j} b_{m n} S_{m n}\right)\right] \\
& +0.8 K\left[\left(b_{i k}^{2} W_{k j}+W_{i k} b_{k j}^{2}\right)\right]
\end{aligned}
$$

Where, $I I=-0.5 b_{m n} b_{m n}, \beta$ and $\gamma$ are functions of the invariants of $b_{i j}$ and the turbulent Reynolds number, $R_{e t}=\frac{2 k}{9 \epsilon}$, [59].

4. Fu, Launder and Tselepidakis, $(F L T)$ [16], [47]

$$
\begin{aligned}
\phi_{i j} & =-C_{1} \epsilon b_{i j}+C_{1} \epsilon\left(b_{i k} b_{k j}-\frac{1}{3} b_{m n} b_{m n}\right) \\
& +C_{2} K S_{i j}+1.2 K\left(b_{i k} S_{k j}+S_{i k} b_{k j}-\frac{2}{3} b_{m n} S_{m n} \delta_{i j}\right) \\
& +\frac{26}{15} K\left(b_{i k} W_{k j}+W_{i k} b_{k j}\right)-\frac{112}{5} K\left[I I\left(b_{i k} W_{k j}+W_{i k} b_{k j}\right)\right] \\
& +\frac{4}{5} K\left(b_{i k} b_{k l} S_{l j}+S_{i l} b_{l k} b_{k j}-2 b_{i k} S_{k l} b_{l j}-3 b_{m n} S_{m n} b_{i j}\right) \\
& +\frac{4}{5} K\left(b_{i k} b_{k l} W_{l j}+W_{i k} b_{k l} b_{l j}\right)-\frac{168}{5} K\left(b_{i k} b_{k l} W_{l m} b_{m j}+b_{i m} W_{m l} b_{l k} b_{k j}\right)
\end{aligned}
$$

Where, $C_{1}=-120 I I \sqrt{F}-2 \sqrt{F}+2, C_{1}=144 I I \sqrt{F}, C_{2}=0.8, F=1+9 I I+27 I I I$, $I I=-0.5 b_{i j}^{2}$ and $I I I=b_{i j}^{3} / 3$

The models are used to calculate the pressure-strain tensor for profiles $P 07, P 13, P 18$, $P 19, P 20$ and $P 32$. These profiles represent all the features present in this experiment. All models are used without wall correction. 
Figures 6.86 .13 show the comparison between elements of the extracted pressurestrain tensor and the models estimates. Figures 6.8a 6.13a show the estimates for the $\phi_{11}$. In general, all models agree well with experimental data for $y^{+} \geq 50$. Close to the wall in the inner layer all models overestimates the pressure-strain correlation; however, the SHL is closer to experimental data.

Figures $6.8 \mathrm{~b} 6.13 \mathrm{~b}$ show the $\phi_{22}$ component. Above $y^{+}=40$ the LRR model agrees very well with the data; all other models differ slightly. Near the wall all models fail to capture the correct value, however they capture the right trend, except for the FLT model.

Figures $6.8 \mathrm{c}-6.13 \mathrm{c}$ show the $\phi_{33}$ component. All models capture the correct sign and trend in for this component; however, the magnitude is significantly different. For profiles $P 07$ and $P 13$ there is good agreement away from the wall, since these two profiles are more two-dimensional than the other profiles. As three-dimensionality increases, the disagreement between the experimental data and the models extends beyond the inner layer up to $y^{+}=500$. The LRR model is consistently closer to the experimental data in the outer layer.

Figures $6.8 \mathrm{~d} 6.13 \mathrm{~d}$ show the $\phi_{12}$ component. The SSG model consistently gives very good agreement with experimental data for this pressure-strain component. The agreement extends to the near wall region down to $y^{+} \approx 11-20$. All other models significantly overestimate this correlation.

Figures $6.8 \mathrm{e}$ 6.13e show the $\phi_{13}$ component. The values of this correlation is an order of magnitude smaller than $\phi_{12}$. For profiles $P 07, P 13$ and $P 33$, the experimental estimates are very close to zero. For the other profiles the flow is three dimensional, hence, $\phi_{13}$ is non-zero. Surprisingly, the models give good estimates of this component down to $y^{+}=40$. Furthermore, the SHL model is in very good agreement with the experimental estimate further down to $y^{+} \approx 12$.

Finally, figures 6.8f 6.13f show the $\phi_{23}$ component. The SSG model gives the best estimates of this component down to $y^{+}=15$. However, for profiles $P 13$ and $P 33$ the agreement is not good up to $y^{+}=40$. All other models overestimate this correlation near the wall in the inner layer.

To summarize, all models need near-wall corrections. The SSG model gives very good estimates for $\phi_{12}$ and $\phi_{23}$ while the SHL model give the best estimate for $\phi_{11}$ and $\phi_{13}$. All models predict the right trend for $\phi_{22}$ and $\phi_{33}$ but all of them give the wrong magnitude. Furthermore, the disagreement between the experimental and model estimates for $\phi_{33}$ extends all the way to the outer layer when the flow is highly three dimensional.

In general, the $\phi_{12}$ component is the most important term for boundary layer flows since it appears in the $\overline{u v^{+}}$stress transport equation. However, for strong three dimensional flow situations the $\phi_{23}$ component might become equally important. This might be the reason why the SSG model gained popularity since it predicts both $\phi_{12}$ and $\phi_{23}$ components to a good extent. The conclusions drawn here are in line with those obtained by 
Ölçmen and Simpson, [43].

\subsection{Reynolds Shear Stress Angle Lag Model}

The flow angle, flow gradient angle and shear stress angle are important modeling parameters. If the lag between the shear stress angle and flow angle can be expressed algebraically it would decrease the computational cost for turbulent boundary layer flow type calculations since we would be solving one less equation. In this section, an expression for shear stress lag is derived and tested with measured data by comparing calculated lag with measured values.

The start point is the flow gradient angle, $\gamma_{g}$, defined by Equation 6.15

$$
\gamma_{g}=\arctan \left(\frac{W_{, y}}{U_{, y}}\right)
$$

The flow gradient angle can be related to the flow angle, $\gamma_{a}$, as follows,

$$
\begin{array}{r}
\gamma_{a}=\arctan \left(\frac{W}{U}\right) \\
\gamma_{a, y}=\frac{1}{1+\left(\frac{W}{U}\right)^{2}}\left[\frac{W_{, y}}{U}-\frac{W U_{, y}}{U^{2}}\right]=\frac{U^{2}}{U^{2}+W^{2}} \frac{U_{, y}}{U}\left[\frac{W_{, y}}{U_{, y}}-\frac{W}{U}\right] \\
\gamma_{g}=\gamma_{a}+\arcsin \left(\frac{Q}{\|S\|} \gamma_{a, y}\right)
\end{array}
$$

Where, $(\bullet)_{, y}$ denotes derivative with respect to the normal to the wall direction and $\|S\|=$ $\sqrt{U_{, y}{ }^{2}+W_{, y}{ }^{2}}$ is the rate of strain.

Figure 6.14 shows Equation 6.18 evaluated using measured flow angles and mean flow strain. Figure 6.14 shows excellent agreement between measured lag for the Flow gradient angle and the calculated lag using Equation 6.18. This give confidence in the measurements and flow angle estimates since Equation 6.18 is an exact analytical result. At the wall the Flow Gradient angle approaches the flow angle asymptotically and at the wall the lag is zero. Away from the wall, inertial effects as well as history effects become more dominant. There are some differences near the wall and around $y^{+}=1000$ which may be due to numerical differencing.

Equation 6.18 gives an analytical expression for the lag between the mean flow angle and the flow gradient angle. The lag term, second term on the right hand side, is invariant with respect to coordinate rotation about the wall-normal direction. The argument of the arcsin function is basically a mean flow length scale multiplied by the rate of change of flow angle normal to the wall. Since the shear stress angle usually follows the same 
trend of the flow gradient angle but with different magnitude and scale, this functional expression can be utilized to describe the lag between the shear stress angle and the flow angle. However, it would be reasonable to use a length scale based on turbulence quantities rather than mean flow ones. Hence, a turbulence length scale $L_{T}$ should be used instead. Moreover, the lag term in Equation 6.18 is zero at the wall since the Flow Gradient Angle is equal to the Flow Angle, in general this is not true for the Reynolds Shear Stress Angle. Hence, a constant term must be added to the lag term in order to account for the lag at the boundary. Therefore the model for the Reynolds Shear Stress Angle can be written in the following form,

$$
\gamma_{s}=\gamma_{a}+\arcsin \left[L_{T} \gamma_{a, y}\right]+\beta
$$

In order to be able to use this model, the length scale, $L_{T}$, and the constant, $\beta$, must be specified. In the next section an analysis is made and an exact expression for $\beta$ is obtained.

\subsubsection{Reynolds Shear Stress Angle Wall Limiting Value}

In order to use 6.19 the value of $\beta$ has to be specified apriori. In order to do that, we will look at the momentum equation and try to obtain a limiting value for the Reynolds Shear Stress Angle consistent with the momentum equation. We start by writing the Reynolds Averaged Navier-Stokes equation in the $x$ and $z$ directions as follows normalized using wall variables, keeping in mind that as $y \rightarrow 0, U_{l}=0, \partial(\bullet) / \partial x=0$ and $\partial(\bullet) / \partial z=0$. All quantities are normalized by wall variables, $u_{\tau}$ and $\nu$.

$$
\begin{aligned}
\frac{D U}{D t} & =-\frac{\partial P}{\partial x}+\frac{\partial^{2} U}{\partial y^{2}}-\frac{\partial \overline{u v}}{\partial y} \\
\frac{D W}{D t} & =-\frac{\partial P}{\partial z}+\frac{\partial^{2} W}{\partial y^{2}}-\frac{\partial \overline{v w}}{\partial y}
\end{aligned}
$$

Also the Reynolds shear stresses $\overline{u v}$ and $\overline{v w}$ are written in terms of the total Reynolds Shear stress, $\tau$, and the Reynolds Shear Stress Angle, $\gamma_{s}$.

$$
\begin{aligned}
& \overline{u v}=-\tau \cos \left[\gamma_{s}\right] \\
& \overline{v w}=-\tau \sin \left[\gamma_{s}\right]
\end{aligned}
$$

Substituting Equations 6.22 and 6.23 into Equations 6.20 and 6.21 we get,

$$
\begin{aligned}
\frac{D U}{D t} & =-\frac{\partial P}{\partial x}+\frac{\partial}{\partial y} \frac{\partial U}{\partial y}+\frac{\partial \tau \cos \left[\gamma_{s}\right]}{\partial y} \\
\frac{D W}{D t} & =-\frac{\partial P}{\partial z}+\frac{\partial}{\partial y} \frac{\partial W}{\partial y}+\frac{\partial \tau \sin \left[\gamma_{s}\right]}{\partial y}
\end{aligned}
$$


Rearranging Equations 6.246 .25 and noting that,

$$
\begin{aligned}
& \frac{\partial U}{\partial y}=\|S\| \cos \left[\gamma_{g}\right] \\
& \frac{\partial W}{\partial y}=\|S\| \sin \left[\gamma_{g}\right] \\
& \frac{\partial P}{\partial x}=\frac{\partial P}{\partial l} \cos \left[\gamma_{a}\right] \\
& \frac{\partial P}{\partial z}=\frac{\partial P}{\partial l} \sin \left[\gamma_{a}\right]
\end{aligned}
$$

Where, $\partial P / \partial l$ is the pressure gradient along the projection of a streamline onto a plane parallel to the wall and at the wall it is equal to the surface pressure gradient along the limiting streamline direction. Hence we get,

$$
\begin{aligned}
& \cos \left[\gamma_{s}\right] \frac{\partial \tau}{\partial y}-\tau \sin \left[\gamma_{s}\right] \frac{\partial \gamma_{s}}{\partial y}=\frac{D U}{D t}+\frac{\partial P}{\partial l} \cos \left[\gamma_{a}\right]-\frac{\partial}{\partial y}\left(\|S\| \cos \left[\gamma_{g}\right]\right) \\
& \sin \left[\gamma_{s}\right] \frac{\partial \tau}{\partial y}+\tau \cos \left[\gamma_{s}\right] \frac{\partial \gamma_{s}}{\partial y}=\frac{D W}{D t}+\frac{\partial P}{\partial l} \sin \left[\gamma_{a}\right]-\frac{\partial}{\partial y}\left(\|S\| \sin \left[\gamma_{g}\right]\right)
\end{aligned}
$$

If Equation 6.31 is divided by Equation 6.30 and taking the limit as $y \rightarrow 0$, we obtain Equation 6.32

$$
\left[\frac{\sin \left[\gamma_{s}\right]}{\cos \left[\gamma_{s}\right]}\right]_{y=0}=\lim _{y \rightarrow 0}\left[\frac{\frac{D(W)}{D t}+\frac{\partial P}{\partial l} \sin \left[\gamma_{a}\right]-\frac{\partial}{\partial y}\left(\|S\| \sin \left[\gamma_{g}\right]\right)}{\frac{D(U)}{D t}+\frac{\partial P}{\partial l} \cos \left[\gamma_{a}\right]-\frac{\partial}{\partial y}\left(\|S\| \cos \left[\gamma_{g}\right]\right)}\right] .
$$

In Equation 6.32, both numerator and denominator are zero at the wall. Hence in order to obtain the limiting value, l'Hôpital's rule is used by differentiating the numerator and denominator with respect to $y$ as follows,

$$
\left[\frac{\sin \left[\gamma_{s}\right]}{\cos \left[\gamma_{s}\right]}\right]_{y=0}=\lim _{y \rightarrow 0} \frac{\left[\frac{D(W)}{D t}+\frac{\partial P}{\partial l} \sin \left[\gamma_{a}\right]-\frac{\partial}{\partial y}\left(\|S\| \sin \left[\gamma_{g}\right]\right)\right]_{, y}}{\left[\frac{D(U)}{D t}+\frac{\partial P}{\partial l} \cos \left[\gamma_{a}\right]-\frac{\partial}{\partial y}\left(\|S\| \cos \left[\gamma_{g}\right]\right)\right]_{, y}}
$$

Equation 6.33 contains three terms that need to be evaluated in the numerator and denominator. Each term will be evaluated below. The first term to be evaluated is the substantial derivative, $D() / D t$, as follows,

$$
\left[\frac{D(W)}{D t}\right]_{, y}=\frac{\partial}{\partial y}\left[\frac{\partial W}{\partial t}+U_{l} \frac{\partial W}{\partial x_{l}}\right]=\frac{\partial W_{, y}}{\partial t}+\frac{\partial U_{l}}{\partial y} \frac{\partial W}{\partial x_{l}}+U_{l} \frac{\partial W_{, y}}{\partial x_{l}}
$$

The right hand side of Equation 6.34 contains three terms, the first term in general is non-zero for unsteady flows. It is also noted that this term is equal to the time rate of change of $x$-direction vorticity flux since $\Omega_{x}=W_{, y}$ at the wall. The second and third 
terms in Equation 6.34 are exactly zero at the wall since, $U_{l}=0, \partial W / \partial x=\partial W / \partial z=0$ and $\partial V / \partial y=0$. Hence, at the wall Equation 6.34 reduces to,

$$
\left[\frac{D(W)}{D t}\right]_{, y}=\left[\frac{\partial \Omega_{x}}{\partial t}\right]_{y=0}
$$

Similarly,

$$
\left[\frac{D(U)}{D t}\right]_{, y}=-\left[\frac{\partial \Omega_{z}}{\partial t}\right]_{y=0}
$$

Next, the limiting value of the second term in Equation 6.33 is obtained as follows,

$$
\left[\frac{\partial P}{\partial l} \sin \left[\gamma_{a}\right]\right]_{, y}=\frac{\partial^{2} P}{\partial l \partial y} \sin \left[\gamma_{a}\right]+\frac{\partial P}{\partial l} \cos \left[\gamma_{a}\right] \gamma_{a, y}
$$

In general, the terms in Equation 6.37 are non-zero at the wall so it will be left in this form, however, for a boundary layer assumption $\partial^{2} P / \partial l \partial y$ can be shown to be equal to zero at the wall. Similarly, in the denominator we obtain,

$$
\left[\frac{\partial P}{\partial l} \cos \left[\gamma_{a}\right]\right]_{, y}=\frac{\partial^{2} P}{\partial l \partial y} \cos \left[\gamma_{a}\right]-\frac{\partial P}{\partial l} \sin \left[\gamma_{a}\right] \gamma_{a, y}
$$

Finally the limiting value of the last term is obtained as follows,

$$
\begin{gathered}
{\left[\frac{\partial}{\partial y}\left(\|S\| \sin \left[\gamma_{g}\right]\right)\right]_{, y}=\left[\|S\|_{, y} \sin \left[\gamma_{g}\right]+\|S\| \cos \left[\gamma_{g}\right] \gamma_{g, y}\right]_{, y}} \\
{\left[\frac{\partial}{\partial y}\left(\|S\| \sin \left[\gamma_{g}\right]\right)\right]_{, y}=\|S\|_{, y y} \sin \left[\gamma_{g}\right]+2\|S\|_{, y} \cos \left[\gamma_{g}\right] \gamma_{g, y}-\|S\| \sin \left[\gamma_{g}\right] \gamma_{g, y}^{2}} \\
+\|S\|_{, y} \cos \left[\gamma_{g}\right] \gamma_{g, y y} \\
{\left[\frac{\partial}{\partial y}\left(\|S\| \sin \left[\gamma_{g}\right]\right)\right]_{, y}=\left(\|S\|_{, y y}-\|S\| \gamma_{g, y}^{2}\right) \sin \left[\gamma_{g}\right]+\left(2\|S\|_{, y} \gamma_{g, y}+\|S\| \gamma_{g, y y}\right) \cos \left[\gamma_{g}\right]}
\end{gathered}
$$

Similarly we can write the last term in the denominator of Equation 6.33 as,

$$
\left[\frac{\partial}{\partial y}\left(\|S\| \cos \left[\gamma_{g}\right]\right)\right]_{, y}=\left(\|S\|_{, y y}-\|S\| \gamma_{g, y}^{2}\right) \cos \left[\gamma_{g}\right]-\left(2\|S\|_{, y} \gamma_{g, y}+\|S\| \gamma_{g, y y}\right) \sin \left[\gamma_{g}\right]
$$

Now we can collect Equations 6.356.37 and 6.41 to form the numerator and Equations 6.36 6.38 and 6.42 to form the denominator. The result is shown in Equation 6.43.

$$
\left[\frac{\sin \left[\gamma_{s}\right]}{\cos \left[\gamma_{s}\right]}\right]_{y=0}=
$$




$$
\left[\frac{\dot{\Omega_{x}}+\sin \left[\gamma_{g}\right]\left(P_{, l y}-\|S\|_{, y y}+\|S\| \gamma_{g, y}^{2}\right)-\cos \left[\gamma_{g}\right]\left(-P_{, l} \gamma_{a, y}+2\|S\|_{, y} \gamma_{g, y}+\|S\| \gamma_{g, y y}\right)}{-\dot{\Omega}_{z}+\cos \left[\gamma_{g}\right]\left(P_{, l y}-\|S\|_{, y y}+\|S\| \gamma_{g, y}^{2}\right)+\sin \left[\gamma_{g}\right]\left(-P_{, l} \gamma_{a, y}+2\|S\|_{, y} \gamma_{g, y}+\|S\| \gamma_{g, y y}\right)}\right]_{y=0}
$$

Finally, the limiting value at a solid wall for the Reynolds Shear Stress Angle is given by Equation 6.44 .

$$
\left[\frac{\sin \left[\gamma_{s}\right]}{\cos \left[\gamma_{s}\right]}\right]_{y=0}=\left[\frac{\dot{\Omega_{x}}+\sin \left[\gamma_{g}-\theta\right]}{-\dot{\Omega}_{z}+\cos \left[\gamma_{g}-\theta\right]}\right]_{y=0}
$$

Where,

$$
\theta=\arctan \left[\frac{-P_{, l} \gamma_{a, y}+2\|S\|_{, y} \gamma_{g, y}+\|S\| \gamma_{g, y y}}{P_{, l y}-\|S\|_{, y y}+\|S\| \gamma_{g, y}^{2}}\right]_{y=0}
$$

At the wall, the value of the Flow Gradient Angle is equal to the Flow Angle as a result of Equation 6.18. Also for steady flow, the time rate of change of vorticity components vanishes.

Equation 6.44 can be viewed as a compatibility condition between the mean flow field and the turbulent shear stress field at a solid boundary. This condition must be met regardless of the turbulence closure model used.

For unsteady flow Equation 6.44 reduces to the following,

$$
\begin{aligned}
{\left[\frac{\sin \left[\gamma_{s}\right]}{\cos \left[\gamma_{s}\right]}\right]_{y=0} } & =\left[\frac{\sin \left[\gamma_{g}-\theta\right]}{\cos \left[\gamma_{g}-\theta\right]}\right]_{y=0} \\
\tan \left[\gamma_{s}\right]_{y=0} & =\left[\tan \left[\gamma_{g}-\theta\right]\right)_{y=0}
\end{aligned}
$$

Hence,

$$
\left(\gamma_{s}\right)_{y=0}=\left(\gamma_{g}-\theta\right)_{y=0}
$$

Comparing Equation 6.19 with Equation 6.48 at the wall for steady flows, it can be seen that the value of the constant $\beta$ is equal to $-\theta$. Hence, substituting Equation 6.45 into Equation 6.19 we can write the Reynolds Shear Stress angle for a steady flow as follows,

$$
\gamma_{s}=\gamma_{a}+\arcsin \left[L_{T} \frac{\partial \gamma_{a}}{\partial y}\right]-\arctan \left[\frac{-P_{, l} \gamma_{a, y}+2\|S\|_{, y} \gamma_{g, y}+\|S\| \gamma_{g, y y}}{P_{, l y}-\|S\|_{, y y}+\|S\| \gamma_{g, y}^{2}}\right]_{y=0}
$$

Equation 6.49 gives an explicit expression for the Reynolds Shear Stress Angle in terms of the Flow Angle, Flow Gradient Angle and their gradient in addition to mean Strain and Pressure gradients at the wall. This expression is also invariant under coordinate axis rotation around the wall normal direction. Moreover, it has the right value at the wall that is consistent with the momentum equation. The other advantage is that it doesn't have any user prescribed constants. 
Table 6.1 shows values of $\beta$ computed from measured values and using Equation 6.45 for some profiles measured. Computed values are very close to measured ones except for profiles $P 18_{1}$ and $P 35$. This is because Equation 6.45 contains multiple first and second derivatives of very small quantities which makes it very hard to compute given the experimental uncertainties.

\begin{tabular}{lccccc}
\hline Profile & $P 16$ & $P 18$ & $P 18_{1}$ & $P 19_{1}$ & $P 35$ \\
\hline$y^{+}$ & 2.9 & 6.8 & 2 & 6.5 & 2.8 \\
\hline$\gamma_{s}-\gamma_{a}[\mathrm{deg}]$ & 19 & 22 & -14 & 24 & 9 \\
\hline$\beta=-\theta[\mathrm{deg}]$ & 20 & 23 & -6 & 23 & 3 \\
\hline
\end{tabular}

Table 6.1: Comparison between measured and computed Reynolds Shear Stress angle, $\gamma_{s}$, at the Wall

If this model is to be used in a numerical computation, the value of $\beta$ need to be estimated. One choice is to assume that at the wall the shear stress angle equals the flow gradient angle. Then in the next iteration, $\beta$ is taken as equal to $-\theta$, where $\theta$ is calculated as defined by Equation 6.45 using values of mean flow field from the previous iteration.

The last step is to define the turbulence length scale, $L_{T}$. One choice is to use a diffusive length scale such that, $L_{T}=k^{1.5} / \epsilon$. The analysis here will be confined to this choice. The next section shows the model results.

\subsubsection{Results}

Figures 6.15a 6.15f show the measured and calculated Reynolds Shear Stress angle using Equations 6.19 and Rotta's model, [55], for Profiles P06, P13, P18, $P 19 p p, P 20_{1}$ and $P 33$.

In order to test the functional form of Equation 6.19 and exclude errors in evaluating $\beta$, the value of $\beta$ is set equal to the measured difference between the shear stress angle and the mean flow angle at the wall. In general, estimates using Equation 6.19 are very close to experimental values near the wall up to about $y^{+}=200$. Above this height Equation 6.19 becomes sensitive to the uncertainties in estimating the turbulence kinetic energy dissipation rate, $\epsilon$. Moreover, the way the model is formulated so far offers no guarantees that the absolute value of the argument of the arcsin function is going to be less than unity. This is the reason why for profiles $P 18_{1}$ and $P 19 p p$, Equation 6.19 gives a different trend from experimental data.

However, the current model captures the right trend near the wall and up to $y^{+}=$ 200. In contrast, Rotta's model, [55], doesn't give the right values nor trends in the same region. For example for profiles $P 18_{1}, P 19 p p$ and $P 20_{1}$, the Shear Stress Angle decreases semi-logarithmically near the wall, Rotta's model shows an increase in the Shear Stress 
Angle for profiles $P 18_{1}, P 20_{1}$ and an almost constant angle for profile $P 19 p p$. The current model gives the right behavior in all cases in the inner layer up to $y^{+} \approx 250$. The discrepancy in Rotta's model is due to the fact that the computed lag in Reynolds Shear Stress Angle is proportional to the lag in the Flow Gradient Angle. This is not correct at the wall since the Flow Gradient Angle approaches the Flow Angle and in general the Reynolds Shear Stress Angle is not equal to the Flow Angle at the wall.

In general, Equation 6.19 gives a very good estimate for the Shear Stress Angle over a wide range of flow situations present at different profiles. It is more consistent and superior to Rotta's model in the inner layer. However, it needs to be modified so that the arcsin argument is guaranteed to be within the interval $[-1,1]$ which probably will improve the performance in the outer layer. 


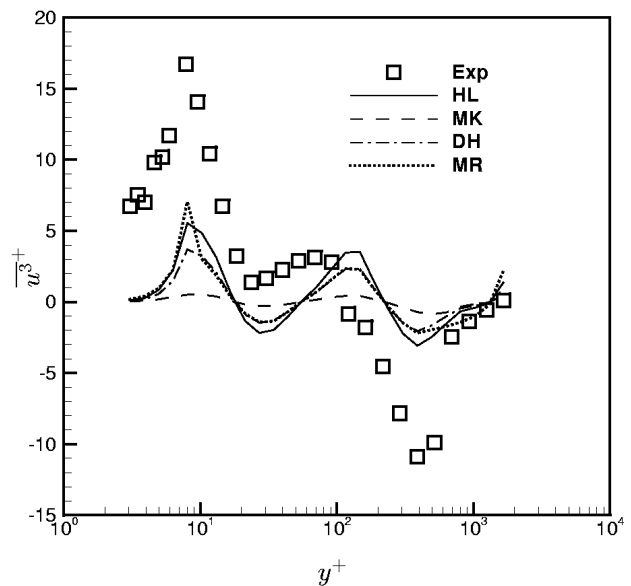

(a)

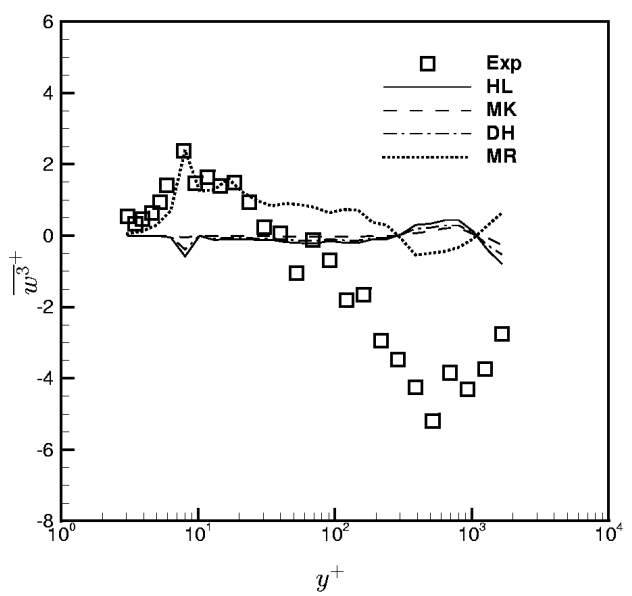

(c)

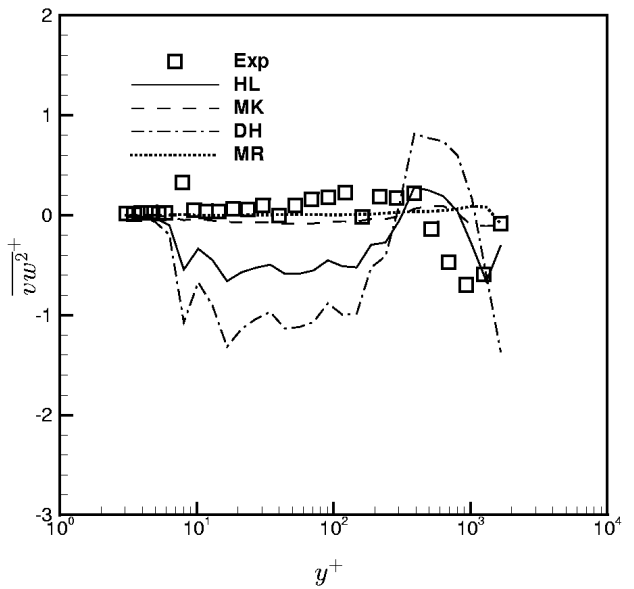

(e)

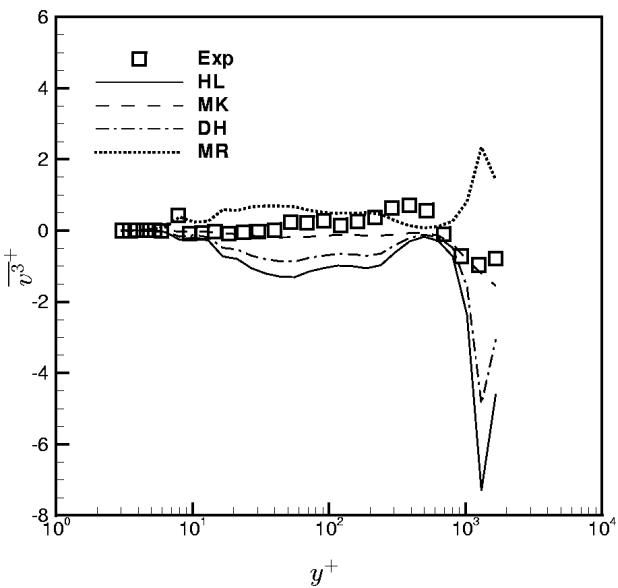

(b)

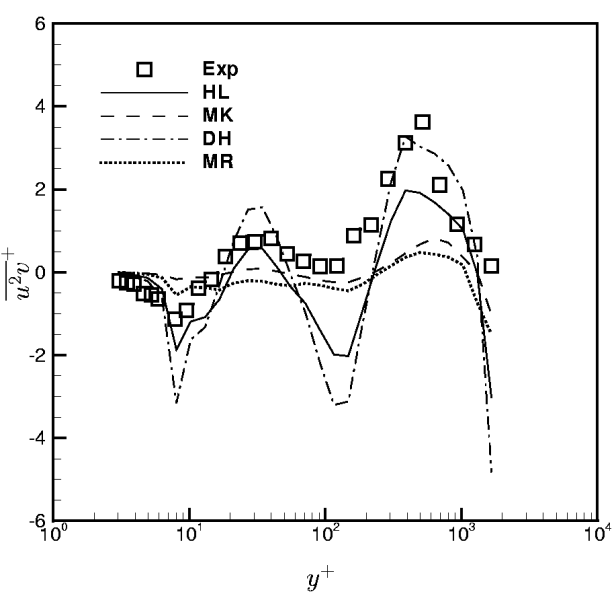

(d)

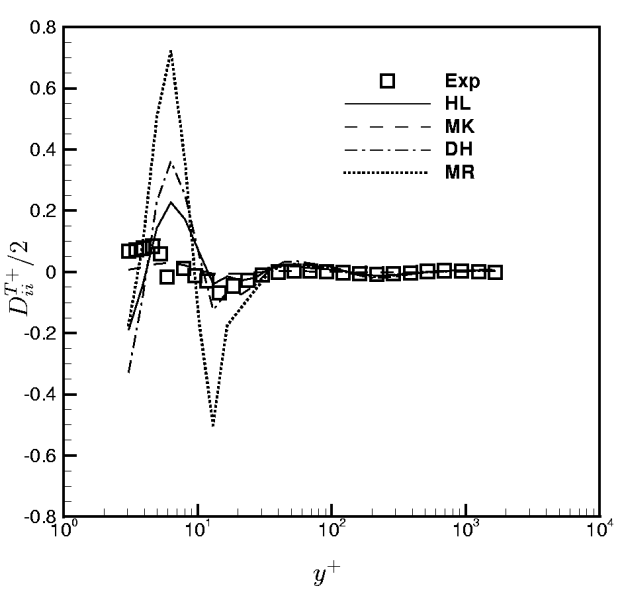

(f)

Figure 6.1: Comparison between measured and modeled triple-velocity correlations for Profile P07, (Exp) Experiment, $(H L)$ Hanjalić and Launder model, $(M k)$ Mackinnon model, $(D H)$ Daly-Harlow model and $(M R)$ Moore's model. $D_{i i}^{T} / 2$ is the Turbulence Kinetic Energy Diffusion. 


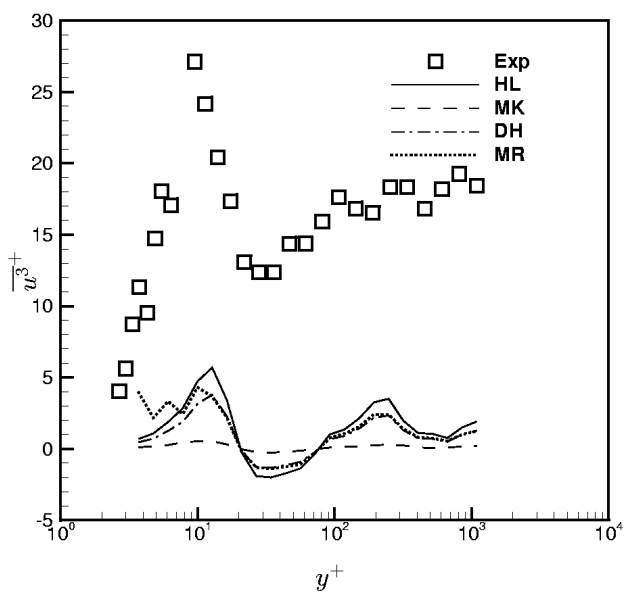

(a)

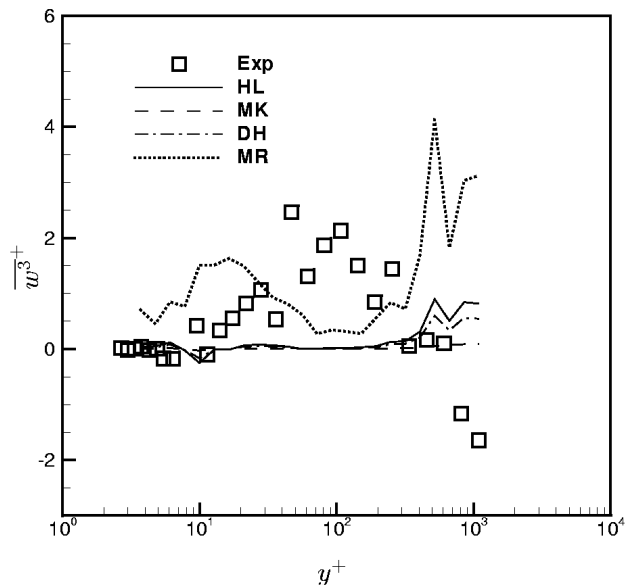

(c)

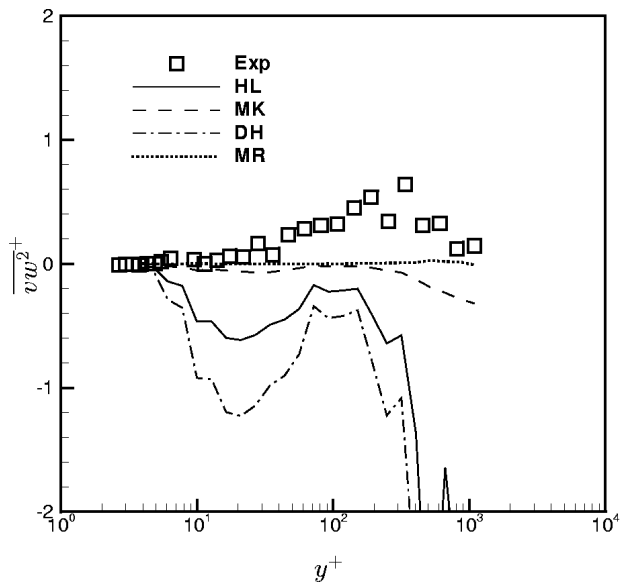

(e)

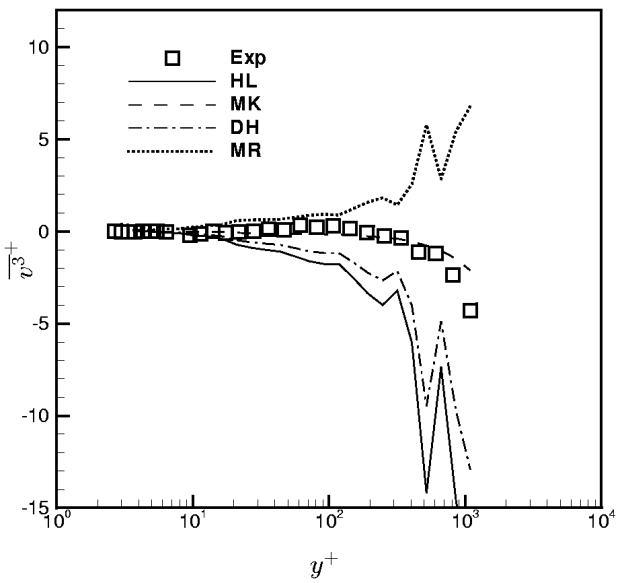

(b)

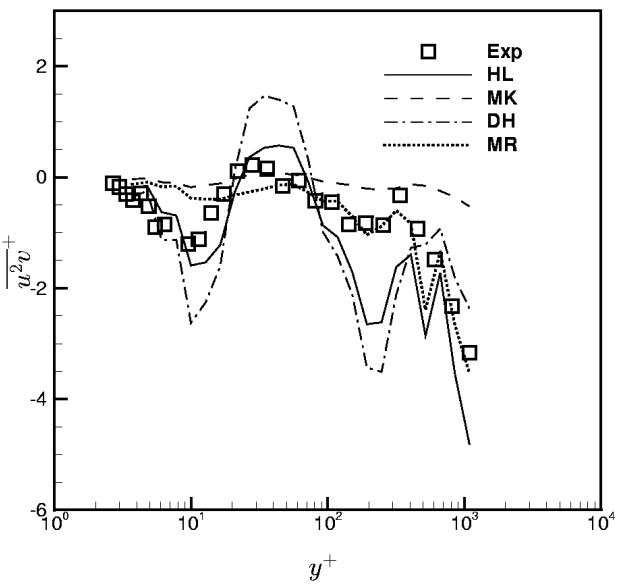

(d)

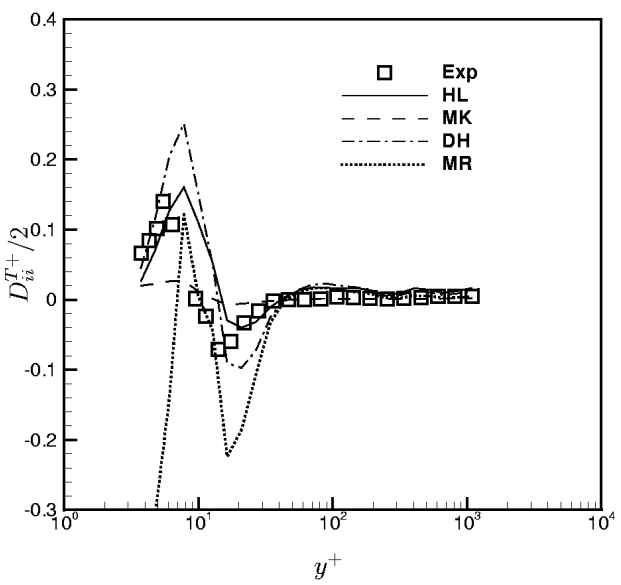

(f)

Figure 6.2: Comparison between measured and modeled triple-velocity correlations for Profile P13, (Exp) Experiment, $(H L)$ Hanjalić and Launder model, $(M k)$ Mackinnon model, $(D H)$ Daly-Harlow model and $(M R)$ Moore's model. $D_{i i}^{T} / 2$ is the Turbulence Kinetic Energy Diffusion. 


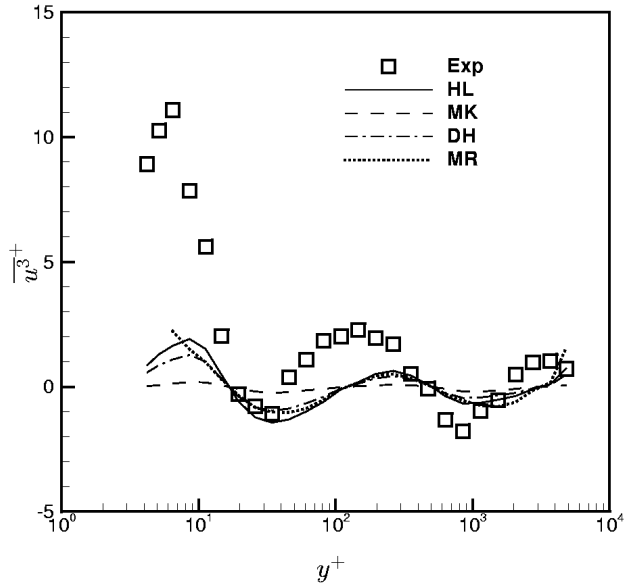

(a)

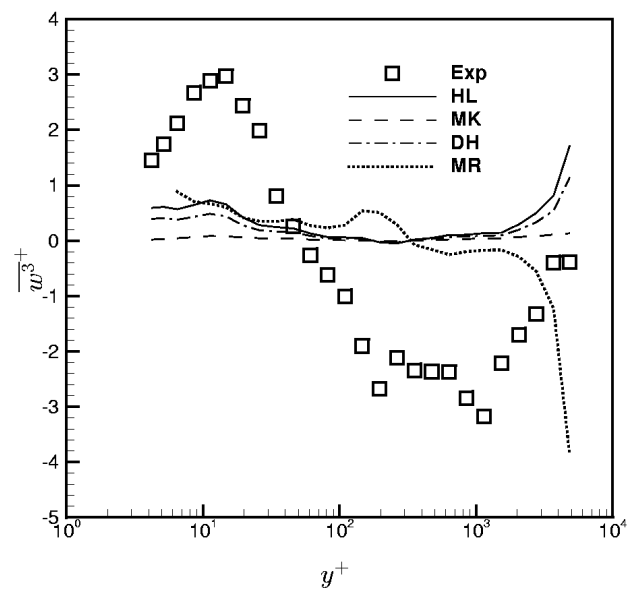

(c)

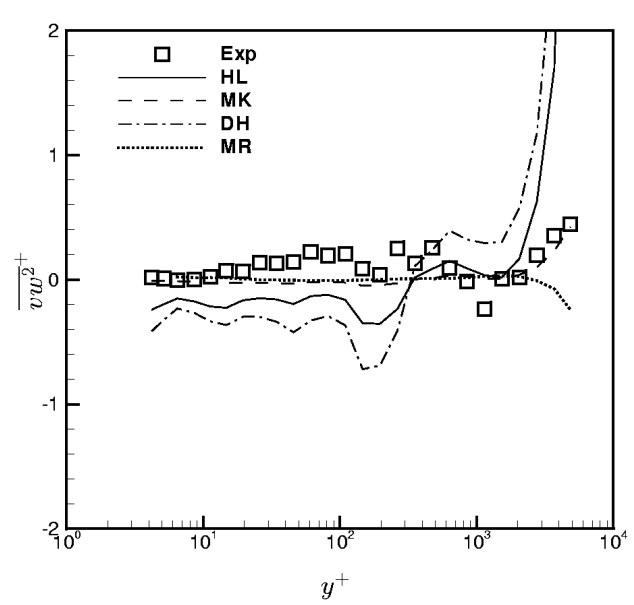

(e)

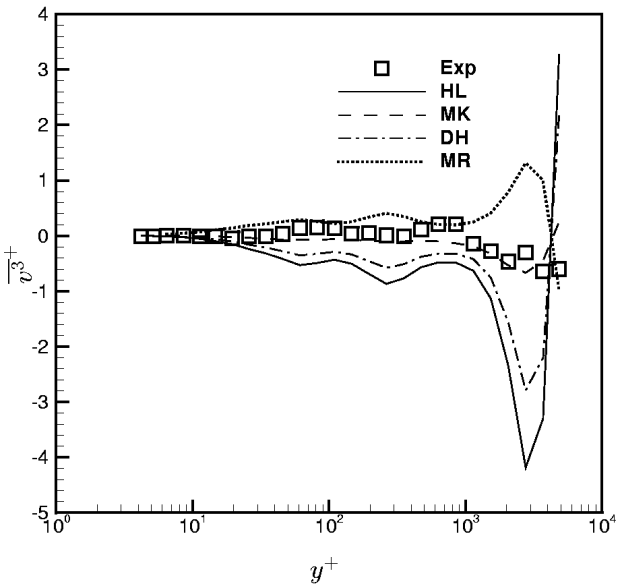

(b)

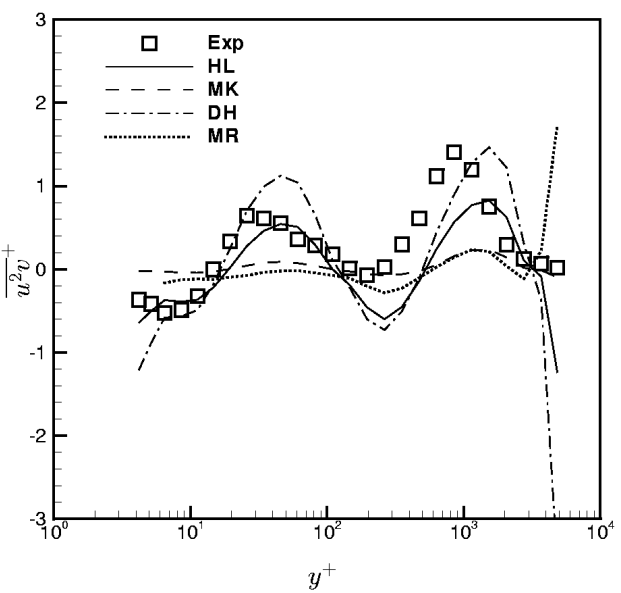

(d)

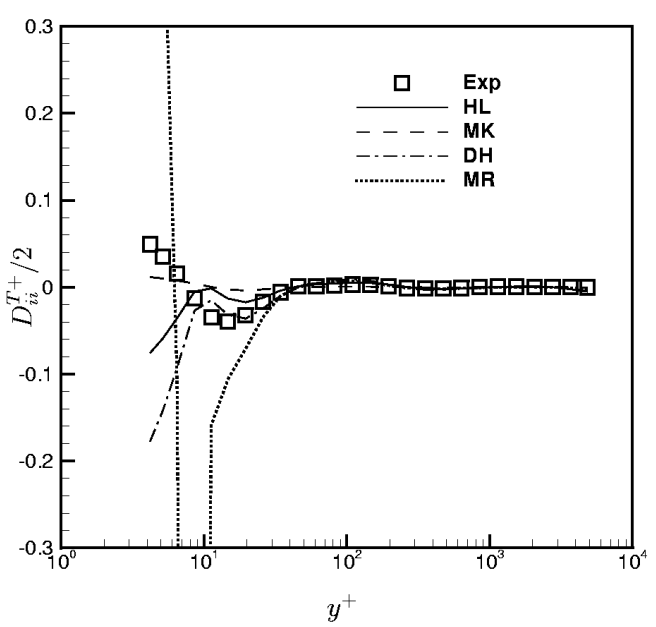

(f)

Figure 6.3: Comparison between measured and modeled triple-velocity correlations for Profile P19pp, (Exp) Experiment, $(H L)$ Hanjalić and Launder model, $(M k)$ Mackinnon model, $(D H)$ Daly-Harlow model and $(M R)$ Moore's model. $D_{i i}^{T} / 2$ is the Turbulence Kinetic Energy Diffusion. 


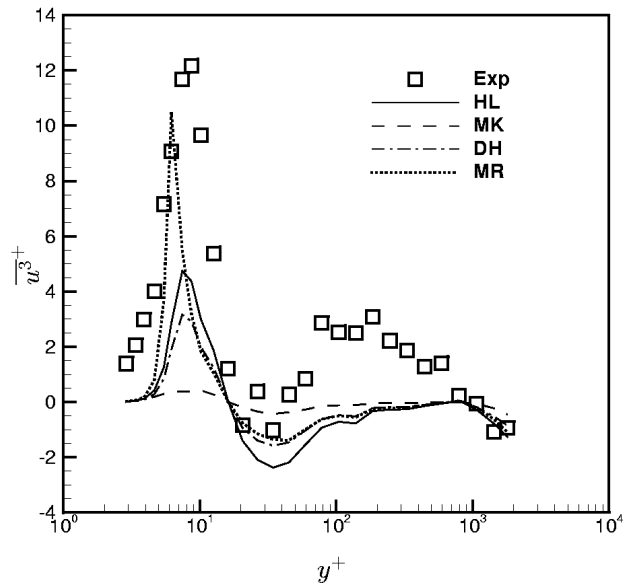

(a)

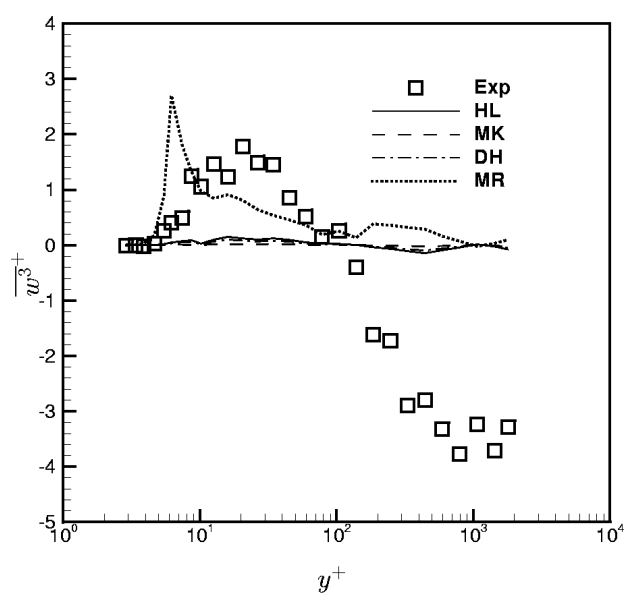

(c)

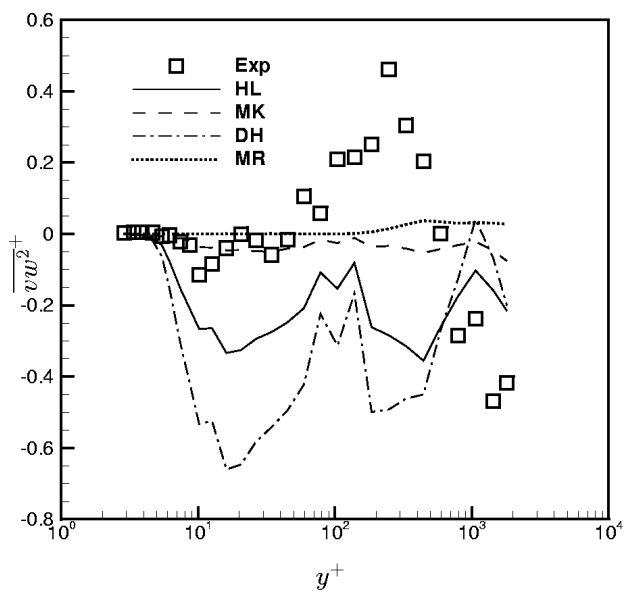

(e)

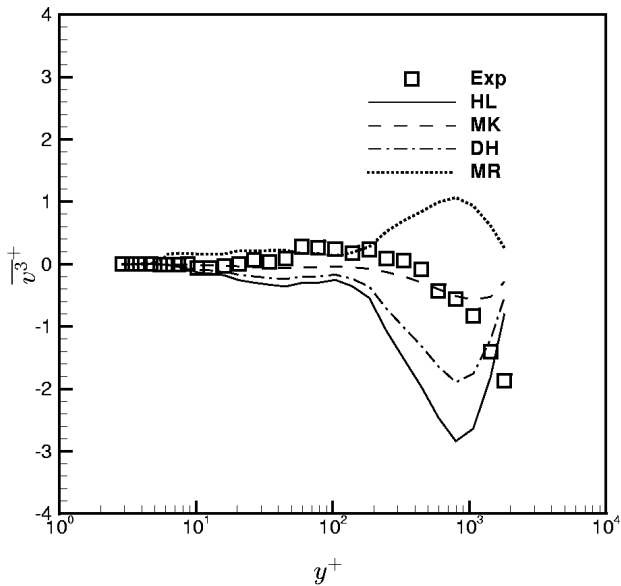

(b)

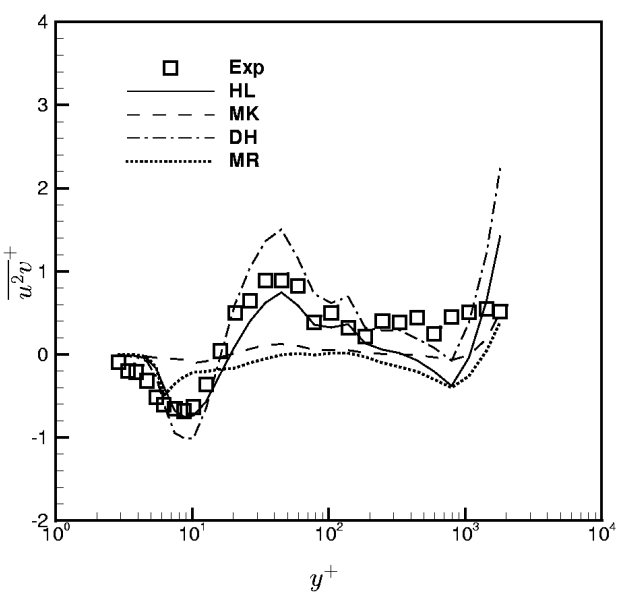

(d)

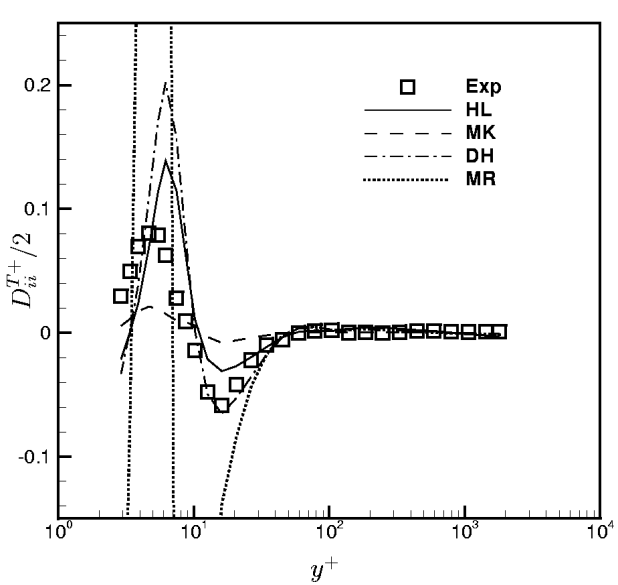

(f)

Figure 6.4: Comparison between measured and modeled triple-velocity correlations for Profile P32, (Exp) Experiment, $(H L)$ Hanjalić and Launder model, $(M k)$ Mackinnon model, $(D H)$ Daly-Harlow model and $(M R)$ Moore's model. $D_{i i}^{T} / 2$ is the Turbulence Kinetic Energy Diffusion. 


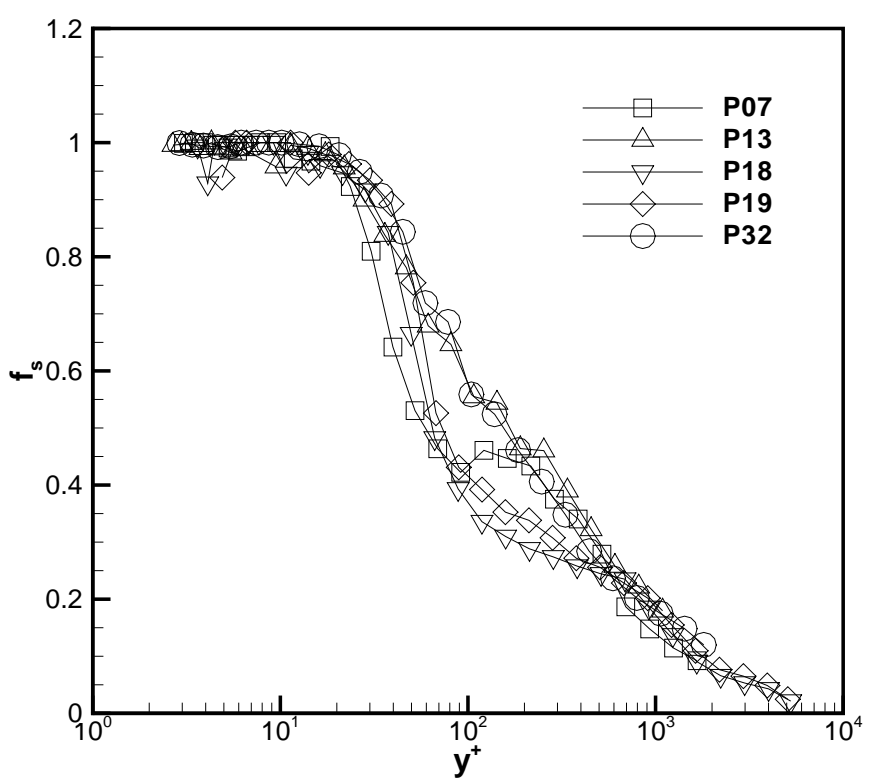

Figure 6.5: Blending function, $f_{s}$, in Jakirlić and Hanjalić dissipation rate tensor anisotropy 


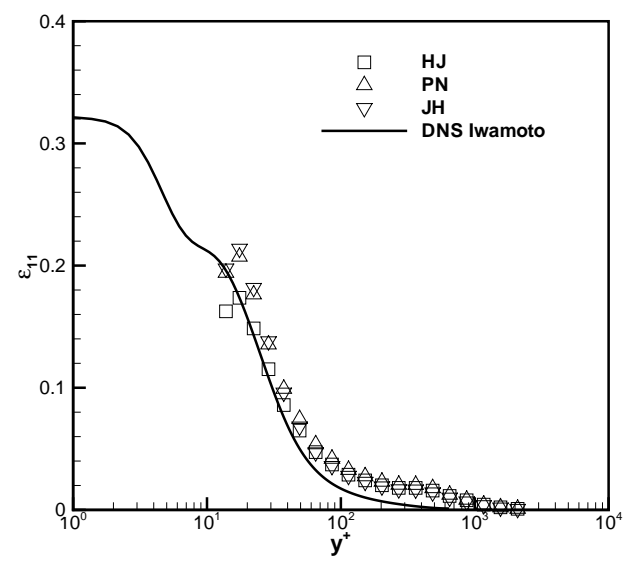

(a)

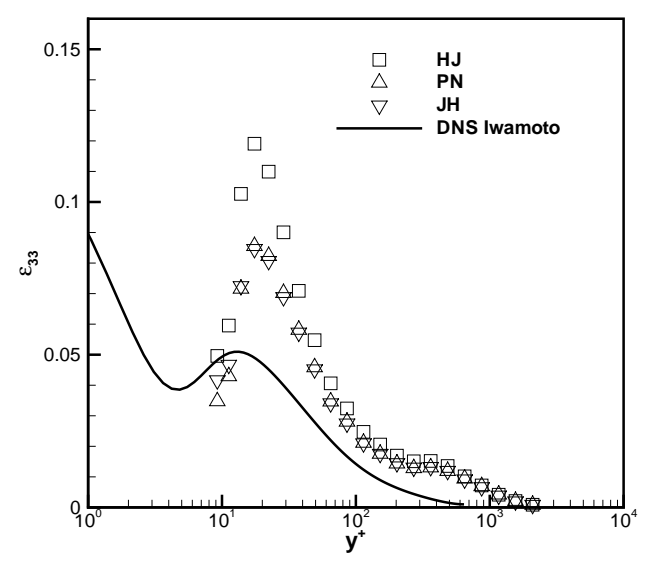

(c)

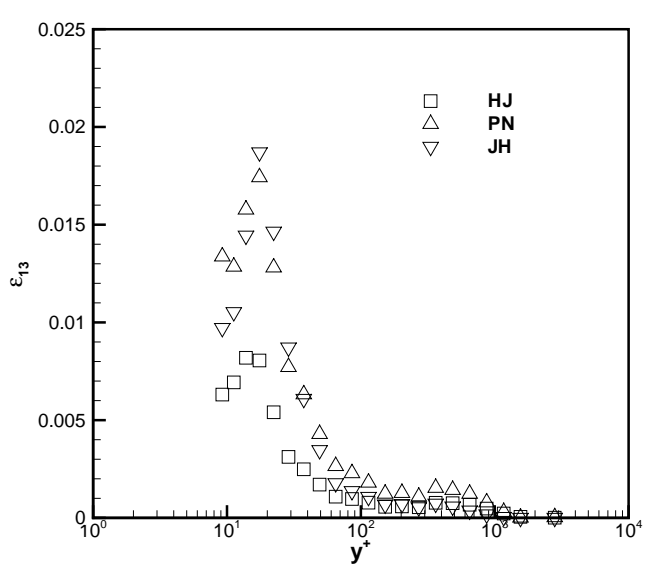

(e)

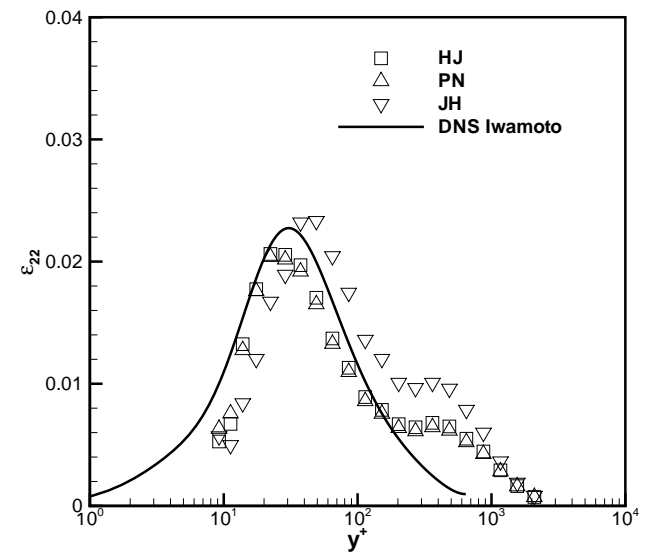

(b)

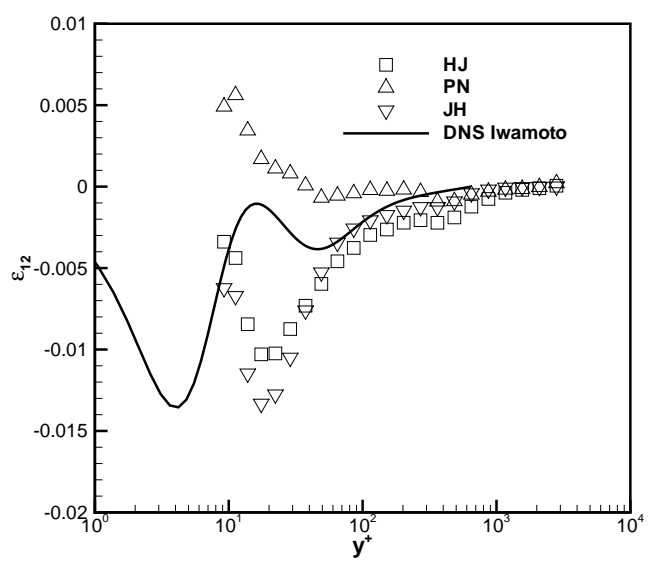

(d)

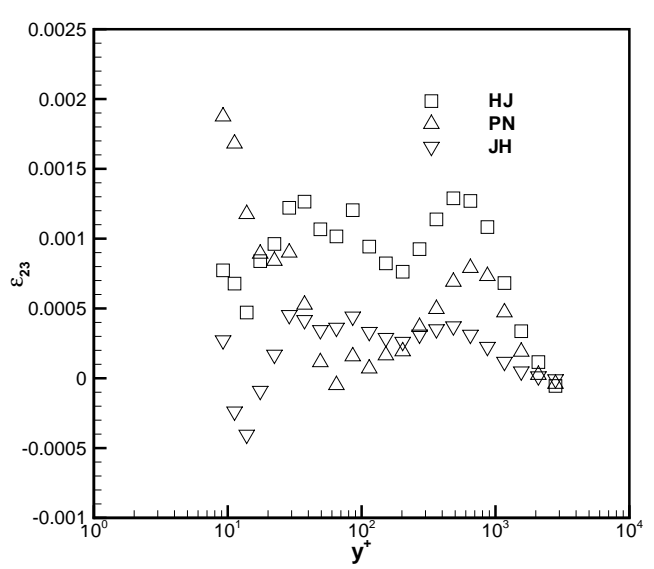

(f)

Figure 6.6: Comparison between dissipation rate models at profile $P 10,(H J)$ Hallback and Johansson model, $(P N)$ Perot and Natu model, $(J H)$ Jakirlić and Hanjalić model 


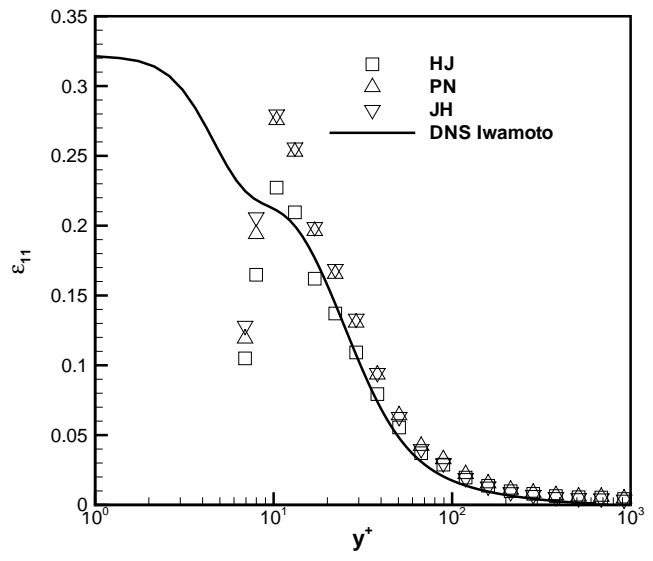

(a)

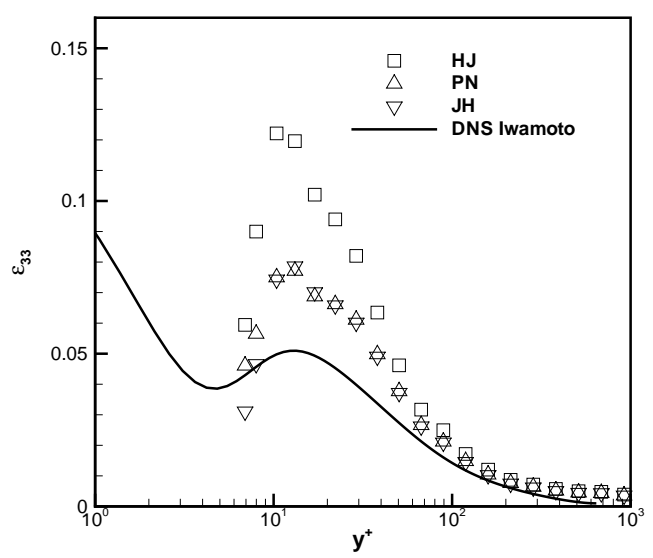

(c)

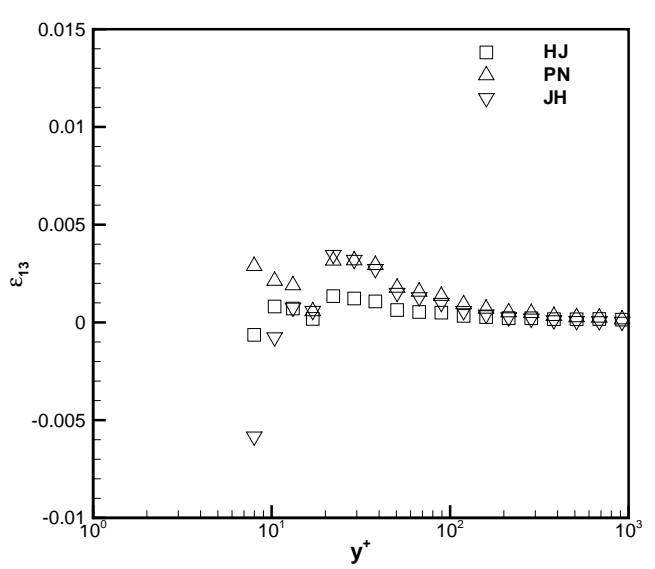

(e)

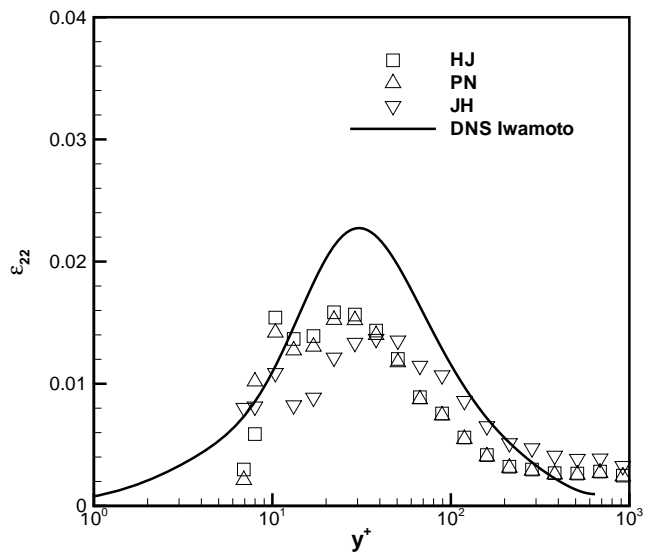

(b)

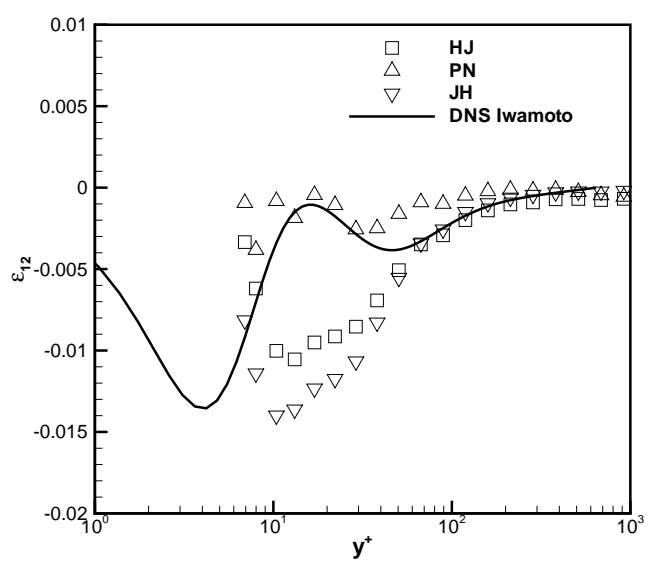

(d)

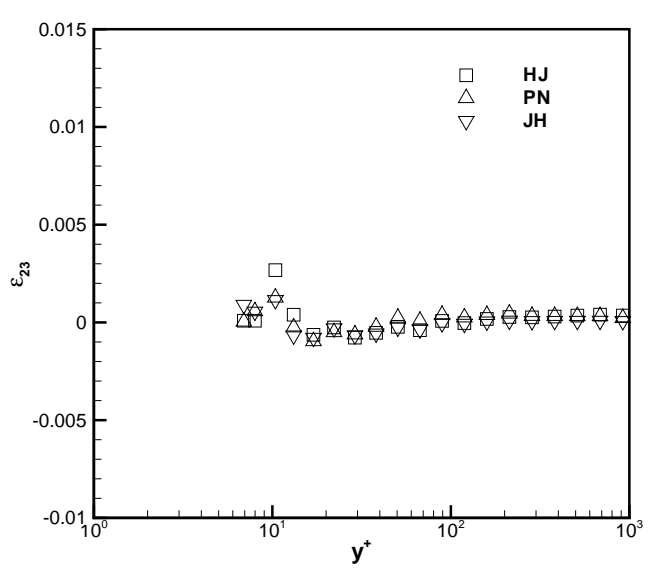

(f)

Figure 6.7: Comparison between dissipation rate models at profile $P 35,(H J)$ Hallback and Johansson model, $(P N)$ Perot and Natu model, $(J H)$ Jakirlić and Hanjalić model 


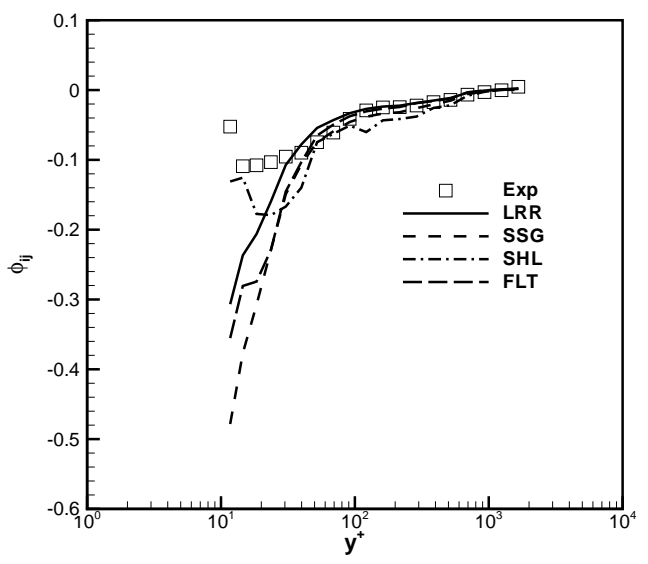

(a) $\phi_{11}$

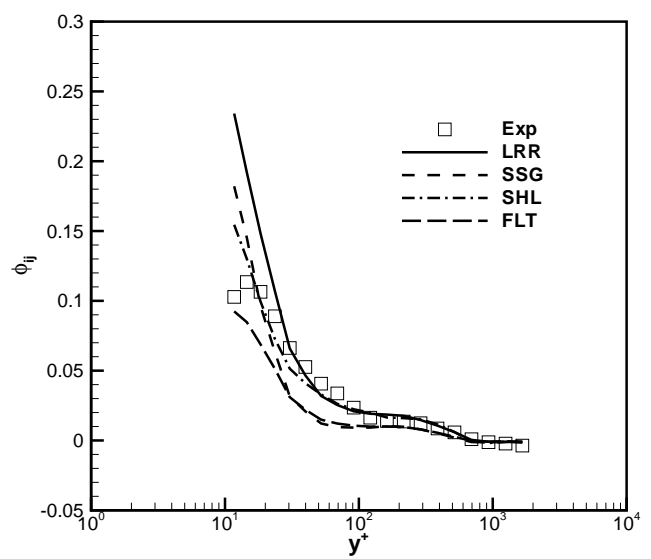

(c) $\phi_{33}$

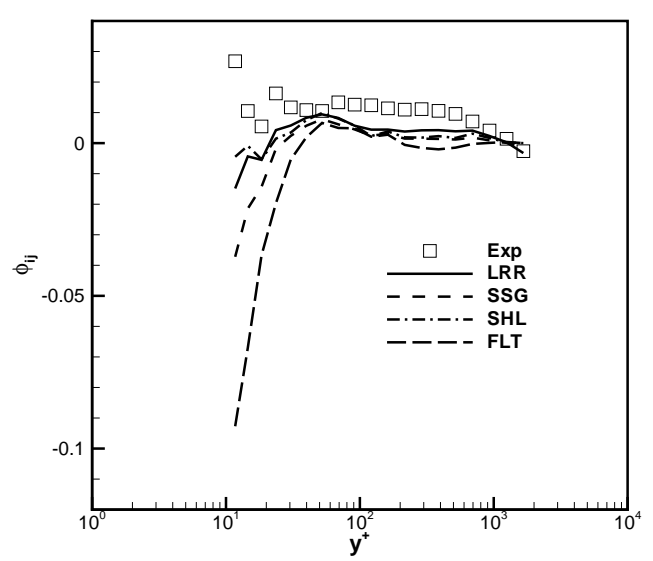

(e) $\phi_{13}$

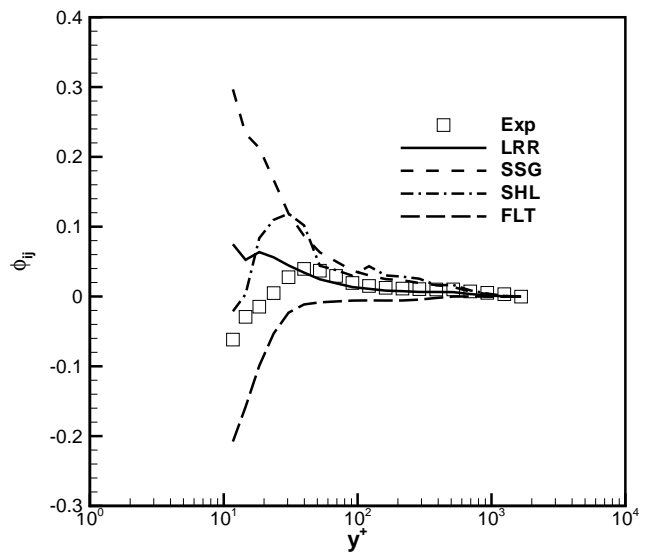

(b) $\phi_{22}$

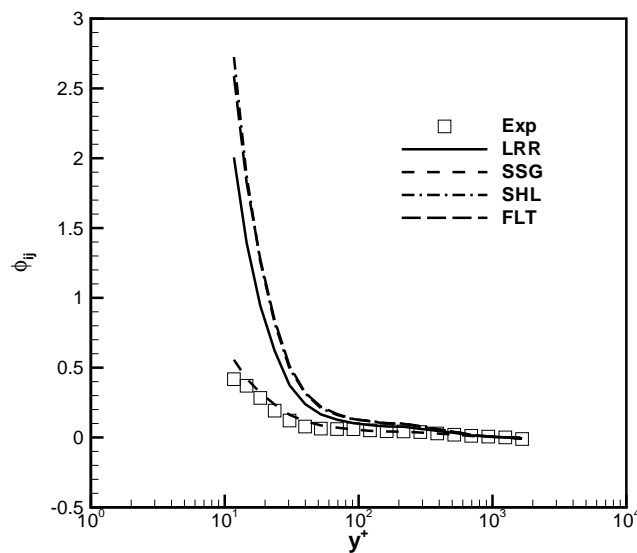

(d) $\phi_{12}$

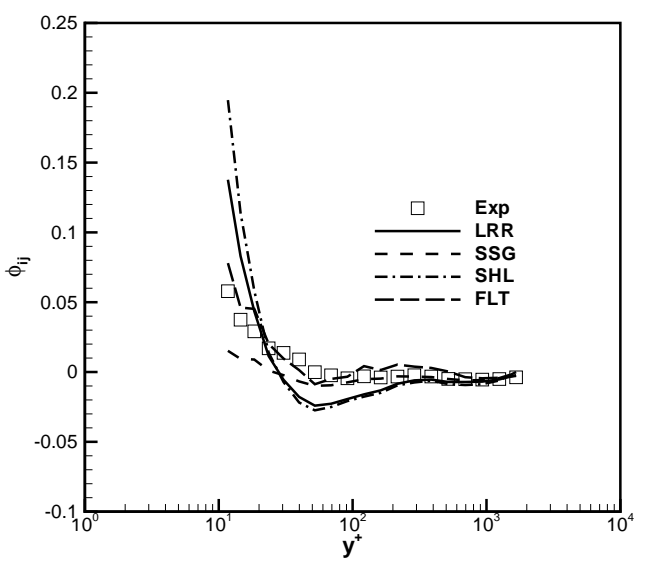

(f) $\phi_{23}$

Figure 6.8: Comparison between Pressure-Strain models at profile $P 07$, (Exp) experiment, $(L R R)$ Launder, Reece and Rodi model, $(S S G)$ Sarkar, Speziale and Gatski model, $(S H L)$ Shih and Lumley model, $(F L T)$ Fu, Launder and Tselepidakis model 


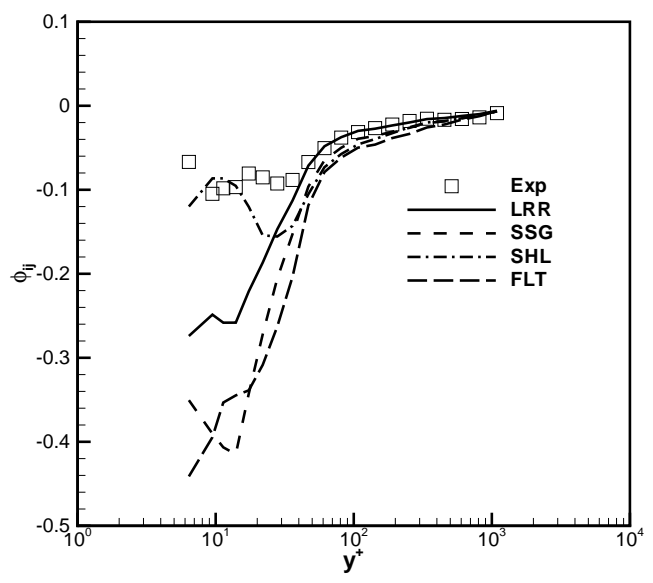

(a) $\phi_{11}$

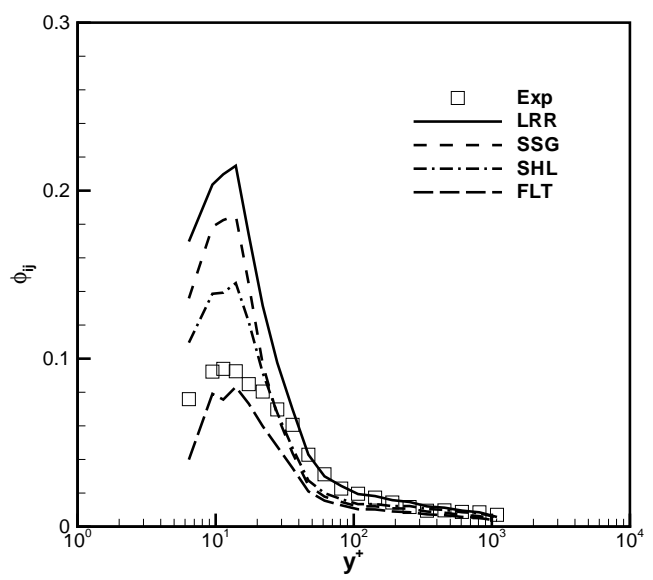

(c) $\phi_{33}$

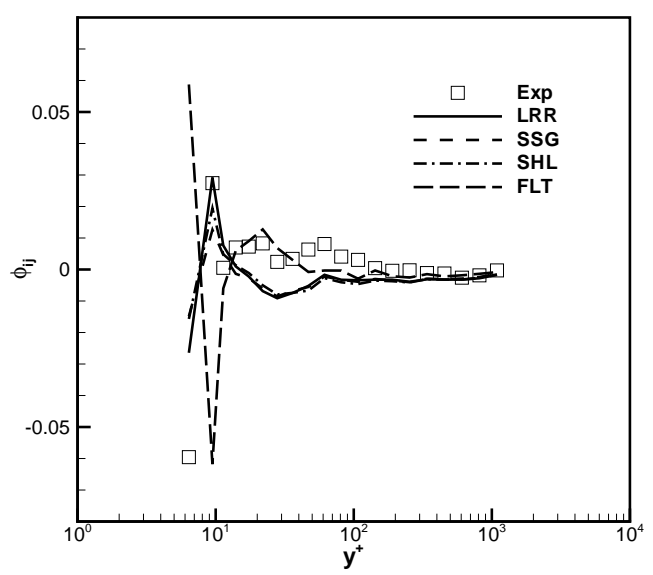

(e) $\phi_{13}$

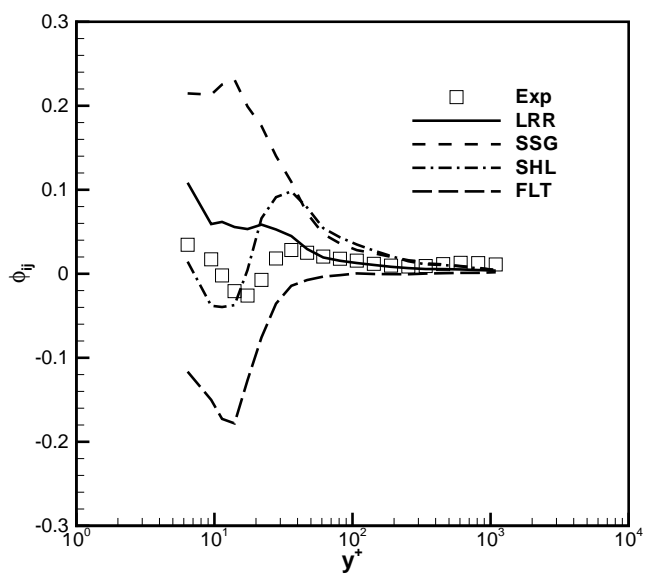

(b) $\phi_{22}$

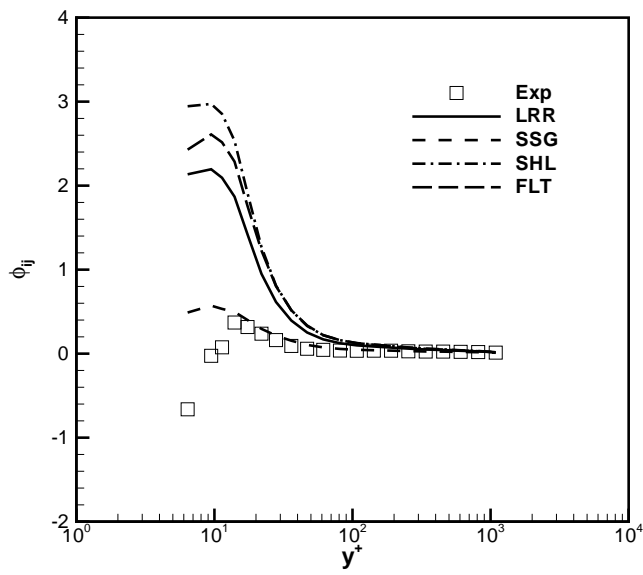

(d) $\phi_{12}$

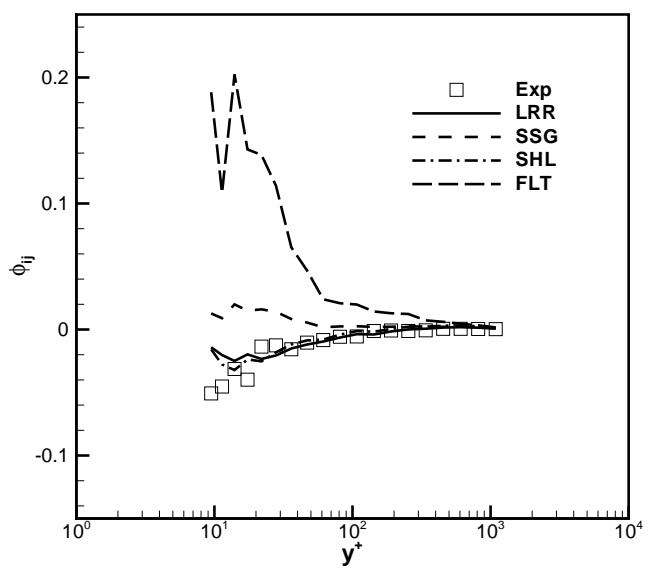

(f) $\phi_{23}$

Figure 6.9: Comparison between Pressure-Strain models at profile $P 13,($ Exp $)$ experiment, $(L R R)$ Launder, Reece and Rodi model, $(S S G)$ Sarkar, Speziale and Gatski model, $(S H L)$ Shih and Lumley model, (FLT) Fu, Launder and Tselepidakis model 


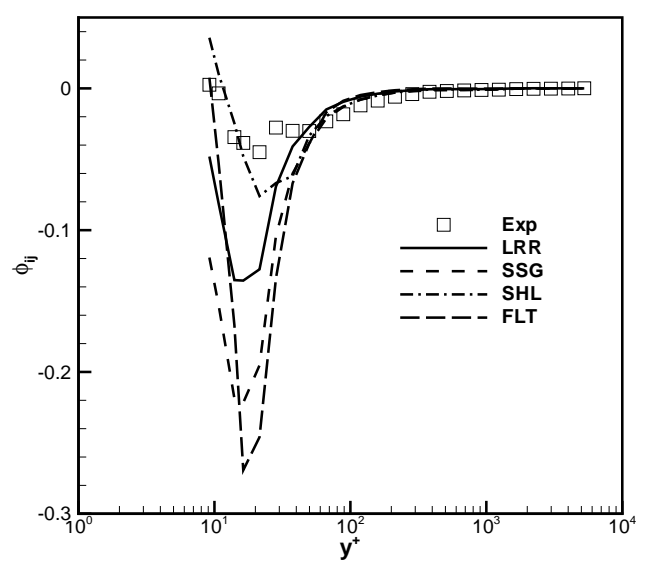

(a) $\phi_{11}$

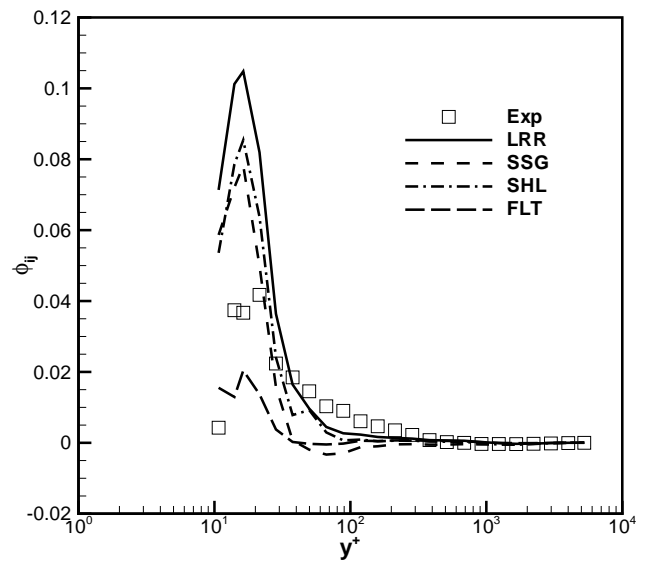

(c) $\phi_{33}$

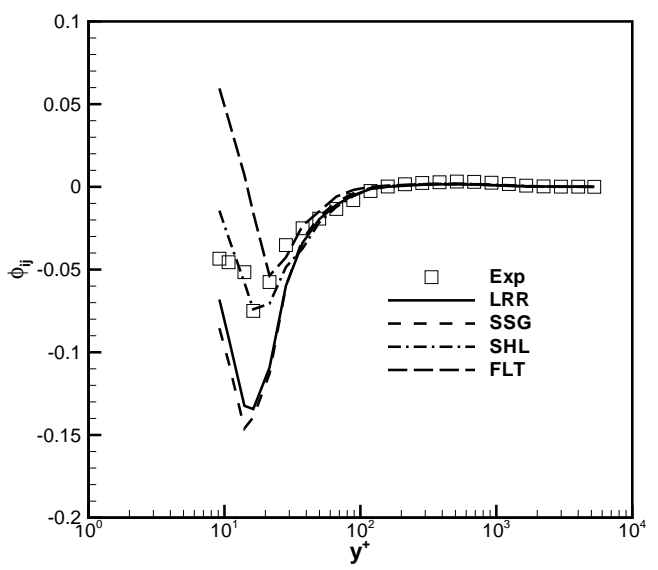

(e) $\phi_{13}$

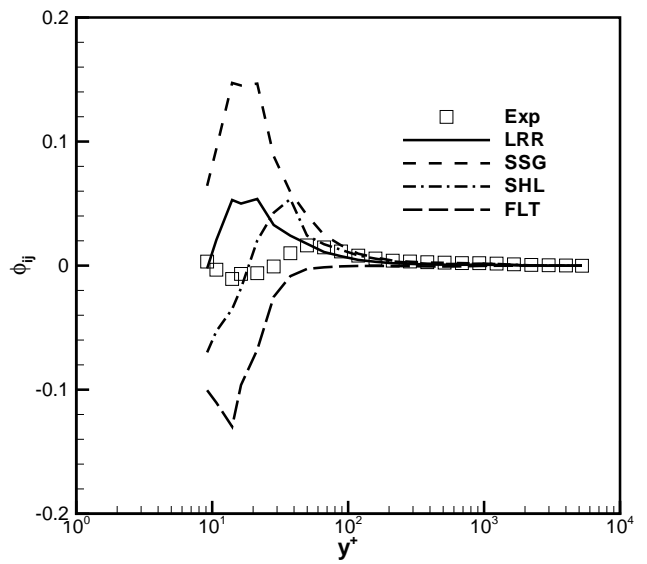

(b) $\phi_{22}$

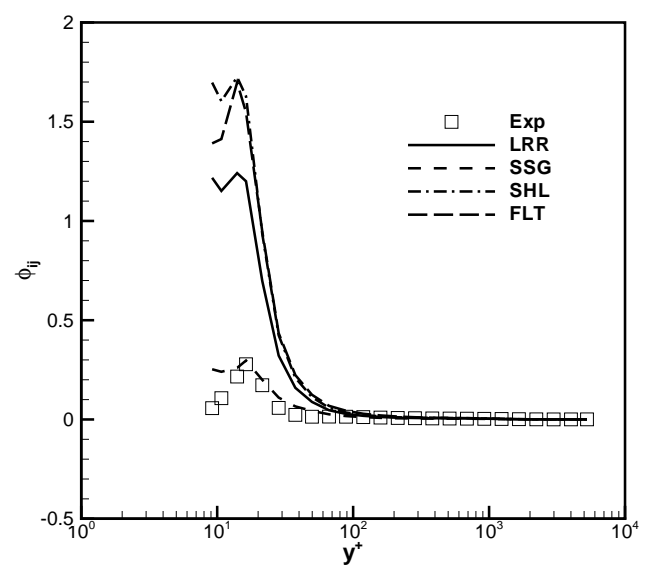

(d) $\phi_{12}$

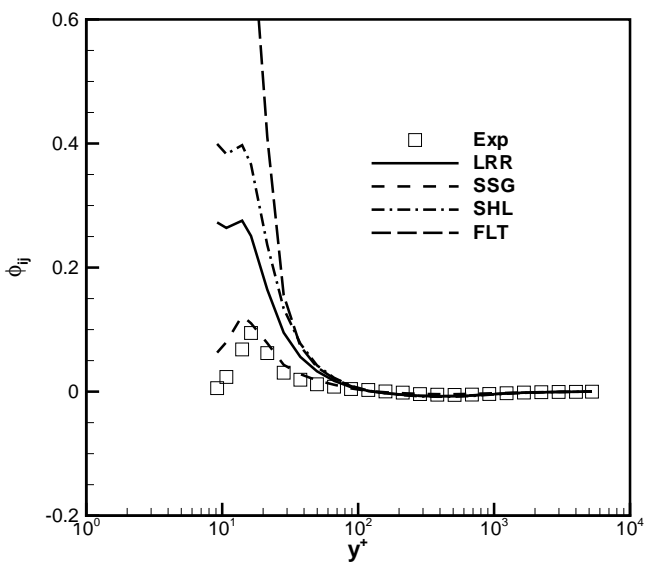

(f) $\phi_{23}$

Figure 6.10: Comparison between Pressure-Strain models at profile $P 18,($ Exp) experiment, $(L R R)$ Launder, Reece and Rodi model, $(S S G)$ Sarkar, Speziale and Gatski model, $(S H L)$ Shih and Lumley model, $(F L T) \mathrm{Fu}$, Launder and Tselepidakis model 


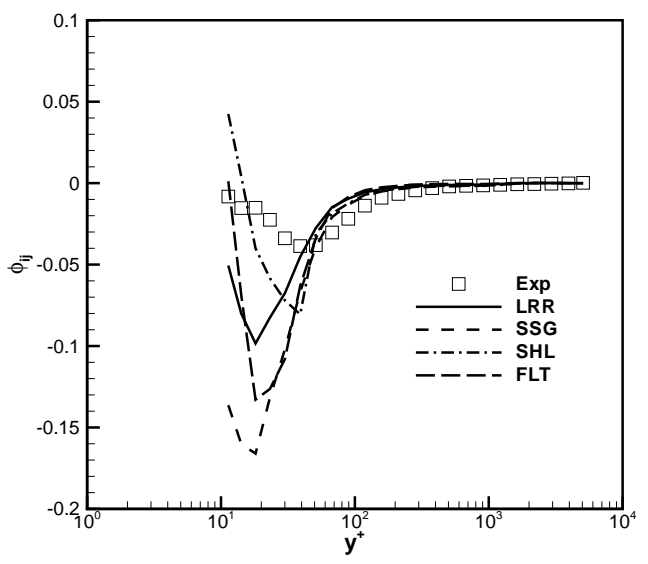

(a) $\phi_{11}$

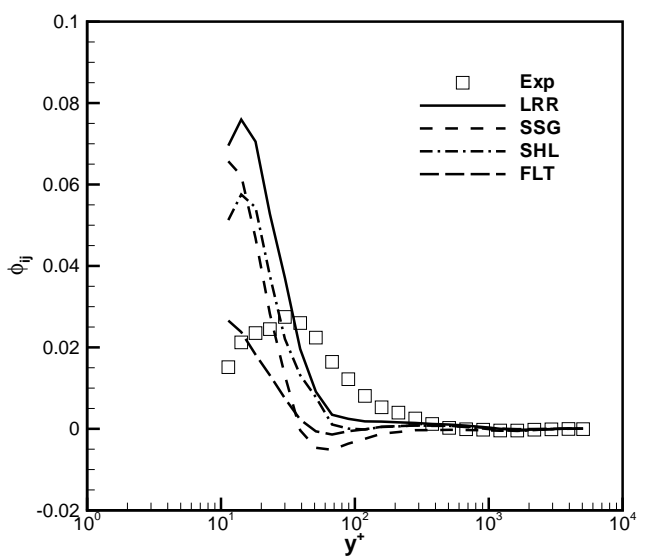

(c) $\phi_{33}$

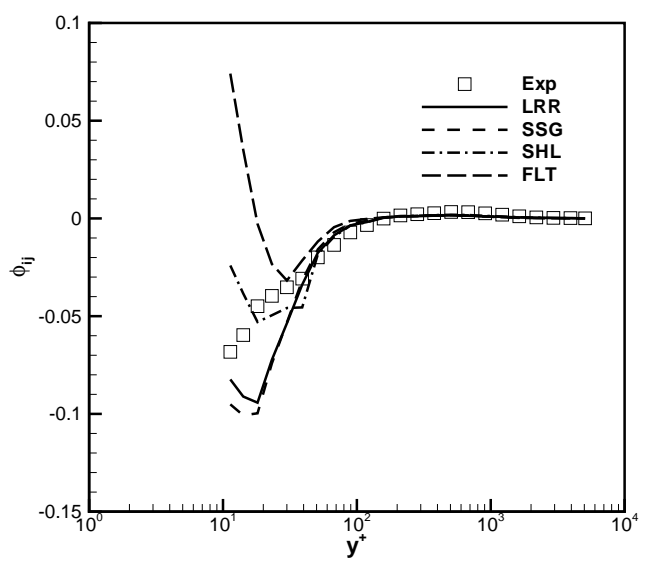

(e) $\phi_{13}$

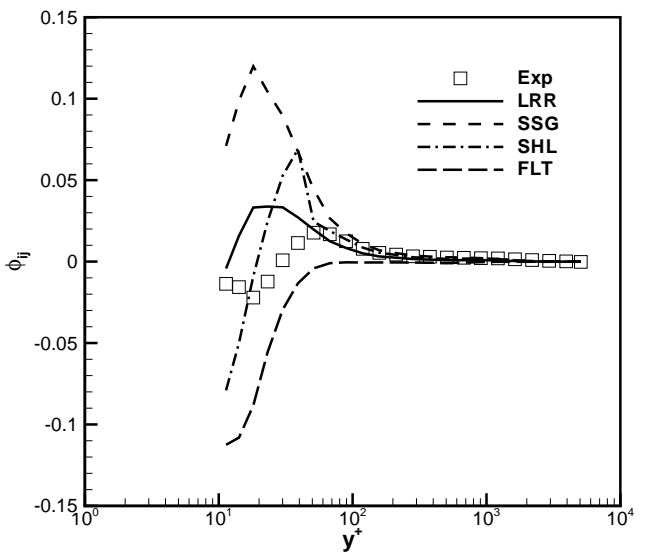

(b) $\phi_{22}$

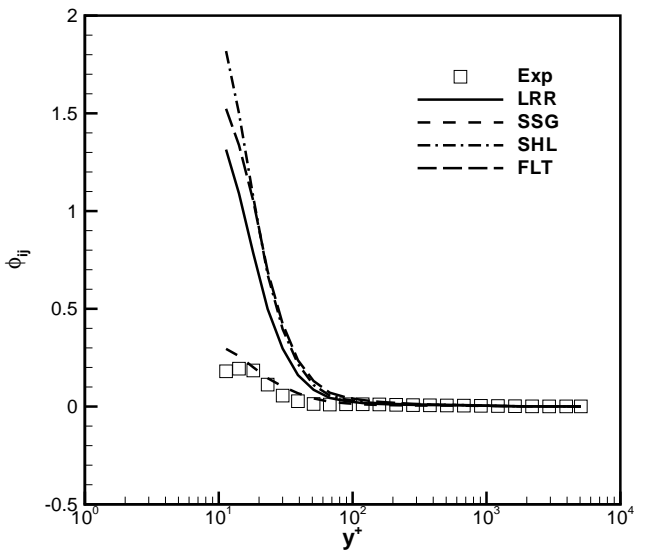

(d) $\phi_{12}$

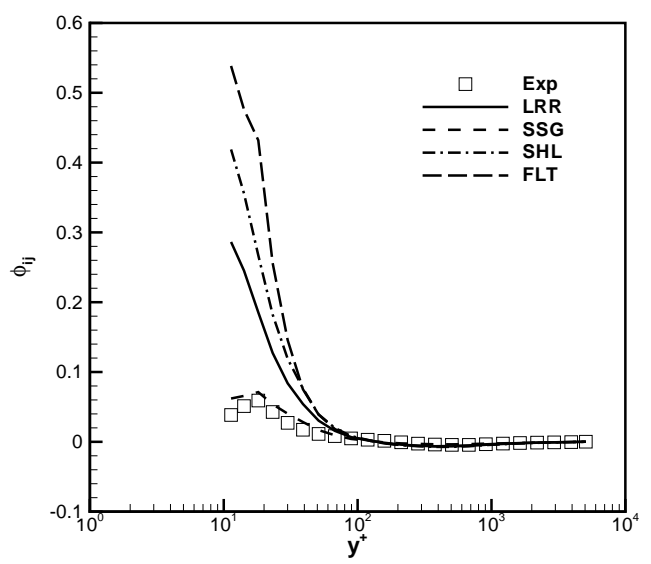

(f) $\phi_{23}$

Figure 6.11: Comparison between Pressure-Strain models at profile P19, (Exp) experiment, $(L R R)$ Launder, Reece and Rodi model, $(S S G)$ Sarkar, Speziale and Gatski model, $(S H L)$ Shih and Lumley model, $(F L T) \mathrm{Fu}$, Launder and Tselepidakis model 


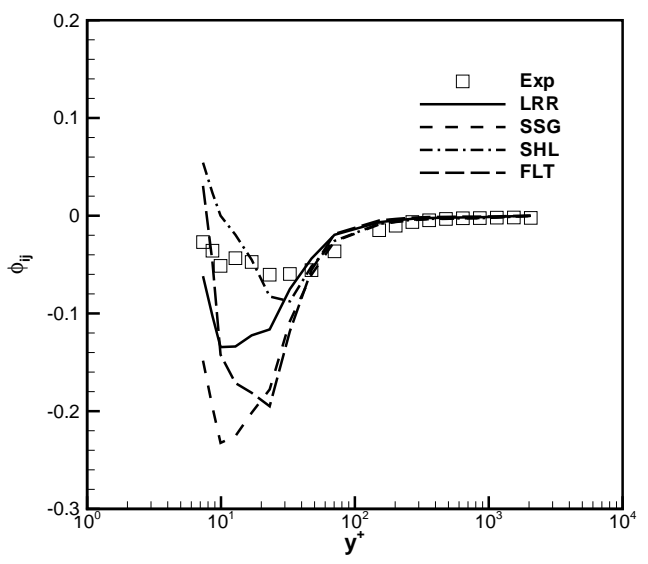

(a) $\phi_{11}$

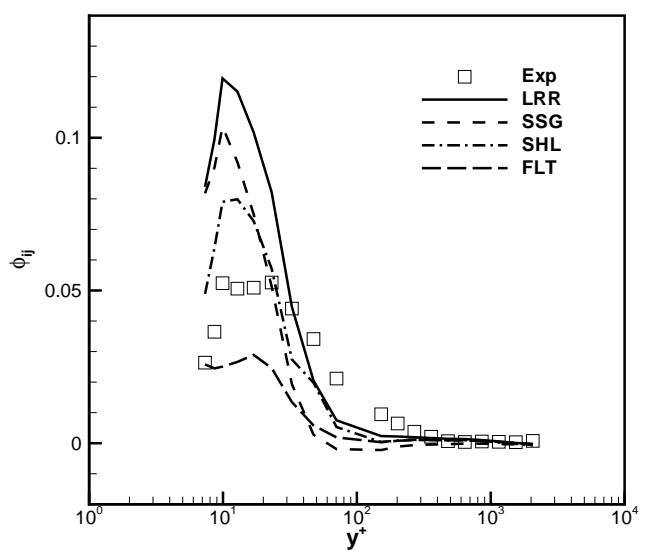

(c) $\phi_{33}$

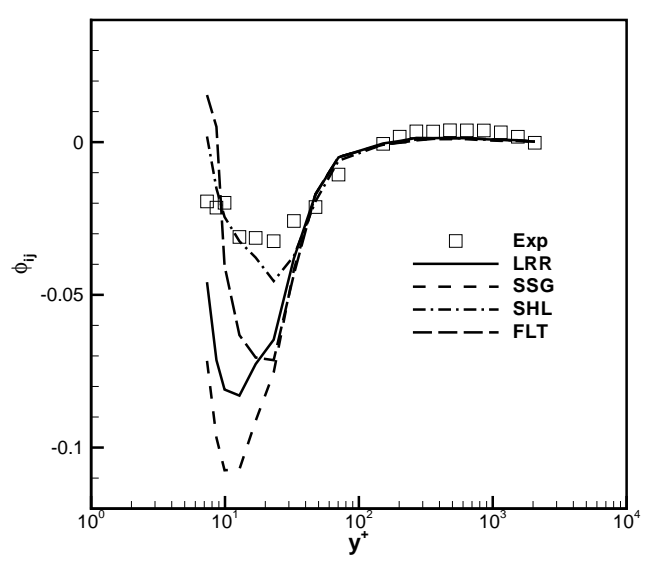

(e) $\phi_{13}$

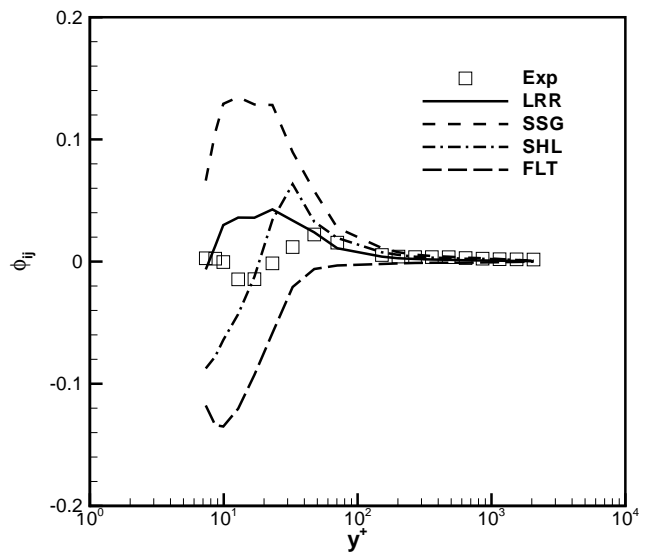

(b) $\phi_{22}$

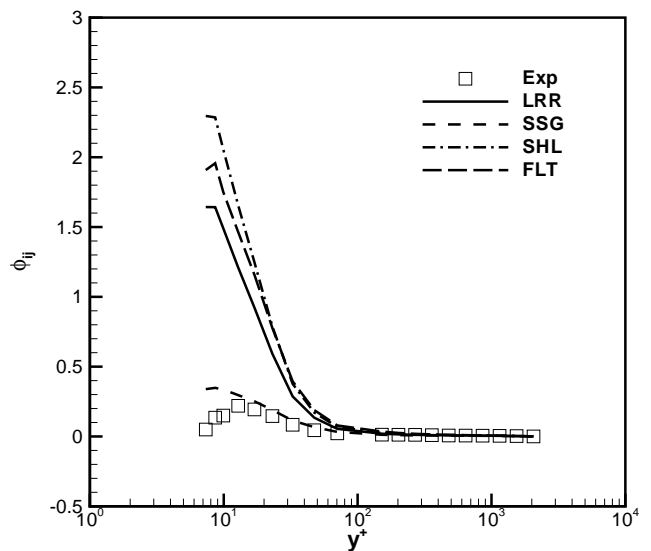

(d) $\phi_{12}$

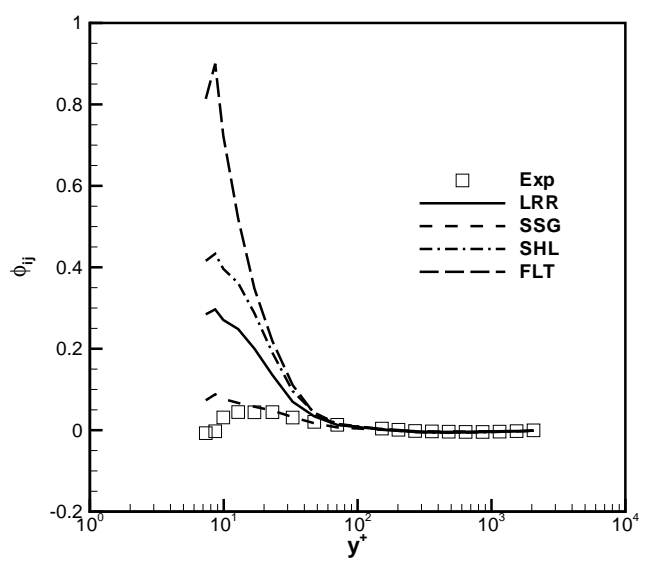

(f) $\phi_{23}$

Figure 6.12: Comparison between Pressure-Strain models at profile P20, (Exp) experiment, $(L R R)$ Launder, Reece and Rodi model, $(S S G)$ Sarkar, Speziale and Gatski model, $(S H L)$ Shih and Lumley model, $(F L T) \mathrm{Fu}$, Launder and Tselepidakis model 


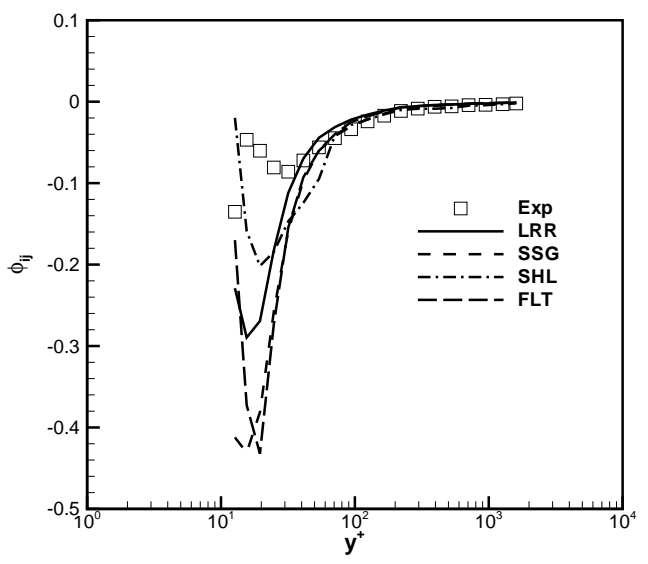

(a) $\phi_{11}$

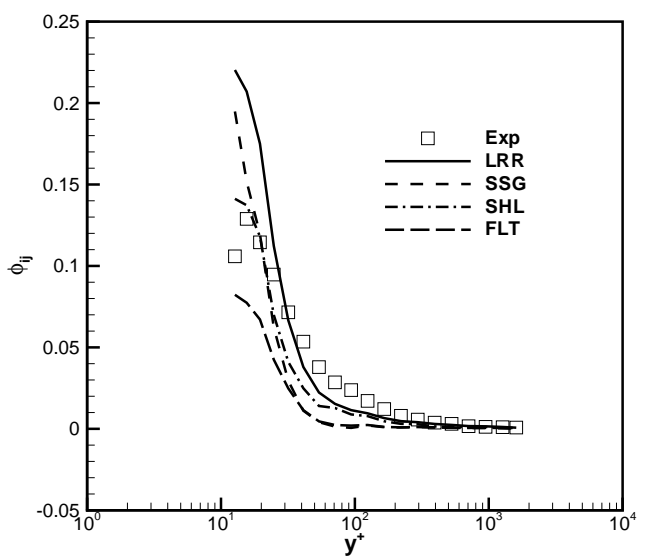

(c) $\phi_{33}$

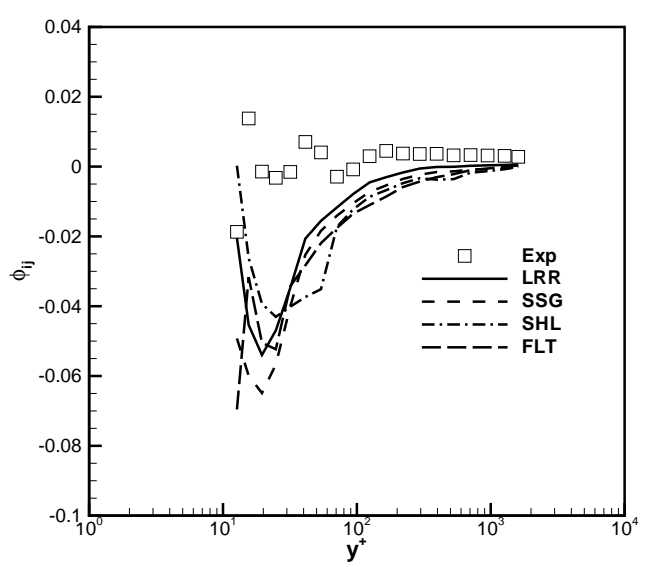

(e) $\phi_{13}$

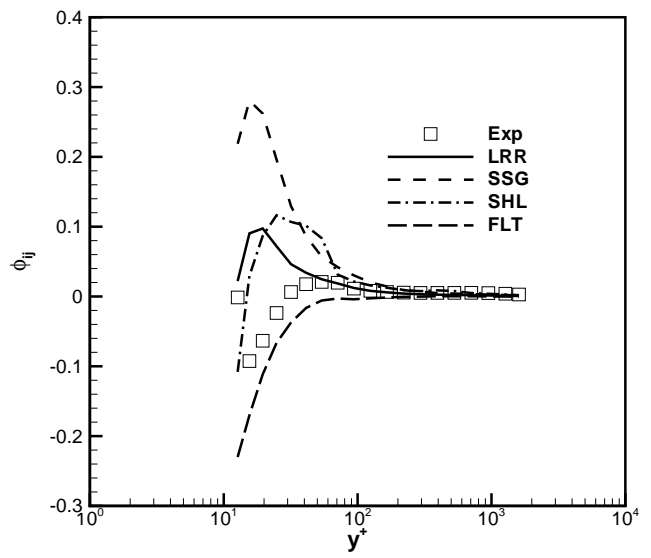

(b) $\phi_{22}$

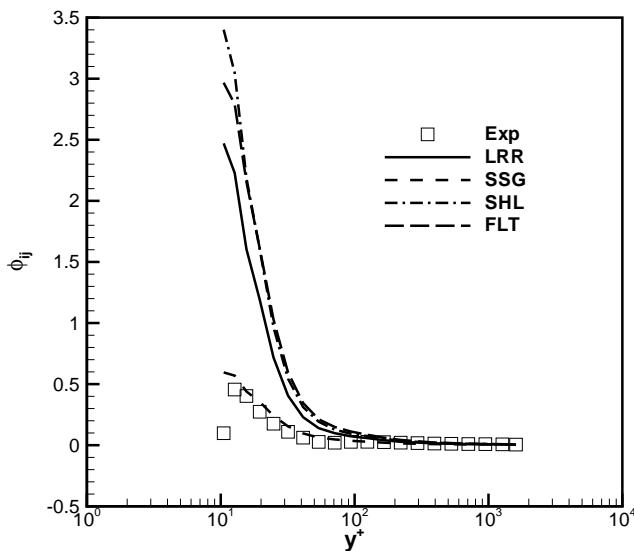

(d) $\phi_{12}$

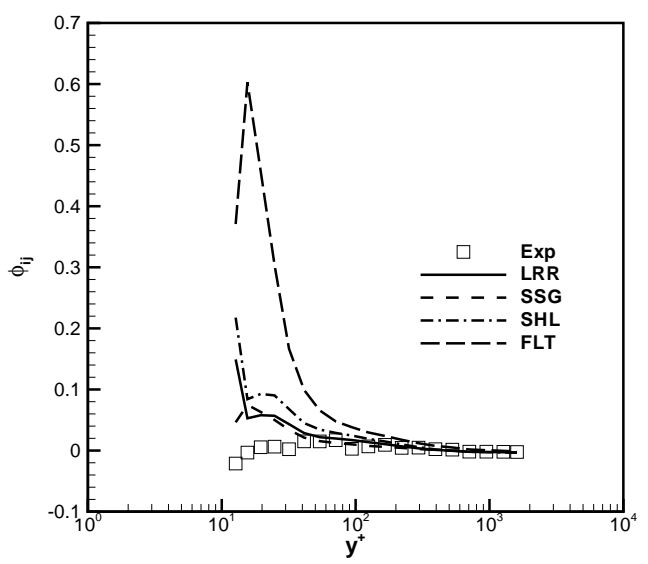

(f) $\phi_{23}$

Figure 6.13: Comparison between Pressure-Strain models at profile P33, (Exp) experiment, $(L R R)$ Launder, Reece and Rodi model, $(S S G)$ Sarkar, Speziale and Gatski model, $(S H L)$ Shih and Lumley model, $(F L T) \mathrm{Fu}$, Launder and Tselepidakis model 


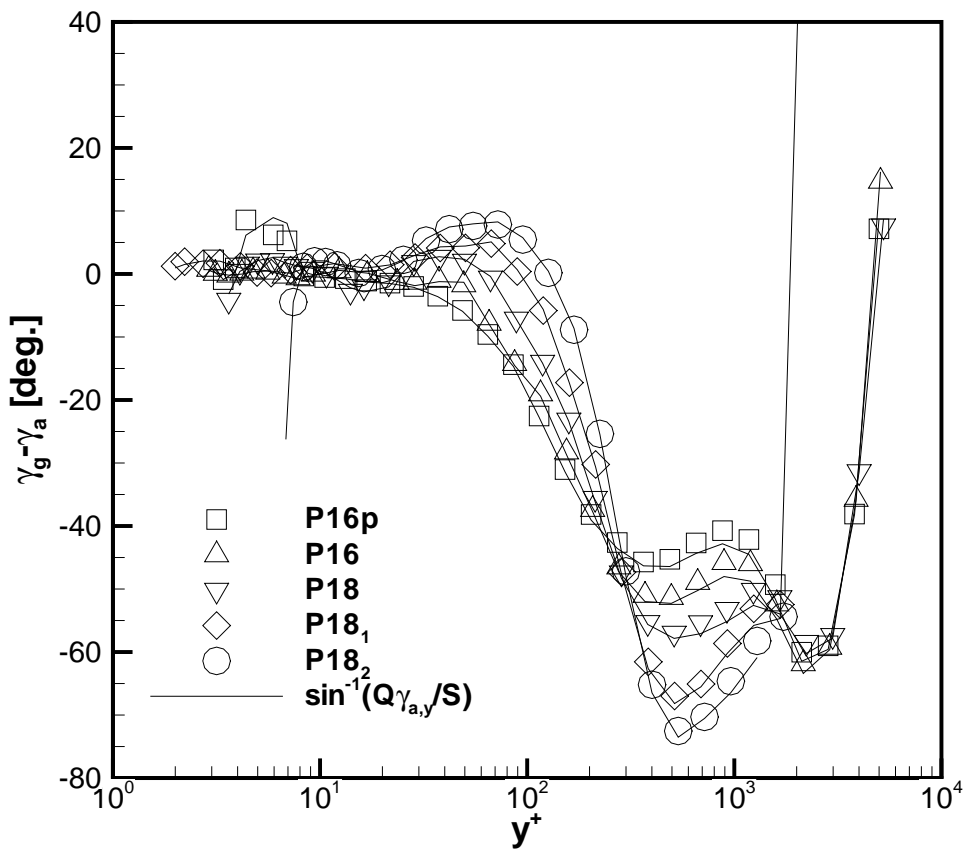

Figure 6.14: Expression for Flow Gradient Angle Lag 


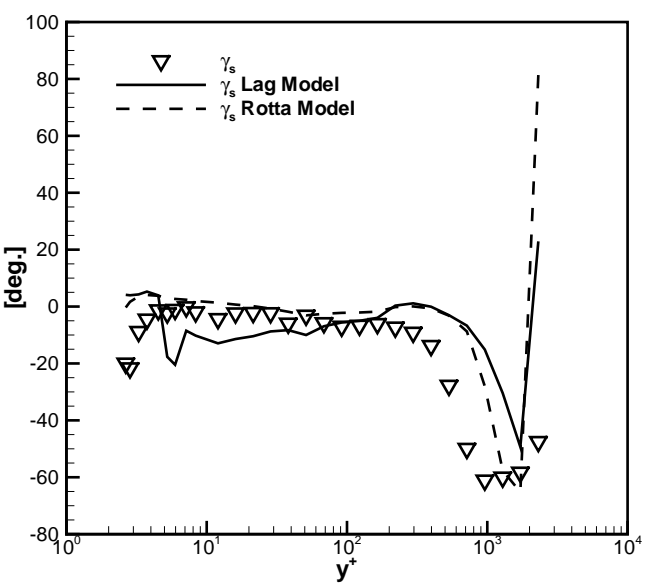

(a) Profile P06

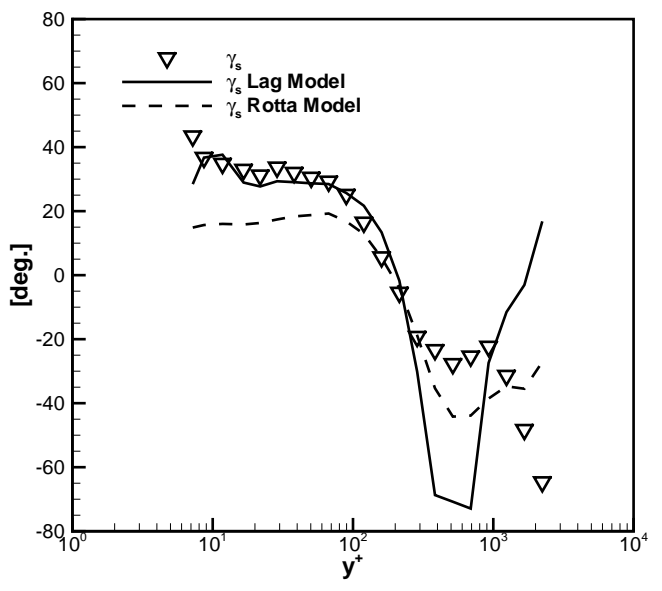

(c) Profile $P 18_{1}$

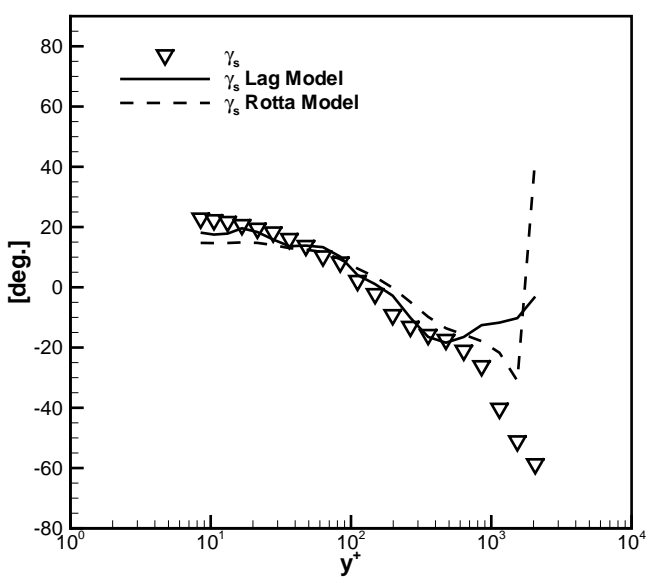

(e) Profile $P 20_{1}$

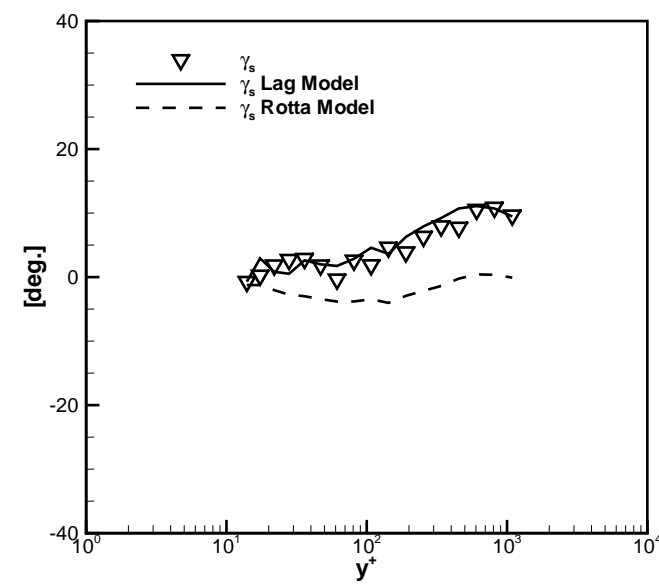

(b) Profile $P 13$

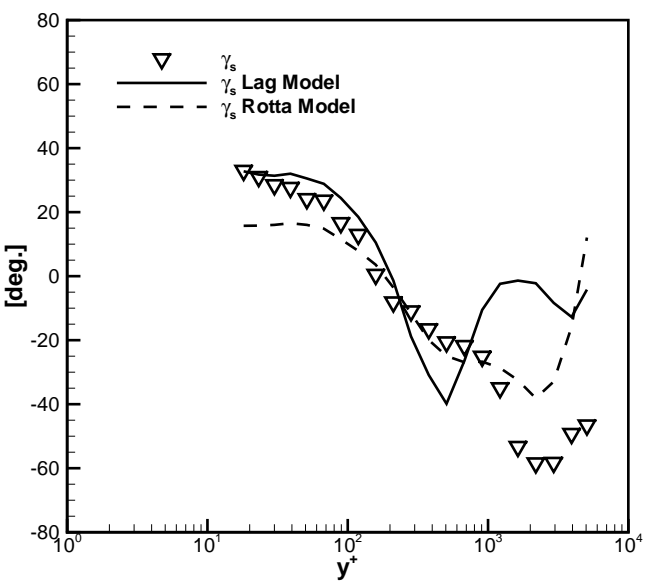

(d) Profile $P 19 p p$

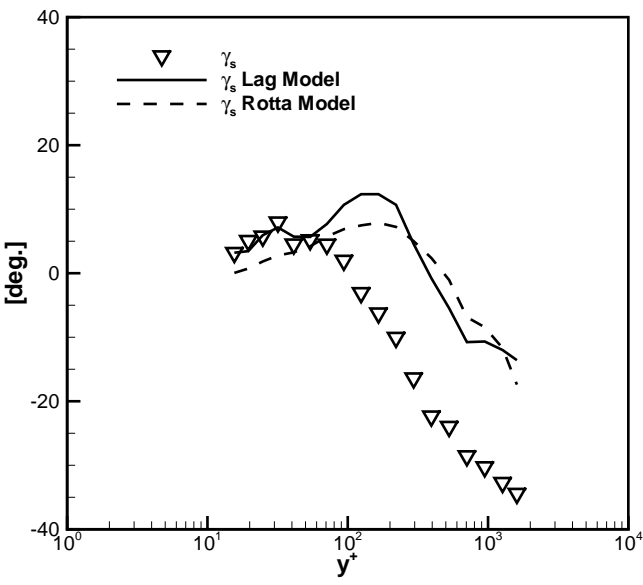

(f) Profile P33

Figure 6.15: Comparison between Measured and modeled Reynolds Shear Stress Angle, $\nabla$ Experiment, - Current Lag Model and - - Rotta's Lag Model 


\section{Chapter 7}

\section{Conclusions}

In the present work, a three-dimensional boundary layer experiment was developed such that span-wise and stream-wise pressure gradients are present with alternating signs. The pressure gradients are responsible for generating an alternating sign vorticity flux at the tunnel's floor and ceiling. Mean flow velocity components as well as static and total pressures were measured at different stream-wise locations along the test-section. Turbulence quantities including Reynolds Stresses, Turbulent transport and dissipation rate were measured at different stations along the last sine wave. Several observations and conclusions can be made,

- Mean flow velocities, Reynolds stresses, turbulent transport and turbulence kinetic energy dissipation rate were measured at selected locations along the test section.

- The data obtained using both LDV probes had very low uncertainty. However, the process of using two probes to measure partial profiles then matching those profiles together induces some uncertainty in the data.

- Mean flow velocities along the test section didn't show any signs of flow separation.

- The entrance mean flow at the test section inlet is a two-dimensional boundary layer. As the flow go through the test section it develops and becomes a complex three-dimensional channel as the boundary layer from the floor and ceiling merge together.

- Turbulent Reynolds stresses reaches a state of equal normal stresses with non-zero shear stresses in the outer layers of all clusters.

- Estimated turbulent kinetic energy dissipation rate from spectral measurements agreed very well with estimates from balancing the turbulent kinetic energy transport equation. 
- The span-wise pressure gradient deforms the boundary layer and creates a flux of stream-wise vorticity at the test section floor as the flow goes around the first sine wave.

- As the flow progresses downstream more vorticity is generated at the wall and older vorticity is diffused away from the wall.

- The vorticity components in the stream-wise and span-wise directions are strengthened due to stretching and tilting terms in the vorticity transport equations.

- The positive-z half of the test section contains large areas that generate positive vorticity flux in the trough region and smaller areas generating negative vorticity around the wave peak. The opposite is true for the negative-z half of the test-section. This results in a large positive stream-wise vorticity in the positive-z half and negative stream-wise vorticity in the negative- $z$ half of the test-section.

- The smaller regions of opposite sign vorticity in each half tend to mix the flow such that as they diffuse away from the wall, the turbulent stresses are more uniform.

- The available turbulent diffusion models don't predict the flow adequately. The magnitude of estimated kinetic energy turbulent diffusion is incorrect for all models tested. Furthermore, the trends for some of the terms are not correct.

- Different pressure-strain models are compared with estimates extracted from experimental data also show poor agreement.

- Each component in the pressure-strain tensor is modeled best by a different model. The SSG model gives very good estimates for $\phi_{12}$ and $\phi_{23}$ while the SHL model give the best estimate for $\phi_{11}$ and $\phi_{13}$. All models predict the right trend for $\phi_{22}$ and $\phi_{33}$ but all of them give the wrong magnitude. Furthermore, the disagreement between the experimental and model estimates for $\phi_{33}$ extends all the way to the outer layer when the flow is highly three-dimensional.

- In order to improve turbulence modeling, in the context of second-moment closure, better pressure-strain models are needed. More importantly, more experimental data for the pressure-strain or velocity-pressure gradient is needed.

- A proposed model for the Reynolds shear stress angle is presented. The model outperforms Rotta's model in the inner layer reproducing the change in the turbulent shear stress angle to a good extent. However, in the outer layer it deviates significantly. The model still needs more improvements. 


\section{Bibliography}

[1] S. Ahn. Unsteady Features of Turbulent Boundary Layers. PhD thesis, Virginia Polytechnic Institute and State University, 1986.

[2] R.S. Amano, P. Goel, and J.C. Chai. Turbulence energy and diffusion transport of third-moments in a separating and reattaching flow. AIAA Journal, 26(3):273-282, 1988.

[3] J. C. Andre, G. De Moor, P. Lacarrere, G. Therry, and R. Du Vachat. The clipping approximation for inhomogeneous turbulence. In 10th Symposium on Turbulent Shear Flows, 1977.

[4] Gwibo Byun. Structure of Three-Dimensional Separated Flow on Symmetric Bumps. PhD thesis, Virginia Polytechnic Institue and State University, 2005.

[5] T. Cebeci and A. M. O. Smith. Analysis of Turbulent Boundary Layers. Academic, 1974.

[6] P. Y. Chou. On velocity correlations and the solutions of the equations of turbulent fluctuation. Quartely of Applied Mathematics, 3:38-54, 1945.

[7] D. E. Cormack, L. G. Leal, and J. H. Seinfeld. An evaluation of mean reynolds stress turbulence models: the triple velocity correlation. Journal of Fluids Engineering, 100:47-54, 1978.

[8] Bart J. Daly and Francis H. Harlow. Transport equations in turbulence. The Physics of Fluids, 13(11):2634-2649, 1970.

[9] B. I. Davydov. On the statistical dynamics of an incompressible turbulent fluid. Soviet Physics, Doklady, pages 769-772, 1959.

[10] D. DeGraff and J. Eaton. Reynolds-number scaling of the flat-plate turbulent boundary layer. Journal of Fluid Mechanics, 2000.

[11] William J. Devenport and Roger L. Simpson. Time-dependent and time-averaged turbulence structure near the nose of a wing-body junction. Journal of Fluid Mechanics, 210:23-55, 1990. 
[12] Thierry Dubois and Roger Temam. Dynamic Multilevel methods. Cambridge University Press, 1999.

[13] W. H. Echols and J. W. Young. Studies of portable air-operated aerosol generators. NRL Rep. 5929, 1963.

[14] H. H. Fernholz and P. J. Finley. The incompressible zero-pressure-gradient turbulent boundary layer: An assessment of the data. Progress in Aerospace Sciences, 32:245-311, 1996.

[15] M. Fischer, J. Jovanovic, and F. Durst. Near-wall behaviour of statistical properties in turbulent flows. International Journal of Heat and Fluid Flow, 21:471-479, 2000.

[16] S. Fu, B. E. Launder, and D. P. Tselepidakis. Accommodating the effects of high strain rates in modelling the pressure-strain correlation, 1987.

[17] M. Hallback, J. Groth, and A. Johansson. An algebraic model for nonisotropic turbulent dissipation rate in reynolds stress closure. Physics of Fluids, 10.

[18] K. Hanjalic and B. E. Launder. A reynolds stress model of turbulence and its application to thin shear flows. Journal of Fluid Mechanics, 52(4):609-638, 1972.

[19] J. O. Hinze. Turbulence. McGraw Hill, 1975.

[20] K. Iwamoto, Y. Suzuki, and N. Kasagi. Reynolds number effect on wall turbulence: Towards effective feedback control. International Journal of Heat and Fluid Flow, 23:678-689, 2002.

[21] S. Jakirlic and K. Hanjalic. A new approach to modelling near-wall turbulence energy and stress dissipation. Journal of fluid Mechanics, 459:139-166, 2002.

[22] A. F. Kurbatskii and S.V. Poroseva. Modeling turbulent diffusion in a rotating cylindrical pipe flow. International Journal of Heat and Fluid Flow, 20:341-348, 1999.

[23] B. E. Launder, G. J. Reece, and W. Rodi. Progress in the development of a reynoldsstress turbulence closure. Journal of Fluid Mechanics, 68:537-566, 1975.

[24] M. Lesieur and O. Metais. New trends in large-eddy simulation of turbulence. Annual Review of Fluid Mechanics, 28:45-82, 1996.

[25] K. T. Lowe. Design and Application of a novel Laser Doppler Velocimeter for Turbulence Structural Measurements in Turbulent Boundary Layers. PhD thesis, Virginia Polytechnic and State University, 2006.

[26] K. T. Lowe and R. L. Simpson. Measurements of velocityacceleration statistics in turbulent boundary layers. International Journal of Heat and Fluid Flow, 27:558-565, 2006. 
[27] John L. Lumley. Computaitonal modeling of turbulent flows. Advances in Applied Mechanics, 18:123-176, 1978.

[28] John L. Lumley and Khajeh-Nouri Bejan. Computational modeling of turbulent transport. Advances in Geophysics, 18A:169-192, 1974.

[29] R. Ma and Roger L. Simpson. Characterization of turbulent flow downstream of a three-dimensional axisymmetric bumo, 27-29 June 2005.

[30] J.C. Mackinnon, M. Renksizbulut, and A.B. Strong. Evaluation of reynolds stress closure for turbulent boundary layer in turbulent freestream. AIAA Journal, 36(6):936945, 1998.

[31] J. Magnaudet. Modelling of inhomogeneous turbulence in the absence of mean velocity gradients. Applied Scientific Research, 51:525-531, 1993.

[32] N. N. Mansour, J. Kim, and P. Moin. Reynolds-stress and dissipation-rate budgets in a turbulent channel flow. Journal of Fluid Mechanics, 194:15-44, 1988.

[33] George L. Mellor and H. James Herring. A survey of the mean turbulent field closure models. AIAA Journal, 2(5):590-599, 1972.

[34] C Meneveau and J. Katz. Scale invariance and turbulence models for large-eddy simulation. Annual Review of Fluid Mechanics, 32:1-32, 2000.

[35] M. D. Millionshchikov. On the theory of homogeneous isotropic functions. C. R. Acad. Sci. SSSR, 32:615-619, 1941.

[36] P. Moin and K. Mahesh. Direct numerical simulation: A tool in turbulence research. Annual Review of Fluid Mechanics, 30:539-578, 1998.

[37] P. Moin and T. Shih. Direct numerical simulation of a three-dimensional turbulent boundary layer. Physics of Fluids, 10:1846-1853, 1990.

[38] J. G. Moore and J. Moore. Functional Reynolds Stress Modeling. Pocahontas Press Inc., 2006.

[39] B. R. Morton. The generation and decay of vorticity. Geophysical and Astrophysical Fluid Dynamics, 28:277-308, 1984.

[40] Y. Nagano and M. Tagawa. A structural turbulence model for triple products of velocity and scalar. Journal of Fluid Mechanics, 215:639-657, 1990.

[41] M. S. Olcmen and R. L. Simpson. An experimental study of a threedimensionalpressure-driven turbulent boundary layer. Journal of Fluid Mechanics, 290:225-262, 1995. 
[42] M. S. Olcmen and R. L. Simpson. A five-velocity-component laser-doppler velocimeter for measurements of a three-dimensional turbulent boundary layer. Measurement Science and Technology, 6:702-716, 1995.

[43] Semih M. Olcmen and Roger L. Simpson. Experimental transport-rate budgets in complex 3-d turbulent flow near a wing-body junction. In AIAA, Fluid Dynamics Conference, 27th, New Orleans, LA, 1996. American Institute of Aeronautics and Astronautics.

[44] Semih M. Olcmen and Roger L. Simpson. Experimental evaluation of turbulent diffusion models in complex 3-d flow near a wing/body junction. In AIAA, Aerospace Sciences Meeting and Exhibit. 35th, pages 97-0650, Reno, NV, 1997. American Institute of Aeronautics and Astronautics.

[45] S. A. Orszag and G. S. Patterson. Numerical simulation of three-dimensional homogeneous isotropic turbulence. Physics Review Letter, 28(76-79), 1972.

[46] B. Perot and S. Natu. A model for the dissipation rate tensor in inhomogeneous and isotropic turbulence. Physics of Fluids, 16(11):4054-4065, 2004.

[47] Roger Peyret, editor. Handbook of Computational Fluid Mechanics. Academic Press, 2000.

[48] E. Piomelli, U. Balaras. Wall-layer models for large-eddy simulation. Annual Review of Fluid Mechanics, 34:349-374, 2002.

[49] U. Piomelli. Large-eddy simulation: Achievements and challenges. Progress in Aerospace Sciences, 35:335-362, 1999.

[50] Kevin Pisterman. Use of a Seven-Hole Pressure Probe in Highly Turbulent Flow-Fields. Electronic, Virginia Polytechinic and State University, 2004.

[51] S. B. Pope. Modeling Complex Turbulent Flows, pages 53-67. ICASE/LaRC Interdisciplinary Series in Science and Engineering. Kluwer Academic Publishers, 1999.

[52] S. B. Pope. Turbulent Flows. Cambridge University Press, 2000.

[53] Spalart P.R. Direct simulation of a turbulent boundary layer up to re=1410. Journal of Fluid Mechanics, 187:61-98, 1988.

[54] Stephen M. Ross. Peirce's criterion for the elimination of suspcet experimental data. Journal of Engineering Technology, 2003.

[55] J. C. Rotta. A family of turbulence models for three-dimensional thin shear layers. In Symposium on Turbulent Shear Flows, 1977. 
[56] K. R. Saripalli and R. L. Simpson. Investigation of blown boundary layers with an improved wall jet system. NASA Contractor Report 3340, Langley Research Center, 1980.

[57] Walter R. Schwarz and Peter Bradshaw. Term-by-term tests of stress-transport turbulence models in a three-dimensional boundary layer. Physics of Fluids, 6(2), 1994.

[58] O. Sendstad and P. Moin. The near-wall mechanics of three-dimensional turbulent boundary layers. Stanford University Dept. Mech Engng Thermosciences Div Rep. TF-57, 1992.

[59] John L. Lumley Shih, Tsan-hsing. Second-order modelling of a variable-density mixing layer. Journal of Fluid Mechanics, 180:93-116, 1987.

[60] Yutaka Shimomura. A theoretical study of the turbulent diffusion in incompressible shear flows and in passive scalars. Physics of Fluids, 10(10), 1998.

[61] C. C. Shir. A preliminary numerical study of atmospheric turbulent flows in the idealised planetary boundary layer. Journal of Atmospheric Science, 30:1327, 1973.

[62] J. Smagorinsky. General circulation experiments with the primitive equations. Monthly Weather Review, 91:99-164, 1963.

[63] G. F. Smith. Constitutive equations for anisotropic and isotropic materials. North-Holland, 1994.

[64] P. R. Spalart. A new version of detached-eddy simulation, resistent to ambiguous grid densities. Theortical and Computational Fluid Dynamics, 20:181-195, 2006.

[65] C. G. Speziale, S. Sarkar, and T.D. Gatski. Modelling the pressure-strain correlation of turbulence: an invariant dynamical systems approach. Journal of Fluid Mechanics, 227:245-272, 1991.

[66] Anthony G. Straatman. A modified model for diffusion in second-moment turbulence closures. Journal of Fluid Engineering, 121:747-756, 1999.

[67] Kazuhiko Suga. Improvement of second moment closure for turbulent obstacle flow and heat transfer. American Journal of Heat and Fluid Flow, 25(5):776-784, 2004.

[68] H. Tennekes and John L. Lumley. A First Course in Turbulence. The MIT Press, 1972.

[69] Q. Tian. Some features of tip gap flow fields of a linear compressor cascade. Master's thesis, Virginia Polytechinic and State University, 2003.

[70] Christian W. Wenger and William J. Devenport. Seven-hole pressure probe calibration method utilizing look-up error tables. AIAA Journal, 37:675-679, 1999. 
[71] J. C. Wyngaard, O. R. Cot, and K. S. Rao. Modeling the atmospheric boundary layer. In Second IUTAM-IUGG Symposium on Turbulent Diffusion and Enviromental Pollution, Charllotesville, VA, 1973.

[72] B. A. Younis, T. B. Gatski, and C.G. Speziale. Towards a rational model for the triple velocity correlations of turbulence. The Royal Society, 456:909-920, 2000. 


\section{Appendix A}

\section{Wavy Wall Geometry}

Accurate measurement of the test section geometry is important for the documentation of the experiment. Thus the geometry of the side walls was measured and compared to the original analytical geometry desired. The surface slope is also compared to the original design.

\section{Measurement Procedure}

The wavy wall test section consists of two side walls and the ceiling. The side walls and the ceiling are divided into two parts each. The plan view of the test section is shown in Figure A.1; flow is from left to right.

The geometry of the side walls was obtained by laying the side walls flat on a flat surface and attaching a 3/4" thick flat Plexiglas plate normal to the bottom surface of the side walls. The geometry of the wavy side walls were measured relative to the Plexiglass plate using a high precision. The setup is shown in Figure A.2.

The wall contours were measured as heights from the Plexiglas plate using a vertical caliper as shown in Figure A.2. The vertical caliper had a resolution of 1/1000". The axial location of each measurement was read off of a tape measure attached to the bottom of the wall. Unfortunately the Plexiglass plate was shorter than the side wall length, so each part geometry was measured in multiple steps (3-4). In order to make sure that the same reference is used for each measurement, overlap regions between different steps were measured to make sure that all measurement had the same reference plane position. In general there were differences of 0.003 " to 0.01 ". However, these differences were constant for each overlap region and a constant shift was added to the measurements to account for these differences. The comparisons between consecutive measurements are shown in Figure. 


\section{Measured Geometry}

The final wall geometries are shown in Figure. Also shown is the departure from the original sine wave geometries as a percentage of the peak to trough amplitude 6 ".

It can also be seen that the agreement between the design and actual geometry is to some extent very good. However the waves seem to be shifted vertically along the axial direction such that the peaks and troughs are slightly higher than the design value which accounts for the $10 \%$ error in the geometry. However the distance between the peaks and troughs is more or less equal to 6 ".

Figure shows the surface slopes of the walls which agree well to the design values except for some points where the geometry deviates from the design.

\section{Uncertainty of Measurement}

The axial position of each height measurement is determined with uncertainty of $1 / 32$ ". For a given sine wave,

$$
z=\alpha \cdot \sin 2 \pi \frac{x}{\lambda}
$$

Where, $\alpha=3 "$ and $\lambda=24 "$

This leads to a maximum uncertainty in z-location of 0.0245 ", which is larger than the resolution of the caliper used to measure the height of the wall. The axial positions were taken each 1", however these locations were modified because the caliper's measuring arm had a large width so that the actual position of measurement was different than the actual position by the width of this arm which was $0.341 "$. This is done by either adding or subtracting the arm's width to or from the x-location depending on the position of the arm relative to the waves. 
Table A.1: Wavy Wall Test Section Geometry

\begin{tabular}{|c|c|c|c|c|c|}
\hline$X_{R} / L$ & $Z_{R} / W_{x=0}$ & $X_{L} / L$ & $Z_{L} / W_{x=0}$ & $X / L$ & $Y / Y_{x=0}$ \\
\hline $0.00 E+00$ & $-5.00 E-01$ & $00 E+00$ & $5.00 E-01$ & $00 E+00$ & $.00 E+00$ \\
\hline $5.21 E-03$ & $-4.97 E-01$ & $21 E-03$ & $97 E-01$ & $21 E-03$ & $99 E-01$ \\
\hline $4 E-02$ & $-4.95 E-01$ & $4 E-02$ & $5 E-01$ & $4 E-02$ & $98 E-01$ \\
\hline $1.56 E-02$ & $-4.93 E-01$ & $6 E-02$ & $4.93 E-01$ & $6 E-02$ & $6 E-01$ \\
\hline $2.08 E-02$ & $-4.90 E-01$ & $.08 E-02$ & $91 E-01$ & $08 E-02$ & $95 E-01$ \\
\hline $2.60 E-02$ & $-4.88 E-01$ & $2.60 E-02$ & $38 E-01$ & $50 E-02$ & $94 E-01$ \\
\hline $3.13 E-02$ & $-4.87 E-01$ & $3.13 E-02$ & $4.86 E-01$ & $13 E-02$ & $93 E-01$ \\
\hline$E-02$ & $E-01$ & $E-02$ & $E-01$ & $5 E-02$ & $1 E-01$ \\
\hline $4.17 E-02$ & $-4.82 E-01$ & $4.17 E-02$ & $4.81 E-01$ & $17 E-02$ & $0 E-01$ \\
\hline $4.69 E-02$ & $E-01$ & $69 E-02$ & $4.79 E-01$ & $69 E-02$ & $39 E-01$ \\
\hline $1 E-02$ & $E-01$ & $E-02$ & $E-01$ & $1 E-02$ & $8 E-01$ \\
\hline $5.73 E-02$ & $-4.75 E-01$ & $5.73 E-02$ & $4.74 E-01$ & $5.73 E-02$ & $9.86 E-01$ \\
\hline $6.25 E-02$ & $E-01$ & $25 E-02$ & $4.72 E-$ & $5 E-02$ & $E-01$ \\
\hline $6.77 E-02$ & -4 & & & 2 & \\
\hline $7.29 E-02$ & $-4.67 E-01$ & $7.29 E-02$ & $4.67 E-01$ & $7.29 E-02$ & $.83 E-01$ \\
\hline $1 E-02$ & $-4.65 E-01$ & $1 E-02$ & $4.64 E-$ & $1 E-$ & $1 E-$ \\
\hline $3 E-02$ & $E-01$ & $8.33 E-02$ & $4.61 E-01$ & $3 E-02$ & $9.80 E-01$ \\
\hline$E-02$ & $E-01$ & $E-02$ & $E-$ & $E-$ & $9.79 E-01$ \\
\hline & -4 & & & & \\
\hline $9.90 E-02$ & $-4.56 E-01$ & $90 E-02$ & $4.54 E-01$ & $90 E-02$ & $9.76 E-01$ \\
\hline $4 E-01$ & $-4.54 E-01$ & $4 E-01$ & $4.52 E-01$ & $4 E-01$ & $.75 E-01$ \\
\hline $1.09 E-01$ & $E-01$ & $09 E-01$ & $4.49 E-$ & $E-01$ & $.74 E-01$ \\
\hline$E-01$ & $-4.49 E-01$ & $1.15 E-01$ & $4.46 E-$ & $1.15 E-01$ & $9.73 E-01$ \\
\hline $1.20 E-01$ & $-4.45 E-01$ & $1.20 E-01$ & $4.44 E-01$ & $1.20 E-01$ & $.71 E-01$ \\
\hline $1.25 E-01$ & $E-01$ & $1.25 E-01$ & $4.42 E-$ & $.25 E-01$ & $.70 E-01$ \\
\hline $1.30 E-01$ & $-4.43 E-01$ & $1.29 E-01$ & $4.43 E-01$ & $1.30 E-01$ & $9.69 E-01$ \\
\hline $1.35 E-01$ & $E-01$ & $1.35 E-01$ & $4.46 E-01$ & $1.35 E-01$ & $.68 E-01$ \\
\hline $1.41 E-01$ & $-4.32 E-01$ & $1.40 E-01$ & $4.50 E-$ & $1.41 E-01$ & $9.67 E-01$ \\
\hline $1.46 E-01$ & $-4.23 E-01$ & $1.45 E-01$ & $4.55 E-01$ & $1.46 E-01$ & $9.65 E-01$ \\
\hline $1.51 E-01$ & $E-01$ & $1.50 E-01$ & $4.63 E-01$ & $1.51 E-01$ & $9.64 E-01$ \\
\hline $1.56 E-01$ & $-3.99 E-01$ & $1.55 E-01$ & $4.71 E-01$ & $1.56 E-01$ & $9.63 E-01$ \\
\hline $1.61 E-01$ & $-3.88 E-01$ & $1.61 E-01$ & $4.79 E-01$ & $1.61 E-01$ & $9.62 E-01$ \\
\hline $1.67 E-01$ & $-3.76 E-01$ & $1.66 E-01$ & $4.87 E-01$ & $1.67 E-01$ & $9.60 E-01$ \\
\hline $1.72 E-01$ & $-3.64 E-01$ & $1.71 E-01$ & $4.93 E-01$ & $1.72 E-01$ & $9.59 E-01$ \\
\hline $1.77 E-01$ & $-3.57 E-01$ & $1.76 E-01$ & $4.97 E-01$ & $1.77 E-01$ & $9.58 E-01$ \\
\hline
\end{tabular}


Table A.1 - continued from previous page

\begin{tabular}{|c|c|c|c|c|c|}
\hline$X_{R} / L$ & $\overline{Z_{R}} / W_{x=0}$ & $X_{L} / L$ & $Z_{L} / W_{x=0}$ & $X / L$ & $Y / Y_{x=0}$ \\
\hline $1.82 E-01$ & $-3.52 E-01$ & $1.81 E-01$ & $5.00 E-01$ & $1.82 E-01$ & $9.57 E-01$ \\
\hline $1.88 E-01$ & $-3.48 E-01$ & $8 E-01$ & $5.01 E-01$ & $38 E-01$ & $9.55 E-01$ \\
\hline $1.93 E-01$ & $-3.46 E-01$ & $1.93 E-01$ & $4.95 E-01$ & $93 E-01$ & $54 E-01$ \\
\hline $1.97 E-01$ & $-3.53 E-01$ & $1.98 E-01$ & $4.85 E-01$ & $1.98 E-01$ & $.53 E-01$ \\
\hline $2.02 E-01$ & $-3.64 E-01$ & $2.03 E-01$ & $4.71 E-01$ & $2.03 E-01$ & $.52 E-01$ \\
\hline $2.07 E-01$ & $3 E-01$ & $2.08 E-01$ & $4.54 E-01$ & $2.08 E-01$ & $9.50 E-01$ \\
\hline $2.13 E-01$ & $-3.96 E-01$ & $2.14 E-01$ & $4 E-01$ & $2.14 E-01$ & $49 E-01$ \\
\hline $2.18 E-01$ & $-4.16 E-01$ & $2.19 E-01$ & $4.08 E-01$ & $2.19 E-01$ & $9.48 E-01$ \\
\hline $2.23 E-01$ & $-4.38 E-01$ & $2.24 E-01$ & $3.90 E-01$ & $2.24 E-01$ & $.47 E-01$ \\
\hline $2.28 E-01$ & $-4.58 E-01$ & $2.29 E-01$ & $3.70 E-01$ & $2.29 E-01$ & $9.45 E-01$ \\
\hline $2.33 E-01$ & $-4.74 E-01$ & $2.34 E-01$ & $3.52 E-01$ & $2.34 E-01$ & $9.44 E-01$ \\
\hline $2.39 E-01$ & $-4.87 E-01$ & $2.40 E-01$ & $3.40 E-$ & $2.40 E-01$ & $9.43 E-01$ \\
\hline $2.44 E-01$ & $-4.95 E-01$ & $2.45 E-01$ & $3.35 E-$ & $2.45 E-01$ & $9.42 E-01$ \\
\hline $2.49 E-01$ & $-5.00 E-01$ & $2.50 E-01$ & $3.34 E-01$ & $2.50 E-01$ & $9.40 E-01$ \\
\hline $2.55 E-01$ & $E-01$ & $2.54 E-01$ & 1 & -01 & \\
\hline $2.60 E-01$ & $-4.90 E-01$ & $2.60 E-01$ & $3.42 E-01$ & $2.60 E-01$ & $9.38 E-01$ \\
\hline $2.66 E-01$ & $-4.78 E-01$ & $2.65 E-01$ & $3.58 E-$ & $2.66 E-01$ & $9.37 E-01$ \\
\hline $2.71 E-01$ & -4 & $2.70 E-01$ & & $2.71 E-01$ & \\
\hline $2.76 E-01$ & $-4.41 E-01$ & $2.75 E-01$ & $3.95 E-01$ & $2.76 E-01$ & $9.34 E-01$ \\
\hline $2.81 E-01$ & $-4.20 E-01$ & $2.80 E-01$ & $4.14 E-01$ & $2.81 E-01$ & $9.33 E-01$ \\
\hline $2.86 E-01$ & $-3.98 E-01$ & $2.86 E-01$ & $4.36 E-01$ & $2.86 E-01$ & $9.32 E-01$ \\
\hline $2.92 E-01$ & $-3.81 E-01$ & $2.91 E-01$ & $4.55 E-01$ & $2.92 E-01$ & $9.31 E-01$ \\
\hline $2.97 E-01$ & $-3.60 E-01$ & $2.96 E-01$ & $4.75 E-01$ & $2.97 E-01$ & $9.29 E-01$ \\
\hline $3.02 E-01$ & $-3.47 E-01$ & $3.01 E-01$ & $4.89 E-01$ & $3.02 E-01$ & $9.28 E-01$ \\
\hline $3.07 E-01$ & $-3.38 E-01$ & $3.06 E-01$ & $4.97 E-01$ & $3.07 E-01$ & $9.27 E-01$ \\
\hline $3.13 E-01$ & $-3.34 E-01$ & $3.13 E-01$ & $5.00 E-01$ & $3.13 E-01$ & $9.26 E-01$ \\
\hline $3.17 E-01$ & $-3.36 E-01$ & $3.18 E-01$ & $4.95 E-01$ & $3.18 E-01$ & $9.24 E-01$ \\
\hline $3.22 E-01$ & $-3.43 E-01$ & $3.23 E-01$ & $4.85 E-01$ & $3.23 E-01$ & $9.23 E-01$ \\
\hline $3.27 E-01$ & $-3.55 E-01$ & $3.28 E-01$ & $4.70 E-01$ & $3.28 E-01$ & $9.22 E-01$ \\
\hline $3.32 E-01$ & $-3.71 E-01$ & $3.33 E-01$ & $4.52 E-01$ & $3.33 E-01$ & $9.21 E-01$ \\
\hline $3.38 E-01$ & $-3.90 E-01$ & $3.39 E-01$ & $4.32 E-01$ & $3.39 E-01$ & $9.19 E-01$ \\
\hline $3.43 E-01$ & $-4.10 E-01$ & $3.44 E-01$ & $4.10 E-01$ & $3.44 E-01$ & $9.18 E-01$ \\
\hline $3.48 E-01$ & $-4.31 E-01$ & $3.49 E-01$ & $3.88 E-01$ & $3.49 E-01$ & $9.17 E-01$ \\
\hline $3.53 E-01$ & $-4.51 E-01$ & $3.54 E-01$ & $3.67 E-01$ & $3.54 E-01$ & $9.16 E-01$ \\
\hline $3.58 E-01$ & $-4.71 E-01$ & $3.59 E-01$ & $3.52 E-01$ & $3.59 E-01$ & $9.14 E-01$ \\
\hline $3.64 E-01$ & $-4.87 E-01$ & $3.65 E-01$ & $3.41 E-01$ & $3.65 E-01$ & $9.13 E-01$ \\
\hline
\end{tabular}


Table A.1 - continued from previous page

\begin{tabular}{|c|c|c|c|c|c|}
\hline$X_{R} / L$ & $Z_{R} / W_{x=0}$ & $X_{L} / L$ & $Z_{L} / W_{x=0}$ & $X / L$ & $Y / Y_{x=0}$ \\
\hline $3.69 E-01$ & $-4.95 E-01$ & $3.70 E-01$ & $3.35 E-01$ & $3.70 E-01$ & $9.12 E-01$ \\
\hline $3.74 E-01$ & $-4.99 E-01$ & $3.75 E-01$ & $3.34 E-01$ & $3.75 E-01$ & $.11 E-01$ \\
\hline $3.80 E-01$ & $-4.97 E-01$ & $3.79 E-01$ & $3.39 E-01$ & $3.80 E-01$ & $09 E-01$ \\
\hline $5 E-01$ & $-4.90 E-01$ & $3.85 E-01$ & $3.45 E-01$ & $3.85 E-01$ & $.08 E-01$ \\
\hline $3.91 E-01$ & $-4.77 E-01$ & $3.90 E-01$ & $3.58 E-01$ & $3.91 E-01$ & $9.07 E-01$ \\
\hline $3.96 E-01$ & $-4.61 E-01$ & $3.95 E-01$ & $3.75 E-01$ & $3.96 E-01$ & $9.06 E-01$ \\
\hline $4.01 E-01$ & $-4.42 E-01$ & $4.00 E-01$ & $5 E-01$ & $4.01 E-01$ & $9.05 E-01$ \\
\hline $4.06 E-01$ & $-4.22 E-01$ & $4.05 E-01$ & $4.16 E-01$ & $4.06 E-01$ & $9.03 E-01$ \\
\hline $4.11 E-01$ & $-3.99 E-01$ & $4.11 E-01$ & $4.38 E-01$ & $4.11 E-01$ & $9.02 E-01$ \\
\hline $4.17 E-01$ & $-3.81 E-01$ & $4.16 E-01$ & $4.56 E-01$ & $4.17 E-01$ & $9.01 E-01$ \\
\hline $4.22 E-01$ & $-3.59 E-01$ & $4.21 E-01$ & $4.75 E-01$ & $4.22 E-01$ & $9.00 E-01$ \\
\hline $4.27 E-01$ & $-3.47 E-01$ & $4.26 E-01$ & $4.89 E-01$ & $4.27 E-01$ & $.98 E-01$ \\
\hline $4.32 E-01$ & $-3.37 E-01$ & $4.31 E-01$ & $4.98 E-01$ & $4.32 E-01$ & $.97 E-01$ \\
\hline $4.38 E-01$ & $-3.33 E-01$ & $4.38 E-01$ & $4.98 E-01$ & $4.38 E-01$ & $8.96 E-01$ \\
\hline $4.42 E-01$ & $-3.34 E-01$ & $4.44 E-01$ & $4.89 E-01$ & $4.43 E-01$ & $.95 E-01$ \\
\hline $4.47 E-01$ & $-3.44 E-01$ & $4.49 E-01$ & $4.78 E-01$ & $4.48 E-01$ & $8.93 E-01$ \\
\hline $4.52 E-01$ & $-3.55 E-01$ & $4.54 E-01$ & $4.62 E-01$ & $4.53 E-01$ & $8.92 E-01$ \\
\hline$E-01$ & $-3.71 E-01$ & $4.59 E-$ & $4.44 E-01$ & $4.58 E-01$ & \\
\hline $4.63 E-01$ & $-3.91 E-01$ & $4.64 E-01$ & $4.24 E-01$ & $4.64 E-01$ & $8.90 E-01$ \\
\hline $4.68 E-01$ & $-4.09 E-01$ & $4.70 E-01$ & $4.02 E-01$ & $4.69 E-01$ & $8.88 E-01$ \\
\hline $4.73 E-01$ & $-4.32 E-01$ & $4.75 E-01$ & $3.81 E-01$ & $4.74 E-01$ & $8.87 E-01$ \\
\hline $4.78 E-01$ & $-4.53 E-01$ & $4.80 E-01$ & $3.60 E-01$ & $4.79 E-01$ & $8.86 E-01$ \\
\hline $4.83 E-01$ & $-4.71 E-01$ & $4.85 E-01$ & $3.46 E-01$ & $4.84 E-01$ & $8.85 E-01$ \\
\hline $4.89 E-01$ & $-4.86 E-01$ & $4.90 E-01$ & $3.36 E-01$ & $4.90 E-01$ & $8.83 E-01$ \\
\hline $4.94 E-01$ & $-4.96 E-01$ & $4.96 E-01$ & $3.31 E-01$ & $4.95 E-01$ & $8.82 E-01$ \\
\hline $5.00 E-01$ & $-4.99 E-01$ & $5.01 E-01$ & $3.32 E-01$ & $5.00 E-01$ & $8.81 E-01$ \\
\hline $5.05 E-01$ & $-4.92 E-01$ & $5.06 E-01$ & $3.35 E-01$ & $5.05 E-01$ & $8.80 E-01$ \\
\hline $5.10 E-01$ & $-4.84 E-01$ & $5.11 E-01$ & $3.42 E-01$ & $5.10 E-01$ & $8.78 E-01$ \\
\hline $5.16 E-01$ & $-4.72 E-01$ & $5.17 E-01$ & $3.55 E-01$ & $5.16 E-01$ & $8.77 E-01$ \\
\hline $5.21 E-01$ & $-4.56 E-01$ & $5.22 E-01$ & $3.72 E-01$ & $5.21 E-01$ & $8.76 E-01$ \\
\hline $5.26 E-01$ & $-4.35 E-01$ & $5.27 E-01$ & $3.92 E-01$ & $5.26 E-01$ & $8.75 E-01$ \\
\hline $5.31 E-01$ & $-4.14 E-01$ & $5.32 E-01$ & $4.13 E-01$ & $5.31 E-01$ & $8.74 E-01$ \\
\hline $5.36 E-01$ & $-3.93 E-01$ & $5.37 E-01$ & $4.33 E-01$ & $5.36 E-01$ & $8.72 E-01$ \\
\hline $5.42 E-01$ & $-3.74 E-01$ & $5.43 E-01$ & $4.53 E-01$ & $5.42 E-01$ & $8.71 E-01$ \\
\hline $5.47 E-01$ & $-3.56 E-01$ & $5.48 E-01$ & $4.69 E-01$ & $5.47 E-01$ & $8.70 E-01$ \\
\hline $5.52 E-01$ & $-3.41 E-01$ & $5.53 E-01$ & $4.84 E-01$ & $5.52 E-01$ & $8.69 E-01$ \\
\hline
\end{tabular}


Table A.1 - continued from previous page

\begin{tabular}{|c|c|c|c|c|c|}
\hline$X_{R} / L$ & $Z_{R} / W_{x=0}$ & $X_{L} / L$ & $Z_{L} / W_{x=0}$ & $X / L$ & $Y / Y_{x=0}$ \\
\hline $5.57 E-01$ & $-3.32 E-01$ & $5.58 E-01$ & $4.92 E-01$ & $5.57 E-01$ & $8.67 E-01$ \\
\hline $5.63 E-01$ & $-3.28 E-01$ & $5.63 E-01$ & $4.96 E-01$ & $5.63 E-01$ & $66 E-01$ \\
\hline $5.67 E-01$ & $-3.31 E-01$ & $5.68 E-01$ & $4.92 E-01$ & $68 E-01$ & $65 E-01$ \\
\hline $5.72 E-01$ & $-3.37 E-01$ & $5.73 E-01$ & $4.81 E-01$ & $3 E-01$ & $4 E-01$ \\
\hline $5.77 E-01$ & $-3.50 E-01$ & $5.78 E-01$ & $4.66 E-01$ & $5.78 E-01$ & $8.62 E-01$ \\
\hline $5.82 E-01$ & $-3.66 E-01$ & $5.83 E-01$ & $4.48 E-01$ & $5.83 E-01$ & $8.61 E-01$ \\
\hline $5.88 E-01$ & $5 E-01$ & $E-01$ & $4.28 E-01$ & $E-01$ & $8.60 E-01$ \\
\hline $5.93 E-01$ & $-4.04 E-01$ & $5.94 E-01$ & $4.07 E-01$ & $5.94 E-01$ & $8.59 E-01$ \\
\hline $8 E-01$ & $E-01$ & $5.99 E-01$ & $3.85 E-01$ & $5.99 E-01$ & $.57 E-01$ \\
\hline $6.03 E-01$ & $-4.47 E-01$ & $6.04 E-01$ & $3.68 E-01$ & $6.04 E-01$ & $.56 E-01$ \\
\hline $6.08 E-01$ & $-4.66 E-01$ & $6.09 E-01$ & $3.51 E-01$ & $6.09 E-01$ & $8.55 E-01$ \\
\hline $6.14 E-01$ & $-4.81 E-01$ & $6.15 E-01$ & $3.40 E-01$ & $6.15 E-01$ & $8.54 E-01$ \\
\hline $6.19 E-01$ & $-4.90 E-01$ & $6.20 E-01$ & $3.33 E-$ & $6.20 E-01$ & $8.52 E-01$ \\
\hline $6.24 E-01$ & $-4.95 E-01$ & $6.25 E-01$ & $3.31 E-01$ & $6.25 E-01$ & $8.51 E-01$ \\
\hline $0 E-01$ & $-4.92 E-01$ & $0 E-01$ & $3.33 E-01$ & $0 E-01$ & $E-01$ \\
\hline $6.35 E-01$ & $-4.86 E-01$ & $6.35 E-01$ & $3.39 E-01$ & $6.35 E-01$ & $8.49 E-01$ \\
\hline $6.41 E-01$ & $-4.74 E-01$ & $6.41 E-01$ & $3.51 E-01$ & $6.41 E-01$ & $8.47 E-01$ \\
\hline $5 E-01$ & $-4.57 E-01$ & & $3.67 E-$ & $6.46 E-01$ & \\
\hline $6.51 E-01$ & $-4.35 E-01$ & $6.51 E-01$ & $3.88 E-01$ & $6.51 E-01$ & $8.45 E-01$ \\
\hline $6.56 E-01$ & $-4.16 E-01$ & $6.56 E-01$ & $4.09 E-01$ & $6.56 E-01$ & $8.44 E-01$ \\
\hline $6.61 E-01$ & $-3.95 E-01$ & $6.61 E-01$ & $4.31 E-01$ & $6.61 E-01$ & $8.43 E-01$ \\
\hline $6.67 E-01$ & $-3.76 E-01$ & $6.67 E-01$ & $4.52 E-01$ & $6.67 E-01$ & $8.41 E-01$ \\
\hline $6.72 E-01$ & $-3.57 E-01$ & $6.72 E-01$ & $4.71 E-01$ & $6.72 E-01$ & $8.40 E-01$ \\
\hline $6.77 E-01$ & $-3.43 E-01$ & $6.77 E-01$ & $4.84 E-01$ & $6.77 E-01$ & $8.39 E-01$ \\
\hline $6.82 E-01$ & $-3.31 E-01$ & $6.82 E-01$ & $4.92 E-01$ & $6.82 E-01$ & $8.38 E-01$ \\
\hline $3 E-01$ & $-3.29 E-01$ & $6.88 E-01$ & $4.96 E-01$ & $6.88 E-01$ & $8.36 E-01$ \\
\hline $6.92 E-01$ & $-3.31 E-01$ & $6.94 E-01$ & $4.91 E-01$ & $6.93 E-01$ & $8.35 E-01$ \\
\hline $6.97 E-01$ & $-3.36 E-01$ & $6.99 E-01$ & $4.82 E-01$ & $6.98 E-01$ & $8.34 E-01$ \\
\hline $7.02 E-01$ & $-3.50 E-01$ & $7.04 E-01$ & $4.67 E-01$ & $7.03 E-01$ & $8.33 E-01$ \\
\hline $7.07 E-01$ & $-3.68 E-01$ & $7.09 E-01$ & $4.49 E-01$ & $7.08 E-01$ & $8.31 E-01$ \\
\hline $7.13 E-01$ & $-3.86 E-01$ & $7.14 E-01$ & $4.27 E-01$ & $7.14 E-01$ & $8.30 E-01$ \\
\hline $7.18 E-01$ & $-4.07 E-01$ & $7.20 E-01$ & $4.06 E-01$ & $7.19 E-01$ & $8.29 E-01$ \\
\hline $7.23 E-01$ & $-4.29 E-01$ & $7.25 E-01$ & $3.85 E-01$ & $7.24 E-01$ & $8.28 E-01$ \\
\hline $7.28 E-01$ & $-4.49 E-01$ & $7.30 E-01$ & $3.65 E-01$ & $7.29 E-01$ & $8.26 E-01$ \\
\hline $7.33 E-01$ & $-4.66 E-01$ & $7.35 E-01$ & $3.49 E-01$ & $7.34 E-01$ & $8.25 E-01$ \\
\hline $7.39 E-01$ & $-4.79 E-01$ & $7.40 E-01$ & $3.39 E-01$ & $7.40 E-01$ & $8.24 E-01$ \\
\hline
\end{tabular}


Table A.1 - continued from previous page

\begin{tabular}{|c|c|c|c|c|c|}
\hline$X_{R} / L$ & $Z_{R} / W_{x=0}$ & $X_{L} / L$ & $Z_{L} / W_{x=0}$ & $X / L$ & $Y / Y_{x=0}$ \\
\hline $7.44 E-01$ & $-4.89 E-01$ & $7.46 E-01$ & $3.31 E-01$ & $7.45 E-01$ & $8.23 E-01$ \\
\hline $7.49 E-01$ & $-4.96 E-01$ & $7.51 E-01$ & $3.28 E-01$ & $7.50 E-01$ & $.21 E-01$ \\
\hline $7.55 E-01$ & $-4.94 E-01$ & $7.56 E-01$ & $3.31 E-01$ & $7.55 E-01$ & $20 E-01$ \\
\hline $7.60 E-01$ & $-4.87 E-01$ & $7.61 E-01$ & $3.38 E-01$ & $7.60 E-01$ & $.19 E-01$ \\
\hline $7.66 E-01$ & $-4.76 E-01$ & $7.67 E-01$ & $3.51 E-01$ & $7.66 E-01$ & $.18 E-01$ \\
\hline $7.71 E-01$ & $-4.59 E-01$ & $7.72 E-01$ & $3.68 E-01$ & $7.71 E-01$ & $8.16 E-01$ \\
\hline $7.76 E-01$ & $-4.39 E-01$ & $7.77 E-01$ & $8 E-01$ & $7.76 E-01$ & $15 E-01$ \\
\hline $7.81 E-01$ & $-4.18 E-01$ & $7.82 E-01$ & $4.09 E-01$ & $7.81 E-01$ & $14 E-01$ \\
\hline $7.86 E-01$ & $-3.97 E-01$ & $7.87 E-01$ & $4.29 E-01$ & $7.86 E-01$ & $.13 E-01$ \\
\hline $7.92 E-01$ & $-3.77 E-01$ & $7.93 E-01$ & $4.51 E-01$ & $7.92 E-01$ & $.12 E-01$ \\
\hline $7.97 E-01$ & $-3.60 E-01$ & $7.98 E-01$ & $4.70 E-01$ & $7.97 E-01$ & $8.10 E-01$ \\
\hline $8.02 E-01$ & $-3.45 E-01$ & $8.03 E-01$ & $4.83 E-01$ & $8.02 E-01$ & $8.09 E-01$ \\
\hline $8.07 E-01$ & $-3.34 E-01$ & $8.08 E-01$ & $4.92 E-01$ & $8.07 E-01$ & $8.08 E-01$ \\
\hline $8.13 E-01$ & $-3.31 E-01$ & $8.13 E-01$ & $4.95 E-01$ & $8.13 E-01$ & $8.07 E-01$ \\
\hline $8.17 E-01$ & $-3.32 E-01$ & $3 E-01$ & $4.92 E-01$ & $E-01$ & $5 E-01$ \\
\hline $8.22 E-01$ & $-3.37 E-01$ & $8.23 E-01$ & $4.81 E-01$ & $8.23 E-01$ & $8.04 E-01$ \\
\hline $8.27 E-01$ & $-3.49 E-01$ & $8.28 E-01$ & $4.66 E-01$ & $8.28 E-01$ & $8.03 E-01$ \\
\hline $8.32 E-01$ & $-3.66 E-$ & $8.33 E-$ & $4.48 E-$ & $8.33 E-01$ & \\
\hline $8.38 E-01$ & $-3.85 E-01$ & $8.39 E-01$ & $4.26 E-01$ & $8.39 E-01$ & $8.00 E-01$ \\
\hline $8.43 E-01$ & $-4.04 E-01$ & $8.44 E-01$ & $4.07 E-01$ & $8.44 E-01$ & $7.99 E-01$ \\
\hline $8.48 E-01$ & $-4.27 E-01$ & $8.49 E-01$ & $3.84 E-01$ & $8.49 E-01$ & $7.98 E-01$ \\
\hline $8.53 E-01$ & $-4.45 E-01$ & $8.54 E-01$ & $3.67 E-01$ & $8.54 E-01$ & $7.97 E-01$ \\
\hline $3 E-01$ & $-4.65 E-01$ & $8.59 E-01$ & $3.49 E-01$ & $8.59 E-01$ & $7.95 E-01$ \\
\hline $4 E-01$ & $-4.80 E-01$ & $8.65 E-01$ & $3.40 E-01$ & $8.65 E-01$ & $7.94 E-01$ \\
\hline $8.69 E-01$ & $-4.90 E-01$ & $8.70 E-01$ & $3.32 E-01$ & $8.70 E-01$ & $7.93 E-01$ \\
\hline $8.74 E-01$ & $-4.96 E-01$ & $8.75 E-01$ & $3.30 E-01$ & $8.75 E-01$ & $7.92 E-01$ \\
\hline $8.80 E-01$ & $-4.93 E-01$ & $8.80 E-01$ & $3.33 E-01$ & $8.80 E-01$ & $7.90 E-01$ \\
\hline $8.85 E-01$ & $-4.86 E-01$ & $8.85 E-01$ & $3.40 E-01$ & $8.85 E-01$ & $7.89 E-01$ \\
\hline $1 E-01$ & $-4.75 E-01$ & $8.91 E-01$ & $3.53 E-01$ & $8.91 E-01$ & $7.88 E-01$ \\
\hline $8.96 E-01$ & $-4.57 E-01$ & $8.96 E-01$ & $3.69 E-01$ & $8.96 E-01$ & $7.87 E-01$ \\
\hline $9.01 E-01$ & $-4.38 E-01$ & $9.01 E-01$ & $3.90 E-01$ & $9.01 E-01$ & $7.85 E-01$ \\
\hline $9.06 E-01$ & $-4.17 E-01$ & $9.06 E-01$ & $4.10 E-01$ & $9.06 E-01$ & $7.84 E-01$ \\
\hline $9.11 E-01$ & $-3.96 E-01$ & $9.11 E-01$ & $4.32 E-01$ & $9.11 E-01$ & $7.83 E-01$ \\
\hline $9.17 E-01$ & $-3.76 E-01$ & $9.17 E-01$ & $4.52 E-01$ & $9.17 E-01$ & $7.82 E-01$ \\
\hline $9.22 E-01$ & $-3.58 E-01$ & $9.22 E-01$ & $4.68 E-01$ & $9.22 E-01$ & $7.81 E-01$ \\
\hline $9.27 E-01$ & $-3.44 E-01$ & $9.27 E-01$ & $4.82 E-01$ & $9.27 E-01$ & $7.79 E-01$ \\
\hline
\end{tabular}


Table A.1 - concluded from previous page

\begin{tabular}{|c|c|c|c|c|c|}
\hline$X_{R} / L$ & $Z_{R} / W_{x=0}$ & $X_{L} / L$ & $Z_{L} / W_{x=0}$ & $X / L$ & $Y / Y_{x=0}$ \\
\hline $9.32 E-01$ & $-3.34 E-01$ & $9.32 E-01$ & $4.91 E-01$ & $9.32 E-01$ & $7.78 E-01$ \\
$9.38 E-01$ & $-3.31 E-01$ & $9.38 E-01$ & $4.93 E-01$ & $9.38 E-01$ & $7.77 E-01$ \\
$9.42 E-01$ & $-3.33 E-01$ & $9.44 E-01$ & $4.88 E-01$ & $9.43 E-01$ & $7.76 E-01$ \\
$9.47 E-01$ & $-3.39 E-01$ & $9.49 E-01$ & $4.80 E-01$ & $9.48 E-01$ & $7.74 E-01$ \\
$9.52 E-01$ & $-3.51 E-01$ & $9.54 E-01$ & $4.66 E-01$ & $9.53 E-01$ & $7.73 E-01$ \\
$9.57 E-01$ & $-3.66 E-01$ & $9.59 E-01$ & $4.49 E-01$ & $9.58 E-01$ & $7.72 E-01$ \\
$9.63 E-01$ & $-3.85 E-01$ & $9.64 E-01$ & $4.29 E-01$ & $9.64 E-01$ & $7.71 E-01$ \\
$9.68 E-01$ & $-4.04 E-01$ & $9.70 E-01$ & $4.07 E-01$ & $9.69 E-01$ & $7.69 E-01$ \\
$9.73 E-01$ & $-4.26 E-01$ & $9.75 E-01$ & $3.87 E-01$ & $9.74 E-01$ & $7.68 E-01$ \\
$9.78 E-01$ & $-4.47 E-01$ & $9.80 E-01$ & $3.68 E-01$ & $9.79 E-01$ & $7.67 E-01$ \\
$9.83 E-01$ & $-4.65 E-01$ & $9.85 E-01$ & $3.50 E-01$ & $9.84 E-01$ & $7.66 E-01$ \\
$9.89 E-01$ & $-4.80 E-01$ & $9.90 E-01$ & $3.40 E-01$ & $9.90 E-01$ & $7.64 E-01$ \\
$9.94 E-01$ & $-4.91 E-01$ & $9.96 E-01$ & $3.34 E-01$ & $9.95 E-01$ & $7.63 E-01$ \\
$1.00 E+00$ & $-5.01 E-01$ & $1.00 E+00$ & $3.28 E-01$ & $1.00 E+00$ & $7.62 E-01$ \\
$1.03 E+00$ & $-5.01 E-01$ & $1.03 E+00$ & $3.28 E-01$ & $1.03 E+00$ & $7.62 E-01$ \\
$1.06 E+00$ & $-5.01 E-01$ & $1.06 E+00$ & $3.28 E-01$ & $1.06 E+00$ & $7.62 E-01$ \\
$1.09 E+00$ & $-5.01 E-01$ & $1.09 E+00$ & $3.28 E-01$ & $1.09 E+00$ & $7.62 E-01$ \\
$1.13 E+00$ & $-5.01 E-01$ & $1.13 E+00$ & $3.28 E-01$ & $1.13 E+00$ & $7.62 E-01$ \\
$1.16 E+00$ & $-5.01 E-01$ & $1.16 E+00$ & $3.28 E-01$ & $1.16 E+00$ & $7.62 E-01$ \\
$1.19 E+00$ & $-5.01 E-01$ & $1.19 E+00$ & $3.28 E-01$ & $1.19 E+00$ & $7.62 E-01$ \\
\hline
\end{tabular}




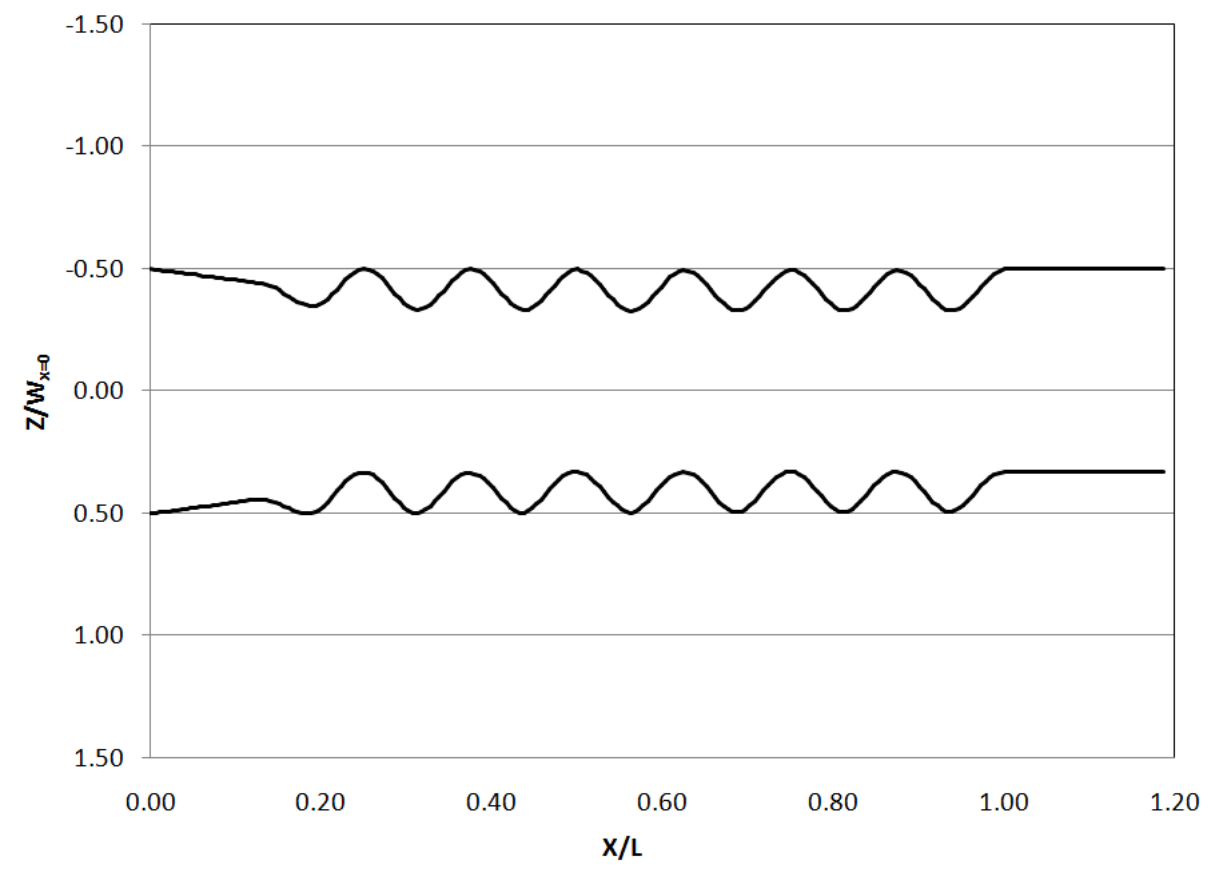

Figure A.1: Wavy Wall Test Section Plan-view

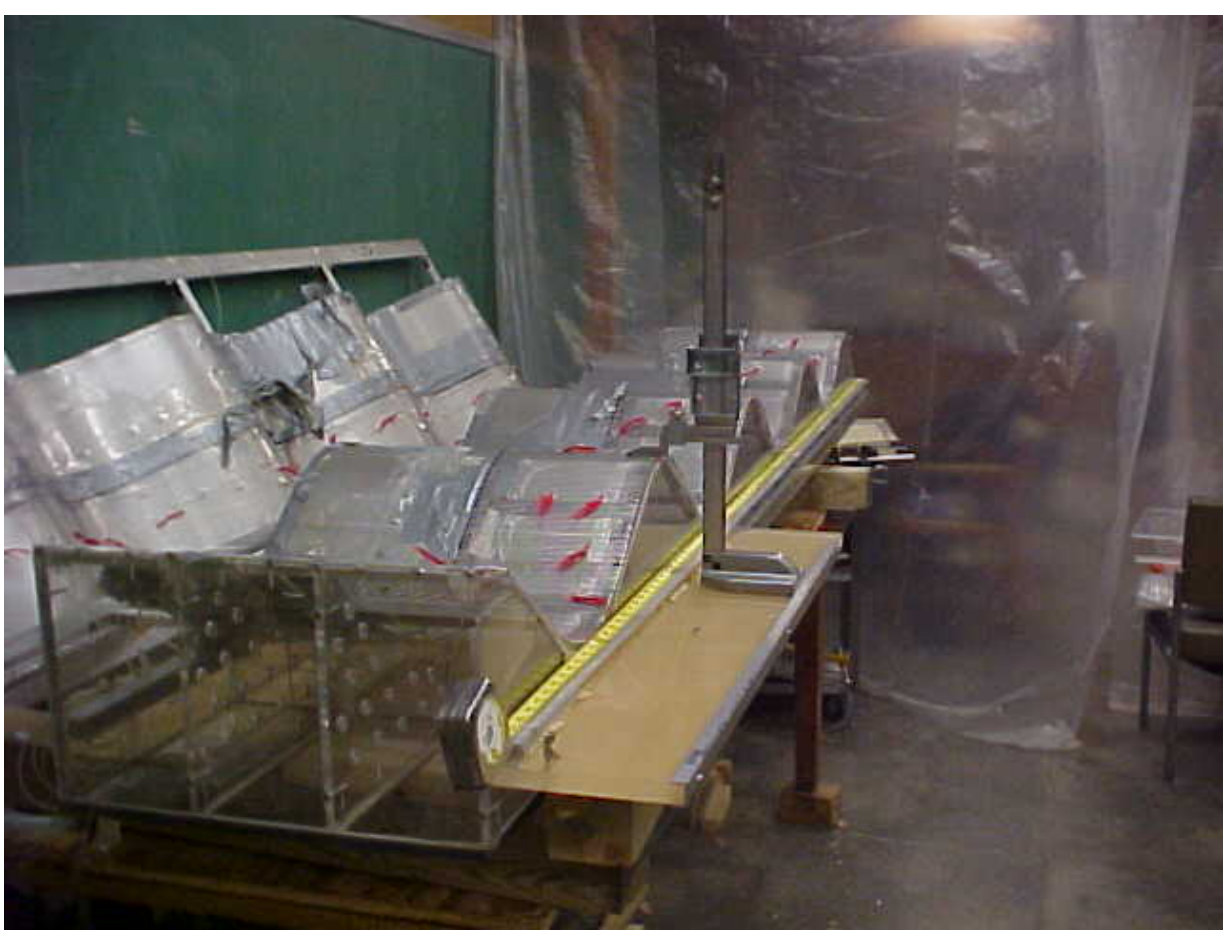

Figure A.2: Side Wall Geometry Measurement Setup 


\section{Appendix B}

\section{Suction Slots Locations and Dimensions}

The perforated aluminum sheets used had an open area of $40 \%$ of total area of perforated material. The hole diameters were 0.125 ", the center to center distance between holes was 0.1835 " staggered. Table B.1 lists the location and dimensions of all the suction slots used along the side walls.

The item listed are, $X, b, h, A, N$ are the streamwise location, width, height, area and number of holes for each suction slot respectively. The streamwise location is measured at the upstream most end of the slot. Subscripts L and R refer to Left and Right side walls.

Table B.1: Suction Slots Location and Size

\begin{tabular}{|c|c|c|c|c|c|c|c|c|c|}
\hline$X_{L}[\mathrm{ft}]$ & $b[\mathrm{in}]$ & $X_{R}[\mathrm{ft}]$ & $b[\mathrm{in}]$ & $h_{L}[\mathrm{in}]$ & $h_{R}[\mathrm{in}]$ & $A_{L}\left[\mathrm{in}^{2}\right]$ & $A_{R}\left[\mathrm{in}^{2}\right]$ & $N_{L}$ & $N_{R}$ \\
\hline 0.88 & 2.50 & 1.21 & 2.50 & 10.36 & 10.31 & 10.36 & 10.31 & 844 & 840 \\
2.96 & 1.00 & 3.06 & 0.50 & 10.04 & 10.02 & 4.02 & 2.00 & 327 & 163 \\
3.13 & 1.00 & 3.23 & 0.50 & 10.01 & 10.00 & 4.00 & 2.00 & 326 & 163 \\
3.40 & 0.50 & 3.44 & 0.50 & 9.97 & 9.96 & 1.99 & 1.99 & 162 & 162 \\
4.02 & 0.50 & 3.73 & 0.50 & 9.87 & 9.92 & 1.97 & 1.98 & 161 & 162 \\
4.19 & 0.50 & 3.85 & 0.50 & 9.85 & 9.90 & 1.97 & 1.98 & 160 & 161 \\
4.41 & 0.25 & 4.02 & 0.50 & 9.81 & 9.87 & 0.98 & 1.97 & 80 & 161 \\
4.73 & 0.50 & 4.19 & 0.50 & 9.76 & 9.85 & 1.95 & 1.97 & 159 & 160 \\
5.02 & 0.50 & 4.39 & 0.25 & 9.72 & 9.81 & 1.94 & 0.98 & 158 & 80 \\
5.32 & 0.30 & 4.47 & 0.25 & 9.67 & 9.80 & 1.16 & 0.98 & 95 & 80 \\
6.02 & 0.50 & 5.02 & 0.50 & 9.56 & 9.72 & 1.91 & 1.94 & 156 & 158 \\
6.19 & 0.50 & 5.31 & 0.50 & 9.53 & 9.67 & 1.91 & 1.93 & 155 & 158 \\
6.41 & 0.25 & 5.56 & 0.50 & 9.50 & 9.63 & 0.95 & 1.93 & 77 & 157 \\
6.73 & 0.50 & 5.80 & 0.25 & 9.45 & 9.59 & 1.89 & 0.96 & 154 & 78 \\
7.02 & 0.50 & 6.02 & 0.50 & 9.40 & 9.56 & 1.88 & 1.91 & 153 & 156 \\
\hline
\end{tabular}


Table B.1 - concluded from previous page

\begin{tabular}{|c|c|c|c|c|c|c|c|c|c|}
\hline$X_{L}[\mathrm{ft}]$ & $b[\mathrm{in}]$ & $X_{R}[\mathrm{ft}]$ & $b[\mathrm{in}]$ & $h_{L}[\mathrm{in}]$ & $h_{R}[\mathrm{in}]$ & $A_{L}\left[\mathrm{in}^{2}\right]$ & $A_{R}\left[\mathrm{in}^{2}\right]$ & $N_{L}$ & $N_{R}$ \\
\hline 7.40 & 0.50 & 6.19 & 0.50 & 9.34 & 9.53 & 1.87 & 1.91 & 152 & 155 \\
8.02 & 0.50 & 6.39 & 0.25 & 9.25 & 9.50 & 1.85 & 0.95 & 151 & 77 \\
8.26 & 0.38 & 6.47 & 0.25 & 9.21 & 9.49 & 1.38 & 0.95 & 113 & 77 \\
8.49 & 0.44 & 7.02 & 0.50 & 9.17 & 9.40 & 1.61 & 1.88 & 131 & 153 \\
8.75 & 0.31 & 7.31 & 0.50 & 9.13 & 9.36 & 1.14 & 1.87 & 93 & 153 \\
9.08 & 0.50 & 7.56 & 0.50 & 9.08 & 9.32 & 1.82 & 1.86 & 148 & 152 \\
9.32 & 0.38 & 7.80 & 0.25 & 9.04 & 9.28 & 1.36 & 0.93 & 111 & 76 \\
10.07 & 0.50 & 8.08 & 0.50 & 8.93 & 9.24 & 1.79 & 1.85 & 145 & 151 \\
10.35 & 0.38 & 8.37 & 0.31 & 8.88 & 9.19 & 1.33 & 1.15 & 109 & 94 \\
10.58 & 0.38 & 9.02 & 0.50 & 8.85 & 9.09 & 1.33 & 1.82 & 108 & 148 \\
10.84 & 0.31 & 9.29 & 0.31 & 8.81 & 9.05 & 1.10 & 1.13 & 90 & 92 \\
11.03 & 0.38 & 9.54 & 0.38 & 8.78 & 9.01 & 1.32 & 1.35 & 107 & 110 \\
11.35 & 0.31 & 9.74 & 0.31 & 8.73 & 8.98 & 1.09 & 1.12 & 89 & 91 \\
12.06 & 0.50 & 10.02 & 0.50 & 8.62 & 8.93 & 1.72 & 1.79 & 140 & 146 \\
12.35 & 0.31 & 10.27 & 0.31 & 8.57 & 8.90 & 1.07 & 1.11 & 87 & 91 \\
12.58 & 0.31 & 11.02 & 0.50 & 8.53 & 8.78 & 1.07 & 1.76 & 87 & 143 \\
12.83 & 0.31 & 11.30 & 0.31 & 8.50 & 8.73 & 1.06 & 1.09 & 87 & 89 \\
13.02 & 0.31 & 11.52 & 0.31 & 8.47 & 8.70 & 1.06 & 1.09 & 86 & 89 \\
13.35 & 0.31 & 11.77 & 0.31 & 8.41 & 8.66 & 1.05 & 1.08 & 86 & 88 \\
14.06 & 0.31 & 12.05 & 0.38 & 8.30 & 8.62 & 1.04 & 1.29 & 85 & 105 \\
14.32 & 0.38 & 12.32 & 0.50 & 8.26 & 8.58 & 1.24 & 1.72 & 101 & 140 \\
14.54 & 0.31 & 13.02 & 0.56 & 8.23 & 8.47 & 1.03 & 1.90 & 84 & 155 \\
14.76 & 0.31 & 13.27 & 0.31 & 8.19 & 8.43 & 1.02 & 1.05 & 83 & 86 \\
15.07 & 0.31 & 13.51 & 0.31 & 8.15 & 8.39 & 1.02 & 1.05 & 83 & 85 \\
& & 13.77 & 0.31 & & 8.35 & & 1.04 & & 85 \\
& & 14.04 & 0.31 & & 8.31 & & 1.04 & & 85 \\
& & 14.31 & 0.31 & & 8.26 & & 1.03 & & 84 \\
& & 15.02 & 0.44 & & 8.15 & & 1.43 & & 116 \\
& & 15.28 & 0.31 & & 8.11 & & 1.01 & & 83 \\
& & 15.55 & 0.38 & & 8.07 & & 1.21 & & 99 \\
& & 15.83 & 0.31 & & 8.03 & & 1.00 & & 82 \\
\hline
\end{tabular}




\section{Appendix C}

\section{Matching Short LDV System and CompLDV Profiles and Uncertainty Estimates}

\section{C.1 Matching Procedure}

Short LDV system measurements were performed to obtain an accurate estimate of friction velocity while the CompLDV was used to obtain turbulence measurements in the semi-log and outer layers. For each measurement location there was an overlap region between the Short LDV system and the CompLDV profiles so that composite profiles can be obtained.

Since two different probes were used with different settings for each group of profiles it is unlikely to get a perfect fit for the data and in general the probes are not expected to be aligned perfectly. In order to determine the orientation of LDV probes relative to the tunnel coordinates a paper template was made that has the locations of all profile locations marked. The probe measurement volume is placed at a given location on the tunnel's floor then was moved vertically a certain distance and the locations where the beams crossed the floor are marked on the template. The beam angles and measurement directions are then calculated. This is done for both LDV probes.

In order to match the Short system and CompLDV measurements, the Short system is used as a reference for the flow directions, hence the CompLDV data were rotated around the $x, y$ and $z$ axes to match the $U, V$ and $W$ velocity components to the Short system values. The rotation angles were limited to $4^{\circ}, 2^{\circ}$ and $4^{\circ}$ in the $x, y$ and $z$ directions respectively. However, for each cluster of profiles the angles didn't change more than $1^{\circ}$.

Values for friction velocities obtained at different locations from Short system mea- 
surements were also used to scale CompLDV data. However, these values were multiplied by a factor to improve the fit between the two sets of data. In general the friction velocities used for each pair of profiles were within $6 \%$; out of 29 profiles, five profiles had a difference between $6 \%$ and $10 \%$ and four profiles were within $15 \%$. Table C.1 shows the estimated friction velocities for each profile along with the average friction velocity for each cluster based on average wall shear stress. In addition, the CompLDV data were also y-shifted to match Short system data. The maximum absolute $y$-shift was equal to $70 \mu m$ which is about half the CompLDV measurement volume.

After aligning both sets of data from Short system and CompLDV system and $y$ shifting and scaling so that the mean velocity components are in agreement, composite profiles are produced from each pair of profiles. Near the wall Short system profiles are expected to be more accurate due to the smaller measurement volume size and the ability to obtain measurement at closer distances to the wall. Near the wall the Short system profiles had the same record length as the CompLDV profiles. However, the record length was halved for the short system profiles at the $8^{\text {th }}$ point in the profile, while the record length was kept constant for the entire CompLDV profile. This leads to a small discontinuity in the Short LDV system profiles that was apparent at some locations. Hence, while generating the composite profiles, the transition from Short LDV system data to CompLDV data was attempted before the $8^{\text {th }}$ point. For profiles that didn't show such discontinuity, the Short LDVsystem data were used further away from the wall. Three profiles, $P 16 p, P 18$ and $P 18_{1}$, didn't have a good match between the Reynolds stresses such that a composite profile was not possible. For these profiles the mean velocities are matched perfectly and only the CompLDV data is used.

Mean velocity profiles as well as Reynolds stresses and triple velocity correlation profiles for both Short system and CompLDV for some profiles are shown in Figures C.1. C.5.

\section{C.2 Uncertainty Estimates}

Uncertainty estimates were made based on the two acquired sets of data from the Short LDV system and CompLDV system. The uncertainties reported here are estimated using the differences between the two data sets. The 20:1 uncertainty in each measured quantity is given as the maximum of the mean absolute difference and $\pm 1.96 \sigma$, where $\sigma$ is the standard deviation of the calculated differences in each quantity. This should be a good estimate of overall uncertainty since data from different probes with different settings (beam angles, fringe spacings and measurement volumes) taken at different times are compared.

For each profile, the differences in each measured quantity are averaged along the overlap region and a single value for the difference in this quantity is obtained for each 
profile. This is done for all profiles. Then, for each measured quantity the averaged differences from all profiles are used as a sample to estimate the overall mean and standard deviation of the difference. For this sample a systematic procedure was chosen to reject outliers and obtain a closer estimate of the true mean and standard deviation. Previous researchers, Olcmen [42], Byun [4] and others, used Chauvenet's criterion to reject outliers from experimental data. In the present work Peirce's criterion was used, Ross [54], which is more rigorous than Chauvenet's criterion and in fact Chauvenet's criterion is a simplification to Peirce's criterion. The main advantage of Peirce's criterion is the ability to handle the rejection of more than one outliers at the same time and that it doesn't make any assumptions regarding the rejection of outliers. The procedure is explained in Ross [54].

To obtain a better feel of the measurement uncertainties, uncertainties related to each probe are also estimated by measuring a single profile twice by the same probe. Then following the same procedure as above, an estimate of uncertainties in each measured quantity is obtained. The uncertainty estimates obtained by matching the two LDV systems and those for each system are shown in Tables C.2.C.4. In general the uncertainty estimates from each probe are lower than those obtained by matching the two systems together.

Finally, uncertainties in derived quantities are shown in Table C.5. These estimates were obtained using uncertainties in primary quantities. Three values are given for each quantity, since these quantities are very sensitive to the primary quantities behavior near or away from the wall. 
Table C.1: Short system and CompLDV estimated friction velocity, $u_{\tau}$ and average value for each cluster

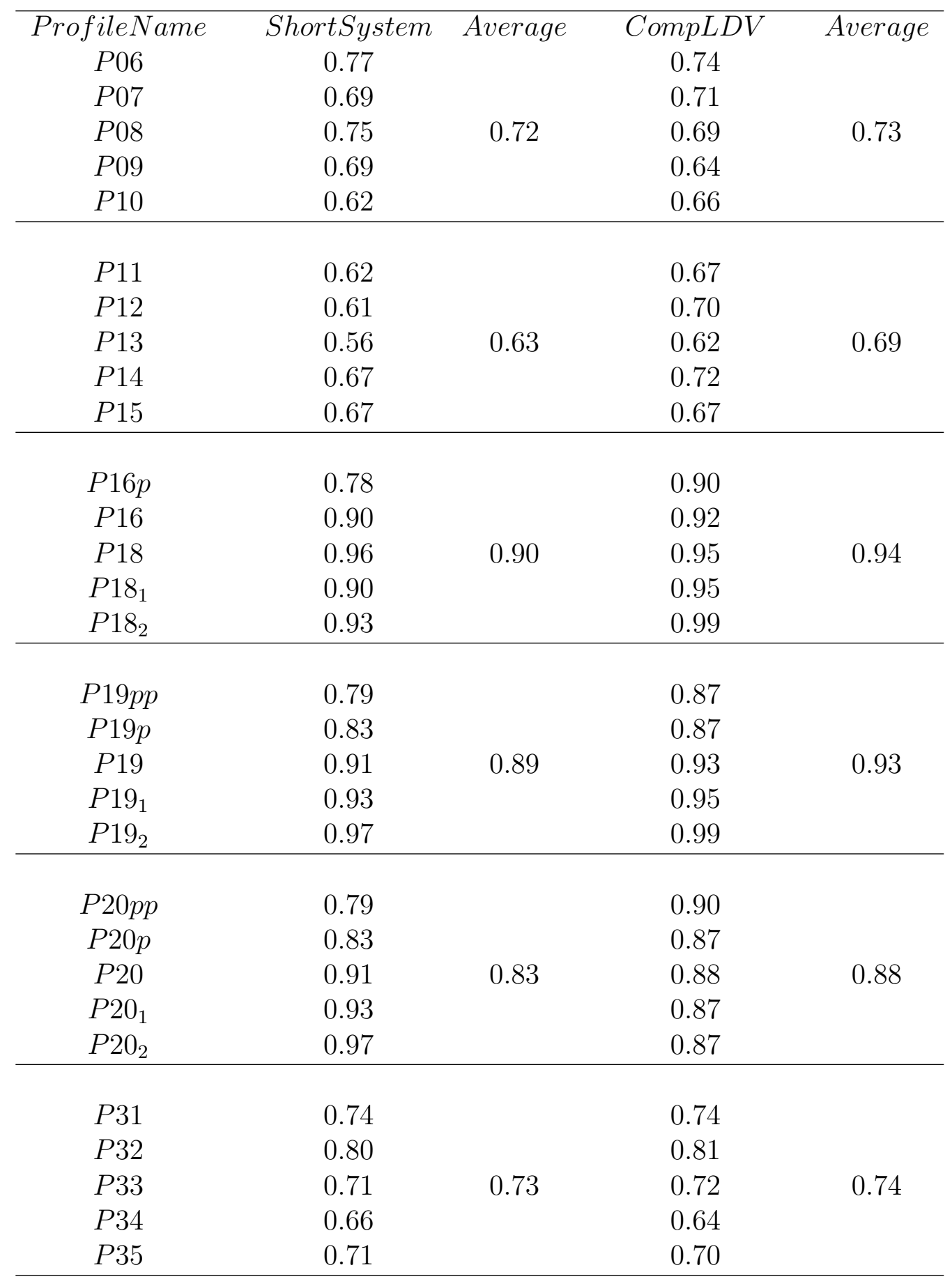


Table C.2: 20:1 Uncertainties by matching Short system and CompLDV profiles using Peirce's Criterion

\begin{tabular}{cccc}
\hline \hline$U^{+}$ & \pm 0.18 & ${\overline{u^{3}}}^{+}$ & \pm 3.67 \\
$V^{+}$ & \pm 0.06 & ${\overline{v^{3}}}^{+}$ & \pm 0.90 \\
$W^{+}$ & \pm 0.12 & ${\overline{w^{3}}}^{+}$ & \pm 2.68 \\
$\overline{u^{2}}$ & \pm 0.64 & $\bar{u}^{2} v$ & \pm 0.24 \\
$\bar{v}^{2}$ & \pm 0.15 & ${\overline{u^{2} w}}^{+}$ & \pm 0.76 \\
$\overline{w^{2}}$ & \pm 0.54 & ${\overline{u v^{2}}}^{+}$ & \pm 0.27 \\
$\overline{u v}$ & \pm 0.07 & ${\overline{u w^{2}}}^{+}$ & \pm 1.60 \\
$\overline{u w^{+}}$ & \pm 0.28 & ${\overline{v^{2} w}}^{+}$ & \pm 0.45 \\
$\overline{v w^{+}}$ & \pm 0.13 & $\overline{v w^{2}}$ & \pm 0.23 \\
& & $\overline{u v w^{+}}$ & \pm 0.17 \\
\hline
\end{tabular}

Table C.3: 20:1 Short system Uncertainties using Peirce's Criterion

\begin{tabular}{cccc}
\hline \hline$U^{+}$ & \pm 0.14 & $\bar{u}^{+}$ & \pm 1.23 \\
$V^{+}$ & \pm 0.11 & ${\overline{v^{3}}}^{+}$ & \pm 0.26 \\
$W^{+}$ & \pm 0.09 & ${\overline{w^{3}}}^{+}$ & \pm 0.46 \\
$\bar{u}^{+}$ & \pm 0.35 & $\bar{u}^{2}{ }^{+}$ & \pm 0.08 \\
${\overline{v^{2}}}^{+}$ & \pm 0.05 & ${\overline{u^{2} w}}^{+}$ & \pm 0.28 \\
$\overline{w^{2}}$ & \pm 0.09 & ${\overline{u v^{2}}}^{+}$ & \pm 0.09 \\
$\overline{u v}$ & \pm 0.05 & ${\overline{u w^{2}}}^{+}$ & \pm 0.25 \\
$\overline{u w^{+}}$ & \pm 0.07 & ${\overline{v^{2} w}}^{+}$ & \pm 0.28 \\
$\overline{v w^{+}}$ & \pm 0.04 & $\overline{v w^{2}}$ & \pm 0.33 \\
& & $\overline{u v w^{+}}$ & \pm 0.11 \\
\hline
\end{tabular}

Table C.4: 20:1 CompLDV system Uncertainties using Peirce's Criterion

\begin{tabular}{cccc}
\hline \hline$U^{+}$ & \pm 0.10 & ${\overline{u^{3}}}^{+}$ & \pm 0.26 \\
$V^{+}$ & \pm 0.04 & $\bar{v}^{3}$ & \pm 0.05 \\
$W^{+}$ & \pm 0.03 & ${\overline{w^{3}}}^{+}$ & \pm 0.21 \\
$\overline{u^{2}}$ & \pm 0.10 & ${\overline{u^{2} v}}^{+}$ & \pm 0.09 \\
$\bar{v}^{2}$ & \pm 0.02 & ${\overline{u^{2} w}}^{+}$ & \pm 0.16 \\
$\overline{w^{2}}$ & \pm 0.06 & ${\overline{u v^{2}}}^{+}$ & \pm 0.04 \\
$\overline{u v}$ & \pm 0.04 & ${\overline{u w^{2}}}^{+}$ & \pm 0.17 \\
$\overline{u w^{+}}$ & \pm 0.06 & ${\overline{v^{2} w}}^{+}$ & \pm 0.04 \\
$\overline{v w^{+}}$ & \pm 0.03 & $\overline{v w^{2}}$ & \pm 0.06 \\
& & $\overline{u v w^{+}}$ & \pm 0.05 \\
\hline
\end{tabular}


Table C.5: Derived quantities uncertainties using formal sensitivity analysis and estimated uncertainties in measured primary quantities.

\begin{tabular}{lccc}
\hline \hline Range & $y^{+} \leq 30$ & $30 \leq y^{+} \leq 100$ & $y^{+} \geq 100$ \\
\hline$\rho_{u v}$ & \pm 0.40 & \pm 0.01 & \pm 0.01 \\
$S^{-1}$ & \pm 12.00 & \pm 0.30 & \pm 0.05 \\
$V_{q x}^{+}$ & \pm 0.47 & \pm 0.05 & \pm 0.04 \\
$V_{q y}^{+}$ & \pm 0.10 & \pm 0.01 & \pm 0.01 \\
$V_{q z}^{+}$ & \pm 0.30 & \pm 0.04 & \pm 0.03 \\
$A_{1}$ & \pm 0.01 & \pm 0.001 & \pm 0.005 \\
$\gamma_{a}[$ deg. $]$ & \pm 0.50 & \pm 0.07 & \pm 0.08 \\
$\gamma_{s}[$ deg. $]$ & \pm 40.10 & \pm 2.00 & \pm 5.50 \\
$\gamma_{g}[$ deg. $]$ & \pm 1.90 & \pm 10.00 & \pm 25.00 \\
$L_{m}^{+}$ & \pm 0.16 & \pm 5.00 & \pm 395.00 \\
$\nu_{T x} / \nu$ & \pm 0.23 & \pm 4.00 & \pm 450.00 \\
\hline
\end{tabular}




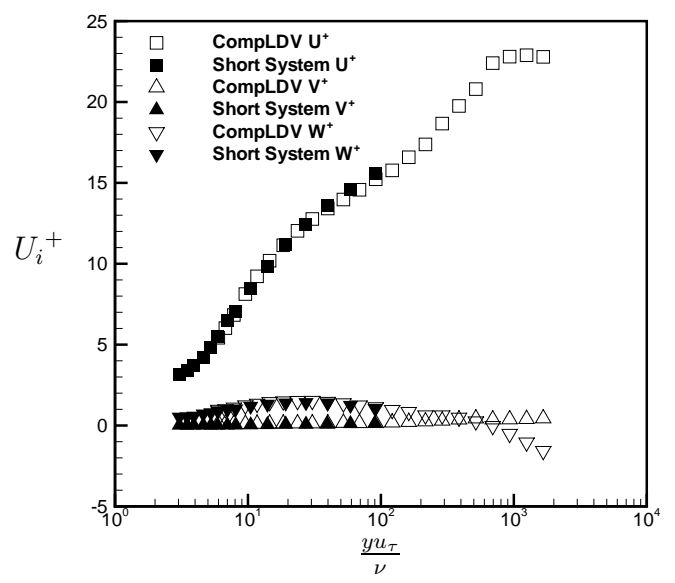

(a) Mean Velocity Components, $U_{i}^{+}$

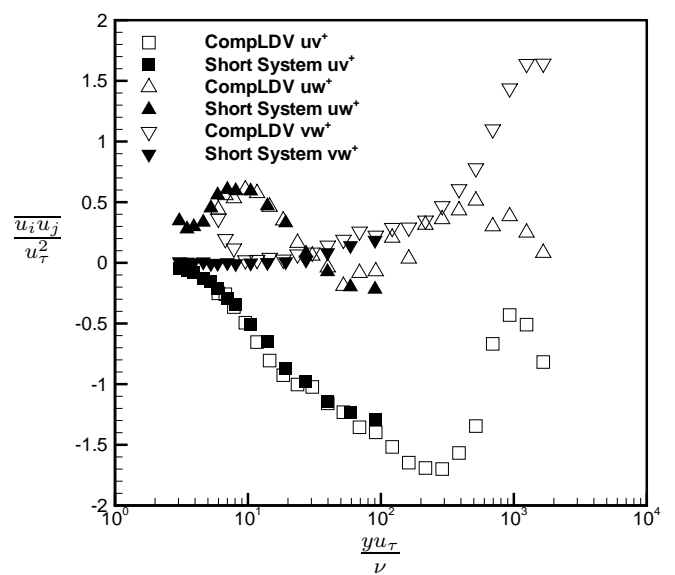

(c) Reynolds Shear Stresses, $\overline{u v}^{+}, \overline{u w}^{+}, \overline{v w}^{+}$

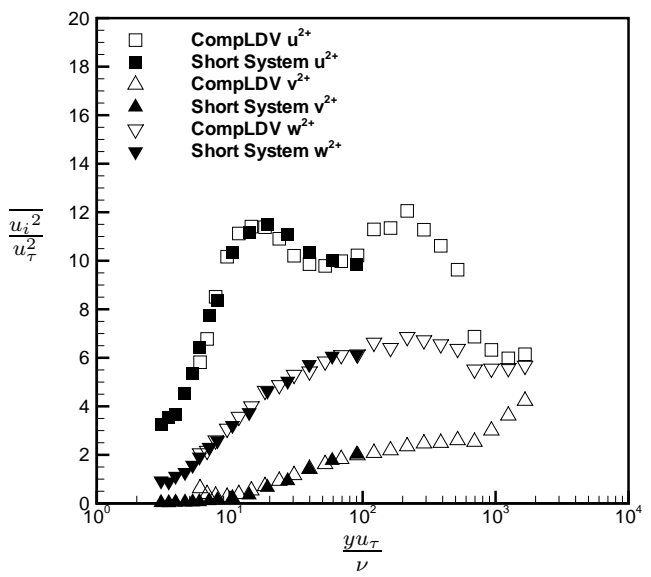

(b) Reynolds Normal Stresses, $\bar{u}^{2},{\overline{v^{2}}}^{+},{\overline{w^{2}}}^{+}$

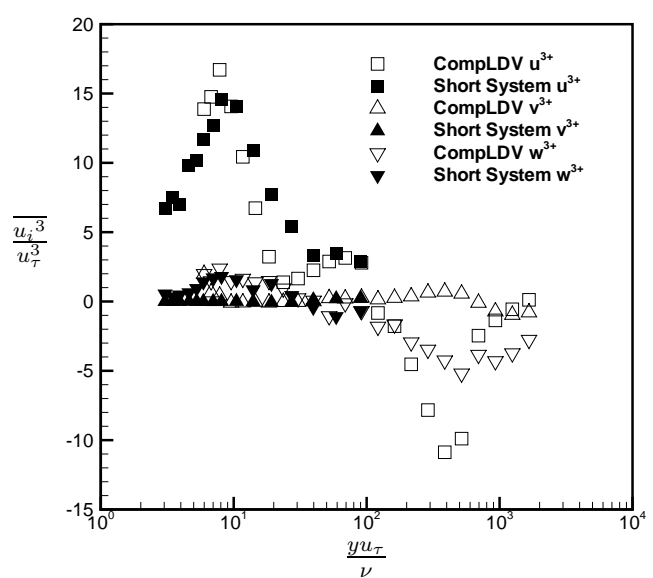

(d) Triple Velocity Correlations, ${\overline{u^{3}}}^{+}, \overline{v^{3}}+, \overline{w^{3}}+$

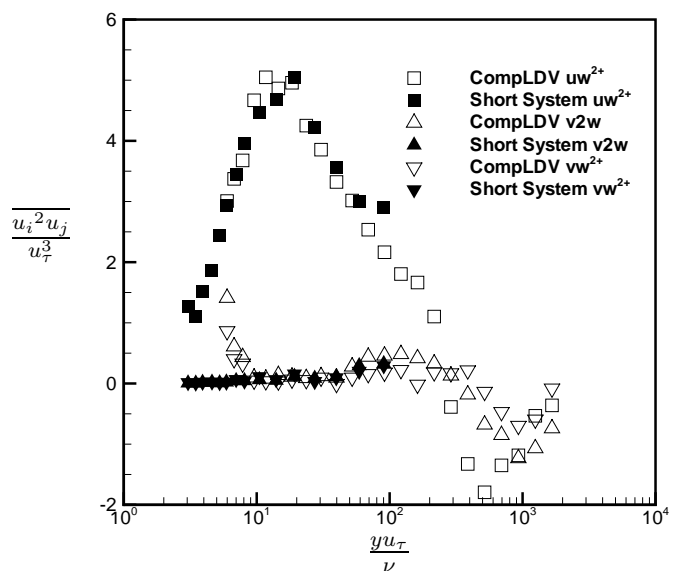
$\frac{\text { (e) }}{u^{2} v}+\frac{\text { Triple }}{u^{2} w}+\frac{u^{2}}{+}+$ Velocity $\quad$ Correlations, $\left(\frac{(\mathrm{f})}{u w^{2}}+\frac{\text { Triple }}{v^{2} w}, \frac{v w^{2}}{}+\right.$

Figure C.1: Matching Short and CompLDV Profiles at P07 


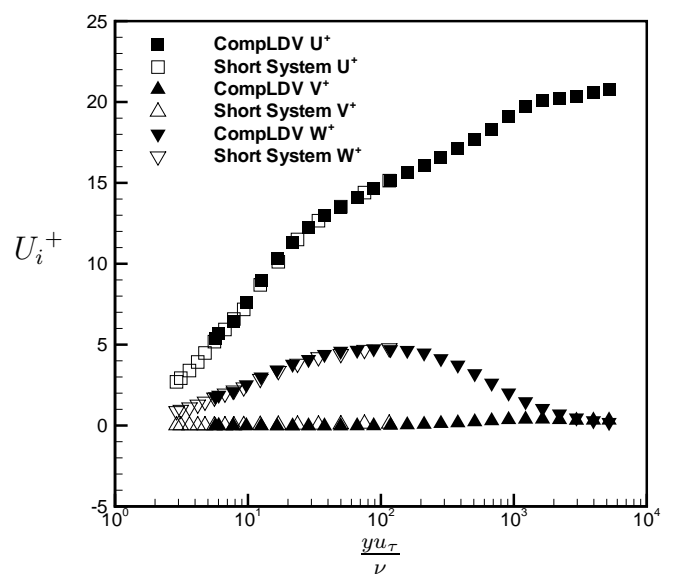

(a) Mean Velocity Components, $U_{i}^{+}$

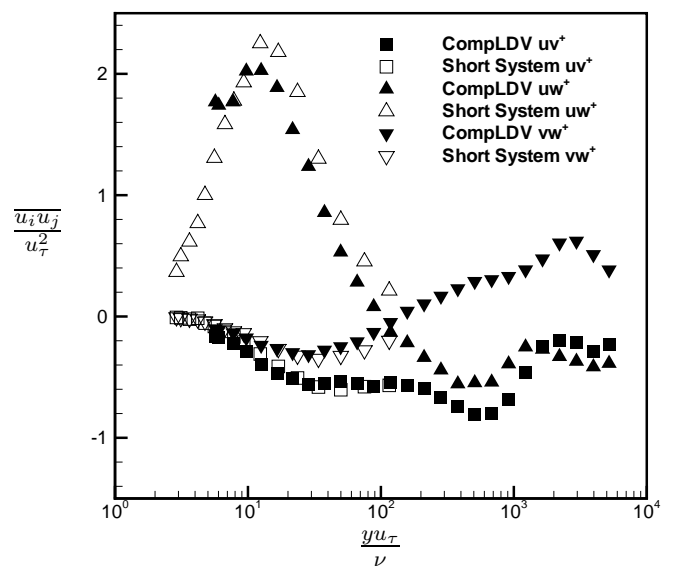

(c) Reynolds Shear Stresses, $\overline{u v}^{+}, \overline{u w}^{+}, \overline{v w}^{+}$

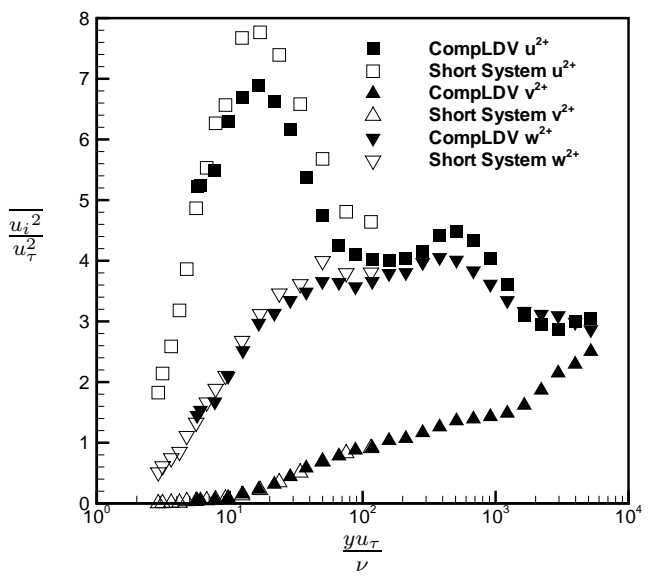

(b) Reynolds Normal Stresses, $\bar{u}^{2},{\overline{v^{2}}}^{+},{\overline{w^{2}}}^{+}$

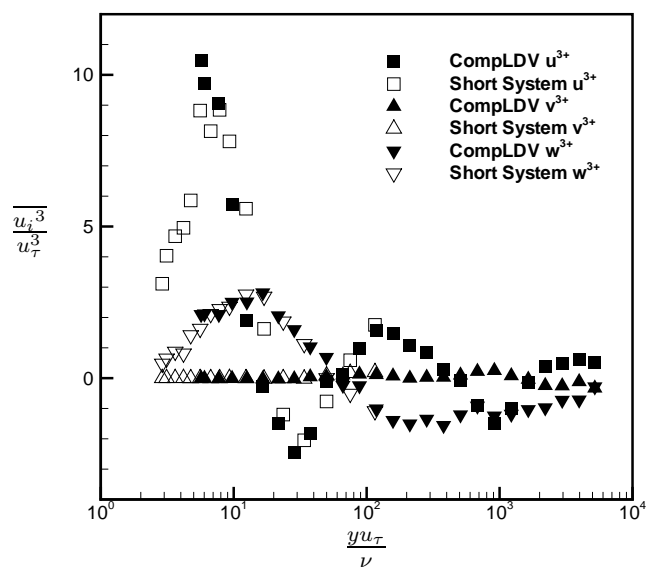

(d) Triple Velocity Correlations, ${\overline{u^{3}}}^{+},{\overline{v^{3}}}^{+}, \overline{w^{3}}+$

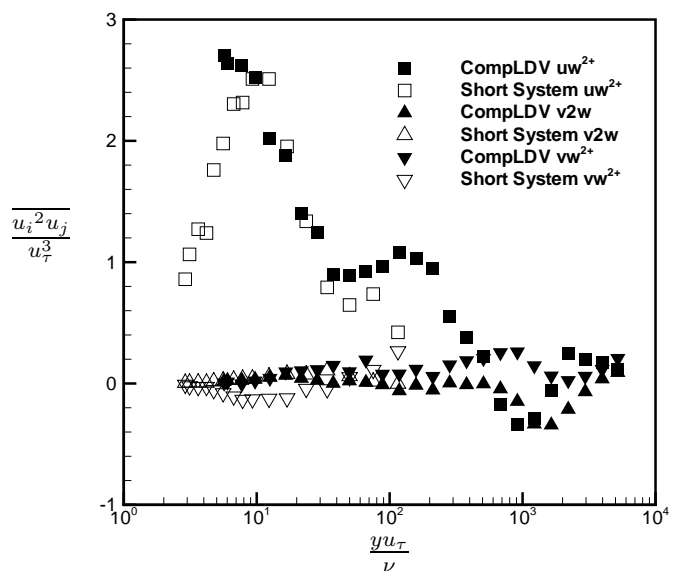

(e) Triple Velocity Correlations, $\frac{u^{2} v}{+}, \bar{u}^{2} w+,{\overline{u v^{2}}}^{+}+\frac{u^{2}}{+}, \frac{\text { Triple }}{v^{2} w},{\overline{v w^{2}}}^{+}$

Figure C.2: Matching Short and CompLDV Profiles at P16 


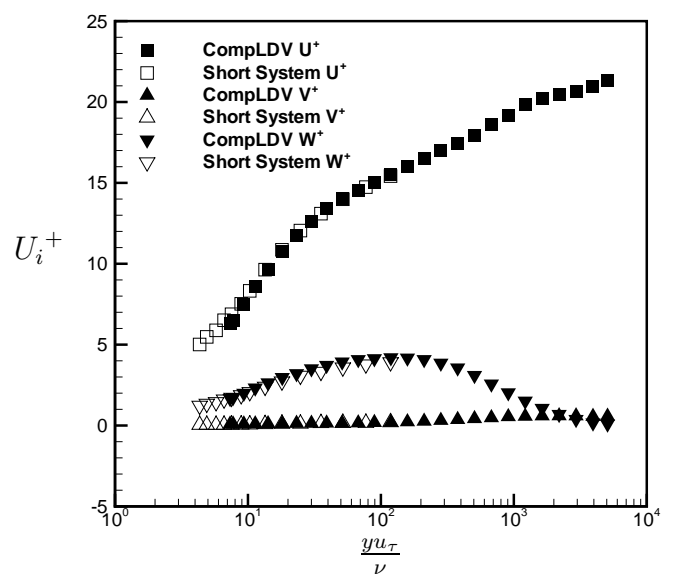

(a) Mean Velocity Components, $U_{i}^{+}$

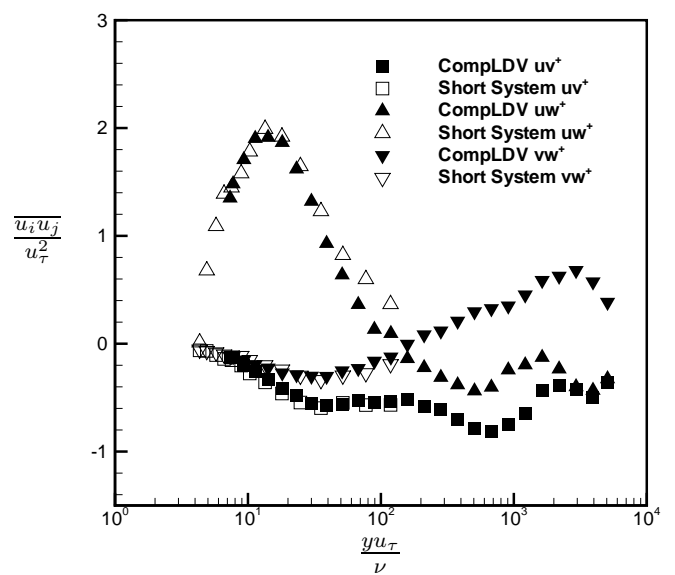

(c) Reynolds Shear Stresses, $\overline{u v}^{+}, \overline{u w}^{+}, \overline{v w}^{+}$

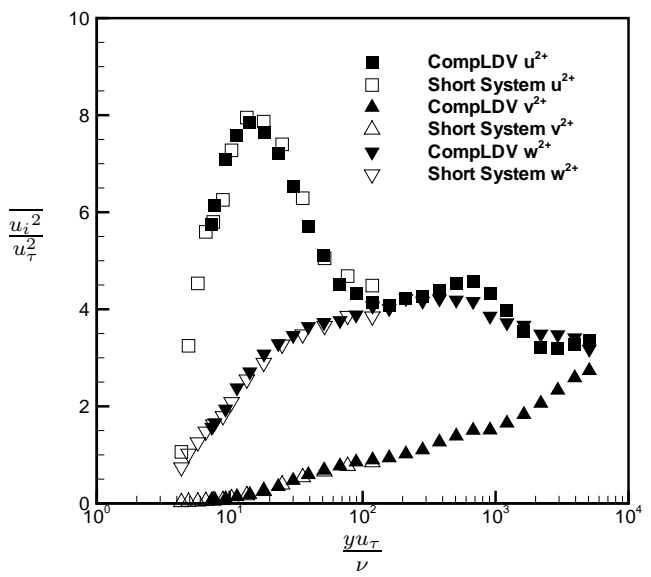

(b) Reynolds Normal Stresses, $\bar{u}^{2},{\overline{v^{2}}}^{+},{\overline{w^{2}}}^{+}$

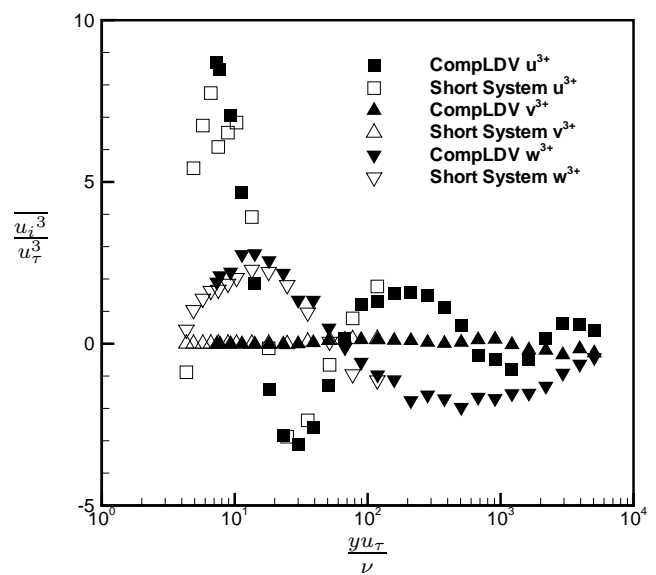

(d) Triple Velocity Correlations, ${\overline{u^{3}}}^{+},{\overline{v^{3}}}^{+},{\overline{w^{3}}}^{+}$

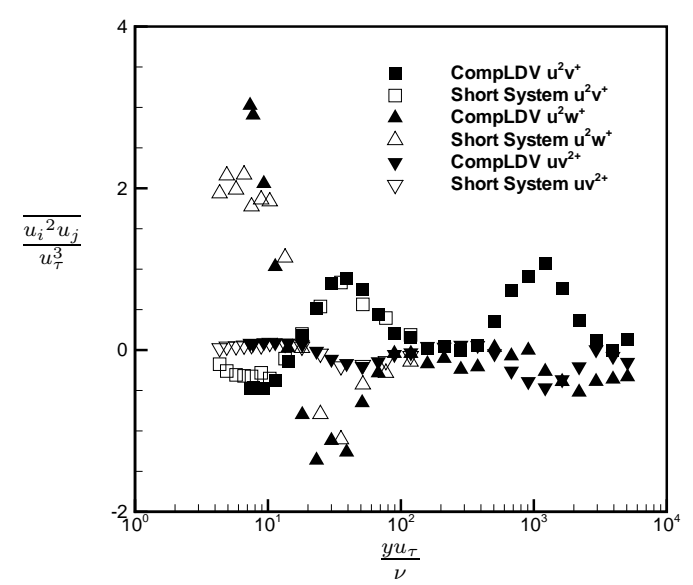

(e) Triple Velocit $\frac{u^{2} v}{+}, \bar{u}^{2} w+\overline{u v}^{2}+$

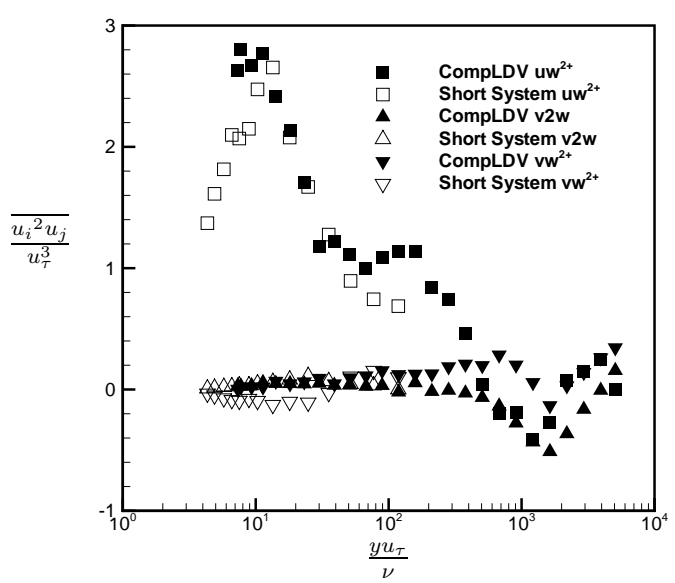

Correlations,

Figure C.3: Matching Short and CompLDV Profiles at P19 


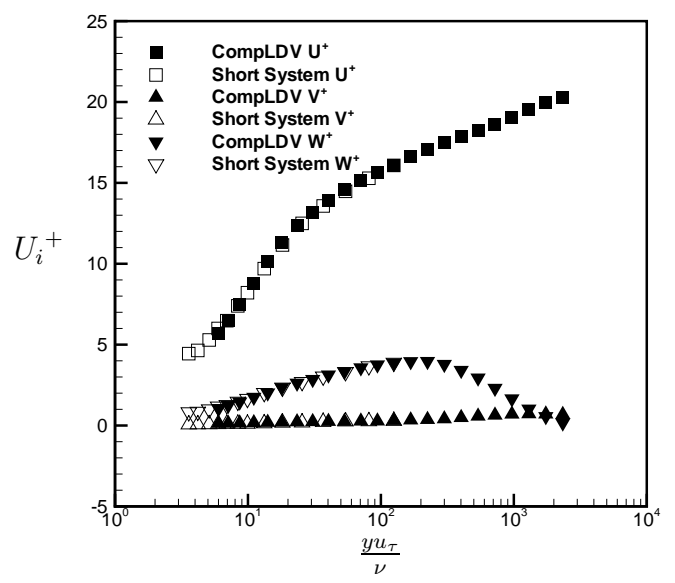

(a) Mean Velocity Components, $U_{i}^{+}$

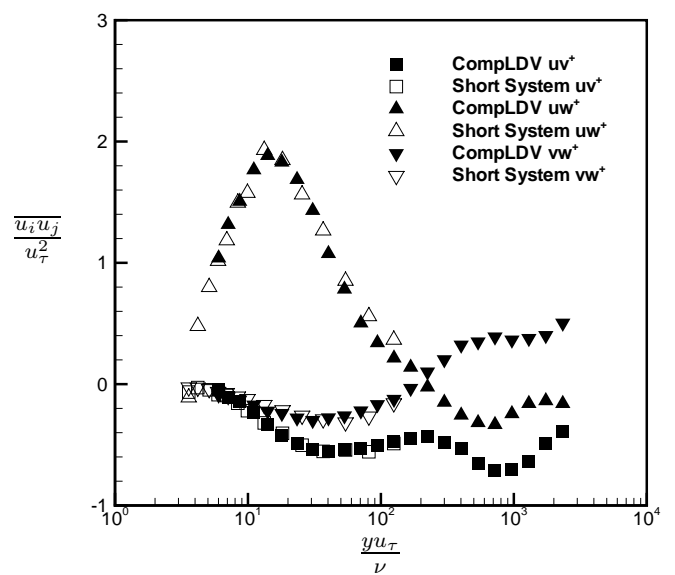

(c) Reynolds Shear Stresses, $\overline{u v}^{+}, \overline{u w}^{+}, \overline{v w}^{+}$

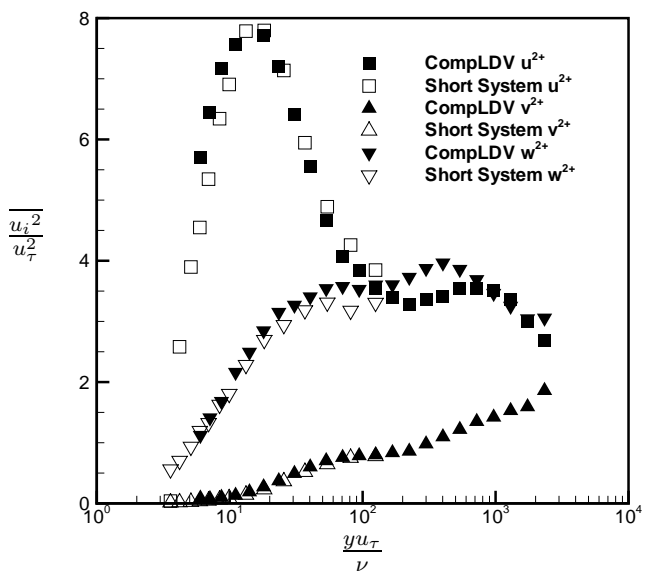

(b) Reynolds Normal Stresses, $\bar{u}^{2},{\overline{v^{2}}}^{+},{\overline{w^{2}}}^{+}$

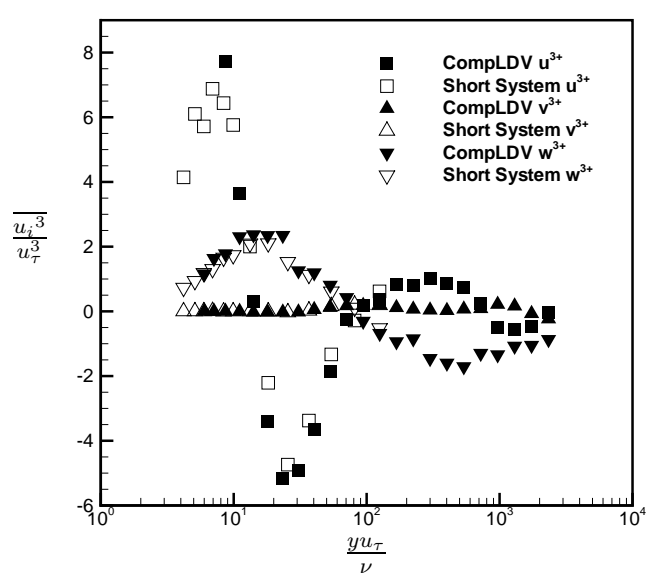

(d) Triple Velocity Correlations, ${\overline{u^{3}}}^{+},{\overline{v^{3}}}^{+},{\overline{w^{3}}}^{+}$
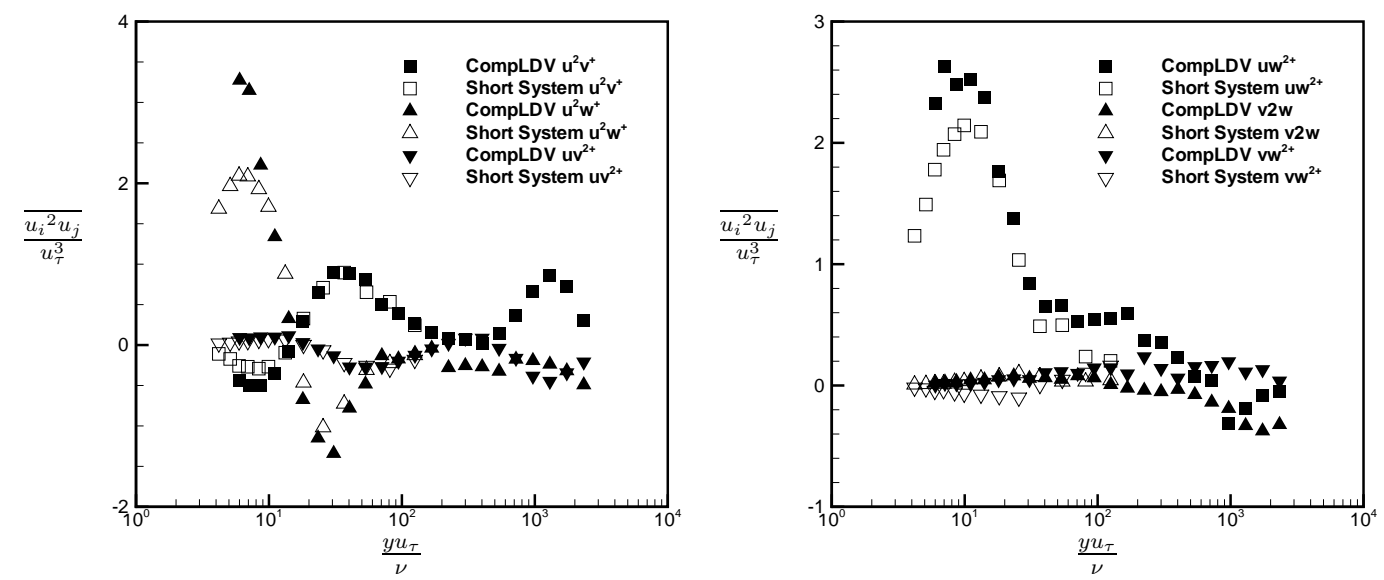

$\frac{\text { (e) }}{u^{2} v}+\frac{\text { Triple }}{u^{2} w}+\frac{u^{2}}{+}$ Velocity Correlations, $\frac{(\mathrm{f})}{u w^{2}}+\frac{\text { Triple }}{v^{2} w}+\frac{v^{2}}{}+{ }^{+}$Velocity Correlations,

Figure C.4: Matching Short and CompLDV Profiles at $P 19_{2}$ 


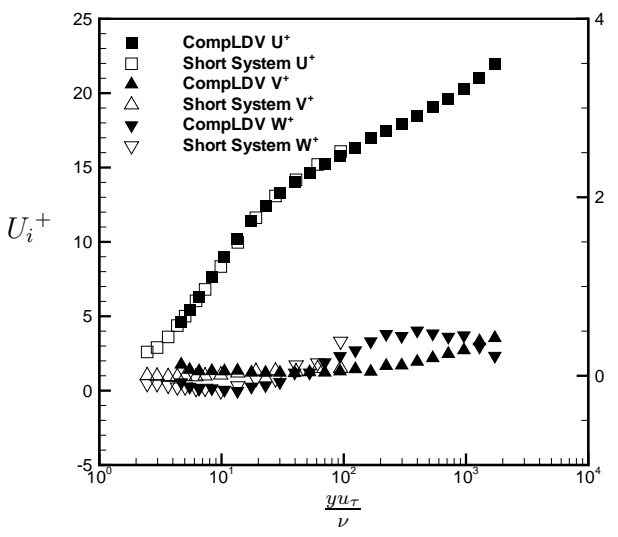

(a) Mean Velocity Components, $U_{i}^{+}$

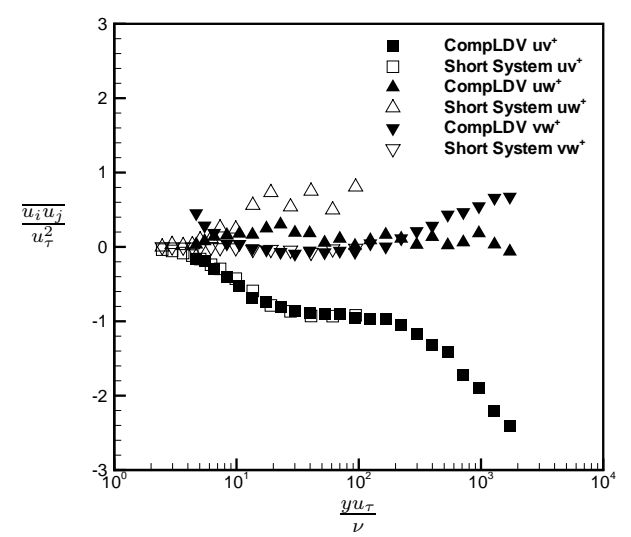

(c) Reynolds Shear Stresses, $\overline{u v}^{+}, \overline{u w}^{+}, \overline{v w}^{+}$

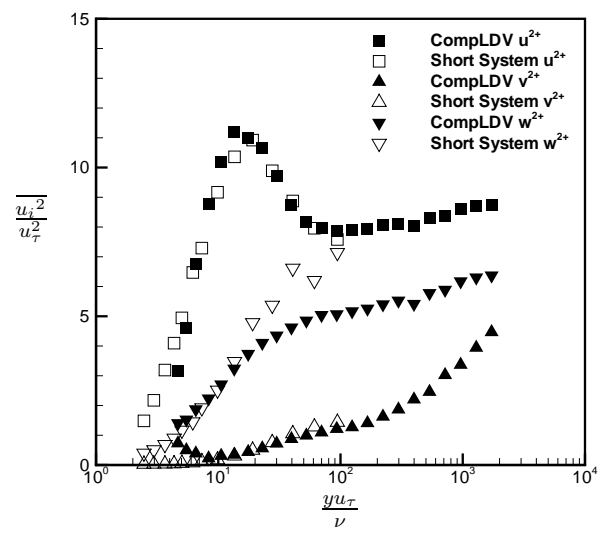

(b) Reynolds Normal Stresses, ${\overline{u^{2}}}^{+},{\overline{v^{2}}}^{+},{\overline{w^{2}}}^{+}$

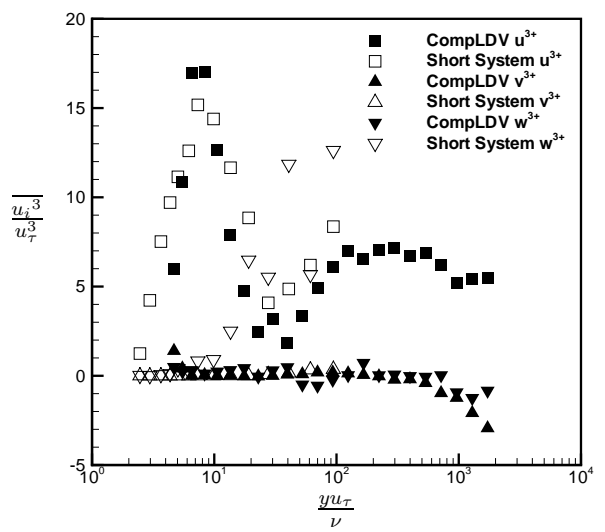

(d) Triple Velocity Correlations, ${\overline{u^{3}}}^{+},{\overline{v^{3}}}^{+},{\overline{w^{3}}}^{+}$

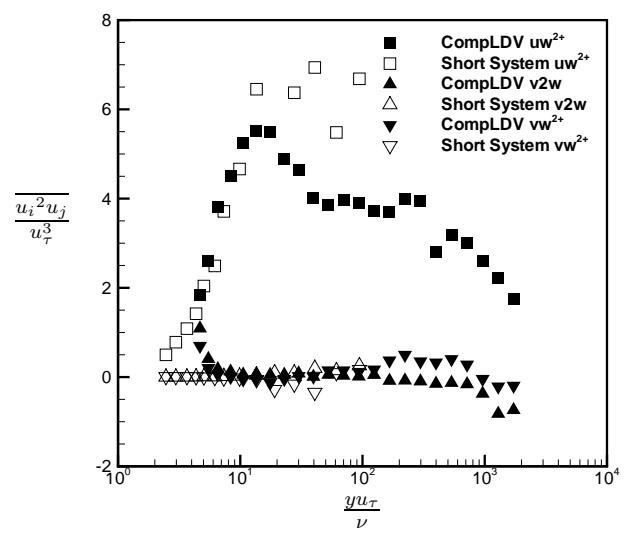

(e) Triple Velocity Correlations, (f) Triple Velocity Correlations, ${\overline{u^{2} v}}^{+},{\overline{u^{2} w}}^{+},{\overline{u v^{2}}}^{+} \quad{\overline{u w^{2}}}^{+},{\overline{v^{2} w}}^{+},{\overline{v w^{2}}}^{+}$

Figure C.5: Matching Short and CompLDV Profiles at P31 


\section{Appendix D}

\section{Inviscid Calculation}

\section{Introduction}

In order to gain more insight and to explain the development of the mean flow field, a generalized coordinates three dimensional potential flow solver was developed. Pressure coefficient, $C_{p}$, as well as mean flow rate of strains $S_{11}, S_{12}$ and $S_{33}$ could be estimated which play a role in strengthening or attenuating the mean vorticity components. The results are not expected to match the measured flow field, however the trends and the mechanism of generating mean vorticity flux at the tunnel floor should be valid. The governing equations and details of the numerical solution are discussed in the next section.

\section{Governing Equations}

Potential flow is governed by Laplace equation, which is very simple to solve in cartesian coordinates. However, since the current geometry is very complicated, boundary fitted grid had to be used. Hence Laplace equation was written in generalized coordinates using as given by Equation D.1 in tensor notation.

$$
A \frac{\partial^{2} \varphi}{\partial \xi^{2}}+B \frac{\partial^{2} \varphi}{\partial \eta^{2}}+C \frac{\partial^{2} \varphi}{\partial \zeta^{2}}+D \frac{\partial^{2} \varphi}{\partial \xi \partial \eta}+E \frac{\partial^{2} \varphi}{\partial \xi \partial \zeta}+F \frac{\partial^{2} \varphi}{\partial \eta \partial \zeta}+G \frac{\partial \varphi}{\partial \xi}+H \frac{\partial \varphi}{\partial \eta}+I \frac{\partial \varphi}{\partial \zeta}=0
$$

Where,

$$
\begin{array}{r}
A=\xi_{, x_{i}}^{2} ; B=\eta_{, x_{i}}^{2} ; C=\zeta_{, x_{i}}^{2} ; D=2 \xi_{, x_{i}} \eta_{, x_{i}} ; E=2 \xi_{, x_{i}} \zeta_{x_{i}} ; F=2 \eta_{, x_{i}} \zeta_{,_{i}} ; \\
G=\xi_{i, x_{j}} \xi_{x_{j} \xi_{i}} ; H=\xi_{i, x_{j}} \eta_{, x_{j} \xi_{i}} ; I=\xi_{i, x_{j}} \zeta_{x_{j}} \xi_{i}
\end{array}
$$


Where, $\varphi$ is the velocity potential, $x, y$ and $z$ are the cartesian spatial coordinates, $\xi, \eta$ and $\zeta$ are the generalized coordinates, $x_{i}$ and $\xi_{i}$ are the cartesian and generalized coordinates in tensor notation respectively. The generalized coordinates are defined as follows,

$$
\xi=\frac{x}{L} ; \eta=\frac{y}{y_{\max }(x)} ; \zeta=\frac{z-z_{\min }(x)}{W(x)}
$$

In order to be able to solve Equation D.1, boundary conditions must be specified. Three kinds of boundary conditions were used in this simulation given as follows,

- Solid Wall

The solid wall or zero-normal velocity component boundary condition is applied to the side walls and to the top and bottom walls of the test section. This condition is specified in Equation D.4.

$$
\begin{aligned}
\hat{n} \cdot \nabla \varphi & =0 \\
\hat{n}_{x_{i}} \cdot \xi_{j, x_{i}} \varphi_{, \xi_{j}} & =0
\end{aligned}
$$

Where, $\hat{n}$ is the normal vector to the boundary.

- Inflow Boundary Condition

At the test section inlet plane, inflow boundary condition is applied. Uniform flow of magnitude $U_{\infty}$ is assumed to be entering the test section. The boundary condition is given by Equation D.5.

$$
\varphi_{, x}=\xi_{i, x} \varphi_{, \xi_{i}}=U_{\infty}
$$

\section{- Outflow Boundary Condition}

At the test section exit, the flow exits and flows without any further changes, hence the stream-wise velocity gradient is set equal to zero. This is given by Equation D.6 In order to simplify the implementation of this boundary condition, an approximation is used and the condition is applied in the computational domain using the generalized variables. This is given by Equation D.7.

$$
\begin{aligned}
& u_{, x}=\varphi_{, x x}=0 \\
& u_{, x} \approx \varphi_{, \xi \xi}=0
\end{aligned}
$$

\section{Numerics}

Since the geometry is relatively simple to grid, an algebraic grid is used with uniform spacing along the $y$ and $z$-coordinate direction. Coordinates in the stream-wise directions were obtained from the measured geometry. Since the measured geometry had 
non-uniform grid spacings and in order not to complicate the discretization procedure, the measured geometry was spline fitted and then sampled uniformly with a suitable number of points to capture all the geometry details and at the same time maintain a relatively low number of points. The number of points used was 129x6x16 points in the $x, y$ and $z$ directions, respectively. All derivatives are discretized using second order central, forward or backward schemes. The discretization results in 12-point stencil. The governing equation is written for all internal points of the domain and solved iteratively using Gauss-Siedel scheme. 